Surveillance for Certain Health Behaviors and Conditions Among States and Selected Local Areas - Behavioral Risk Factor Surveillance System, United States, 2013 and 2014 


\section{CONTENTS}

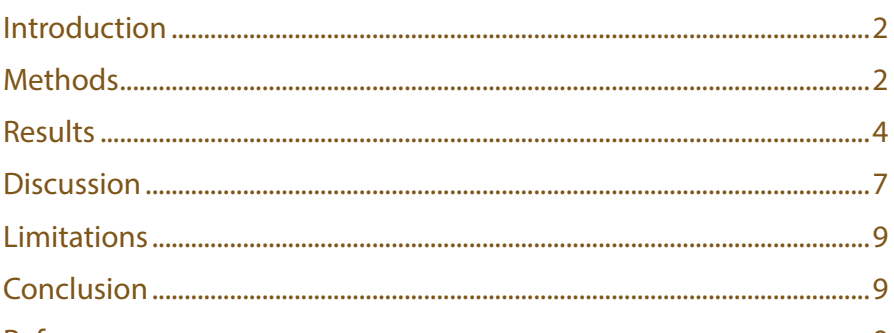

References

The MMWR series of publications is published by the Center for Surveillance, Epidemiology, and Laboratory Services, Centers for Disease Control and Prevention (CDC), U.S. Department of Health and Human Services, Atlanta, GA 30329-4027.

Suggested citation: [Author names; first three, then et al., if more than six.] [Title]. MMWR Surveill Summ 2017;66(No. SS-\#):[inclusive page numbers].

\section{Centers for Disease Control and Prevention}

Brenda Fitzgerald, MD, Director

William R. Mac Kenzie, MD, Acting Associate Director for Science

Joanne Cono, MD, ScM, Director, Office of Science Quality

Chesley L. Richards, MD, MPH, Deputy Director for Public Health Scientific Services

Michael F. Iademarco, MD, MPH, Director, Center for Surveillance, Epidemiology, and Laboratory Services

Sonja A. Rasmussen, MD, MS, Editor-in-Chief

MMWR Editorial and Production Staff (Serials)

Charlotte K. Kent, PhD, MPH, Executive Editor

Christine G. Casey, MD, Editor

Teresa F. Rutledge, Managing Editor

David C. Johnson, Lead Technical Writer-Editor

Marella Meadows, Project Editor
Martha F. Boyd, Lead Visual Information Specialist Maureen A. Leahy, Julia C. Martinroe, Stephen R. Spriggs, Tong Yang, Visual Information Specialists

Quang M. Doan, MBA, Phyllis H. King,

Paul D. Maitland, Terraye M. Starr, Moua Yang, Information Technology Specialists
Timothy F. Jones, MD, Chairman

Matthew L. Boulton, MD, MPH

Virginia A. Caine, MD

Katherine Lyon Daniel, PhD

Jonathan E. Fielding, MD, MPH, MBA

David W. Fleming, MD
MMWR Editorial Board

William E. Halperin, MD, DrPH, MPH

King K. Holmes, MD, PhD

Robin Ikeda, MD, MPH

Rima F. Khabbaz, MD

Phyllis Meadows, $\mathrm{PhD}, \mathrm{MSN}, \mathrm{RN}$

Jewel Mullen, MD, MPH, MPA
Jeff Niederdeppe, $\mathrm{PhD}$

Patricia Quinlisk, MD, MPH

Patrick L. Remington, MD, MPH Carlos Roig, MS, MA

William L. Roper, MD, MPH

William Schaffner, MD 


\title{
Surveillance for Certain Health Behaviors and Conditions Among States and Selected Local Areas - Behavioral Risk Factor Surveillance System, United States, 2013 and 2014
}

\author{
Sonya Gamble, MS ${ }^{1}$ \\ Tebitha Mawokomatanda, MSPH${ }^{1}$ \\ Fang $\mathrm{Xu}, \mathrm{PhD}^{1}$ \\ Pranesh P. Chowdhury, $\mathrm{MD}^{1}$ \\ Carol Pierannunzi, $\mathrm{PhD}^{1}$ \\ David Flegel, $\mathrm{MS}^{2}$ \\ William Garvin ${ }^{1}$ \\ Machell Town, $\mathrm{PhD}^{1}$ \\ ${ }^{1}$ Division of Population Health, National Center for Chronic Disease Prevention and Health Promotion, CDC \\ ${ }^{2}$ Northrop Grumman Corporation, Atlanta, Georgia
}

\begin{abstract}
Problem: Chronic diseases and conditions (e.g., heart diseases, stroke, arthritis, and diabetes) are the leading causes of morbidity and mortality in the United States. These conditions are costly to the U.S. economy, yet they are often preventable or controllable. Behavioral risk factors (e.g., excessive alcohol consumption, tobacco use, poor diet, frequent mental distress, and insufficient sleep) are linked to the leading causes of morbidity and mortality. Adopting positive health behaviors (e.g., staying physically active, quitting tobacco use, obtaining routine physical checkups, and checking blood pressure and cholesterol levels) can reduce morbidity and mortality from chronic diseases and conditions. Monitoring the health risk behaviors, chronic diseases and conditions, access to health care, and use of preventive health services at multilevel public health points (states, territories, and metropolitan and micropolitan statistical areas [MMSA]) can provide important information for development and evaluation of health intervention programs.
\end{abstract}

Reporting Period: 2013 and 2014.

Description of the System: The Behavioral Risk Factor Surveillance System (BRFSS) is an ongoing, state-based, random-digitdialed telephone survey of noninstitutionalized adults aged $\geq 18$ years residing in the United States. BRFSS collects data on health risk behaviors, chronic diseases and conditions, access to health care, and use of preventive health services and practices related to the leading causes of death and disability in the United States and participating territories. This is the first BRFSS report to include age-adjusted prevalence estimates. For 2013 and 2014, these age-adjusted prevalence estimates are presented for all 50 states, the District of Columbia, the Commonwealth of Puerto Rico, Guam, and selected MMSA.

Results: Age-adjusted prevalence estimates of health status indicators, health care access and preventive practices, health risk behaviors, chronic diseases and conditions, and cardiovascular conditions vary by state, territory, and MMSA. Each set of proportions presented refers to the range of age-adjusted prevalence estimates of selected BRFSS measures as reported by survey respondents.

The following are estimates for 2013. Adults reporting frequent mental distress: $7.7 \%-15.2 \%$ in states and territories and $6.3 \%-$ $19.4 \%$ in MMSA. Adults with inadequate sleep: $27.6 \%-49.2 \%$ in states and territories and $26.5 \%-44.4 \%$ in MMSA. Adults aged 18-64 years having health care coverage: $66.9 \%-92.4 \%$ in states and territories and $60.5 \%-97.6 \%$ in MMSA. Adults identifying as current cigarette smokers: $10.1 \%-28.8 \%$ in states and territories and $6.1 \%-33.6 \%$ in MMSA. Adults reporting binge drinking during the past month: $10.5 \%-25.2 \%$ in states and territories and $7.2 \%-25.3 \%$ in MMSA. Adults with obesity: $21.0 \%-35.2 \%$ in states and territories and $12.1 \%-37.1 \%$ in MMSA. Adults aged $\geq 45$ years with some form of arthritis: $30.6 \%-51.0 \%$ in states and territories and $27.6 \%-52.4 \%$ in MMSA. Adults aged $\geq 45$ years who have had coronary heart disease: $7.4 \%-17.5 \%$ in states and territories and $6.2 \%-20.9 \%$ in MMSA. Adults aged $\geq 45$ years who have had a stroke: $3.1 \%-7.5 \%$ in states and territories and 2.3\%-9.4\% in MMSA. Adults with high blood pressure: $25.2 \%-40.1 \%$ in states and territories and 22.2\%-42.2\% in MMSA. Adults with high blood cholesterol: $28.8 \%-38.4 \%$ in states

Corresponding author: Tebitha Mawokomatanda, MSPH, Division of Population Health, National Center for Chronic Disease Prevention and Health Promotion, CDC. Telephone: 770-488-4561; E-mail: chn4@cdc.gov. and territories and $26.3 \%-39.6 \%$ in MMSA.

The following are estimates for 2014 . Adults reporting frequent physical distress: $7.8 \%-16.0 \%$ in states and territories and $6.2 \%-18.5 \%$ in MMSA. Women aged $21-65$ years who had 
a Papanicolaou test during the past 3 years: $67.7 \%-87.8 \%$ in states and territories and $68.0 \%-94.3 \%$ in MMSA. Adults aged 50-75 years who received colorectal cancer screening on the basis of the 2008 U.S. Preventive Services Task Force recommendation: $42.8 \%-76.7 \%$ in states and territories and $49.1 \%-79.6 \%$ in MMSA. Adults with inadequate sleep: $28.4 \%-48.6 \%$ in states and territories and 25.4\%-45.3\% in MMSA. Adults reporting binge drinking during the past month: $10.7 \%-25.1 \%$ in states and territories and 6.7\%-26.3\% in MMSA. Adults aged $\geq 45$ years who have had coronary heart disease: $8.0 \%-17.1 \%$ in states and territories and $7.6 \%-19.2 \%$ in MMSA. Adults aged $\geq 45$ years with some form of arthritis: $31.2 \%-54.7 \%$ in states and territories and 28.4\%-54.7\% in MMSA. Adults with obesity: 21.0\%-35.9\% in states and territories and 19.7\%-42.5\% in MMSA.

Interpretation: Prevalence of certain chronic diseases and conditions, health risk behaviors, and use of preventive health services varies among states, territories, and MMSA. The findings of this report highlight the need for continued monitoring of health status, health care access, health behaviors, and chronic diseases and conditions at state and local levels.

Public Health Action: State and local health departments and agencies can continue to use BRFSS data to identify populations at risk for certain unhealthy behaviors and chronic diseases and conditions. Data also can be used to design, monitor, and evaluate public health programs at state and local levels.

\section{Introduction}

Chronic diseases and conditions (e.g., cardiovascular disease, cancer, chronic lower respiratory diseases, and diabetes) are among the top 10 leading causes of death in the United States (1). Practicing healthy behaviors (e.g., quitting smoking, being more physically active, limiting alcohol intake, eating a nutritious diet, and maintaining a healthy weight) and using preventive health services (e.g., regular checks for high blood pressure and high blood cholesterol, screening for cancer on recommended schedules, and obtaining regular physical checkups) can reduce morbidity and premature mortality from chronic diseases and conditions (2).

BRFSS is an ongoing, state-based, random-digit-dialed cellular and landline telephone survey of noninstitutionalized adults aged $\geq 18$ years in each U.S. state and participating territory. Since 1984, CDC has assisted state and territorial health departments in conducting the BRFSS survey each year. The survey is one of the main data sources that public health officials and practitioners use to track chronic diseases and conditions, health risk behaviors, use of preventive health services, and emerging health problems at state and local levels. The data are frequently used to set health goals as well as to monitor progress and success of public health programs and policy implementation at national, state, and local levels. BRFSS data collection is conducted by state health departments with assistance from CDC. The estimates in this report are calculated from BRFSS data sets, which are aggregates of the combined landline and cellular telephone data submitted during 2013 and 2014. Beginning in 2002, BRFSS data have been used to generate prevalence estimates from metropolitan and micropolitan statistical areas (MMSA) that meet the system's inclusion criteria. This report includes BRFSS findings related to selected chronic diseases and conditions, health risk behaviors, health care access, and use of preventive health services.

\section{Methods}

BRFSS is conducted in all 50 states, the District of Columbia, the Commonwealth of Puerto Rico, and Guam. BRFSS uses a multistage sampling design and random-digitdialing methods to select a representative sample from the noninstitutionalized adult population aged $\geq 18$ years in each state and participating territory $(3,4)$. Details on methodology, random sampling procedures, design (5), and reliability and validity of measures $(\sigma)$ used in BRFSS have been described in previous publications. Estimates are from 53 states and territories for both years, 145 MMSA for 2013, and 132 MMSA for 2014. A list of MMSA for each year is available on the BRFSS SMART website (https://www.cdc.gov/brfss/ smart/smart_data.htm). The 2013 and 2014 questionnaires and all related supporting documents are available at the BRFSS website (https://www.cdc.gov/brfss/).

MMSA are defined by the Office of Management and Budget; respondents are assigned MMSA according to their county Federal Information Processing Standards code. MMSA were included in the data set if they met the selection criterion of $\geq 500$ participants. Data are submitted monthly to CDC by the states or their designees. Data cleaning and weighting are conducted by CDC. Complete documentation of the BRFSS methodology is available at https://www.cdc.gov/brfss.

\section{Questionnaire}

The BRFSS questionnaire is designed to collect uniform, state-specific, self-reported data on a range of health behaviors and conditions $(3,4)$. All questions undergo cognitive and field testing. The standard questionnaire consists of three parts: 1) core questions, 2) optional BRFSS modules, and 3) stateadded questions. The core consists of a set of demographic and standard health-related questions used by all participating states 
and includes some topics that appear biennially. Topics and number of optional modules vary by year and are adopted by states depending upon their programmatic needs. State-added questions are developed, added, and used by the authoring state, specifically for their own residents; CDC does not develop, track, or record state-added questions. All BRFSS questionnaires are available at https://www.cdc.gov/brfss/ questionnaires/index.htm.

\section{Data Collection and Processing}

Data collection for BRFSS is conducted using a computerassisted telephone interviewing system. Data are collected monthly by each state and territory according to BRFSS standard protocol. After the monthly interviewing cycle concludes, data are submitted to CDC to be edited, processed, weighted, checked for reliability, and prepared for analysis. At the end of the survey year, CDC processes and aggregates the monthly data files to create a year-end data file for each state and territory.

\section{Sampling}

In 2013, BRFSS used a partially overlapping sample that, in addition to other eligibility requirements, screened out cellular telephone respondents who received more than 10\% of all incoming calls on a landline telephone. In 2014, BRFSS adopted the use of a fully overlapping sample of landline and cellular telephone respondents aged $\geq 18$ years. No minor children are included in the BRFSS sample. States designed samples using substate regions (e.g., public health districts or other jurisdictions) to ensure geographic representation within the sample. CDC assisted states with sample design and set minimum sample sizes for substate regions, split-sample versions of the questionnaire, and oversampling of populations that are hard to reach.

\section{Data Weighting}

BRFSS data were used to create direct estimates for each geographic area (i.e., state or MMSA). Data were weighted using a raking method. Raking (iterative proportional fitting) was applied using each demographic factor individually in an iterative process until demographic estimates matched control totals based on U.S. Census estimates for that year. Raking has improved the precision with which the BRFSS sample reflects the sociodemographic profile at the state level. Details of the BRFSS raking method are provided in the BRFSS weighting documents for 2013 and $2014(7,8)$. The 2014 sampling overlap also prompted an adjustment to the BRFSS design weights. To account for overlap of the two samples, a composite factor was multiplied by the design weight for each dual user to create an adjustment that addressed and corrected for the respondent's probability of being selected in both frames. More information about the composite factor calculation is available at https://www.cdc.gov/brfss/annual_data/2014/pdf/ compare_2014.pdf.

\section{Statistical Analysis}

The analysis was conducted using statistical software, SASCallable SUDAAN release 11.0 (Research Triangle Institute, Cary, North Carolina), to account for the complex sampling design and calculate age-adjusted prevalence estimates, standard errors, and 95\% confidence intervals. Sample sizes are unweighted in this report. Data with sample sizes $<50$ or having a relative standard error $>30 \%$ were deemed unstable and less reliable and were suppressed in the tables, as noted by N/A (not available). Responses coded as do not know or refused were excluded from the analysis. Several chronic diseases and conditions (i.e., diabetes, arthritis, coronary heart disease, and stroke) were limited to participants aged $\geq 45$ years (9).

This is the first BRFSS report to include age-adjusted prevalence estimates. Age adjustment is a standard analytical technique used to compare estimates between populations with different age distributions (e.g., states) and over time. In this report, the estimates were age adjusted so that data could be compared across states, MMSA, and time, each having different age distributions. BRFSS age-adjusted estimates were standardized to the 2000 U.S. population using distribution No. 8, consistent with the current National Center for Health Statistics recommendations and practice (10).

Crude prevalence estimates for each individual state and MMSA are provided at https://www.cdc.gov/brfss/ brfssprevalence/index.html. Results should not be compared with those in previous BRFSS reports, which provided nonageadjusted estimates by state, territory, and MMSA. The ageadjusted prevalence estimates were calculated from results of BRFSS and might differ from those derived by other methods.

\section{About This Report}

This report presents age-adjusted prevalence estimates and discussion of five topics. The topics are 1) health status indicators (self-rated general health status, frequent mental distress, and frequent physical distress); 2) health care access and preventive practices (health care coverage, recent routine physical checkup, Papanicolaou [Pap] test, colorectal cancer screening, and blood cholesterol check); 3) health risk behaviors (no leisure-time physical activity, inadequate sleep, current cigarette smoking, and binge drinking); 4) chronic diseases and conditions (obesity, diabetes, arthritis, and depression); 
and 5) cardiovascular conditions (coronary heart disease and stroke for adults aged $\geq 45$ years and high blood pressure and high blood cholesterol for adults aged $\geq 18$ years).

\section{Results}

In 2013, a total of 491,773 adults completed BRFSS interviews on landline and cellular telephones. Results were from 53 states and territories and 145 MMSA with sufficient sample sizes. A total of 360,079 respondents completed the interview by landline telephone (range: 1,461 in Guam to 27,763 in Florida; median: 5,668). A total of 131,694 respondents completed the interview by cellular telephone (range: 445 in Guam to 7,620 in Kansas; median: 2,291). In 2014, a total of 464,662 adults completed the interview on landline and cellular telephones. Results were from 53 states and territories and 132 MMSA with sufficient sample size. A total of 298,568 respondents completed the interview by landline telephone (range: 1,852 in Guam to 12,962 in Nebraska; median: 4,973). A total of 166,094 respondents completed the interview by cellular telephone (range: 686 in Guam to 9,952 in Nebraska; median: 2,868).

BRFSS uses the American Association of Public Opinion Research Response Rate 4 (defined as the number of complete and partial interviews divided by the number of contacted and eligible respondents) (11) as a method for calculating response. In 2013, landline response rates ranged from $28.0 \%$ in Alabama to $63.7 \%$ in Puerto Rico (median: $49.6 \%$ ) and cellular response rates ranged from $19.1 \%$ in Washington to 62.6\% in Alaska (median: 37.8\%). The combined (landline and cellular) response rates ranged from $29.0 \%$ in Alabama to $60.3 \%$ in Puerto Rico (median: $46.4 \%$ ). In 2014, landline response rates ranged from $26.7 \%$ in California to $61.6 \%$ in Kentucky (median: $48.7 \%$ ) and cellular response rates ranged from $22.2 \%$ in California to $60.0 \%$ in Alaska (median: $40.5 \%$ ). Overall, the combined response rates ranged from $25.1 \%$ in California to $60.1 \%$ in South Dakota (median: $47.0 \%$ ). BRFSS Summary Data Quality Reports for 2013 (12) and 2014 (13) have detailed information on response, cooperation, and refusal rates.

Increasing use of cellular telephones (14) prompted BRFSS to move to an overlapping sample in 2014. This was a change from the 2013 screening process, which restricted the eligibility of cellular telephone respondents who also used landline telephones. Effects of the 2014 sample change include larger proportions of completed interviews among persons aged 18-44 years, men, and Hispanics. Moving to an overlapping sample increased the proportion of cellular telephone respondents eligible to participate in the survey (15).

\section{Health Status Indicators}

\section{Health Status}

All respondents were asked if their general health was excellent, very good, good, fair, or poor. Respondents were then divided into two groups: those reporting their health was excellent, very good, or good and those reporting their health was fair or poor. In 2013, age-adjusted prevalence estimates for good or better health ranged from $66.4 \%$ in Puerto Rico to $88.7 \%$ in Vermont (median: 84.0\%) (Table 1). Among selected MMSA, estimated age-adjusted prevalence ranged from $66.7 \%$ in San Juan-Carolina-Caguas, Puerto Rico, to $91.2 \%$ in Sioux Falls, South Dakota (median: 83.8\%) (Table 2). In 2014, age-adjusted prevalence estimates of persons with good or better health ranged from $66.9 \%$ in Puerto Rico to $89.1 \%$ in Vermont (median: 84.1\%) (Table 3). Among selected MMSA, age-adjusted prevalence estimates ranged from $63.3 \%$ in Ponce, Puerto Rico, to $92.2 \%$ in Logan, Utah-Idaho (median: $83.7 \%$ ) (Table 4).

\section{Frequent Mental Distress}

All respondents were asked to determine how many days during the past 30 days their mental health status (e.g., stress, depression, and problems with emotions) was not good. The respondents were divided into two groups: those who reported frequent mental distress ( $\geq 14$ mentally unhealthy days during the past 30 days) and those who reported no frequent mental distress ( $<14$ mentally unhealthy days during the past 30 days). In 2013, age-adjusted prevalence estimates of frequent mental distress ranged from $7.7 \%$ in North Dakota to $15.2 \%$ in West Virginia (median: 11.3\%) (Table 5). Among selected MMSA, estimated age-adjusted prevalence ranged from $6.3 \%$ in Grand Forks, North Dakota-Minnesota, and Minot, North Dakota, to $19.4 \%$ in Akron, Ohio (median: 10.9\%) (Table 6).

\section{Frequent Physical Distress}

Frequent physical distress included respondents who reported $\geq 14$ days of poor physical health (e.g., physical illness or injury) during the past 30 days. In 2014, age-adjusted prevalence estimates of frequent physical distress ranged from $7.8 \%$ in North Dakota to $16.0 \%$ in Kentucky (median: $10.9 \%$ ) (Table 7). Among selected MMSA, age-adjusted prevalence estimates ranged from 6.2\% in Logan, Utah-Idaho, to $18.5 \%$ in Aguadilla-Isabela, Puerto Rico (median: 11.1\%) (Table 8). 


\section{Health Care Access and Preventive Practices}

\section{Health Care Coverage}

Health care coverage was defined as respondents aged 18-64 years having any form of coverage, including private health insurance, prepaid plans (e.g., health maintenance organizations), or a government plan (e.g., Medicare or Medicaid) at the time of the interview. In 2013, age-adjusted prevalence estimates of health care coverage ranged from 66.9\% in Texas to $92.4 \%$ in Massachusetts (median: $79.6 \%$ ) (Table 9). Among selected MMSA, estimated age-adjusted prevalence ranged from $60.5 \%$ in El Paso, Texas, to $97.6 \%$ in Aguadilla-Isabela, Puerto Rico (median: 80.6\%) (Table 10). In 2014, age-adjusted prevalence estimates ranged from $70.5 \%$ in Texas to $94.5 \%$ in Massachusetts (median: 84.2\%) (Table 11). Among selected MMSA, age-adjusted prevalence estimates ranged from $64.7 \%$ in El Paso, Texas, to $96.6 \%$ in Ponce, Puerto Rico (median: 84.3\%) (Table 12).

\section{Recent Routine Physical Checkup}

A recent routine physical checkup was defined as a visit that occurred during the past 12 months to a doctor for a general physical examination rather than for a specific injury, illness, or condition. In 2013, age-adjusted prevalence estimates of a routine physical checkup during the past 12 months ranged from $57.0 \%$ in Oregon to $77.7 \%$ in Rhode Island (median: 67.9\%) (Table 13). Among selected MMSA, age-adjusted prevalence estimates ranged from $52.6 \%$ in Logan, UtahIdaho, to $79.5 \%$ in Memphis, Tennessee-Mississippi-Arkansas (median: 68.9\%) (Table 14). In 2014, age-adjusted prevalence estimates ranged from $57.2 \%$ in Idaho to $79.2 \%$ in Rhode Island (median: 69.2\%) (Table 15). Among selected MMSA, age-adjusted prevalence estimates ranged from $55.9 \%$ in Scottsbluff, Nebraska, to $80.8 \%$ in Providence-Warwick, Rhode Island-Massachusetts (median: 69.8\%) (Table 16).

\section{Pap Test}

A Pap test detects cancer of the cervix. The U.S. Preventive Services Task Force (USPSTF) recommends that women aged $\geq 21$ years should receive a Pap test to screen for cervical cancer at least every 3 years until aged 65 years or a Pap test in combination with a human papillomavirus test every 5 years for women aged 30-65 years (16). Women aged 21-65 years who self-reported ever having a Pap test were included in this report. In 2014, age-adjusted prevalence estimates of a Pap test among women aged 21-65 years ranged from $67.7 \%$ in Guam to $87.8 \%$ in Massachusetts (median: 82.4\%) (Table 17).
Among selected MMSA, age-adjusted prevalence estimates ranged from $68.0 \%$ in Wichita Falls, Texas, to $94.3 \%$ in Knoxville, Tennessee (median: 83.1\%) (Table 18).

\section{Colorectal Cancer Screening}

USPSTF recommends colorectal cancer screening for adults aged $50-75$ years using a blood stool test (also known as fecal occult blood test [FOBT]) every year, a colonoscopy every 10 years, or a flexible sigmoidoscopy every 5 years with an FOBT every 3 years (17). Adults aged $50-75$ years who self-reported ever having a colorectal cancer screening were included in this report. In 2014, age-adjusted prevalence estimates of colorectal cancer screening among adults aged $50-75$ years ranged from $42.8 \%$ in Guam to $76.7 \%$ in Massachusetts (median: 66.3\%) (Table 19). Among selected MMSA, age-adjusted prevalence estimates ranged from $49.1 \%$ in Ponce, Puerto Rico, to $79.6 \%$ in Madison, Wisconsin (median: 68.1\%) (Table 20).

\section{Blood Cholesterol Check}

Respondents were categorized as having had a blood cholesterol check if they had their blood cholesterol checked during the past 5 years. In 2013, age-adjusted prevalence estimates of a blood cholesterol check during the past 5 years ranged from $65.9 \%$ in Guam to $81.9 \%$ in Massachusetts (median: 74.3\%) (Table 21). Among selected MMSA, ageadjusted prevalence estimates ranged from $62.5 \%$ in Logan, Utah-Idaho, to $83.5 \%$ in Nassau County-Suffolk County, New York (median: 75.6\%) (Table 22).

\section{Health Risk Behaviors}

\section{No Leisure-time Physical Activity}

Respondents were categorized as having no leisure-time physical activity if they did not participate in any physical activity or exercise (e.g., running, calisthenics, golf, gardening, or walking for exercise) other than their regular job during the preceding month. In 2013, age-adjusted prevalence estimates of no leisure-time physical activity ranged from $17.9 \%$ in Colorado to $47.4 \%$ in Puerto Rico (median: $25.1 \%$ ) (Table 23). Among selected MMSA, age-adjusted prevalence estimates ranged from $14.8 \%$ in San Francisco-Redwood City-South San Francisco, California, to $48.3 \%$ in San Juan-Carolina-Caguas, Puerto Rico (median: 25.1\%) (Table 24). In 2014, age-adjusted prevalence estimates ranged from $15.9 \%$ in Oregon to $39.7 \%$ in Puerto Rico (median: $22.4 \%$ ) (Table 25). Among selected MMSA, age-adjusted prevalence estimates ranged from $11.7 \%$ in Logan, Utah-Idaho, to $42.1 \%$ in Aguadilla-Isabela, Puerto Rico (median: 22.6\%) (Table 26). 


\section{Inadequate Sleep}

Respondents were asked to determine the average number of hours of sleep they usually get during a 24-hour period. Those having $<7$ hours of sleep were classified as having inadequate sleep and those having $>7$ hours of sleep as having adequate sleep. In 2013, age-adjusted prevalence estimates of inadequate sleep ranged from $27.6 \%$ in South Dakota to $49.2 \%$ in Guam (median: 35.3\%) (Table 27). Among selected MMSA, ageadjusted prevalence estimates ranged from $26.5 \%$ in Sioux Falls, South Dakota, to $44.4 \%$ in Augusta-Richmond County, Georgia-South Carolina (median: 34.9\%) (Table 28). In 2014, age-adjusted prevalence estimates ranged from $28.4 \%$ in South Dakota to $48.6 \%$ in Guam (median: $34.7 \%$ ) (Table 29). Among selected MMSA, age-adjusted prevalence estimates ranged from $25.4 \%$ in Fargo, North Dakota-Minnesota, to $45.3 \%$ in Kingsport-Bristol-Bristol, Tennessee-Virginia (median: 34.8\%) (Table 30).

\section{Current Cigarette Smoking}

Current cigarette smokers were defined as respondents who reported having smoked at least 100 cigarettes in their lifetime and who smoked every day or some days at the time of the interview. In 2013, age-adjusted prevalence estimates of current cigarette smoking ranged from $10.1 \%$ in Utah to $28.8 \%$ in West Virginia (median: 19.3\%) (Table 31). Among selected MMSA, age-adjusted prevalence estimates ranged from $6.1 \%$ in Provo-Orem, Utah, to 33.6\% in Kingsport-Bristol-Bristol, Tennessee-Virginia (median: 19.5\%) (Table 32). In 2014, ageadjusted prevalence estimates ranged from $9.5 \%$ in Utah to $28.1 \%$ in West Virginia (median: $18.7 \%$ ) (Table 33). Among selected MMSA, age-adjusted prevalence estimates ranged from $5.0 \%$ in Logan, Utah-Idaho, to $29.3 \%$ in Charleston, West Virginia (median: 18.6\%) (Table 34).

\section{Binge Drinking}

Respondents were considered to be binge drinkers if during the past 30 days a man had five or more drinks on one occasion and a woman had four or more drinks on one occasion. In 2013, age-adjusted prevalence estimates of binge drinking among both men and women ranged from $10.5 \%$ in Tennessee to $25.2 \%$ in North Dakota (median: 17.7\%) (Table 35). Among selected MMSA, age-adjusted prevalence estimates ranged from $7.2 \%$ in Provo-Orem, Utah, to $25.3 \%$ in Buffalo-Cheektowaga-Niagara Falls, New York (median: 17.8\%) (Table 36). In 2014, age-adjusted prevalence estimates ranged from $10.7 \%$ in West Virginia to $25.1 \%$ in North Dakota (median: 17.0\%) (Table 37). Among selected MMSA, age-adjusted prevalence estimates ranged from $6.7 \%$ in Provo-Orem, Utah, to $26.3 \%$ in Fargo, North DakotaMinnesota (median: 17.0\%) (Table 38).

\section{Chronic Diseases and Conditions}

\section{Obesity}

Obesity, calculated from self-reported height and weight, was defined as having a body mass index of $\geq 30$ (weight $[\mathrm{kg}] /$ height $\left[\mathrm{m}^{2}\right]$ ). In 2013, age-adjusted prevalence estimates of obesity ranged from $21.0 \%$ in Colorado to $35.2 \%$ in West Virginia (median: 28.2\%) (Table 39). Among selected MMSA, age-adjusted prevalence estimates ranged from 12.1\% in San Francisco-Redwood City-South San Francisco, California, to $37.1 \%$ in Huntington-Ashland, West VirginiaKentucky-Ohio (median: 28.3\%) (Table 40). In 2014, ageadjusted prevalence estimates ranged from $21.0 \%$ in Colorado to $35.9 \%$ in Arkansas (median: 29.0\%) (Table 41). Among selected MMSA, age-adjusted prevalence estimates ranged from $19.7 \%$ in Reno, Nevada, to $42.5 \%$ in Corpus Christi, Texas (median: 29.3\%) (Table 42).

\section{Diabetes}

Diabetes was defined as respondents aged $\geq 45$ years who reported having ever been told by a doctor, nurse, or other health professional they have diabetes, excluding prediabetes or borderline diabetes and diabetes during pregnancy for women. In 2013, age-adjusted prevalence estimates of diabetes among adults aged $\geq 45$ years ranged from $11.1 \%$ in Colorado to 27.5\% in Guam (median: 16.2\%) (Table 43). Among selected MMSA, age-adjusted prevalence estimates ranged from 10.6\% in Buffalo-Cheektowaga-Niagara Falls, New York, to 24.8\% in Aguadilla-Isabela, Puerto Rico (median: 15.8\%) (Table 44).

\section{Arthritis}

Arthritis was defined as respondents aged $\geq 45$ years who reported having ever been told by a doctor, nurse, or other health professional they have some form of arthritis, including rheumatoid arthritis, gout, lupus, or fibromyalgia. In 2013, age-adjusted prevalence estimates of arthritis among adults aged $\geq 45$ years ranged from $30.6 \%$ in Hawaii to $51.0 \%$ in West Virginia (median: 39.4\%) (Table 45). Among selected MMSA, age-adjusted prevalence estimates ranged from $27.6 \%$ in Dallas-Plano-Irving, Texas, to $52.4 \%$ in Huntington-Ashland, West Virginia-Kentucky-Ohio (median: 39.4\%) (Table 46). In 2014, age-adjusted prevalence estimates ranged from $31.2 \%$ in Hawaii to $54.7 \%$ in West Virginia (median: 39.8\%) (Table 47). Among selected MMSA, age-adjusted prevalence ranged from $28.4 \%$ in College Station-Bryan, Texas, to $54.7 \%$ in Montgomery, Alabama (median: 40.1\%) (Table 48). 


\section{Depression}

Respondents were asked if they had ever been told by a doctor, nurse, or other health professional they have a depressive disorder, including depression, major depression, dysthymia, or minor depression. In 2013, age-adjusted prevalence estimates of depression ranged from $8.6 \%$ in Guam to $26.8 \%$ in Oregon (median: $18.6 \%$ ) (Table 49). Among selected MMSA, age-adjusted prevalence estimates ranged from $7.7 \%$ in San Jose-Sunnyvale-Santa Clara, California, to $28.5 \%$ in Fort Smith, Arkansas-Oklahoma (median: 18.7\%) (Table 50). In 2014, age-adjusted prevalence estimates ranged from $8.9 \%$ in Guam to $24.2 \%$ in Maine (median: $18.8 \%$ ) (Table 51). Among selected MMSA, age-adjusted prevalence estimates ranged from $10.5 \%$ in Riverside-San BernardinoOntario, California, to $29.5 \%$ in Springfield, Massachusetts (median: 18.8\%) (Table 52).

\section{Cardiovascular Conditions}

\section{Coronary Heart Disease}

Respondents aged $\geq 45$ years were categorized as having coronary heart disease if they reported having ever been told by a doctor, nurse, or other health professional they have had a heart attack (myocardial infarction) or angina. In 2013, age-adjusted prevalence estimates of coronary heart disease among adults aged $\geq 45$ years ranged from $7.4 \%$ in Hawaii to $17.5 \%$ in West Virginia (median: $11.0 \%$ ) (Table 53). Among selected MMSA, age-adjusted prevalence estimates ranged from 6.2\% in San Francisco-Redwood City-South San Francisco, California, to $20.9 \%$ in Ponce, Puerto Rico (median: $11.1 \%$ ) (Table 54). In 2014, age-adjusted prevalence estimates ranged from $8.0 \%$ in Hawaii to $17.1 \%$ in Puerto Rico (median: $11.0 \%$ ) (Table 55). Among selected MMSA, the age-adjusted prevalence estimates ranged from $7.6 \%$ in Provo-Orem, Utah, to $19.2 \%$ in Ponce, Puerto Rico (median: 11.3\%) (Table 56).

\section{Stroke}

Stroke was defined as respondents aged $\geq 45$ years having ever been told by a doctor, nurse, or other health professional they have had a stroke. In 2013, age-adjusted prevalence estimates for stroke among adults aged $\geq 45$ years ranged from $3.1 \%$ in Puerto Rico to $7.5 \%$ in Mississippi (median: 4.7\%) (Table 57). Among selected MMSA, age-adjusted prevalence estimates ranged from 2.3\% in Grand Island, Nebraska, to $9.4 \%$ in Spartanburg, South Carolina (median: 4.9\%) (Table 58). In 2014, age-adjusted prevalence estimates ranged from 3.3\% in Colorado to $8.0 \%$ in Guam (median: $4.8 \%$ ) (Table 59). Among selected MMSA, age-adjusted prevalence estimates ranged from $2.3 \%$ in Sacramento-Roseville-Arden-Arcade,
California, to $9.0 \%$ in Berlin, New Hampshire-Vermont (median: 5.0\%) (Table 60).

\section{High Blood Pressure}

High blood pressure was defined as respondents who reported having ever been told by a doctor, nurse, or other health professional they have high blood pressure. In 2013, age-adjusted prevalence estimates of high blood pressure ranged from $25.2 \%$ in Minnesota to $40.1 \%$ in Puerto Rico (median: 29.5\%) (Table 61). Among selected MMSA, ageadjusted prevalence estimates ranged from $22.2 \%$ in Duluth, Minnesota-Wisconsin, to $42.2 \%$ in Fort Smith, ArkansasOklahoma (median: 30.4\%) (Table 62).

\section{High Blood Cholesterol}

Respondents were categorized as having high blood cholesterol if, after having their cholesterol checked, they had ever been told by a doctor, nurse, or other health professional it was high. In 2013, age-adjusted prevalence estimates of high blood cholesterol ranged from $28.8 \%$ in Vermont to $38.4 \%$ in Alabama (median: 33.9\%) (Table 63). Among selected MMSA, age-adjusted prevalence estimates ranged from 26.3\% in Burlington-South Burlington, Vermont, to $39.6 \%$ in Baton Rouge, Louisiana (median: 33.5\%) (Table 64).

\section{Discussion}

Considerable variation exists at the levels of state, territory, and MMSA in age-adjusted prevalence estimates of health status, health care access, health risk behaviors, use of preventive practices, and chronic diseases and conditions among U.S. adults. The variations might reflect differences in demographic factors of respondents, including race and sex distribution of the population; socioeconomic conditions, including education level, income level, and employment status; state laws and local ordinances relating to health policy; availability of and access to health care services; use of preventive health services; and patterns of reimbursement for preventive services.

\section{Health Status Indicators}

Use of a single question to measure self-rated health status is complex because it includes a person's physical health, mental health, and functional capacity (18). Health status is a measure of the perceived effects of acute and chronic health conditions (19). In this report, variations of prevalence estimates of good or better health across states, territories, and MMSA suggest differences in patterns of chronic disease, health care access, and health behaviors. 
Frequent mental distress assesses both the effects of chronic disease and self-reported mental distress (20). Persons with frequent mental distress are at a higher risk for certain health risk behaviors (e.g., physical inactivity, inadequate sleep, smoking, and drinking) and chronic diseases and conditions (e.g., diabetes, high blood pressure, heart disease, stroke, asthma, and arthritis) $(21,22)$. Similarly, frequent physical distress is a measure of physical symptoms related to chronic diseases and conditions (e.g., cancer, diabetes, obesity, and arthritis) and health risk factors (e.g., body mass index, physical inactivity, and smoking status) (19). The questions related to frequent physical distress have demonstrated validity and reliability for population health surveillance (23). Both frequent mental distress and frequent physical distress are measured and tracked by the health-related quality of life question (19). The wide variation in frequent mental distress and frequent physical distress indicates the continued need for surveillance of symptoms related to mental and physical unhealthy days at state and local levels (23).

\section{Health Care Access and Preventive Practices}

Health care coverage is associated with access to preventive health care, and lack of health insurance can often lead to adverse health outcomes (24). In 2010, one in four adults did not have health care coverage, and those who had a chronic illness and did not have health insurance were more likely to skip or delay medical care because of cost (25).

In the United States, cancer is a major public health problem and is the second-leading cause of death (26). In 2014, having a Pap test among women aged 21-65 years varied among states. This difference might be associated with lack of access to health care and lack of health insurance (27). Evidencebased public health approaches can improve cervical cancer screening among women in this age group. Colorectal cancer is the second leading cause of death from cancers that affect both men and women (28). Screening is key to finding precancerous polyps, and early detection makes colorectal cancer easier to treat. USPSTF recommends colorectal cancer screening for adults aged 50-75 years. The 2014 estimates suggest the need for continued population-level efforts to identify groups that are not receiving colorectal cancer screening.

\section{Health Risk Behaviors}

Staying physically active is an important part of improving health; it helps maintain a healthy weight, improves cardiorespiratory efficiency, strengthens muscles and bones, lowers stress, and can improve one's mental health and mood
(29). Physical inactivity is a risk factor for chronic diseases and conditions (e.g., diabetes, heart disease, and arthritis) (30). BRFSS measured physical inactivity or lack of exercise as no leisure-time activity during the past 30 days. The varying prevalence of no leisure-time physical activity among states and MMSA indicates the need to implement strategies outlined in the CDC Guide to Strategies to Increase Physical Activity in the Community (31).

Good sleep is critical for good health and overall quality of life (32). The American Academy of Sleep Medicine and Sleep Research Society recommends that adults aged $\geq 18$ years get at least 7 hours of sleep each night (33). Inadequate sleep ( $<7$ hours) is associated with high blood pressure, asthma, arthritis, obesity, diabetes, coronary heart disease, stroke, depression, and other chronic diseases and conditions (34). The range of prevalence estimates for 2013 and 2014 shows greater efforts are needed to develop and implement interventions that address multiple health risk factors and conditions associated with insufficient sleep.

Tobacco use continues to be the single most preventable cause of morbidity and mortality worldwide; it is responsible for approximately 6 million deaths per year (35). Smoking causes various types of cancer, cardiovascular and pulmonary diseases, and reproductive and developmental disorders (36). Nicotine, found in tobacco products, is acutely toxic, and smoking has been linked to diseases of nearly every organ of the body (37). Implementing comprehensive tobacco control programs, which can include a combination of smoke-free laws, cigarette price increases, access to proven smoking cessation treatments and services, and direct media campaigns, can help reduce current smoking prevalence (38).

Binge drinking is the most common pattern of excessive alcohol use in the United States (39). It is a major risk factor for morbidity and mortality and other societal costs $(40,41)$. Health-related risks extend beyond those stemming from alcohol abuse; binge drinking can lead to risky sexual activity, unintentional injuries, violence, fetal alcohol disorders, and suicide (42). In this report, estimated prevalence of binge drinking varies across the United States and might be associated with socioeconomic and demographic factors and alcoholrelated policies in states and MMSA (43).

\section{Chronic Diseases and Conditions}

Obesity is a national epidemic and a contributing factor in many health problems, including heart disease, stroke, diabetes, and certain types of cancer (44). It lowers quality of life and results in higher medical costs (45). A 2013 study on obesity reported that prevalence had increased during 1999-2002 and 2007-2010 among both men and women and substantial 
disparity persisted among certain population groups (45). Access to healthy food and regular physical activity, knowledge about healthy servings and portions, community-based social capital, and guidance from health care providers can help persons maintain a healthy weight (46).

Arthritis affects $20 \%$ (53 million) of the adult population in the United States (47), and it is the major contributor to falls among elderly persons (48). Physical activity and selfmanagement education interventions can reduce pain and improve function and quality of life for adults with arthritis and for adults with other chronic conditions who might be limited by their arthritis (49). In 2013 and 2014, approximately 40\% of adults aged $\geq 45$ years had some form of arthritis in each year.

Depression is one of the top five causes of disability; it can cause fatigue, decrease one's ability to work or attend school, and increase risk for suicide (50). The Global Burden of Disease Study 2010 identified depression as a leading cause of morbidity and mortality worldwide (51). Varying prevalence estimates among states and MMSA underscore the need for prevention and intervention efforts at state and local levels.

\section{Cardiovascular Conditions}

Heart disease is the leading cause of death in the United States, accounting for $23.4 \%$ of all deaths in 2014; stroke is the fifth-leading cause of death, accounting for $5.1 \%$ of all deaths in 2014 (52). Both heart disease and stroke are major causes of disability among adults $(52,53)$.

High blood pressure and high cholesterol are primary contributors to heart disease and stroke (54). In addition, adults aged $\geq 45$ years with frequent mental distress have been found to have a higher likelihood of heart disease (55). High blood pressure and high cholesterol often can be controlled or prevented with medication, regular exercise, and a healthy diet, as well as by quitting smoking, reducing alcohol use, and monitoring blood pressure and cholesterol. Because high blood pressure and high cholesterol are contributors to stroke and heart disease, strategies for prevention and control can help prevent cardiovascular complications.

\section{Limitations}

The findings of this report are subject to at least five limitations. First, because it is a household telephone survey BRFSS excludes information from persons in institutions, military installations, nursing homes, long-term care facilities, and correctional institutions. Second, the questionnaire is administered only to persons who speak English, Spanish, Mandarin, or Portuguese. Persons who do not speak these languages would not be able to participate in the survey.
Third, the BRFSS survey collects self-reported data that are subject to recall bias and social desirability effects. Fourth, because of small sample size or unreliable estimates, certain estimates could not be obtained for some MMSA. Finally, persons without a landline or cellular telephone are not able to participate.

Overall, BRFSS is a cost-effective, timely, and flexible surveillance system that provides state health departments and local communities with reliable estimates to monitor and track health status, health risk behaviors, chronic diseases and conditions, and access to preventive health care. Crude estimates obtained using BRFSS are comparable and consistent with other U.S. survey estimates (56). BRFSS questions have been shown to be valid and reliable ( $)$.

\section{Conclusion}

Although chronic diseases and conditions are a challenge to the overall health of the U.S. population, prevalence of morbidity and mortality can be estimated and reduced by monitoring trends, promoting healthy behaviors, identifying emerging diseases, and building effective and sustainable public health community interventions. Results from this report reflect variations in health status, health care access, health behaviors, and chronic diseases and conditions at state and MMSA levels. Identifying areas with populations at risk can help public health officials address health needs and use limited resources more effectively. BRFSS results can be used to identify emerging health problems, support health-related legislative efforts, and develop and evaluate public health policies and programs at state and local levels. CDC will continue to work with states and territories to collect data, identify populations that are underserved and at risk, monitor chronic diseases and conditions and access to health care, and encourage the U.S. population to adopt healthy behaviors.

\section{Acknowledgement}

The authors acknowledge the BRFSS coordinators in states and territories and the Population Health Surveillance Branch, Division of Population Health, CDC.

\section{References}

1. Xu J, Murphy SL, Kochanek KD, Bastian BA. Deaths: final data for 2013. Natl Vital Stat Rep 2016;64:1-119.

2. CDC. Chronic diseases overview. Atlanta, GA: US Department of Health and Human Services, CDC; 2012. https://www.cdc.gov/chronicdisease/overview

3. CDC. Behavioral Risk Factor Surveillance System—overview: BRFSS 2013. Atlanta, GA: US Department of Health and Human Services, CDC; 2016. https://www.cdc.gov/brfss/annual_data/2013/pdf/ overview_2013.pdf 
4. CDC. Behavioral Risk Factor Surveillance System-overview: BRFSS 2014. Atlanta, GA: US Department of Health and Human Services, CDC; 2016. https://www.cdc.gov/brfss/annual_data/2014/pdf/ overview_2014.pdf

5. Chowdhury PP, Mawokomatanda T, Xu F, et al. Surveillance for certain health behaviors, chronic diseases, and conditions, access to health care, and use of preventive health services among states and selected local areas-Behavioral Risk Factor Surveillance System, United States, 2012. MMWR Surveill Summ 2016;65(No. SS-4). https://doi.org/10.15585/ mmwr.ss6504a1

6. Pierannunzi C, Hu SS, Balluz L. A systematic review of publications assessing reliability and validity of the Behavioral Risk Factor Surveillance System (BRFSS), 2004-2011. BMC Med Res Methodol 2013;13:49. https://doi.org/10.1186/1471-2288-13-49

7. CDC. Behavioral Risk Factor Surveillance System-weighting BRFSS data, BRFSS 2013. Atlanta, GA: US Department of Health and Human Services, CDC; 2016. https://www.cdc.gov/brfss/annual_data/2013/ pdf/Weighting_Data.pdf

8. CDC. Behavioral Risk Factor Surveillance System-weighting BRFSS data, BRFSS 2014. Atlanta, GA: US Department of Health and Human Services, CDC; 2016. https://www.cdc.gov/brfss/annual_data/2014/ pdf/weighting-data.pdf

9. CDC, National Center for Health Statistics. Tables of summary health statistics for US adults: 2015 National Health Interview Survey. Atlanta, GA: US Department of Health and Human Services, CDC, National Center for Health Statistics; 2016. https://www.cdc.gov/ nchs/nhis/SHS/tables.htm

10. US Department of Health and Human Services. Healthy people 2010: age adjustment using the 2000 projected U.S. population. Washington, DC: US Department of Health and Human Services; 2001. https:// www.cdc.gov/nchs/data/statnt/statnt20.pdf

11. The American Association for Public Opinion Research. Standard definitions: final dispositions of case codes and outcome rates for surveys. 9th ed. Oakbrook Terrace, IL: AAPOR; 2016. http://www.aapor.org/AAPOR_Main/media/ publications/Standard-Definitions20169theditionfinal.pdf

12. CDC. Behavioral Risk Factor Surveillance System, 2013 summary data quality report. Atlanta, GA: US Department of Health and Human Services, CDC; 2014. https://www.cdc.gov/brfss/annual_data/2013/ pdf/2013_dqr.pdf

13. CDC. Behavioral Risk Factor Surveillance System, 2014 summary data quality report. Atlanta, GA: US Department of Health and Human Services, CDC; 2015. https://www.cdc.gov/brfss/annual_data/2014/ pdf/2014_dqr.pdf

14. Pew Research Center [Internet]. Mobile fact sheet. Washington, DC: Pew Research Center; 2016. http://www.pewinternet.org/fact-sheet/mobile/

15. Pierannunzi C, Chowdhury P, Town M. The effects of overlapping RDD sampling on the BRFSS. Presented at the American Association for Public Opinion Research Annual Conference; May 14-17, 2015; Hollywood, Florida.

16. Moyer VA; US Preventive Services Task Force. Screening for cervical cancer: U.S. Preventive Services Task Force recommendation statement. Ann Intern Med 2012;156:880-91, W312. https://doi.org/10.7326/ 0003-4819-156-12-201206190-00424

17. Zauber AG, Lansdorp-Vogelaar I, Knudsen AB, Wilschut J, van Ballegooijen M, Kuntz KM. Evaluating test strategies for colorectal cancer screening: a decision analysis for the U.S. Preventive Services Task Force. Ann Intern Med 2008;149:659-69. https://doi.org/10.7326/ 0003-4819-149-9-200811040-00244

18. Simon JG, De Boer JB, Joung IMA, Bosma H, Mackenbach JP. How is your health in general? A qualitative study on self-assessed health. Eur J Public Health 2005;15:200-8. https://doi.org/10.1093/eurpub/cki102

19. CDC. Measuring healthy days: population assessment of health-related quality of life. Atlanta, GA: US Department of Health and Human Services, CDC, National Center for Chronic Disease Prevention and Health Promotion; 2000. https://www.cdc.gov/hrqol/pdfs/mhd.pdf
20. Strine TW, Balluz L, Chapman DP, Moriarty DG, Owens M, Mokdad $\mathrm{AH}$. Risk behaviors and healthcare coverage among adults by frequent mental distress status, 2001. Am J Prev Med 2004;26:213-6. https:// doi.org/10.1016/j.amepre.2003.11.002

21. Liu Y, Croft JB, Wheaton AG, et al. Association between perceived insufficient sleep, frequent mental distress, obesity and chronic diseases among US adults, 2009 Behavioral Risk Factor Surveillance System. BMC Public Health 2013;13:84. https://doi.org/10.1186/1471-2458-13-84

22. Zack MM, Moriarty DG, Stroup DF, Ford ES, Mokdad AH. Worsening trends in adult health-related quality of life and self-rated health-United States, 1993-2001. Public Health Rep 2004;119:493-505. https://doi. org/10.1016/j.phr.2004.07.007

23. Zack MM. Health-related quality of life-United States, 2006 and 2010. In: CDC. CDC health disparities and inequalities report-United States, 2013. MMWR Suppl 2013;62(No. Suppl 3).

24. Hoffman C, Paradise J. Health insurance and access to health care in the United States. Ann N Y Acad Sci 2008;1136:149-60. https://doi. org/10.1196/annals. 1425.007

25. Fox JB, Richards CL. Vital signs: health insurance coverage and health care utilization-United States, 2006-2009 and January-March 2010. MMWR Morb Mortal Wkly Rep 2010;59:1448-54.

26. Heron M. Deaths: leading causes for 2014. Natl Vital Stat Rep 2016;65:1-96.

27. Benard VB, Thomas CC, King J, Massetti GM, Doria-Rose VP, Saraiya M. Vital signs: cervical cancer incidence, mortality, and screeningUnited States, 2007-2012. MMWR Morb Mortal Wkly Rep 2014;63:1004-9.

28. Klabunde CN, Joseph DA, King JB. Vital signs: colorectal cancer screening test use-United States, 2012. MMWR Morb Mortal Wkly Rep 2013;62:881-8.

29. CDC. Physical activity and health. Atlanta, GA: US Department of Health and Human Services, CDC; 2016. https://www.cdc.gov/ physicalactivity/basics/pa-health/index.htm

30. Lee IM, Shiroma EJ, Lobelo F, Puska P, Blair SN, Katzmarzyk PT; Lancet Physical Activity Series Working Group. Effect of physical inactivity on major non-communicable diseases worldwide: an analysis of burden of disease and life expectancy. Lancet 2012;380:219-29. https://doi. org/10.1016/S0140-6736(12)61031-9

31. CDC. Strategies to prevent obesity and other chronic diseases: the CDC guide to strategies to increase physical activity in the community. Atlanta, GA: US Department of Health and Human Services; 2011.

32. Watson NF, Badr MS, Belenky G, et al. Recommended amount of sleep for a healthy adult: a joint consensus statement of the American Academy of Sleep Medicine and Sleep Research Society. Sleep 2015;38:843-4.

33. Watson NF, Badr MS, Belenky G, et al.; Consensus Conference Panel. Joint consensus statement of the American Academy of Sleep Medicine and Sleep Research Society on the recommended amount of sleep for a healthy adult: methodology and discussion. Sleep 2015;38:1161-83. https://doi.org/10.5665/sleep.4886

34. Liu Y, Croft JB, Wheaton AG, et al. Association between perceived insufficient sleep, frequent mental distress, obesity and chronic diseases among US adults, 2009 behavioral risk factor surveillance system. BMC Public Health 2013;13:84. https://doi.org/10.1186/1471-2458-13-84

35 . World Health Organization. WHO report on the global tobacco epidemic, 2013: enforcing bans on tobacco advertising, promotion and sponsorship. Geneva, Switzerland: World Health Organization; 2013.

36. CDC, National Center for Chronic Disease Prevention and Health Promotion. How tobacco smoke causes disease: the biology and behavioral basis for smoking-attributable disease: a report of the Surgeon General. Atlanta, GA: US Department of Health and Human Services, CDC, National Center for Chronic Disease Prevention and Health Promotion; 2010. 
37. CDC, National Center for Chronic Disease Prevention and Health Promotion. The health consequences of smoking - 50 years of progress: a report of the Surgeon General. Atlanta, GA: US Department of Health and Human Services, CDC, National Center for Chronic Disease Prevention and Health Promotion; 2014.

38. King B, Dube S, Kaufmann R, et al. Vital signs: adult smoking in the US. MMWR Morb Mortal Wkly Rep 2011;60:1207-12.

39. CDC. Fact sheets: binge drinking. Atlanta, GA: US Department of Health and Human Services, CDC; 2017. https://www.cdc.gov/alcohol/ fact-sheets/binge-drinking.htm

40. Dwyer-Lindgren L, Flaxman AD, Ng M, Hansen GM, Murray CJL, Mokdad AH. Drinking patterns in US counties from 2002 to 2012. Am J Public Health 2015;105:1120-7. https://doi.org/10.2105/ AJPH.2014.302313

41. Bouchery EE, Harwood HJ, Sacks JJ, Simon CJ, Brewer RD. Economic costs of excessive alcohol consumption in the U.S., 2006. Am J Prev Med 2011;41:516-24. https://doi.org/10.1016/j.amepre.2011.06.045

42. Kanny D, Liu Y, Brewer RD, et al. Vital signs: binge drinking nationwide problems, local solutions. MMWR Morb Mortal Wkly Rep 2012;61:14-9.

43. Xuan Z, Blanchette J, Nelson TF, Heeren T, Oussayef N, Naimi TS. The alcohol policy environment and policy subgroups as predictors of binge drinking measures among US adults. Am J Public Health 2015;105:816-22. https://doi.org/10.2105/AJPH.2014.302112

44. CDC. Adult obesity facts. Atlanta, GA: US Department of Health and Human Services, CDC; 2016. https://www.cdc.gov/obesity/data/adult.html

45. Sherry B, Blanck HM, Galuska DA, et al. Vital signs: adult obesity: obesity rises among adults. MMWR Morb Mortal Wkly Rep 2010;59:951-5.

46. May AL, Freedman D, Sherry B, Blanck HM. Obesity-United States, 1999-2010. In: CDC. CDC health disparities and inequalities reportUnited States, 2013. MMWR Suppl 2013;62(No. Suppl 3).

47. CDC. Arthritis awareness month. Atlanta, GA: US Department of Health and Human Services, CDC; 2017. https://www.cdc.gov/features/ arthritisawareness/

48. Stevens JA, Corso PS, Finkelstein EA, Miller TR. The costs of fatal and non-fatal falls among older adults. Inj Prev 2006;12:290-5. https://doi. org/10.1136/ip.2005.011015
49. Barbour KE, Helmick CG, Boring M, Brady TJ. Vital signs: prevalence of doctor-diagnosed arthritis and arthritis-attributable activity limitation-United States, 2013-2015. MMWR Morb Mortal Wkly Rep 2017;66:246-53. https://dx.doi.org/10.15585/mmwr.mm6609e1

50. Whiteford HA, Degenhardt L, Rehm J, et al. Global burden of disease attributable to mental and substance use disorders: findings from the Global Burden of Disease Study 2010. Lancet 2013;382:1575-86. https://doi.org/10.1016/S0140-6736(13)61611-6

51. Ferrari AJ, Charlson FJ, Norman RE, et al. Burden of depressive disorders by country, sex, age, and year: findings from the global burden of disease study 2010. PLoS Med 2013;10:e1001547. https://doi.org/10.1371/ journal.pmed.1001547

52. Kochanek KD, Murphy SL, Xu J, Tejada-Vera B. Deaths: final data for 2014. Natl Vital Stat Rep 2016;65:1-122.

53. Mozaffarian D, Benjamin EJ, Go AS, et al.; American Heart Association Statistics Committee and Stroke Statistics Subcommittee. Heart disease and stroke statistics - 2015 update: a report from the American Heart Association. Circulation 2015;131:e29-322. https://doi.org/10.1161/ CIR.0000000000000152

54. Mozaffarian D, Benjamin EJ, Go AS, et al.; Writing Group Members; American Heart Association Statistics Committee and Stroke Statistics Subcommittee. Executive summary: heart disease and stroke statistics - 2016 update: a report from the American Heart Association. Circulation 2016;133:447-54. https://doi.org/10.1161/ CIR.0000000000000366

55. Strine TW, Greenlund KJ, Brown DW, Mokdad A, Balluz L. Characteristics of people aged 45 years or older with heart disease by frequent mental distress status, 2001. Prev Med 2004;39:191-6. https:// doi.org/10.1016/j.ypmed.2004.01.022

56. Li C, Balluz LS, Ford ES, Okoro CA, Zhao G, Pierannunzi C. A comparison of prevalence estimates for selected health indicators and chronic diseases or conditions from the Behavioral Risk Factor Surveillance System, the National Health Interview Survey, and the National Health and Nutrition Examination Survey, 2007-2008. Prev Med 2012;54:381-7. https://doi.org/10.1016/j.ypmed.2012.04.003 
TABLE 1. Age-adjusted* prevalence estimates of adults aged $\geq 18$ years who reported good or better health, ${ }^{\dagger}$ by state/territory Behavioral Risk Factor Surveillance System, United States, 2013

\begin{tabular}{|c|c|c|c|c|}
\hline State/Territory & $\begin{array}{l}\text { Sample } \\
\text { size }\end{array}$ & $\%$ & SE & $95 \% \mathrm{Cl}$ \\
\hline Alabama & 6,476 & 78.6 & 0.7 & (77.3-79.9) \\
\hline Alaska & 4,561 & 85.1 & 0.7 & $(83.6-86.5)$ \\
\hline Arizona & 4,242 & 84.1 & 1.0 & $(82.1-86.1)$ \\
\hline Arkansas & 5,243 & 77.1 & 0.8 & (75.5-78.8) \\
\hline California & 11,508 & 81.4 & 0.5 & $(80.4-82.4)$ \\
\hline Colorado & 13,617 & 87.0 & 0.4 & (86.3-87.8) \\
\hline Connecticut & 7,691 & 87.6 & 0.5 & $(86.6-88.7)$ \\
\hline Delaware & 5,200 & 83.5 & 0.7 & (82.0-84.9) \\
\hline District of Columbia & 4,920 & 86.6 & 0.7 & $(85.2-88.0)$ \\
\hline Florida & 33,822 & 81.7 & 0.5 & $(80.7-82.7)$ \\
\hline Georgia & 8,113 & 81.2 & 0.6 & $(80.0-82.4)$ \\
\hline Hawaii & 7,850 & 86.9 & 0.5 & (85.9-88.0) \\
\hline Idaho & 5,598 & 86.2 & 0.7 & (84.9-87.6) \\
\hline Illinois & 5,605 & 83.6 & 0.7 & (82.2-85.0) \\
\hline Indiana & 10,206 & 82.6 & 0.5 & (81.7-83.6) \\
\hline lowa & 8,145 & 86.4 & 0.5 & (85.4-87.5) \\
\hline Kansas & 23,228 & 85.2 & 0.3 & (84.6-85.8) \\
\hline Kentucky & 10,995 & 77.9 & 0.6 & (76.7-79.1) \\
\hline Louisiana & 5,241 & 78.1 & 0.9 & (76.4-79.8) \\
\hline Maine & 8,033 & 86.5 & 0.5 & $(85.5-87.5)$ \\
\hline Maryland & 12,972 & 85.4 & 0.5 & (84.4-86.5) \\
\hline Massachusetts & 15,042 & 86.8 & 0.5 & (85.9-87.7) \\
\hline Michigan & 12,745 & 83.2 & 0.5 & (82.3-84.2) \\
\hline Minnesota & 14,299 & 88.1 & 0.5 & $(87.1-89.1)$ \\
\hline Mississippi & 7,424 & 76.5 & 0.7 & (75.2-77.9) \\
\hline Missouri & 7,103 & 82.4 & 0.7 & (81.1-83.8) \\
\hline Montana & 9,674 & 85.8 & 0.5 & (84.9-86.8) \\
\hline Nebraska & 17,106 & 86.7 & 0.4 & (85.9-87.5) \\
\hline Nevada & 5,087 & 83.1 & 1.0 & $(81.2-85.1)$ \\
\hline New Hampshire & 6,449 & 88.0 & 0.6 & (86.9-89.1) \\
\hline New Jersey & 13,351 & 84.0 & 0.5 & $(83.0-85.0)$ \\
\hline New Mexico & 9,295 & 80.1 & 0.6 & (78.9-81.3) \\
\hline New York & 8,886 & 82.8 & 0.5 & (81.7-83.8) \\
\hline North Carolina & 8,827 & 81.5 & 0.5 & $(80.5-82.6)$ \\
\hline North Dakota & 7,775 & 86.1 & 0.6 & $(85.0-87.2)$ \\
\hline Ohio & 11,940 & 82.8 & 0.5 & (81.8-83.9) \\
\hline Oklahoma & 8,204 & 80.1 & 0.6 & (78.9-81.2) \\
\hline Oregon & 5,934 & 83.2 & 0.7 & (81.7-84.6) \\
\hline Pennsylvania & 11,352 & 84.2 & 0.5 & (83.2-85.1) \\
\hline Rhode Island & 6,508 & 84.3 & 0.6 & $(83.1-85.6)$ \\
\hline South Carolina & 10,667 & 81.4 & 0.5 & (80.4-82.4) \\
\hline South Dakota & 6,887 & 88.2 & 0.6 & (87.1-89.3) \\
\hline Tennessee & 5,785 & 78.1 & 0.8 & (76.6-79.6) \\
\hline Texas & 10,708 & 80.8 & 0.6 & (79.7-82.0) \\
\hline Utah & 12,737 & 87.1 & 0.4 & (86.3-87.8) \\
\hline Vermont & 6,383 & 88.7 & 0.5 & (87.7-89.8) \\
\hline Virginia & 8,431 & 84.7 & 0.5 & $(83.7-85.6)$ \\
\hline Washington & 11,129 & 84.7 & 0.5 & (83.7-85.6) \\
\hline West Virginia & 5,885 & 76.4 & 0.6 & (75.1-77.6) \\
\hline Wisconsin & 6,582 & 85.2 & 0.7 & $(83.8-86.7)$ \\
\hline Wyoming & 6,433 & 85.4 & 0.6 & $(84.2-86.6)$ \\
\hline Guam & 1,896 & 79.0 & 1.3 & (76.6-81.5) \\
\hline Puerto Rico & 6,003 & 66.4 & 0.8 & (64.9-67.9) \\
\hline Median & & 84.0 & & \\
\hline Range & & $66.4-88.7$ & & \\
\hline
\end{tabular}

Abbreviations: $\mathrm{Cl}=$ confidence interval; $\mathrm{SE}=$ standard error

${ }^{*}$ Age adjusted to the 2000 U.S. standard population.

${ }^{\dagger}$ Respondents were asked to rate their general health as poor, fair, good, very good, or excellent. Respondents were classified into two groups: those who reported poor or fair health and those who reported good, very good, or excellent health. 
TABLE 2. Age-adjusted* prevalence estimates of adults aged $\geq 18$ years who reported good or better health, ${ }^{\dagger}$ by metropolitan and micropolitan statistical area - Behavioral Risk Factor Surveillance System, United States, 2013

\begin{tabular}{|c|c|c|c|c|}
\hline MMSA & $\begin{array}{l}\text { Sample } \\
\text { size }\end{array}$ & $\%$ & SE & $95 \% \mathrm{Cl}$ \\
\hline Aguadilla-Isabela, Puerto Rico & 593 & 67.8 & 2.5 & $(62.9-72.7)$ \\
\hline Akron, Ohio & 687 & 79.8 & 2.6 & $(74.8-84.8)$ \\
\hline Albuquerque, New Mexico & 2,081 & 81.4 & 1.2 & (79.1-83.7) \\
\hline Allentown-Bethlehem-Easton, Pennsylvania-New Jersey & 1,027 & 80.1 & 2.0 & $(76.1-84.1)$ \\
\hline Anchorage, Alaska & 1,523 & 86.1 & 1.1 & $(83.9-88.3)$ \\
\hline Atlanta-Sandy Springs-Roswell, Georgia & 3,508 & 83.6 & 0.8 & $(82.0-85.3)$ \\
\hline Augusta-Richmond County, Georgia-South Carolina & 913 & 79.4 & 2.0 & $(75.5-83.4)$ \\
\hline Austin-Round Rock, Texas & 924 & 84.9 & 1.7 & $(81.6-88.2)$ \\
\hline Baltimore-Columbia-Towson, Maryland & 4,757 & 85.0 & 0.8 & $(83.4-86.6)$ \\
\hline Baton Rouge, Louisiana & 928 & 80.8 & 1.9 & $(77.1-84.4)$ \\
\hline Billings, Montana & 816 & 83.6 & 1.5 & $(80.7-86.4)$ \\
\hline Birmingham-Hoover, Alabama & 1,349 & 80.8 & 1.3 & $(78.3-83.3)$ \\
\hline Bismarck, North Dakota & 1,032 & 86.1 & 1.4 & (83.4-88.9) \\
\hline Boise City, Idaho & 1,485 & 88.5 & 1.1 & $(86.3-90.7)$ \\
\hline Boston, Massachusetts $\S$ & 4,067 & 87.3 & 0.8 & $(85.6-88.9)$ \\
\hline Buffalo-Cheektowaga-Niagara Falls, New York & 505 & 84.9 & 2.5 & $(80.0-89.7)$ \\
\hline Burlington-South Burlington, Vermont & 1,632 & 89.7 & 0.9 & $(87.8-91.5)$ \\
\hline Cambridge-Newton-Framingham, Massachusetts ${ }^{\S}$ & 4,888 & 88.0 & 0.7 & $(86.6-89.4)$ \\
\hline Camden, New Jersey $§$ & 1,866 & 83.2 & 1.3 & $(80.8-85.7)$ \\
\hline Cedar Rapids, lowa & 648 & 85.1 & 2.1 & $(80.9-89.2)$ \\
\hline Charleston, West Virginia & 819 & 77.6 & 1.7 & $(74.2-81.0)$ \\
\hline Charleston-North Charleston, South Carolina & 1,547 & 84.1 & 1.2 & $(81.7-86.5)$ \\
\hline Charlotte-Concord-Gastonia, North Carolina-South Carolina & 1,948 & 82.4 & 1.1 & $(80.3-84.5)$ \\
\hline Chattanooga, Tennessee-Georgia & 577 & 78.9 & 2.5 & $(74.0-83.9)$ \\
\hline Chicago-Naperville-Elgin, Illinois-Indiana-Wisconsin & 3,330 & 83.2 & 0.9 & $(81.4-85.1)$ \\
\hline Cincinnati, Ohio-Kentucky-Indiana & 2,601 & 84.6 & 1.0 & $(82.5-86.6)$ \\
\hline Claremont-Lebanon, New Hampshire-Vermont & 1,688 & 86.7 & 1.5 & $(83.8-89.6)$ \\
\hline Cleveland-Elyria, Ohio & 1,105 & 81.6 & 1.8 & $(78.2-85.1)$ \\
\hline Colorado Springs, Colorado & 1,376 & 87.8 & 1.0 & (85.7-89.8) \\
\hline Columbia, South Carolina & 1,442 & 85.1 & 1.1 & $(82.9-87.4)$ \\
\hline Columbus, Ohio & 1,861 & 84.2 & 1.1 & $(82.1-86.4)$ \\
\hline Crestview-Fort Walton Beach-Destin, Florida & 1,069 & 82.1 & 1.7 & (78.7-85.5) \\
\hline Dallas-Plano-Irving, Texas $\S$ & 887 & 83.8 & 1.4 & $(81.0-86.5)$ \\
\hline Davenport-Moline-Rock Island, lowa-Illinois & 672 & 83.0 & 2.3 & $(78.5-87.4)$ \\
\hline Dayton, Ohio & 837 & 87.6 & 1.4 & $(85.0-90.3)$ \\
\hline Deltona-Daytona Beach-Ormond Beach, Florida & 1,103 & 85.1 & 1.6 & $(81.9-88.3)$ \\
\hline Denver-Aurora-Lakewood, Colorado & 5,702 & 87.0 & 0.6 & $(85.9-88.1)$ \\
\hline Des Moines-West Des Moines, lowa & 1,343 & 88.8 & 1.0 & $(86.8-90.9)$ \\
\hline Duluth, Minnesota-Wisconsin & 699 & 87.5 & 1.7 & $(84.1-90.9)$ \\
\hline Durham-Chapel Hill, North Carolina & 617 & 84.0 & 1.9 & $(80.2-87.8)$ \\
\hline El Paso, Texas & 743 & 79.5 & 1.7 & $(76.1-82.9)$ \\
\hline Evansville, Indiana-Kentucky & 571 & 80.4 & 2.5 & $(75.6-85.3)$ \\
\hline Fargo, North Dakota-Minnesota & 1,179 & 86.1 & 1.4 & $(83.3-88.9)$ \\
\hline Fayetteville-Springdale-Rogers, Arkansas-Missouri & 820 & 79.4 & 2.0 & $(75.5-83.3)$ \\
\hline Fort Smith, Arkansas-Oklahoma & 497 & 69.7 & 3.3 & $(63.2-76.2)$ \\
\hline Fort Wayne, Indiana & 772 & 86.1 & 1.7 & $(82.8-89.4)$ \\
\hline Fort Worth-Arlington, Texas ${ }^{\S}$ & 802 & 85.7 & 1.6 & $(82.6-88.9)$ \\
\hline Gainesville, Florida & 1,018 & 87.0 & 1.6 & $(83.8-90.2)$ \\
\hline Grand Forks, North Dakota-Minnesota & 503 & 86.0 & 2.4 & $(81.2-90.7)$ \\
\hline Grand Island, Nebraska & 798 & 82.6 & 1.9 & $(78.9-86.3)$ \\
\hline Grand Rapids-Wyoming, Michigan & 1,346 & 86.8 & 1.4 & $(84.1-89.4)$ \\
\hline Greensboro-High Point, North Carolina & 662 & 82.0 & 1.9 & $(78.2-85.7)$ \\
\hline Greenville-Anderson-Mauldin, South Carolina & 1,339 & 83.1 & 1.4 & $(80.3-85.9)$ \\
\hline Gulfport-Biloxi-Pascagoula, Mississippi & 771 & 75.6 & 2.0 & $(71.6-79.6)$ \\
\hline Hagerstown-Martinsburg, Maryland-West Virginia & 766 & 82.2 & 1.9 & $(78.4-86.0)$ \\
\hline Hartford-West Hartford-East Hartford, Connecticut & 2,831 & 88.2 & 0.8 & $(86.6-89.9)$ \\
\hline Hilton Head Island-Bluffton-Beaufort, South Carolina & 825 & 89.5 & 1.5 & $(86.7-92.4)$ \\
\hline Houston-The Woodlands-Sugar Land, Texas & 1,363 & 81.9 & 1.5 & (78.9-84.9) \\
\hline Huntington-Ashland, West Virginia-Kentucky-Ohio & 1,175 & 75.6 & 1.5 & $(72.6-78.5)$ \\
\hline Idaho Falls, Idaho & 506 & 80.0 & 2.6 & $(74.9-85.0)$ \\
\hline Indianapolis-Carmel-Anderson, Indiana & 2,516 & 82.9 & 1.0 & $(80.9-84.9)$ \\
\hline Jackson, Mississippi & 802 & 81.8 & 1.7 & $(78.5-85.2)$ \\
\hline
\end{tabular}

See table footnotes on page 15. 
TABLE 2. (Continued) Age-adjusted* prevalence estimates of adults aged $\geq 18$ years who reported good or better health, ${ }^{\dagger}$ by metropolitan and micropolitan statistical area - Behavioral Risk Factor Surveillance System, United States, 2013

\begin{tabular}{|c|c|c|c|c|}
\hline MMSA & $\begin{array}{l}\text { Sample } \\
\text { size }\end{array}$ & $\%$ & SE & $95 \% \mathrm{Cl}$ \\
\hline Jacksonville, Florida & 2,852 & 82.4 & 1.0 & $(80.3-84.4)$ \\
\hline Kansas City, Missouri-Kansas & 7,411 & 85.2 & 0.9 & $(83.5-86.9)$ \\
\hline Kingsport-Bristol-Bristol, Tennessee-Virginia & 536 & 73.7 & 3.0 & $(67.8-79.7)$ \\
\hline Knoxville, Tennessee & 651 & 78.2 & 2.1 & $(74.1-82.2)$ \\
\hline Lansing-East Lansing, Michigan & 687 & 86.3 & 1.5 & $(83.3-89.2)$ \\
\hline Lexington-Fayette, Kentucky & 637 & 80.8 & 2.0 & $(76.8-84.8)$ \\
\hline Lincoln, Nebraska & 1,875 & 88.8 & 0.8 & $(87.2-90.5)$ \\
\hline Little Rock-North Little Rock-Conway, Arkansas & 1,140 & 79.0 & 1.6 & $(75.8-82.1)$ \\
\hline Logan, Utah-Idaho & 641 & 86.5 & 1.8 & $(83.0-89.9)$ \\
\hline Los Angeles-Long Beach-Anaheim, California & 3,041 & 80.6 & 0.9 & $(78.8-82.4)$ \\
\hline Louisville/Jefferson County, Kentucky-Indiana & 2,142 & 81.7 & 1.3 & $(79.2-84.2)$ \\
\hline Lubbock, Texas & 525 & 80.0 & 2.6 & $(74.9-85.0)$ \\
\hline Manhattan, Kansas & 663 & 90.2 & 1.2 & $(87.8-92.5)$ \\
\hline Memphis, Tennessee-Mississippi-Arkansas & 1,206 & 81.4 & 1.5 & $(78.6-84.3)$ \\
\hline Miami-Fort Lauderdale-West Palm Beach, Florida & 2,193 & 82.1 & 1.3 & $(79.5-84.7)$ \\
\hline Milwaukee-Waukesha-West Allis, Wisconsin & 1,271 & 81.2 & 1.9 & $(77.6-84.9)$ \\
\hline Minneapolis-St. Paul-Bloomington, Minnesota-Wisconsin & 9,113 & 88.7 & 0.6 & $(87.4-89.9)$ \\
\hline Minot, North Dakota & 650 & 87.8 & 1.4 & $(85.0-90.6)$ \\
\hline $\begin{array}{l}\text { Montgomery County-Bucks County-Chester County, } \\
\text { Pennsylvania§ }\end{array}$ & 963 & 88.5 & 1.2 & $(86.1-90.9)$ \\
\hline $\begin{array}{l}\text { Myrtle Beach-Conway-North Myrtle Beach, South Carolina- } \\
\text { North Carolina }\end{array}$ & 775 & 81.8 & 1.7 & $(78.4-85.1)$ \\
\hline Nashville-Davidson County-Murfreesboro-Franklin, Tennessee & 1,055 & 84.5 & 1.4 & $(81.8-87.2)$ \\
\hline Nassau County-Suffolk County, New York ${ }^{\S}$ & 936 & 85.5 & 1.4 & $(82.7-88.3)$ \\
\hline Newark, New Jersey-Pennsylvania§ & 4,113 & 85.3 & 0.8 & $(83.7-86.8)$ \\
\hline New Orleans-Metairie, Louisiana & 1,284 & 79.7 & 1.8 & $(76.2-83.2)$ \\
\hline New York-Jersey City-White Plains, New York-New Jersey $§$ & 8,889 & 80.7 & 0.6 & $(79.5-81.9)$ \\
\hline Norfolk, Nebraska & 673 & 81.5 & 1.9 & $(77.8-85.1)$ \\
\hline North Platte, Nebraska & 718 & 85.4 & 1.8 & $(81.8-89.0)$ \\
\hline North Port-Sarasota-Bradenton, Florida & 1,082 & 81.2 & 2.0 & $(77.4-85.1)$ \\
\hline Oakland-Hayward-Berkeley, California ${ }^{\S}$ & 700 & 87.0 & 1.5 & $(84.1-89.8)$ \\
\hline Ogden-Clearfield, Utah & 2,454 & 88.3 & 0.8 & $(86.8-89.8)$ \\
\hline Oklahoma City, Oklahoma & 2,634 & 83.0 & 0.8 & $(81.4-84.7)$ \\
\hline Omaha-Council Bluffs, Nebraska-lowa & 3,123 & 86.8 & 0.8 & $(85.2-88.4)$ \\
\hline Orlando-Kissimmee-Sanford, Florida & 2,264 & 80.0 & 1.3 & $(77.5-82.5)$ \\
\hline Panama City, Florida & 1,021 & 80.8 & 1.9 & $(77.1-84.5)$ \\
\hline Pensacola-Ferry Pass-Brent, Florida & 1,307 & 82.7 & 1.3 & $(80.1-85.2)$ \\
\hline Philadelphia, Pennsylvania ${ }^{\S}$ & 1,765 & 81.5 & 1.2 & $(79.2-83.9)$ \\
\hline Phoenix-Mesa-Scottsdale, Arizona & 1,545 & 85.2 & 1.4 & $(82.4-88.0)$ \\
\hline Pittsburgh, Pennsylvania & 2,344 & 85.5 & 0.9 & $(83.7-87.3)$ \\
\hline Ponce, Puerto Rico & 530 & 68.3 & 2.1 & $(64.3-72.4)$ \\
\hline Portland-South Portland, Maine & 2,618 & 90.0 & 0.7 & $(88.5-91.4)$ \\
\hline Portland-Vancouver-Hillsboro, Oregon-Washington & 3,239 & 84.6 & 0.9 & $(82.8-86.5)$ \\
\hline Port St. Lucie, Florida & 1,020 & 82.6 & 2.3 & $(78.1-87.1)$ \\
\hline Providence-Warwick, Rhode Island-Massachusetts & 8,282 & 84.0 & 0.7 & $(82.5-85.4)$ \\
\hline Provo-Orem, Utah & 1,854 & 88.2 & 0.9 & $(86.5-90.0)$ \\
\hline Raleigh, North Carolina & 673 & 84.8 & 1.6 & $(81.7-87.9)$ \\
\hline Rapid City, South Dakota & 871 & 87.1 & 1.3 & $(84.5-89.7)$ \\
\hline Reno, Nevada & 1,818 & 82.5 & 1.3 & $(80.0-85.0)$ \\
\hline Richmond, Virginia & 1,308 & 85.1 & 1.2 & $(82.8-87.4)$ \\
\hline Riverside-San Bernardino-Ontario, California & 1,370 & 78.6 & 1.4 & $(75.9-81.3)$ \\
\hline Rochester, New York & 504 & 88.5 & 1.5 & $(85.5-91.5)$ \\
\hline Rockingham County-Strafford County, New Hampshire & 1,660 & 88.8 & 1.0 & $(86.7-90.8)$ \\
\hline Sacramento-Roseville-Arden-Arcade, California & 888 & 83.5 & 1.9 & $(79.8-87.3)$ \\
\hline St. Louis, Missouri-Illinois & 2,058 & 83.7 & 1.2 & $(81.3-86.0)$ \\
\hline Salem, Oregon & 527 & 83.5 & 2.5 & $(78.6-88.5)$ \\
\hline Salina, Kansas & 523 & 83.1 & 2.2 & $(78.8-87.4)$ \\
\hline Salisbury, Maryland-Delaware & 2,060 & 84.4 & 1.2 & $(82.1-86.8)$ \\
\hline Salt Lake City, Utah & 4,662 & 86.5 & 0.6 & $(85.2-87.7)$ \\
\hline San Antonio-New Braunfels, Texas & 928 & 76.7 & 1.8 & $(73.2-80.3)$ \\
\hline San Francisco-Redwood City-South San Francisco, California§ & 539 & 84.4 & 2.0 & $(80.4-88.4)$ \\
\hline San Jose-Sunnyvale-Santa Clara, California & 627 & 85.4 & 2.1 & $(81.3-89.5)$ \\
\hline
\end{tabular}

See table footnotes on page 15. 
TABLE 2. (Continued) Age-adjusted* prevalence estimates of adults aged $\geq 18$ years who reported good or better health, ${ }^{\dagger}$ by metropolitan and micropolitan statistical area — Behavioral Risk Factor Surveillance System, United States, 2013

\begin{tabular}{|c|c|c|c|c|}
\hline MMSA & $\begin{array}{l}\text { Sample } \\
\text { size }\end{array}$ & $\%$ & SE & $95 \% \mathrm{Cl}$ \\
\hline San Juan-Carolina-Caguas, Puerto Rico & 3,652 & 66.7 & 1.0 & $(64.8-68.6)$ \\
\hline Scottsbluff, Nebraska & 712 & 82.0 & 2.0 & $(78.0-86.0)$ \\
\hline Scranton-Wilkes-Barre-Hazleton, Pennsylvania & 564 & 84.7 & 2.1 & $(80.5-88.8)$ \\
\hline Seattle-Bellevue-Everett, Washington ${ }^{\S}$ & 3,762 & 87.2 & 0.7 & $(85.7-88.6)$ \\
\hline Shreveport-Bossier City, Louisiana & 571 & 79.2 & 2.5 & $(74.3-84.0)$ \\
\hline Silver Spring-Frederick-Rockville, Maryland ${ }^{\S}$ & 2,417 & 88.4 & 1.1 & $(86.2-90.6)$ \\
\hline Sioux City, lowa-Nebraska-South Dakota & 1,055 & 85.4 & 2.2 & $(81.0-89.8)$ \\
\hline Sioux Falls, South Dakota & 1,007 & 91.2 & 1.1 & $(89.0-93.4)$ \\
\hline Spartanburg, South Carolina & 592 & 79.2 & 2.6 & $(74.2-84.2)$ \\
\hline Spokane-Spokane Valley, Washington & 857 & 84.7 & 1.8 & $(81.3-88.2)$ \\
\hline Springfield, Massachusetts & 1,573 & 83.1 & 1.5 & $(80.1-86.1)$ \\
\hline Tallahassee, Florida & 1,840 & 85.7 & 1.3 & $(83.1-88.2)$ \\
\hline Tampa-St. Petersburg-Clearwater, Florida & 2,193 & 82.1 & 1.1 & $(79.9-84.4)$ \\
\hline Toledo, Ohio & 998 & 83.2 & 1.8 & $(79.7-86.7)$ \\
\hline Topeka, Kansas & 2,399 & 83.0 & 1.0 & $(81.1-84.9)$ \\
\hline Tulsa, Oklahoma & 1,988 & 81.1 & 1.2 & $(78.8-83.4)$ \\
\hline Virginia Beach-Norfolk-Newport News, Virginia-North Carolina & 1,679 & 84.8 & 1.1 & $(82.7-87.0)$ \\
\hline Warren-Troy-Farmington Hills, Michigan ${ }^{\S}$ & 2,259 & 85.6 & 0.9 & $(83.7-87.4)$ \\
\hline $\begin{array}{l}\text { Washington-Arlington-Alexandria, District of Columbia-Virginia- } \\
\text { Maryland-West Virginia§ }\end{array}$ & 9,005 & 87.4 & 0.6 & $(86.2-88.7)$ \\
\hline Wichita, Kansas & 4,921 & 84.5 & 0.6 & $(83.3-85.8)$ \\
\hline Wilmington, Delaware-Maryland-New Jersey ${ }^{\S}$ & 3,276 & 83.2 & 0.9 & $(81.4-85.1)$ \\
\hline Winston-Salem, North Carolina & 694 & 82.6 & 2.0 & $(78.7-86.6)$ \\
\hline Worcester, Massachusetts-Connecticut & 2,760 & 85.3 & 1.1 & $(83.0-87.5)$ \\
\hline Median & & 83.8 & & \\
\hline Range & & $66.7-91.2$ & & \\
\hline
\end{tabular}

Abbreviations: $\mathrm{Cl}$ = confidence interval; MMSA = metropolitan and micropolitan statistical areas; $\mathrm{SE}=$ standard error.

* Age adjusted to the 2000 U.S. standard population.

† Respondents were asked to rate their general health as poor, fair, good, very good, or excellent. Respondents were classified into two groups: those who reported poor or fair health and those who reported good, very good, or excellent health.

$\S$ Metropolitan division. 
TABLE 3. Age-adjusted* prevalence estimates of adults aged $\geq 18$ years who reported good or better health, ${ }^{\dagger}$ by state/territory Behavioral Risk Factor Surveillance System, United States, 2014

\begin{tabular}{|c|c|c|c|c|}
\hline State/Territory & $\begin{array}{l}\text { Sample } \\
\text { size }\end{array}$ & $\%$ & SE & $95 \% \mathrm{Cl}$ \\
\hline Alabama & 8,624 & 78.5 & 0.6 & $(77.3-79.6)$ \\
\hline Alaska & 4,371 & 86.6 & 0.7 & $(85.2-87.9)$ \\
\hline Arizona & 14,832 & 81.6 & 0.6 & $(80.5-82.7)$ \\
\hline Arkansas & 5,238 & 77.7 & 0.9 & $(75.9-79.5)$ \\
\hline California & 8,819 & 82.3 & 0.5 & $(81.2-83.3)$ \\
\hline Colorado & 13,352 & 87.1 & 0.4 & $(86.3-87.8)$ \\
\hline Connecticut & 7,935 & 86.2 & 0.6 & $(85.1-87.4)$ \\
\hline Delaware & 4,285 & 85.3 & 0.8 & $(83.8-86.8)$ \\
\hline District of Columbia & 4,067 & 86.5 & 0.9 & $(84.8-88.2)$ \\
\hline Florida & 9,749 & 82.1 & 0.6 & $(81.0-83.2)$ \\
\hline Georgia & 6,332 & 81.7 & 0.7 & $(80.4-83.0)$ \\
\hline Hawaii & 7,244 & 86.0 & 0.6 & $(84.9-87.1)$ \\
\hline Idaho & 5,468 & 87.2 & 0.7 & $(85.9-88.5)$ \\
\hline Illinois & 5,051 & 83.1 & 0.8 & $(81.6-84.6)$ \\
\hline Indiana & 11,431 & 81.8 & 0.5 & (80.9-82.8) \\
\hline lowa & 8,114 & 87.3 & 0.5 & $(86.3-88.2)$ \\
\hline Kansas & 13,705 & 85.2 & 0.4 & $(84.5-85.9)$ \\
\hline Kentucky & 11,179 & 76.8 & 0.7 & $(75.4-78.2)$ \\
\hline Louisiana & 6,765 & 79.1 & 0.6 & (77.9-80.3) \\
\hline Maine & 9,101 & 85.9 & 0.6 & $(84.8-87.0)$ \\
\hline Maryland & 12,543 & 86.2 & 0.6 & $(84.9-87.4)$ \\
\hline Massachusetts & 15,614 & 86.2 & 0.5 & $(85.3-87.1)$ \\
\hline Michigan & 8,454 & 84.1 & 0.5 & $(83.1-85.2)$ \\
\hline Minnesota & 16,375 & 88.4 & 0.3 & $(87.8-89.1)$ \\
\hline Mississippi & 4,183 & 79.0 & 0.8 & $(77.4-80.6)$ \\
\hline Missouri & 7,065 & 84.2 & 0.6 & $(83.0-85.4)$ \\
\hline Montana & 7,474 & 85.7 & 0.6 & (84.4-86.9) \\
\hline Nebraska & 22,370 & 87.4 & 0.4 & $(86.7-88.1)$ \\
\hline Nevada & 3,756 & 81.9 & 1.0 & (79.9-83.9) \\
\hline New Hampshire & 6,171 & 87.1 & 0.7 & $(85.6-88.5)$ \\
\hline New Jersey & 13,000 & 83.9 & 0.5 & (82.9-84.9) \\
\hline New Mexico & 8,915 & 80.2 & 0.7 & $(78.8-81.5)$ \\
\hline New York & 6,802 & 83.5 & 0.6 & $(82.3-84.7)$ \\
\hline North Carolina & 7,256 & 81.8 & 0.6 & $(80.7-82.9)$ \\
\hline North Dakota & 7,734 & 86.5 & 0.6 & $(85.3-87.7)$ \\
\hline Ohio & 10,907 & 83.1 & 0.6 & $(82.0-84.3)$ \\
\hline Oklahoma & 8,431 & 80.4 & 0.5 & $(79.3-81.4)$ \\
\hline Oregon & 5,206 & 85.1 & 0.7 & $(83.8-86.4)$ \\
\hline Pennsylvania & 10,923 & 84.0 & 0.5 & $(83.0-85.0)$ \\
\hline Rhode Island & 6,435 & 85.6 & 0.6 & (84.4-86.8) \\
\hline South Carolina & 10,990 & 81.3 & 0.5 & $(80.3-82.3)$ \\
\hline South Dakota & 7,391 & 86.8 & 0.7 & (85.4-88.2) \\
\hline Tennessee & 5,128 & 77.4 & 0.8 & $(75.8-79.1)$ \\
\hline Texas & 15,122 & 80.7 & 0.5 & (79.6-81.7) \\
\hline Utah & 14,977 & 87.5 & 0.3 & (86.9-88.1) \\
\hline Vermont & 6,458 & 89.1 & 0.5 & (88.1-90.0) \\
\hline Virginia & 9,444 & 83.6 & 0.5 & $(82.6-84.7)$ \\
\hline Washington & 10,073 & 84.3 & 0.5 & $(83.3-85.4)$ \\
\hline West Virginia & 6,186 & 6.5 & 0.7 & (75.2-77.8) \\
\hline Wisconsin & 7,035 & 85.2 & 0.7 & (83.9-86.5) \\
\hline Wyoming & 6,393 & 86.7 & 0.7 & $(85.3-88.2)$ \\
\hline Guam & 2,516 & 78.8 & 1.1 & (76.6-81.0) \\
\hline Puerto Rico & 5,983 & 66.9 & 0.7 & $(65.5-68.2)$ \\
\hline Median & & 84.1 & & \\
\hline Range & & $66.9-89.1$ & & \\
\hline
\end{tabular}

Abbreviations: $\mathrm{Cl}=$ confidence interval; $\mathrm{SE}=$ standard error.

* Age adjusted to the 2000 U.S. standard population.

† Respondents were asked to rate their general health as poor, fair, good, very good, or excellent. Respondents were classified into two groups: those who reported fair or poor health and those who reported good, very good, or excellent health. 
TABLE 4. Age-adjusted* prevalence estimates of adults aged $\geq 18$ years who reported good or better health, ${ }^{\dagger}$ by metropolitan and micropolitan statistical area - Behavioral Risk Factor Surveillance System, United States, 2014

\begin{tabular}{|c|c|c|c|c|}
\hline MMSA & $\begin{array}{l}\text { Sample } \\
\text { size }\end{array}$ & $\%$ & SE & $95 \% \mathrm{Cl}$ \\
\hline Aberdeen, South Dakota & 620 & 85.2 & 2.1 & $(81.1-89.2)$ \\
\hline Aguadilla-Isabela, Puerto Rico & 544 & 69.9 & 2.1 & $(65.8-73.9)$ \\
\hline Albuquerque, New Mexico & 1,786 & 81.7 & 1.3 & $(79.3-84.2)$ \\
\hline Allentown-Bethlehem-Easton, Pennsylvania-New Jersey & 1,088 & 85.3 & 1.8 & $(81.8-88.8)$ \\
\hline Anchorage, Alaska & 1,781 & 86.7 & 1.0 & $(84.7-88.7)$ \\
\hline Atlanta-Sandy Springs-Roswell, Georgia & 2,768 & 84.8 & 0.9 & $(83.0-86.6)$ \\
\hline Augusta-Richmond County, Georgia-South Carolina & 887 & 80.0 & 2.7 & $(74.7-85.2)$ \\
\hline Austin-Round Rock, Texas & 2,221 & 85.3 & 0.9 & $(83.5-87.2)$ \\
\hline Baltimore-Columbia-Towson, Maryland & 4,608 & 85.8 & 1.0 & $(83.9-87.8)$ \\
\hline Baton Rouge, Louisiana & 923 & 80.0 & 1.6 & $(76.8-83.2)$ \\
\hline Berlin, New Hampshire-Vermont & 538 & 87.8 & 1.4 & $(85.0-90.6)$ \\
\hline Billings, Montana & 804 & 83.6 & 1.7 & $(80.4-86.9)$ \\
\hline Birmingham-Hoover, Alabama & 1,568 & 80.9 & 1.3 & (78.4-83.4) \\
\hline Bismarck, North Dakota & 1,032 & 86.5 & 1.7 & $(83.2-89.9)$ \\
\hline Boise City, Idaho & 1,353 & 87.8 & 1.3 & $(85.3-90.3)$ \\
\hline Boston, Massachusetts $§$ & 4,539 & 87.0 & 0.9 & $(85.2-88.7)$ \\
\hline Burlington-South Burlington, Vermont & 1,982 & 90.5 & 0.8 & $(88.9-92.2)$ \\
\hline Cambridge-Newton-Framingham, Massachusetts $\S$ & 5,167 & 87.0 & 0.8 & $(85.5-88.5)$ \\
\hline Camden, New Jersey ${ }^{\S}$ & 1,714 & 84.8 & 1.4 & $(82.0-87.5)$ \\
\hline Cedar Rapids, lowa & 638 & 87.9 & 1.7 & $(84.5-91.3)$ \\
\hline Charleston, West Virginia & 875 & 77.5 & 1.8 & $(74.0-81.0)$ \\
\hline Charleston-North Charleston, South Carolina & 1,402 & 83.7 & 1.4 & $(81.0-86.5)$ \\
\hline Charlotte-Concord-Gastonia, North Carolina-South Carolina & 2,152 & 82.8 & 1.0 & $(80.8-84.9)$ \\
\hline Chicago-Naperville-Elgin, Illinois-Indiana-Wisconsin & 4,123 & 82.8 & 0.9 & $(80.9-84.6)$ \\
\hline Cincinnati, Ohio-Kentucky-Indiana & 2,041 & 83.2 & 1.4 & $(80.6-85.9)$ \\
\hline Claremont-Lebanon, New Hampshire-Vermont & 1,683 & 87.5 & 1.5 & $(84.6-90.3)$ \\
\hline Cleveland-Elyria, Ohio & 962 & 84.8 & 1.7 & $(81.4-88.1)$ \\
\hline College Station-Bryan, Texas & 565 & 83.4 & 2.6 & $(78.4-88.4)$ \\
\hline Colorado Springs, Colorado & 1,298 & 88.4 & 1.0 & $(86.3-90.4)$ \\
\hline Columbia, South Carolina & 1,206 & 82.2 & 1.4 & $(79.5-84.9)$ \\
\hline Columbus, Ohio & 1,651 & 83.4 & 1.4 & $(80.7-86.2)$ \\
\hline Corpus Christi, Texas & 606 & 75.7 & 3.5 & $(68.7-82.6)$ \\
\hline Dallas-Plano-Irving, Texas ${ }^{\S}$ & 1,269 & 84.3 & 1.5 & $(81.3-87.3)$ \\
\hline Dayton, Ohio & 564 & 81.6 & 2.3 & $(77.1-86.0)$ \\
\hline Denver-Aurora-Lakewood, Colorado & 5,782 & 87.2 & 0.5 & $(86.1-88.2)$ \\
\hline Des Moines-West Des Moines, lowa & 1,357 & 87.9 & 1.2 & $(85.6-90.2)$ \\
\hline Duluth, Minnesota-Wisconsin & 945 & 85.4 & 1.5 & $(82.4-88.4)$ \\
\hline El Paso, Texas & 695 & 74.3 & 2.1 & $(70.2-78.5)$ \\
\hline Evansville, Indiana-Kentucky & 652 & 83.4 & 2.1 & $(79.2-87.5)$ \\
\hline Fargo, North Dakota-Minnesota & 1,150 & 87.7 & 1.2 & $(85.2-90.1)$ \\
\hline Fayetteville-Springdale-Rogers, Arkansas-Missouri & 810 & 80.7 & 2.1 & $(76.7-84.8)$ \\
\hline Fort Wayne, Indiana & 860 & 83.3 & 2.1 & $(79.2-87.4)$ \\
\hline Fort Worth-Arlington, Texas ${ }^{\S}$ & 742 & 80.4 & 2.2 & $(76.0-84.8)$ \\
\hline Grand Island, Nebraska & 1,058 & 80.3 & 2.0 & $(76.3-84.3)$ \\
\hline Grand Rapids-Wyoming, Michigan & 897 & 87.9 & 1.7 & $(84.6-91.3)$ \\
\hline Greensboro-High Point, North Carolina & 519 & 81.1 & 2.1 & $(77.0-85.3)$ \\
\hline Greenville-Anderson-Mauldin, South Carolina & 1,490 & 83.3 & 1.3 & $(80.8-85.7)$ \\
\hline Hagerstown-Martinsburg, Maryland-West Virginia & 780 & 82.3 & 2.2 & $(78.0-86.6)$ \\
\hline Hartford-West Hartford-East Hartford, Connecticut & 2,639 & 87.0 & 0.9 & $(85.2-88.7)$ \\
\hline Hilton Head Island-Bluffton-Beaufort, South Carolina & 550 & 85.1 & 2.6 & $(80.1-90.1)$ \\
\hline Houston-The Woodlands-Sugar Land, Texas & 2,115 & 81.2 & 1.4 & $(78.6-83.9)$ \\
\hline Huntington-Ashland, West Virginia-Kentucky-Ohio & 1,246 & 77.6 & 1.4 & $(74.8-80.4)$ \\
\hline Idaho Falls, Idaho & 516 & 86.4 & 1.7 & $(83.0-89.8)$ \\
\hline Indianapolis-Carmel-Anderson, Indiana & 3,576 & 82.8 & 0.9 & $(81.1-84.5)$ \\
\hline Jacksonville, Florida & 670 & 83.8 & 1.9 & $(80.0-87.6)$ \\
\hline Kansas City, Missouri-Kansas & 4,873 & 85.1 & 0.9 & $(83.4-86.9)$ \\
\hline Kingsport-Bristol-Bristol, Tennessee-Virginia & 505 & 76.7 & 2.8 & $(71.1-82.2)$ \\
\hline Knoxville, Tennessee & 564 & 73.0 & 3.1 & $(66.9-79.1)$ \\
\hline Lafayette, Louisiana & 561 & 79.8 & 2.0 & $(75.8-83.7)$ \\
\hline Lexington-Fayette, Kentucky & 624 & 82.5 & 2.0 & $(78.6-86.4)$ \\
\hline Lincoln, Nebraska & 2,007 & 90.8 & 0.8 & $(89.2-92.3)$ \\
\hline
\end{tabular}

See table footnotes on page 19. 
TABLE 4. (Continued) Age-adjusted* prevalence estimates of adults aged $\geq 18$ years who reported good or better health, ${ }^{\dagger}$ by metropolitan and micropolitan statistical area - Behavioral Risk Factor Surveillance System, United States, 2014

\begin{tabular}{|c|c|c|c|c|}
\hline MMSA & $\begin{array}{l}\text { Sample } \\
\text { size }\end{array}$ & $\%$ & SE & $95 \% \mathrm{Cl}$ \\
\hline Little Rock-North Little Rock-Conway, Arkansas & 1,179 & 81.5 & 1.7 & $(78.2-84.8)$ \\
\hline Logan, Utah-Idaho & 624 & 92.2 & 1.1 & $(90.0-94.3)$ \\
\hline Los Angeles-Long Beach-Anaheim, California & 2,449 & 80.1 & 1.0 & $(78.2-82.1)$ \\
\hline Louisville-Jefferson County, Kentucky-Indiana & 2,454 & 79.6 & 1.6 & $(76.5-82.7)$ \\
\hline Madison, Wisconsin & 549 & 90.7 & 1.5 & $(87.8-93.6)$ \\
\hline Memphis, Tennessee-Mississippi-Arkansas & 881 & 82.0 & 2.0 & $(78.0-86.0)$ \\
\hline Miami-Fort Lauderdale-West Palm Beach, Florida & 2,212 & 81.3 & 1.1 & $(79.1-83.6)$ \\
\hline Milwaukee-Waukesha-West Allis, Wisconsin & 1,353 & 80.9 & 1.8 & $(77.3-84.5)$ \\
\hline Minneapolis-St. Paul-Bloomington, Minnesota-Wisconsin & 8,745 & 89.1 & 0.4 & $(88.3-89.9)$ \\
\hline Minot, North Dakota & 591 & 86.9 & 1.9 & $(83.2-90.6)$ \\
\hline Montgomery, Alabama & 513 & 78.1 & 2.3 & $(73.6-82.6)$ \\
\hline $\begin{array}{l}\text { Montgomery County-Bucks County-Chester County, } \\
\text { Pennsylvania }\end{array}$ & 796 & 90.9 & 1.2 & $(88.5-93.3)$ \\
\hline $\begin{array}{l}\text { Myrtle Beach-Conway-North Myrtle Beach, South Carolina- } \\
\text { North Carolina }\end{array}$ & 995 & 81.2 & 1.4 & $(78.4-84.0)$ \\
\hline Nashville-Davidson County-Murfreesboro-Franklin, Tennessee & 803 & 81.3 & 1.7 & $(77.9-84.6)$ \\
\hline Nassau County-Suffolk County, New York ${ }^{\S}$ & 765 & 84.8 & 1.8 & $(81.2-88.4)$ \\
\hline Newark, New Jersey-Pennsylvania ${ }^{\S}$ & 4,141 & 85.1 & 0.9 & $(83.4-86.8)$ \\
\hline New Orleans-Metairie, Louisiana & 1,914 & 82.5 & 1.0 & $(80.6-84.4)$ \\
\hline New York-Jersey City-White Plains, New York-New Jersey ${ }^{\S}$ & 7,486 & 81.5 & 0.7 & $(80.1-82.8)$ \\
\hline Norfolk, Nebraska & 995 & 84.0 & 1.4 & $(81.3-86.8)$ \\
\hline North Platte, Nebraska & 964 & 84.7 & 1.4 & $(81.9-87.4)$ \\
\hline North Port-Sarasota-Bradenton, Florida & 506 & 83.6 & 3.0 & $(77.8-89.5)$ \\
\hline Oakland-Hayward-Berkeley, Californias & 699 & 86.8 & 1.5 & $(83.9-89.7)$ \\
\hline Ogden-Clearfield, Utah & 2,922 & 87.6 & 0.7 & $(86.2-89.0)$ \\
\hline Oklahoma City, Oklahoma & 2,432 & 83.3 & 0.9 & $(81.6-85.1)$ \\
\hline Omaha-Council Bluffs, Nebraska-lowa & 4,861 & 87.6 & 0.7 & $(86.3-88.9)$ \\
\hline Orlando-Kissimmee-Sanford, Florida & 952 & 81.6 & 1.8 & $(78.1-85.1)$ \\
\hline Philadelphia, Pennsylvania ${ }^{\S}$ & 1,509 & 78.4 & 1.5 & $(75.5-81.4)$ \\
\hline Phoenix-Mesa-Scottsdale, Arizona & 9,380 & 82.3 & 0.6 & $(81.1-83.6)$ \\
\hline Pittsburgh, Pennsylvania & 2,402 & 84.4 & 1.0 & $(82.5-86.3)$ \\
\hline Ponce, Puerto Rico & 530 & 63.3 & 2.4 & $(58.6-68.1)$ \\
\hline Portland-South Portland, Maine & 2,746 & 87.9 & 1.0 & $(86.0-89.8)$ \\
\hline Portland-Vancouver-Hillsboro, Oregon-Washington & 2,840 & 87.1 & 0.8 & $(85.5-88.7)$ \\
\hline Providence-Warwick, Rhode Island-Massachusetts & 8,092 & 84.8 & 0.7 & $(83.4-86.2)$ \\
\hline Provo-Orem, Utah & 2,146 & 88.2 & 0.8 & $(86.7-89.8)$ \\
\hline Raleigh, North Carolina & 720 & 85.6 & 1.5 & $(82.7-88.6)$ \\
\hline Rapid City, South Dakota & 1,420 & 85.7 & 1.4 & $(82.9-88.5)$ \\
\hline Reno, Nevada & 1,201 & 83.4 & 1.5 & $(80.4-86.3)$ \\
\hline Richmond, Virginia & 1,462 & 85.9 & 1.1 & $(83.7-88.2)$ \\
\hline Riverside-San Bernardino-Ontario, California & 940 & 80.5 & 1.6 & $(77.4-83.7)$ \\
\hline Roanoke, Virginia & 531 & 86.6 & 1.7 & $(83.2-89.9)$ \\
\hline Rochester, Minnesota & 699 & 90.6 & 1.3 & $(88.0-93.2)$ \\
\hline Rockingham County-Strafford County, New Hampshire ${ }^{\S}$ & 1,441 & 87.2 & 1.3 & $(84.7-89.7)$ \\
\hline Sacramento-Roseville-Arden-Arcade, California & 642 & 84.2 & 1.7 & $(80.8-87.6)$ \\
\hline St. Cloud, Minnesota & 559 & 90.3 & 1.5 & $(87.4-93.3)$ \\
\hline St. Louis, Missouri-Illinois & 1,924 & 85.4 & 1.1 & $(83.1-87.6)$ \\
\hline Salisbury, Maryland-Delaware & 1,954 & 82.9 & 1.4 & $(80.1-85.7)$ \\
\hline Salt Lake City, Utah & 5,406 & 86.8 & 0.5 & $(85.7-87.8)$ \\
\hline San Antonio-New Braunfels, Texas & 2,240 & 81.4 & 1.0 & (79.4-83.5) \\
\hline San Juan-Carolina-Caguas, Puerto Rico & 3,745 & 67.2 & 0.9 & $(65.4-68.9)$ \\
\hline Scottsbluff, Nebraska & 903 & 80.6 & 1.9 & (76.9-84.3) \\
\hline Seattle-Bellevue-Everett, Washington $\S$ & 3,689 & 86.5 & 0.8 & $(84.8-88.1)$ \\
\hline Shreveport-Bossier City, Louisiana & 549 & 75.1 & 2.3 & $(70.6-79.7)$ \\
\hline Silver Spring-Frederick-Rockville, Maryland ${ }^{\S}$ & 2,379 & 86.8 & 1.6 & $(83.7-89.8)$ \\
\hline Sioux City, lowa-Nebraska-South Dakota & 1,142 & 83.3 & 2.4 & $(78.6-88.0)$ \\
\hline Sioux Falls, South Dakota & 1,344 & 87.0 & 1.5 & $(84.0-89.9)$ \\
\hline Spartanburg, South Carolina & 561 & 79.8 & 2.5 & $(74.9-84.7)$ \\
\hline Spokane-Spokane Valley, Washington & 726 & 83.2 & 2.0 & $(79.3-87.2)$ \\
\hline Springfield, Massachusetts & 1,105 & 81.3 & 1.7 & $(78.0-84.6)$ \\
\hline Tampa-St. Petersburg-Clearwater, Florida & 1,571 & 82.1 & 1.5 & $(79.2-84.9)$ \\
\hline
\end{tabular}

See table footnotes on page 19. 
TABLE 4. (Continued) Age-adjusted* prevalence estimates of adults aged $\geq 18$ years who reported good or better health, ${ }^{\dagger}$ by metropolitan and micropolitan statistical area - Behavioral Risk Factor Surveillance System, United States, 2014

\begin{tabular}{|c|c|c|c|c|}
\hline MMSA & $\begin{array}{l}\text { Sample } \\
\text { size }\end{array}$ & $\%$ & SE & $95 \% \mathrm{Cl}$ \\
\hline Toledo, Ohio & 649 & 76.6 & 2.6 & $(71.6-81.7)$ \\
\hline Topeka, Kansas & 1,442 & 82.8 & 1.3 & $(80.2-85.3)$ \\
\hline Tulsa, Oklahoma & 2,028 & 82.0 & 1.1 & $(79.8-84.1)$ \\
\hline Tuscaloosa, Alabama & 717 & 78.5 & 1.9 & $(74.7-82.2)$ \\
\hline Virginia Beach-Norfolk-Newport News, Virginia-North Carolina & 1,877 & 83.0 & 1.3 & $(80.5-85.5)$ \\
\hline Warren-Troy-Farmington Hills, Michigan $§$ & 2,115 & 85.1 & 1.1 & $(83.0-87.2)$ \\
\hline $\begin{array}{l}\text { Washington-Arlington-Alexandria, District of Columbia-Virginia- } \\
\text { Maryland-West Virginia§ }\end{array}$ & 8,287 & 88.1 & 0.7 & $(86.8-89.4)$ \\
\hline Wichita, Kansas & 2,732 & 84.5 & 0.8 & $(82.9-86.1)$ \\
\hline Wichita Falls, Texas & 539 & 82.1 & 2.7 & $(76.8-87.3)$ \\
\hline Wilmington, Delaware-Maryland-New Jersey ${ }^{\S}$ & 2,760 & 85.2 & 1.0 & $(83.3-87.1)$ \\
\hline Worcester, Massachusetts-Connecticut & 2,461 & 86.0 & 1.1 & $(83.9-88.2)$ \\
\hline Youngstown-Warren-Boardman, Ohio-Pennsylvania & 525 & 84.6 & 2.0 & $(80.7-88.5)$ \\
\hline Median & & 83.7 & & \\
\hline Range & & $63.3-92.2$ & & \\
\hline
\end{tabular}

Abbreviations: $\mathrm{Cl}=$ confidence interval; $\mathrm{MMSA}=$ metropolitan and micropolitan statistical areas; $\mathrm{SE}=$ standard error.

* Age adjusted to the 2000 U.S. standard population.

† Respondents were asked to rate their general health as poor, fair, good, very good, or excellent. Respondents were classified into two groups: those who reported fair or poor health and those who reported good, very good, or excellent health.

$\S$ Metropolitan division. 
TABLE 5. Age adjusted* prevalence estimates of adults aged $\geq 18$ years who reported $\geq 14$ days of frequent mental distress during the past 30 days, ${ }^{\dagger}$ by state/territory - Behavioral Risk Factor Surveillance System, United States, 2013

\begin{tabular}{|c|c|c|c|c|}
\hline State/Territory & $\begin{array}{l}\text { Sample } \\
\text { size }\end{array}$ & $\%$ & SE & $95 \% \mathrm{Cl}$ \\
\hline Alabama & 6,334 & 14.4 & 0.7 & $(13.1-15.7)$ \\
\hline Alaska & 4,505 & 9.1 & 0.6 & $(7.8-10.3)$ \\
\hline Arizona & 4,180 & 12.3 & 1.1 & $(10.2-14.4)$ \\
\hline Arkansas & 5,139 & 14.9 & 0.8 & $(13.3-16.4)$ \\
\hline California & 11,410 & 11.6 & 0.4 & $(10.8-12.4)$ \\
\hline Colorado & 13,453 & 9.6 & 0.4 & $(8.9-10.3)$ \\
\hline Connecticut & 7,592 & 10.4 & 0.5 & $(9.4-11.5)$ \\
\hline Delaware & 5,134 & 11.2 & 0.7 & $(9.9-12.5)$ \\
\hline District of Columbia & 4,832 & 10.2 & 0.8 & (8.8-11.7) \\
\hline Florida & 33,227 & 12.8 & 0.5 & $(11.9-13.7)$ \\
\hline Georgia & 7,982 & 10.5 & 0.5 & (9.6-11.5) \\
\hline Hawaii & 7,783 & 8.3 & 0.4 & $(7.4-9.2)$ \\
\hline Idaho & 5,523 & 11.4 & 0.7 & $(10.1-12.7)$ \\
\hline Illinois & 5,564 & 11.0 & 0.7 & (9.7-12.3) \\
\hline Indiana & 10,088 & 12.5 & 0.5 & $(11.5-13.5)$ \\
\hline lowa & 8,058 & 9.0 & 0.5 & $(8.1-10.0)$ \\
\hline Kansas & 22,972 & 9.8 & 0.3 & $(9.3-10.3)$ \\
\hline Kentucky & 10,851 & 14.3 & 0.6 & (13.3-15.4) \\
\hline Louisiana & 5,147 & 13.7 & 0.9 & $(12.0-15.5)$ \\
\hline Maine & 7,950 & 12.7 & 0.6 & (11.5-13.9) \\
\hline Maryland & 12,779 & 10.0 & 0.5 & (9.1-10.9) \\
\hline Massachusetts & 14,792 & 12.0 & 0.5 & $(11.1-13.0)$ \\
\hline Michigan & 12,601 & 12.0 & 0.4 & $(11.1-12.9)$ \\
\hline Minnesota & 14,158 & 8.5 & 0.4 & (7.6-9.3) \\
\hline Mississippi & 7,298 & 14.2 & 0.6 & $(13.0-15.5)$ \\
\hline Missouri & 7,028 & 12.3 & 0.6 & $(11.1-13.5)$ \\
\hline Montana & 9,566 & 10.2 & 0.4 & $(9.3-11.0)$ \\
\hline Nebraska & 16,889 & 9.0 & 0.4 & (8.2-9.8) \\
\hline Nevada & 5,003 & 11.0 & 0.9 & $(9.3-12.6)$ \\
\hline New Hampshire & 6,378 & 11.1 & 0.6 & $(9.9-12.2)$ \\
\hline New Jersey & 12,996 & 9.7 & 0.4 & $(8.9-10.5)$ \\
\hline New Mexico & 9,164 & 12.2 & 0.5 & $(11.2-13.3)$ \\
\hline New York & 8,730 & 11.3 & 0.5 & $(10.3-12.3)$ \\
\hline North Carolina & 8,702 & 11.7 & 0.5 & $(10.7-12.7)$ \\
\hline North Dakota & 7,617 & 7.7 & 0.5 & (6.7-8.7) \\
\hline Ohio & 11,751 & 12.2 & 0.5 & $(11.3-13.2)$ \\
\hline Oklahoma & 8,114 & 14.3 & 0.6 & $(13.2-15.4)$ \\
\hline Oregon & 5,861 & 13.5 & 0.7 & $(12.1-14.8)$ \\
\hline Pennsylvania & 11,193 & 12.8 & 0.5 & $(11.8-13.7)$ \\
\hline Rhode Island & 6,428 & 11.6 & 0.6 & (10.4-12.9) \\
\hline South Carolina & 10,501 & 11.7 & 0.5 & $(10.7-12.6)$ \\
\hline South Dakota & 6,818 & 7.9 & 0.6 & (6.7-9.0) \\
\hline Tennessee & 5,703 & 12.1 & 0.6 & $(10.8-13.3)$ \\
\hline Texas & 10,604 & 10.0 & 0.5 & (9.1-10.9) \\
\hline Utah & 12,591 & 10.4 & 0.4 & $(9.7-11.1)$ \\
\hline Vermont & 6,303 & 9.7 & 0.6 & (8.7-10.8) \\
\hline Virginia & 8,311 & 10.5 & 0.5 & (9.5-11.4) \\
\hline Washington & 11,018 & 11.7 & 0.5 & $(10.8-12.6)$ \\
\hline West Virginia & 5,798 & 15.2 & 0.6 & $(14.0-16.4)$ \\
\hline Wisconsin & 6,525 & 10.4 & 0.7 & (9.0-11.7) \\
\hline Wyoming & 6,356 & 9.8 & 0.6 & $(8.6-11.0)$ \\
\hline Guam & 1,879 & 10.1 & 0.9 & (8.4-11.8) \\
\hline Puerto Rico & 5,959 & 13.8 & 0.6 & $(12.6-15.0)$ \\
\hline Median & & 11.3 & & \\
\hline Range & & $7.7-15.2$ & & \\
\hline
\end{tabular}

Abbreviations: $\mathrm{Cl}=$ confidence interval; $\mathrm{SE}=$ standard error.

${ }^{*}$ Age adjusted to the 2000 U.S. standard population.

† Respondents were asked "How many days in the past month was your mental health (including stress, depression, and problems with emotions) not good?" 
TABLE 6. Age-adjusted* prevalence estimates of adults aged $\geq 18$ years who reported $\geq 14$ days of frequent mental distress during the past 30 days $^{\dagger}{ }^{\dagger}$ by metropolitan and micropolitan statistical area - Behavioral Risk Factor Surveillance System, United States, 2013

\begin{tabular}{|c|c|c|c|c|}
\hline MMSA & $\begin{array}{l}\text { Sample } \\
\text { size }\end{array}$ & $\%$ & SE & $95 \% \mathrm{Cl}$ \\
\hline Aguadilla-Isabela, Puerto Rico & 586 & 12.6 & 1.8 & $(9.1-16.0)$ \\
\hline Akron, Ohio & 680 & 19.4 & 2.9 & $(13.7-25.1)$ \\
\hline Albuquerque, New Mexico & 2,055 & 12.4 & 1.0 & $(10.4-14.4)$ \\
\hline Allentown-Bethlehem-Easton, Pennsylvania-New Jersey & 1,011 & 12.9 & 1.8 & $(9.4-16.4)$ \\
\hline Anchorage, Alaska & 1,501 & 8.9 & 1.0 & $(6.9-10.8)$ \\
\hline Atlanta-Sandy Springs-Roswell, Georgia & 3,451 & 9.2 & 0.7 & $(7.9-10.5)$ \\
\hline Augusta-Richmond County, Georgia-South Carolina & 893 & 16.3 & 2.4 & $(11.6-21.0)$ \\
\hline Austin-Round Rock, Texas & 915 & 11.7 & 1.5 & $(8.8-14.6)$ \\
\hline Baltimore-Columbia-Towson, Maryland & 4,679 & 11.0 & 0.8 & $(9.4-12.5)$ \\
\hline Baton Rouge, Louisiana & 914 & 8.8 & 1.3 & $(6.2-11.4)$ \\
\hline Billings, Montana & 813 & 10.2 & 1.2 & $(7.9-12.6)$ \\
\hline Birmingham-Hoover, Alabama & 1,335 & 14.0 & 1.2 & $(11.5-16.4)$ \\
\hline Bismarck, North Dakota & 1,011 & 7.5 & 1.2 & $(5.1-9.8)$ \\
\hline Boise City, Idaho & 1,470 & 10.8 & 1.1 & $(8.6-13.1)$ \\
\hline Boston, Massachusetts $\S$ & 4,003 & 11.9 & 0.9 & $(10.2-13.7)$ \\
\hline Buffalo-Cheektowaga-Niagara Falls, New York & 497 & 11.4 & 2.2 & $(7.1-15.8)$ \\
\hline Burlington-South Burlington, Vermont & 1,617 & 8.7 & 0.9 & $(7.0-10.4)$ \\
\hline Cambridge-Newton-Framingham, Massachusetts ${ }^{\S}$ & 4,821 & 10.6 & 0.8 & $(9.1-12.2)$ \\
\hline Camden, New Jersey ${ }^{\S}$ & 1,823 & 11.0 & 1.0 & $(9.0-13.0)$ \\
\hline Cedar Rapids, lowa & 641 & 10.0 & 1.9 & $(6.2-13.8)$ \\
\hline Charleston, West Virginia & 810 & 15.2 & 1.6 & $(12.1-18.4)$ \\
\hline Charleston-North Charleston, South Carolina & 1,527 & 10.6 & 1.1 & $(8.4-12.8)$ \\
\hline Charlotte-Concord-Gastonia, North Carolina-South Carolina & 1,918 & 12.0 & 1.1 & $(9.9-14.1)$ \\
\hline Chattanooga, Tennessee-Georgia & 572 & 11.1 & 2.1 & $(7.0-15.2)$ \\
\hline Chicago-Naperville-Elgin, Illinois-Indiana-Wisconsin & 3,308 & 12.4 & 0.9 & $(10.7-14.1)$ \\
\hline Cincinnati, Ohio-Kentucky-Indiana & 2,566 & 10.8 & 1.0 & $(8.8-12.8)$ \\
\hline Claremont-Lebanon, New Hampshire-Vermont & 1,656 & 10.0 & 1.2 & $(7.7-12.3)$ \\
\hline Cleveland-Elyria, Ohio & 1,099 & 9.4 & 1.2 & $(7.1-11.7)$ \\
\hline Colorado Springs, Colorado & 1,361 & 11.5 & 1.2 & $(9.2-13.8)$ \\
\hline Columbia, South Carolina & 1,411 & 9.6 & 1.1 & $(7.4-11.7)$ \\
\hline Columbus, Ohio & 1,834 & 13.5 & 1.1 & $(11.4-15.6)$ \\
\hline Crestview-Fort Walton Beach-Destin, Florida & 1,052 & 10.8 & 1.3 & $(8.2-13.5)$ \\
\hline Dallas-Plano-Irving, Texas $§$ & 877 & 9.7 & 1.3 & $(7.1-12.2)$ \\
\hline Davenport-Moline-Rock Island, lowa-Illinois & 665 & 10.1 & 1.9 & $(6.5-13.8)$ \\
\hline Dayton, Ohio & 829 & 12.4 & 1.7 & $(9.0-15.8)$ \\
\hline Deltona-Daytona Beach-Ormond Beach, Florida & 1,082 & 15.4 & 2.1 & $(11.2-19.5)$ \\
\hline Denver-Aurora-Lakewood, Colorado & 5,633 & 9.0 & 0.5 & $(8.1-10.0)$ \\
\hline Des Moines-West Des Moines, lowa & 1,332 & 10.4 & 1.2 & $(8.0-12.8)$ \\
\hline Duluth, Minnesota-Wisconsin & 694 & 11.2 & 2.2 & $(6.9-15.5)$ \\
\hline Durham-Chapel Hill, North Carolina & 606 & 7.3 & 1.7 & $(4.0-10.6)$ \\
\hline El Paso, Texas & 730 & 9.4 & 1.3 & $(6.8-11.9)$ \\
\hline Evansville, Indiana-Kentucky & 564 & 14.7 & 2.2 & $(10.3-19.1)$ \\
\hline Fargo, North Dakota-Minnesota & 1,164 & 10.6 & 1.8 & $(7.1-14.1)$ \\
\hline Fayetteville-Springdale-Rogers, Arkansas-Missouri & 808 & 15.8 & 2.2 & $(11.4-20.2)$ \\
\hline Fort Smith, Arkansas-Oklahoma & 490 & 17.6 & 2.5 & $(12.8-22.4)$ \\
\hline Fort Wayne, Indiana & 766 & 10.9 & 1.7 & $(7.5-14.2)$ \\
\hline Fort Worth-Arlington, Texas $§$ & 793 & 10.6 & 1.6 & $(7.5-13.8)$ \\
\hline Gainesville, Florida & 992 & 11.5 & 1.7 & $(8.3-14.7)$ \\
\hline Grand Forks, North Dakota-Minnesota & 495 & 6.3 & 1.5 & $(3.4-9.2)$ \\
\hline Grand Island, Nebraska & 785 & 12.2 & 1.9 & $(8.5-15.9)$ \\
\hline Grand Rapids-Wyoming, Michigan & 1,334 & 10.7 & 1.3 & $(8.2-13.2)$ \\
\hline Greensboro-High Point, North Carolina & 655 & 10.5 & 1.5 & $(7.4-13.5)$ \\
\hline Greenville-Anderson-Mauldin, South Carolina & 1,327 & 13.5 & 1.4 & $(10.7-16.2)$ \\
\hline Gulfport-Biloxi-Pascagoula, Mississippi & 756 & 16.6 & 1.9 & $(12.8-20.3)$ \\
\hline Hagerstown-Martinsburg, Maryland-West Virginia & 754 & 11.2 & 1.5 & $(8.2-14.2)$ \\
\hline Hartford-West Hartford-East Hartford, Connecticut & 2,811 & 10.2 & 0.9 & $(8.5-11.9)$ \\
\hline Hilton Head Island-Bluffton-Beaufort, South Carolina & 817 & 10.4 & 2.1 & $(6.4-14.5)$ \\
\hline Houston-The Woodlands-Sugar Land, Texas & 1,359 & 8.9 & 1.1 & $(6.8-11.0)$ \\
\hline Huntington-Ashland, West Virginia-Kentucky-Ohio & 1,155 & 16.2 & 1.5 & $(13.3-19.1)$ \\
\hline Idaho Falls, Idaho & 499 & 13.5 & 2.2 & $(9.2-17.7)$ \\
\hline Indianapolis-Carmel-Anderson, Indiana & 2,490 & 11.7 & 0.9 & $(10.0-13.4)$ \\
\hline Jackson, Mississippi & 795 & 11.5 & 1.5 & $(8.5-14.4)$ \\
\hline
\end{tabular}

See table footnotes on page 23. 
TABLE 6. (Continued) Age-adjusted* prevalence estimates of adults aged $\geq 18$ years who reported $\geq 14$ days of frequent mental distress during the past 30 days $^{\dagger}{ }^{\dagger}$ by metropolitan and micropolitan statistical area - Behavioral Risk Factor Surveillance System, United States, 2013

\begin{tabular}{|c|c|c|c|c|}
\hline MMSA & $\begin{array}{l}\text { Sample } \\
\text { size }\end{array}$ & $\%$ & SE & $95 \% \mathrm{Cl}$ \\
\hline Jacksonville, Florida & 2,820 & 13.7 & 1.0 & $(11.7-15.7)$ \\
\hline Kansas City, Missouri-Kansas & 7,350 & 10.4 & 0.8 & $(8.8-11.9)$ \\
\hline Kingsport-Bristol-Bristol, Tennessee-Virginia & 524 & 18.0 & 3.0 & $(12.1-23.9)$ \\
\hline Knoxville, Tennessee & 643 & 14.6 & 1.9 & $(11.0-18.3)$ \\
\hline Lansing-East Lansing, Michigan & 680 & 11.3 & 1.6 & $(8.3-14.4)$ \\
\hline Lexington-Fayette, Kentucky & 633 & 12.3 & 1.6 & $(9.1-15.5)$ \\
\hline Lincoln, Nebraska & 1,851 & 9.3 & 0.9 & $(7.6-11.0)$ \\
\hline Little Rock-North Little Rock-Conway, Arkansas & 1,120 & 13.5 & 1.5 & $(10.6-16.5)$ \\
\hline Logan, Utah-Idaho & 628 & 9.9 & 1.6 & $(6.7-13.1)$ \\
\hline Los Angeles-Long Beach-Anaheim, California & 3,011 & 10.6 & 0.8 & $(9.1-12.1)$ \\
\hline Louisville/Jefferson County, Kentucky-Indiana & 2,120 & 14.3 & 1.3 & $(11.7-16.8)$ \\
\hline Lubbock, Texas & 520 & 9.0 & 2.0 & $(5.1-13.0)$ \\
\hline Manhattan, Kansas & 651 & 9.9 & 1.4 & $(7.2-12.6)$ \\
\hline Memphis, Tennessee-Mississippi-Arkansas & 1,185 & 8.5 & 1.1 & $(6.4-10.6)$ \\
\hline Miami-Fort Lauderdale-West Palm Beach, Florida & 2,146 & 13.0 & 1.2 & $(10.6-15.4)$ \\
\hline Milwaukee-Waukesha-West Allis, Wisconsin & 1,251 & 14.0 & 1.9 & $(10.3-17.7)$ \\
\hline Minneapolis-St. Paul-Bloomington, Minnesota-Wisconsin & 9,016 & 8.1 & 0.5 & $(7.1-9.2)$ \\
\hline Minot, North Dakota & 638 & 6.3 & 1.5 & $(3.4-9.2)$ \\
\hline $\begin{array}{l}\text { Montgomery County-Bucks County-Chester County, } \\
\text { Pennsylvania }\end{array}$ & 957 & 11.7 & 1.4 & $(8.9-14.4)$ \\
\hline $\begin{array}{l}\text { Myrtle Beach-Conway-North Myrtle Beach, South Carolina- } \\
\text { North Carolina }\end{array}$ & 762 & 12.0 & 1.8 & $(8.4-15.6)$ \\
\hline Nashville-Davidson County-Murfreesboro-Franklin, Tennessee & 1,043 & 9.5 & 1.3 & $(7.0-12.0)$ \\
\hline Nassau County-Suffolk County, New York ${ }^{\S}$ & 916 & 8.8 & 1.1 & $(6.7-11.0)$ \\
\hline Newark, New Jersey-Pennsylvania ${ }^{\S}$ & 4,005 & 10.1 & 0.8 & $(8.6-11.6)$ \\
\hline New Orleans-Metairie, Louisiana & 1,267 & 16.1 & 2.0 & $(12.2-20.1)$ \\
\hline New York-Jersey City-White Plains, New York-New Jersey $\$$ & 8,688 & 11.0 & 0.6 & $(9.9-12.1)$ \\
\hline Norfolk, Nebraska & 664 & 9.3 & 1.4 & $(6.6-11.9)$ \\
\hline North Platte, Nebraska & 704 & 8.9 & 1.9 & $(5.2-12.5)$ \\
\hline North Port-Sarasota-Bradenton, Florida & 1,061 & 13.8 & 1.7 & $(10.5-17.1)$ \\
\hline Oakland-Hayward-Berkeley, California ${ }^{\S}$ & 694 & 9.9 & 1.5 & $(6.8-12.9)$ \\
\hline Ogden-Clearfield, Utah & 2,430 & 10.0 & 0.7 & $(8.5-11.5)$ \\
\hline Oklahoma City, Oklahoma & 2,612 & 11.8 & 0.9 & $(10.1-13.4)$ \\
\hline Omaha-Council Bluffs, Nebraska-lowa & 3,090 & 8.9 & 0.7 & $(7.6-10.3)$ \\
\hline Orlando-Kissimmee-Sanford, Florida & 2,231 & 11.1 & 1.0 & $(9.0-13.1)$ \\
\hline Panama City, Florida & 1,001 & 15.3 & 1.9 & $(11.7-19.0)$ \\
\hline Pensacola-Ferry Pass-Brent, Florida & 1,291 & 10.5 & 1.1 & $(8.4-12.6)$ \\
\hline Philadelphia, Pennsylvania $\$$ & 1,733 & 14.1 & 1.3 & $(11.7-16.6)$ \\
\hline Phoenix-Mesa-Scottsdale, Arizona & 1,521 & 13.0 & 1.5 & $(10.1-15.9)$ \\
\hline Pittsburgh, Pennsylvania & 2,324 & 12.3 & 1.0 & $(10.3-14.3)$ \\
\hline Ponce, Puerto Rico & 528 & 12.8 & 2.2 & $(8.5-17.1)$ \\
\hline Portland-South Portland, Maine & 2,597 & 13.5 & 1.1 & $(11.4-15.5)$ \\
\hline Portland-Vancouver-Hillsboro, Oregon-Washington & 3,205 & 12.0 & 0.8 & $(10.4-13.6)$ \\
\hline Port St. Lucie, Florida & 996 & 12.3 & 1.9 & $(8.6-16.0)$ \\
\hline Providence-Warwick, Rhode Island-Massachusetts & 8,165 & 12.9 & 0.8 & $(11.4-14.4)$ \\
\hline Provo-Orem, Utah & 1,832 & 8.6 & 0.8 & $(7.1-10.1)$ \\
\hline Raleigh, North Carolina & 667 & 9.2 & 1.2 & $(6.8-11.6)$ \\
\hline Rapid City, South Dakota & 865 & 9.6 & 1.4 & $(6.8-12.4)$ \\
\hline Reno, Nevada & 1,783 & 11.9 & 1.2 & $(9.5-14.3)$ \\
\hline Richmond, Virginia & 1,293 & 11.5 & 1.3 & $(9.0-14.0)$ \\
\hline Riverside-San Bernardino-Ontario, California & 1,355 & 13.4 & 1.2 & $(10.9-15.8)$ \\
\hline Rochester, New York & 498 & 10.7 & 1.8 & $(7.3-14.1)$ \\
\hline Rockingham County-Strafford County, New Hampshire ${ }^{\S}$ & 1,641 & 10.7 & 1.1 & $(8.5-12.9)$ \\
\hline Sacramento-Roseville-Arden-Arcade, California & 885 & 14.6 & 1.9 & $(11.0-18.3)$ \\
\hline St. Louis, Missouri-Illinois & 2,038 & 10.7 & 1.0 & $(8.7-12.6)$ \\
\hline Salem, Oregon & 520 & 12.1 & 2.5 & $(7.2-17.1)$ \\
\hline Salina, Kansas & 519 & 9.2 & 1.7 & $(5.7-12.6)$ \\
\hline Salisbury, Maryland-Delaware & 2,029 & 9.9 & 1.2 & $(7.5-12.3)$ \\
\hline Salt Lake City, Utah & 4,626 & 10.6 & 0.6 & $(9.5-11.7)$ \\
\hline San Antonio-New Braunfels, Texas & 918 & 10.6 & 1.4 & $(7.8-13.3)$ \\
\hline San Francisco-Redwood City-South San Francisco, California & 532 & 10.0 & 1.7 & $(6.7-13.3)$ \\
\hline
\end{tabular}

See table footnotes on page 23. 
TABLE 6. (Continued) Age-adjusted* prevalence estimates of adults aged $\geq 18$ years who reported $\geq 14$ days of frequent mental distress during the past 30 days, ${ }^{\dagger}$ by metropolitan and micropolitan statistical area - Behavioral Risk Factor Surveillance System, United States, 2013

\begin{tabular}{|c|c|c|c|c|}
\hline MMSA & $\begin{array}{l}\text { Sample } \\
\text { size }\end{array}$ & $\%$ & SE & $95 \% \mathrm{Cl}$ \\
\hline San Jose-Sunnyvale-Santa Clara, California & 622 & 8.2 & 1.4 & $(5.4-11.0)$ \\
\hline San Juan-Carolina-Caguas, Puerto Rico & 3,626 & 14.3 & 0.8 & $(12.8-15.9)$ \\
\hline Scottsbluff, Nebraska & 703 & 8.7 & 1.4 & $(5.9-11.5)$ \\
\hline Scranton-Wilkes-Barre-Hazleton, Pennsylvania & 554 & 12.8 & 2.1 & $(8.6-17.0)$ \\
\hline Seattle-Bellevue-Everett, Washington $\S$ & 3,734 & 11.1 & 0.7 & $(9.7-12.6)$ \\
\hline Shreveport-Bossier City, Louisiana & 561 & 10.7 & 1.9 & $(7.0-14.5)$ \\
\hline Silver Spring-Frederick-Rockville, Maryland ${ }^{\S}$ & 2,385 & 8.1 & 1.0 & $(6.2-10.1)$ \\
\hline Sioux City, lowa-Nebraska-South Dakota & 1,041 & 7.1 & 1.5 & $(4.1-10.0)$ \\
\hline Sioux Falls, South Dakota & 1,003 & 6.5 & 1.3 & $(4.1-9.0)$ \\
\hline Spartanburg, South Carolina & 580 & 8.7 & 1.7 & $(5.5-12.0)$ \\
\hline Spokane-Spokane Valley, Washington & 851 & 10.7 & 1.6 & $(7.6-13.9)$ \\
\hline Springfield, Massachusetts & 1,541 & 13.1 & 1.6 & $(10.0-16.2)$ \\
\hline Tallahassee, Florida & 1,805 & 9.6 & 1.2 & $(7.3-11.9)$ \\
\hline Tampa-St. Petersburg-Clearwater, Florida & 2,163 & 13.9 & 1.2 & $(11.6-16.1)$ \\
\hline Toledo, Ohio & 986 & 12.7 & 1.8 & $(9.2-16.2)$ \\
\hline Topeka, Kansas & 2,378 & 10.4 & 0.9 & $(8.7-12.1)$ \\
\hline Tulsa, Oklahoma & 1,973 & 14.6 & 1.2 & $(12.4-16.9)$ \\
\hline Virginia Beach-Norfolk-Newport News, Virginia-North Carolina & 1,657 & 9.5 & 1.0 & $(7.5-11.5)$ \\
\hline Warren-Troy-Farmington Hills, Michigan $\S$ & 2,237 & 9.7 & 0.9 & $(8.1-11.4)$ \\
\hline $\begin{array}{l}\text { Washington-Arlington-Alexandria, District of Columbia-Virginia- } \\
\text { Maryland-West Virginia }\end{array}$ & 8,870 & 8.7 & 0.6 & $(7.5-10.0)$ \\
\hline Wichita, Kansas & 4,867 & 10.5 & 0.6 & $(9.3-11.7)$ \\
\hline Wilmington, Delaware-Maryland-New Jersey ${ }^{\S}$ & 3,233 & 11.7 & 0.8 & $(10.1-13.2)$ \\
\hline Winston-Salem, North Carolina & 684 & 16.8 & 2.4 & $(12.1-21.4)$ \\
\hline Worcester, Massachusetts-Connecticut & 2,712 & 12.0 & 1.1 & $(9.7-14.2)$ \\
\hline Median & & 10.9 & & \\
\hline Range & & $6.3-19.4$ & & \\
\hline
\end{tabular}

Abbreviations: $\mathrm{Cl}=$ confidence interval; $\mathrm{MMSA}=$ metropolitan and micropolitan statistical areas; $\mathrm{SE}=$ standard error.

* Age adjusted to the 2000 U.S. standard population.

† Respondents were asked "How many days in the past month was your mental health (including stress, depression, and problems with emotions) not good?"

$\S$ Metropolitan division. 
TABLE 7. Age-adjusted* prevalence estimates of adults aged $\geq 18$ years who reported $\geq 14$ days of frequent physical distress ${ }^{\dagger}$ during the past 30 days, by state/territory - Behavioral Risk Factor Surveillance System, United States, 2014

\begin{tabular}{|c|c|c|c|c|}
\hline State/Territory & $\begin{array}{l}\text { Sample } \\
\text { size }\end{array}$ & $\%$ & SE & $95 \% \mathrm{Cl}$ \\
\hline Alabama & 8,394 & 14.6 & 0.5 & $(13.6-15.6)$ \\
\hline Alaska & 4,248 & 10.1 & 0.6 & $(8.8-11.3)$ \\
\hline Arizona & 14,540 & 11.9 & 0.4 & $(11.1-12.8)$ \\
\hline Arkansas & 5,088 & 14.6 & 0.7 & $(13.1-16.0)$ \\
\hline California & 8,755 & 12.5 & 0.5 & $(11.6-13.4)$ \\
\hline Colorado & 13,153 & 9.5 & 0.3 & $(8.9-10.1)$ \\
\hline Connecticut & 7,835 & 9.2 & 0.5 & $(8.2-10.1)$ \\
\hline Delaware & 4,178 & 10.5 & 0.6 & $(9.3-11.8)$ \\
\hline District of Columbia & 3,956 & 9.3 & 0.8 & $(7.8-10.8)$ \\
\hline Florida & 9,430 & 11.7 & 0.5 & $(10.8-12.6)$ \\
\hline Georgia & 6,211 & 11.9 & 0.5 & $(10.9-12.9)$ \\
\hline Hawaii & 7,196 & 8.4 & 0.5 & (7.4-9.3) \\
\hline Idaho & 5,327 & 9.3 & 0.5 & $(8.3-10.3)$ \\
\hline Illinois & 5,018 & 11.1 & 0.6 & $(9.9-12.3)$ \\
\hline Indiana & 11,152 & 12.6 & 0.4 & $(11.8-13.4)$ \\
\hline lowa & 7,993 & 9.5 & 0.4 & (8.7-10.4) \\
\hline Kansas & 13,389 & 9.6 & 0.3 & $(9.0-10.1)$ \\
\hline Kentucky & 10,967 & 16.0 & 0.6 & $(14.8-17.3)$ \\
\hline Louisiana & 6,614 & 13.5 & 0.5 & $(12.5-14.5)$ \\
\hline Maine & 8,885 & 10.9 & 0.5 & $(10.0-11.8)$ \\
\hline Maryland & 12,278 & 9.9 & 0.5 & (8.9-11.0) \\
\hline Massachusetts & 15,234 & 10.2 & 0.4 & (9.4-11.0) \\
\hline Michigan & 8,340 & 11.8 & 0.5 & $(10.9-12.8)$ \\
\hline Minnesota & 16,135 & 8.2 & 0.3 & (7.7-8.7) \\
\hline Mississippi & 4,098 & 12.9 & 0.7 & $(11.5-14.2)$ \\
\hline Missouri & 6,976 & 12.4 & 0.5 & $(11.4-13.5)$ \\
\hline Montana & 7,337 & 11.7 & 0.6 & $(10.6-12.8)$ \\
\hline Nebraska & 22,024 & 8.6 & 0.3 & (8.0-9.1) \\
\hline Nevada & 3,675 & 10.9 & 0.8 & $(9.4-12.4)$ \\
\hline New Hampshire & 6,073 & 8.9 & 0.5 & (7.9-9.9) \\
\hline New Jersey & 12,733 & 9.9 & 0.4 & $(9.1-10.6)$ \\
\hline New Mexico & 8,775 & 13.5 & 0.6 & $(12.4-14.6)$ \\
\hline New York & 6,631 & 10.8 & 0.5 & $(9.8-11.7)$ \\
\hline North Carolina & 7,134 & 11.8 & 0.5 & $(10.9-12.7)$ \\
\hline North Dakota & 7,560 & 7.8 & 0.5 & (6.9-8.7) \\
\hline Ohio & 10,661 & 11.9 & 0.5 & $(10.9-12.9)$ \\
\hline Oklahoma & 8,291 & 13.8 & 0.5 & $(12.9-14.7)$ \\
\hline Oregon & 5,093 & 13.3 & 0.6 & $(12.0-14.5)$ \\
\hline Pennsylvania & 10,668 & 11.3 & 0.4 & $(10.5-12.1)$ \\
\hline Rhode Island & 6,316 & 10.9 & 0.6 & $(9.8-12.0)$ \\
\hline South Carolina & 10,693 & 12.3 & 0.4 & $(11.5-13.1)$ \\
\hline South Dakota & 7,299 & 9.1 & 0.6 & (7.9-10.3) \\
\hline Tennessee & 4,965 & 14.2 & 0.7 & $(12.9-15.5)$ \\
\hline Texas & 14,846 & 10.8 & 0.4 & $(10.0-11.6)$ \\
\hline Utah & 14,816 & 9.9 & 0.3 & $(9.4-10.5)$ \\
\hline Vermont & 6,377 & 10.0 & 0.5 & (9.1-10.9) \\
\hline Virginia & 9,239 & 10.4 & 0.4 & $(9.6-11.2)$ \\
\hline Washington & 9,928 & 11.5 & 0.5 & $(10.6-12.4)$ \\
\hline West Virginia & 6,108 & 15.6 & 0.5 & $(14.6-16.7)$ \\
\hline Wisconsin & 6,985 & 11.1 & 0.6 & $(9.9-12.2)$ \\
\hline Wyoming & 6,292 & 9.8 & 0.6 & $(8.7-11.0)$ \\
\hline Guam & 2,493 & 9.1 & 0.8 & (7.5-10.8) \\
\hline Puerto Rico & 5,959 & 15.2 & 0.6 & $(14.1-16.3)$ \\
\hline Median & & 10.9 & & \\
\hline Range & & $7.8-16.0$ & & \\
\hline
\end{tabular}

Abbreviations: $\mathrm{Cl}=$ confidence interval; $\mathrm{SE}=$ standard error.

* Age adjusted to the 2000 U.S. standard population.

† Respondents were asked "How many days in the past month was your physical health (including physical illness and injury) not good?" 
TABLE 8. Age-adjusted* prevalence estimates of adults aged $\geq 18$ years who reported $\geq 14$ days of frequent physical distress ${ }^{\dagger}$ during the past 30 days, by metropolitan and micropolitan statistical area - Behavioral Risk Factor Surveillance System, United States, 2014

\begin{tabular}{|c|c|c|c|c|}
\hline MMSA & $\begin{array}{l}\text { Sample } \\
\text { size }\end{array}$ & $\%$ & SE & $95 \% \mathrm{Cl}$ \\
\hline Aberdeen, South Dakota & 614 & 8.7 & 1.5 & $(5.8-11.5)$ \\
\hline Aguadilla-Isabela, Puerto Rico & 544 & 18.5 & 2.0 & $(14.5-22.5)$ \\
\hline Albuquerque, New Mexico & 1,771 & 12.8 & 1.1 & $(10.7-14.9)$ \\
\hline Allentown-Bethlehem-Easton, Pennsylvania-New Jersey & 1,060 & 10.1 & 1.3 & $(7.5-12.7)$ \\
\hline Anchorage, Alaska & 1,737 & 9.7 & 0.9 & $(7.9-11.5)$ \\
\hline Atlanta-Sandy Springs-Roswell, Georgia & 2,725 & 10.1 & 0.7 & $(8.7-11.4)$ \\
\hline Augusta-Richmond County, Georgia-South Carolina & 863 & 14.7 & 2.6 & $(9.6-19.7)$ \\
\hline Austin-Round Rock, Texas & 2,195 & 8.8 & 0.7 & $(7.4-10.2)$ \\
\hline Baltimore-Columbia-Towson, Maryland & 4,509 & 10.1 & 0.9 & $(8.4-11.9)$ \\
\hline Baton Rouge, Louisiana & 900 & 11.3 & 1.2 & $(9.0-13.6)$ \\
\hline Berlin, New Hampshire-Vermont & 534 & 12.6 & 2.8 & $(7.1-18.1)$ \\
\hline Billings, Montana & 796 & 13.4 & 1.7 & $(10.2-16.7)$ \\
\hline Birmingham-Hoover, Alabama & 1,543 & 11.9 & 0.9 & $(10.0-13.7)$ \\
\hline Bismarck, North Dakota & 1,009 & 6.9 & 0.9 & $(5.1-8.7)$ \\
\hline Boise City, Idaho & 1,315 & 7.7 & 0.9 & $(5.9-9.4)$ \\
\hline Boston, Massachusetts $§$ & 4,452 & 8.8 & 0.7 & $(7.5-10.2)$ \\
\hline Burlington-South Burlington, Vermont & 1,960 & 8.3 & 0.7 & $(6.9-9.8)$ \\
\hline Cambridge-Newton-Framingham, Massachusetts ${ }^{\S}$ & 5,026 & 9.6 & 0.6 & $(8.3-10.8)$ \\
\hline Camden, New Jersey ${ }^{\S}$ & 1,684 & 10.5 & 1.1 & $(8.4-12.7)$ \\
\hline Cedar Rapids, lowa & 624 & 11.0 & 1.6 & $(7.8-14.2)$ \\
\hline Charleston, West Virginia & 865 & 16.7 & 1.5 & $(13.7-19.6)$ \\
\hline Charleston-North Charleston, South Carolina & 1,370 & 10.3 & 1.2 & $(8.0-12.6)$ \\
\hline Charlotte-Concord-Gastonia, North Carolina-South Carolina & 2,116 & 11.7 & 0.9 & $(10.0-13.5)$ \\
\hline Chicago-Naperville-Elgin, Illinois-Indiana-Wisconsin & 4,080 & 11.2 & 0.7 & $(9.8-12.7)$ \\
\hline Cincinnati, Ohio-Kentucky-Indiana & 2,017 & 12.4 & 1.1 & $(10.1-14.6)$ \\
\hline Claremont-Lebanon, New Hampshire-Vermont & 1,654 & 9.7 & 1.1 & $(7.6-11.8)$ \\
\hline Cleveland-Elyria, Ohio & 949 & 11.3 & 1.6 & $(8.2-14.4)$ \\
\hline College Station-Bryan, Texas & 556 & 12.3 & 2.7 & $(7.1-17.5)$ \\
\hline Colorado Springs, Colorado & 1,275 & 10.4 & 1.1 & $(8.2-12.6)$ \\
\hline Columbia, South Carolina & 1,184 & 11.1 & 1.0 & $(9.1-13.1)$ \\
\hline Columbus, Ohio & 1,626 & 10.9 & 1.0 & $(8.8-12.9)$ \\
\hline Corpus Christi, Texas & 588 & 12.1 & 2.0 & $(8.1-16.1)$ \\
\hline Dallas-Plano-Irving, Texas ${ }^{\S}$ & 1,255 & 8.9 & 1.1 & $(6.7-11.1)$ \\
\hline Dayton, Ohio & 552 & 13.9 & 2.1 & $(9.9-18.0)$ \\
\hline Denver-Aurora-Lakewood, Colorado & 5,724 & 9.0 & 0.4 & $(8.1-9.8)$ \\
\hline Des Moines-West Des Moines, lowa & 1,343 & 9.7 & 1.1 & $(7.6-11.9)$ \\
\hline Duluth, Minnesota-Wisconsin & 931 & 13.2 & 1.6 & $(10.2-16.3)$ \\
\hline El Paso, Texas & 679 & 11.0 & 1.3 & $(8.4-13.6)$ \\
\hline Evansville, Indiana-Kentucky & 635 & 16.7 & 2.9 & $(11.0-22.3)$ \\
\hline Fargo, North Dakota-Minnesota & 1,131 & 7.4 & 0.9 & $(5.6-9.1)$ \\
\hline Fayetteville-Springdale-Rogers, Arkansas-Missouri & 796 & 12.7 & 1.7 & $(9.4-16.0)$ \\
\hline Fort Wayne, Indiana & 843 & 11.7 & 1.8 & $(8.1-15.3)$ \\
\hline Fort Worth-Arlington, Texas ${ }^{\S}$ & 734 & 10.5 & 1.7 & $(7.2-13.8)$ \\
\hline Grand Island, Nebraska & 1,038 & 9.5 & 1.4 & $(6.8-12.3)$ \\
\hline Grand Rapids-Wyoming, Michigan & 883 & 10.7 & 1.4 & $(8.0-13.3)$ \\
\hline Greensboro-High Point, North Carolina & 506 & 12.7 & 2.4 & $(8.0-17.5)$ \\
\hline Greenville-Anderson-Mauldin, South Carolina & 1,459 & 11.6 & 1.0 & $(9.6-13.6)$ \\
\hline Hagerstown-Martinsburg, Maryland-West Virginia & 766 & 17.2 & 2.9 & $(11.6-22.9)$ \\
\hline Hartford-West Hartford-East Hartford, Connecticut & 2,600 & 9.6 & 0.8 & $(8.0-11.2)$ \\
\hline Hilton Head Island-Bluffton-Beaufort, South Carolina & 542 & 11.6 & 2.0 & $(7.7-15.6)$ \\
\hline Houston-The Woodlands-Sugar Land, Texas & 2,080 & 8.9 & 1.0 & $(7.0-10.8)$ \\
\hline Huntington-Ashland, West Virginia-Kentucky-Ohio & 1,227 & 15.3 & 1.2 & $(12.8-17.7)$ \\
\hline Idaho Falls, Idaho & 507 & 11.4 & 1.7 & $(8.0-14.8)$ \\
\hline Indianapolis-Carmel-Anderson, Indiana & 3,505 & 12.0 & 0.7 & $(10.5-13.4)$ \\
\hline Jacksonville, Florida & 650 & 10.5 & 1.5 & $(7.5-13.5)$ \\
\hline Kansas City, Missouri-Kansas & 4,780 & 10.8 & 0.8 & $(9.3-12.3)$ \\
\hline Kingsport-Bristol-Bristol, Tennessee-Virginia & 488 & 16.9 & 2.3 & $(12.3-21.5)$ \\
\hline Knoxville, Tennessee & 546 & 15.4 & 2.1 & $(11.3-19.6)$ \\
\hline Lafayette, Louisiana & 545 & 14.0 & 1.8 & $(10.4-17.6)$ \\
\hline Lexington-Fayette, Kentucky & 616 & 11.1 & 1.7 & $(7.9-14.4)$ \\
\hline Lincoln, Nebraska & 1,987 & 7.7 & 0.7 & $(6.3-9.2)$ \\
\hline
\end{tabular}

See table footnotes on page 27. 
TABLE 8. (Continued) Age-adjusted* prevalence estimates of adults aged $\geq 18$ years who reported $\geq 14$ days of frequent physical distress ${ }^{\dagger}$ during the past 30 days, by metropolitan and micropolitan statistical area - Behavioral Risk Factor Surveillance System, United States, 2014

\begin{tabular}{|c|c|c|c|c|}
\hline MMSA & $\begin{array}{l}\text { Sample } \\
\text { size }\end{array}$ & $\%$ & SE & $95 \% \mathrm{Cl}$ \\
\hline Little Rock-North Little Rock-Conway, Arkansas & 1,153 & 11.4 & 1.3 & $(8.8-13.9)$ \\
\hline Logan, Utah-Idaho & 620 & 6.2 & 1.0 & $(4.2-8.1)$ \\
\hline Los Angeles-Long Beach-Anaheim, California & 2,421 & 12.5 & 0.8 & $(10.8-14.1)$ \\
\hline Louisville-Jefferson County, Kentucky-Indiana & 2,404 & 13.4 & 1.3 & $(10.8-15.9)$ \\
\hline Madison, Wisconsin & 549 & 9.0 & 1.5 & $(6.0-12.0)$ \\
\hline Memphis, Tennessee-Mississippi-Arkansas & 862 & 10.6 & 1.8 & $(7.1-14.0)$ \\
\hline Miami-Fort Lauderdale-West Palm Beach, Florida & 2,118 & 11.0 & 0.9 & $(9.2-12.7)$ \\
\hline Milwaukee-Waukesha-West Allis, Wisconsin & 1,346 & 12.2 & 1.3 & $(9.6-14.8)$ \\
\hline Minneapolis-St. Paul-Bloomington, Minnesota-Wisconsin & 8,631 & 7.7 & 0.4 & $(7.0-8.4)$ \\
\hline Minot, North Dakota & 585 & 7.5 & 1.3 & $(5.0-10.0)$ \\
\hline Montgomery, Alabama & 497 & 14.6 & 1.9 & $(10.9-18.3)$ \\
\hline $\begin{array}{l}\text { Montgomery County-Bucks County-Chester County, } \\
\text { Pennsylvania }\end{array}$ & 791 & 7.7 & 1.2 & $(5.4-10.0)$ \\
\hline $\begin{array}{l}\text { Myrtle Beach-Conway-North Myrtle Beach, South Carolina- } \\
\text { North Carolina }\end{array}$ & 979 & 16.6 & 1.6 & $(13.4-19.8)$ \\
\hline Nashville-Davidson County-Murfreesboro-Franklin, Tennessee & 786 & 14.1 & 1.5 & $(11.1-17.0)$ \\
\hline Nassau County-Suffolk County, New York ${ }^{\S}$ & 752 & 9.4 & 1.6 & $(6.2-12.6)$ \\
\hline Newark, New Jersey-Pennsylvania§ & 4,060 & 9.1 & 0.7 & $(7.8-10.4)$ \\
\hline New Orleans-Metairie, Louisiana & 1,873 & 12.1 & 0.9 & $(10.4-13.9)$ \\
\hline New York-Jersey City-White Plains, New York-New Jersey ${ }^{\S}$ & 7,307 & 11.2 & 0.5 & $(10.2-12.3)$ \\
\hline Norfolk, Nebraska & 980 & 9.0 & 1.1 & $(6.9-11.1)$ \\
\hline North Platte, Nebraska & 945 & 11.7 & 1.3 & $(9.1-14.2)$ \\
\hline North Port-Sarasota-Bradenton, Florida & 497 & 13.0 & 2.9 & $(7.3-18.7)$ \\
\hline Oakland-Hayward-Berkeley, California§ & 698 & 12.2 & 1.5 & $(9.2-15.2)$ \\
\hline Ogden-Clearfield, Utah & 2,890 & 10.3 & 0.7 & $(9.0-11.7)$ \\
\hline Oklahoma City, Oklahoma & 2,403 & 13.4 & 0.8 & $(11.7-15.0)$ \\
\hline Omaha-Council Bluffs, Nebraska-lowa & 4,803 & 8.3 & 0.5 & $(7.3-9.4)$ \\
\hline Orlando-Kissimmee-Sanford, Florida & 923 & 12.8 & 1.4 & $(10.1-15.5)$ \\
\hline Philadelphia, Pennsylvania ${ }^{\S}$ & 1,462 & 14.4 & 1.3 & $(11.9-16.8)$ \\
\hline Phoenix-Mesa-Scottsdale, Arizona & 9,218 & 11.7 & 0.5 & $(10.7-12.7)$ \\
\hline Pittsburgh, Pennsylvania & 2,335 & 11.9 & 0.8 & $(10.3-13.5)$ \\
\hline Ponce, Puerto Rico & 525 & 15.3 & 2.1 & $(11.2-19.4)$ \\
\hline Portland-South Portland, Maine & 2,694 & 8.7 & 0.8 & $(7.2-10.2)$ \\
\hline Portland-Vancouver-Hillsboro, Oregon-Washington & 2,786 & 11.6 & 0.8 & $(10.1-13.2)$ \\
\hline Providence-Warwick, Rhode Island-Massachusetts & 7,914 & 11.6 & 0.6 & $(10.4-12.8)$ \\
\hline Provo-Orem, Utah & 2,127 & 8.5 & 0.7 & $(7.2-9.8)$ \\
\hline Raleigh, North Carolina & 712 & 10.0 & 1.3 & $(7.5-12.6)$ \\
\hline Rapid City, South Dakota & 1,408 & 10.6 & 1.3 & $(8.1-13.2)$ \\
\hline Reno, Nevada & 1,164 & 10.6 & 1.1 & $(8.4-12.9)$ \\
\hline Richmond, Virginia & 1,437 & 10.2 & 1.0 & $(8.1-12.2)$ \\
\hline Riverside-San Bernardino-Ontario, California & 932 & 15.6 & 1.5 & $(12.6-18.6)$ \\
\hline Roanoke, Virginia & 522 & 9.1 & 1.5 & $(6.2-12.1)$ \\
\hline Rochester, Minnesota & 691 & 7.4 & 1.3 & $(4.8-9.9)$ \\
\hline Rockingham County-Strafford County, New Hampshire ${ }^{\S}$ & 1,424 & 8.5 & 0.8 & $(6.9-10.2)$ \\
\hline Sacramento-Roseville-Arden-Arcade, California & 641 & 12.8 & 1.6 & $(9.7-15.9)$ \\
\hline St. Cloud, Minnesota & 556 & 7.5 & 1.3 & $(5.0-10.1)$ \\
\hline St. Louis, Missouri-Illinois & 1,906 & 11.2 & 1.0 & $(9.2-13.2)$ \\
\hline Salisbury, Maryland-Delaware & 1,893 & 10.8 & 1.3 & $(8.3-13.3)$ \\
\hline Salt Lake City, Utah & 5,340 & 10.2 & 0.5 & $(9.3-11.1)$ \\
\hline San Antonio-New Braunfels, Texas & 2,194 & 10.8 & 0.9 & $(9.1-12.5)$ \\
\hline San Juan-Carolina-Caguas, Puerto Rico & 3,729 & 14.8 & 0.7 & $(13.5-16.2)$ \\
\hline Scottsbluff, Nebraska & 892 & 13.9 & 1.7 & $(10.7-17.2)$ \\
\hline Seattle-Bellevue-Everett, Washington $\S$ & 3,646 & 10.0 & 0.6 & $(8.8-11.3)$ \\
\hline Shreveport-Bossier City, Louisiana & 535 & 16.2 & 2.1 & $(12.2-20.3)$ \\
\hline Silver Spring-Frederick-Rockville, Maryland ${ }^{\S}$ & 2,331 & 7.7 & 1.0 & (5.7-9.6) \\
\hline Sioux City, lowa-Nebraska-South Dakota & 1,111 & 12.8 & 2.1 & $(8.6-17.0)$ \\
\hline Sioux Falls, South Dakota & 1,318 & 7.3 & 1.2 & $(5.0-9.7)$ \\
\hline Spartanburg, South Carolina & 537 & 11.0 & 1.5 & $(8.1-13.9)$ \\
\hline Spokane-Spokane Valley, Washington & 716 & 11.3 & 1.5 & $(8.4-14.2)$ \\
\hline Springfield, Massachusetts & 1,080 & 17.3 & 2.0 & $(13.5-21.2)$ \\
\hline Tampa-St. Petersburg-Clearwater, Florida & 1,523 & 11.8 & 1.1 & $(9.7-13.9)$ \\
\hline
\end{tabular}

See table footnotes on page 27. 
TABLE 8. (Continued) Age-adjusted* prevalence estimates of adults aged $\geq 18$ years who reported $\geq 14$ days of frequent physical distress ${ }^{\dagger}$ during the past 30 days, by metropolitan and micropolitan statistical area - Behavioral Risk Factor Surveillance System, United States, 2014

\begin{tabular}{|c|c|c|c|c|}
\hline MMSA & $\begin{array}{l}\text { Sample } \\
\text { size }\end{array}$ & $\%$ & SE & $95 \% \mathrm{Cl}$ \\
\hline Toledo, Ohio & 631 & 14.7 & 2.1 & $(10.6-18.7)$ \\
\hline Topeka, Kansas & 1,408 & 9.0 & 1.0 & $(7.1-10.9)$ \\
\hline Tulsa, Oklahoma & 2,006 & 12.8 & 0.9 & $(11.0-14.7)$ \\
\hline Tuscaloosa, Alabama & 704 & 14.9 & 1.6 & $(11.7-18.1)$ \\
\hline Virginia Beach-Norfolk-Newport News, Virginia-North Carolina & 1,839 & 10.0 & 0.9 & $(8.2-11.9)$ \\
\hline Warren-Troy-Farmington Hills, Michigan $\S$ & 2,094 & 9.7 & 0.7 & $(8.2-11.1)$ \\
\hline $\begin{array}{l}\text { Washington-Arlington-Alexandria, District of Columbia-Virginia- } \\
\text { Maryland-West Virginia } \$\end{array}$ & 8,109 & 8.4 & 0.6 & $(7.3-9.5)$ \\
\hline Wichita, Kansas & 2,675 & 10.3 & 0.7 & $(9.0-11.6)$ \\
\hline Wichita Falls, Texas & 521 & $N / A^{9}$ & $N / A^{9}$ & $(\mathrm{~N} / \mathrm{A}-\mathrm{N} / \mathrm{A})^{\text {9 }}$ \\
\hline Wilmington, Delaware-Maryland-New Jersey ${ }^{\S}$ & 2,705 & 11.5 & 0.9 & $(9.8-13.2)$ \\
\hline Worcester, Massachusetts-Connecticut & 2,406 & 10.5 & 0.9 & $(8.6-12.3)$ \\
\hline Youngstown-Warren-Boardman, Ohio-Pennsylvania & 512 & 13.2 & 2.0 & $(9.3-17.1)$ \\
\hline Median & & 11.1 & & \\
\hline Range & & $6.2-18.5$ & & \\
\hline
\end{tabular}

Abbreviations: $\mathrm{Cl}=$ confidence interval; $\mathrm{MMSA}=$ metropolitan and micropolitan statistical areas; $\mathrm{N} / \mathrm{A}=$ not available; $\mathrm{SE}=$ standard error.

* Age adjusted to the 2000 U.S. standard population.

† Respondents were asked "How many days in the past month was your physical health (including physical illness and injury) not good?"

$\S$ Metropolitan division.

" Estimate not available if the unweighted sample size for the denominator was $<50$ or if the relative standard error is $>30 \%$. 
TABLE 9. Age-adjusted* prevalence estimates of adults aged 18-64 years who have health care coverage, ${ }^{\dagger}$ by state/territory Behavioral Risk Factor Surveillance System, United States, 2013

\begin{tabular}{|c|c|c|c|c|}
\hline State/Territory & $\begin{array}{l}\text { Sample } \\
\text { size }\end{array}$ & $\%$ & SE & $95 \% \mathrm{Cl}$ \\
\hline Alabama & 4,031 & 77.9 & 1.0 & (75.8-79.9) \\
\hline Alaska & 3,643 & 79.5 & 1.0 & (77.6-81.5) \\
\hline Arizona & 2,615 & 74.6 & 1.6 & (71.4-77.7) \\
\hline Arkansas & 3,127 & 70.8 & 1.2 & $(68.5-73.2)$ \\
\hline California & 8,376 & 79.4 & 0.6 & (78.2-80.6) \\
\hline Colorado & 9,377 & 79.6 & 0.6 & (78.5-80.8) \\
\hline Connecticut & 5,240 & 87.5 & 0.7 & $(86.0-88.9)$ \\
\hline Delaware & 3,351 & 85.0 & 0.9 & $(83.3-86.7)$ \\
\hline District of Columbia & 3,150 & 90.2 & 0.9 & (88.4-92.0) \\
\hline Florida & 19,195 & 70.1 & 0.7 & $(68.6-71.5)$ \\
\hline Georgia & 5,729 & 72.2 & 0.8 & (70.5-73.8) \\
\hline Hawaii & 5,519 & 89.7 & 0.6 & $(88.5-90.8)$ \\
\hline Idaho & 3,607 & 75.6 & 1.1 & $(73.5-77.7)$ \\
\hline Illinois & 3,744 & 80.0 & 1.1 & (77.9-82.1) \\
\hline Indiana & 6,704 & 78.5 & 0.7 & (77.1-79.9) \\
\hline lowa & 5,148 & 86.7 & 0.7 & $(85.3-88.1)$ \\
\hline Kansas & 15,459 & 78.5 & 0.4 & (77.7-79.4) \\
\hline Kentucky & 7,462 & 77.1 & 0.8 & (75.6-78.7) \\
\hline Louisiana & 3,316 & 73.4 & 1.4 & $(70.8-76.1)$ \\
\hline Maine & 5,220 & 84.2 & 0.8 & $(82.7-85.7)$ \\
\hline Maryland & 8,531 & 83.7 & 0.7 & $(82.3-85.1)$ \\
\hline Massachusetts & 10,292 & 92.4 & 0.4 & (91.6-93.3) \\
\hline Michigan & 8,411 & 81.7 & 0.6 & $(80.5-83.0)$ \\
\hline Minnesota & 10,056 & 86.8 & 0.6 & (85.6-88.0) \\
\hline Mississippi & 4,645 & 71.4 & 1.0 & (69.4-73.4) \\
\hline Missouri & 4,387 & 80.5 & 0.9 & $(78.8-82.2)$ \\
\hline Montana & 6,437 & 78.0 & 0.7 & (76.6-79.5) \\
\hline Nebraska & 10,939 & 81.8 & 0.7 & $(80.5-83.1)$ \\
\hline Nevada & 3,431 & 71.9 & 1.5 & (69.1-74.8) \\
\hline New Hampshire & 4,221 & 83.7 & 0.9 & $(82.0-85.4)$ \\
\hline New Jersey & 9,674 & 80.0 & 0.7 & (78.7-81.3) \\
\hline New Mexico & 6,334 & 73.0 & 0.8 & (71.4-74.7) \\
\hline New York & 6,242 & 82.3 & 0.7 & (80.9-83.6) \\
\hline North Carolina & 5,818 & 75.2 & 0.8 & (73.7-76.7) \\
\hline North Dakota & 5,271 & 87.4 & 0.7 & (86.0-88.8) \\
\hline Ohio & 8,077 & 82.8 & 0.7 & (81.5-84.2) \\
\hline Oklahoma & 5,239 & 76.9 & 0.8 & (75.3-78.4) \\
\hline Oregon & 3,847 & 74.3 & 1.1 & $(72.2-76.4)$ \\
\hline Pennsylvania & 7,473 & 84.1 & 0.6 & (82.9-85.3) \\
\hline Rhode Island & 4,367 & 80.7 & 0.9 & (78.9-82.5) \\
\hline South Carolina & 6,745 & 75.4 & 0.8 & (73.9-76.9) \\
\hline South Dakota & 4,673 & 84.6 & 0.9 & $(82.7-86.4)$ \\
\hline Tennessee & 3,788 & 78.2 & 1.0 & (76.2-80.2) \\
\hline Texas & 7,305 & 66.9 & 0.8 & (65.3-68.5) \\
\hline Utah & 9,368 & 81.0 & 0.6 & (79.9-82.1) \\
\hline Vermont & 4,232 & 87.8 & 0.7 & (86.4-89.3) \\
\hline Virginia & 5,907 & 81.0 & 0.7 & $(79.6-82.5)$ \\
\hline Washington & 7,273 & 78.7 & 0.7 & (77.4-80.1) \\
\hline West Virginia & 4,068 & 75.0 & 0.9 & (73.2-76.8) \\
\hline Wisconsin & 4,496 & 85.2 & 0.9 & (83.5-87.0) \\
\hline Wyoming & 3,845 & 75.7 & 1.0 & (73.7-77.8) \\
\hline Guam & 1,667 & 72.3 & 1.4 & $(69.5-75.1)$ \\
\hline Puerto Rico & 3,974 & 91.5 & 0.6 & $(90.3-92.6)$ \\
\hline Median & & 79.6 & & \\
\hline Range & & $66.9-92.4$ & & \\
\hline
\end{tabular}

Abbreviations: $\mathrm{Cl}=$ confidence interval; $\mathrm{SE}=$ standard error.

* Age adjusted to the 2000 U.S. standard population.

† Including health insurance, prepaid plans (e.g., health maintenance organizations), or government plans (e.g., Medicare). 
TABLE 10. Age-adjusted* prevalence estimates of adults aged 18-64 years who have health care coverage, ${ }^{\dagger}$ by metropolitan and micropolitan statistical area - Behavioral Risk Factor Surveillance System, United States, 2013

\begin{tabular}{|c|c|c|c|c|}
\hline MMSA & $\begin{array}{l}\text { Sample } \\
\text { size }\end{array}$ & $\%$ & SE & $95 \% \mathrm{Cl}$ \\
\hline Aguadilla-Isabela, Puerto Rico & 388 & 97.6 & 0.9 & $(95.8-99.4)$ \\
\hline Akron, Ohio & 426 & 87.7 & 2.1 & $(83.5-91.9)$ \\
\hline Albuquerque, New Mexico & 1,511 & 75.4 & 1.6 & $(72.4-78.5)$ \\
\hline Allentown-Bethlehem-Easton, Pennsylvania-New Jersey & 732 & 83.8 & 2.3 & $(79.2-88.4)$ \\
\hline Anchorage, Alaska & 1,216 & 80.9 & 1.5 & $(77.9-83.9)$ \\
\hline Atlanta-Sandy Springs-Roswell, Georgia & 2,631 & 73.9 & 1.2 & $(71.4-76.3)$ \\
\hline Augusta-Richmond County, Georgia-South Carolina & 584 & 81.8 & 2.4 & $(77.1-86.6)$ \\
\hline Austin-Round Rock, Texas & 672 & 74.1 & 2.3 & $(69.6-78.6)$ \\
\hline Baltimore-Columbia-Towson, Maryland & 3,166 & 85.5 & 1.1 & $(83.4-87.7)$ \\
\hline Baton Rouge, Louisiana & 614 & 76.3 & 2.9 & $(70.6-81.9)$ \\
\hline Billings, Montana & 604 & 80.6 & 1.9 & $(76.8-84.4)$ \\
\hline Birmingham-Hoover, Alabama & 898 & 80.4 & 2.0 & $(76.5-84.3)$ \\
\hline Bismarck, North Dakota & 710 & 88.1 & 1.6 & $(84.9-91.2)$ \\
\hline Boise City, Idaho & 1,003 & 76.6 & 1.9 & $(73.0-80.3)$ \\
\hline Boston, Massachusetts ${ }^{\S}$ & 2,790 & 91.8 & 0.9 & $(90.0-93.5)$ \\
\hline Buffalo-Cheektowaga-Niagara Falls, New York & 316 & 89.7 & 2.1 & $(85.5-93.9)$ \\
\hline Burlington-South Burlington, Vermont & 1,209 & 90.8 & 1.2 & $(88.5-93.2)$ \\
\hline Cambridge-Newton-Framingham, Massachusetts $§$ & 3,453 & 93.5 & 0.7 & $(92.1-95.0)$ \\
\hline Camden, New Jersey ${ }^{\S}$ & 1,345 & 83.5 & 1.6 & $(80.3-86.7)$ \\
\hline Cedar Rapids, lowa & 419 & 85.1 & 2.3 & $(80.5-89.6)$ \\
\hline Charleston, West Virginia & 566 & 72.8 & 2.6 & $(67.8-77.9)$ \\
\hline Charleston-North Charleston, South Carolina & 1,017 & 76.6 & 1.8 & $(73.0-80.2)$ \\
\hline Charlotte-Concord-Gastonia, North Carolina-South Carolina & 1,334 & 76.5 & 1.5 & $(73.5-79.5)$ \\
\hline Chattanooga, Tennessee-Georgia & 354 & 77.2 & 3.8 & $(69.8-84.6)$ \\
\hline Chicago-Naperville-Elgin, Illinois-Indiana-Wisconsin & 2,360 & 78.1 & 1.4 & $(75.4-80.8)$ \\
\hline Cincinnati, Ohio-Kentucky-Indiana & 1,853 & 81.0 & 1.5 & $(78.1-84.0)$ \\
\hline Claremont-Lebanon, New Hampshire-Vermont & 1,079 & 82.8 & 1.8 & $(79.2-86.4)$ \\
\hline Cleveland-Elyria, Ohio & 756 & 85.6 & 1.9 & $(82.0-89.3)$ \\
\hline Colorado Springs, Colorado & 973 & 82.5 & 1.6 & $(79.4-85.7)$ \\
\hline Columbia, South Carolina & 990 & 77.8 & 1.9 & $(74.1-81.4)$ \\
\hline Columbus, Ohio & 1,344 & 83.3 & 1.4 & $(80.7-86.0)$ \\
\hline Crestview-Fort Walton Beach-Destin, Florida & 685 & 74.2 & 2.5 & $(69.3-79.0)$ \\
\hline Dallas-Plano-Irving, Texas $§$ & 665 & 71.0 & 2.2 & $(66.6-75.3)$ \\
\hline Davenport-Moline-Rock Island, lowa-Illinois & 413 & 86.9 & 2.5 & $(82.0-91.9)$ \\
\hline Dayton, Ohio & 526 & 82.4 & 2.7 & $(77.1-87.8)$ \\
\hline Deltona-Daytona Beach-Ormond Beach, Florida & 534 & 74.0 & 2.9 & $(68.3-79.8)$ \\
\hline Denver-Aurora-Lakewood, Colorado & 4,164 & 80.4 & 0.8 & $(78.8-81.9)$ \\
\hline Des Moines-West Des Moines, lowa & 882 & 89.5 & 1.6 & $(86.4-92.6)$ \\
\hline Duluth, Minnesota-Wisconsin & 481 & 88.0 & 2.9 & $(82.3-93.8)$ \\
\hline Durham-Chapel Hill, North Carolina & 419 & 74.2 & 2.8 & $(68.7-79.8)$ \\
\hline El Paso, Texas & 501 & 60.5 & 2.8 & $(55.1-66.0)$ \\
\hline Evansville, Indiana-Kentucky & 360 & 84.6 & 2.6 & $(79.6-89.6)$ \\
\hline Fargo, North Dakota-Minnesota & 867 & 89.0 & 1.4 & $(86.3-91.8)$ \\
\hline Fayetteville-Springdale-Rogers, Arkansas-Missouri & 502 & 69.6 & 3.0 & $(63.8-75.4)$ \\
\hline Fort Smith, Arkansas-Oklahoma & 311 & 73.3 & 3.5 & $(66.4-80.2)$ \\
\hline Fort Wayne, Indiana & 503 & 76.6 & 2.6 & $(71.6-81.7)$ \\
\hline Fort Worth-Arlington, Texas ${ }^{\S}$ & 505 & 69.9 & 3.0 & $(64.1-75.7)$ \\
\hline Gainesville, Florida & 626 & 77.5 & 2.7 & $(72.3-82.7)$ \\
\hline Grand Forks, North Dakota-Minnesota & 345 & 88.3 & 2.7 & $(83.1-93.6)$ \\
\hline Grand Island, Nebraska & 467 & 76.9 & 3.2 & $(70.7-83.1)$ \\
\hline Grand Rapids-Wyoming, Michigan & 938 & 81.5 & 2.0 & $(77.5-85.4)$ \\
\hline Greensboro-High Point, North Carolina & 448 & 75.1 & 2.8 & $(69.6-80.6)$ \\
\hline Greenville-Anderson-Mauldin, South Carolina & 865 & 76.3 & 2.0 & $(72.4-80.2)$ \\
\hline Gulfport-Biloxi-Pascagoula, Mississippi & 524 & 70.0 & 2.7 & $(64.8-75.2)$ \\
\hline Hagerstown-Martinsburg, Maryland-West Virginia & 492 & 80.4 & 2.8 & $(75.0-85.8)$ \\
\hline Hartford-West Hartford-East Hartford, Connecticut & 1,917 & 88.3 & 1.1 & $(86.0-90.5)$ \\
\hline Hilton Head Island-Bluffton-Beaufort, South Carolina & 406 & 71.8 & 3.7 & $(64.6-79.0)$ \\
\hline Houston-The Woodlands-Sugar Land, Texas & 1,062 & 66.4 & 2.0 & $(62.4-70.3)$ \\
\hline Huntington-Ashland, West Virginia-Kentucky-Ohio & 798 & 75.8 & 2.0 & $(71.8-79.7)$ \\
\hline Idaho Falls, Idaho & 343 & 76.4 & 3.1 & $(70.3-82.4)$ \\
\hline Indianapolis-Carmel-Anderson, Indiana & 1,738 & 77.5 & 1.4 & $(74.8-80.2)$ \\
\hline
\end{tabular}

See table footnotes on page 31 . 
TABLE 10. (Continued) Age-adjusted* prevalence estimates of adults aged 18-64 years who have health care coverage, ${ }^{\dagger}$ by metropolitan and micropolitan statistical area - Behavioral Risk Factor Surveillance System, United States, 2013

\begin{tabular}{|c|c|c|c|c|}
\hline MMSA & $\begin{array}{l}\text { Sample } \\
\text { size }\end{array}$ & $\%$ & SE & $95 \% \mathrm{Cl}$ \\
\hline Jackson, Mississippi & 542 & 71.2 & 2.7 & $(65.9-76.5)$ \\
\hline Jacksonville, Florida & 1,863 & 78.1 & 1.5 & $(75.2-81.0)$ \\
\hline Kansas City, Missouri-Kansas & 4,987 & 80.5 & 1.1 & $(78.2-82.7)$ \\
\hline Kingsport-Bristol-Bristol, Tennessee-Virginia & 322 & 81.9 & 3.3 & $(75.5-88.4)$ \\
\hline Knoxville, Tennessee & 442 & 76.7 & 2.8 & $(71.3-82.1)$ \\
\hline Lansing-East Lansing, Michigan & 465 & 85.1 & 2.1 & $(81.1-89.2)$ \\
\hline Lexington-Fayette, Kentucky & 478 & 78.9 & 2.4 & $(74.1-83.7)$ \\
\hline Lincoln, Nebraska & 1,402 & 82.2 & 1.3 & $(79.7-84.8)$ \\
\hline Little Rock-North Little Rock-Conway, Arkansas & 716 & 77.7 & 2.2 & $(73.4-82.1)$ \\
\hline Logan, Utah-Idaho & 461 & 76.6 & 2.5 & $(71.7-81.6)$ \\
\hline Los Angeles-Long Beach-Anaheim, California & 2,283 & 75.8 & 1.2 & $(73.4-78.1)$ \\
\hline Louisville-Jefferson County, Kentucky-Indiana & 1,416 & 81.0 & 1.7 & $(77.8-84.3)$ \\
\hline Lubbock, Texas & 315 & 72.2 & 3.5 & $(65.2-79.1)$ \\
\hline Manhattan, Kansas & 487 & 85.5 & 1.9 & $(81.7-89.3)$ \\
\hline Memphis, Tennessee-Mississippi-Arkansas & 821 & 79.5 & 2.1 & $(75.4-83.6)$ \\
\hline Miami-Fort Lauderdale-West Palm Beach, Florida & 1,290 & 66.8 & 2.0 & $(62.9-70.6)$ \\
\hline Milwaukee-Waukesha-West Allis, Wisconsin & 889 & 82.4 & 2.1 & $(78.4-86.5)$ \\
\hline Minneapolis-St. Paul-Bloomington, Minnesota-Wisconsin & 6,521 & 87.4 & 0.8 & $(85.9-89.0)$ \\
\hline Minot, North Dakota & 468 & 90.1 & 1.8 & $(86.5-93.7)$ \\
\hline $\begin{array}{l}\text { Montgomery County-Bucks County-Chester County, } \\
\text { Pennsylvania }\end{array}$ & 683 & 87.5 & 1.6 & $(84.4-90.6)$ \\
\hline $\begin{array}{l}\text { Myrtle Beach-Conway-North Myrtle Beach, South Carolina- } \\
\text { North Carolina }\end{array}$ & 478 & 68.3 & 3.0 & $(62.5-74.1)$ \\
\hline Nashville-Davidson County-Murfreesboro-Franklin, Tennessee & 758 & 82.0 & 1.9 & $(78.3-85.7)$ \\
\hline Nassau County-Suffolk County, New York ${ }^{\S}$ & 675 & 81.5 & 2.0 & $(77.6-85.4)$ \\
\hline Newark, New Jersey-Pennsylvania ${ }^{\S}$ & 3,023 & 80.6 & 1.1 & $(78.4-82.7)$ \\
\hline New Orleans-Metairie, Louisiana & 790 & 73.5 & 2.8 & $(68.0-78.9)$ \\
\hline New York-Jersey City-White Plains, New York-New Jersey $\$$ & 6,451 & 79.6 & 0.8 & $(78.1-81.1)$ \\
\hline Norfolk, Nebraska & 480 & 83.0 & 2.2 & $(78.8-87.3)$ \\
\hline North Platte, Nebraska & 443 & 79.6 & 3.1 & $(73.6-85.6)$ \\
\hline North Port-Sarasota-Bradenton, Florida & 554 & 66.1 & 2.8 & $(60.7-71.5)$ \\
\hline Oakland-Hayward-Berkeley, California§ & 528 & 87.1 & 1.8 & $(83.7-90.6)$ \\
\hline Ogden-Clearfield, Utah & 1,826 & 85.3 & 1.1 & $(83.2-87.5)$ \\
\hline Oklahoma City, Oklahoma & 1,759 & 75.6 & 1.4 & (72.9-78.3) \\
\hline Omaha-Council Bluffs, Nebraska-lowa & 2,255 & 83.3 & 1.1 & $(81.0-85.5)$ \\
\hline Orlando-Kissimmee-Sanford, Florida & 1,411 & 69.1 & 1.8 & $(65.6-72.6)$ \\
\hline Panama City, Florida & 599 & 69.8 & 2.8 & $(64.3-75.3)$ \\
\hline Pensacola-Ferry Pass-Brent, Florida & 885 & 73.4 & 2.0 & $(69.5-77.3)$ \\
\hline Philadelphia, Pennsylvanias & 1,198 & 81.4 & 1.6 & $(78.3-84.4)$ \\
\hline Phoenix-Mesa-Scottsdale, Arizona & 1,026 & 73.7 & 2.2 & $(69.3-78.0)$ \\
\hline Pittsburgh, Pennsylvania & 1,486 & 86.7 & 1.2 & $(84.2-89.1)$ \\
\hline Ponce, Puerto Rico & 365 & 94.0 & 1.5 & $(91.0-97.0)$ \\
\hline Portland-South Portland, Maine & 1,716 & 86.5 & 1.2 & $(84.1-88.9)$ \\
\hline Portland-Vancouver-Hillsboro, Oregon-Washington & 2,180 & 79.1 & 1.3 & $(76.6-81.7)$ \\
\hline Port St. Lucie, Florida & 493 & 67.5 & 3.4 & $(60.9-74.1)$ \\
\hline Providence-Warwick, Rhode Island-Massachusetts & 5,539 & 84.5 & 0.8 & $(82.9-86.2)$ \\
\hline Provo-Orem, Utah & 1,485 & 79.8 & 1.3 & $(77.2-82.4)$ \\
\hline Raleigh, North Carolina & 551 & 81.6 & 1.8 & $(78.0-85.1)$ \\
\hline Rapid City, South Dakota & 579 & 87.6 & 1.8 & $(84.2-91.1)$ \\
\hline Reno, Nevada & 1,249 & 76.7 & 1.6 & $(73.5-79.8)$ \\
\hline Richmond, Virginia & 949 & 83.1 & 1.7 & $(79.9-86.4)$ \\
\hline Riverside-San Bernardino-Ontario, California & 1,027 & 78.9 & 1.7 & $(75.6-82.1)$ \\
\hline Rochester, New York & 349 & 87.9 & 2.3 & $(83.3-92.5)$ \\
\hline Rockingham County-Strafford County, New Hampshire ${ }^{\S}$ & 1,147 & 85.5 & 1.5 & $(82.5-88.4)$ \\
\hline Sacramento-Roseville-Arden-Arcade, California & 625 & 81.1 & 2.1 & $(77.1-85.2)$ \\
\hline St. Louis, Missouri-Illinois & 1,314 & 83.7 & 1.4 & $(80.9-86.5)$ \\
\hline Salem, Oregon & 327 & 73.5 & 3.9 & $(65.8-81.2)$ \\
\hline Salina, Kansas & 353 & 74.2 & 3.1 & $(68.1-80.2)$ \\
\hline Salisbury, Maryland-Delaware & 1,154 & 82.1 & 2.0 & $(78.2-86.1)$ \\
\hline Salt Lake City, Utah & 3,508 & 80.9 & 0.9 & $(79.2-82.7)$ \\
\hline San Antonio-New Braunfels, Texas & 623 & 65.7 & 2.5 & $(60.9-70.6)$ \\
\hline San Francisco-Redwood City-South San Francisco, California§ & 405 & 92.6 & 1.5 & $(89.8-95.5)$ \\
\hline
\end{tabular}
See table footnotes on page 31 . 
TABLE 10. (Continued) Age-adjusted* prevalence estimates of adults aged 18-64 years who have health care coverage, ${ }^{\dagger}$ by metropolitan and micropolitan statistical area - Behavioral Risk Factor Surveillance System, United States, 2013

\begin{tabular}{|c|c|c|c|c|}
\hline MMSA & $\begin{array}{l}\text { Sample } \\
\text { size }\end{array}$ & $\%$ & SE & $95 \% \mathrm{Cl}$ \\
\hline San Jose-Sunnyvale-Santa Clara, California & 487 & 81.3 & 2.6 & $(76.3-86.3)$ \\
\hline San Juan-Carolina-Caguas, Puerto Rico & 2,442 & 89.7 & 0.8 & $(88.2-91.3)$ \\
\hline Scottsbluff, Nebraska & 424 & 76.5 & 2.9 & $(70.9-82.2)$ \\
\hline Scranton-Wilkes-Barre-Hazleton, Pennsylvania & 347 & 85.8 & 2.7 & $(80.5-91.0)$ \\
\hline Seattle-Bellevue-Everett, Washington ${ }^{\S}$ & 2,665 & 83.0 & 1.0 & $(81.0-85.1)$ \\
\hline Shreveport-Bossier City, Louisiana & 353 & 68.9 & 4.0 & $(61.0-76.8)$ \\
\hline Silver Spring-Frederick-Rockville, Maryland $\S$ & 1,683 & 84.6 & 1.5 & $(81.7-87.5)$ \\
\hline Sioux City, lowa-Nebraska-South Dakota & 653 & 81.5 & 3.2 & $(75.2-87.9)$ \\
\hline Sioux Falls, South Dakota & 752 & 84.1 & 2.0 & $(80.2-88.0)$ \\
\hline Spartanburg, South Carolina & 370 & 76.3 & 3.2 & $(70.0-82.5)$ \\
\hline Spokane-Spokane Valley, Washington & 555 & 77.1 & 2.6 & $(72.1-82.2)$ \\
\hline Springfield, Massachusetts & 1,055 & 91.6 & 1.6 & $(88.4-94.8)$ \\
\hline Tallahassee, Florida & 1,160 & 79.1 & 2.4 & $(74.3-83.9)$ \\
\hline Tampa-St. Petersburg-Clearwater, Florida & 1,313 & 75.4 & 1.7 & $(72.1-78.7)$ \\
\hline Toledo, Ohio & 681 & 77.8 & 3.1 & $(71.7-83.8)$ \\
\hline Topeka, Kansas & 1,610 & 81.7 & 1.3 & $(79.1-84.3)$ \\
\hline Tulsa, Oklahoma & 1,267 & 79.1 & 1.5 & $(76.1-82.1)$ \\
\hline Virginia Beach-Norfolk-Newport News, Virginia-North Carolina & 1,170 & 85.0 & 1.5 & $(82.0-87.9)$ \\
\hline Warren-Troy-Farmington Hills, Michigan ${ }^{\S}$ & 1,520 & 83.0 & 1.5 & $(80.1-85.9)$ \\
\hline $\begin{array}{l}\text { Washington-Arlington-Alexandria, District of Columbia- } \\
\text { Virginia-Maryland-West Virginia }{ }^{\S}\end{array}$ & 6,219 & 82.5 & 1.0 & $(80.5-84.5)$ \\
\hline Wichita, Kansas & 3,314 & 76.2 & 1.0 & $(74.2-78.2)$ \\
\hline Wilmington, Delaware-Maryland-New Jersey ${ }^{\S}$ & 2,267 & 85.7 & 1.1 & $(83.6-87.9)$ \\
\hline Winston-Salem, North Carolina & 416 & 70.3 & 3.3 & $(63.9-76.7)$ \\
\hline Worcester, Massachusetts-Connecticut & 1,923 & 91.4 & 1.1 & $(89.2-93.7)$ \\
\hline Median & & 80.6 & & \\
\hline Range & & $60.5-97.6$ & & \\
\hline
\end{tabular}

Abbreviations: $\mathrm{Cl}=$ confidence interval; $\mathrm{MMSA}=$ metropolitan and micropolitan statistical areas; $\mathrm{SE}=$ standard error.

* Age adjusted to the 2000 U.S. standard population.

† Including health insurance, prepaid plans (e.g., health maintenance organizations), or government plans (e.g., Medicare).

$\S$ Metropolitan division. 
TABLE 11. Age-adjusted* prevalence estimates of adults aged 18-64 years who have health care coverage, ${ }^{\dagger}$ by state/territory Behavioral Risk Factor Surveillance System, United States, 2014

\begin{tabular}{|c|c|c|c|c|}
\hline State/Territory & $\begin{array}{c}\text { Sample } \\
\text { size }\end{array}$ & $\%$ & SE & $95 \% \mathrm{Cl}$ \\
\hline Alabama & 5,440 & 81.9 & 0.8 & $(80.3-83.4)$ \\
\hline Alaska & 3,312 & 82.5 & 1.0 & $(80.6-84.5)$ \\
\hline Arizona & 8,036 & 81.8 & 0.7 & $(80.3-83.2)$ \\
\hline Arkansas & 2,778 & 79.2 & 1.2 & $(76.8-81.6)$ \\
\hline California & 6,447 & 82.0 & 0.6 & $(80.7-83.2)$ \\
\hline Colorado & 8,874 & 84.3 & 0.5 & $(83.2-85.3)$ \\
\hline Connecticut & 5,132 & 88.7 & 0.7 & $(87.3-90.1)$ \\
\hline Delaware & 2,665 & 88.6 & 0.9 & $(86.8-90.4)$ \\
\hline District of Columbia & 2,421 & 90.6 & 1.1 & $(88.4-92.8)$ \\
\hline Florida & 5,408 & 76.6 & 0.8 & $(74.9-78.2)$ \\
\hline Georgia & 4,064 & 74.4 & 1.0 & $(72.5-76.4)$ \\
\hline Hawaii & 5,003 & 89.9 & 0.7 & $(88.6-91.2)$ \\
\hline Idaho & 3,490 & 79.4 & 1.0 & $(77.4-81.4)$ \\
\hline Illinois & 3,384 & 85.1 & 0.9 & $(83.3-86.9)$ \\
\hline Indiana & 7,183 & 81.3 & 0.7 & $(80.0-82.6)$ \\
\hline lowa & 5,091 & 90.0 & 0.6 & $(88.8-91.2)$ \\
\hline Kansas & 9,155 & 81.8 & 0.6 & $(80.7-82.9)$ \\
\hline Kentucky & 7,110 & 87.3 & 0.8 & $(85.8-88.8)$ \\
\hline Louisiana & 4,616 & 76.8 & 0.8 & $(75.1-78.4)$ \\
\hline Maine & 5,696 & 85.5 & 0.7 & (84.1-86.9) \\
\hline Maryland & 7,663 & 88.2 & 0.8 & $(86.5-89.8)$ \\
\hline Massachusetts & 9,615 & 94.5 & 0.4 & (93.7-95.3) \\
\hline Michigan & 5,447 & 86.8 & 0.6 & $(85.6-88.1)$ \\
\hline Minnesota & 11,656 & 90.8 & 0.4 & $(90.0-91.5)$ \\
\hline Mississippi & 2,598 & 76.6 & 1.2 & $(74.2-79.0)$ \\
\hline Missouri & 4,219 & 83.1 & 0.9 & $(81.3-84.9)$ \\
\hline Montana & 4,590 & 83.5 & 0.8 & $(81.9-85.2)$ \\
\hline Nebraska & 14,323 & 84.1 & 0.6 & $(83.0-85.2)$ \\
\hline Nevada & 2,408 & 78.7 & 1.4 & (76.0-81.4) \\
\hline New Hampshire & 3,868 & 85.0 & 0.9 & $(83.1-86.8)$ \\
\hline New Jersey & 8,831 & 84.2 & 0.6 & $(83.0-85.5)$ \\
\hline New Mexico & 5,853 & 80.4 & 0.9 & $(78.7-82.1)$ \\
\hline New York & 4,651 & 84.8 & 0.7 & $(83.4-86.2)$ \\
\hline North Carolina & 4,831 & 79.3 & 0.7 & $(77.9-80.7)$ \\
\hline North Dakota & 4,897 & 89.3 & 0.8 & $(87.8-90.9)$ \\
\hline Ohio & 6,818 & 87.1 & 0.7 & $(85.6-88.5)$ \\
\hline Oklahoma & 5,356 & 82.5 & 0.7 & $(81.1-83.9)$ \\
\hline Oregon & 3,138 & 85.9 & 0.9 & $(84.1-87.7)$ \\
\hline Pennsylvania & 6,807 & 86.7 & 0.7 & $(85.4-88.1)$ \\
\hline Rhode Island & 4,154 & 89.7 & 0.8 & (88.1-91.4) \\
\hline South Carolina & 6,896 & 78.1 & 0.7 & (76.7-79.5) \\
\hline South Dakota & 4,782 & 87.6 & 1.0 & $(85.6-89.5)$ \\
\hline Tennessee & 3,064 & 81.7 & 1.1 & $(79.5-83.8)$ \\
\hline Texas & 9,879 & 70.5 & 0.7 & $(69.1-72.0)$ \\
\hline Utah & 11,236 & 84.0 & 0.4 & $(83.2-84.9)$ \\
\hline Vermont & 4,574 & 91.2 & 0.6 & $(90.0-92.3)$ \\
\hline Virginia & 6,444 & 83.7 & 0.7 & $(82.3-85.1)$ \\
\hline Washington & 6,294 & 86.6 & 0.7 & $(85.3-87.9)$ \\
\hline West Virginia & 4,109 & 86.3 & 0.7 & $(84.8-87.7)$ \\
\hline Wisconsin & 4,703 & 89.0 & 0.8 & $(87.5-90.5)$ \\
\hline Wyoming & 3,495 & 81.0 & 1.2 & (78.6-83.3) \\
\hline Guam & 2,185 & 73.3 & 1.5 & $(70.4-76.1)$ \\
\hline Puerto Rico & 3,923 & 92.6 & 0.5 & (91.5-93.6) \\
\hline Median & & 84.2 & & \\
\hline Range & & $70.5-94.5$ & & \\
\hline
\end{tabular}

Abbreviations: $\mathrm{Cl}$ = confidence interval; $\mathrm{SE}=$ standard error.

* Age adjusted to the 2000 U.S. standard population.

† Including health insurance, prepaid plans (e.g., health maintenance organizations), or government plans (e.g., Medicare). 
TABLE 12. Age-adjusted* prevalence estimates of adults aged 18-64 years who have health care coverage, ${ }^{\dagger}$ by metropolitan and micropolitan statistical area - Behavioral Risk Factor Surveillance System, United States, 2014

\begin{tabular}{|c|c|c|c|c|}
\hline MMSA & $\begin{array}{l}\text { Sample } \\
\text { size }\end{array}$ & $\%$ & SE & $95 \% \mathrm{Cl}$ \\
\hline Aberdeen, South Dakota & 379 & 90.3 & 2.9 & $(84.5-96.0)$ \\
\hline Aguadilla-Isabela, Puerto Rico & 361 & 89.0 & 2.1 & $(84.9-93.1)$ \\
\hline Albuquerque, New Mexico & 1,213 & 82.8 & 1.6 & $(79.7-86.0)$ \\
\hline Allentown-Bethlehem-Easton, Pennsylvania-New Jersey & 651 & 84.6 & 2.4 & $(79.8-89.4)$ \\
\hline Anchorage, Alaska & 1,362 & 83.0 & 1.4 & $(80.3-85.7)$ \\
\hline Atlanta-Sandy Springs-Roswell, Georgia & 1,878 & 77.4 & 1.3 & $(74.8-80.0)$ \\
\hline Augusta-Richmond County, Georgia-South Carolina & 578 & 76.5 & 3.5 & $(69.6-83.3)$ \\
\hline Austin-Round Rock, Texas & 1,507 & 79.1 & 1.5 & $(76.2-81.9)$ \\
\hline Baltimore-Columbia-Towson, Maryland & 2,900 & 90.8 & 1.1 & $(88.6-92.9)$ \\
\hline Baton Rouge, Louisiana & 656 & 78.7 & 2.1 & $(74.5-82.9)$ \\
\hline Berlin, New Hampshire-Vermont & 325 & 79.0 & 5.2 & $(68.9-89.1)$ \\
\hline Billings, Montana & 521 & 82.9 & 2.2 & $(78.6-87.3)$ \\
\hline Birmingham-Hoover, Alabama & 1,053 & 83.0 & 1.7 & $(79.7-86.3)$ \\
\hline Bismarck, North Dakota & 624 & 88.8 & 2.1 & $(84.7-93.0)$ \\
\hline Boise City, Idaho & 845 & 80.1 & 1.9 & $(76.5-83.8)$ \\
\hline Boston, Massachusetts $\S$ & 2,810 & 94.1 & 0.8 & $(92.6-95.6)$ \\
\hline Burlington-South Burlington, Vermont & 1,493 & 93.2 & 0.8 & $(91.6-94.9)$ \\
\hline Cambridge-Newton-Framingham, Massachusetts $\S$ & 3,183 & 94.5 & 0.7 & $(93.1-95.9)$ \\
\hline Camden, New Jersey ${ }^{\S}$ & 1,126 & 92.8 & 1.3 & $(90.2-95.4)$ \\
\hline Cedar Rapids, lowa & 401 & 89.3 & 2.4 & $(84.7-94.0)$ \\
\hline Charleston, West Virginia & 588 & 88.8 & 1.8 & $(85.3-92.4)$ \\
\hline Charleston-North Charleston, South Carolina & 918 & 78.2 & 2.0 & $(74.3-82.1)$ \\
\hline Charlotte-Concord-Gastonia, North Carolina-South Carolina & 1,421 & 78.7 & 1.4 & $(75.9-81.5)$ \\
\hline Chicago-Naperville-Elgin, Illinois-Indiana-Wisconsin & 2,859 & 83.3 & 1.1 & $(81.1-85.4)$ \\
\hline Cincinnati, Ohio-Kentucky-Indiana & 1,347 & 85.2 & 1.9 & $(81.5-88.9)$ \\
\hline Claremont-Lebanon, New Hampshire-Vermont & 1,077 & 86.8 & 2.0 & $(82.9-90.7)$ \\
\hline Cleveland-Elyria, Ohio & 618 & 88.5 & 2.0 & $(84.6-92.3)$ \\
\hline College Station-Bryan, Texas & 308 & 82.0 & 3.8 & $(74.5-89.5)$ \\
\hline Colorado Springs, Colorado & 885 & 85.4 & 1.6 & $(82.2-88.5)$ \\
\hline Columbia, South Carolina & 851 & 81.4 & 1.9 & $(77.8-85.1)$ \\
\hline Columbus, Ohio & 1,160 & 90.6 & 1.4 & $(87.9-93.3)$ \\
\hline Corpus Christi, Texas & 307 & 83.0 & 2.9 & $(77.3-88.6)$ \\
\hline Dallas-Plano-Irving, Texas ${ }^{\S}$ & 825 & 74.2 & 2.2 & $(69.9-78.5)$ \\
\hline Dayton, Ohio & 340 & 88.3 & 2.2 & $(83.9-92.7)$ \\
\hline Denver-Aurora-Lakewood, Colorado & 4,051 & 84.1 & 0.7 & $(82.6-85.5)$ \\
\hline Des Moines-West Des Moines, lowa & 861 & 90.6 & 1.5 & $(87.7-93.5)$ \\
\hline Duluth, Minnesota-Wisconsin & 634 & 89.0 & 1.8 & $(85.6-92.5)$ \\
\hline El Paso, Texas & 439 & 64.7 & 2.9 & $(59.1-70.4)$ \\
\hline Evansville, Indiana-Kentucky & 376 & 89.4 & 2.6 & $(84.3-94.6)$ \\
\hline Fargo, North Dakota-Minnesota & 739 & 90.9 & 1.6 & $(87.7-94.0)$ \\
\hline Fayetteville-Springdale-Rogers, Arkansas-Missouri & 442 & 81.0 & 3.2 & $(74.8-87.2)$ \\
\hline Fort Wayne, Indiana & 510 & 80.3 & 2.7 & $(75.0-85.6)$ \\
\hline Fort Worth-Arlington, Texas $§$ & 480 & 71.9 & 3.0 & $(66.0-77.7)$ \\
\hline Grand Island, Nebraska & 639 & 81.4 & 2.6 & $(76.4-86.5)$ \\
\hline Grand Rapids-Wyoming, Michigan & 598 & 86.9 & 2.1 & $(82.8-91.0)$ \\
\hline Greensboro-High Point, North Carolina & 344 & 79.0 & 2.8 & $(73.5-84.6)$ \\
\hline Greenville-Anderson-Mauldin, South Carolina & 951 & 80.4 & 1.7 & $(77.0-83.8)$ \\
\hline Hagerstown-Martinsburg, Maryland-West Virginia & 443 & 88.0 & 3.0 & $(82.2-93.8)$ \\
\hline Hartford-West Hartford-East Hartford, Connecticut & 1,692 & 91.0 & 1.1 & $(88.9-93.1)$ \\
\hline Hilton Head Island-Bluffton-Beaufort, South Carolina & 264 & 78.5 & 3.5 & $(71.6-85.4)$ \\
\hline Houston-The Woodlands-Sugar Land, Texas & 1,428 & 69.4 & 1.9 & $(65.7-73.0)$ \\
\hline Huntington-Ashland, West Virginia-Kentucky-Ohio & 833 & 85.9 & 2.1 & $(81.8-90.0)$ \\
\hline Idaho Falls, Idaho & 355 & 84.4 & 2.4 & $(79.7-89.2)$ \\
\hline Indianapolis-Carmel-Anderson, Indiana & 2,304 & 83.6 & 1.1 & $(81.4-85.9)$ \\
\hline Jacksonville, Florida & 404 & 80.9 & 3.0 & $(75.1-86.7)$ \\
\hline Kansas City, Missouri-Kansas & 3,192 & 83.0 & 1.3 & $(80.5-85.4)$ \\
\hline Kingsport-Bristol-Bristol, Tennessee-Virginia & 276 & 80.8 & 4.2 & $(72.6-89.1)$ \\
\hline Knoxville, Tennessee & 316 & 83.8 & 3.3 & $(77.4-90.2)$ \\
\hline Lafayette, Louisiana & 399 & 79.8 & 2.5 & $(74.9-84.7)$ \\
\hline Lexington-Fayette, Kentucky & 452 & 83.5 & 2.6 & $(78.4-88.5)$ \\
\hline Lincoln, Nebraska & 1,423 & 85.1 & 1.4 & $(82.4-87.9)$ \\
\hline
\end{tabular}

\footnotetext{
See table footnotes on page 35 .
} 
TABLE 12. (Continued) Age-adjusted* prevalence estimates of adults aged 18-64 years who have health care coverage, ${ }^{\dagger}$ by metropolitan and micropolitan statistical area - Behavioral Risk Factor Surveillance System, United States, 2014

\begin{tabular}{|c|c|c|c|c|}
\hline MMSA & $\begin{array}{l}\text { Sample } \\
\text { size }\end{array}$ & $\%$ & SE & $95 \% \mathrm{Cl}$ \\
\hline Little Rock-North Little Rock-Conway, Arkansas & 685 & 80.0 & 2.4 & $(75.2-84.7)$ \\
\hline Logan, Utah-Idaho & 463 & 87.0 & 1.8 & $(83.4-90.5)$ \\
\hline Los Angeles-Long Beach-Anaheim, California & 1,871 & 78.2 & 1.2 & $(75.8-80.6)$ \\
\hline Louisville-Jefferson County, Kentucky-Indiana & 1,492 & 87.6 & 1.6 & $(84.5-90.6)$ \\
\hline Madison, Wisconsin & 400 & 90.2 & 2.2 & $(85.9-94.5)$ \\
\hline Memphis, Tennessee-Mississippi-Arkansas & 540 & 79.1 & 3.0 & $(73.3-84.9)$ \\
\hline Miami-Fort Lauderdale-West Palm Beach, Florida & 1,291 & 74.8 & 1.7 & $(71.5-78.1)$ \\
\hline Milwaukee-Waukesha-West Allis, Wisconsin & 888 & 89.3 & 1.7 & $(85.9-92.6)$ \\
\hline Minneapolis-St. Paul-Bloomington, Minnesota-Wisconsin & 6,385 & 91.3 & 0.5 & $(90.4-92.3)$ \\
\hline Minot, North Dakota & 380 & 88.9 & 2.1 & $(84.7-93.1)$ \\
\hline Montgomery, Alabama & 331 & 90.3 & 2.1 & $(86.2-94.3)$ \\
\hline $\begin{array}{l}\text { Montgomery County-Bucks County-Chester County, } \\
\text { Pennsylvania§ }\end{array}$ & 531 & 91.5 & 1.9 & $(87.7-95.2)$ \\
\hline $\begin{array}{l}\text { Myrtle Beach-Conway-North Myrtle Beach, South Carolina- } \\
\text { North Carolina }\end{array}$ & 591 & 74.3 & 2.4 & $(69.6-79.0)$ \\
\hline Nashville-Davidson County-Murfreesboro-Franklin, Tennessee & 547 & 87.0 & 2.0 & $(83.0-91.0)$ \\
\hline Nassau County-Suffolk County, New York ${ }^{\S}$ & 519 & 86.7 & 2.2 & $(82.3-91.0)$ \\
\hline Newark, New Jersey-Pennsylvania§ & 2,845 & 82.2 & 1.1 & $(80.0-84.5)$ \\
\hline New Orleans-Metairie, Louisiana & 1,287 & 80.0 & 1.5 & $(77.1-82.9)$ \\
\hline New York-Jersey City-White Plains, New York-New Jersey $§$ & 5,373 & 81.7 & 0.8 & $(80.1-83.3)$ \\
\hline Norfolk, Nebraska & 687 & 85.6 & 1.8 & $(82.0-89.2)$ \\
\hline North Platte, Nebraska & 612 & 81.4 & 2.2 & $(77.2-85.7)$ \\
\hline North Port-Sarasota-Bradenton, Florida & 216 & 76.0 & 4.6 & $(67.0-84.9)$ \\
\hline Oakland-Hayward-Berkeley, California $\$$ & 523 & 86.8 & 1.9 & $(83.2-90.5)$ \\
\hline Ogden-Clearfield, Utah & 2,211 & 85.4 & 0.9 & $(83.6-87.2)$ \\
\hline Oklahoma City, Oklahoma & 1,602 & 81.4 & 1.3 & $(79.0-83.9)$ \\
\hline Omaha-Council Bluffs, Nebraska-lowa & 3,305 & 85.7 & 0.9 & $(83.8-87.5)$ \\
\hline Orlando-Kissimmee-Sanford, Florida & 597 & 79.7 & 2.3 & $(75.1-84.2)$ \\
\hline Philadelphia, Pennsylvania ${ }^{\S}$ & 945 & 81.9 & 1.8 & $(78.3-85.5)$ \\
\hline Phoenix-Mesa-Scottsdale, Arizona & 5,289 & 81.7 & 0.9 & $(79.9-83.4)$ \\
\hline Pittsburgh, Pennsylvania & 1,498 & 89.9 & 1.2 & $(87.4-92.3)$ \\
\hline Ponce, Puerto Rico & 357 & 96.6 & 1.2 & $(94.2-99.0)$ \\
\hline Portland-South Portland, Maine & 1,672 & 88.7 & 1.3 & $(86.3-91.2)$ \\
\hline Portland-Vancouver-Hillsboro, Oregon-Washington & 1,829 & 89.6 & 1.0 & $(87.5-91.6)$ \\
\hline Providence-Warwick, Rhode Island-Massachusetts & 5,164 & 92.0 & 0.7 & $(90.6-93.4)$ \\
\hline Provo-Orem, Utah & 1,760 & 84.4 & 1.0 & $(82.4-86.3)$ \\
\hline Raleigh, North Carolina & 553 & 83.7 & 1.8 & $(80.2-87.2)$ \\
\hline Rapid City, South Dakota & 868 & 85.9 & 1.8 & $(82.4-89.4)$ \\
\hline Reno, Nevada & 772 & 81.9 & 2.0 & $(78.0-85.8)$ \\
\hline Richmond, Virginia & 1,025 & 86.1 & 1.6 & $(83.0-89.1)$ \\
\hline Riverside-San Bernardino-Ontario, California & 684 & 82.1 & 1.8 & $(78.5-85.6)$ \\
\hline Roanoke, Virginia & 322 & 81.3 & 3.3 & $(74.8-87.7)$ \\
\hline Rochester, Minnesota & 489 & 91.1 & 2.1 & $(87.1-95.2)$ \\
\hline Rockingham County-Strafford County, New Hampshire ${ }^{\S}$ & 924 & 87.0 & 1.7 & $(83.6-90.3)$ \\
\hline Sacramento-Roseville-Arden-Arcade, California & 459 & 90.2 & 1.9 & $(86.4-94.0)$ \\
\hline St. Cloud, Minnesota & 439 & 93.8 & 1.4 & $(91.0-96.5)$ \\
\hline St. Louis, Missouri-Illinois & 1,235 & 86.8 & 1.7 & $(83.6-90.1)$ \\
\hline Salisbury, Maryland-Delaware & 1,060 & 84.1 & 2.1 & $(80.0-88.1)$ \\
\hline Salt Lake City, Utah & 4,207 & 83.8 & 0.7 & $(82.4-85.2)$ \\
\hline San Antonio-New Braunfels, Texas & 1,416 & 72.9 & 1.6 & $(69.8-75.9)$ \\
\hline San Juan-Carolina-Caguas, Puerto Rico & 2,430 & 91.2 & 0.8 & $(89.7-92.7)$ \\
\hline Scottsbluff, Nebraska & 505 & 81.6 & 2.3 & $(77.1-86.2)$ \\
\hline Seattle-Bellevue-Everett, Washington ${ }^{\S}$ & 2,418 & 86.7 & 1.1 & $(84.6-88.8)$ \\
\hline Shreveport-Bossier City, Louisiana & 377 & 73.9 & 3.0 & $(68.0-79.7)$ \\
\hline Silver Spring-Frederick-Rockville, Maryland ${ }^{\S}$ & 1,516 & 86.0 & 2.0 & $(82.0-89.9)$ \\
\hline Sioux City, lowa-Nebraska-South Dakota & 673 & 83.6 & 2.9 & $(77.9-89.3)$ \\
\hline Sioux Falls, South Dakota & 877 & 87.7 & 2.2 & $(83.5-92.0)$ \\
\hline Spartanburg, South Carolina & 321 & 77.2 & 3.4 & $(70.6-83.8)$ \\
\hline Spokane-Spokane Valley, Washington & 442 & 85.0 & 2.6 & (79.9-90.1) \\
\hline Springfield, Massachusetts & 688 & 91.8 & 1.5 & $(88.8-94.8)$ \\
\hline Tampa-St. Petersburg-Clearwater, Florida & 899 & 81.3 & 1.9 & $(77.5-85.0)$ \\
\hline
\end{tabular}

See table footnotes on page 35 . 


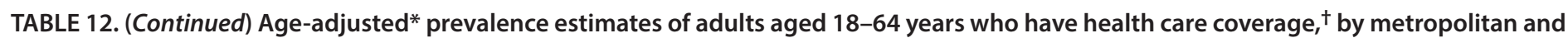
micropolitan statistical area - Behavioral Risk Factor Surveillance System, United States, 2014

\begin{tabular}{|c|c|c|c|c|}
\hline MMSA & $\begin{array}{l}\text { Sample } \\
\text { size }\end{array}$ & $\%$ & SE & $95 \% \mathrm{Cl}$ \\
\hline Toledo, Ohio & 427 & 88.7 & 2.6 & $(83.5-93.8)$ \\
\hline Topeka, Kansas & 942 & 80.8 & 1.7 & $(77.4-84.2)$ \\
\hline Tulsa, Oklahoma & 1,323 & 84.0 & 1.3 & $(81.4-86.6)$ \\
\hline Tuscaloosa, Alabama & 493 & 84.0 & 2.2 & $(79.6-88.4)$ \\
\hline Virginia Beach-Norfolk-Newport News, Virginia-North Carolina & 1,287 & 86.0 & 1.4 & $(83.2-88.7)$ \\
\hline Warren-Troy-Farmington Hills, Michigan ${ }^{\S}$ & 1,362 & 88.4 & 1.2 & $(86.0-90.8)$ \\
\hline $\begin{array}{l}\text { Washington-Arlington-Alexandria, District of Columbia- } \\
\text { Virginia-Maryland-West Virginia§ }\end{array}$ & 5,411 & 85.9 & 1.0 & $(84.0-87.9)$ \\
\hline Wichita, Kansas & 1,843 & 81.5 & 1.2 & $(79.1-83.8)$ \\
\hline Wichita Falls, Texas & 256 & 80.5 & 6.4 & $(68.0-93.1)$ \\
\hline Wilmington, Delaware-Maryland-New Jersey $\S$ & 1,817 & 90.9 & 1.0 & $(88.9-93.0)$ \\
\hline Worcester, Massachusetts-Connecticut & 1,580 & 95.5 & 0.8 & $(94.0-97.1)$ \\
\hline Youngstown-Warren-Boardman, Ohio-Pennsylvania & 314 & 82.9 & 3.9 & $(75.4-90.5)$ \\
\hline $\begin{array}{l}\text { Median } \\
\text { Range }\end{array}$ & & $\begin{array}{c}84.3 \\
64.7-96.6\end{array}$ & & \\
\hline
\end{tabular}

Abbreviations: $\mathrm{Cl}=$ confidence interval; $\mathrm{MMSA}=$ metropolitan and micropolitan statistical areas; $\mathrm{SE}=$ standard error.

${ }^{*}$ Age adjusted to the 2000 U.S. standard population.

† Including health insurance, prepaid plans (e.g., health maintenance organizations), or government plans (e.g., Medicare).

$\S$ Metropolitan division. 
TABLE 13. Age-adjusted* prevalence estimates of adults aged $\geq 18$ years who visited a doctor for a routine physical checkup during the past 12 months, by state/territory - Behavioral Risk Factor Surveillance System, United States, 2013

\begin{tabular}{|c|c|c|c|c|}
\hline State/Territory & $\begin{array}{c}\text { Sample } \\
\text { size }\end{array}$ & $\%$ & SE & $95 \% \mathrm{Cl}$ \\
\hline Alabama & 6,388 & 71.6 & 0.9 & (69.7-73.4) \\
\hline Alaska & 4,536 & 57.8 & 1.1 & $(55.7-60.0)$ \\
\hline Arizona & 4,192 & 63.8 & 1.4 & $(61.0-66.5)$ \\
\hline Arkansas & 5,165 & 65.0 & 1.1 & $(62.9-67.1)$ \\
\hline California & 11,464 & 63.8 & 0.6 & $(62.6-65.1)$ \\
\hline Colorado & 13,435 & 61.5 & 0.6 & $(60.4-62.7)$ \\
\hline Connecticut & 7,665 & 70.9 & 0.9 & $(69.2-72.6)$ \\
\hline Delaware & 5,165 & 75.3 & 0.9 & $(73.5-77.1)$ \\
\hline District of Columbia & 4,890 & 74.0 & 1.0 & $(72.0-76.1)$ \\
\hline Florida & 33,809 & 67.9 & 0.6 & $(66.6-69.1)$ \\
\hline Georgia & 8,050 & 72.6 & 0.7 & $(71.1-74.0)$ \\
\hline Hawaii & 7,828 & 66.9 & 0.8 & $(65.3-68.5)$ \\
\hline Idaho & 5,583 & 57.9 & 1.0 & $(55.9-59.8)$ \\
\hline Illinois & 5,597 & 66.2 & 1.0 & $(64.3-68.1)$ \\
\hline Indiana & 10,220 & 64.0 & 0.7 & $(62.7-65.3)$ \\
\hline lowa & 8,073 & 68.6 & 0.8 & $(67.1-70.1)$ \\
\hline Kansas & 22,855 & 69.6 & 0.4 & $(68.8-70.4)$ \\
\hline Kentucky & 10,800 & 68.6 & 0.7 & $(67.1-70.0)$ \\
\hline Louisiana & 5,159 & 74.8 & 1.1 & $(72.6-76.9)$ \\
\hline Maine & 8,044 & 69.8 & 0.8 & $(68.2-71.4)$ \\
\hline Maryland & 12,887 & 74.6 & 0.7 & $(73.3-76.0)$ \\
\hline Massachusetts & 14,963 & 76.9 & 0.6 & $(75.7-78.1)$ \\
\hline Michigan & 12,602 & 69.2 & 0.6 & $(68.0-70.4)$ \\
\hline Minnesota & 14,189 & 67.9 & 0.7 & $(66.4-69.3)$ \\
\hline Mississippi & 7,299 & 70.6 & 0.9 & $(68.9-72.3)$ \\
\hline Missouri & 7,007 & 66.6 & 0.9 & $(64.8-68.4)$ \\
\hline Montana & 9,559 & 60.7 & 0.8 & $(59.2-62.2)$ \\
\hline Nebraska & 16,919 & 61.4 & 0.7 & $(60.1-62.7)$ \\
\hline Nevada & 5,038 & 66.0 & 1.3 & $(63.5-68.5)$ \\
\hline New Hampshire & 6,413 & 70.2 & 0.9 & $(68.5-72.0)$ \\
\hline New Jersey & 13,195 & 74.7 & 0.6 & $(73.5-75.9)$ \\
\hline New Mexico & 9,177 & 62.5 & 0.8 & $(61.0-64.1)$ \\
\hline New York & 8,906 & 74.4 & 0.7 & $(73.1-75.7)$ \\
\hline North Carolina & 8,759 & 73.0 & 0.7 & $(71.6-74.4)$ \\
\hline North Dakota & 7,720 & 62.7 & 0.8 & $(61.0-64.3)$ \\
\hline Ohio & 11,819 & 69.8 & 0.7 & $(68.5-71.2)$ \\
\hline Oklahoma & 8,090 & 61.5 & 0.8 & $(60.0-63.0)$ \\
\hline Oregon & 5,783 & 57.0 & 0.9 & $(55.2-58.9)$ \\
\hline Pennsylvania & 11,316 & 69.9 & 0.6 & $(68.7-71.2)$ \\
\hline Rhode Island & 6,489 & 77.7 & 0.8 & $(76.1-79.3)$ \\
\hline South Carolina & 10,568 & 65.6 & 0.7 & $(64.2-67.0)$ \\
\hline South Dakota & 6,812 & 67.3 & 1.0 & $(65.3-69.3)$ \\
\hline Tennessee & 5,718 & 74.9 & 0.9 & $(73.1-76.7)$ \\
\hline Texas & 10,673 & 69.9 & 0.7 & $(68.5-71.3)$ \\
\hline Utah & 12,451 & 59.6 & 0.6 & $(58.5-60.7)$ \\
\hline Vermont & 6,331 & 66.1 & 0.9 & $(64.3-67.8)$ \\
\hline Virginia & 8,359 & 72.5 & 0.7 & (71.1-73.9) \\
\hline Washington & 10,986 & 61.7 & 0.7 & $(60.3-63.0)$ \\
\hline West Virginia & 5,833 & 72.5 & 0.8 & $(70.9-74.1)$ \\
\hline Wisconsin & 6,552 & 67.1 & 1.0 & $(65.2-69.0)$ \\
\hline Wyoming & 6,350 & 58.6 & 1.0 & $(56.7-60.5)$ \\
\hline Guam & 1,881 & 65.1 & 1.5 & $(62.2-68.0)$ \\
\hline Puerto Rico & 5,928 & 76.0 & 0.8 & $(74.4-77.5)$ \\
\hline Median & & 67.9 & & \\
\hline Range & & $57.0-77.7$ & & \\
\hline
\end{tabular}

Abbreviations: $\mathrm{Cl}=$ confidence interval; $\mathrm{SE}=$ standard error.

* Age adjusted to the 2000 U.S. standard population. 
TABLE 14. Age-adjusted* prevalence estimates of adults aged $\geq 18$ years who visited a doctor for a routine physical checkup during the past 12 months, by metropolitan and micropolitan statistical area - Behavioral Risk Factor Surveillance System, United States, 2013

\begin{tabular}{|c|c|c|c|c|}
\hline MMSA & $\begin{array}{l}\text { Sample } \\
\text { size }\end{array}$ & $\%$ & SE & $95 \% \mathrm{Cl}$ \\
\hline Aguadilla-Isabela, Puerto Rico & 587 & 78.5 & 2.5 & $(73.6-83.3)$ \\
\hline Akron, Ohio & 683 & 78.2 & 2.5 & $(73.3-83.2)$ \\
\hline Albuquerque, New Mexico & 2,050 & 61.2 & 1.5 & $(58.3-64.0)$ \\
\hline Allentown-Bethlehem-Easton, Pennsylvania-New Jersey & 1,020 & 70.5 & 2.3 & $(65.9-75.1)$ \\
\hline Anchorage, Alaska & 1,517 & 58.7 & 1.7 & $(55.4-62.0)$ \\
\hline Atlanta-Sandy Springs-Roswell, Georgia & 3,484 & 72.0 & 1.1 & $(69.9-74.1)$ \\
\hline Augusta-Richmond County, Georgia-South Carolina & 899 & 78.6 & 2.1 & $(74.5-82.8)$ \\
\hline Austin-Round Rock, Texas & 922 & 68.3 & 2.1 & $(64.2-72.3)$ \\
\hline Baltimore-Columbia-Towson, Maryland & 4,730 & 74.5 & 1.1 & $(72.4-76.6)$ \\
\hline Baton Rouge, Louisiana & 916 & 72.7 & 2.4 & $(68.0-77.5)$ \\
\hline Billings, Montana & 805 & 64.0 & 2.0 & $(60.1-67.9)$ \\
\hline Birmingham-Hoover, Alabama & 1,338 & 72.7 & 1.8 & $(69.1-76.3)$ \\
\hline Bismarck, North Dakota & 1,023 & 61.2 & 2.1 & $(57.1-65.2)$ \\
\hline Boise City, Idaho & 1,480 & 60.8 & 1.8 & $(57.3-64.3)$ \\
\hline Boston, Massachusetts ${ }^{\dagger}$ & 4,031 & 78.4 & 1.0 & $(76.3-80.4)$ \\
\hline Buffalo-Cheektowaga-Niagara Falls, New York & 502 & 73.3 & 2.8 & $(67.8-78.8)$ \\
\hline Burlington-South Burlington, Vermont & 1,620 & 66.7 & 1.6 & $(63.7-69.8)$ \\
\hline Cambridge-Newton-Framingham, Massachusetts ${ }^{\dagger}$ & 4,876 & 75.4 & 1.0 & (73.4-77.5) \\
\hline Camden, New Jersey ${ }^{\dagger}$ & 1,838 & 74.6 & 1.5 & $(71.6-77.6)$ \\
\hline Cedar Rapids, lowa & 641 & 68.2 & 2.6 & $(63.1-73.3)$ \\
\hline Charleston, West Virginia & 813 & 72.8 & 2.2 & $(68.4-77.1)$ \\
\hline Charleston-North Charleston, South Carolina & 1,527 & 67.4 & 1.7 & $(64.0-70.7)$ \\
\hline Charlotte-Concord-Gastonia, North Carolina-South Carolina & 1,927 & 70.1 & 1.5 & $(67.2-73.1)$ \\
\hline Chattanooga, Tennessee-Georgia & 573 & 79.1 & 3.1 & $(73.1-85.2)$ \\
\hline Chicago-Naperville-Elgin, Illinois-Indiana-Wisconsin & 3,328 & 66.2 & 1.2 & $(63.8-68.6)$ \\
\hline Cincinnati, Ohio-Kentucky-Indiana & 2,570 & 69.5 & 1.5 & $(66.6-72.3)$ \\
\hline Claremont-Lebanon, New Hampshire-Vermont & 1,671 & 69.1 & 1.9 & $(65.4-72.7)$ \\
\hline Cleveland-Elyria, Ohio & 1,102 & 73.3 & 2.0 & $(69.4-77.1)$ \\
\hline Colorado Springs, Colorado & 1,345 & 65.7 & 1.7 & $(62.4-69.0)$ \\
\hline Columbia, South Carolina & 1,432 & 65.8 & 1.8 & $(62.2-69.3)$ \\
\hline Columbus, Ohio & 1,831 & 71.2 & 1.4 & $(68.5-73.9)$ \\
\hline Crestview-Fort Walton Beach-Destin, Florida & 1,069 & 64.4 & 2.2 & $(60.0-68.8)$ \\
\hline Dallas-Plano-Irving, Texas ${ }^{\dagger}$ & 885 & 73.1 & 2.0 & $(69.3-77.0)$ \\
\hline Davenport-Moline-Rock Island, lowa-Illinois & 666 & 66.8 & 2.9 & $(61.1-72.5)$ \\
\hline Dayton, Ohio & 833 & 67.0 & 2.6 & $(61.9-72.1)$ \\
\hline Deltona-Daytona Beach-Ormond Beach, Florida & 1,101 & 66.1 & 2.8 & $(60.6-71.6)$ \\
\hline Denver-Aurora-Lakewood, Colorado & 5,633 & 62.3 & 0.8 & $(60.7-63.9)$ \\
\hline Des Moines-West Des Moines, lowa & 1,333 & 71.0 & 1.8 & $(67.5-74.5)$ \\
\hline Duluth, Minnesota-Wisconsin & 700 & 71.5 & 3.1 & $(65.4-77.6)$ \\
\hline Durham-Chapel Hill, North Carolina & 616 & 73.2 & 2.6 & $(68.1-78.2)$ \\
\hline El Paso, Texas & 749 & 65.8 & 2.4 & $(61.1-70.6)$ \\
\hline Evansville, Indiana-Kentucky & 569 & 65.6 & 3.1 & $(59.6-71.6)$ \\
\hline Fargo, North Dakota-Minnesota & 1,173 & 66.7 & 2.1 & $(62.6-70.7)$ \\
\hline Fayetteville-Springdale-Rogers, Arkansas-Missouri & 796 & 63.8 & 2.6 & $(58.7-69.0)$ \\
\hline Fort Smith, Arkansas-Oklahoma & 489 & 64.4 & 3.5 & $(57.6-71.3)$ \\
\hline Fort Wayne, Indiana & 776 & 62.1 & 2.4 & $(57.4-66.8)$ \\
\hline Fort Worth-Arlington, Texas ${ }^{\dagger}$ & 798 & 67.6 & 2.6 & $(62.6-72.6)$ \\
\hline Gainesville, Florida & 1,025 & 64.6 & 2.7 & $(59.4-69.8)$ \\
\hline Grand Forks, North Dakota-Minnesota & 500 & 66.9 & 3.3 & $(60.4-73.5)$ \\
\hline Grand Island, Nebraska & 790 & 56.0 & 2.7 & $(50.7-61.3)$ \\
\hline Grand Rapids-Wyoming, Michigan & 1,333 & 70.7 & 1.7 & $(67.4-74.1)$ \\
\hline Greensboro-High Point, North Carolina & 658 & 73.2 & 2.5 & $(68.3-78.1)$ \\
\hline Greenville-Anderson-Mauldin, South Carolina & 1,337 & 61.5 & 1.9 & $(57.8-65.3)$ \\
\hline Gulfport-Biloxi-Pascagoula, Mississippi & 759 & 65.6 & 2.5 & $(60.8-70.5)$ \\
\hline Hagerstown-Martinsburg, Maryland-West Virginia & 756 & 72.0 & 2.8 & $(66.6-77.5)$ \\
\hline Hartford-West Hartford-East Hartford, Connecticut & 2,820 & 73.2 & 1.3 & $(70.6-75.8)$ \\
\hline Hilton Head Island-Bluffton-Beaufort, South Carolina & 817 & 59.6 & 3.1 & $(53.5-65.7)$ \\
\hline Houston-The Woodlands-Sugar Land, Texas & 1,364 & 72.1 & 1.7 & $(68.8-75.4)$ \\
\hline Huntington-Ashland, West Virginia-Kentucky-Ohio & 1,159 & 72.6 & 1.8 & $(69.1-76.1)$ \\
\hline Idaho Falls, Idaho & 506 & 59.0 & 3.0 & $(53.2-64.8)$ \\
\hline Indianapolis-Carmel-Anderson, Indiana & 2,518 & 66.5 & 1.3 & $(64.0-69.1)$ \\
\hline
\end{tabular}

See table footnotes on page 39. 
TABLE 14. (Continued) Age-adjusted* prevalence estimates of adults aged $\geq 18$ years who visited a doctor for a routine physical checkup during the past 12 months, by metropolitan and micropolitan statistical area - Behavioral Risk Factor Surveillance System, United States, 2013

\begin{tabular}{|c|c|c|c|c|}
\hline MMSA & $\begin{array}{l}\text { Sample } \\
\text { size }\end{array}$ & $\%$ & SE & $95 \% \mathrm{Cl}$ \\
\hline Jackson, Mississippi & 792 & 78.0 & 2.1 & $(73.8-82.2)$ \\
\hline Jacksonville, Florida & 2,852 & 68.9 & 1.4 & $(66.1-71.6)$ \\
\hline Kansas City, Missouri-Kansas & 7,341 & 68.5 & 1.1 & $(66.3-70.8)$ \\
\hline Kingsport-Bristol-Bristol, Tennessee-Virginia & 528 & 74.0 & 3.6 & $(66.8-81.1)$ \\
\hline Knoxville, Tennessee & 643 & 70.9 & 2.5 & $(65.9-75.8)$ \\
\hline Lansing-East Lansing, Michigan & 679 & 69.0 & 2.4 & $(64.3-73.6)$ \\
\hline Lexington-Fayette, Kentucky & 631 & 67.4 & 2.3 & $(62.9-71.9)$ \\
\hline Lincoln, Nebraska & 1,854 & 59.9 & 1.4 & $(57.2-62.5)$ \\
\hline Little Rock-North Little Rock-Conway, Arkansas & 1,123 & 68.2 & 2.1 & $(64.2-72.3)$ \\
\hline Logan, Utah-Idaho & 630 & 52.6 & 2.4 & $(47.9-57.3)$ \\
\hline Los Angeles-Long Beach-Anaheim, California & 3,025 & 64.8 & 1.2 & $(62.5-67.1)$ \\
\hline Louisville-Jefferson County, Kentucky-Indiana & 2,128 & 70.3 & 1.7 & $(67.0-73.6)$ \\
\hline Lubbock, Texas & 516 & 68.4 & 3.2 & $(62.2-74.6)$ \\
\hline Manhattan, Kansas & 653 & 70.8 & 2.1 & $(66.6-75.0)$ \\
\hline Memphis, Tennessee-Mississippi-Arkansas & 1,190 & 79.5 & 1.9 & $(75.7-83.3)$ \\
\hline Miami-Fort Lauderdale-West Palm Beach, Florida & 2,196 & 68.8 & 1.7 & $(65.5-72.1)$ \\
\hline Milwaukee-Waukesha-West Allis, Wisconsin & 1,262 & 69.8 & 2.1 & $(65.7-73.9)$ \\
\hline Minneapolis-St. Paul-Bloomington, Minnesota-Wisconsin & 9,040 & 68.0 & 0.9 & $(66.2-69.9)$ \\
\hline Minot, North Dakota & 642 & 61.4 & 2.5 & $(56.6-66.3)$ \\
\hline $\begin{array}{l}\text { Montgomery County-Bucks County-Chester County, } \\
\text { Pennsylvania§ }\end{array}$ & 964 & 70.9 & 1.9 & $(67.2-74.5)$ \\
\hline $\begin{array}{l}\text { Myrtle Beach-Conway-North Myrtle Beach, South Carolina- } \\
\text { North Carolina }\end{array}$ & 766 & 64.5 & 2.6 & $(59.3-69.6)$ \\
\hline Nashville-Davidson County-Murfreesboro-Franklin, Tennessee & 1,049 & 73.4 & 1.8 & $(69.8-76.9)$ \\
\hline Nassau County-Suffolk County, New York ${ }^{\dagger}$ & 940 & 75.1 & 1.9 & $(71.3-78.9)$ \\
\hline Newark, New Jersey-Pennsylvania ${ }^{\dagger}$ & 4,076 & 75.9 & 1.0 & $(74.0-77.9)$ \\
\hline New Orleans-Metairie, Louisiana & 1,265 & 73.4 & 2.3 & $(68.9-77.8)$ \\
\hline New York-Jersey City-White Plains, New York-New Jersey ${ }^{\dagger}$ & 8,847 & 74.6 & 0.7 & $(73.2-76.1)$ \\
\hline Norfolk, Nebraska & 666 & 65.4 & 2.3 & $(60.9-69.9)$ \\
\hline North Platte, Nebraska & 712 & 56.5 & 2.8 & $(51.1-61.9)$ \\
\hline North Port-Sarasota-Bradenton, Florida & 1,081 & 64.1 & 2.3 & $(59.5-68.7)$ \\
\hline Oakland-Hayward-Berkeley, California ${ }^{\dagger}$ & 699 & 61.8 & 2.3 & $(57.2-66.4)$ \\
\hline Ogden-Clearfield, Utah & 2,390 & 63.7 & 1.2 & $(61.4-66.1)$ \\
\hline Oklahoma City, Oklahoma & 2,598 & 61.0 & 1.3 & $(58.4-63.5)$ \\
\hline Omaha-Council Bluffs, Nebraska-lowa & 3,104 & 64.7 & 1.2 & $(62.3-67.0)$ \\
\hline Orlando-Kissimmee-Sanford, Florida & 2,259 & 65.5 & 1.6 & $(62.5-68.6)$ \\
\hline Panama City, Florida & 1,014 & 62.6 & 2.5 & $(57.8-67.5)$ \\
\hline Pensacola-Ferry Pass-Brent, Florida & 1,307 & 69.4 & 1.8 & $(66.0-72.9)$ \\
\hline Philadelphia, Pennsylvania ${ }^{\dagger}$ & 1,757 & 75.6 & 1.4 & $(72.8-78.4)$ \\
\hline Phoenix-Mesa-Scottsdale, Arizona & 1,536 & 64.1 & 1.9 & $(60.4-67.8)$ \\
\hline Pittsburgh, Pennsylvania & 2,349 & 69.8 & 1.4 & $(67.1-72.5)$ \\
\hline Ponce, Puerto Rico & 523 & 72.5 & 2.7 & $(67.1-77.8)$ \\
\hline Portland-South Portland, Maine & 2,627 & 70.4 & 1.4 & $(67.8-73.1)$ \\
\hline Portland-Vancouver-Hillsboro, Oregon-Washington & 3,172 & 61.0 & 1.2 & $(58.6-63.4)$ \\
\hline Port St. Lucie, Florida & 1,013 & 67.6 & 2.8 & $(62.1-73.1)$ \\
\hline Providence-Warwick, Rhode Island-Massachusetts & 8,250 & 78.8 & 0.9 & $(77.0-80.6)$ \\
\hline Provo-Orem, Utah & 1,803 & 56.7 & 1.4 & $(54.1-59.4)$ \\
\hline Raleigh, North Carolina & 669 & 74.4 & 2.0 & $(70.6-78.3)$ \\
\hline Rapid City, South Dakota & 866 & 65.5 & 2.3 & $(61.1-70.0)$ \\
\hline Reno, Nevada & 1,803 & 63.5 & 1.6 & $(60.3-66.7)$ \\
\hline Richmond, Virginia & 1,300 & 72.5 & 1.7 & $(69.2-75.8)$ \\
\hline Riverside-San Bernardino-Ontario, California & 1,363 & 66.5 & 1.7 & $(63.2-69.9)$ \\
\hline Rochester, New York & 508 & 70.9 & 2.7 & $(65.7-76.1)$ \\
\hline Rockingham County-Strafford County, New Hampshire ${ }^{\S}$ & 1,654 & 70.1 & 1.7 & $(66.9-73.4)$ \\
\hline Sacramento-Roseville-Arden-Arcade, California & 891 & 63.9 & 2.2 & $(59.5-68.2)$ \\
\hline St. Louis, Missouri-Illinois & 2,039 & 70.2 & 1.6 & $(67.0-73.4)$ \\
\hline Salem, Oregon & 515 & 54.2 & 3.4 & $(47.6-60.8)$ \\
\hline Salina, Kansas & 518 & 69.8 & 2.7 & $(64.4-75.1)$ \\
\hline Salisbury, Maryland-Delaware & 2,047 & 75.4 & 1.9 & $(71.6-79.1)$ \\
\hline Salt Lake City, Utah & 4,571 & 60.7 & 0.9 & $(58.9-62.5)$ \\
\hline San Antonio-New Braunfels, Texas & 922 & 65.4 & 2.1 & $(61.3-69.5)$ \\
\hline
\end{tabular}

See table footnotes on page 39. 
TABLE 14. (Continued) Age-adjusted* prevalence estimates of adults aged $\geq 18$ years who visited a doctor for a routine physical checkup during the past 12 months, by metropolitan and micropolitan statistical area - Behavioral Risk Factor Surveillance System, United States, 2013

\begin{tabular}{|c|c|c|c|c|}
\hline MMSA & $\begin{array}{l}\text { Sample } \\
\text { size }\end{array}$ & $\%$ & SE & $95 \% \mathrm{Cl}$ \\
\hline San Francisco-Redwood City-South San Francisco, California ${ }^{\dagger}$ & 538 & 66.4 & 2.8 & $(60.9-71.8)$ \\
\hline San Jose-Sunnyvale-Santa Clara, California & 626 & 63.2 & 2.6 & $(58.2-68.2)$ \\
\hline San Juan-Carolina-Caguas, Puerto Rico & 3,609 & 75.5 & 1.0 & $(73.5-77.4)$ \\
\hline Scottsbluff, Nebraska & 698 & 54.3 & 2.8 & $(48.7-59.8)$ \\
\hline Scranton-Wilkes-Barre-Hazleton, Pennsylvania & 563 & 71.5 & 3.0 & $(65.7-77.4)$ \\
\hline Seattle-Bellevue-Everett, Washington ${ }^{\dagger}$ & 3,717 & 61.7 & 1.1 & $(59.6-63.8)$ \\
\hline Shreveport-Bossier City, Louisiana & 567 & 73.9 & 3.2 & $(67.6-80.3)$ \\
\hline Silver Spring-Frederick-Rockville, Maryland ${ }^{\dagger}$ & 2,410 & 73.4 & 1.5 & $(70.6-76.3)$ \\
\hline Sioux City, lowa-Nebraska-South Dakota & 1,044 & 69.7 & 3.0 & $(63.8-75.7)$ \\
\hline Sioux Falls, South Dakota & 1,002 & 69.8 & 2.0 & $(65.8-73.7)$ \\
\hline Spartanburg, South Carolina & 586 & 64.6 & 3.1 & $(58.6-70.7)$ \\
\hline Spokane-Spokane Valley, Washington & 852 & 60.5 & 2.4 & $(55.8-65.2)$ \\
\hline Springfield, Massachusetts & 1,564 & 75.8 & 2.1 & $(71.8-79.9)$ \\
\hline Tallahassee, Florida & 1,843 & 70.4 & 2.2 & $(66.0-74.8)$ \\
\hline Tampa-St. Petersburg-Clearwater, Florida & 2,191 & 68.2 & 1.5 & $(65.2-71.2)$ \\
\hline Toledo, Ohio & 985 & 68.6 & 2.5 & $(63.6-73.5)$ \\
\hline Topeka, Kansas & 2,383 & 70.1 & 1.3 & $(67.5-72.6)$ \\
\hline Tulsa, Oklahoma & 1,969 & 61.0 & 1.6 & $(57.9-64.2)$ \\
\hline Virginia Beach-Norfolk-Newport News, Virginia-North Carolina & 1,662 & 74.5 & 1.6 & $(71.3-77.6)$ \\
\hline Warren-Troy-Farmington Hills, Michigan ${ }^{\dagger}$ & 2,239 & 70.3 & 1.4 & $(67.6-73.1)$ \\
\hline $\begin{array}{l}\text { Washington-Arlington-Alexandria, District of Columbia- } \\
\text { Virginia-Maryland-West Virginia }{ }^{\dagger}\end{array}$ & 8,946 & 74.7 & 0.9 & $(72.9-76.5)$ \\
\hline Wichita, Kansas & 4,842 & 69.5 & 0.9 & $(67.7-71.2)$ \\
\hline Wilmington, Delaware-Maryland-New Jersey ${ }^{\dagger}$ & 3,244 & 75.1 & 1.1 & $(72.9-77.3)$ \\
\hline Winston-Salem, North Carolina & 691 & 72.6 & 2.8 & $(67.1-78.2)$ \\
\hline Worcester, Massachusetts-Connecticut & 2,750 & 75.9 & 1.4 & $(73.1-78.7)$ \\
\hline Median & & 68.9 & & \\
\hline Range & & $52.6-79.5$ & & \\
\hline
\end{tabular}

Abbreviations: $\mathrm{Cl}=$ confidence interval; $\mathrm{MMSA}=$ metropolitan and micropolitan statistical areas; $\mathrm{SE}=$ standard error.

* Age adjusted to the 2000 U.S. standard population.

+ Metropolitan division. 
TABLE 15. Age-adjusted* prevalence estimates of adults aged $\geq 18$ years who visited a doctor for a routine checkup during the past 12 months, by state/territory - Behavioral Risk Factor Surveillance System, United States, 2014

\begin{tabular}{|c|c|c|c|c|}
\hline State/Territory & $\begin{array}{c}\text { Sample } \\
\text { size }\end{array}$ & $\%$ & SE & $95 \% \mathrm{Cl}$ \\
\hline Alabama & 8,477 & 71.4 & 0.8 & $(69.8-73.0)$ \\
\hline Alaska & 4,334 & 58.7 & 1.1 & $(56.6-60.8)$ \\
\hline Arizona & 14,664 & 63.4 & 0.7 & (61.9-64.8) \\
\hline Arkansas & 5,127 & 66.2 & 1.2 & $(63.8-68.5)$ \\
\hline California & 8,762 & 67.3 & 0.7 & $(66.0-68.6)$ \\
\hline Colorado & 13,141 & 63.1 & 0.6 & $(62.0-64.2)$ \\
\hline Connecticut & 7,885 & 71.3 & 0.8 & $(69.6-72.9)$ \\
\hline Delaware & 4,240 & 72.9 & 1.1 & $(70.7-75.2)$ \\
\hline District of Columbia & 4,033 & 75.0 & 1.2 & (72.7-77.3) \\
\hline Florida & 9,733 & 70.9 & 0.7 & $(69.4-72.3)$ \\
\hline Georgia & 6,262 & 72.3 & 0.8 & $(70.7-74.0)$ \\
\hline Hawaii & 7,207 & 66.7 & 0.8 & $(65.1-68.3)$ \\
\hline Idaho & 5,433 & 57.2 & 1.0 & $(55.2-59.2)$ \\
\hline Illinois & 5,045 & 69.0 & 0.9 & $(67.2-70.8)$ \\
\hline Indiana & 11,415 & 64.2 & 0.7 & $(62.9-65.5)$ \\
\hline lowa & 8,028 & 70.3 & 0.7 & $(68.8-71.7)$ \\
\hline Kansas & 13,395 & 67.7 & 0.6 & $(66.6-68.8)$ \\
\hline Kentucky & 11,009 & 71.4 & 0.8 & $(69.8-73.1)$ \\
\hline Louisiana & 6,684 & 75.2 & 0.7 & (73.8-76.6) \\
\hline Maine & 9,090 & 69.2 & 0.8 & $(67.6-70.8)$ \\
\hline Maryland & 12,466 & 75.0 & 0.8 & (73.4-76.6) \\
\hline Massachusetts & 15,457 & 77.7 & 0.6 & (76.5-78.8) \\
\hline Michigan & 8,370 & 70.7 & 0.7 & $(69.3-72.1)$ \\
\hline Minnesota & 16,220 & 69.2 & 0.5 & $(68.2-70.1)$ \\
\hline Mississippi & 4,131 & 72.3 & 1.1 & $(70.1-74.4)$ \\
\hline Missouri & 6,952 & 66.4 & 0.9 & $(64.5-68.2)$ \\
\hline Montana & 7,382 & 62.4 & 0.9 & $(60.6-64.2)$ \\
\hline Nebraska & 22,090 & 62.9 & 0.6 & $(61.8-64.1)$ \\
\hline Nevada & 3,700 & 64.0 & 1.3 & (61.4-66.6) \\
\hline New Hampshire & 6,114 & 66.8 & 1.0 & $(64.8-68.8)$ \\
\hline New Jersey & 12,876 & 75.8 & 0.6 & $(74.6-77.1)$ \\
\hline New Mexico & 8,785 & 62.0 & 0.9 & $(60.2-63.7)$ \\
\hline New York & 6,801 & 74.4 & 0.7 & $(73.0-75.8)$ \\
\hline North Carolina & 7,192 & 75.1 & 0.7 & (73.8-76.4) \\
\hline North Dakota & 7,720 & 63.8 & 1.0 & $(61.9-65.7)$ \\
\hline Ohio & 10,777 & 69.6 & 0.8 & $(68.0-71.1)$ \\
\hline Oklahoma & 8,303 & 64.0 & 0.7 & $(62.5-65.4)$ \\
\hline Oregon & 5,066 & 61.8 & 1.0 & (59.9-63.8) \\
\hline Pennsylvania & 10,904 & 71.0 & 0.7 & $(69.6-72.4)$ \\
\hline Rhode Island & 6,406 & 79.2 & 0.9 & (77.5-80.9) \\
\hline South Carolina & 10,882 & 66.9 & 0.7 & $(65.5-68.2)$ \\
\hline South Dakota & 7,290 & 69.4 & 1.1 & $(67.3-71.5)$ \\
\hline Tennessee & 5,075 & 73.8 & 1.0 & (71.7-75.8) \\
\hline Texas & 15,130 & 69.1 & 0.7 & $(67.8-70.4)$ \\
\hline Utah & 14,714 & 60.2 & 0.5 & $(59.2-61.2)$ \\
\hline Vermont & 6,412 & 66.1 & 0.8 & $(64.5-67.7)$ \\
\hline Virginia & 9,383 & 73.1 & 0.7 & $(71.8-74.5)$ \\
\hline Washington & 9,944 & 63.7 & 0.7 & $(62.2-65.1)$ \\
\hline West Virginia & 6,129 & 75.3 & 0.8 & (73.7-76.9) \\
\hline Wisconsin & 7,013 & 69.2 & 0.9 & $(67.5-70.9)$ \\
\hline Wyoming & 6,302 & 58.0 & 1.2 & $(55.7-60.4)$ \\
\hline Guam & 2,503 & 61.6 & 1.4 & (58.9-64.3) \\
\hline Puerto Rico & 5,906 & 76.0 & 0.8 & $(74.5-77.6)$ \\
\hline Median & & 69.2 & & \\
\hline Range & & $57.2-79.2$ & & \\
\hline
\end{tabular}

Abbreviations: $\mathrm{Cl}=$ confidence interval; $\mathrm{SE}=$ standard error

* Age adjusted to the 2000 U.S. standard population. 
TABLE 16. Age-adjusted* prevalence estimates of adults aged $\geq 18$ years who visited a doctor for a routine checkup during the past 12 months, by metropolitan and micropolitan statistical area - Behavioral Risk Factor Surveillance System, United States, 2014

\begin{tabular}{|c|c|c|c|c|}
\hline MMSA & $\begin{array}{l}\text { Sample } \\
\text { size }\end{array}$ & $\%$ & SE & $95 \% \mathrm{Cl}$ \\
\hline Aberdeen, South Dakota & 609 & 66.3 & 3.2 & $(60.1-72.5)$ \\
\hline Aguadilla-Isabela, Puerto Rico & 535 & 78.6 & 2.4 & $(73.9-83.2)$ \\
\hline Albuquerque, New Mexico & 1,765 & 62.8 & 1.6 & $(59.6-66.0)$ \\
\hline Allentown-Bethlehem-Easton, Pennsylvania-New Jersey & 1,082 & 68.2 & 2.6 & $(63.0-73.3)$ \\
\hline Anchorage, Alaska & 1,767 & 59.4 & 1.5 & $(56.5-62.4)$ \\
\hline Atlanta-Sandy Springs-Roswell, Georgia & 2,752 & 72.1 & 1.2 & $(69.8-74.4)$ \\
\hline Augusta-Richmond County, Georgia-South Carolina & 876 & 67.5 & 2.8 & $(61.9-73.0)$ \\
\hline Austin-Round Rock, Texas & 2,226 & 69.3 & 1.4 & $(66.6-72.0)$ \\
\hline Baltimore-Columbia-Towson, Maryland & 4,585 & 75.5 & 1.3 & (73.0-77.9) \\
\hline Baton Rouge, Louisiana & 909 & 77.4 & 1.8 & $(73.9-80.9)$ \\
\hline Berlin, New Hampshire-Vermont & 537 & 64.7 & 4.7 & $(55.5-73.8)$ \\
\hline Billings, Montana & 796 & 63.8 & 2.4 & $(59.1-68.4)$ \\
\hline Birmingham-Hoover, Alabama & 1,553 & 73.7 & 1.6 & $(70.6-76.9)$ \\
\hline Bismarck, North Dakota & 1,023 & 60.4 & 2.4 & $(55.7-65.0)$ \\
\hline Boise City, Idaho & 1,343 & 60.6 & 2.0 & $(56.7-64.4)$ \\
\hline Boston, Massachusetts ${ }^{\dagger}$ & 4,491 & 77.6 & 1.1 & $(75.4-79.8)$ \\
\hline Burlington-South Burlington, Vermont & 1,961 & 65.8 & 1.4 & $(63.2-68.5)$ \\
\hline Cambridge-Newton-Framingham, Massachusetts ${ }^{\dagger}$ & 5,108 & 76.4 & 1.0 & $(74.4-78.4)$ \\
\hline Camden, New Jersey ${ }^{\dagger}$ & 1,705 & 76.9 & 1.8 & $(73.4-80.5)$ \\
\hline Cedar Rapids, lowa & 632 & 68.6 & 2.7 & $(63.4-73.8)$ \\
\hline Charleston, West Virginia & 870 & 79.6 & 1.9 & $(76.0-83.3)$ \\
\hline Charleston-North Charleston, South Carolina & 1,380 & 68.6 & 1.8 & $(65.1-72.1)$ \\
\hline Charlotte-Concord-Gastonia, North Carolina-South Carolina & 2,126 & 71.7 & 1.4 & $(69.1-74.4)$ \\
\hline Chicago-Naperville-Elgin, Illinois-Indiana-Wisconsin & 4,109 & 69.6 & 1.1 & $(67.5-71.7)$ \\
\hline Cincinnati, Ohio-Kentucky-Indiana & 2,019 & 69.5 & 1.9 & $(65.8-73.1)$ \\
\hline Claremont-Lebanon, New Hampshire-Vermont & 1,672 & 63.4 & 2.1 & $(59.3-67.6)$ \\
\hline Cleveland-Elyria, Ohio & 957 & 71.9 & 2.2 & $(67.6-76.1)$ \\
\hline College Station-Bryan, Texas & 572 & 70.1 & 3.8 & $(62.7-77.4)$ \\
\hline Colorado Springs, Colorado & 1,276 & 68.3 & 1.8 & $(64.9-71.8)$ \\
\hline Columbia, South Carolina & 1,195 & 68.3 & 1.9 & $(64.7-72.0)$ \\
\hline Columbus, Ohio & 1,641 & 70.7 & 1.8 & $(67.2-74.1)$ \\
\hline Corpus Christi, Texas & 611 & 69.5 & 3.6 & $(62.4-76.6)$ \\
\hline Dallas-Plano-Irving, Texas ${ }^{\dagger}$ & 1,273 & 72.1 & 1.9 & $(68.3-75.9)$ \\
\hline Dayton, Ohio & 553 & 67.5 & 3.0 & $(61.6-73.4)$ \\
\hline Denver-Aurora-Lakewood, Colorado & 5,705 & 62.6 & 0.8 & $(61.0-64.2)$ \\
\hline Des Moines-West Des Moines, lowa & 1,340 & 71.9 & 1.8 & $(68.4-75.5)$ \\
\hline Duluth, Minnesota-Wisconsin & 938 & 69.7 & 2.1 & $(65.5-73.9)$ \\
\hline El Paso, Texas & 713 & 63.8 & 2.6 & $(58.7-68.8)$ \\
\hline Evansville, Indiana-Kentucky & 652 & 74.4 & 3.3 & $(68.0-80.9)$ \\
\hline Fargo, North Dakota-Minnesota & 1,142 & 70.0 & 1.9 & $(66.2-73.8)$ \\
\hline Fayetteville-Springdale-Rogers, Arkansas-Missouri & 797 & 65.2 & 3.0 & $(59.3-71.1)$ \\
\hline Fort Wayne, Indiana & 856 & 60.8 & 2.6 & $(55.7-65.9)$ \\
\hline Fort Worth-Arlington, Texas ${ }^{\dagger}$ & 752 & 67.6 & 2.6 & $(62.5-72.7)$ \\
\hline Grand Island, Nebraska & 1,051 & 63.7 & 2.5 & $(58.9-68.6)$ \\
\hline Grand Rapids-Wyoming, Michigan & 886 & 70.9 & 2.2 & $(66.6-75.2)$ \\
\hline Greensboro-High Point, North Carolina & 510 & 73.6 & 2.7 & $(68.2-78.9)$ \\
\hline Greenville-Anderson-Mauldin, South Carolina & 1,474 & 63.5 & 1.7 & $(60.1-66.9)$ \\
\hline Hagerstown-Martinsburg, Maryland-West Virginia & 774 & 73.8 & 3.0 & $(67.9-79.6)$ \\
\hline Hartford-West Hartford-East Hartford, Connecticut & 2,625 & 72.3 & 1.4 & $(69.6-74.9)$ \\
\hline Hilton Head Island-Bluffton-Beaufort, South Carolina & 550 & 69.8 & 3.4 & $(63.2-76.4)$ \\
\hline Houston-The Woodlands-Sugar Land, Texas & 2,117 & 70.2 & 1.6 & $(67.1-73.4)$ \\
\hline Huntington-Ashland, West Virginia-Kentucky-Ohio & 1,230 & 73.8 & 1.9 & $(70.1-77.6)$ \\
\hline Idaho Falls, Idaho & 514 & 60.4 & 2.8 & $(55.0-65.8)$ \\
\hline Indianapolis-Carmel-Anderson, Indiana & 3,585 & 65.2 & 1.2 & $(62.8-67.5)$ \\
\hline Jacksonville, Florida & 668 & 73.7 & 2.5 & $(68.7-78.6)$ \\
\hline Kansas City, Missouri-Kansas & 4,804 & 66.4 & 1.3 & $(63.9-69.0)$ \\
\hline Kingsport-Bristol-Bristol, Tennessee-Virginia & 497 & 75.2 & 3.7 & $(68.0-82.5)$ \\
\hline Knoxville, Tennessee & 559 & 77.0 & 3.3 & $(70.5-83.4)$ \\
\hline Lafayette, Louisiana & 553 & 76.1 & 2.3 & $(71.6-80.6)$ \\
\hline Lexington-Fayette, Kentucky & 614 & 70.6 & 2.5 & $(65.7-75.4)$ \\
\hline Lincoln, Nebraska & 1,983 & 59.9 & 1.5 & $(57.1-62.8)$ \\
\hline
\end{tabular}

See table footnotes on page 43. 
TABLE 16. (Continued) Age-adjusted* prevalence estimates of adults aged $\geq 18$ years who visited a doctor for a routine checkup during the past 12 months, by metropolitan and micropolitan statistical area - Behavioral Risk Factor Surveillance System, United States, 2014

\begin{tabular}{|c|c|c|c|c|}
\hline MMSA & $\begin{array}{l}\text { Sample } \\
\text { size }\end{array}$ & $\%$ & SE & $95 \% \mathrm{Cl}$ \\
\hline Little Rock-North Little Rock-Conway, Arkansas & 1,167 & 69.7 & 2.1 & $(65.5-73.8)$ \\
\hline Logan, Utah-Idaho & 614 & 58.0 & 2.2 & $(53.6-62.4)$ \\
\hline Los Angeles-Long Beach-Anaheim, California & 2,434 & 68.4 & 1.2 & $(66.0-70.7)$ \\
\hline Louisville-Jefferson County, Kentucky-Indiana & 2,436 & 71.8 & 1.8 & $(68.2-75.4)$ \\
\hline Madison, Wisconsin & 549 & 72.8 & 2.5 & $(68.0-77.7)$ \\
\hline Memphis, Tennessee-Mississippi-Arkansas & 870 & 76.7 & 2.7 & $(71.4-82.0)$ \\
\hline Miami-Fort Lauderdale-West Palm Beach, Florida & 2,200 & 73.8 & 1.4 & $(71.0-76.6)$ \\
\hline Milwaukee-Waukesha-West Allis, Wisconsin & 1,354 & 71.8 & 1.9 & $(68.1-75.6)$ \\
\hline Minneapolis-St. Paul-Bloomington, Minnesota-Wisconsin & 8,655 & 69.0 & 0.6 & $(67.8-70.3)$ \\
\hline Minot, North Dakota & 594 & 67.2 & 2.8 & $(61.7-72.8)$ \\
\hline Montgomery, Alabama & 508 & 76.2 & 3.0 & $(70.2-82.1)$ \\
\hline $\begin{array}{l}\text { Montgomery County-Bucks County-Chester County, } \\
\text { Pennsylvania }^{\dagger}\end{array}$ & 799 & 68.8 & 2.4 & $(64.1-73.5)$ \\
\hline $\begin{array}{l}\text { Myrtle Beach-Conway-North Myrtle Beach, South Carolina- } \\
\text { North Carolina }\end{array}$ & 991 & 68.7 & 2.2 & $(64.5-73.0)$ \\
\hline Nashville-Davidson County-Murfreesboro-Franklin, Tennessee & 795 & 74.5 & 2.2 & $(70.1-78.9)$ \\
\hline Nassau County-Suffolk County, New York ${ }^{\dagger}$ & 767 & 71.7 & 2.3 & $(67.2-76.1)$ \\
\hline Newark, New Jersey-Pennsylvania ${ }^{\dagger}$ & 4,093 & 75.7 & 1.1 & $(73.6-77.8)$ \\
\hline New Orleans-Metairie, Louisiana & 1,888 & 75.3 & 1.4 & $(72.6-77.9)$ \\
\hline New York-Jersey City-White Plains, New York-New Jersey ${ }^{\dagger}$ & 7,453 & 75.7 & 0.8 & $(74.2-77.2)$ \\
\hline Norfolk, Nebraska & 982 & 57.5 & 2.0 & $(53.5-61.4)$ \\
\hline North Platte, Nebraska & 948 & 56.8 & 2.2 & $(52.6-61.1)$ \\
\hline North Port-Sarasota-Bradenton, Florida & 510 & 61.7 & 4.2 & $(53.5-69.9)$ \\
\hline Oakland-Hayward-Berkeley, California ${ }^{\dagger}$ & 696 & 69.0 & 2.4 & $(64.3-73.6)$ \\
\hline Ogden-Clearfield, Utah & 2,873 & 62.3 & 1.0 & $(60.2-64.3)$ \\
\hline Oklahoma City, Oklahoma & 2,397 & 64.0 & 1.3 & $(61.5-66.6)$ \\
\hline Omaha-Council Bluffs, Nebraska-lowa & 4,824 & 67.6 & 1.0 & $(65.7-69.6)$ \\
\hline Orlando-Kissimmee-Sanford, Florida & 949 & 70.7 & 2.1 & $(66.5-74.9)$ \\
\hline Philadelphia, Pennsylvania $^{\dagger}$ & 1,509 & 72.4 & 1.7 & $(69.0-75.8)$ \\
\hline Phoenix-Mesa-Scottsdale, Arizona & 9,277 & 63.5 & 0.9 & $(61.8-65.2)$ \\
\hline Pittsburgh, Pennsylvania & 2,398 & 72.1 & 1.4 & $(69.4-74.8)$ \\
\hline Ponce, Puerto Rico & 524 & 75.8 & 2.8 & (70.4-81.3) \\
\hline Portland-South Portland, Maine & 2,746 & 70.3 & 1.4 & $(67.5-73.0)$ \\
\hline Portland-Vancouver-Hillsboro, Oregon-Washington & 2,765 & 63.0 & 1.4 & $(60.4-65.7)$ \\
\hline Providence-Warwick, Rhode Island-Massachusetts & 8,051 & 80.8 & 0.9 & $(79.1-82.5)$ \\
\hline Provo-Orem, Utah & 2,107 & 57.8 & 1.2 & $(55.4-60.2)$ \\
\hline Raleigh, North Carolina & 714 & 73.5 & 2.0 & $(69.6-77.4)$ \\
\hline Rapid City, South Dakota & 1,403 & 66.5 & 2.1 & $(62.3-70.7)$ \\
\hline Reno, Nevada & 1,193 & 62.3 & 2.0 & $(58.4-66.2)$ \\
\hline Richmond, Virginia & 1,451 & 72.4 & 1.7 & $(69.0-75.8)$ \\
\hline Riverside-San Bernardino-Ontario, California & 937 & 69.5 & 1.9 & $(65.8-73.3)$ \\
\hline Roanoke, Virginia & 524 & 74.0 & 3.0 & $(68.2-79.8)$ \\
\hline Rochester, Minnesota & 699 & 64.5 & 2.3 & $(60.0-69.0)$ \\
\hline Rockingham County-Strafford County, New Hampshire ${ }^{\dagger}$ & 1,424 & 68.9 & 1.9 & $(65.1-72.7)$ \\
\hline Sacramento-Roseville-Arden-Arcade, California & 639 & 65.9 & 2.4 & $(61.2-70.7)$ \\
\hline St. Cloud, Minnesota & 558 & 72.3 & 2.3 & $(67.9-76.8)$ \\
\hline St. Louis, Missouri-Illinois & 1,911 & 71.5 & 1.8 & $(68.1-75.0)$ \\
\hline Salisbury, Maryland-Delaware & 1,937 & 74.0 & 2.3 & $(69.5-78.4)$ \\
\hline Salt Lake City, Utah & 5,326 & 60.6 & 0.8 & $(59.1-62.1)$ \\
\hline San Antonio-New Braunfels, Texas & 2,241 & 69.6 & 1.4 & $(66.9-72.3)$ \\
\hline San Juan-Carolina-Caguas, Puerto Rico & 3,699 & 75.0 & 1.0 & (73.1-77.0) \\
\hline Scottsbluff, Nebraska & 884 & 55.9 & 2.4 & $(51.2-60.6)$ \\
\hline Seattle-Bellevue-Everett, Washington ${ }^{\dagger}$ & 3,653 & 63.7 & 1.1 & $(61.5-66.0)$ \\
\hline Shreveport-Bossier City, Louisiana & 547 & 72.8 & 2.7 & $(67.4-78.1)$ \\
\hline Silver Spring-Frederick-Rockville, Maryland ${ }^{\dagger}$ & 2,357 & 72.4 & 1.7 & $(69.0-75.8)$ \\
\hline Sioux City, lowa-Nebraska-South Dakota & 1,126 & 76.4 & 2.6 & (71.4-81.4) \\
\hline Sioux Falls, South Dakota & 1,337 & 73.7 & 2.1 & $(69.7-77.8)$ \\
\hline Spartanburg, South Carolina & 562 & 63.9 & 3.3 & $(57.4-70.4)$ \\
\hline Spokane-Spokane Valley, Washington & 712 & 62.1 & 2.7 & $(56.9-67.3)$ \\
\hline Springfield, Massachusetts & 1,095 & 78.6 & 2.0 & $(74.7-82.5)$ \\
\hline Tampa-St. Petersburg-Clearwater, Florida & 1,574 & 69.6 & 1.8 & $(66.0-73.2)$ \\
\hline
\end{tabular}

See table footnotes on page 43. 
TABLE 16. (Continued) Age-adjusted* prevalence estimates of adults aged $\geq 18$ years who visited a doctor for a routine checkup during the past 12 months, by metropolitan and micropolitan statistical area - Behavioral Risk Factor Surveillance System, United States, 2014

\begin{tabular}{|c|c|c|c|c|}
\hline MMSA & $\begin{array}{l}\text { Sample } \\
\text { size }\end{array}$ & $\%$ & SE & $95 \% \mathrm{Cl}$ \\
\hline Toledo, Ohio & 639 & 65.8 & 3.1 & $(59.7-71.9)$ \\
\hline Topeka, Kansas & 1,425 & 67.3 & 1.7 & $(64.0-70.6)$ \\
\hline Tulsa, Oklahoma & 2,002 & 61.8 & 1.4 & $(59.0-64.6)$ \\
\hline Tuscaloosa, Alabama & 708 & 72.1 & 2.4 & $(67.4-76.9)$ \\
\hline Virginia Beach-Norfolk-Newport News, Virginia-North Carolina & 1,872 & 77.8 & 1.5 & $(74.9-80.6)$ \\
\hline Warren-Troy-Farmington Hills, Michigan ${ }^{\dagger}$ & 2,095 & 71.0 & 1.4 & $(68.2-73.8)$ \\
\hline $\begin{array}{l}\text { Washington-Arlington-Alexandria, District of Columbia-Virginia- } \\
\text { Maryland-West Virginia }{ }^{\dagger}\end{array}$ & 8,231 & 73.8 & 0.9 & $(71.9-75.6)$ \\
\hline Wichita, Kansas & 2,667 & 69.5 & 1.2 & $(67.2-71.8)$ \\
\hline Wichita Falls, Texas & 540 & 76.7 & 4.2 & $(68.5-84.8)$ \\
\hline Wilmington, Delaware-Maryland-New Jersey ${ }^{\dagger}$ & 2,733 & 73.5 & 1.4 & $(70.8-76.3)$ \\
\hline Worcester, Massachusetts-Connecticut & 2,437 & 77.1 & 1.4 & $(74.4-79.8)$ \\
\hline Youngstown-Warren-Boardman, Ohio-Pennsylvania & 523 & 72.3 & 3.4 & $(65.6-79.1)$ \\
\hline $\begin{array}{l}\text { Median } \\
\text { Range }\end{array}$ & & $\begin{array}{c}69.8 \\
55.9-80.8\end{array}$ & & \\
\hline
\end{tabular}

Abbreviations: $\mathrm{Cl}=$ confidence interval; $\mathrm{MMSA}=$ metropolitan and micropolitan statistical areas; $\mathrm{SE}=$ standard error.

* Age adjusted to the 2000 U.S. standard population.

${ }^{\dagger}$ Metropolitan division. 
TABLE 17. Age-adjusted* prevalence estimates of women aged 21-65 years who reported having a Pap ${ }^{\dagger}$ test, by state/territory Behavioral Risk Factor Surveillance System, United States, 2014

\begin{tabular}{|c|c|c|c|c|}
\hline State/Territory & $\begin{array}{c}\text { Sample } \\
\text { size }\end{array}$ & $\%$ & SE & $95 \% \mathrm{Cl}$ \\
\hline Alabama & 2,056 & 82.9 & 1.1 & $(80.8-85.0)$ \\
\hline Alaska & 1,420 & 78.6 & 1.6 & $(75.5-81.8)$ \\
\hline Arizona & 3,342 & 79.8 & 1.1 & (77.6-81.9) \\
\hline Arkansas & 1,042 & 77.7 & 1.8 & $(74.1-81.3)$ \\
\hline California & 2,120 & 83.0 & 1.1 & $(80.9-85.1)$ \\
\hline Colorado & 3,460 & 84.6 & 0.8 & $(83.1-86.2)$ \\
\hline Connecticut & 2,142 & 87.5 & 1.0 & $(85.5-89.5)$ \\
\hline Delaware & 1,184 & 86.4 & 1.5 & $(83.5-89.3)$ \\
\hline District of Columbia & 1,108 & 85.1 & 1.7 & $(81.8-88.5)$ \\
\hline Florida & 2,304 & 79.6 & 1.1 & (77.4-81.8) \\
\hline Georgia & 1,563 & 84.6 & 1.1 & $(82.3-86.8)$ \\
\hline Hawaii & 2,010 & 77.8 & 1.4 & $(75.1-80.6)$ \\
\hline Idaho & 1,353 & 76.0 & 1.5 & (73.0-78.9) \\
\hline Illinois & 1,460 & 81.5 & 1.4 & $(78.7-84.2)$ \\
\hline Indiana & 2,811 & 78.0 & 1.0 & $(75.9-80.0)$ \\
\hline lowa & 2,118 & 84.5 & 1.1 & $(82.4-86.6)$ \\
\hline Kansas & 3,401 & 81.8 & 0.8 & $(80.2-83.5)$ \\
\hline Kentucky & 2,993 & 81.4 & 1.2 & (79.1-83.7) \\
\hline Louisiana & 1,761 & 83.7 & 1.1 & $(81.5-85.8)$ \\
\hline Maine & 2,493 & 85.2 & 1.0 & $(83.1-87.2)$ \\
\hline Maryland & 3,676 & 86.7 & 1.2 & $(84.3-89.0)$ \\
\hline Massachusetts & 4,424 & 87.8 & 0.8 & $(86.1-89.4)$ \\
\hline Michigan & 2,233 & 83.4 & 1.0 & $(81.4-85.4)$ \\
\hline Minnesota & 4,746 & 86.1 & 0.6 & (84.9-87.3) \\
\hline Mississippi & 1,011 & 83.0 & 1.5 & $(80.1-85.9)$ \\
\hline Missouri & 1,737 & 80.8 & 1.3 & $(78.2-83.3)$ \\
\hline Montana & 1,778 & 81.5 & 1.3 & $(78.9-84.1)$ \\
\hline Nebraska & 5,779 & 81.7 & 0.8 & $(80.1-83.4)$ \\
\hline Nevada & 993 & 78.1 & 2.1 & $(73.9-82.2)$ \\
\hline New Hampshire & 1,765 & 85.1 & 1.4 & $(82.3-87.9)$ \\
\hline New Jersey & 3,976 & 83.8 & 0.9 & $(82.1-85.6)$ \\
\hline New Mexico & 2,394 & 79.0 & 1.3 & $(76.5-81.5)$ \\
\hline New York & 2,073 & 82.6 & 1.1 & $(80.3-84.8)$ \\
\hline North Carolina & 1,898 & 85.8 & 0.9 & $(84.0-87.6)$ \\
\hline North Dakota & 1,959 & 81.5 & 1.5 & $(78.6-84.3)$ \\
\hline Ohio & 2,943 & 81.6 & 1.1 & $(79.5-83.7)$ \\
\hline Oklahoma & 2,070 & 77.0 & 1.2 & $(74.7-79.3)$ \\
\hline Oregon & 1,226 & 83.0 & 1.3 & (80.4-85.6) \\
\hline Pennsylvania & 2,918 & 80.8 & 1.1 & $(78.5-83.0)$ \\
\hline Rhode Island & 1,925 & 85.9 & 1.2 & $(83.4-88.3)$ \\
\hline South Carolina & 2,655 & 82.4 & 1.0 & $(80.5-84.4)$ \\
\hline South Dakota & 2,012 & 84.8 & 1.5 & $(81.8-87.7)$ \\
\hline Tennessee & 1,210 & 85.4 & 1.4 & $(82.7-88.1)$ \\
\hline Texas & 3,959 & 77.7 & 1.1 & $(75.6-79.8)$ \\
\hline Utah & 4,205 & 77.2 & 0.8 & $(75.6-78.8)$ \\
\hline Vermont & 2,038 & 85.8 & 1.0 & (83.8-87.8) \\
\hline Virginia & 2,773 & 85.2 & 1.0 & $(83.3-87.1)$ \\
\hline Washington & 2,657 & 80.9 & 1.1 & $(78.8-83.1)$ \\
\hline West Virginia & 1,656 & 80.5 & 1.2 & (78.2-82.8) \\
\hline Wisconsin & 1,831 & 86.7 & 1.1 & $(84.4-88.9)$ \\
\hline Wyoming & 1,352 & 81.2 & 1.5 & $(78.2-84.2)$ \\
\hline Guam & 955 & 67.7 & 2.2 & $(63.5-72.0)$ \\
\hline Puerto Rico & 1,880 & 77.4 & 1.2 & $(75.1-79.7)$ \\
\hline Median & & 82.4 & & \\
\hline Range & & $67.7-87.8$ & & \\
\hline
\end{tabular}

Abbreviations: $\mathrm{Cl}=$ confidence interval; $\mathrm{Pap}=$ Papanicolaou; $\mathrm{SE}=$ standard error.

* Age adjusted to the 2000 U.S. standard population.

$\dagger$ Test for cancer of the cervix. 
TABLE 18. Age-adjusted* prevalence estimates of women aged 21-65 years who reported having a Pap ${ }^{\dagger}$ test, by metropolitan and micropolitan statistical area - Behavioral Risk Factor Surveillance System, United States, 2014

\begin{tabular}{|c|c|c|c|c|}
\hline MMSA & $\begin{array}{l}\text { Sample } \\
\text { size }\end{array}$ & $\%$ & SE & $95 \% \mathrm{Cl}$ \\
\hline Aberdeen, South Dakota & 161 & 84.8 & 4.7 & $(75.6-94.0)$ \\
\hline Aguadilla-Isabela, Puerto Rico & 176 & 68.2 & 4.3 & $(59.7-76.6)$ \\
\hline Albuquerque, New Mexico & 509 & 80.8 & 2.3 & $(76.2-85.4)$ \\
\hline Allentown-Bethlehem-Easton, Pennsylvania-New Jersey & 302 & 88.7 & 2.4 & $(84.1-93.4)$ \\
\hline Anchorage, Alaska & 568 & 78.2 & 2.4 & $(73.5-82.8)$ \\
\hline Atlanta-Sandy Springs-Roswell, Georgia & 747 & 86.6 & 1.5 & $(83.7-89.6)$ \\
\hline Augusta-Richmond County, Georgia-South Carolina & 209 & 88.1 & 3.2 & $(81.8-94.5)$ \\
\hline Austin-Round Rock, Texas & 664 & 83.3 & 2.0 & $(79.4-87.1)$ \\
\hline Baltimore-Columbia-Towson, Maryland & 1,374 & 87.3 & 2.0 & $(83.3-91.2)$ \\
\hline Baton Rouge, Louisiana & 248 & 82.6 & 2.9 & $(77.0-88.2)$ \\
\hline Berlin, New Hampshire-Vermont & 140 & 90.5 & 3.3 & $(84.0-96.9)$ \\
\hline Billings, Montana & 186 & 82.9 & 3.5 & $(76.0-89.8)$ \\
\hline Birmingham-Hoover, Alabama & 417 & 83.7 & 2.2 & $(79.3-88.0)$ \\
\hline Bismarck, North Dakota & 259 & 86.2 & 2.5 & $(81.3-91.2)$ \\
\hline Boise City, Idaho & 323 & 80.6 & 2.7 & $(75.4-85.8)$ \\
\hline Boston, Massachusetts $§$ & 1,325 & 86.3 & 1.7 & $(83.0-89.6)$ \\
\hline Burlington-South Burlington, Vermont & 648 & 86.0 & 1.8 & $(82.5-89.4)$ \\
\hline Cambridge-Newton-Framingham, Massachusetts $§$ & 1,461 & 88.3 & 1.4 & $(85.6-91.0)$ \\
\hline Camden, New Jersey ${ }^{\S}$ & 541 & 87.4 & 2.2 & $(83.0-91.8)$ \\
\hline Cedar Rapids, lowa & 177 & 87.1 & 3.6 & $(80.0-94.2)$ \\
\hline Charleston, West Virginia & 227 & 78.5 & 3.3 & $(71.9-85.0)$ \\
\hline Charleston-North Charleston, South Carolina & 382 & 81.7 & 2.6 & $(76.6-86.8)$ \\
\hline Charlotte-Concord-Gastonia, North Carolina-South Carolina & 541 & 84.7 & 1.9 & $(81.1-88.4)$ \\
\hline Chicago-Naperville-Elgin, Illinois-Indiana-Wisconsin & 1,217 & 81.5 & 1.7 & $(78.2-84.7)$ \\
\hline Cincinnati, Ohio-Kentucky-Indiana & 568 & 82.9 & 2.5 & $(78.1-87.8)$ \\
\hline Claremont-Lebanon, New Hampshire-Vermont & 502 & 84.8 & 2.5 & $(79.9-89.7)$ \\
\hline Cleveland-Elyria, Ohio & 283 & 83.5 & 2.9 & $(77.8-89.1)$ \\
\hline College Station-Bryan, Texas & 120 & 78.5 & 5.9 & $(66.9-90.2)$ \\
\hline Colorado Springs, Colorado & 322 & 82.8 & 2.6 & $(77.7-87.9)$ \\
\hline Columbia, South Carolina & 369 & 86.8 & 2.0 & $(82.9-90.7)$ \\
\hline Columbus, Ohio & 526 & 83.7 & 2.2 & $(79.3-88.1)$ \\
\hline Corpus Christi, Texas & 119 & 84.1 & 4.4 & $(75.5-92.7)$ \\
\hline Dallas-Plano-Irving, Texas ${ }^{\S}$ & 349 & 77.4 & 3.1 & $(71.3-83.5)$ \\
\hline Dayton, Ohio & 146 & 87.2 & 4.0 & $(79.4-95.0)$ \\
\hline Denver-Aurora-Lakewood, Colorado & 1,601 & 84.3 & 1.1 & $(82.0-86.5)$ \\
\hline Des Moines-West Des Moines, lowa & 374 & 88.0 & 2.6 & $(82.9-93.2)$ \\
\hline Duluth, Minnesota-Wisconsin & 263 & 81.5 & 3.0 & $(75.7-87.3)$ \\
\hline El Paso, Texas & 187 & 80.2 & 3.6 & $(73.2-87.3)$ \\
\hline Evansville, Indiana-Kentucky & 119 & 85.1 & 6.1 & (73.0-97.1) \\
\hline Fargo, North Dakota-Minnesota & 288 & 81.2 & 3.3 & $(74.7-87.7)$ \\
\hline Fayetteville-Springdale-Rogers, Arkansas-Missouri & 176 & 71.6 & 4.9 & $(62.0-81.3)$ \\
\hline Fort Wayne, Indiana & 222 & 73.2 & 4.1 & $(65.1-81.3)$ \\
\hline Fort Worth-Arlington, Texas ${ }^{\S}$ & 209 & 82.3 & 3.3 & $(75.8-88.8)$ \\
\hline Grand Island, Nebraska & 277 & 84.2 & 3.0 & $(78.4-90.0)$ \\
\hline Grand Rapids-Wyoming, Michigan & 218 & 86.1 & 3.4 & $(79.3-92.8)$ \\
\hline Greensboro-High Point, North Carolina & 144 & 78.8 & 4.1 & $(70.8-86.7)$ \\
\hline Greenville-Anderson-Mauldin, South Carolina & 314 & 80.9 & 2.6 & $(75.7-86.0)$ \\
\hline Hagerstown-Martinsburg, Maryland-West Virginia & 183 & 82.2 & 5.4 & $(71.7-92.7)$ \\
\hline Hartford-West Hartford-East Hartford, Connecticut & 669 & 90.4 & 1.4 & $(87.7-93.1)$ \\
\hline Hilton Head Island-Bluffton-Beaufort, South Carolina & 114 & 84.8 & 4.1 & $(76.8-92.7)$ \\
\hline Houston-The Woodlands-Sugar Land, Texas & 522 & 79.2 & 2.8 & $(73.7-84.8)$ \\
\hline Huntington-Ashland, West Virginia-Kentucky-Ohio & 327 & 75.8 & 3.4 & $(69.2-82.4)$ \\
\hline Idaho Falls, Idaho & 129 & 73.2 & 4.6 & $(64.2-82.2)$ \\
\hline Indianapolis-Carmel-Anderson, Indiana & 927 & 81.1 & 1.8 & $(77.6-84.5)$ \\
\hline Jacksonville, Florida & 184 & 81.4 & 3.6 & $(74.4-88.5)$ \\
\hline Kansas City, Missouri-Kansas & 1,283 & 81.9 & 2.0 & $(78.0-85.8)$ \\
\hline Kingsport-Bristol-Bristol, Tennessee-Virginia & 108 & 86.1 & 3.4 & $(79.5-92.7)$ \\
\hline Knoxville, Tennessee & 117 & 94.3 & 2.7 & $(89.0-99.7)$ \\
\hline Lafayette, Louisiana & 143 & 81.8 & 3.8 & $(74.4-89.2)$ \\
\hline Lexington-Fayette, Kentucky & 176 & 83.9 & 3.3 & $(77.5-90.3)$ \\
\hline Lincoln, Nebraska & 577 & 78.8 & 2.3 & $(74.3-83.2)$ \\
\hline
\end{tabular}

\footnotetext{
See table footnotes on page 47.
} 
TABLE 18. (Continued) Age-adjusted* prevalence estimates of women aged 21-65 years who reported having a Pap ${ }^{\dagger}$ test, by metropolitan and micropolitan statistical area - Behavioral Risk Factor Surveillance System, United States, 2014

\begin{tabular}{|c|c|c|c|c|}
\hline MMSA & $\begin{array}{l}\text { Sample } \\
\text { size }\end{array}$ & $\%$ & SE & $95 \% \mathrm{Cl}$ \\
\hline Little Rock-North Little Rock-Conway, Arkansas & 263 & 85.9 & 2.9 & $(80.3-91.6)$ \\
\hline Logan, Utah-Idaho & 186 & 72.2 & 3.9 & $(64.6-79.8)$ \\
\hline Los Angeles-Long Beach-Anaheim, California & 570 & 82.1 & 2.0 & $(78.1-86.1)$ \\
\hline Louisville/Jefferson County, Kentucky-Indiana & 631 & 82.2 & 2.5 & $(77.2-87.1)$ \\
\hline Madison, Wisconsin & 145 & 90.6 & 3.3 & $(84.2-97.1)$ \\
\hline Memphis, Tennessee-Mississippi-Arkansas & 230 & 90.1 & 2.3 & $(85.6-94.7)$ \\
\hline Miami-Fort Lauderdale-West Palm Beach, Florida & 547 & 81.0 & 2.2 & $(76.7-85.3)$ \\
\hline Milwaukee-Waukesha-West Allis, Wisconsin & 353 & 90.3 & 2.0 & $(86.4-94.1)$ \\
\hline Minneapolis-St. Paul-Bloomington, Minnesota-Wisconsin & 2,716 & 86.9 & 0.8 & $(85.3-88.5)$ \\
\hline Minot, North Dakota & 148 & 75.7 & 5.0 & $(65.8-85.5)$ \\
\hline Montgomery, Alabama & 132 & 86.4 & 3.7 & $(79.1-93.6)$ \\
\hline $\begin{array}{l}\text { Montgomery County-Bucks County-Chester County, } \\
\text { Pennsylvania§ }\end{array}$ & 244 & 80.4 & 4.5 & $(71.7-89.2)$ \\
\hline $\begin{array}{l}\text { Myrtle Beach-Conway-North Myrtle Beach, South Carolina- } \\
\text { North Carolina }\end{array}$ & 206 & 75.3 & 4.2 & $(67.0-83.6)$ \\
\hline Nashville-Davidson County-Murfreesboro-Franklin, Tennessee & 220 & 86.0 & 2.8 & $(80.5-91.5)$ \\
\hline Nassau County-Suffolk County, New York ${ }^{\S}$ & 235 & 81.7 & 3.2 & $(75.4-88.0)$ \\
\hline Newark, New Jersey-Pennsylvania ${ }^{\S}$ & 1,326 & 84.1 & 1.5 & $(81.2-87.0)$ \\
\hline New Orleans-Metairie, Louisiana & 513 & 86.9 & 1.9 & $(83.1-90.6)$ \\
\hline New York-Jersey City-White Plains, New York-New Jersey $§$ & 2,369 & 82.0 & 1.3 & $(79.5-84.4)$ \\
\hline Norfolk, Nebraska & 261 & 79.9 & 3.5 & $(73.1-86.7)$ \\
\hline North Platte, Nebraska & 232 & 70.9 & 3.4 & $(64.2-77.7)$ \\
\hline North Port-Sarasota-Bradenton, Florida & 95 & 81.2 & 5.2 & $(71.1-91.4)$ \\
\hline Oakland-Hayward-Berkeley, California ${ }^{\S}$ & 193 & 86.7 & 3.5 & (79.9-93.5) \\
\hline Ogden-Clearfield, Utah & 805 & 76.7 & 1.8 & $(73.2-80.1)$ \\
\hline Oklahoma City, Oklahoma & 688 & 80.9 & 1.7 & $(77.5-84.3)$ \\
\hline Omaha-Council Bluffs, Nebraska-lowa & 1,365 & 85.0 & 1.5 & $(82.1-87.9)$ \\
\hline Orlando-Kissimmee-Sanford, Florida & 236 & 82.2 & 3.4 & $(75.6-88.8)$ \\
\hline Philadelphia, Pennsylvania§ & 445 & 76.6 & 3.1 & $(70.6-82.6)$ \\
\hline Phoenix-Mesa-Scottsdale, Arizona & 2,173 & 79.9 & 1.3 & $(77.3-82.5)$ \\
\hline Pittsburgh, Pennsylvania & 645 & 81.1 & 2.0 & $(77.2-84.9)$ \\
\hline Ponce, Puerto Rico & 183 & 73.1 & 3.9 & $(65.4-80.8)$ \\
\hline Portland-South Portland, Maine & 774 & 85.0 & 2.1 & $(80.9-89.0)$ \\
\hline Portland-Vancouver-Hillsboro, Oregon-Washington & 734 & 86.5 & 1.7 & $(83.2-89.9)$ \\
\hline Providence-Warwick, Rhode Island-Massachusetts & 2,406 & 87.1 & 1.1 & $(84.9-89.4)$ \\
\hline Provo-Orem, Utah & 640 & 72.1 & 2.1 & $(67.9-76.3)$ \\
\hline Raleigh, North Carolina & 232 & 89.3 & 2.6 & $(84.3-94.3)$ \\
\hline Rapid City, South Dakota & 365 & 81.5 & 3.2 & $(75.2-87.7)$ \\
\hline Reno, Nevada & 337 & 77.4 & 3.3 & $(70.9-84.0)$ \\
\hline Richmond, Virginia & 452 & 90.4 & 1.6 & $(87.2-93.5)$ \\
\hline Riverside-San Bernardino-Ontario, California & 219 & 84.4 & 2.8 & $(78.9-89.9)$ \\
\hline Roanoke, Virginia & 134 & 79.1 & 4.4 & $(70.5-87.7)$ \\
\hline Rochester, Minnesota & 193 & 87.7 & 2.9 & $(82.1-93.3)$ \\
\hline Rockingham County-Strafford County, New Hampshire & 429 & 86.6 & 2.7 & $(81.4-91.8)$ \\
\hline Sacramento-Roseville-Arden-Arcade, California & 152 & 86.1 & 3.5 & $(79.2-93.0)$ \\
\hline St. Cloud, Minnesota & 147 & 86.3 & 3.1 & $(80.2-92.5)$ \\
\hline St. Louis, Missouri-Illinois & 515 & 83.7 & 2.3 & $(79.1-88.3)$ \\
\hline Salisbury, Maryland-Delaware & 436 & 85.5 & 2.7 & $(80.3-90.7)$ \\
\hline Salt Lake City, Utah & 1,555 & 81.2 & 1.2 & $(78.8-83.5)$ \\
\hline San Antonio-New Braunfels, Texas & 568 & 75.8 & 2.4 & $(71.1-80.6)$ \\
\hline San Juan-Carolina-Caguas, Puerto Rico & 1,180 & 80.5 & 1.4 & $(77.8-83.3)$ \\
\hline Scottsbluff, Nebraska & 199 & 76.2 & 3.7 & $(69.0-83.5)$ \\
\hline Seattle-Bellevue-Everett, Washington $\S$ & 1,022 & 81.3 & 1.8 & (77.9-84.8) \\
\hline Shreveport-Bossier City, Louisiana & 180 & 80.5 & 4.2 & $(72.3-88.6)$ \\
\hline Silver Spring-Frederick-Rockville, Maryland ${ }^{\S}$ & 731 & 84.5 & 2.7 & $(79.3-89.7)$ \\
\hline Sioux City, lowa-Nebraska-South Dakota & 261 & 83.7 & 4.0 & $(75.8-91.6)$ \\
\hline Sioux Falls, South Dakota & 383 & 88.3 & 2.4 & $(83.6-92.9)$ \\
\hline Spartanburg, South Carolina & 112 & 83.2 & 6.7 & $(70.0-96.4)$ \\
\hline Spokane-Spokane Valley, Washington & 191 & 82.7 & 3.7 & $(75.4-89.9)$ \\
\hline Springfield, Massachusetts & 302 & 86.3 & 3.0 & $(80.4-92.2)$ \\
\hline
\end{tabular}
See table footnotes on page 47. 
TABLE 18. (Continued) Age-adjusted* prevalence estimates of women aged 21-65 years who reported having a Pap ${ }^{\dagger}$ test, by metropolitan and micropolitan statistical area - Behavioral Risk Factor Surveillance System, United States, 2014

\begin{tabular}{|c|c|c|c|c|}
\hline MMSA & $\begin{array}{l}\text { Sample } \\
\text { size }\end{array}$ & $\%$ & SE & $95 \% \mathrm{Cl}$ \\
\hline Tampa-St. Petersburg-Clearwater, Florida & 417 & 78.7 & 2.7 & $(73.5-83.9)$ \\
\hline Toledo, Ohio & 176 & 81.0 & 4.1 & $(73.0-89.0)$ \\
\hline Topeka, Kansas & 379 & 81.2 & 2.6 & $(76.1-86.3)$ \\
\hline Tulsa, Oklahoma & 483 & 77.9 & 2.2 & $(73.6-82.2)$ \\
\hline Tuscaloosa, Alabama & 192 & 83.4 & 3.5 & $(76.6-90.3)$ \\
\hline Virginia Beach-Norfolk-Newport News, Virginia-North Carolina & 551 & 85.3 & 2.1 & $(81.2-89.5)$ \\
\hline Warren-Troy-Farmington Hills, Michigan $\$$ & 587 & 82.0 & 1.9 & $(78.2-85.8)$ \\
\hline $\begin{array}{l}\text { Washington-Arlington-Alexandria, District of Columbia- } \\
\text { Virginia-Maryland-West Virginia\$ }\end{array}$ & 2,495 & 85.8 & 1.5 & $(82.8-88.7)$ \\
\hline Wichita, Kansas & 660 & 84.9 & 1.6 & $(81.7-88.0)$ \\
\hline Wichita Falls, Texas & 85 & 68.0 & 9.6 & $(49.3-86.8)$ \\
\hline Wilmington, Delaware-Maryland-New Jersey§ & 818 & 87.1 & 1.7 & $(83.9-90.4)$ \\
\hline Worcester, Massachusetts-Connecticut & 690 & 86.6 & 2.4 & $(81.8-91.3)$ \\
\hline Youngstown-Warren-Boardman, Ohio-Pennsylvania & 142 & 83.8 & 5.0 & $(74.0-93.7)$ \\
\hline $\begin{array}{l}\text { Median } \\
\text { Range }\end{array}$ & & $\begin{array}{c}83.1 \\
68.0-94.3\end{array}$ & & \\
\hline
\end{tabular}

Abbreviations: $\mathrm{Cl}$ = confidence interval; $\mathrm{MMSA}=$ metropolitan and micropolitan statistical areas; $\mathrm{Pap}=$ Papanicolaou; $\mathrm{SE}=$ standard error.

${ }^{*}$ Age adjusted to the 2000 U.S. standard population.

† Test for cancer of the cervix.

$\S$ Metropolitan division. 
TABLE 19. Age-adjusted* prevalence estimates of adults aged 50-75 years who received colorectal cancer screening according to most recent guidelines, ${ }^{\dagger}$ by state/territory - Behavioral Risk Factor Surveillance System, United States, 2014

\begin{tabular}{|c|c|c|c|c|}
\hline State/Territory & $\begin{array}{l}\text { Sample } \\
\text { size }\end{array}$ & $\%$ & SE & $95 \% \mathrm{Cl}$ \\
\hline Alabama & 4,450 & 64.8 & 1.0 & $(62.9-66.7)$ \\
\hline Alaska & 2,052 & 61.5 & 1.5 & (58.6-64.4) \\
\hline Arizona & 7,311 & 63.3 & 0.8 & $(61.7-65.0)$ \\
\hline Arkansas & 2,556 & 60.5 & 1.4 & $(57.9-63.2)$ \\
\hline California & 2,909 & 66.3 & 1.1 & $(64.1-68.6)$ \\
\hline Colorado & 6,267 & 67.2 & 0.7 & $(65.8-68.7)$ \\
\hline Connecticut & 3,771 & 73.3 & 1.0 & (71.4-75.2) \\
\hline Delaware & 2,215 & 71.7 & 1.2 & $(69.2-74.1)$ \\
\hline District of Columbia & 1,904 & 68.1 & 1.7 & $(64.8-71.5)$ \\
\hline Florida & 4,565 & 65.9 & 0.9 & (64.1-67.7) \\
\hline Georgia & 3,005 & 66.8 & 1.1 & $(64.6-69.1)$ \\
\hline Hawaii & 3,198 & 69.5 & 1.2 & $(67.2-71.8)$ \\
\hline Idaho & 2,643 & 60.8 & 1.2 & (58.4-63.3) \\
\hline Illinois & 2,385 & 61.9 & 1.3 & $(59.3-64.4)$ \\
\hline Indiana & 5,518 & 61.9 & 0.8 & $(60.3-63.5)$ \\
\hline lowa & 3,876 & 67.6 & 0.9 & $(65.8-69.4)$ \\
\hline Kansas & 6,398 & 64.9 & 0.7 & $(63.5-66.2)$ \\
\hline Kentucky & 5,839 & 67.1 & 1.0 & $(65.2-69.1)$ \\
\hline Louisiana & 3,172 & 64.5 & 1.0 & $(62.5-66.4)$ \\
\hline Maine & 4,939 & 75.2 & 0.8 & (73.6-76.9) \\
\hline Maryland & 6,641 & 71.1 & 0.9 & $(69.3-73.0)$ \\
\hline Massachusetts & 7,350 & 76.7 & 0.8 & $(75.2-78.2)$ \\
\hline Michigan & 4,329 & 71.2 & 0.9 & $(69.5-72.9)$ \\
\hline Minnesota & 7,514 & 71.8 & 0.6 & $(70.6-73.0)$ \\
\hline Mississippi & 2,120 & 60.3 & 1.4 & $(57.5-63.0)$ \\
\hline Missouri & 3,564 & 61.9 & 1.1 & $(59.7-64.2)$ \\
\hline Montana & 3,877 & 62.3 & 1.1 & $(60.1-64.4)$ \\
\hline Nebraska & 10,646 & 64.4 & 0.7 & $(63.0-65.7)$ \\
\hline Nevada & 1,788 & 59.3 & 1.8 & (55.7-62.8) \\
\hline New Hampshire & 3,109 & 74.1 & 1.0 & $(72.0-76.1)$ \\
\hline New Jersey & 5,973 & 65.4 & 0.9 & $(63.6-67.3)$ \\
\hline New Mexico & 4,279 & 61.3 & 1.0 & (59.2-63.3) \\
\hline New York & 2,974 & 68.3 & 1.1 & $(66.2-70.4)$ \\
\hline North Carolina & 3,273 & 70.7 & 0.9 & $(68.9-72.5)$ \\
\hline North Dakota & 3,817 & 62.3 & 1.1 & $(60.2-64.5)$ \\
\hline Ohio & 5,653 & 65.2 & 0.9 & $(63.4-67.0)$ \\
\hline Oklahoma & 4,125 & 58.0 & 0.9 & $(56.2-59.8)$ \\
\hline Oregon & 2,528 & 66.8 & 1.2 & $(64.5-69.1)$ \\
\hline Pennsylvania & 5,370 & 67.0 & 0.9 & $(65.3-68.7)$ \\
\hline Rhode Island & 3,184 & 74.7 & 1.0 & (72.8-76.6) \\
\hline South Carolina & 5,545 & 67.0 & 0.8 & $(65.4-68.7)$ \\
\hline South Dakota & 3,431 & 67.0 & 1.4 & (64.2-69.8) \\
\hline Tennessee & 2,554 & 64.8 & 1.3 & $(62.3-67.2)$ \\
\hline Texas & 6,669 & 61.4 & 1.1 & $(59.3-63.5)$ \\
\hline Utah & 5,694 & 70.5 & 0.7 & $(69.1-72.0)$ \\
\hline Vermont & 3,166 & 70.8 & 0.9 & $(69.0-72.6)$ \\
\hline Virginia & 4,630 & 69.2 & 0.9 & $(67.5-71.0)$ \\
\hline Washington & 5,052 & 69.9 & 0.9 & (68.1-71.6) \\
\hline West Virginia & 3,195 & 64.1 & 1.0 & $(62.1-66.0)$ \\
\hline Wisconsin & 3,388 & 73.7 & 1.0 & $(71.7-75.7)$ \\
\hline Wyoming & 3,360 & 57.3 & 1.2 & $(54.9-59.7)$ \\
\hline Guam & 763 & 42.8 & 2.3 & $(38.3-47.3)$ \\
\hline Puerto Rico & 2,842 & 52.2 & 1.2 & $(49.9-54.6)$ \\
\hline Median & & 66.3 & & \\
\hline Range & & $42.8-76.7$ & & \\
\hline
\end{tabular}

Abbreviations: $\mathrm{Cl}=$ confidence interval; $\mathrm{SE}=$ standard error.

* Age adjusted to the 2000 U.S. standard population.

+ Adults aged 50-75 years who had a blood stool test during the past year, sigmoidoscopy during the past 5 years and blood stool test during the past 3 years, or a colonoscopy during the past 10 years. 
TABLE 20. Age-adjusted* prevalence estimates of adults aged 50-75 years who received colorectal cancer screening according to most recent guidelines, ${ }^{\dagger}$ by metropolitan and micropolitan statistical area - Behavioral Risk Factor Surveillance System, United States, 2014

\begin{tabular}{|c|c|c|c|c|}
\hline MMSA & $\begin{array}{l}\text { Sample } \\
\text { size }\end{array}$ & $\%$ & SE & $95 \% \mathrm{Cl}$ \\
\hline Aberdeen, South Dakota & 306 & 75.7 & 2.8 & $(70.3-81.2)$ \\
\hline Aguadilla-Isabela, Puerto Rico & 258 & 53.4 & 3.9 & $(45.8-61.0)$ \\
\hline Albuquerque, New Mexico & 832 & 69.8 & 2.0 & $(65.9-73.8)$ \\
\hline Allentown-Bethlehem-Easton, Pennsylvania-New Jersey & 557 & 68.0 & 3.0 & $(62.2-73.8)$ \\
\hline Anchorage, Alaska & 794 & 63.7 & 2.2 & $(59.4-67.9)$ \\
\hline Atlanta-Sandy Springs-Roswell, Georgia & 1,229 & 69.5 & 1.7 & $(66.2-72.8)$ \\
\hline Augusta-Richmond County, Georgia-South Carolina & 406 & 69.0 & 4.0 & $(61.2-76.8)$ \\
\hline Austin-Round Rock, Texas & 988 & 60.1 & 2.1 & $(55.9-64.2)$ \\
\hline Baltimore-Columbia-Towson, Maryland & 2,392 & 70.9 & 1.5 & $(68.0-73.8)$ \\
\hline Baton Rouge, Louisiana & 389 & 69.5 & 2.7 & $(64.3-74.7)$ \\
\hline Berlin, New Hampshire-Vermont & 314 & 67.4 & 3.3 & $(60.9-73.9)$ \\
\hline Billings, Montana & 378 & 67.2 & 2.8 & $(61.7-72.8)$ \\
\hline Birmingham-Hoover, Alabama & 777 & 67.5 & 2.0 & $(63.5-71.5)$ \\
\hline Bismarck, North Dakota & 533 & 62.0 & 2.6 & $(56.9-67.0)$ \\
\hline Boise City, Idaho & 645 & 63.8 & 2.5 & $(58.9-68.7)$ \\
\hline Boston, Massachusetts $\S$ & 2,073 & 74.6 & 1.5 & $(71.6-77.6)$ \\
\hline Burlington-South Burlington, Vermont & 898 & 76.5 & 1.7 & $(73.2-79.7)$ \\
\hline Cambridge-Newton-Framingham, Massachusetts ${ }^{\S}$ & 2,406 & 78.6 & 1.3 & $(76.0-81.2)$ \\
\hline Camden, New Jersey ${ }^{\S}$ & 839 & 70.4 & 2.2 & $(66.0-74.8)$ \\
\hline Cedar Rapids, lowa & 300 & 72.0 & 3.2 & $(65.6-78.3)$ \\
\hline Charleston, West Virginia & 431 & 69.6 & 2.7 & $(64.4-74.8)$ \\
\hline Charleston-North Charleston, South Carolina & 661 & 71.2 & 2.3 & $(66.6-75.7)$ \\
\hline Charlotte-Concord-Gastonia, North Carolina-South Carolina & 950 & 70.2 & 2.0 & $(66.3-74.1)$ \\
\hline Chicago-Naperville-Elgin, Illinois-Indiana-Wisconsin & 1,904 & 60.9 & 1.6 & $(57.8-64.0)$ \\
\hline Cincinnati, Ohio-Kentucky-Indiana & 971 & 66.9 & 2.2 & $(62.7-71.2)$ \\
\hline Claremont-Lebanon, New Hampshire-Vermont & 875 & 69.8 & 2.0 & $(65.9-73.7)$ \\
\hline Cleveland-Elyria, Ohio & 507 & 66.1 & 2.6 & $(60.9-71.2)$ \\
\hline College Station-Bryan, Texas & 252 & 68.2 & 6.1 & $(56.2-80.3)$ \\
\hline Colorado Springs, Colorado & 604 & 71.1 & 2.2 & $(66.7-75.5)$ \\
\hline Columbia, South Carolina & 598 & 69.5 & 2.2 & $(65.2-73.7)$ \\
\hline Columbus, Ohio & 806 & 67.9 & 2.3 & $(63.3-72.4)$ \\
\hline Corpus Christi, Texas & 313 & 68.9 & 3.9 & $(61.2-76.5)$ \\
\hline Dallas-Plano-Irving, Texas & 584 & 64.5 & 3.2 & $(58.2-70.9)$ \\
\hline Dayton, Ohio & 276 & 69.7 & 3.7 & $(62.5-76.9)$ \\
\hline Denver-Aurora-Lakewood, Colorado & 2,556 & 68.8 & 1.1 & $(66.7-70.9)$ \\
\hline Des Moines-West Des Moines, lowa & 623 & 68.3 & 2.4 & $(63.7-73.0)$ \\
\hline Duluth, Minnesota-Wisconsin & 473 & 67.4 & 2.7 & $(62.1-72.7)$ \\
\hline El Paso, Texas & 311 & 55.7 & 3.7 & $(48.5-62.9)$ \\
\hline Evansville, Indiana-Kentucky & 360 & 73.8 & 3.0 & $(67.9-79.7)$ \\
\hline Fargo, North Dakota-Minnesota & 534 & 71.4 & 2.5 & $(66.5-76.3)$ \\
\hline Fayetteville-Springdale-Rogers, Arkansas-Missouri & 386 & 60.2 & 3.3 & $(53.8-66.7)$ \\
\hline Fort Wayne, Indiana & 417 & 61.6 & 2.9 & $(55.8-67.3)$ \\
\hline Fort Worth-Arlington, Texas ${ }^{\S}$ & 332 & 70.8 & 3.8 & $(63.4-78.2)$ \\
\hline Grand Island, Nebraska & 507 & 67.5 & 2.7 & $(62.1-72.8)$ \\
\hline Grand Rapids-Wyoming, Michigan & 451 & 75.0 & 2.6 & $(69.9-80.2)$ \\
\hline Greensboro-High Point, North Carolina & 244 & 74.2 & 3.2 & $(68.0-80.5)$ \\
\hline Greenville-Anderson-Mauldin, South Carolina & 714 & 8.8 & 2.4 & $(64.1-73.4)$ \\
\hline Hagerstown-Martinsburg, Maryland-West Virginia & 411 & 64.2 & 3.0 & $(58.3-70.0)$ \\
\hline Hartford-West Hartford-East Hartford, Connecticut & 1,226 & 73.5 & 1.8 & $(70.0-77.0)$ \\
\hline Hilton Head Island-Bluffton-Beaufort, South Carolina & 288 & 70.9 & 3.5 & $(64.1-77.8)$ \\
\hline Houston-The Woodlands-Sugar Land, Texas & 914 & 61.7 & 2.9 & $(56.1-67.3)$ \\
\hline Huntington-Ashland, West Virginia-Kentucky-Ohio & 688 & 66.5 & 2.2 & $(62.3-70.8)$ \\
\hline Idaho Falls, Idaho & 218 & 68.7 & 3.7 & $(61.4-76.0)$ \\
\hline Indianapolis-Carmel-Anderson, Indiana & 1,711 & 65.8 & 1.5 & $(62.9-68.6)$ \\
\hline Jacksonville, Florida & 302 & 70.3 & 3.4 & $(63.6-77.0)$ \\
\hline Kansas City, Missouri-Kansas & 2,364 & 66.3 & 1.7 & $(63.0-69.6)$ \\
\hline Kingsport-Bristol-Bristol, Tennessee-Virginia & 269 & 64.6 & 4.4 & $(55.9-73.3)$ \\
\hline Knoxville, Tennessee & 298 & 66.5 & 3.7 & $(59.2-73.8)$ \\
\hline Lafayette, Louisiana & 266 & 63.7 & 3.4 & $(57.1-70.4)$ \\
\hline Lexington-Fayette, Kentucky & 274 & 75.1 & 3.4 & $(68.4-81.8)$ \\
\hline Lincoln, Nebraska & 850 & 68.1 & 1.9 & $(64.4-71.9)$ \\
\hline
\end{tabular}

See table footnotes on page 51. 
TABLE 20. (Continued) Age-adjusted* prevalence estimates of adults aged 50-75 years who received colorectal cancer screening according to most recent guidelines, ${ }^{\dagger}$ by metropolitan and micropolitan statistical area - Behavioral Risk Factor Surveillance System, United States, 2014

\begin{tabular}{|c|c|c|c|c|}
\hline MMSA & $\begin{array}{l}\text { Sample } \\
\text { size }\end{array}$ & $\%$ & SE & $95 \% \mathrm{Cl}$ \\
\hline Little Rock-North Little Rock-Conway, Arkansas & 573 & 71.3 & 2.6 & $(66.2-76.5)$ \\
\hline Logan, Utah-Idaho & 218 & 66.2 & 3.6 & $(59.1-73.2)$ \\
\hline Los Angeles-Long Beach-Anaheim, California & 687 & 64.0 & 2.3 & $(59.5-68.5)$ \\
\hline Louisville-Jefferson County, Kentucky-Indiana & 1,337 & 67.7 & 2.1 & $(63.5-71.9)$ \\
\hline Madison, Wisconsin & 235 & 79.6 & 3.2 & (73.4-85.8) \\
\hline Memphis, Tennessee-Mississippi-Arkansas & 408 & 72.2 & 3.0 & $(66.3-78.2)$ \\
\hline Miami-Fort Lauderdale-West Palm Beach, Florida & 936 & 63.4 & 2.0 & $(59.5-67.4)$ \\
\hline Milwaukee-Waukesha-West Allis, Wisconsin & 640 & 72.5 & 2.5 & $(67.5-77.4)$ \\
\hline Minneapolis-St. Paul-Bloomington, Minnesota-Wisconsin & 3,856 & 72.9 & 0.8 & $(71.3-74.6)$ \\
\hline Minot, North Dakota & 285 & 56.3 & 3.6 & $(49.2-63.4)$ \\
\hline Montgomery, Alabama & 243 & 66.8 & 4.1 & $(58.9-74.8)$ \\
\hline $\begin{array}{l}\text { Montgomery County-Bucks County-Chester County, } \\
\text { Pennsylvania§ }\end{array}$ & 387 & 63.5 & 2.8 & $(58.1-69.0)$ \\
\hline $\begin{array}{l}\text { Myrtle Beach-Conway-North Myrtle Beach, South Carolina- } \\
\text { North Carolina }\end{array}$ & 545 & 66.3 & 2.5 & $(61.5-71.2)$ \\
\hline Nashville-Davidson County-Murfreesboro-Franklin, Tennessee & 367 & 65.9 & 3.1 & $(59.9-71.9)$ \\
\hline Nassau County-Suffolk County, New York ${ }^{\S}$ & 345 & 62.1 & 3.0 & $(56.2-68.0)$ \\
\hline Newark, New Jersey-Pennsylvania§ & 1,928 & 66.2 & 1.5 & $(63.3-69.2)$ \\
\hline New Orleans-Metairie, Louisiana & 931 & 65.5 & 1.9 & $(61.8-69.3)$ \\
\hline New York-Jersey City-White Plains, New York-New Jersey ${ }^{\S}$ & 2,989 & 67.1 & 1.3 & $(64.5-69.6)$ \\
\hline Norfolk, Nebraska & 475 & 61.9 & 2.5 & $(57.0-66.7)$ \\
\hline North Platte, Nebraska & 488 & 55.5 & 2.6 & $(50.4-60.5)$ \\
\hline North Port-Sarasota-Bradenton, Florida & 239 & 65.4 & 4.1 & $(57.4-73.3)$ \\
\hline Oakland-Hayward-Berkeley, California ${ }^{\S}$ & 245 & 75.2 & 3.9 & $(67.5-82.9)$ \\
\hline Ogden-Clearfield, Utah & 1,050 & 75.1 & 1.5 & $(72.2-78.0)$ \\
\hline Oklahoma City, Oklahoma & 1,138 & 60.9 & 1.8 & $(57.4-64.3)$ \\
\hline Omaha-Council Bluffs, Nebraska-lowa & 2,242 & 69.1 & 1.4 & $(66.4-71.7)$ \\
\hline Orlando-Kissimmee-Sanford, Florida & 414 & 66.8 & 2.9 & $(61.2-72.4)$ \\
\hline Philadelphia, Pennsylvania ${ }^{\S}$ & 690 & 63.0 & 2.8 & $(57.6-68.4)$ \\
\hline Phoenix-Mesa-Scottsdale, Arizona & 4,546 & 64.6 & 1.0 & $(62.7-66.5)$ \\
\hline Pittsburgh, Pennsylvania & 1,183 & 69.1 & 1.6 & $(66.1-72.2)$ \\
\hline Ponce, Puerto Rico & 273 & 49.1 & 3.6 & $(41.9-56.2)$ \\
\hline Portland-South Portland, Maine & 1,469 & 77.0 & 1.5 & (74.1-79.8) \\
\hline Portland-Vancouver-Hillsboro, Oregon-Washington & 1,355 & 70.0 & 1.6 & $(66.9-73.1)$ \\
\hline Providence-Warwick, Rhode Island-Massachusetts & 4,008 & 75.7 & 1.1 & $(73.6-77.8)$ \\
\hline Provo-Orem, Utah & 625 & 68.1 & 2.1 & $(64.1-72.2)$ \\
\hline Raleigh, North Carolina & 273 & 74.3 & 3.0 & $(68.5-80.2)$ \\
\hline Rapid City, South Dakota & 703 & 64.8 & 2.7 & $(59.5-70.1)$ \\
\hline Reno, Nevada & 585 & 65.8 & 2.4 & $(61.1-70.5)$ \\
\hline Richmond, Virginia & 710 & 69.5 & 2.2 & $(65.1-73.9)$ \\
\hline Riverside-San Bernardino-Ontario, California & 322 & 61.8 & 3.3 & $(55.3-68.3)$ \\
\hline Roanoke, Virginia & 276 & 67.9 & 3.5 & $(61.0-74.9)$ \\
\hline Rochester, Minnesota & 314 & 77.5 & 2.5 & $(72.6-82.5)$ \\
\hline Rockingham County-Strafford County, New Hampshire ${ }^{\S}$ & 703 & 75.1 & 2.0 & $(71.1-79.1)$ \\
\hline Sacramento-Roseville-Arden-Arcade, California & 227 & 68.3 & 4.3 & $(59.9-76.6)$ \\
\hline St. Cloud, Minnesota & 233 & 72.5 & 3.5 & $(65.7-79.3)$ \\
\hline St. Louis, Missouri-Illinois & 901 & 66.1 & 2.3 & $(61.6-70.6)$ \\
\hline Salisbury, Maryland-Delaware & 1,104 & 71.8 & 1.9 & $(68.1-75.4)$ \\
\hline Salt Lake City, Utah & 2,051 & 71.3 & 1.2 & $(69.0-73.6)$ \\
\hline San Antonio-New Braunfels, Texas & 1,033 & 65.2 & 1.9 & $(61.5-69.0)$ \\
\hline San Juan-Carolina-Caguas, Puerto Rico & 1,772 & 53.3 & 1.5 & $(50.4-56.3)$ \\
\hline Scottsbluff, Nebraska & 456 & 53.0 & 2.8 & $(47.6-58.5)$ \\
\hline Seattle-Bellevue-Everett, Washington $§$ & 1,757 & 69.5 & 1.5 & $(66.6-72.4)$ \\
\hline Shreveport-Bossier City, Louisiana & 243 & 55.4 & 3.5 & $(48.5-62.4)$ \\
\hline Silver Spring-Frederick-Rockville, Maryland ${ }^{\S}$ & 1,198 & 70.7 & 2.3 & $(66.1-75.3)$ \\
\hline Sioux City, lowa-Nebraska-South Dakota & 539 & 73.2 & 3.2 & $(66.9-79.5)$ \\
\hline Sioux Falls, South Dakota & 575 & 69.4 & 3.0 & $(63.5-75.2)$ \\
\hline Spartanburg, South Carolina & 312 & 61.8 & 3.7 & $(54.6-69.0)$ \\
\hline Spokane-Spokane Valley, Washington & 369 & 75.1 & 2.9 & $(69.4-80.7)$ \\
\hline Springfield, Massachusetts & 481 & 77.6 & 2.8 & $(72.2-83.0)$ \\
\hline Tampa-St. Petersburg-Clearwater, Florida & 744 & 63.8 & 2.4 & $(59.1-68.4)$ \\
\hline
\end{tabular}

See table footnotes on page 51. 
TABLE 20. (Continued) Age-adjusted* prevalence estimates of adults aged 50-75 years who received colorectal cancer screening according to most recent guidelines, ${ }^{\dagger}$ by metropolitan and micropolitan statistical area - Behavioral Risk Factor Surveillance System, United States, 2014

\begin{tabular}{|c|c|c|c|c|}
\hline MMSA & $\begin{array}{l}\text { Sample } \\
\text { size }\end{array}$ & $\%$ & SE & $95 \% \mathrm{Cl}$ \\
\hline Toledo, Ohio & 340 & 66.2 & 3.0 & $(60.3-72.1)$ \\
\hline Topeka, Kansas & 742 & 68.0 & 2.0 & $(64.0-72.0)$ \\
\hline Tulsa, Oklahoma & 1,022 & 63.6 & 1.7 & $(60.2-67.0)$ \\
\hline Tuscaloosa, Alabama & 364 & 70.4 & 2.8 & $(64.9-75.8)$ \\
\hline Virginia Beach-Norfolk-Newport News, Virginia-North Carolina & 930 & 77.9 & 1.7 & $(74.5-81.3)$ \\
\hline Warren-Troy-Farmington Hills, Michigan $\$$ & 1,082 & 74.2 & 1.6 & $(71.0-77.4)$ \\
\hline $\begin{array}{l}\text { Washington-Arlington-Alexandria, District of Columbia-Virginia- } \\
\text { Maryland-West Virginia§ }\end{array}$ & 3,904 & 70.4 & 1.3 & $(67.8-73.0)$ \\
\hline Wichita, Kansas & 1,248 & 67.4 & 1.6 & $(64.3-70.5)$ \\
\hline Wichita Falls, Texas & 273 & 62.2 & 4.3 & $(53.8-70.7)$ \\
\hline Wilmington, Delaware-Maryland-New Jersey $§$ & 1,400 & 70.0 & 1.6 & $(66.9-73.2)$ \\
\hline Worcester, Massachusetts-Connecticut & 1,177 & 77.5 & 1.9 & $(73.8-81.1)$ \\
\hline Youngstown-Warren-Boardman, Ohio-Pennsylvania & 268 & 64.2 & 3.6 & $(57.1-71.4)$ \\
\hline Median & & 68.1 & & \\
\hline Range & & $49.1-79.6$ & & \\
\hline
\end{tabular}

Abbreviations: $\mathrm{Cl}=$ confidence interval; $\mathrm{MMSA}=$ metropolitan and micropolitan statistical areas; $\mathrm{SE}=$ standard error.

* Age adjusted to the 2000 U.S. standard population.

${ }^{\dagger}$ Adults aged $50-75$ years who had a blood stool test during the past year, sigmoidoscopy during the past 5 years and blood stool test during the past 3 years, or a colonoscopy during the past 10 years.

$\S$ Metropolitan division. 
TABLE 21. Age-adjusted* prevalence estimates of adults aged $\geq 18$ years who have had their blood cholesterol checked during the past 5 years, by state/territory - Behavioral Risk Factor Surveillance System, United States, 2013

\begin{tabular}{|c|c|c|c|c|}
\hline State/Territory & $\begin{array}{l}\text { Sample } \\
\text { size }\end{array}$ & $\%$ & SE & $95 \% \mathrm{Cl}$ \\
\hline Alabama & 6,252 & 75.2 & 1.0 & $(73.3-77.1)$ \\
\hline Alaska & 4,450 & 69.5 & 1.0 & $(67.6-71.5)$ \\
\hline Arizona & 4,115 & 70.7 & 1.4 & $(68.0-73.5)$ \\
\hline Arkansas & 5,102 & 69.3 & 1.0 & $(67.3-71.4)$ \\
\hline California & 11,315 & 74.4 & 0.6 & $(73.2-75.5)$ \\
\hline Colorado & 13,179 & 75.0 & 0.5 & (73.9-76.0) \\
\hline Connecticut & 7,491 & 80.3 & 0.8 & (78.6-81.9) \\
\hline Delaware & 5,078 & 78.4 & 0.9 & $(76.5-80.2)$ \\
\hline District of Columbia & 4,805 & 81.5 & 1.0 & $(79.5-83.6)$ \\
\hline Florida & 33,365 & 76.3 & 0.6 & $(75.1-77.5)$ \\
\hline Georgia & 7,901 & 76.6 & 0.7 & $(75.2-78.0)$ \\
\hline Hawaii & 7,685 & 73.2 & 0.8 & (71.7-74.7) \\
\hline Idaho & 5,462 & 67.2 & 1.0 & $(65.3-69.1)$ \\
\hline Illinois & 5,553 & 72.2 & 0.9 & (70.3-74.0) \\
\hline Indiana & 10,045 & 72.1 & 0.7 & (70.8-73.4) \\
\hline lowa & 7,950 & 72.8 & 0.8 & $(71.3-74.3)$ \\
\hline Kansas & 22,455 & 70.9 & 0.4 & (70.1-71.8) \\
\hline Kentucky & 10,727 & 74.6 & 0.7 & $(73.2-76.0)$ \\
\hline Louisiana & 5,055 & 74.3 & 1.1 & $(72.1-76.5)$ \\
\hline Maine & 7,904 & 77.1 & 0.8 & (75.6-78.7) \\
\hline Maryland & 12,696 & 79.2 & 0.7 & (77.9-80.5) \\
\hline Massachusetts & 14,591 & 81.9 & 0.6 & $(80.7-83.1)$ \\
\hline Michigan & 12,394 & 76.5 & 0.6 & (75.3-77.7) \\
\hline Minnesota & 13,957 & 74.7 & 0.7 & $(73.3-76.0)$ \\
\hline Mississippi & 7,160 & 72.4 & 0.9 & (70.7-74.1) \\
\hline Missouri & 6,859 & 71.2 & 0.9 & $(69.5-73.0)$ \\
\hline Montana & 9,413 & 70.2 & 0.7 & $(68.8-71.6)$ \\
\hline Nebraska & 16,649 & 71.8 & 0.6 & $(70.6-73.1)$ \\
\hline Nevada & 4,923 & 72.3 & 1.3 & $(69.8-74.9)$ \\
\hline New Hampshire & 6,306 & 78.4 & 0.9 & $(76.7-80.1)$ \\
\hline New Jersey & 12,948 & 78.8 & 0.6 & $(77.6-80.0)$ \\
\hline New Mexico & 9,080 & 68.5 & 0.8 & $(67.0-70.0)$ \\
\hline New York & 8,678 & 79.6 & 0.7 & (78.3-80.9) \\
\hline North Carolina & 8,548 & 77.2 & 0.7 & (75.9-78.6) \\
\hline North Dakota & 7,561 & 71.1 & 0.8 & $(69.5-72.7)$ \\
\hline Ohio & 11,632 & 75.3 & 0.7 & (74.0-76.6) \\
\hline Oklahoma & 7,952 & 71.4 & 0.7 & (70.0-72.9) \\
\hline Oregon & 5,714 & 71.6 & 0.9 & (69.8-73.4) \\
\hline Pennsylvania & 11,097 & 74.1 & 0.6 & $(72.9-75.3)$ \\
\hline Rhode Island & 6,381 & 79.4 & 0.9 & $(77.7-81.2)$ \\
\hline South Carolina & 10,435 & 76.2 & 0.7 & (74.8-77.5) \\
\hline South Dakota & 6,698 & 69.9 & 1.0 & $(68.0-71.8)$ \\
\hline Tennessee & 5,598 & 78.9 & 0.9 & $(77.1-80.6)$ \\
\hline Texas & 10,582 & 74.2 & 0.7 & (72.9-75.6) \\
\hline Utah & 12,337 & 69.6 & 0.5 & $(68.6-70.6)$ \\
\hline Vermont & 6,210 & 74.7 & 0.9 & $(73.0-76.5)$ \\
\hline Virginia & 8,261 & 78.4 & 0.7 & $(77.1-79.8)$ \\
\hline Washington & 10,823 & 72.5 & 0.6 & $(71.2-73.7)$ \\
\hline West Virginia & 5,705 & 74.9 & 0.8 & $(73.3-76.5)$ \\
\hline Wisconsin & 6,488 & 73.9 & 0.9 & $(72.0-75.7)$ \\
\hline Wyoming & 6,268 & 71.5 & 0.9 & (69.7-73.4) \\
\hline Guam & 1,857 & 65.9 & 1.4 & $(63.2-68.6)$ \\
\hline Puerto Rico & 5,827 & 78.3 & 0.8 & $(76.7-79.8)$ \\
\hline Median & & 74.3 & & \\
\hline Range & & $65.9-81.9$ & & \\
\hline
\end{tabular}

Abbreviations: $\mathrm{Cl}=$ confidence interval; $\mathrm{SE}=$ standard error.

* Age adjusted to the 2000 U.S. standard population. 
TABLE 22. Age-adjusted* prevalence estimates of adults aged $\geq 18$ years who have had their blood cholesterol checked during the past 5 years, metropolitan and micropolitan statistical area-Behavioral Risk Factor Surveillance System, United States, 2013

\begin{tabular}{|c|c|c|c|c|}
\hline MMSA & $\begin{array}{l}\text { Sample } \\
\text { size }\end{array}$ & $\%$ & SE & $95 \% \mathrm{Cl}$ \\
\hline Aguadilla-Isabela, Puerto Rico & 580 & 79.0 & 2.5 & $(74.0-84.0)$ \\
\hline Akron, Ohio & 681 & 74.0 & 3.0 & $(68.0-79.9)$ \\
\hline Albuquerque, New Mexico & 2,032 & 69.6 & 1.4 & $(67.0-72.3)$ \\
\hline Allentown-Bethlehem-Easton, Pennsylvania-New Jersey & 993 & 74.6 & 2.4 & $(69.9-79.3)$ \\
\hline Anchorage, Alaska & 1,483 & 70.7 & 1.5 & $(67.7-73.7)$ \\
\hline Atlanta-Sandy Springs-Roswell, Georgia & 3,417 & 77.5 & 1.1 & $(75.4-79.6)$ \\
\hline Augusta-Richmond County, Georgia-South Carolina & 896 & 82.4 & 2.2 & $(78.1-86.8)$ \\
\hline Austin-Round Rock, Texas & 892 & 78.4 & 1.9 & $(74.6-82.1)$ \\
\hline Baltimore-Columbia-Towson, Maryland & 4,659 & 80.7 & 1.0 & $(78.7-82.7)$ \\
\hline Baton Rouge, Louisiana & 892 & 72.7 & 2.4 & $(68.0-77.5)$ \\
\hline Billings, Montana & 796 & 72.2 & 1.8 & $(68.6-75.8)$ \\
\hline Birmingham-Hoover, Alabama & 1,294 & 77.9 & 1.8 & $(74.5-81.3)$ \\
\hline Bismarck, North Dakota & 1,016 & 73.4 & 1.9 & $(69.7-77.1)$ \\
\hline Boise City, Idaho & 1,443 & 71.1 & 1.7 & $(67.7-74.4)$ \\
\hline Boston, Massachusetts ${ }^{\dagger}$ & 3,936 & 81.9 & 1.1 & $(79.8-84.0)$ \\
\hline Buffalo-Cheektowaga-Niagara Falls, New York & 486 & 80.2 & 2.7 & $(74.9-85.5)$ \\
\hline Burlington-South Burlington, Vermont & 1,587 & 76.4 & 1.5 & (73.4-79.3) \\
\hline Cambridge-Newton-Framingham, Massachusetts ${ }^{\dagger}$ & 4,741 & 82.6 & 1.0 & $(80.6-84.6)$ \\
\hline Camden, New Jersey ${ }^{\dagger}$ & 1,802 & 80.7 & 1.5 & $(77.8-83.5)$ \\
\hline Cedar Rapids, lowa & 630 & 74.0 & 2.6 & $(68.9-79.1)$ \\
\hline Charleston, West Virginia & 794 & 78.8 & 2.1 & $(74.7-82.8)$ \\
\hline Charleston-North Charleston, South Carolina & 1,503 & 78.7 & 1.6 & $(75.6-81.8)$ \\
\hline Charlotte-Concord-Gastonia, North Carolina-South Carolina & 1,876 & 80.0 & 1.4 & $(77.3-82.7)$ \\
\hline Chattanooga, Tennessee-Georgia & 566 & 78.9 & 3.3 & $(72.4-85.4)$ \\
\hline Chicago-Naperville-Elgin, Illinois-Indiana-Wisconsin & 3,289 & 73.1 & 1.2 & $(70.7-75.5)$ \\
\hline Cincinnati, Ohio-Kentucky-Indiana & 2,540 & 76.1 & 1.4 & $(73.3-78.9)$ \\
\hline Claremont-Lebanon, New Hampshire-Vermont & 1,638 & 73.8 & 2.0 & $(69.9-77.7)$ \\
\hline Cleveland-Elyria, Ohio & 1,089 & 78.7 & 1.9 & $(74.9-82.4)$ \\
\hline Colorado Springs, Colorado & 1,335 & 74.7 & 1.6 & $(71.5-77.8)$ \\
\hline Columbia, South Carolina & 1,411 & 79.3 & 1.6 & $(76.1-82.5)$ \\
\hline Columbus, Ohio & 1,806 & 76.9 & 1.3 & $(74.4-79.5)$ \\
\hline Crestview-Fort Walton Beach-Destin, Florida & 1,058 & 72.8 & 2.1 & $(68.6-77.0)$ \\
\hline Dallas-Plano-Irving, Texas ${ }^{\dagger}$ & 872 & 76.6 & 1.9 & $(72.9-80.2)$ \\
\hline Davenport-Moline-Rock Island, lowa-Illinois & 662 & 71.1 & 2.8 & $(65.6-76.6)$ \\
\hline Dayton, Ohio & 818 & 78.7 & 2.4 & $(73.9-83.4)$ \\
\hline Deltona-Daytona Beach-Ormond Beach, Florida & 1,095 & 73.9 & 2.7 & $(68.5-79.2)$ \\
\hline Denver-Aurora-Lakewood, Colorado & 5,517 & 77.1 & 0.8 & $(75.6-78.6)$ \\
\hline Des Moines-West Des Moines, lowa & 1,308 & 81.2 & 1.6 & $(78.0-84.3)$ \\
\hline Duluth, Minnesota-Wisconsin & 679 & 77.7 & 3.1 & $(71.6-83.8)$ \\
\hline Durham-Chapel Hill, North Carolina & 607 & 79.3 & 2.5 & $(74.5-84.2)$ \\
\hline El Paso, Texas & 736 & 69.7 & 2.4 & $(65.0-74.5)$ \\
\hline Evansville, Indiana-Kentucky & 558 & 71.4 & 3.0 & $(65.5-77.4)$ \\
\hline Fargo, North Dakota-Minnesota & 1,151 & 73.4 & 2.0 & $(69.5-77.3)$ \\
\hline Fayetteville-Springdale-Rogers, Arkansas-Missouri & 789 & 64.2 & 2.4 & $(59.5-69.0)$ \\
\hline Fort Smith, Arkansas-Oklahoma & 481 & 70.3 & 3.3 & $(63.8-76.8)$ \\
\hline Fort Wayne, Indiana & 756 & 71.1 & 2.3 & $(66.5-75.7)$ \\
\hline Fort Worth-Arlington, Texas ${ }^{\dagger}$ & 792 & 74.4 & 2.3 & $(69.8-79.0)$ \\
\hline Gainesville, Florida & 1,008 & 78.2 & 2.4 & $(73.5-82.9)$ \\
\hline Grand Forks, North Dakota-Minnesota & 485 & 76.2 & 3.0 & $(70.3-82.2)$ \\
\hline Grand Island, Nebraska & 777 & 72.9 & 2.6 & $(67.8-78.0)$ \\
\hline Grand Rapids-Wyoming, Michigan & 1,314 & 78.6 & 1.7 & $(75.3-81.8)$ \\
\hline Greensboro-High Point, North Carolina & 644 & 76.3 & 2.5 & $(71.5-81.1)$ \\
\hline Greenville-Anderson-Mauldin, South Carolina & 1,320 & 74.7 & 1.8 & $(71.3-78.2)$ \\
\hline Gulfport-Biloxi-Pascagoula, Mississippi & 746 & 69.5 & 2.4 & $(64.7-74.2)$ \\
\hline Hagerstown-Martinsburg, Maryland-West Virginia & 750 & 78.6 & 2.7 & $(73.3-83.8)$ \\
\hline Hartford-West Hartford-East Hartford, Connecticut & 2,761 & 80.6 & 1.4 & (77.9-83.3) \\
\hline Hilton Head Island-Bluffton-Beaufort, South Carolina & 804 & 74.4 & 3.3 & $(68.0-80.8)$ \\
\hline Houston-The Woodlands-Sugar Land, Texas & 1,354 & 76.7 & 1.7 & $(73.4-80.1)$ \\
\hline Huntington-Ashland, West Virginia-Kentucky-Ohio & 1,136 & 74.9 & 1.8 & $(71.5-78.4)$ \\
\hline Idaho Falls, Idaho & 496 & 66.8 & 2.8 & $(61.3-72.2)$ \\
\hline Indianapolis-Carmel-Anderson, Indiana & 2,480 & 74.2 & 1.2 & $(71.9-76.6)$ \\
\hline
\end{tabular}

See table footnotes on page 55 . 
TABLE 22. (Continued) Age-adjusted* prevalence estimates of adults aged $\geq 18$ years who have had their blood cholesterol checked during the past 5 years, metropolitan and micropolitan statistical area-Behavioral Risk Factor Surveillance System, United States, 2013

\begin{tabular}{|c|c|c|c|c|}
\hline MMSA & $\begin{array}{l}\text { Sample } \\
\text { size }\end{array}$ & $\%$ & SE & $95 \% \mathrm{Cl}$ \\
\hline Jackson, Mississippi & 772 & 74.6 & 2.2 & $(70.3-79.0)$ \\
\hline Jacksonville, Florida & 2,823 & 75.4 & 1.3 & $(72.8-78.0)$ \\
\hline Kansas City, Missouri-Kansas & 7,214 & 75.7 & 1.1 & $(73.6-77.7)$ \\
\hline Kingsport-Bristol-Bristol, Tennessee-Virginia & 520 & 82.7 & 3.3 & $(76.3-89.1)$ \\
\hline Knoxville, Tennessee & 629 & 77.2 & 2.5 & $(72.3-82.1)$ \\
\hline Lansing-East Lansing, Michigan & 664 & 76.2 & 2.3 & $(71.7-80.6)$ \\
\hline Lexington-Fayette, Kentucky & 616 & 72.4 & 2.3 & $(67.9-76.8)$ \\
\hline Lincoln, Nebraska & 1,825 & 72.4 & 1.3 & $(69.9-74.9)$ \\
\hline Little Rock-North Little Rock-Conway, Arkansas & 1,111 & 75.8 & 2.0 & $(72.0-79.6)$ \\
\hline Logan, Utah-Idaho & 619 & 62.5 & 2.2 & $(58.1-66.9)$ \\
\hline Los Angeles-Long Beach-Anaheim, California & 2,983 & 75.9 & 1.1 & $(73.8-77.9)$ \\
\hline Louisville-Jefferson County, Kentucky-Indiana & 2,093 & 75.9 & 1.6 & $(72.7-79.2)$ \\
\hline Lubbock, Texas & 514 & 71.0 & 3.1 & $(64.9-77.1)$ \\
\hline Manhattan, Kansas & 639 & 68.6 & 2.1 & $(64.5-72.7)$ \\
\hline Memphis, Tennessee-Mississippi-Arkansas & 1,166 & 77.5 & 2.1 & $(73.5-81.6)$ \\
\hline Miami-Fort Lauderdale-West Palm Beach, Florida & 2,165 & 77.3 & 1.6 & $(74.2-80.4)$ \\
\hline Milwaukee-Waukesha-West Allis, Wisconsin & 1,253 & 72.5 & 2.0 & $(68.5-76.4)$ \\
\hline Minneapolis-St. Paul-Bloomington, Minnesota-Wisconsin & 8,891 & 76.7 & 0.9 & $(75.0-78.4)$ \\
\hline Minot, North Dakota & 629 & 70.9 & 2.4 & $(66.2-75.6)$ \\
\hline $\begin{array}{l}\text { Montgomery County-Bucks County-Chester County, } \\
\text { Pennsylvania }{ }^{\dagger}\end{array}$ & 944 & 80.4 & 1.8 & $(76.8-84.0)$ \\
\hline $\begin{array}{l}\text { Myrtle Beach-Conway-North Myrtle Beach, South Carolina- } \\
\text { North Carolina }\end{array}$ & 754 & 72.5 & 2.5 & $(67.5-77.5)$ \\
\hline Nashville-Davidson County-Murfreesboro-Franklin, Tennessee & 1,017 & 80.4 & 1.6 & $(77.1-83.6)$ \\
\hline Nassau County-Suffolk County, New York ${ }^{\dagger}$ & 920 & 83.5 & 1.7 & $(80.1-86.9)$ \\
\hline Newark, New Jersey-Pennsylvania ${ }^{\dagger}$ & 3,978 & 79.4 & 1.0 & $(77.3-81.4)$ \\
\hline New Orleans-Metairie, Louisiana & 1,241 & 76.4 & 2.3 & (71.9-80.9) \\
\hline New York-Jersey City-White Plains, New York-New Jersey ${ }^{\dagger}$ & 8,668 & 79.9 & 0.7 & (78.6-81.3) \\
\hline Norfolk, Nebraska & 657 & 73.5 & 2.2 & $(69.2-77.8)$ \\
\hline North Platte, Nebraska & 698 & 67.9 & 2.8 & $(62.5-73.3)$ \\
\hline North Port-Sarasota-Bradenton, Florida & 1,063 & 71.4 & 2.3 & $(66.8-76.0)$ \\
\hline Oakland-Hayward-Berkeley, California $^{\dagger}$ & 684 & 74.5 & 2.2 & $(70.2-78.9)$ \\
\hline Ogden-Clearfield, Utah & 2,393 & 73.4 & 1.1 & $(71.2-75.6)$ \\
\hline Oklahoma City, Oklahoma & 2,563 & 73.1 & 1.2 & $(70.7-75.5)$ \\
\hline Omaha-Council Bluffs, Nebraska-lowa & 3,047 & 74.4 & 1.2 & $(72.2-76.7)$ \\
\hline Orlando-Kissimmee-Sanford, Florida & 2,234 & 75.6 & 1.5 & $(72.7-78.5)$ \\
\hline Panama City, Florida & 1,001 & 74.7 & 2.3 & $(70.1-79.2)$ \\
\hline Pensacola-Ferry Pass-Brent, Florida & 1,281 & 74.3 & 1.7 & (70.9-77.7) \\
\hline Philadelphia, Pennsylvania ${ }^{\dagger}$ & 1,723 & 76.5 & 1.5 & $(73.7-79.4)$ \\
\hline Phoenix-Mesa-Scottsdale, Arizona & 1,502 & 71.3 & 1.9 & $(67.6-75.0)$ \\
\hline Pittsburgh, Pennsylvania & 2,305 & 73.8 & 1.3 & $(71.2-76.4)$ \\
\hline Ponce, Puerto Rico & 514 & 79.4 & 2.4 & $(74.6-84.1)$ \\
\hline Portland-South Portland, Maine & 2,583 & 78.0 & 1.3 & $(75.4-80.6)$ \\
\hline Portland-Vancouver-Hillsboro, Oregon-Washington & 3,118 & 74.7 & 1.2 & $(72.4-77.0)$ \\
\hline Port St. Lucie, Florida & 1,010 & 78.3 & 2.7 & $(73.0-83.5)$ \\
\hline Providence-Warwick, Rhode Island-Massachusetts & 8,107 & 81.1 & 0.9 & $(79.3-82.8)$ \\
\hline Provo-Orem, Utah & 1,770 & 67.7 & 1.2 & $(65.2-70.1)$ \\
\hline Raleigh, North Carolina & 646 & 79.1 & 1.8 & $(75.5-82.7)$ \\
\hline Rapid City, South Dakota & 849 & 72.5 & 2.2 & $(68.2-76.8)$ \\
\hline Reno, Nevada & 1,762 & 72.5 & 1.7 & $(69.3-75.8)$ \\
\hline Richmond, Virginia & 1,284 & 77.5 & 1.7 & $(74.2-80.8)$ \\
\hline Riverside-San Bernardino-Ontario, California & 1,349 & 74.1 & 1.6 & $(71.0-77.3)$ \\
\hline Rochester, New York & 495 & 81.3 & 2.5 & $(76.3-86.2)$ \\
\hline Rockingham County-Strafford County, New Hampshire ${ }^{\dagger}$ & 1,625 & 79.2 & 1.6 & $(76.0-82.5)$ \\
\hline Sacramento-Roseville-Arden-Arcade, California & 874 & 73.5 & 2.0 & $(69.5-77.5)$ \\
\hline St. Louis, Missouri-Illinois & 1,997 & 74.1 & 1.6 & $(71.0-77.2)$ \\
\hline Salem, Oregon & 509 & 71.8 & 3.3 & $(65.2-78.3)$ \\
\hline Salina, Kansas & 512 & 68.9 & 2.7 & $(63.5-74.2)$ \\
\hline Salisbury, Maryland-Delaware & 2,014 & 75.3 & 2.0 & (71.4-79.2) \\
\hline Salt Lake City, Utah & 4,530 & 71.1 & 0.8 & $(69.4-72.7)$ \\
\hline San Antonio-New Braunfels, Texas & 917 & 72.2 & 2.0 & $(68.3-76.1)$ \\
\hline
\end{tabular}

See table footnotes on page 55 . 
TABLE 22. (Continued) Age-adjusted* prevalence estimates of adults aged $\geq 18$ years who have had their blood cholesterol checked during the past 5 years, metropolitan and micropolitan statistical area-Behavioral Risk Factor Surveillance System, United States, 2013

\begin{tabular}{|c|c|c|c|c|}
\hline MMSA & $\begin{array}{l}\text { Sample } \\
\text { size }\end{array}$ & $\%$ & SE & $95 \% \mathrm{Cl}$ \\
\hline San Francisco-Redwood City-South San Francisco, California $^{\dagger}$ & 526 & 77.6 & 2.5 & $(72.6-82.6)$ \\
\hline San Jose-Sunnyvale-Santa Clara, California & 616 & 76.3 & 2.4 & $(71.6-81.0)$ \\
\hline San Juan-Carolina-Caguas, Puerto Rico & 3,535 & 77.7 & 1.0 & $(75.7-79.7)$ \\
\hline Scottsbluff, Nebraska & 684 & 67.9 & 2.8 & $(62.4-73.4)$ \\
\hline Scranton-Wilkes-Barre-Hazleton, Pennsylvania & 551 & 78.5 & 2.9 & $(72.9-84.1)$ \\
\hline Seattle-Bellevue-Everett, Washington ${ }^{\dagger}$ & 3,664 & 75.8 & 1.0 & $(73.9-77.7)$ \\
\hline Shreveport-Bossier City, Louisiana & 552 & 74.9 & 3.4 & $(68.1-81.6)$ \\
\hline Silver Spring-Frederick-Rockville, Maryland ${ }^{\dagger}$ & 2,379 & 79.0 & 1.4 & $(76.2-81.7)$ \\
\hline Sioux City, lowa-Nebraska-South Dakota & 1,035 & 67.2 & 2.9 & $(61.6-72.9)$ \\
\hline Sioux Falls, South Dakota & 979 & 72.5 & 2.0 & $(68.6-76.4)$ \\
\hline Spartanburg, South Carolina & 584 & 68.9 & 3.0 & $(63.1-74.7)$ \\
\hline Spokane-Spokane Valley, Washington & 833 & 70.1 & 2.3 & $(65.5-74.6)$ \\
\hline Springfield, Massachusetts & 1,523 & 80.0 & 2.0 & $(76.0-83.9)$ \\
\hline Tallahassee, Florida & 1,813 & 77.4 & 2.2 & $(73.1-81.7)$ \\
\hline Tampa-St. Petersburg-Clearwater, Florida & 2,165 & 78.9 & 1.4 & $(76.2-81.7)$ \\
\hline Toledo, Ohio & 966 & 72.4 & 2.5 & $(67.4-77.3)$ \\
\hline Topeka, Kansas & 2,308 & 70.5 & 1.3 & $(67.9-73.1)$ \\
\hline Tulsa, Oklahoma & 1,933 & 71.1 & 1.5 & $(68.1-74.1)$ \\
\hline Virginia Beach-Norfolk-Newport News, Virginia-North Carolina & 1,629 & 78.4 & 1.6 & $(75.2-81.6)$ \\
\hline Warren-Troy-Farmington Hills, Michigan ${ }^{\dagger}$ & 2,213 & 79.0 & 1.4 & $(76.3-81.7)$ \\
\hline $\begin{array}{l}\text { Washington-Arlington-Alexandria, District of Columbia- } \\
\text { Virginia-Maryland-West Virginia }{ }^{\dagger}\end{array}$ & 8,806 & 79.9 & 0.9 & $(78.1-81.7)$ \\
\hline Wichita, Kansas & 4,766 & 71.3 & 0.9 & $(69.5-73.1)$ \\
\hline Wilmington, Delaware-Maryland-New Jersey ${ }^{\dagger}$ & 3,187 & 78.2 & 1.2 & $(75.9-80.5)$ \\
\hline Winston-Salem, North Carolina & 671 & 79.3 & 2.7 & $(74.0-84.6)$ \\
\hline Worcester, Massachusetts-Connecticut & 2,692 & 81.2 & 1.4 & $(78.5-83.9)$ \\
\hline Median & & 75.6 & & \\
\hline Range & & $62.5-83.5$ & & \\
\hline
\end{tabular}

Abbreviations: $\mathrm{Cl}=$ confidence interval; $\mathrm{MMSA}=$ metropolitan and micropolitan statistical areas; $\mathrm{SE}=$ standard error.

* Age adjusted to the 2000 U.S. standard population.

+ Metropolitan division. 
TABLE 23. Age-adjusted* prevalence estimates of adults aged $\geq 18$ years who reported no leisure-time physical activity ${ }^{\dagger}$ during the past 30 days, by state/territory - Behavioral Risk Factor Surveillance System, United States, 2013

\begin{tabular}{|c|c|c|c|c|}
\hline State/Territory & $\begin{array}{l}\text { Sample } \\
\text { size }\end{array}$ & $\%$ & SE & $95 \% \mathrm{Cl}$ \\
\hline Alabama & 6,164 & 30.7 & 0.9 & $(28.9-32.5)$ \\
\hline Alaska & 4,275 & 22.4 & 0.9 & $(20.5-24.2)$ \\
\hline Arizona & 3,910 & 24.8 & 1.3 & $(22.2-27.4)$ \\
\hline Arkansas & 4,871 & 33.4 & 1.0 & (31.4-35.4) \\
\hline California & 10,190 & 21.3 & 0.6 & $(20.2-22.5)$ \\
\hline Colorado & 12,370 & 17.9 & 0.5 & $(17.0-18.8)$ \\
\hline Connecticut & 7,121 & 24.2 & 0.8 & $(22.6-25.8)$ \\
\hline Delaware & 4,926 & 27.0 & 0.9 & $(25.2-28.7)$ \\
\hline District of Columbia & 4,429 & 19.7 & 1.0 & $(17.8-21.7)$ \\
\hline Florida & 31,527 & 27.0 & 0.6 & $(25.8-28.2)$ \\
\hline Georgia & 7,381 & 27.0 & 0.7 & $(25.5-28.4)$ \\
\hline Hawaii & 7,368 & 21.6 & 0.8 & $(20.1-23.1)$ \\
\hline Idaho & 5,260 & 23.3 & 0.9 & $(21.6-25.1)$ \\
\hline Illinois & 5,305 & 24.7 & 0.9 & $(23.0-26.4)$ \\
\hline Indiana & 9,584 & 30.5 & 0.7 & $(29.2-31.8)$ \\
\hline lowa & 7,678 & 27.7 & 0.7 & $(26.2-29.1)$ \\
\hline Kansas & 22,177 & 26.1 & 0.4 & $(25.4-26.9)$ \\
\hline Kentucky & 10,156 & 29.4 & 0.7 & $(27.9-30.8)$ \\
\hline Louisiana & 5,026 & 31.4 & 1.1 & $(29.3-33.6)$ \\
\hline Maine & 7,724 & 22.0 & 0.7 & $(20.6-23.4)$ \\
\hline Maryland & 12,261 & 24.7 & 0.6 & $(23.5-26.0)$ \\
\hline Massachusetts & 13,532 & 23.0 & 0.6 & $(21.8-24.2)$ \\
\hline Michigan & 12,214 & 23.7 & 0.6 & $(22.6-24.9)$ \\
\hline Minnesota & 13,323 & 22.9 & 0.7 & $(21.6-24.3)$ \\
\hline Mississippi & 7,009 & 37.3 & 0.9 & $(35.6-39.1)$ \\
\hline Missouri & 6,793 & 27.7 & 0.8 & $(26.0-29.3)$ \\
\hline Montana & 9,218 & 21.5 & 0.6 & $(20.3-22.7)$ \\
\hline Nebraska & 16,158 & 24.9 & 0.6 & $(23.7-26.0)$ \\
\hline Nevada & 4,799 & 23.2 & 1.1 & $(21.0-25.5)$ \\
\hline New Hampshire & 6,010 & 21.7 & 0.8 & $(20.1-23.3)$ \\
\hline New Jersey & 12,049 & 26.4 & 0.7 & $(25.1-27.7)$ \\
\hline New Mexico & 8,512 & 23.9 & 0.7 & $(22.5-25.2)$ \\
\hline New York & 8,164 & 26.2 & 0.7 & $(24.9-27.6)$ \\
\hline North Carolina & 8,401 & 26.0 & 0.7 & $(24.7-27.3)$ \\
\hline North Dakota & 7,380 & 27.1 & 0.8 & $(25.6-28.6)$ \\
\hline Ohio & 11,117 & 27.6 & 0.7 & $(26.3-28.9)$ \\
\hline Oklahoma & 7,831 & 32.2 & 0.7 & $(30.8-33.6)$ \\
\hline Oregon & 5,407 & 18.1 & 0.8 & $(16.6-19.7)$ \\
\hline Pennsylvania & 10,564 & 25.1 & 0.6 & $(24.0-26.3)$ \\
\hline Rhode Island & 5,922 & 26.2 & 0.8 & $(24.6-27.9)$ \\
\hline South Carolina & 10,067 & 26.2 & 0.7 & $(24.9-27.5)$ \\
\hline South Dakota & 6,553 & 23.2 & 0.9 & $(21.4-24.9)$ \\
\hline Tennessee & 5,196 & 36.6 & 1.0 & $(34.6-38.6)$ \\
\hline Texas & 10,100 & 30.0 & 0.7 & $(28.5-31.4)$ \\
\hline Utah & 11,937 & 20.9 & 0.5 & (19.9-21.8) \\
\hline Vermont & 5,986 & 19.4 & 0.7 & $(18.1-20.8)$ \\
\hline Virginia & 7,767 & 25.1 & 0.7 & $(23.7-26.5)$ \\
\hline Washington & 10,734 & 19.7 & 0.6 & $(18.5-20.8)$ \\
\hline West Virginia & 5,769 & 30.1 & 0.8 & $(28.6-31.6)$ \\
\hline Wisconsin & 5,823 & 22.9 & 0.9 & $(21.2-24.6)$ \\
\hline Wyoming & 6,055 & 24.7 & 0.9 & $(23.0-26.4)$ \\
\hline Guam & 1,728 & 33.3 & 1.5 & $(30.3-36.3)$ \\
\hline Puerto Rico & 5,922 & 47.4 & 0.9 & $(45.7-49.1)$ \\
\hline Median & & 25.1 & & \\
\hline Range & & $17.9-47.4$ & & \\
\hline
\end{tabular}

Abbreviations: $\mathrm{Cl}=$ confidence interval; $\mathrm{SE}=$ standard error.

* Age adjusted to the 2000 U.S. standard population.

† Any physical activity or exercise (e.g., running, calisthenics, golf, gardening, or walking for exercise). 
TABLE 24. Age-adjusted* prevalence of adults aged $\geq 18$ years who reported no leisure-time physical activity ${ }^{\dagger}$ during the past 30 days, by metropolitan and micropolitan statistical area - Behavioral Risk Factor Surveillance System, United States, 2013

\begin{tabular}{|c|c|c|c|c|}
\hline MMSA & $\begin{array}{l}\text { Sample } \\
\text { size }\end{array}$ & $\%$ & SE & $95 \% \mathrm{Cl}$ \\
\hline Aguadilla-Isabela, Puerto Rico & 585 & 42.5 & 2.7 & $(37.2-47.7)$ \\
\hline Akron, Ohio & 642 & 22.7 & 2.6 & $(17.7-27.8)$ \\
\hline Albuquerque, New Mexico & 1,918 & 22.4 & 1.3 & $(19.9-24.8)$ \\
\hline Allentown-Bethlehem-Easton, Pennsylvania-New Jersey & 949 & 25.2 & 2.1 & $(21.1-29.2)$ \\
\hline Anchorage, Alaska & 1,423 & 21.2 & 1.4 & $(18.4-24.0)$ \\
\hline Atlanta-Sandy Springs-Roswell, Georgia & 3,193 & 24.1 & 1.0 & $(22.1-26.2)$ \\
\hline Augusta-Richmond County, Georgia-South Carolina & 852 & 24.5 & 2.2 & $(20.1-28.9)$ \\
\hline Austin-Round Rock, Texas & 879 & 22.0 & 2.0 & $(18.1-25.9)$ \\
\hline Baltimore-Columbia-Towson, Maryland & 4,474 & 25.0 & 1.0 & $(23.1-26.9)$ \\
\hline Baton Rouge, Louisiana & 879 & 33.1 & 2.5 & $(28.1-38.0)$ \\
\hline Billings, Montana & 774 & 24.3 & 1.8 & $(20.7-27.9)$ \\
\hline Birmingham-Hoover, Alabama & 1,288 & 31.8 & 1.9 & $(28.2-35.5)$ \\
\hline Bismarck, North Dakota & 984 & 28.5 & 2.0 & $(24.6-32.5)$ \\
\hline Boise City, Idaho & 1,393 & 23.0 & 1.6 & $(19.8-26.2)$ \\
\hline Boston, Massachusetts ${ }^{\S}$ & 3,653 & 21.0 & 1.1 & $(18.9-23.2)$ \\
\hline Buffalo-Cheektowaga-Niagara Falls, New York & 468 & 24.5 & 2.9 & $(18.8-30.2)$ \\
\hline Burlington-South Burlington, Vermont & 1,517 & 18.8 & 1.3 & $(16.4-21.3)$ \\
\hline Cambridge-Newton-Framingham, Massachusetts ${ }^{\S}$ & 4,393 & 21.9 & 1.0 & $(19.9-23.9)$ \\
\hline Camden, New Jersey ${ }^{\S}$ & 1,700 & 25.3 & 1.5 & $(22.3-28.2)$ \\
\hline Cedar Rapids, lowa & 612 & 24.7 & 2.3 & $(20.1-29.3)$ \\
\hline Charleston, West Virginia & 803 & 32.9 & 2.3 & $(28.4-37.3)$ \\
\hline Charleston-North Charleston, South Carolina & 1,462 & 21.6 & 1.5 & $(18.7-24.5)$ \\
\hline Charlotte-Concord-Gastonia, North Carolina-South Carolina & 1,852 & 23.6 & 1.3 & $(21.1-26.2)$ \\
\hline Chattanooga, Tennessee-Georgia & 507 & 32.5 & 3.4 & $(25.9-39.1)$ \\
\hline Chicago-Naperville-Elgin, Illinois-Indiana-Wisconsin & 3,124 & 24.0 & 1.1 & $(21.8-26.3)$ \\
\hline Cincinnati, Ohio-Kentucky-Indiana & 2,405 & 25.8 & 1.4 & $(23.1-28.5)$ \\
\hline Claremont-Lebanon, New Hampshire-Vermont & 1,578 & 20.2 & 1.6 & $(17.1-23.4)$ \\
\hline Cleveland-Elyria, Ohio & 1,006 & 25.4 & 1.9 & $(21.7-29.0)$ \\
\hline Colorado Springs, Colorado & 1,254 & 16.3 & 1.3 & $(13.8-18.8)$ \\
\hline Columbia, South Carolina & 1,367 & 23.6 & 1.6 & $(20.4-26.7)$ \\
\hline Columbus, Ohio & 1,724 & 28.7 & 1.5 & $(25.9-31.6)$ \\
\hline Crestview-Fort Walton Beach-Destin, Florida & 997 & 24.7 & 2.0 & $(20.7-28.7)$ \\
\hline Dallas-Plano-Irving, Texas ${ }^{\S}$ & 830 & 29.3 & 2.0 & $(25.3-33.3)$ \\
\hline Davenport-Moline-Rock Island, lowa-Illinois & 642 & 26.9 & 2.7 & $(21.6-32.2)$ \\
\hline Dayton, Ohio & 792 & 27.1 & 2.3 & $(22.5-31.7)$ \\
\hline Deltona-Daytona Beach-Ormond Beach, Florida & 1,027 & 23.6 & 2.4 & $(18.8-28.4)$ \\
\hline Denver-Aurora-Lakewood, Colorado & 5,168 & 17.9 & 0.7 & $(16.6-19.3)$ \\
\hline Des Moines-West Des Moines, lowa & 1,271 & 25.0 & 1.8 & $(21.5-28.5)$ \\
\hline Duluth, Minnesota-Wisconsin & 665 & 22.5 & 2.7 & $(17.2-27.7)$ \\
\hline Durham-Chapel Hill, North Carolina & 597 & 22.2 & 2.3 & $(17.6-26.8)$ \\
\hline El Paso, Texas & 706 & 31.2 & 2.3 & $(26.7-35.7)$ \\
\hline Evansville, Indiana-Kentucky & 542 & 31.3 & 3.0 & $(25.4-37.2)$ \\
\hline Fargo, North Dakota-Minnesota & 1,126 & 25.1 & 2.1 & $(21.1-29.2)$ \\
\hline Fayetteville-Springdale-Rogers, Arkansas-Missouri & 768 & 28.2 & 2.3 & $(23.7-32.8)$ \\
\hline Fort Smith, Arkansas-Oklahoma & 473 & 28.5 & 2.9 & $(22.7-34.2)$ \\
\hline Fort Wayne, Indiana & 730 & 29.4 & 2.4 & $(24.7-34.0)$ \\
\hline Fort Worth-Arlington, Texas ${ }^{\S}$ & 755 & 31.1 & 2.5 & $(26.2-35.9)$ \\
\hline Gainesville, Florida & 966 & 21.5 & 2.2 & $(17.2-25.8)$ \\
\hline Grand Forks, North Dakota-Minnesota & 477 & 23.1 & 3.1 & $(16.9-29.2)$ \\
\hline Grand Island, Nebraska & 751 & 33.9 & 2.8 & $(28.5-39.3)$ \\
\hline Grand Rapids-Wyoming, Michigan & 1,296 & 20.4 & 1.5 & $(17.5-23.3)$ \\
\hline Greensboro-High Point, North Carolina & 629 & 31.0 & 2.5 & $(26.0-35.9)$ \\
\hline Greenville-Anderson-Mauldin, South Carolina & 1,254 & 27.8 & 1.7 & $(24.4-31.2)$ \\
\hline Gulfport-Biloxi-Pascagoula, Mississippi & 718 & 29.8 & 2.2 & $(25.5-34.1)$ \\
\hline Hagerstown-Martinsburg, Maryland-West Virginia & 729 & 27.5 & 2.9 & $(21.9-33.1)$ \\
\hline Hartford-West Hartford-East Hartford, Connecticut & 2,653 & 23.0 & 1.3 & $(20.5-25.5)$ \\
\hline Hilton Head Island-Bluffton-Beaufort, South Carolina & 764 & 19.7 & 2.7 & $(14.4-25.0)$ \\
\hline Houston-The Woodlands-Sugar Land, Texas & 1,268 & 28.7 & 2.0 & $(24.8-32.5)$ \\
\hline Huntington-Ashland, West Virginia-Kentucky-Ohio & 1,123 & 30.0 & 1.7 & $(26.7-33.3)$ \\
\hline Idaho Falls, Idaho & 476 & 24.1 & 2.6 & $(19.0-29.3)$ \\
\hline Indianapolis-Carmel-Anderson, Indiana & 2,352 & 28.8 & 1.2 & $(26.4-31.2)$ \\
\hline
\end{tabular}

See table footnotes on page 59. 
TABLE 24. (Continued) Age-adjusted* prevalence of adults aged $\geq 18$ years who reported no leisure-time physical activity ${ }^{\dagger}$ during the past 30 days, by metropolitan and micropolitan statistical area — Behavioral Risk Factor Surveillance System, United States, 2013

\begin{tabular}{|c|c|c|c|c|}
\hline MMSA & $\begin{array}{l}\text { Sample } \\
\text { size }\end{array}$ & $\%$ & SE & $95 \% \mathrm{Cl}$ \\
\hline Jackson, Mississippi & 751 & 35.1 & 2.4 & $(30.5-39.8)$ \\
\hline Jacksonville, Florida & 2,658 & 25.4 & 1.4 & $(22.7-28.0)$ \\
\hline Kansas City, Missouri-Kansas & 7,063 & 24.7 & 1.1 & $(22.6-26.8)$ \\
\hline Kingsport-Bristol-Bristol, Tennessee-Virginia & 488 & 38.2 & 3.7 & $(30.9-45.5)$ \\
\hline Knoxville, Tennessee & 591 & 33.6 & 2.7 & $(28.4-38.8)$ \\
\hline Lansing-East Lansing, Michigan & 665 & 20.0 & 1.9 & $(16.2-23.8)$ \\
\hline Lexington-Fayette, Kentucky & 599 & 25.1 & 2.4 & $(20.5-29.8)$ \\
\hline Lincoln, Nebraska & 1,774 & 19.8 & 1.1 & $(17.6-22.1)$ \\
\hline Little Rock-North Little Rock-Conway, Arkansas & 1,054 & 30.6 & 2.0 & $(26.7-34.5)$ \\
\hline Logan, Utah-Idaho & 598 & 17.9 & 2.0 & $(14.0-21.8)$ \\
\hline Los Angeles-Long Beach-Anaheim, California & 2,651 & 23.4 & 1.1 & $(21.2-25.7)$ \\
\hline Louisville-Jefferson County, Kentucky-Indiana & 1,986 & 28.2 & 1.7 & $(24.9-31.5)$ \\
\hline Lubbock, Texas & 503 & 27.2 & 2.9 & $(21.6-32.8)$ \\
\hline Manhattan, Kansas & 629 & 21.1 & 1.9 & $(17.3-24.9)$ \\
\hline Memphis, Tennessee-Mississippi-Arkansas & 1,102 & 36.2 & 2.2 & $(31.8-40.6)$ \\
\hline Miami-Fort Lauderdale-West Palm Beach, Florida & 2,034 & 29.9 & 1.7 & $(26.6-33.1)$ \\
\hline Milwaukee-Waukesha-West Allis, Wisconsin & 1,098 & 19.6 & 1.8 & $(16.0-23.2)$ \\
\hline Minneapolis-St. Paul-Bloomington, Minnesota-Wisconsin & 8,458 & 19.9 & 0.8 & $(18.2-21.5)$ \\
\hline Minot, North Dakota & 606 & 27.2 & 2.4 & $(22.5-31.9)$ \\
\hline $\begin{array}{l}\text { Montgomery County-Bucks County-Chester County, } \\
\text { Pennsylvania§ }\end{array}$ & 904 & 20.8 & 1.7 & $(17.5-24.2)$ \\
\hline $\begin{array}{l}\text { Myrtle Beach-Conway-North Myrtle Beach, South Carolina- } \\
\text { North Carolina }\end{array}$ & 729 & 24.2 & 2.3 & $(19.6-28.8)$ \\
\hline Nashville-Davidson County-Murfreesboro-Franklin, Tennessee & 967 & 31.7 & 2.0 & $(27.9-35.6)$ \\
\hline Nassau County-Suffolk County, New York ${ }^{\S}$ & 852 & 27.3 & 2.0 & $(23.4-31.3)$ \\
\hline Newark, New Jersey-Pennsylvania§ & 3,713 & 25.3 & 1.1 & $(23.2-27.4)$ \\
\hline New Orleans-Metairie, Louisiana & 1,230 & 27.6 & 2.1 & $(23.4-31.7)$ \\
\hline New York-Jersey City-White Plains, New York-New Jersey ${ }^{\S}$ & 8,014 & 27.2 & 0.7 & $(25.8-28.7)$ \\
\hline Norfolk, Nebraska & 635 & 24.0 & 2.0 & $(20.0-28.0)$ \\
\hline North Platte, Nebraska & 679 & 27.3 & 2.6 & $(22.3-32.3)$ \\
\hline North Port-Sarasota-Bradenton, Florida & 983 & 22.0 & 2.0 & $(18.0-26.0)$ \\
\hline Oakland-Hayward-Berkeley, California§ & 622 & 20.6 & 2.2 & $(16.3-24.9)$ \\
\hline Ogden-Clearfield, Utah & 2,312 & 20.1 & 1.0 & $(18.1-22.1)$ \\
\hline Oklahoma City, Oklahoma & 2,506 & 31.1 & 1.2 & $(28.7-33.5)$ \\
\hline Omaha-Council Bluffs, Nebraska-lowa & 2,937 & 24.7 & 1.1 & $(22.6-26.9)$ \\
\hline Orlando-Kissimmee-Sanford, Florida & 2,118 & 26.6 & 1.5 & $(23.8-29.5)$ \\
\hline Panama City, Florida & 954 & 28.0 & 2.3 & $(23.5-32.5)$ \\
\hline Pensacola-Ferry Pass-Brent, Florida & 1,216 & 26.6 & 1.7 & $(23.2-30.0)$ \\
\hline Philadelphia, Pennsylvania§ & 1,624 & 27.3 & 1.5 & $(24.3-30.3)$ \\
\hline Phoenix-Mesa-Scottsdale, Arizona & 1,428 & 25.3 & 1.9 & $(21.7-29.0)$ \\
\hline Pittsburgh, Pennsylvania & 2,174 & 24.1 & 1.2 & $(21.6-26.5)$ \\
\hline Ponce, Puerto Rico & 526 & 46.6 & 2.9 & $(40.9-52.2)$ \\
\hline Portland-South Portland, Maine & 2,534 & 19.3 & 1.2 & $(17.0-21.6)$ \\
\hline Portland-Vancouver-Hillsboro, Oregon-Washington & 3,007 & 17.6 & 1.1 & $(15.5-19.8)$ \\
\hline Port St. Lucie, Florida & 932 & 27.7 & 2.5 & $(22.8-32.5)$ \\
\hline Providence-Warwick, Rhode Island-Massachusetts & 7,505 & 27.7 & 1.0 & $(25.7-29.6)$ \\
\hline Provo-Orem, Utah & 1,734 & 19.5 & 1.1 & $(17.3-21.8)$ \\
\hline Raleigh, North Carolina & 628 & 20.9 & 1.9 & $(17.3-24.6)$ \\
\hline Rapid City, South Dakota & 828 & 20.3 & 1.9 & $(16.6-24.0)$ \\
\hline Reno, Nevada & 1,697 & 17.6 & 1.2 & $(15.3-20.0)$ \\
\hline Richmond, Virginia & 1,182 & 21.5 & 1.5 & $(18.6-24.5)$ \\
\hline Riverside-San Bernardino-Ontario, California & 1,226 & 23.3 & 1.6 & $(20.3-26.4)$ \\
\hline Rochester, New York & 469 & 20.8 & 2.6 & $(15.6-25.9)$ \\
\hline Rockingham County-Strafford County, New Hampshire ${ }^{\S}$ & 1,537 & 21.8 & 1.5 & $(18.8-24.7)$ \\
\hline Sacramento-Roseville-Arden-Arcade, California & 809 & 17.0 & 2.0 & $(13.1-20.9)$ \\
\hline St. Louis, Missouri-Illinois & 1,948 & 25.8 & 1.5 & $(22.9-28.7)$ \\
\hline Salem, Oregon & 463 & 18.5 & 2.7 & $(13.2-23.7)$ \\
\hline Salina, Kansas & 498 & 29.7 & 2.6 & $(24.5-34.8)$ \\
\hline Salisbury, Maryland-Delaware & 1,934 & 27.9 & 1.7 & $(24.5-31.3)$ \\
\hline Salt Lake City, Utah & 4,331 & 21.4 & 0.8 & $(19.8-23.0)$ \\
\hline San Antonio-New Braunfels, Texas & 879 & 29.3 & 2.0 & $(25.3-33.2)$ \\
\hline
\end{tabular}

See table footnotes on page 59. 
TABLE 24. (Continued) Age-adjusted* prevalence of adults aged $\geq 18$ years who reported no leisure-time physical activity ${ }^{\dagger}$ during the past 30 days, by metropolitan and micropolitan statistical area - Behavioral Risk Factor Surveillance System, United States, 2013

\begin{tabular}{|c|c|c|c|c|}
\hline MMSA & $\begin{array}{l}\text { Sample } \\
\text { size }\end{array}$ & $\%$ & SE & $95 \% \mathrm{Cl}$ \\
\hline San Francisco-Redwood City-South San Francisco, California ${ }^{\S}$ & 473 & 14.8 & 2.1 & $(10.7-18.9)$ \\
\hline San Jose-Sunnyvale-Santa Clara, California & 543 & 21.2 & 2.7 & $(15.9-26.5)$ \\
\hline San Juan-Carolina-Caguas, Puerto Rico & 3,601 & 48.3 & 1.1 & $(46.1-50.4)$ \\
\hline Scottsbluff, Nebraska & 682 & 28.8 & 2.6 & $(23.6-33.9)$ \\
\hline Scranton-Wilkes-Barre-Hazleton, Pennsylvania & 517 & 24.3 & 2.6 & $(19.2-29.5)$ \\
\hline Seattle-Bellevue-Everett, Washington $\S$ & 3,610 & 18.0 & 0.9 & $(16.3-19.8)$ \\
\hline Shreveport-Bossier City, Louisiana & 555 & 31.3 & 3.2 & $(25.0-37.6)$ \\
\hline Silver Spring-Frederick-Rockville, Maryland ${ }^{\S}$ & 2,304 & 20.5 & 1.5 & $(17.6-23.4)$ \\
\hline Sioux City, lowa-Nebraska-South Dakota & 997 & 32.7 & 3.3 & $(26.3-39.1)$ \\
\hline Sioux Falls, South Dakota & 951 & 21.1 & 1.8 & $(17.6-24.7)$ \\
\hline Spartanburg, South Carolina & 562 & 27.3 & 2.7 & $(22.0-32.6)$ \\
\hline Spokane-Spokane Valley, Washington & 830 & 17.4 & 2.0 & $(13.5-21.3)$ \\
\hline Springfield, Massachusetts & 1,418 & 26.5 & 2.2 & $(22.2-30.8)$ \\
\hline Tallahassee, Florida & 1,730 & 21.2 & 1.9 & $(17.5-24.9)$ \\
\hline Tampa-St. Petersburg-Clearwater, Florida & 2,028 & 26.5 & 1.5 & $(23.6-29.5)$ \\
\hline Toledo, Ohio & 939 & 29.5 & 2.7 & $(24.2-34.9)$ \\
\hline Topeka, Kansas & 2,288 & 26.2 & 1.2 & $(23.8-28.7)$ \\
\hline Tulsa, Oklahoma & 1,913 & 32.1 & 1.5 & $(29.3-35.0)$ \\
\hline Virginia Beach-Norfolk-Newport News, Virginia-North Carolina & 1,541 & 26.2 & 1.6 & $(23.0-29.4)$ \\
\hline Warren-Troy-Farmington Hills, Michigan $\$$ & 2,152 & 21.3 & 1.2 & $(18.8-23.7)$ \\
\hline $\begin{array}{l}\text { Washington-Arlington-Alexandria, District of Columbia- } \\
\text { Virginia-Maryland-West Virginia }\end{array}$ & 8,270 & 22.6 & 1.0 & $(20.7-24.5)$ \\
\hline Wichita, Kansas & 4,730 & 26.5 & 0.8 & $(24.9-28.2)$ \\
\hline Wilmington, Delaware-Maryland-New Jersey ${ }^{\S}$ & 3,090 & 27.1 & 1.1 & $(24.9-29.3)$ \\
\hline Winston-Salem, North Carolina & 658 & 25.8 & 2.3 & $(21.2-30.4)$ \\
\hline Worcester, Massachusetts-Connecticut & 2,517 & 24.9 & 1.4 & $(22.1-27.7)$ \\
\hline Median & & 25.1 & & \\
\hline Range & & $14.8-48.3$ & & \\
\hline
\end{tabular}

Abbreviations: $\mathrm{Cl}$ = confidence interval; MMSA = metropolitan and micropolitan statistical areas; $\mathrm{SE}=$ standard error.

* Age adjusted to the 2000 U.S. standard population.

${ }^{\dagger}$ Any physical activity or exercise (e.g., running, calisthenics, golf, gardening, or walking for exercise).

$\S$ Metropolitan division. 
TABLE 25. Age-adjusted* prevalence estimates of adults aged $\geq 18$ years who reported no leisure-time physical activity ${ }^{\dagger}$ during the past 30 days, by state/territory - Behavioral Risk Factor Surveillance System, United States, 2014

\begin{tabular}{|c|c|c|c|c|}
\hline State/Territory & $\begin{array}{l}\text { Sample } \\
\text { size }\end{array}$ & $\%$ & SE & $95 \% \mathrm{Cl}$ \\
\hline Alabama & 8,639 & 26.8 & 0.7 & $(25.4-28.2)$ \\
\hline Alaska & 4,378 & 19.2 & 0.8 & $(17.5-20.8)$ \\
\hline Arizona & 14,829 & 20.8 & 0.6 & $(19.6-22.0)$ \\
\hline Arkansas & 5,250 & 29.4 & 1.0 & $(27.5-31.4)$ \\
\hline California & 7,822 & 21.5 & 0.6 & $(20.3-22.7)$ \\
\hline Colorado & 13,383 & 16.4 & 0.4 & $(15.5-17.2)$ \\
\hline Connecticut & 7,943 & 19.9 & 0.7 & $(18.5-21.3)$ \\
\hline Delaware & 4,293 & 24.1 & 1.0 & $(22.2-26.1)$ \\
\hline District of Columbia & 4,059 & 21.2 & 1.1 & $(19.1-23.4)$ \\
\hline Florida & 9,796 & 22.7 & 0.7 & (21.4-24.0) \\
\hline Georgia & 6,347 & 23.3 & 0.7 & $(21.9-24.8)$ \\
\hline Hawaii & 7,245 & 19.0 & 0.7 & $(17.7-20.3)$ \\
\hline Idaho & 5,468 & 18.4 & 0.8 & $(16.8-20.0)$ \\
\hline Illinois & 5,050 & 23.6 & 0.8 & $(22.0-25.2)$ \\
\hline Indiana & 11,510 & 25.4 & 0.6 & $(24.2-26.5)$ \\
\hline lowa & 8,121 & 21.5 & 0.6 & $(20.3-22.6)$ \\
\hline Kansas & 13,727 & 23.1 & 0.5 & $(22.2-24.0)$ \\
\hline Kentucky & 11,189 & 27.1 & 0.7 & $(25.7-28.5)$ \\
\hline Louisiana & 6,772 & 29.0 & 0.7 & $(27.6-30.4)$ \\
\hline Maine & 9,118 & 18.3 & 0.6 & $(17.1-19.5)$ \\
\hline Maryland & 12,556 & 21.1 & 0.7 & $(19.7-22.6)$ \\
\hline Massachusetts & 15,632 & 19.6 & 0.5 & (18.5-20.6) \\
\hline Michigan & 8,455 & 24.8 & 0.7 & $(23.5-26.1)$ \\
\hline Minnesota & 16,395 & 19.8 & 0.4 & (19.0-20.6) \\
\hline Mississippi & 4,203 & 31.1 & 1.0 & $(29.0-33.1)$ \\
\hline Missouri & 7,076 & 24.2 & 0.8 & $(22.6-25.8)$ \\
\hline Montana & 7,493 & 18.5 & 0.7 & $(17.1-19.8)$ \\
\hline Nebraska & 22,397 & 20.7 & 0.5 & (19.8-21.6) \\
\hline Nevada & 3,754 & 22.0 & 1.1 & $(19.9-24.1)$ \\
\hline New Hampshire & 6,174 & 18.3 & 0.8 & (16.8-19.8) \\
\hline New Jersey & 13,025 & 22.8 & 0.6 & $(21.7-24.0)$ \\
\hline New Mexico & 8,927 & 23.0 & 0.7 & $(21.6-24.5)$ \\
\hline New York & 6,845 & 25.4 & 0.7 & $(24.0-26.8)$ \\
\hline North Carolina & 7,281 & 22.6 & 0.6 & (21.4-23.8) \\
\hline North Dakota & 7,764 & 20.7 & 0.7 & $(19.2-22.1)$ \\
\hline Ohio & 10,904 & 24.0 & 0.7 & $(22.6-25.3)$ \\
\hline Oklahoma & 8,444 & 27.5 & 0.6 & $(26.3-28.8)$ \\
\hline Oregon & 5,214 & 15.9 & 0.7 & $(14.5-17.3)$ \\
\hline Pennsylvania & 10,977 & 22.4 & 0.6 & (21.2-23.6) \\
\hline Rhode Island & 6,436 & 21.8 & 0.8 & $(20.3-23.4)$ \\
\hline South Carolina & 11,009 & 24.6 & 0.6 & $(23.4-25.8)$ \\
\hline South Dakota & 7,399 & 20.7 & 0.9 & $(18.9-22.5)$ \\
\hline Tennessee & 5,139 & 25.7 & 0.9 & $(24.0-27.4)$ \\
\hline Texas & 15,394 & 27.5 & 0.6 & $(26.3-28.7)$ \\
\hline Utah & 14,993 & 17.1 & 0.4 & $(16.3-17.8)$ \\
\hline Vermont & 6,458 & 18.1 & 0.6 & $(17.0-19.3)$ \\
\hline Virginia & 9,452 & 23.1 & 0.6 & (21.9-24.3) \\
\hline Washington & 10,074 & 17.9 & 0.6 & $(16.7-19.0)$ \\
\hline West Virginia & 6,190 & 27.1 & 0.7 & $(25.6-28.5)$ \\
\hline Wisconsin & 7,036 & 20.7 & 0.8 & $(19.2-22.2)$ \\
\hline Wyoming & 6,396 & 21.3 & 0.9 & $(19.6-23.1)$ \\
\hline Guam & 2,518 & 28.6 & 1.3 & $(26.1-31.1)$ \\
\hline Puerto Rico & 5,988 & 39.7 & 0.8 & $(38.1-41.3)$ \\
\hline Median & & 22.4 & & \\
\hline Range & & $15.9-39.7$ & & \\
\hline
\end{tabular}

Abbreviations: $\mathrm{Cl}=$ confidence interval; $\mathrm{SE}=$ standard error.

* Age adjusted to the 2000 U.S. standard population.

† Any physical activity or exercise (e.g., running, calisthenics, golf, gardening, or walking for exercise). 
TABLE 26. Age-adjusted* prevalence estimates of adults aged $\geq 18$ years who reported no leisure-time physical activity ${ }^{\dagger}$ during the past $30^{*}$ days, by metropolitan and micropolitan statistical area - Behavioral Risk Factor Surveillance System, United States, 2014

\begin{tabular}{|c|c|c|c|c|}
\hline MMSA & $\begin{array}{l}\text { Sample } \\
\text { size }\end{array}$ & $\%$ & SE & $95 \% \mathrm{Cl}$ \\
\hline Aberdeen, South Dakota & 620 & 25.6 & 3.1 & $(19.5-31.7)$ \\
\hline Aguadilla-Isabela, Puerto Rico & 544 & 42.1 & 2.7 & $(36.9-47.3)$ \\
\hline Albuquerque, New Mexico & 1,787 & 21.3 & 1.4 & $(18.6-24.1)$ \\
\hline Allentown-Bethlehem-Easton, Pennsylvania-New Jersey & 1,092 & 22.5 & 2.1 & $(18.5-26.6)$ \\
\hline Anchorage, Alaska & 1,784 & 17.7 & 1.1 & $(15.5-19.9)$ \\
\hline Atlanta-Sandy Springs-Roswell, Georgia & 2,776 & 20.1 & 1.0 & $(18.1-22.0)$ \\
\hline Augusta-Richmond County, Georgia-South Carolina & 888 & 23.3 & 2.4 & $(18.5-28.0)$ \\
\hline Austin-Round Rock, Texas & 2,254 & 19.4 & 1.1 & $(17.3-21.6)$ \\
\hline Baltimore-Columbia-Towson, Maryland & 4,612 & 21.5 & 1.1 & $(19.2-23.7)$ \\
\hline Baton Rouge, Louisiana & 922 & 27.4 & 1.8 & $(23.9-30.8)$ \\
\hline Berlin, New Hampshire-Vermont & 543 & 20.5 & 2.9 & $(14.9-26.1)$ \\
\hline Billings, Montana & 808 & 21.2 & 2.0 & $(17.2-25.2)$ \\
\hline Birmingham-Hoover, Alabama & 1,571 & 25.2 & 1.5 & $(22.3-28.1)$ \\
\hline Bismarck, North Dakota & 1,037 & 20.0 & 1.9 & $(16.3-23.8)$ \\
\hline Boise City, Idaho & 1,349 & 18.2 & 1.6 & $(15.1-21.2)$ \\
\hline Boston, Massachusetts $§$ & 4,545 & 18.7 & 1.0 & $(16.7-20.6)$ \\
\hline Burlington-South Burlington, Vermont & 1,978 & 16.3 & 1.0 & $(14.3-18.3)$ \\
\hline Cambridge-Newton-Framingham, Massachusetts ${ }^{\S}$ & 5,171 & 18.4 & 0.9 & $(16.6-20.1)$ \\
\hline Camden, New Jersey ${ }^{\S}$ & 1,717 & 24.8 & 1.7 & $(21.4-28.2)$ \\
\hline Cedar Rapids, lowa & 640 & 22.6 & 2.2 & $(18.4-26.9)$ \\
\hline Charleston, West Virginia & 876 & 26.0 & 1.9 & $(22.3-29.7)$ \\
\hline Charleston-North Charleston, South Carolina & 1,402 & 20.7 & 1.4 & $(18.0-23.5)$ \\
\hline Charlotte-Concord-Gastonia, North Carolina-South Carolina & 2,156 & 20.7 & 1.1 & $(18.5-22.9)$ \\
\hline Chicago-Naperville-Elgin, Illinois-Indiana-Wisconsin & 4,119 & 22.9 & 1.0 & $(21.0-24.8)$ \\
\hline Cincinnati, Ohio-Kentucky-Indiana & 2,043 & 22.8 & 1.6 & $(19.7-25.9)$ \\
\hline Claremont-Lebanon, New Hampshire-Vermont & 1,685 & 20.9 & 1.9 & $(17.2-24.5)$ \\
\hline Cleveland-Elyria, Ohio & 962 & 23.7 & 2.2 & $(19.4-27.9)$ \\
\hline College Station-Bryan, Texas & 573 & 31.9 & 3.8 & $(24.5-39.3)$ \\
\hline Colorado Springs, Colorado & 1,299 & 19.1 & 1.5 & $(16.1-22.0)$ \\
\hline Columbia, South Carolina & 1,206 & 21.3 & 1.5 & $(18.4-24.3)$ \\
\hline Columbus, Ohio & 1,652 & 22.1 & 1.6 & $(19.1-25.2)$ \\
\hline Corpus Christi, Texas & 623 & 25.9 & 2.9 & $(20.3-31.5)$ \\
\hline Dallas-Plano-Irving, Texas $§$ & 1,297 & 26.1 & 1.9 & $(22.4-29.8)$ \\
\hline Dayton, Ohio & 564 & 22.6 & 2.5 & $(17.7-27.5)$ \\
\hline Denver-Aurora-Lakewood, Colorado & 5,797 & 15.7 & 0.6 & $(14.5-16.9)$ \\
\hline Des Moines-West Des Moines, lowa & 1,358 & 18.5 & 1.4 & $(15.7-21.2)$ \\
\hline Duluth, Minnesota-Wisconsin & 947 & 19.3 & 1.8 & $(15.8-22.7)$ \\
\hline El Paso, Texas & 722 & 30.0 & 2.4 & $(25.4-34.7)$ \\
\hline Evansville, Indiana-Kentucky & 656 & 20.9 & 2.2 & $(16.7-25.2)$ \\
\hline Fargo, North Dakota-Minnesota & 1,149 & 16.8 & 1.4 & $(14.1-19.6)$ \\
\hline Fayetteville-Springdale-Rogers, Arkansas-Missouri & 815 & 27.8 & 2.6 & $(22.8-32.8)$ \\
\hline Fort Wayne, Indiana & 863 & 24.2 & 2.2 & $(19.8-28.6)$ \\
\hline Fort Worth-Arlington, Texas $§$ & 759 & 22.3 & 2.2 & $(18.0-26.7)$ \\
\hline Grand Island, Nebraska & 1,059 & 27.1 & 2.2 & $(22.7-31.5)$ \\
\hline Grand Rapids-Wyoming, Michigan & 896 & 23.6 & 2.1 & $(19.6-27.7)$ \\
\hline Greensboro-High Point, North Carolina & 521 & 23.2 & 2.2 & $(18.8-27.6)$ \\
\hline Greenville-Anderson-Mauldin, South Carolina & 1,491 & 24.6 & 1.6 & $(21.5-27.7)$ \\
\hline Hagerstown-Martinsburg, Maryland-West Virginia & 782 & 30.1 & 3.3 & $(23.7-36.5)$ \\
\hline Hartford-West Hartford-East Hartford, Connecticut & 2,640 & 19.4 & 1.1 & $(17.2-21.5)$ \\
\hline Hilton Head Island-Bluffton-Beaufort, South Carolina & 554 & 16.7 & 2.2 & $(12.4-21.1)$ \\
\hline Houston-The Woodlands-Sugar Land, Texas & 2,159 & 26.5 & 1.5 & $(23.5-29.5)$ \\
\hline Huntington-Ashland, West Virginia-Kentucky-Ohio & 1,245 & 26.4 & 1.8 & $(22.8-30.0)$ \\
\hline Idaho Falls, Idaho & 518 & 20.9 & 2.4 & $(16.1-25.7)$ \\
\hline Indianapolis-Carmel-Anderson, Indiana & 3,598 & 25.3 & 1.1 & $(23.2-27.5)$ \\
\hline Jacksonville, Florida & 672 & 22.5 & 2.3 & $(18.0-26.9)$ \\
\hline Kansas City, Missouri-Kansas & 4,878 & 21.7 & 1.0 & $(19.6-23.7)$ \\
\hline Kingsport-Bristol-Bristol, Tennessee-Virginia & 507 & 26.3 & 2.7 & $(21.0-31.5)$ \\
\hline Knoxville, Tennessee & 564 & 23.9 & 2.6 & $(18.9-29.0)$ \\
\hline Lafayette, Louisiana & 562 & 32.5 & 2.6 & $(27.5-37.5)$ \\
\hline Lexington-Fayette, Kentucky & 623 & 27.3 & 2.4 & $(22.6-31.9)$ \\
\hline Lincoln, Nebraska & 2,008 & 18.3 & 1.1 & $(16.1-20.6)$ \\
\hline
\end{tabular}

See table footnotes on page 63. 
TABLE 26. (Continued) Age-adjusted* prevalence estimates of adults aged $\geq 18$ years who reported no leisure-time physical activity ${ }^{\dagger}$ during $^{*}$ the past 30 days, by metropolitan and micropolitan statistical area - Behavioral Risk Factor Surveillance System, United States, 2014

\begin{tabular}{|c|c|c|c|c|}
\hline MMSA & $\begin{array}{l}\text { Sample } \\
\text { size }\end{array}$ & $\%$ & SE & $95 \% \mathrm{Cl}$ \\
\hline Little Rock-North Little Rock-Conway, Arkansas & 1,179 & 27.1 & 1.8 & $(23.5-30.7)$ \\
\hline Logan, Utah-Idaho & 622 & 11.7 & 1.4 & $(9.0-14.5)$ \\
\hline Los Angeles-Long Beach-Anaheim, California & 2,037 & 20.9 & 1.1 & $(18.7-23.0)$ \\
\hline Louisville-Jefferson County, Kentucky-Indiana & 2,460 & 24.4 & 1.6 & $(21.2-27.5)$ \\
\hline Madison, Wisconsin & 551 & 17.9 & 2.1 & $(13.8-22.0)$ \\
\hline Memphis, Tennessee-Mississippi-Arkansas & 882 & 21.2 & 1.8 & $(17.7-24.7)$ \\
\hline Miami-Fort Lauderdale-West Palm Beach, Florida & 2,220 & 22.7 & 1.2 & $(20.3-25.2)$ \\
\hline Milwaukee-Waukesha-West Allis, Wisconsin & 1,355 & 21.8 & 1.8 & $(18.3-25.4)$ \\
\hline Minneapolis-St. Paul-Bloomington, Minnesota-Wisconsin & 8,756 & 18.3 & 0.5 & $(17.3-19.4)$ \\
\hline Minot, North Dakota & 594 & 23.8 & 2.7 & $(18.6-29.0)$ \\
\hline Montgomery, Alabama & 513 & 23.3 & 2.4 & $(18.6-28.0)$ \\
\hline $\begin{array}{l}\text { Montgomery County-Bucks County-Chester County, } \\
\text { Pennsylvania } \$\end{array}$ & 805 & 15.9 & 1.7 & $(12.6-19.1)$ \\
\hline $\begin{array}{l}\text { Myrtle Beach-Conway-North Myrtle Beach, South Carolina- } \\
\text { North Carolina }\end{array}$ & 999 & 26.3 & 2.1 & $(22.2-30.4)$ \\
\hline Nashville-Davidson County-Murfreesboro-Franklin, Tennessee & 803 & 21.9 & 2.0 & $(17.9-25.9)$ \\
\hline Nassau County-Suffolk County, New York ${ }^{\S}$ & 770 & 25.9 & 2.2 & $(21.5-30.3)$ \\
\hline Newark, New Jersey-Pennsylvania ${ }^{\S}$ & 4,144 & 22.6 & 1.1 & $(20.5-24.7)$ \\
\hline New Orleans-Metairie, Louisiana & 1,913 & 25.7 & 1.3 & $(23.1-28.3)$ \\
\hline New York-Jersey City-White Plains, New York-New Jersey ${ }^{\S}$ & 7,531 & 26.6 & 0.8 & $(25.1-28.2)$ \\
\hline Norfolk, Nebraska & 996 & 22.9 & 1.7 & $(19.5-26.3)$ \\
\hline North Platte, Nebraska & 967 & 22.8 & 1.7 & $(19.4-26.1)$ \\
\hline North Port-Sarasota-Bradenton, Florida & 513 & 22.9 & 3.7 & $(15.6-30.2)$ \\
\hline Oakland-Hayward-Berkeley, California§ & 638 & 21.3 & 2.2 & $(16.9-25.7)$ \\
\hline Ogden-Clearfield, Utah & 2,922 & 16.2 & 0.8 & $(14.7-17.8)$ \\
\hline Oklahoma City, Oklahoma & 2,436 & 25.1 & 1.1 & $(23.0-27.3)$ \\
\hline Omaha-Council Bluffs, Nebraska-lowa & 4,869 & 19.1 & 0.8 & $(17.5-20.7)$ \\
\hline Orlando-Kissimmee-Sanford, Florida & 956 & 23.3 & 2.0 & $(19.4-27.2)$ \\
\hline Philadelphia, Pennsylvania ${ }^{\S}$ & 1,520 & 23.9 & 1.6 & $(20.8-27.1)$ \\
\hline Phoenix-Mesa-Scottsdale, Arizona & 9,377 & 21.0 & 0.7 & $(19.6-22.4)$ \\
\hline Pittsburgh, Pennsylvania & 2,410 & 22.5 & 1.2 & $(20.2-24.8)$ \\
\hline Ponce, Puerto Rico & 530 & 37.1 & 2.8 & $(31.5-42.6)$ \\
\hline Portland-South Portland, Maine & 2,755 & 15.2 & 1.0 & $(13.2-17.2)$ \\
\hline Portland-Vancouver-Hillsboro, Oregon-Washington & 2,839 & 15.5 & 1.0 & $(13.5-17.5)$ \\
\hline Providence-Warwick, Rhode Island-Massachusetts & 8,100 & 22.4 & 0.9 & $(20.7-24.1)$ \\
\hline Provo-Orem, Utah & 2,147 & 15.1 & 0.9 & $(13.3-16.8)$ \\
\hline Raleigh, North Carolina & 720 & 16.1 & 1.6 & $(12.9-19.2)$ \\
\hline Rapid City, South Dakota & 1,420 & 20.3 & 1.8 & $(16.8-23.8)$ \\
\hline Reno, Nevada & 1,200 & 15.6 & 1.4 & $(12.9-18.3)$ \\
\hline Richmond, Virginia & 1,465 & 22.3 & 1.5 & $(19.4-25.2)$ \\
\hline Riverside-San Bernardino-Ontario, California & 840 & 21.5 & 1.7 & $(18.1-24.8)$ \\
\hline Roanoke, Virginia & 532 & 22.5 & 2.4 & $(17.7-27.2)$ \\
\hline Rochester, Minnesota & 699 & 22.6 & 2.2 & $(18.3-26.9)$ \\
\hline Rockingham County-Strafford County, New Hampshire $§$ & 1,439 & 18.7 & 1.5 & $(15.8-21.6)$ \\
\hline Sacramento-Roseville-Arden-Arcade, California & 583 & 19.9 & 2.2 & $(15.6-24.3)$ \\
\hline St. Cloud, Minnesota & 561 & 22.7 & 2.3 & $(18.1-27.3)$ \\
\hline St. Louis, Missouri-Illinois & 1,926 & 24.0 & 1.6 & $(20.9-27.1)$ \\
\hline Salisbury, Maryland-Delaware & 1,956 & 29.7 & 2.3 & $(25.1-34.2)$ \\
\hline Salt Lake City, Utah & 5,418 & 18.2 & 0.6 & $(17.0-19.5)$ \\
\hline San Antonio-New Braunfels, Texas & 2,276 & 27.8 & 1.3 & $(25.2-30.4)$ \\
\hline San Juan-Carolina-Caguas, Puerto Rico & 3,749 & 39.9 & 1.0 & $(37.9-41.9)$ \\
\hline Scottsbluff, Nebraska & 909 & 26.1 & 2.1 & $(22.0-30.2)$ \\
\hline Seattle-Bellevue-Everett, Washington ${ }^{\S}$ & 3,690 & 15.8 & 0.9 & $(14.0-17.6)$ \\
\hline Shreveport-Bossier City, Louisiana & 552 & 27.4 & 2.3 & $(22.9-32.0)$ \\
\hline Silver Spring-Frederick-Rockville, Maryland ${ }^{\S}$ & 2,378 & 17.5 & 1.6 & $(14.3-20.6)$ \\
\hline Sioux City, lowa-Nebraska-South Dakota & 1,143 & 26.6 & 2.6 & $(21.6-31.6)$ \\
\hline Sioux Falls, South Dakota & 1,348 & 20.5 & 1.8 & $(16.9-24.1)$ \\
\hline Spartanburg, South Carolina & 562 & 28.0 & 3.1 & $(21.9-34.2)$ \\
\hline Spokane-Spokane Valley, Washington & 725 & 16.6 & 2.0 & $(12.7-20.5)$ \\
\hline Springfield, Massachusetts & 1,106 & 24.1 & 2.1 & $(20.0-28.2)$ \\
\hline Tampa-St. Petersburg-Clearwater, Florida & 1,576 & 20.7 & 1.6 & $(17.6-23.7)$ \\
\hline
\end{tabular}

See table footnotes on page 63. 
TABLE 26. (Continued) Age-adjusted* prevalence estimates of adults aged $\geq 18$ years who reported no leisure-time physical activity ${ }^{\dagger}$ during the past 30 days, by metropolitan and micropolitan statistical area - Behavioral Risk Factor Surveillance System, United States, 2014

\begin{tabular}{|c|c|c|c|c|}
\hline MMSA & $\begin{array}{l}\text { Sample } \\
\text { size }\end{array}$ & $\%$ & SE & $95 \% \mathrm{Cl}$ \\
\hline Toledo, Ohio & 649 & 27.1 & 2.7 & $(21.9-32.3)$ \\
\hline Topeka, Kansas & 1,441 & 22.2 & 1.4 & $(19.4-24.9)$ \\
\hline Tulsa, Oklahoma & 2,031 & 24.0 & 1.2 & $(21.7-26.4)$ \\
\hline Tuscaloosa, Alabama & 720 & 29.7 & 2.6 & $(24.7-34.7)$ \\
\hline Virginia Beach-Norfolk-Newport News, Virginia-North Carolina & 1,879 & 23.3 & 1.4 & $(20.6-26.0)$ \\
\hline Warren-Troy-Farmington Hills, Michigan ${ }^{\S}$ & 2,116 & 21.8 & 1.2 & $(19.3-24.2)$ \\
\hline $\begin{array}{l}\text { Washington-Arlington-Alexandria, District of Columbia- } \\
\text { Virginia-Maryland-West Virginia } \$\end{array}$ & 8,280 & 19.7 & 0.8 & $(18.1-21.3)$ \\
\hline Wichita, Kansas & 2,735 & 23.2 & 1.0 & $(21.3-25.1)$ \\
\hline Wichita Falls, Texas & 545 & 26.7 & 3.8 & $(19.2-34.1)$ \\
\hline Wilmington, Delaware-Maryland-New Jersey ${ }^{\S}$ & 2,765 & 23.4 & 1.2 & $(21.0-25.9)$ \\
\hline Worcester, Massachusetts-Connecticut & 2,459 & 20.6 & 1.3 & $(18.1-23.1)$ \\
\hline Youngstown-Warren-Boardman, Ohio-Pennsylvania & 522 & 23.0 & 2.8 & $(17.6-28.5)$ \\
\hline Median & & 22.6 & & \\
\hline Range & & $11.7-42.1$ & & \\
\hline
\end{tabular}

Abbreviations: $\mathrm{Cl}$ = confidence interval; $\mathrm{MMSA}=$ metropolitan and micropolitan statistical areas; $\mathrm{SE}=$ standard error.

* Age adjusted to the 2000 U.S. standard population.

${ }^{\dagger}$ Any physical activity or exercise (e.g., running, calisthenics, golf, gardening, or walking for exercise).

$\S$ Metropolitan division. 
TABLE 27. Age-adjusted* prevalence estimates of adults aged $\geq 18$ years who reported having inadequate sleep ${ }^{\dagger}$ during a 24 -hour period, by state/territory - Behavioral Risk Factor Surveillance System, United States, 2013

\begin{tabular}{|c|c|c|c|c|}
\hline State/Territory & $\begin{array}{l}\text { Sample } \\
\text { size }\end{array}$ & $\%$ & SE & $95 \% \mathrm{Cl}$ \\
\hline Alabama & 6,319 & 37.6 & 1.0 & $(35.7-39.5)$ \\
\hline Alaska & 4,545 & 34.9 & 1.1 & $(32.8-36.9)$ \\
\hline Arizona & 4,195 & 35.2 & 1.4 & $(32.4-38.0)$ \\
\hline Arkansas & 5,166 & 36.6 & 1.1 & $(34.5-38.7)$ \\
\hline California & 11,461 & 33.5 & 0.6 & $(32.3-34.8)$ \\
\hline Colorado & 13,457 & 28.9 & 0.5 & $(27.9-30.0)$ \\
\hline Connecticut & 7,614 & 34.9 & 0.9 & $(33.2-36.5)$ \\
\hline Delaware & 5,133 & 36.9 & 1.0 & $(34.9-38.8)$ \\
\hline District of Columbia & 4,828 & 35.6 & 1.1 & $(33.4-37.7)$ \\
\hline Florida & 33,620 & 37.6 & 0.7 & $(36.3-38.9)$ \\
\hline Georgia & 7,981 & 38.2 & 0.8 & $(36.6-39.7)$ \\
\hline Hawaii & 7,816 & 41.2 & 0.8 & $(39.5-42.8)$ \\
\hline Idaho & 5,563 & 31.7 & 1.0 & $(29.8-33.6)$ \\
\hline Illinois & 5,601 & 33.9 & 1.0 & $(32.1-35.8)$ \\
\hline Indiana & 10,203 & 36.4 & 0.7 & $(35.0-37.7)$ \\
\hline lowa & 8,075 & 31.4 & 0.8 & $(29.9-32.9)$ \\
\hline Kansas & 22,995 & 29.4 & 0.4 & $(28.7-30.2)$ \\
\hline Kentucky & 10,838 & 41.6 & 0.8 & $(40.0-43.1)$ \\
\hline Louisiana & 5,106 & 37.0 & 1.2 & $(34.7-39.3)$ \\
\hline Maine & 8,003 & 32.6 & 0.8 & $(31.0-34.1)$ \\
\hline Maryland & 12,751 & 38.9 & 0.7 & (37.4-40.3) \\
\hline Massachusetts & 14,857 & 34.6 & 0.6 & (33.3-35.9) \\
\hline Michigan & 12,628 & 38.4 & 0.7 & (37.1-39.6) \\
\hline Minnesota & 14,176 & 31.9 & 0.7 & $(30.5-33.4)$ \\
\hline Mississippi & 7,180 & 36.2 & 0.9 & $(34.5-38.0)$ \\
\hline Missouri & 6,942 & 33.2 & 0.9 & $(31.4-35.0)$ \\
\hline Montana & 9,588 & 31.4 & 0.7 & $(30.0-32.8)$ \\
\hline Nebraska & 16,972 & 32.1 & 0.6 & $(30.9-33.4)$ \\
\hline Nevada & 5,035 & 35.3 & 1.3 & $(32.8-37.9)$ \\
\hline New Hampshire & 6,398 & 32.7 & 0.9 & $(30.9-34.4)$ \\
\hline New Jersey & 13,121 & 39.1 & 0.7 & $(37.8-40.4)$ \\
\hline New Mexico & 9,199 & 32.5 & 0.8 & $(31.0-34.0)$ \\
\hline New York & 8,832 & 40.1 & 0.7 & $(38.6-41.5)$ \\
\hline North Carolina & 8,644 & 35.8 & 0.7 & $(34.3-37.2)$ \\
\hline North Dakota & 7,717 & 29.6 & 0.8 & $(28.0-31.1)$ \\
\hline Ohio & 11,758 & 40.2 & 0.7 & (38.8-41.6) \\
\hline Oklahoma & 8,059 & 37.6 & 0.8 & $(36.1-39.1)$ \\
\hline Oregon & 5,886 & 31.2 & 0.9 & $(29.4-32.9)$ \\
\hline Pennsylvania & 11,291 & 37.8 & 0.7 & $(36.5-39.1)$ \\
\hline Rhode Island & 6,424 & 38.0 & 0.9 & $(36.3-39.8)$ \\
\hline South Carolina & 10,408 & 38.0 & 0.7 & $(36.5-39.4)$ \\
\hline South Dakota & 6,840 & 27.6 & 1.0 & $(25.7-29.5)$ \\
\hline Tennessee & 5,659 & 38.5 & 1.0 & $(36.5-40.4)$ \\
\hline Texas & 10,687 & 32.5 & 0.7 & (31.1-33.9) \\
\hline Utah & 12,638 & 32.6 & 0.5 & $(31.6-33.7)$ \\
\hline Vermont & 6,322 & 31.5 & 0.9 & $(29.8-33.1)$ \\
\hline Virginia & 8,321 & 37.4 & 0.8 & $(35.9-38.9)$ \\
\hline Washington & 11,025 & 32.9 & 0.6 & $(31.7-34.2)$ \\
\hline West Virginia & 5,810 & 40.5 & 0.9 & $(38.9-42.2)$ \\
\hline Wisconsin & 6,558 & 31.7 & 1.0 & $(29.8-33.5)$ \\
\hline Wyoming & 6,360 & 29.3 & 0.9 & $(27.5-31.0)$ \\
\hline Guam & 1,879 & 49.2 & 1.5 & $(46.2-52.1)$ \\
\hline Puerto Rico & 5,917 & 37.0 & 0.8 & $(35.4-38.7)$ \\
\hline Median & & 35.3 & & \\
\hline Range & & $27.6-49.2$ & & \\
\hline
\end{tabular}

Abbreviations: $\mathrm{Cl}=$ confidence interval; $\mathrm{SE}=$ standard error.

* Age adjusted to the 2000 U.S. standard population.

$\dagger$ Inadequate sleep is defined as $<7$ hours of sleep during a 24 -hour period on average. 
TABLE 28. Age-adjusted* prevalence estimates of adults aged $\geq 18$ years who reported having inadequate sleep ${ }^{\dagger}$ during a 24 -hour period, by metropolitan and micropolitan statistical area - Behavioral Risk Factor Surveillance System, United States, 2013

\begin{tabular}{|c|c|c|c|c|}
\hline MMSA & $\begin{array}{l}\text { Sample } \\
\text { size }\end{array}$ & $\%$ & SE & $95 \% \mathrm{Cl}$ \\
\hline Aguadilla-Isabela, Puerto Rico & 583 & 34.3 & 2.7 & $(29.1-39.6)$ \\
\hline Akron, Ohio & 686 & 42.9 & 3.2 & $(36.6-49.1)$ \\
\hline Albuquerque, New Mexico & 2,063 & 32.9 & 1.4 & $(30.2-35.6)$ \\
\hline Allentown-Bethlehem-Easton, Pennsylvania-New Jersey & 1,016 & 39.2 & 2.5 & $(34.4-44.1)$ \\
\hline Anchorage, Alaska & 1,511 & 36.3 & 1.6 & $(33.1-39.5)$ \\
\hline Atlanta-Sandy Springs-Roswell, Georgia & 3,458 & 37.0 & 1.1 & $(34.8-39.2)$ \\
\hline Augusta-Richmond County, Georgia-South Carolina & 897 & 44.4 & 2.7 & $(39.1-49.8)$ \\
\hline Austin-Round Rock, Texas & 931 & 33.4 & 2.1 & $(29.2-37.5)$ \\
\hline Baltimore-Columbia-Towson, Maryland & 4,685 & 39.1 & 1.1 & $(36.9-41.3)$ \\
\hline Baton Rouge, Louisiana & 914 & 34.2 & 2.4 & $(29.4-38.9)$ \\
\hline Billings, Montana & 810 & 34.2 & 1.9 & $(30.4-38.0)$ \\
\hline Birmingham-Hoover, Alabama & 1,324 & 35.3 & 1.8 & $(31.7-38.9)$ \\
\hline Bismarck, North Dakota & 1,030 & 29.9 & 1.9 & $(26.1-33.7)$ \\
\hline Boise City, Idaho & 1,476 & 31.0 & 1.7 & $(27.6-34.3)$ \\
\hline Boston, Massachusetts $§$ & 4,012 & 37.3 & 1.2 & $(34.8-39.7)$ \\
\hline Buffalo-Cheektowaga-Niagara Falls, New York & 497 & 38.6 & 3.1 & $(32.5-44.8)$ \\
\hline Burlington-South Burlington, Vermont & 1,620 & 31.6 & 1.5 & $(28.6-34.6)$ \\
\hline Cambridge-Newton-Framingham, Massachusetts ${ }^{\S}$ & 4,842 & 32.1 & 1.1 & $(29.9-34.2)$ \\
\hline Camden, New Jersey ${ }^{\S}$ & 1,831 & 40.7 & 1.6 & $(37.4-43.9)$ \\
\hline Cedar Rapids, lowa & 641 & 29.9 & 2.6 & $(24.9-35.0)$ \\
\hline Charleston, West Virginia & 806 & 43.7 & 2.3 & $(39.1-48.2)$ \\
\hline Charleston-North Charleston, South Carolina & 1,520 & 34.9 & 1.7 & $(31.5-38.3)$ \\
\hline Charlotte-Concord-Gastonia, North Carolina-South Carolina & 1,915 & 34.9 & 1.5 & $(32.0-37.9)$ \\
\hline Chattanooga, Tennessee-Georgia & 563 & 31.3 & 3.0 & $(25.5-37.2)$ \\
\hline Chicago-Naperville-Elgin, Illinois-Indiana-Wisconsin & 3,333 & 34.3 & 1.2 & $(31.9-36.7)$ \\
\hline Cincinnati, Ohio-Kentucky-Indiana & 2,564 & 38.7 & 1.5 & $(35.8-41.7)$ \\
\hline Claremont-Lebanon, New Hampshire-Vermont & 1,667 & 29.3 & 1.8 & $(25.9-32.8)$ \\
\hline Cleveland-Elyria, Ohio & 1,088 & 42.8 & 2.2 & $(38.6-47.1)$ \\
\hline Colorado Springs, Colorado & 1,363 & 32.0 & 1.7 & $(28.7-35.2)$ \\
\hline Columbia, South Carolina & 1,411 & 38.5 & 1.8 & $(34.9-42.1)$ \\
\hline Columbus, Ohio & 1,836 & 40.4 & 1.5 & $(37.5-43.3)$ \\
\hline Crestview-Fort Walton Beach-Destin, Florida & 1,074 & 36.7 & 2.3 & $(32.3-41.2)$ \\
\hline Dallas-Plano-Irving, Texas ${ }^{\S}$ & 893 & 33.0 & 2.0 & $(29.1-36.8)$ \\
\hline Davenport-Moline-Rock Island, lowa-Illinois & 669 & 33.0 & 2.8 & $(27.5-38.4)$ \\
\hline Dayton, Ohio & 833 & 36.0 & 2.5 & $(31.1-40.9)$ \\
\hline Deltona-Daytona Beach-Ormond Beach, Florida & 1,094 & 39.1 & 2.8 & (33.7-44.6) \\
\hline Denver-Aurora-Lakewood, Colorado & 5,642 & 29.9 & 0.8 & $(28.4-31.4)$ \\
\hline Des Moines-West Des Moines, lowa & 1,334 & 27.5 & 1.7 & $(24.1-30.8)$ \\
\hline Duluth, Minnesota-Wisconsin & 697 & 37.5 & 3.4 & $(30.8-44.2)$ \\
\hline Durham-Chapel Hill, North Carolina & 606 & 29.5 & 2.4 & $(24.7-34.2)$ \\
\hline El Paso, Texas & 740 & 37.1 & 2.4 & $(32.4-41.8)$ \\
\hline Evansville, Indiana-Kentucky & 570 & 40.7 & 3.1 & $(34.6-46.9)$ \\
\hline Fargo, North Dakota-Minnesota & 1,171 & 27.0 & 1.9 & $(23.4-30.6)$ \\
\hline Fayetteville-Springdale-Rogers, Arkansas-Missouri & 812 & 32.5 & 2.5 & $(27.7-37.4)$ \\
\hline Fort Smith, Arkansas-Oklahoma & 490 & 34.3 & 3.3 & $(27.9-40.8)$ \\
\hline Fort Wayne, Indiana & 776 & 32.7 & 2.3 & $(28.2-37.2)$ \\
\hline Fort Worth-Arlington, Texas $§$ & 791 & 36.9 & 2.5 & $(32.0-41.8)$ \\
\hline Gainesville, Florida & 1,011 & 37.2 & 2.7 & $(31.9-42.4)$ \\
\hline Grand Forks, North Dakota-Minnesota & 499 & 32.0 & 3.5 & $(25.2-38.9)$ \\
\hline Grand Island, Nebraska & 785 & 32.1 & 2.6 & $(27.1-37.2)$ \\
\hline Grand Rapids-Wyoming, Michigan & 1,338 & 35.7 & 1.8 & $(32.2-39.3)$ \\
\hline Greensboro-High Point, North Carolina & 645 & 37.5 & 2.6 & $(32.4-42.6)$ \\
\hline Greenville-Anderson-Mauldin, South Carolina & 1,310 & 37.7 & 1.9 & $(34.1-41.4)$ \\
\hline Gulfport-Biloxi-Pascagoula, Mississippi & 750 & 44.1 & 2.5 & $(39.2-49.0)$ \\
\hline Hagerstown-Martinsburg, Maryland-West Virginia & 753 & 39.5 & 3.0 & $(33.7-45.3)$ \\
\hline Hartford-West Hartford-East Hartford, Connecticut & 2,804 & 34.6 & 1.4 & $(31.9-37.4)$ \\
\hline Hilton Head Island-Bluffton-Beaufort, South Carolina & 807 & 36.2 & 3.1 & $(30.1-42.4)$ \\
\hline Houston-The Woodlands-Sugar Land, Texas & 1,365 & 31.5 & 1.8 & $(28.0-34.9)$ \\
\hline Huntington-Ashland, West Virginia-Kentucky-Ohio & 1,170 & 41.7 & 1.9 & $(38.0-45.4)$ \\
\hline Idaho Falls, Idaho & 502 & 31.6 & 2.9 & $(25.9-37.2)$ \\
\hline Indianapolis-Carmel-Anderson, Indiana & 2,515 & 34.8 & 1.3 & $(32.3-37.3)$ \\
\hline
\end{tabular}

See table footnotes on page 67. 
TABLE 28. (Continued) Age-adjusted* prevalence estimates of adults aged $\geq 18$ years who reported having inadequate sleep ${ }^{\dagger}$ during a $24-$ hour $^{*}$ period, by metropolitan and micropolitan statistical area - Behavioral Risk Factor Surveillance System, United States, 2013

\begin{tabular}{|c|c|c|c|c|}
\hline MMSA & $\begin{array}{l}\text { Sample } \\
\text { size }\end{array}$ & $\%$ & SE & $95 \% \mathrm{Cl}$ \\
\hline Jackson, Mississippi & 776 & 32.3 & 2.3 & $(27.8-36.9)$ \\
\hline Jacksonville, Florida & 2,845 & 38.8 & 1.5 & $(36.0-41.7)$ \\
\hline Kansas City, Missouri-Kansas & 7,324 & 30.2 & 1.1 & $(28.0-32.5)$ \\
\hline Kingsport-Bristol-Bristol, Tennessee-Virginia & 526 & 33.6 & 3.4 & $(26.9-40.2)$ \\
\hline Knoxville, Tennessee & 640 & 37.2 & 2.7 & $(31.8-42.6)$ \\
\hline Lansing-East Lansing, Michigan & 685 & 34.6 & 2.4 & $(29.9-39.2)$ \\
\hline Lexington-Fayette, Kentucky & 632 & 35.5 & 2.4 & $(30.8-40.1)$ \\
\hline Lincoln, Nebraska & 1,864 & 30.9 & 1.3 & $(28.4-33.5)$ \\
\hline Little Rock-North Little Rock-Conway, Arkansas & 1,134 & 37.8 & 2.1 & $(33.7-41.9)$ \\
\hline Logan, Utah-Idaho & 634 & 29.5 & 2.3 & $(25.0-33.9)$ \\
\hline Los Angeles-Long Beach-Anaheim, California & 3,022 & 34.9 & 1.2 & $(32.5-37.3)$ \\
\hline Louisville/Jefferson County, Kentucky-Indiana & 2,110 & 40.9 & 1.7 & $(37.6-44.3)$ \\
\hline Lubbock, Texas & 522 & 38.2 & 3.2 & $(31.9-44.6)$ \\
\hline Manhattan, Kansas & 655 & 26.8 & 2.1 & $(22.8-30.9)$ \\
\hline Memphis, Tennessee-Mississippi-Arkansas & 1,180 & 36.0 & 2.2 & $(31.7-40.2)$ \\
\hline Miami-Fort Lauderdale-West Palm Beach, Florida & 2,182 & 39.0 & 1.7 & $(35.6-42.3)$ \\
\hline Milwaukee-Waukesha-West Allis, Wisconsin & 1,262 & 32.6 & 2.2 & $(28.3-36.8)$ \\
\hline Minneapolis-St. Paul-Bloomington, Minnesota-Wisconsin & 9,036 & 30.9 & 1.0 & $(29.1-32.8)$ \\
\hline Minot, North Dakota & 647 & 32.2 & 2.4 & $(27.5-36.9)$ \\
\hline $\begin{array}{l}\text { Montgomery County-Bucks County-Chester County, } \\
\text { Pennsylvania§ }\end{array}$ & 964 & 36.4 & 2.0 & $(32.4-40.4)$ \\
\hline $\begin{array}{l}\text { Myrtle Beach-Conway-North Myrtle Beach, South Carolina- } \\
\text { North Carolina }\end{array}$ & 758 & 36.1 & 2.6 & $(31.0-41.3)$ \\
\hline Nashville-Davidson County-Murfreesboro-Franklin, Tennessee & 1,034 & 34.2 & 1.9 & $(30.4-38.0)$ \\
\hline Nassau County-Suffolk County, New York ${ }^{\S}$ & 932 & 40.1 & 2.1 & $(36.0-44.2)$ \\
\hline Newark, New Jersey-Pennsylvania ${ }^{\S}$ & 4,048 & 37.7 & 1.1 & $(35.5-40.0)$ \\
\hline New Orleans-Metairie, Louisiana & 1,250 & 38.3 & 2.4 & $(33.7-43.0)$ \\
\hline New York-Jersey City-White Plains, New York-New Jersey ${ }^{\S}$ & 8,768 & 40.9 & 0.8 & $(39.3-42.5)$ \\
\hline Norfolk, Nebraska & 664 & 33.7 & 2.2 & $(29.3-38.1)$ \\
\hline North Platte, Nebraska & 708 & 33.8 & 2.7 & $(28.6-39.1)$ \\
\hline North Port-Sarasota-Bradenton, Florida & 1,070 & 32.9 & 2.3 & $(28.5-37.4)$ \\
\hline Oakland-Hayward-Berkeley, California§ & 699 & 35.2 & 2.4 & $(30.6-39.8)$ \\
\hline Ogden-Clearfield, Utah & 2,441 & 33.6 & 1.2 & $(31.3-35.9)$ \\
\hline Oklahoma City, Oklahoma & 2,599 & 38.3 & 1.3 & $(35.8-40.8)$ \\
\hline Omaha-Council Bluffs, Nebraska-lowa & 3,108 & 34.5 & 1.2 & $(32.2-36.9)$ \\
\hline Orlando-Kissimmee-Sanford, Florida & 2,237 & 37.1 & 1.6 & $(34.0-40.2)$ \\
\hline Panama City, Florida & 1,011 & 41.3 & 2.5 & $(36.4-46.2)$ \\
\hline Pensacola-Ferry Pass-Brent, Florida & 1,294 & 39.1 & 1.8 & $(35.4-42.7)$ \\
\hline Philadelphia, Pennsylvania $§$ & 1,752 & 42.5 & 1.7 & $(39.3-45.8)$ \\
\hline Phoenix-Mesa-Scottsdale, Arizona & 1,537 & 36.5 & 1.9 & $(32.6-40.3)$ \\
\hline Pittsburgh, Pennsylvania & 2,337 & 37.9 & 1.4 & $(35.1-40.7)$ \\
\hline Ponce, Puerto Rico & 523 & 30.7 & 2.5 & $(25.8-35.5)$ \\
\hline Portland-South Portland, Maine & 2,611 & 30.1 & 1.3 & $(27.5-32.6)$ \\
\hline Portland-Vancouver-Hillsboro, Oregon-Washington & 3,202 & 28.3 & 1.2 & $(26.1-30.6)$ \\
\hline Port St. Lucie, Florida & 1,014 & 35.4 & 2.7 & $(30.1-40.7)$ \\
\hline Providence-Warwick, Rhode Island-Massachusetts & 8,181 & 37.5 & 1.0 & $(35.5-39.5)$ \\
\hline Provo-Orem, Utah & 1,841 & 31.5 & 1.3 & $(29.0-33.9)$ \\
\hline Raleigh, North Carolina & 665 & 29.5 & 2.0 & $(25.5-33.5)$ \\
\hline Rapid City, South Dakota & 865 & 30.8 & 2.2 & $(26.5-35.0)$ \\
\hline Reno, Nevada & 1,805 & 32.8 & 1.5 & $(29.8-35.8)$ \\
\hline Richmond, Virginia & 1,298 & 38.5 & 1.8 & $(35.0-42.1)$ \\
\hline Riverside-San Bernardino-Ontario, California & 1,362 & 36.4 & 1.7 & $(33.2-39.7)$ \\
\hline Rochester, New York & 504 & 34.5 & 3.0 & $(28.5-40.4)$ \\
\hline Rockingham County-Strafford County, New Hampshire $§$ & 1,645 & 32.5 & 1.7 & $(29.2-35.8)$ \\
\hline Sacramento-Roseville-Arden-Arcade, California & 888 & 30.1 & 2.1 & $(26.1-34.2)$ \\
\hline St. Louis, Missouri-Illinois & 2,024 & 34.8 & 1.6 & $(31.7-38.0)$ \\
\hline Salem, Oregon & 526 & 34.0 & 3.2 & $(27.7-40.4)$ \\
\hline Salina, Kansas & 521 & 32.0 & 2.8 & $(26.5-37.5)$ \\
\hline Salisbury, Maryland-Delaware & 2,032 & 34.9 & 2.0 & $(31.1-38.8)$ \\
\hline Salt Lake City, Utah & 4,625 & 33.6 & 0.9 & $(31.9-35.4)$ \\
\hline San Antonio-New Braunfels, Texas & 923 & 31.3 & 2.0 & $(27.3-35.3)$ \\
\hline
\end{tabular}

See table footnotes on page 67. 
TABLE 28. (Continued) Age-adjusted* prevalence estimates of adults aged $\geq 18$ years who reported having inadequate sleep ${ }^{\dagger}$ during a $24-$ hour $^{*}$ period, by metropolitan and micropolitan statistical area - Behavioral Risk Factor Surveillance System, United States, 2013

\begin{tabular}{|c|c|c|c|c|}
\hline MMSA & $\begin{array}{l}\text { Sample } \\
\text { size }\end{array}$ & $\%$ & SE & $95 \% \mathrm{Cl}$ \\
\hline San Francisco-Redwood City-South San Francisco, California§ & 535 & 32.4 & 2.9 & $(26.7-38.2)$ \\
\hline San Jose-Sunnyvale-Santa Clara, California & 624 & 28.0 & 2.5 & $(23.2-32.9)$ \\
\hline San Juan-Carolina-Caguas, Puerto Rico & 3,603 & 39.2 & 1.1 & $(37.1-41.3)$ \\
\hline Scottsbluff, Nebraska & 705 & 35.6 & 2.7 & $(30.3-41.0)$ \\
\hline Scranton-Wilkes-Barre-Hazleton, Pennsylvania & 558 & 43.7 & 3.1 & $(37.5-49.8)$ \\
\hline Seattle-Bellevue-Everett, Washington ${ }^{\S}$ & 3,739 & 33.8 & 1.0 & $(31.8-35.9)$ \\
\hline Shreveport-Bossier City, Louisiana & 560 & 27.8 & 2.7 & $(22.4-33.2)$ \\
\hline Silver Spring-Frederick-Rockville, Maryland ${ }^{\S}$ & 2,387 & 36.3 & 1.6 & $(33.2-39.3)$ \\
\hline Sioux City, lowa-Nebraska-South Dakota & 1,042 & 27.1 & 2.7 & $(21.8-32.5)$ \\
\hline Sioux Falls, South Dakota & 1,005 & 26.5 & 2.0 & $(22.5-30.5)$ \\
\hline Spartanburg, South Carolina & 581 & 38.7 & 3.1 & $(32.6-44.8)$ \\
\hline Spokane-Spokane Valley, Washington & 851 & 27.6 & 2.2 & $(23.3-32.0)$ \\
\hline Springfield, Massachusetts & 1,539 & 36.3 & 2.2 & $(32.1-40.5)$ \\
\hline Tallahassee, Florida & 1,833 & 35.7 & 2.3 & $(31.3-40.2)$ \\
\hline Tampa-St. Petersburg-Clearwater, Florida & 2,186 & 36.2 & 1.6 & $(33.2-39.3)$ \\
\hline Toledo, Ohio & 985 & 37.1 & 2.5 & $(32.2-42.0)$ \\
\hline Topeka, Kansas & 2,371 & 30.7 & 1.3 & $(28.1-33.2)$ \\
\hline Tulsa, Oklahoma & 1,955 & 36.4 & 1.6 & $(33.3-39.5)$ \\
\hline Virginia Beach-Norfolk-Newport News, Virginia-North Carolina & 1,656 & 38.0 & 1.7 & $(34.6-41.3)$ \\
\hline Warren-Troy-Farmington Hills, Michigan $§$ & 2,244 & 37.0 & 1.4 & $(34.1-39.8)$ \\
\hline $\begin{array}{l}\text { Washington-Arlington-Alexandria, District of Columbia- } \\
\text { Virginia-Maryland-West Virginia } \$\end{array}$ & 8,861 & 35.9 & 1.0 & $(33.9-37.8)$ \\
\hline Wichita, Kansas & 4,887 & 30.9 & 0.9 & $(29.1-32.6)$ \\
\hline Wilmington, Delaware-Maryland-New Jersey ${ }^{\S}$ & 3,223 & 39.2 & 1.2 & $(36.8-41.7)$ \\
\hline Winston-Salem, North Carolina & 681 & 36.5 & 2.8 & $(31.0-41.9)$ \\
\hline Worcester, Massachusetts-Connecticut & 2,729 & 35.1 & 1.5 & $(32.1-38.0)$ \\
\hline Median & & 34.9 & & \\
\hline Range & & $26.5-44.4$ & & \\
\hline
\end{tabular}

Abbreviations: $\mathrm{Cl}$ = confidence interval; MMSA = metropolitan and micropolitan statistical areas; $\mathrm{SE}=$ standard error.

* Age adjusted to the 2000 U.S. standard population.

+ Inadequate sleep is defined as $<7$ hours of sleep during a 24-hour period on average.

$\S$ Metropolitan division. 
TABLE 29. Age-adjusted* prevalence estimates of adults aged $\geq 18$ years who reported receiving inadequate ${ }^{\dagger}$ sleep during a 24-hour period, by state/territory - Behavioral Risk Factor Surveillance System, United States, 2014

\begin{tabular}{|c|c|c|c|c|}
\hline State/Territory & $\begin{array}{l}\text { Sample } \\
\text { size }\end{array}$ & $\%$ & SE & $95 \% \mathrm{Cl}$ \\
\hline Alabama & 8,405 & 38.6 & 0.8 & $(37.0-40.2)$ \\
\hline Alaska & 4,345 & 34.9 & 1.1 & $(32.8-37.0)$ \\
\hline Arizona & 14,685 & 33.0 & 0.7 & $(31.6-34.4)$ \\
\hline Arkansas & 5,144 & 37.1 & 1.2 & $(34.8-39.4)$ \\
\hline California & 8,788 & 33.5 & 0.7 & $(32.2-34.8)$ \\
\hline Colorado & 13,218 & 28.5 & 0.5 & $(27.4-29.5)$ \\
\hline Connecticut & 7,862 & 34.7 & 0.9 & $(33.0-36.4)$ \\
\hline Delaware & 4,212 & 37.7 & 1.2 & $(35.3-40.0)$ \\
\hline District of Columbia & 3,959 & 32.0 & 1.2 & $(29.6-34.4)$ \\
\hline Florida & 9,703 & 35.7 & 0.8 & $(34.2-37.2)$ \\
\hline Georgia & 6,243 & 38.6 & 0.9 & $(36.9-40.3)$ \\
\hline Hawaii & 7,220 & 43.6 & 0.9 & $(41.9-45.3)$ \\
\hline Idaho & 5,426 & 30.6 & 1.0 & $(28.7-32.5)$ \\
\hline Illinois & 5,042 & 34.3 & 0.9 & $(32.5-36.2)$ \\
\hline Indiana & 11,384 & 38.1 & 0.7 & $(36.8-39.4)$ \\
\hline lowa & 8,046 & 30.8 & 0.7 & $(29.3-32.2)$ \\
\hline Kansas & 13,547 & 30.7 & 0.5 & (29.7-31.8) \\
\hline Kentucky & 11,006 & 39.4 & 0.8 & $(37.8-41.1)$ \\
\hline Louisiana & 6,665 & 36.0 & 0.8 & $(34.4-37.5)$ \\
\hline Maine & 9,050 & 32.6 & 0.8 & $(31.1-34.2)$ \\
\hline Maryland & 12,361 & 38.7 & 0.9 & $(37.0-40.4)$ \\
\hline Massachusetts & 15,399 & 34.0 & 0.7 & $(32.7-35.3)$ \\
\hline Michigan & 8,381 & 38.2 & 0.8 & $(36.7-39.7)$ \\
\hline Minnesota & 16,236 & 29.2 & 0.5 & $(28.3-30.1)$ \\
\hline Mississippi & 4,069 & 36.7 & 1.1 & $(34.5-38.9)$ \\
\hline Missouri & 6,953 & 33.6 & 0.9 & $(31.7-35.4)$ \\
\hline Montana & 7,377 & 30.5 & 0.9 & $(28.7-32.2)$ \\
\hline Nebraska & 22,172 & 30.3 & 0.6 & $(29.2-31.4)$ \\
\hline Nevada & 3,693 & 36.2 & 1.3 & $(33.7-38.7)$ \\
\hline New Hampshire & 6,109 & 32.3 & 1.0 & $(30.4-34.2)$ \\
\hline New Jersey & 12,838 & 37.0 & 0.7 & $(35.6-38.3)$ \\
\hline New Mexico & 8,835 & 31.9 & 0.8 & $(30.3-33.5)$ \\
\hline New York & 6,751 & 38.2 & 0.8 & $(36.7-39.8)$ \\
\hline North Carolina & 7,135 & 32.4 & 0.7 & $(31.0-33.8)$ \\
\hline North Dakota & 7,712 & 31.4 & 0.9 & $(29.6-33.2)$ \\
\hline Ohio & 10,805 & 37.6 & 0.8 & $(36.0-39.2)$ \\
\hline Oklahoma & 8,291 & 35.5 & 0.7 & $(34.1-37.0)$ \\
\hline Oregon & 5,154 & 31.5 & 0.9 & $(29.6-33.3)$ \\
\hline Pennsylvania & 10,875 & 37.3 & 0.7 & $(35.9-38.8)$ \\
\hline Rhode Island & 6,340 & 36.5 & 1.0 & $(34.6-38.4)$ \\
\hline South Carolina & 10,747 & 38.2 & 0.7 & (36.8-39.6) \\
\hline South Dakota & 7,338 & 28.4 & 1.0 & $(26.4-30.4)$ \\
\hline Tennessee & 5,026 & 37.1 & 1.1 & $(34.9-39.2)$ \\
\hline Texas & 15,121 & 33.0 & 0.7 & $(31.7-34.3)$ \\
\hline Utah & 14,875 & 30.7 & 0.5 & $(29.8-31.6)$ \\
\hline Vermont & 6,423 & 30.6 & 0.8 & $(29.1-32.1)$ \\
\hline Virginia & 9,333 & 35.9 & 0.7 & $(34.5-37.3)$ \\
\hline Washington & 10,005 & 31.7 & 0.7 & $(30.3-33.0)$ \\
\hline West Virginia & 6,093 & 38.0 & 0.8 & (36.3-39.6) \\
\hline Wisconsin & 7,019 & 31.8 & 0.9 & $(30.1-33.5)$ \\
\hline Wyoming & 6,339 & 31.3 & 1.1 & $(29.1-33.5)$ \\
\hline Guam & 2,513 & 48.6 & 1.4 & $(45.8-51.4)$ \\
\hline Puerto Rico & 5,904 & 36.2 & 0.8 & $(34.6-37.7)$ \\
\hline Median & & 34.7 & & \\
\hline Range & & $28.4-48.6$ & & \\
\hline
\end{tabular}

Abbreviations: $\mathrm{Cl}=$ confidence interval; $\mathrm{SE}=$ standard error

* Age adjusted to the 2000 U.S. standard population.

+ Inadequate sleep is defined as $<7$ hours of sleep during a 24 -hour period on average. 
TABLE 30. Age-adjusted* prevalence estimates of adults aged $\geq 18$ years who reported receiving inadequate sleep ${ }^{\dagger}$ during a 24 -hour period, by metropolitan and micropolitan statistical area - Behavioral Risk Factor Surveillance System, United States, 2014

\begin{tabular}{|c|c|c|c|c|}
\hline MMSA & $\begin{array}{l}\text { Sample } \\
\text { size }\end{array}$ & $\%$ & SE & $95 \% \mathrm{Cl}$ \\
\hline Aberdeen, South Dakota & 611 & 30.9 & 3.0 & $(25.0-36.9)$ \\
\hline Aguadilla-Isabela, Puerto Rico & 534 & 33.6 & 2.5 & $(28.7-38.6)$ \\
\hline Albuquerque, New Mexico & 1,774 & 32.2 & 1.5 & $(29.2-35.2)$ \\
\hline Allentown-Bethlehem-Easton, Pennsylvania-New Jersey & 1,084 & 41.2 & 2.7 & $(36.0-46.5)$ \\
\hline Anchorage, Alaska & 1,764 & 36.3 & 1.5 & $(33.3-39.3)$ \\
\hline Atlanta-Sandy Springs-Roswell, Georgia & 2,741 & 37.8 & 1.2 & $(35.4-40.2)$ \\
\hline Augusta-Richmond County, Georgia-South Carolina & 876 & 39.3 & 3.1 & $(33.3-45.4)$ \\
\hline Austin-Round Rock, Texas & 2,228 & 27.6 & 1.3 & $(25.1-30.2)$ \\
\hline Baltimore-Columbia-Towson, Maryland & 4,547 & 38.1 & 1.3 & $(35.5-40.7)$ \\
\hline Baton Rouge, Louisiana & 905 & 36.4 & 2.0 & $(32.4-40.4)$ \\
\hline Berlin, New Hampshire-Vermont & 534 & 35.7 & 4.8 & $(26.3-45.1)$ \\
\hline Billings, Montana & 800 & 31.5 & 2.3 & $(27.0-36.0)$ \\
\hline Birmingham-Hoover, Alabama & 1,543 & 42.4 & 1.7 & $(39.0-45.8)$ \\
\hline Bismarck, North Dakota & 1,030 & 32.7 & 2.4 & $(28.0-37.3)$ \\
\hline Boise City, Idaho & 1,343 & 28.8 & 1.8 & $(25.2-32.4)$ \\
\hline Boston, Massachusetts $§$ & 4,470 & 33.7 & 1.2 & $(31.3-36.0)$ \\
\hline Burlington-South Burlington, Vermont & 1,969 & 28.4 & 1.3 & $(25.8-30.9)$ \\
\hline Cambridge-Newton-Framingham, Massachusetts ${ }^{\S}$ & 5,109 & 32.8 & 1.1 & $(30.6-34.9)$ \\
\hline Camden, New Jersey ${ }^{\S}$ & 1,698 & 35.7 & 1.8 & $(32.1-39.3)$ \\
\hline Cedar Rapids, lowa & 636 & 33.1 & 2.7 & $(27.8-38.4)$ \\
\hline Charleston, West Virginia & 861 & 37.5 & 2.2 & $(33.2-41.9)$ \\
\hline Charleston-North Charleston, South Carolina & 1,374 & 38.0 & 1.8 & $(34.4-41.5)$ \\
\hline Charlotte-Concord-Gastonia, North Carolina-South Carolina & 2,104 & 32.9 & 1.4 & $(30.2-35.6)$ \\
\hline Chicago-Naperville-Elgin, Illinois-Indiana-Wisconsin & 4,110 & 34.6 & 1.1 & $(32.4-36.8)$ \\
\hline Cincinnati, Ohio-Kentucky-Indiana & 2,019 & 38.0 & 1.9 & $(34.2-41.7)$ \\
\hline Claremont-Lebanon, New Hampshire-Vermont & 1,670 & 32.2 & 2.0 & $(28.2-36.2)$ \\
\hline Cleveland-Elyria, Ohio & 957 & 33.8 & 2.2 & $(29.4-38.2)$ \\
\hline College Station-Bryan, Texas & 560 & 36.0 & 3.9 & $(28.3-43.7)$ \\
\hline Colorado Springs, Colorado & 1,278 & 31.7 & 1.7 & $(28.3-35.1)$ \\
\hline Columbia, South Carolina & 1,183 & 40.5 & 1.9 & $(36.8-44.3)$ \\
\hline Columbus, Ohio & 1,648 & 39.7 & 1.9 & $(36.1-43.4)$ \\
\hline Corpus Christi, Texas & 604 & 39.9 & 3.9 & $(32.2-47.6)$ \\
\hline Dallas-Plano-Irving, Texas ${ }^{\S}$ & 1,275 & 30.1 & 1.9 & $(26.4-33.8)$ \\
\hline Dayton, Ohio & 561 & 37.0 & 3.0 & $(31.2-42.9)$ \\
\hline Denver-Aurora-Lakewood, Colorado & 5,733 & 28.5 & 0.8 & $(27.0-30.0)$ \\
\hline Des Moines-West Des Moines, lowa & 1,338 & 27.2 & 1.8 & $(23.6-30.8)$ \\
\hline Duluth, Minnesota-Wisconsin & 934 & 30.6 & 2.1 & $(26.4-34.8)$ \\
\hline El Paso, Texas & 712 & 33.1 & 2.5 & $(28.2-38.0)$ \\
\hline Evansville, Indiana-Kentucky & 649 & 37.9 & 3.4 & $(31.3-44.5)$ \\
\hline Fargo, North Dakota-Minnesota & 1,143 & 25.4 & 1.8 & $(22.0-28.9)$ \\
\hline Fayetteville-Springdale-Rogers, Arkansas-Missouri & 807 & 34.8 & 2.9 & $(29.2-40.4)$ \\
\hline Fort Wayne, Indiana & 854 & 34.7 & 2.5 & $(29.7-39.6)$ \\
\hline Fort Worth-Arlington, Texas $§$ & 748 & 35.7 & 2.6 & $(30.7-40.7)$ \\
\hline Grand Island, Nebraska & 1,045 & 27.4 & 2.1 & $(23.2-31.5)$ \\
\hline Grand Rapids-Wyoming, Michigan & 894 & 34.8 & 2.2 & $(30.5-39.2)$ \\
\hline Greensboro-High Point, North Carolina & 513 & 35.9 & 3.0 & $(30.0-41.7)$ \\
\hline Greenville-Anderson-Mauldin, South Carolina & 1,456 & 35.7 & 1.7 & $(32.4-39.0)$ \\
\hline Hagerstown-Martinsburg, Maryland-West Virginia & 772 & 40.7 & 3.4 & $(33.9-47.4)$ \\
\hline Hartford-West Hartford-East Hartford, Connecticut & 2,612 & 33.7 & 1.4 & $(31.0-36.5)$ \\
\hline Hilton Head Island-Bluffton-Beaufort, South Carolina & 542 & 38.7 & 3.6 & $(31.7-45.7)$ \\
\hline Houston-The Woodlands-Sugar Land, Texas & 2,129 & 33.9 & 1.6 & $(30.7-37.1)$ \\
\hline Huntington-Ashland, West Virginia-Kentucky-Ohio & 1,227 & 40.3 & 2.1 & $(36.2-44.5)$ \\
\hline Idaho Falls, Idaho & 510 & 34.6 & 2.8 & $(29.2-40.0)$ \\
\hline Indianapolis-Carmel-Anderson, Indiana & 3,559 & 37.2 & 1.2 & $(34.8-39.6)$ \\
\hline Jacksonville, Florida & 663 & 39.4 & 2.8 & $(34.0-44.9)$ \\
\hline Kansas City, Missouri-Kansas & 4,818 & 30.7 & 1.2 & $(28.3-33.0)$ \\
\hline Kingsport-Bristol-Bristol, Tennessee-Virginia & 493 & 45.3 & 3.9 & $(37.7-52.9)$ \\
\hline Knoxville, Tennessee & 557 & 36.7 & 3.3 & $(30.3-43.2)$ \\
\hline Lafayette, Louisiana & 557 & 33.6 & 2.5 & $(28.7-38.6)$ \\
\hline Lexington-Fayette, Kentucky & 615 & 37.1 & 2.6 & $(32.0-42.1)$ \\
\hline Lincoln, Nebraska & 1,999 & 32.3 & 1.4 & $(29.5-35.1)$ \\
\hline
\end{tabular}

See table footnotes on page 71. 
TABLE 30. (Continued) Age-adjusted* prevalence estimates of adults aged $\geq 18$ years who reported receiving inadequate sleep ${ }^{\dagger}$ during a 24 -hour period, by metropolitan and micropolitan statistical area - Behavioral Risk Factor Surveillance System, United States, 2014

\begin{tabular}{|c|c|c|c|c|}
\hline MMSA & $\begin{array}{l}\text { Sample } \\
\text { size }\end{array}$ & $\%$ & SE & $95 \% \mathrm{Cl}$ \\
\hline Little Rock-North Little Rock-Conway, Arkansas & 1,167 & 35.1 & 2.1 & $(31.0-39.3)$ \\
\hline Logan, Utah-Idaho & 622 & 28.6 & 2.1 & $(24.5-32.6)$ \\
\hline Los Angeles-Long Beach-Anaheim, California & 2,442 & 34.6 & 1.2 & $(32.1-37.0)$ \\
\hline Louisville-Jefferson County, Kentucky-Indiana & 2,417 & 37.0 & 1.9 & $(33.3-40.7)$ \\
\hline Madison, Wisconsin & 550 & 29.9 & 2.5 & $(25.0-34.8)$ \\
\hline Memphis, Tennessee-Mississippi-Arkansas & 861 & 30.3 & 2.6 & $(25.1-35.4)$ \\
\hline Miami-Fort Lauderdale-West Palm Beach, Florida & 2,194 & 35.7 & 1.5 & $(32.8-38.7)$ \\
\hline Milwaukee-Waukesha-West Allis, Wisconsin & 1,347 & 33.0 & 2.0 & $(29.0-36.9)$ \\
\hline Minneapolis-St. Paul-Bloomington, Minnesota-Wisconsin & 8,675 & 29.4 & 0.6 & $(28.1-30.6)$ \\
\hline Minot, North Dakota & 594 & 32.0 & 2.9 & $(26.4-37.7)$ \\
\hline Montgomery, Alabama & 498 & 35.9 & 3.0 & $(30.0-41.7)$ \\
\hline $\begin{array}{l}\text { Montgomery County-Bucks County-Chester County, } \\
\text { Pennsylvania§ }\end{array}$ & 799 & 30.7 & 2.3 & $(26.2-35.2)$ \\
\hline $\begin{array}{l}\text { Myrtle Beach-Conway-North Myrtle Beach, South Carolina- } \\
\text { North Carolina }\end{array}$ & 981 & 35.2 & 2.3 & $(30.7-39.6)$ \\
\hline Nashville-Davidson County-Murfreesboro-Franklin, Tennessee & 794 & 39.2 & 2.4 & $(34.4-44.0)$ \\
\hline Nassau County-Suffolk County, New York ${ }^{\S}$ & 762 & 40.9 & 2.4 & $(36.1-45.7)$ \\
\hline Newark, New Jersey-Pennsylvania§ & 4,087 & 35.8 & 1.2 & $(33.5-38.1)$ \\
\hline New Orleans-Metairie, Louisiana & 1,889 & 36.4 & 1.5 & $(33.5-39.3)$ \\
\hline New York-Jersey City-White Plains, New York-New Jersey ${ }^{\S}$ & 7,405 & 39.4 & 0.9 & $(37.7-41.1)$ \\
\hline Norfolk, Nebraska & 985 & 32.4 & 2.0 & $(28.5-36.2)$ \\
\hline North Platte, Nebraska & 953 & 31.3 & 2.1 & $(27.2-35.4)$ \\
\hline North Port-Sarasota-Bradenton, Florida & 510 & 43.1 & 4.2 & $(34.9-51.4)$ \\
\hline Oakland-Hayward-Berkeley, California§ & 698 & 34.1 & 2.5 & $(29.2-38.9)$ \\
\hline Ogden-Clearfield, Utah & 2,903 & 32.7 & 1.0 & $(30.8-34.7)$ \\
\hline Oklahoma City, Oklahoma & 2,406 & 35.1 & 1.2 & $(32.7-37.6)$ \\
\hline Omaha-Council Bluffs, Nebraska-lowa & 4,830 & 29.6 & 0.9 & $(27.7-31.4)$ \\
\hline Orlando-Kissimmee-Sanford, Florida & 946 & 34.1 & 2.1 & $(29.9-38.3)$ \\
\hline Philadelphia, Pennsylvania§ & 1,494 & 40.9 & 1.9 & $(37.2-44.6)$ \\
\hline Phoenix-Mesa-Scottsdale, Arizona & 9,304 & 33.0 & 0.8 & $(31.4-34.6)$ \\
\hline Pittsburgh, Pennsylvania & 2,395 & 39.9 & 1.5 & $(37.0-42.7)$ \\
\hline Ponce, Puerto Rico & 521 & 35.0 & 2.8 & $(29.5-40.5)$ \\
\hline Portland-South Portland, Maine & 2,732 & 28.7 & 1.4 & $(26.0-31.3)$ \\
\hline Portland-Vancouver-Hillsboro, Oregon-Washington & 2,810 & 31.5 & 1.3 & $(29.0-34.0)$ \\
\hline Providence-Warwick, Rhode Island-Massachusetts & 7,968 & 36.9 & 1.0 & $(34.9-39.0)$ \\
\hline Provo-Orem, Utah & 2,133 & 29.1 & 1.1 & $(26.9-31.3)$ \\
\hline Raleigh, North Carolina & 718 & 28.1 & 2.0 & $(24.2-32.0)$ \\
\hline Rapid City, South Dakota & 1,407 & 29.1 & 2.0 & $(25.2-33.0)$ \\
\hline Reno, Nevada & 1,188 & 32.4 & 2.0 & $(28.5-36.3)$ \\
\hline Richmond, Virginia & 1,446 & 33.0 & 1.7 & $(29.6-36.4)$ \\
\hline Riverside-San Bernardino-Ontario, California & 934 & 36.3 & 2.0 & $(32.5-40.2)$ \\
\hline Roanoke, Virginia & 528 & 33.5 & 3.0 & $(27.6-39.4)$ \\
\hline Rochester, Minnesota & 691 & 31.7 & 2.3 & $(27.1-36.3)$ \\
\hline Rockingham County-Strafford County, New Hampshire ${ }^{\S}$ & 1,421 & 28.7 & 1.8 & $(25.2-32.2)$ \\
\hline Sacramento-Roseville-Arden-Arcade, California & 641 & 30.2 & 2.3 & $(25.7-34.8)$ \\
\hline St. Cloud, Minnesota & 560 & 25.6 & 2.2 & $(21.2-29.9)$ \\
\hline St. Louis, Missouri-Illinois & 1,880 & 34.7 & 1.8 & $(31.2-38.3)$ \\
\hline Salisbury, Maryland-Delaware & 1,914 & 39.2 & 2.3 & $(34.6-43.7)$ \\
\hline Salt Lake City, Utah & 5,368 & 31.0 & 0.7 & $(29.6-32.5)$ \\
\hline San Antonio-New Braunfels, Texas & 2,246 & 35.5 & 1.4 & $(32.8-38.2)$ \\
\hline San Juan-Carolina-Caguas, Puerto Rico & 3,698 & 37.1 & 1.0 & $(35.1-39.2)$ \\
\hline Scottsbluff, Nebraska & 889 & 35.8 & 2.3 & $(31.2-40.4)$ \\
\hline Seattle-Bellevue-Everett, Washington ${ }^{\S}$ & 3,667 & 30.7 & 1.1 & $(28.6-32.8)$ \\
\hline Shreveport-Bossier City, Louisiana & 538 & 38.8 & 2.8 & $(33.3-44.3)$ \\
\hline Silver Spring-Frederick-Rockville, Maryland $\S^{\S}$ & 2,344 & 34.7 & 1.9 & $(31.1-38.4)$ \\
\hline Sioux City, lowa-Nebraska-South Dakota & 1,130 & 37.3 & 3.0 & $(31.4-43.1)$ \\
\hline Sioux Falls, South Dakota & 1,342 & 29.8 & 2.1 & $(25.7-33.9)$ \\
\hline Spartanburg, South Carolina & 549 & 38.1 & 3.4 & $(31.4-44.7)$ \\
\hline Spokane-Spokane Valley, Washington & 716 & 28.6 & 2.4 & $(23.9-33.3)$ \\
\hline Springfield, Massachusetts & 1,092 & 36.0 & 2.3 & $(31.6-40.5)$ \\
\hline Tampa-St. Petersburg-Clearwater, Florida & 1,570 & 40.1 & 1.9 & $(36.3-43.9)$ \\
\hline
\end{tabular}

See table footnotes on page 71. 
TABLE 30. (Continued) Age-adjusted* prevalence estimates of adults aged $\geq 18$ years who reported receiving inadequate sleep ${ }^{\dagger}$ during a 24 -hour period, by metropolitan and micropolitan statistical area — Behavioral Risk Factor Surveillance System, United States, 2014

\begin{tabular}{|c|c|c|c|c|}
\hline MMSA & $\begin{array}{l}\text { Sample } \\
\text { size }\end{array}$ & $\%$ & SE & $95 \% \mathrm{Cl}$ \\
\hline Toledo, Ohio & 643 & 37.2 & 3.1 & $(31.1-43.4)$ \\
\hline Topeka, Kansas & 1,425 & 31.9 & 1.7 & $(28.6-35.2)$ \\
\hline Tulsa, Oklahoma & 1,998 & 35.4 & 1.4 & $(32.7-38.2)$ \\
\hline Tuscaloosa, Alabama & 706 & 37.3 & 2.5 & $(32.5-42.2)$ \\
\hline Virginia Beach-Norfolk-Newport News, Virginia-North Carolina & 1,853 & 39.0 & 1.7 & $(35.7-42.2)$ \\
\hline Warren-Troy-Farmington Hills, Michigan ${ }^{\S}$ & 2,096 & 39.4 & 1.5 & $(36.4-42.4)$ \\
\hline $\begin{array}{l}\text { Washington-Arlington-Alexandria, District of Columbia- } \\
\text { Virginia-Maryland-West Virginia } \$\end{array}$ & 8,131 & 35.8 & 1.0 & $(33.8-37.7)$ \\
\hline Wichita, Kansas & 2,705 & 32.7 & 1.1 & $(30.5-34.9)$ \\
\hline Wichita Falls, Texas & 530 & 30.8 & 5.2 & $(20.7-41.0)$ \\
\hline Wilmington, Delaware-Maryland-New Jersey ${ }^{\S}$ & 2,720 & 38.1 & 1.5 & $(35.1-41.0)$ \\
\hline Worcester, Massachusetts-Connecticut & 2,426 & 37.5 & 1.6 & $(34.4-40.6)$ \\
\hline Youngstown-Warren-Boardman, Ohio-Pennsylvania & 517 & 40.0 & 3.7 & $(32.7-47.3)$ \\
\hline Median & & 34.8 & & \\
\hline Range & & $25.4-45.3$ & & \\
\hline
\end{tabular}

Abbreviations: $\mathrm{Cl}=$ confidence interval; $\mathrm{MMSA}=$ metropolitan and micropolitan statistical areas; $\mathrm{SE}=$ standard error.

${ }^{*}$ Age adjusted to the 2000 U.S. standard population.

+ Inadequate sleep is defined as $<7$ hours of sleep during a 24-hour period on average.

$\S$ Metropolitan division. 
TABLE 31. Age-adjusted* prevalence estimates of current smoking ${ }^{\dagger}$ among adults aged $\geq 18$ years, by state/territory - Behavioral Risk Factor Surveillance System, United States, 2013

\begin{tabular}{|c|c|c|c|c|}
\hline State/Territory & $\begin{array}{c}\text { Sample } \\
\text { size }\end{array}$ & $\%$ & SE & $95 \% \mathrm{Cl}$ \\
\hline Alabama & 6,383 & 21.9 & 0.9 & $(20.2-23.6)$ \\
\hline Alaska & 4,476 & 22.0 & 0.9 & $(20.3-23.8)$ \\
\hline Arizona & 4,111 & 16.4 & 1.0 & $(14.4-18.5)$ \\
\hline Arkansas & 5,101 & 26.7 & 1.0 & $(24.7-28.7)$ \\
\hline California & 10,622 & 12.5 & 0.5 & (11.6-13.4) \\
\hline Colorado & 12,774 & 17.7 & 0.5 & $(16.8-18.7)$ \\
\hline Connecticut & 7,479 & 15.9 & 0.7 & $(14.6-17.2)$ \\
\hline Delaware & 5,052 & 20.1 & 0.9 & (18.4-21.9) \\
\hline District of Columbia & 4,702 & 18.7 & 1.0 & $(16.8-20.6)$ \\
\hline Florida & 33,028 & 17.5 & 0.5 & $(16.5-18.5)$ \\
\hline Georgia & 7,725 & 18.8 & 0.7 & $(17.5-20.1)$ \\
\hline Hawaii & 7,676 & 13.9 & 0.6 & $(12.6-15.1)$ \\
\hline Idaho & 5,491 & 17.5 & 0.8 & (15.9-19.1) \\
\hline Illinois & 5,526 & 18.3 & 0.8 & $(16.7-19.8)$ \\
\hline Indiana & 10,068 & 22.4 & 0.6 & $(21.2-23.6)$ \\
\hline lowa & 7,983 & 20.1 & 0.7 & $(18.7-21.5)$ \\
\hline Kansas & 22,891 & 20.4 & 0.4 & (19.7-21.1) \\
\hline Kentucky & 10,584 & 27.2 & 0.7 & $(25.8-28.7)$ \\
\hline Louisiana & 5,162 & 23.7 & 1.1 & $(21.6-25.9)$ \\
\hline Maine & 7,962 & 21.9 & 0.8 & $(20.4-23.4)$ \\
\hline Maryland & 12,593 & 16.6 & 0.6 & $(15.4-17.7)$ \\
\hline Massachusetts & 14,274 & 16.9 & 0.6 & $(15.8-18.0)$ \\
\hline Michigan & 12,519 & 22.1 & 0.6 & $(20.9-23.2)$ \\
\hline Minnesota & 13,794 & 18.4 & 0.6 & $(17.2-19.6)$ \\
\hline Mississippi & 7,243 & 25.2 & 0.8 & $(23.5-26.8)$ \\
\hline Missouri & 6,988 & 22.6 & 0.8 & $(21.0-24.2)$ \\
\hline Montana & 9,526 & 19.7 & 0.6 & (18.5-20.9) \\
\hline Nebraska & 16,687 & 18.9 & 0.5 & (17.8-19.9) \\
\hline Nevada & 4,984 & 19.3 & 1.1 & $(17.2-21.4)$ \\
\hline New Hampshire & 6,291 & 16.9 & 0.7 & $(15.5-18.4)$ \\
\hline New Jersey & 12,590 & 15.9 & 0.5 & $(14.9-16.9)$ \\
\hline New Mexico & 9,096 & 19.4 & 0.7 & $(18.1-20.7)$ \\
\hline New York & 8,659 & 17.0 & 0.6 & $(15.8-18.2)$ \\
\hline North Carolina & 8,698 & 20.5 & 0.6 & (19.3-21.7) \\
\hline North Dakota & 7,655 & 21.7 & 0.8 & $(20.2-23.2)$ \\
\hline Ohio & 11,490 & 24.1 & 0.6 & $(22.9-25.4)$ \\
\hline Oklahoma & 8,133 & 24.2 & 0.7 & $(22.8-25.5)$ \\
\hline Oregon & 5,784 & 17.7 & 0.8 & $(16.2-19.2)$ \\
\hline Pennsylvania & 11,109 & 21.8 & 0.6 & $(20.6-23.0)$ \\
\hline Rhode Island & 6,246 & 18.1 & 0.8 & (16.6-19.6) \\
\hline South Carolina & 10,481 & 22.6 & 0.7 & (21.3-23.9) \\
\hline South Dakota & 6,769 & 20.4 & 0.8 & $(18.8-22.1)$ \\
\hline Tennessee & 5,467 & 24.6 & 0.9 & $(22.8-26.4)$ \\
\hline Texas & 10,594 & 15.8 & 0.6 & $(14.7-16.9)$ \\
\hline Utah & 12,451 & 10.1 & 0.4 & $(9.4-10.8)$ \\
\hline Vermont & 6,241 & 17.5 & 0.7 & $(16.1-19.0)$ \\
\hline Virginia & 8,091 & 19.1 & 0.6 & (17.9-20.3) \\
\hline Washington & 10,996 & 16.2 & 0.5 & $(15.2-17.3)$ \\
\hline West Virginia & 5,841 & 28.8 & 0.8 & $(27.2-30.4)$ \\
\hline Wisconsin & 6,249 & 19.2 & 0.9 & $(17.5-20.9)$ \\
\hline Wyoming & 6,315 & 21.0 & 0.9 & $(19.3-22.7)$ \\
\hline Guam & 1,835 & 25.8 & 1.4 & $(23.1-28.5)$ \\
\hline Puerto Rico & 5,966 & 11.1 & 0.6 & $(9.9-12.3)$ \\
\hline Median & & 19.3 & & \\
\hline Range & & $10.1-28.8$ & & \\
\hline
\end{tabular}

Abbreviations: $\mathrm{Cl}=$ confidence interval; $\mathrm{SE}=$ standard error.

* Age adjusted to the 2000 U.S. standard population.

+ Current smoking is defined as having smoked at least 100 cigarettes and smoking daily or some days during the period of the survey. 
TABLE 32. Age-adjusted* prevalence estimates of current smoking ${ }^{\dagger}$ among adults aged $\geq 18$ years, by metropolitan and micropolitan statistical area - Behavioral Risk Factor Surveillance System, United States, 2013

\begin{tabular}{|c|c|c|c|c|}
\hline MMSA & $\begin{array}{l}\text { Sample } \\
\text { size }\end{array}$ & $\%$ & SE & $95 \% \mathrm{Cl}$ \\
\hline Aguadilla-Isabela, Puerto Rico & 586 & 12.9 & 2.2 & $(8.7-17.2)$ \\
\hline Akron, Ohio & 663 & 20.4 & 2.5 & $(15.5-25.3)$ \\
\hline Albuquerque, New Mexico & 2,037 & 19.2 & 1.2 & $(16.8-21.5)$ \\
\hline Allentown-Bethlehem-Easton, Pennsylvania-New Jersey & 993 & 21.2 & 2.2 & $(16.9-25.5)$ \\
\hline Anchorage, Alaska & 1,486 & 18.9 & 1.3 & $(16.3-21.5)$ \\
\hline Atlanta-Sandy Springs-Roswell, Georgia & 3,331 & 16.0 & 0.9 & $(14.2-17.8)$ \\
\hline Augusta-Richmond County, Georgia-South Carolina & 888 & 19.0 & 2.2 & $(14.7-23.4)$ \\
\hline Austin-Round Rock, Texas & 919 & 13.5 & 1.5 & $(10.6-16.5)$ \\
\hline Baltimore-Columbia-Towson, Maryland & 4,610 & 18.8 & 1.0 & $(16.9-20.7)$ \\
\hline Baton Rouge, Louisiana & 911 & 22.6 & 2.3 & $(18.0-27.2)$ \\
\hline Billings, Montana & 802 & 19.0 & 1.6 & $(15.9-22.1)$ \\
\hline Birmingham-Hoover, Alabama & 1,325 & 22.1 & 1.7 & $(18.8-25.5)$ \\
\hline Bismarck, North Dakota & 1,009 & 23.2 & 2.0 & $(19.3-27.1)$ \\
\hline Boise City, Idaho & 1,451 & 18.6 & 1.5 & $(15.7-21.6)$ \\
\hline Boston, Massachusetts $§$ & 3,849 & 15.7 & 1.0 & $(13.7-17.8)$ \\
\hline Buffalo-Cheektowaga-Niagara Falls, New York & 495 & 24.9 & 3.1 & $(18.9-30.9)$ \\
\hline Burlington-South Burlington, Vermont & 1,596 & 15.5 & 1.2 & $(13.1-17.9)$ \\
\hline Cambridge-Newton-Framingham, Massachusetts $§$ & 4,632 & 14.0 & 0.9 & $(12.3-15.8)$ \\
\hline Camden, New Jersey ${ }^{\S}$ & 1,761 & 20.9 & 1.4 & $(18.1-23.6)$ \\
\hline Cedar Rapids, lowa & 634 & 18.1 & 2.2 & $(13.8-22.3)$ \\
\hline Charleston, West Virginia & 811 & 28.3 & 2.2 & $(24.0-32.6)$ \\
\hline Charleston-North Charleston, South Carolina & 1,518 & 20.4 & 1.5 & $(17.5-23.4)$ \\
\hline Charlotte-Concord-Gastonia, North Carolina-South Carolina & 1,923 & 19.6 & 1.2 & $(17.1-22.0)$ \\
\hline Chattanooga, Tennessee-Georgia & 531 & 25.4 & 3.4 & $(18.7-32.1)$ \\
\hline Chicago-Naperville-Elgin, Illinois-Indiana-Wisconsin & 3,273 & 16.7 & 1.0 & $(14.7-18.6)$ \\
\hline Cincinnati, Ohio-Kentucky-Indiana & 2,504 & 22.0 & 1.4 & $(19.3-24.6)$ \\
\hline Claremont-Lebanon, New Hampshire-Vermont & 1,648 & 15.2 & 1.4 & $(12.5-18.0)$ \\
\hline Cleveland-Elyria, Ohio & 1,053 & 23.4 & 1.9 & $(19.7-27.2)$ \\
\hline Colorado Springs, Colorado & 1,293 & 18.1 & 1.5 & $(15.2-20.9)$ \\
\hline Columbia, South Carolina & 1,423 & 21.5 & 1.6 & $(18.4-24.5)$ \\
\hline Columbus, Ohio & 1,794 & 23.1 & 1.3 & $(20.5-25.7)$ \\
\hline Crestview-Fort Walton Beach-Destin, Florida & 1,042 & 22.8 & 2.0 & $(19.0-26.7)$ \\
\hline Dallas-Plano-Irving, Texas ${ }^{\S}$ & 866 & 12.7 & 1.4 & $(10.0-15.3)$ \\
\hline Davenport-Moline-Rock Island, lowa-Illinois & 664 & 19.8 & 2.6 & $(14.6-24.9)$ \\
\hline Dayton, Ohio & 805 & 22.3 & 2.4 & $(17.6-27.0)$ \\
\hline Deltona-Daytona Beach-Ormond Beach, Florida & 1,078 & 21.3 & 2.5 & $(16.4-26.2)$ \\
\hline Denver-Aurora-Lakewood, Colorado & 5,366 & 17.3 & 0.7 & $(16.0-18.7)$ \\
\hline Des Moines-West Des Moines, lowa & 1,320 & 18.2 & 1.6 & $(15.1-21.3)$ \\
\hline Duluth, Minnesota-Wisconsin & 681 & 27.9 & 3.1 & $(21.8-33.9)$ \\
\hline Durham-Chapel Hill, North Carolina & 609 & 14.5 & 2.0 & $(10.5-18.5)$ \\
\hline El Paso, Texas & 731 & 14.0 & 1.8 & $(10.4-17.5)$ \\
\hline Evansville, Indiana-Kentucky & 564 & 23.6 & 2.7 & $(18.3-29.0)$ \\
\hline Fargo, North Dakota-Minnesota & 1,167 & 20.3 & 1.7 & $(16.9-23.7)$ \\
\hline Fayetteville-Springdale-Rogers, Arkansas-Missouri & 799 & 23.5 & 2.4 & $(18.7-28.2)$ \\
\hline Fort Smith, Arkansas-Oklahoma & 486 & 26.3 & 3.1 & $(20.1-32.4)$ \\
\hline Fort Wayne, Indiana & 763 & 18.9 & 2.0 & $(15.0-22.7)$ \\
\hline Fort Worth-Arlington, Texas ${ }^{\S}$ & 793 & 16.5 & 1.9 & $(12.7-20.3)$ \\
\hline Gainesville, Florida & 1,005 & 16.5 & 2.4 & $(11.8-21.3)$ \\
\hline Grand Forks, North Dakota-Minnesota & 493 & 14.5 & 2.4 & $(9.7-19.3)$ \\
\hline Grand Island, Nebraska & 779 & 20.5 & 2.3 & $(15.9-25.1)$ \\
\hline Grand Rapids-Wyoming, Michigan & 1,325 & 19.6 & 1.7 & $(16.3-22.8)$ \\
\hline Greensboro-High Point, North Carolina & 649 & 20.7 & 2.3 & $(16.3-25.2)$ \\
\hline Greenville-Anderson-Mauldin, South Carolina & 1,315 & 22.6 & 1.8 & $(19.1-26.0)$ \\
\hline Gulfport-Biloxi-Pascagoula, Mississippi & 742 & 26.7 & 2.3 & $(22.2-31.1)$ \\
\hline Hagerstown-Martinsburg, Maryland-West Virginia & 750 & 25.4 & 2.7 & $(20.1-30.6)$ \\
\hline Hartford-West Hartford-East Hartford, Connecticut & 2,763 & 14.8 & 1.1 & $(12.7-17.0)$ \\
\hline Hilton Head Island-Bluffton-Beaufort, South Carolina & 802 & 18.3 & 2.7 & $(13.1-23.5)$ \\
\hline Houston-The Woodlands-Sugar Land, Texas & 1,343 & 13.6 & 1.4 & $(11.0-16.3)$ \\
\hline Huntington-Ashland, West Virginia-Kentucky-Ohio & 1,145 & 30.4 & 1.9 & $(26.7-34.0)$ \\
\hline Idaho Falls, Idaho & 494 & 18.0 & 2.6 & $(12.9-23.1)$ \\
\hline Indianapolis-Carmel-Anderson, Indiana & 2,474 & 20.8 & 1.1 & $(18.6-23.1)$ \\
\hline
\end{tabular}

See table footnotes on page 75 . 
TABLE 32. (Continued) Age-adjusted* prevalence estimates of current smoking ${ }^{\dagger}$ among adults aged $\geq 18$ years, by metropolitan and micropolitan statistical area - Behavioral Risk Factor Surveillance System, United States, 2013

\begin{tabular}{|c|c|c|c|c|}
\hline MMSA & $\begin{array}{l}\text { Sample } \\
\text { size }\end{array}$ & $\%$ & SE & $95 \% \mathrm{Cl}$ \\
\hline Jackson, Mississippi & 778 & 21.5 & 2.2 & $(17.3-25.8)$ \\
\hline Jacksonville, Florida & 2,789 & 17.5 & 1.1 & $(15.3-19.8)$ \\
\hline Kansas City, Missouri-Kansas & 7,293 & 19.7 & 1.0 & $(17.6-21.7)$ \\
\hline Kingsport-Bristol-Bristol, Tennessee-Virginia & 511 & 33.6 & 3.8 & $(26.2-41.0)$ \\
\hline Knoxville, Tennessee & 622 & 22.0 & 2.2 & $(17.7-26.3)$ \\
\hline Lansing-East Lansing, Michigan & 669 & 19.3 & 2.1 & $(15.2-23.3)$ \\
\hline Lexington-Fayette, Kentucky & 619 & 21.9 & 2.1 & $(17.7-26.1)$ \\
\hline Lincoln, Nebraska & 1,828 & 18.9 & 1.2 & $(16.6-21.2)$ \\
\hline Little Rock-North Little Rock-Conway, Arkansas & 1,109 & 21.6 & 1.8 & $(18.1-25.1)$ \\
\hline Logan, Utah-Idaho & 624 & 8.7 & 1.6 & $(5.6-11.8)$ \\
\hline Los Angeles-Long Beach-Anaheim, California & 2,770 & 11.3 & 0.8 & $(9.6-12.9)$ \\
\hline Louisville-Jefferson County, Kentucky-Indiana & 2,071 & 23.5 & 1.5 & $(20.5-26.5)$ \\
\hline Lubbock, Texas & 522 & 19.4 & 2.6 & $(14.3-24.5)$ \\
\hline Manhattan, Kansas & 654 & 18.0 & 1.8 & $(14.4-21.6)$ \\
\hline Memphis, Tennessee-Mississippi-Arkansas & 1,150 & 21.6 & 1.9 & $(17.8-25.4)$ \\
\hline Miami-Fort Lauderdale-West Palm Beach, Florida & 2,127 & 12.7 & 1.2 & $(10.3-15.0)$ \\
\hline Milwaukee-Waukesha-West Allis, Wisconsin & 1,194 & 20.8 & 1.9 & $(17.0-24.5)$ \\
\hline Minneapolis-St. Paul-Bloomington, Minnesota-Wisconsin & 8,756 & 15.7 & 0.7 & $(14.3-17.1)$ \\
\hline Minot, North Dakota & 640 & 22.0 & 2.3 & $(17.5-26.5)$ \\
\hline $\begin{array}{l}\text { Montgomery County-Bucks County-Chester County, } \\
\text { Pennsylvania§ }\end{array}$ & 946 & 19.7 & 1.7 & $(16.4-23.0)$ \\
\hline $\begin{array}{l}\text { Myrtle Beach-Conway-North Myrtle Beach, South Carolina- } \\
\text { North Carolina }\end{array}$ & 760 & 24.2 & 2.4 & $(19.4-29.0)$ \\
\hline Nashville-Davidson County-Murfreesboro-Franklin, Tennessee & 1,015 & 19.4 & 1.7 & $(16.0-22.8)$ \\
\hline Nassau County-Suffolk County, New York $§$ & 906 & 17.7 & 1.9 & $(14.1-21.4)$ \\
\hline Newark, New Jersey-Pennsylvania ${ }^{\S}$ & 3,877 & 13.7 & 0.9 & $(12.0-15.4)$ \\
\hline New Orleans-Metairie, Louisiana & 1,259 & 24.0 & 2.3 & $(19.6-28.5)$ \\
\hline New York-Jersey City-White Plains, New York-New Jersey $\$$ & 8,481 & 15.0 & 0.6 & $(13.8-16.2)$ \\
\hline Norfolk, Nebraska & 649 & 19.5 & 2.0 & $(15.7-23.4)$ \\
\hline North Platte, Nebraska & 697 & 19.4 & 2.2 & $(15.0-23.8)$ \\
\hline North Port-Sarasota-Bradenton, Florida & 1,039 & 22.1 & 2.3 & $(17.7-26.6)$ \\
\hline Oakland-Hayward-Berkeley, California ${ }^{\S}$ & 652 & 11.5 & 1.5 & $(8.5-14.5)$ \\
\hline Ogden-Clearfield, Utah & 2,415 & 10.4 & 0.8 & $(8.8-11.9)$ \\
\hline Oklahoma City, Oklahoma & 2,610 & 21.1 & 1.1 & $(19.0-23.3)$ \\
\hline Omaha-Council Bluffs, Nebraska-lowa & 3,045 & 19.3 & 1.0 & $(17.2-21.3)$ \\
\hline Orlando-Kissimmee-Sanford, Florida & 2,198 & 16.0 & 1.2 & $(13.5-18.4)$ \\
\hline Panama City, Florida & 996 & 26.4 & 2.4 & $(21.6-31.2)$ \\
\hline Pensacola-Ferry Pass-Brent, Florida & 1,266 & 24.0 & 1.6 & $(20.8-27.2)$ \\
\hline Philadelphia, Pennsylvania ${ }^{\S}$ & 1,708 & 21.9 & 1.5 & $(19.0-24.7)$ \\
\hline Phoenix-Mesa-Scottsdale, Arizona & 1,501 & 15.7 & 1.4 & $(12.9-18.4)$ \\
\hline Pittsburgh, Pennsylvania & 2,301 & 21.5 & 1.3 & $(18.9-24.1)$ \\
\hline Ponce, Puerto Rico & 527 & 8.4 & 1.8 & $(5.0-11.9)$ \\
\hline Portland-South Portland, Maine & 2,595 & 19.7 & 1.2 & $(17.3-22.2)$ \\
\hline Portland-Vancouver-Hillsboro, Oregon-Washington & 3,178 & 16.2 & 0.9 & $(14.3-18.0)$ \\
\hline Port St. Lucie, Florida & 985 & 19.5 & 2.3 & $(15.0-24.0)$ \\
\hline Providence-Warwick, Rhode Island-Massachusetts & 7,926 & 18.7 & 0.8 & $(17.1-20.4)$ \\
\hline Provo-Orem, Utah & 1,811 & 6.1 & 0.7 & $(4.8-7.4)$ \\
\hline Raleigh, North Carolina & 659 & 13.8 & 1.6 & $(10.7-16.8)$ \\
\hline Rapid City, South Dakota & 855 & 24.1 & 2.1 & $(19.9-28.2)$ \\
\hline Reno, Nevada & 1,773 & 15.4 & 1.1 & $(13.1-17.6)$ \\
\hline Richmond, Virginia & 1,247 & 21.0 & 1.6 & $(17.9-24.1)$ \\
\hline Riverside-San Bernardino-Ontario, California & 1,279 & 14.8 & 1.3 & $(12.4-17.3)$ \\
\hline Rochester, New York & 494 & 16.0 & 2.4 & $(11.3-20.6)$ \\
\hline Rockingham County-Strafford County, New Hampshire ${ }^{\S}$ & 1,614 & 16.6 & 1.4 & $(13.9-19.2)$ \\
\hline Sacramento-Roseville-Arden-Arcade, California & 839 & 16.4 & 1.9 & $(12.7-20.0)$ \\
\hline St. Louis, Missouri-Illinois & 2,015 & 21.4 & 1.5 & $(18.5-24.2)$ \\
\hline Salem, Oregon & 507 & 15.8 & 2.7 & $(10.5-21.2)$ \\
\hline Salina, Kansas & 516 & 20.3 & 2.4 & $(15.5-25.0)$ \\
\hline Salisbury, Maryland-Delaware & 1,983 & 19.5 & 1.6 & $(16.4-22.6)$ \\
\hline Salt Lake City, Utah & 4,537 & 10.9 & 0.6 & $(9.8-12.1)$ \\
\hline San Antonio-New Braunfels, Texas & 914 & 15.2 & 1.7 & $(11.9-18.4)$ \\
\hline San Francisco-Redwood City-South San Francisco, California $§$ & 499 & 11.7 & 2.3 & $(7.1-16.3)$ \\
\hline
\end{tabular}

See table footnotes on page 75 . 
TABLE 32. (Continued) Age-adjusted* prevalence estimates of current smoking ${ }^{\dagger}$ among adults aged $\geq 18$ years, by metropolitan and micropolitan statistical area - Behavioral Risk Factor Surveillance System, United States, 2013

\begin{tabular}{|c|c|c|c|c|}
\hline MMSA & $\begin{array}{l}\text { Sample } \\
\text { size }\end{array}$ & $\%$ & SE & $95 \% \mathrm{Cl}$ \\
\hline San Jose-Sunnyvale-Santa Clara, California & 574 & 9.4 & 2.0 & $(5.5-13.3)$ \\
\hline San Juan-Carolina-Caguas, Puerto Rico & 3,632 & 11.7 & 0.8 & $(10.2-13.2)$ \\
\hline Scottsbluff, Nebraska & 700 & 23.8 & 2.6 & $(18.8-28.9)$ \\
\hline Scranton-Wilkes-Barre-Hazleton, Pennsylvania & 554 & 24.3 & 2.7 & $(19.1-29.6)$ \\
\hline Seattle-Bellevue-Everett, Washington ${ }^{\S}$ & 3,707 & 13.3 & 0.8 & $(11.8-14.8)$ \\
\hline Shreveport-Bossier City, Louisiana & 565 & 20.7 & 2.7 & $(15.4-26.0)$ \\
\hline Silver Spring-Frederick-Rockville, Maryland $§$ & 2,350 & 10.9 & 1.1 & $(8.7-13.0)$ \\
\hline Sioux City, lowa-Nebraska-South Dakota & 1,031 & 22.6 & 2.9 & $(16.9-28.3)$ \\
\hline Sioux Falls, South Dakota & 991 & 16.3 & 1.7 & $(13.0-19.5)$ \\
\hline Spartanburg, South Carolina & 581 & 25.2 & 2.9 & $(19.6-30.8)$ \\
\hline Spokane-Spokane Valley, Washington & 848 & 20.4 & 2.1 & $(16.2-24.6)$ \\
\hline Springfield, Massachusetts & 1,496 & 21.3 & 2.1 & $(17.2-25.5)$ \\
\hline Tallahassee, Florida & 1,800 & 14.5 & 1.6 & $(11.4-17.6)$ \\
\hline Tampa-St. Petersburg-Clearwater, Florida & 2,129 & 20.9 & 1.4 & $(18.1-23.6)$ \\
\hline Toledo, Ohio & 963 & 24.7 & 2.5 & $(19.8-29.6)$ \\
\hline Topeka, Kansas & 2,365 & 20.5 & 1.1 & $(18.3-22.8)$ \\
\hline Tulsa, Oklahoma & 1,979 & 22.3 & 1.4 & $(19.6-25.0)$ \\
\hline Virginia Beach-Norfolk-Newport News, Virginia-North Carolina & 1,604 & 21.2 & 1.6 & $(18.0-24.4)$ \\
\hline Warren-Troy-Farmington Hills, Michigan $\S$ & 2,212 & 21.2 & 1.4 & $(18.5-23.8)$ \\
\hline $\begin{array}{l}\text { Washington-Arlington-Alexandria, District of Columbia- } \\
\text { Virginia-Maryland-West Virginia§ }\end{array}$ & 8,660 & 14.1 & 0.7 & $(12.7-15.5)$ \\
\hline Wichita, Kansas & 4,857 & 22.3 & 0.8 & $(20.7-23.9)$ \\
\hline Wilmington, Delaware-Maryland-New Jersey ${ }^{\S}$ & 3,179 & 19.3 & 1.1 & $(17.2-21.4)$ \\
\hline Winston-Salem, North Carolina & 679 & 28.0 & 2.8 & $(22.6-33.4)$ \\
\hline Worcester, Massachusetts-Connecticut & 2,656 & 20.5 & 1.4 & $(17.8-23.3)$ \\
\hline Median & & 19.5 & & \\
\hline Range & & $6.1-33.6$ & & \\
\hline
\end{tabular}

Abbreviations: $\mathrm{Cl}=$ confidence interval; $\mathrm{MMSA}=$ metropolitan and micropolitan statistical areas; $\mathrm{SE}=$ standard error.

* Age adjusted to the 2000 U.S. standard population.

${ }^{\dagger}$ Current smoking is defined as having smoked at least 100 cigarettes and smoking daily or some days during the period of the survey.

$\S$ Metropolitan division. 
TABLE 33. Age-adjusted* prevalence estimates of current smoking ${ }^{\dagger}$ among adults aged $\geq 18$ years, by state/territory - Behavioral Risk Factor Surveillance System, United States, 2014

\begin{tabular}{|c|c|c|c|c|}
\hline State/Territory & $\begin{array}{l}\text { Sample } \\
\text { size }\end{array}$ & $\%$ & SE & $95 \% \mathrm{Cl}$ \\
\hline Alabama & 8,368 & 21.7 & 0.8 & $(20.2-23.2)$ \\
\hline Alaska & 4,226 & 19.5 & 0.9 & $(17.8-21.2)$ \\
\hline Arizona & 14,002 & 16.9 & 0.6 & $(15.7-18.1)$ \\
\hline Arkansas & 4,925 & 25.4 & 1.1 & $(23.3-27.6)$ \\
\hline California & 8,195 & 12.8 & 0.5 & (11.8-13.8) \\
\hline Colorado & 12,253 & 15.9 & 0.5 & $(14.9-16.8)$ \\
\hline Connecticut & 7,447 & 16.0 & 0.7 & $(14.7-17.4)$ \\
\hline Delaware & 4,148 & 20.6 & 1.1 & $(18.5-22.7)$ \\
\hline District of Columbia & 3,832 & 16.4 & 1.1 & $(14.3-18.5)$ \\
\hline Florida & 9,207 & 18.7 & 0.7 & $(17.3-20.0)$ \\
\hline Georgia & 5,952 & 17.4 & 0.7 & $(15.9-18.8)$ \\
\hline Hawaii & 6,890 & 14.6 & 0.6 & $(13.4-15.9)$ \\
\hline Idaho & 5,257 & 16.3 & 0.8 & $(14.7-17.9)$ \\
\hline Illinois & 4,844 & 16.6 & 0.8 & $(15.1-18.1)$ \\
\hline Indiana & 10,978 & 23.4 & 0.6 & $(22.2-24.6)$ \\
\hline lowa & 7,751 & 19.1 & 0.7 & $(17.8-20.4)$ \\
\hline Kansas & 13,163 & 18.4 & 0.5 & $(17.5-19.3)$ \\
\hline Kentucky & 10,767 & 26.8 & 0.8 & $(25.2-28.4)$ \\
\hline Louisiana & 6,514 & 24.3 & 0.7 & $(22.9-25.7)$ \\
\hline Maine & 8,808 & 21.2 & 0.8 & $(19.7-22.7)$ \\
\hline Maryland & 12,126 & 14.6 & 0.7 & $(13.3-15.9)$ \\
\hline Massachusetts & 14,686 & 14.9 & 0.5 & $(13.8-15.9)$ \\
\hline Michigan & 8,231 & 22.0 & 0.7 & $(20.7-23.4)$ \\
\hline Minnesota & 15,842 & 16.6 & 0.4 & $(15.8-17.4)$ \\
\hline Mississippi & 4,073 & 23.6 & 1.0 & $(21.5-25.6)$ \\
\hline Missouri & 6,832 & 21.1 & 0.8 & $(19.5-22.7)$ \\
\hline Montana & 7,299 & 21.0 & 0.8 & $(19.4-22.6)$ \\
\hline Nebraska & 21,729 & 17.8 & 0.5 & $(16.8-18.7)$ \\
\hline Nevada & 3,651 & 17.1 & 1.1 & $(15.0-19.2)$ \\
\hline New Hampshire & 5,855 & 18.6 & 0.9 & $(16.8-20.3)$ \\
\hline New Jersey & 12,194 & 15.4 & 0.5 & $(14.4-16.5)$ \\
\hline New Mexico & 8,365 & 19.7 & 0.8 & $(18.2-21.2)$ \\
\hline New York & 6,408 & 14.6 & 0.6 & $(13.4-15.8)$ \\
\hline North Carolina & 6,982 & 19.4 & 0.6 & $(18.2-20.6)$ \\
\hline North Dakota & 7,499 & 20.3 & 0.8 & $(18.7-22.0)$ \\
\hline Ohio & 10,616 & 21.7 & 0.7 & $(20.2-23.1)$ \\
\hline Oklahoma & 8,194 & 21.5 & 0.7 & $(20.2-22.8)$ \\
\hline Oregon & 4,968 & 17.5 & 0.8 & $(15.9-19.0)$ \\
\hline Pennsylvania & 10,489 & 20.7 & 0.6 & $(19.4-21.9)$ \\
\hline Rhode Island & 6,097 & 16.8 & 0.8 & $(15.2-18.5)$ \\
\hline South Carolina & 10,635 & 22.2 & 0.6 & $(21.0-23.5)$ \\
\hline South Dakota & 7,186 & 19.1 & 0.9 & (17.4-20.8) \\
\hline Tennessee & 4,851 & 24.8 & 1.0 & $(22.8-26.8)$ \\
\hline Texas & 14,536 & 14.5 & 0.5 & $(13.5-15.4)$ \\
\hline Utah & 14,459 & 9.5 & 0.3 & (8.9-10.1) \\
\hline Vermont & 6,168 & 17.8 & 0.7 & $(16.4-19.1)$ \\
\hline Virginia & 9,100 & 19.9 & 0.6 & $(18.6-21.1)$ \\
\hline Washington & 9,727 & 15.5 & 0.6 & $(14.4-16.7)$ \\
\hline West Virginia & 6,086 & 28.1 & 0.8 & $(26.5-29.7)$ \\
\hline Wisconsin & 6,727 & 17.8 & 0.8 & $(16.3-19.3)$ \\
\hline Wyoming & 6,078 & 19.9 & 1.0 & $(17.9-21.9)$ \\
\hline Guam & 2,370 & 27.8 & 1.3 & $(25.2-30.4)$ \\
\hline Puerto Rico & 5,907 & 11.6 & 0.6 & $(10.5-12.8)$ \\
\hline Median & & 18.7 & & \\
\hline Range & & $9.5-28.1$ & & \\
\hline
\end{tabular}

Abbreviations: $\mathrm{Cl}=$ confidence interval; $\mathrm{SE}=$ standard error.

* Age adjusted to the 2000 U.S. standard population.

† Current smoking is defined as having smoked at least 100 cigarettes and smoking daily or some days during the period of the survey. 
TABLE 34. Age-adjusted* prevalence estimates of current smoking ${ }^{\dagger}$ among adults aged $\geq 18$ years, by metropolitan and micropolitan statistical area - Behavioral Risk Factor Surveillance System, United States, 2014

\begin{tabular}{|c|c|c|c|c|}
\hline MMSA & $\begin{array}{c}\text { Sample } \\
\text { size }\end{array}$ & $\%$ & SE & $95 \% \mathrm{Cl}$ \\
\hline Aberdeen, South Dakota & 603 & 20.7 & 3.1 & $(14.7-26.7)$ \\
\hline Aguadilla-Isabela, Puerto Rico & 542 & 10.8 & 1.7 & $(7.4-14.2)$ \\
\hline Albuquerque, New Mexico & 1,669 & 19.8 & 1.5 & $(17.0-22.7)$ \\
\hline Allentown-Bethlehem-Easton, Pennsylvania-New Jersey & 1,040 & 21.6 & 2.4 & $(17.0-26.3)$ \\
\hline Anchorage, Alaska & 1,716 & 17.6 & 1.2 & $(15.3-19.9)$ \\
\hline Atlanta-Sandy Springs-Roswell, Georgia & 2,610 & 14.4 & 1.0 & $(12.5-16.3)$ \\
\hline Augusta-Richmond County, Georgia-South Carolina & 844 & 21.1 & 2.6 & $(16.1-26.2)$ \\
\hline Austin-Round Rock, Texas & 2,140 & 11.1 & 1.0 & $(9.2-13.1)$ \\
\hline Baltimore-Columbia-Towson, Maryland & 4,437 & 17.2 & 1.1 & $(15.0-19.4)$ \\
\hline Baton Rouge, Louisiana & 894 & 22.5 & 1.9 & $(18.7-26.2)$ \\
\hline Berlin, New Hampshire-Vermont & 521 & 20.3 & 3.7 & $(13.0-27.6)$ \\
\hline Billings, Montana & 779 & 25.5 & 2.3 & $(21.0-30.1)$ \\
\hline Birmingham-Hoover, Alabama & 1,519 & 18.4 & 1.5 & $(15.6-21.3)$ \\
\hline Bismarck, North Dakota & 1,006 & 21.4 & 2.2 & $(17.1-25.7)$ \\
\hline Boise City, Idaho & 1,290 & 14.6 & 1.5 & $(11.7-17.5)$ \\
\hline Boston, Massachusetts ${ }^{\S}$ & 4,274 & 14.5 & 1.0 & $(12.6-16.5)$ \\
\hline Burlington-South Burlington, Vermont & 1,890 & 15.3 & 1.1 & $(13.1-17.4)$ \\
\hline Cambridge-Newton-Framingham, Massachusetts $\S$ & 4,877 & 11.6 & 0.8 & $(10.1-13.1)$ \\
\hline Camden, New Jersey ${ }^{\S}$ & 1,627 & 14.1 & 1.5 & $(11.3-17.0)$ \\
\hline Cedar Rapids, lowa & 611 & 18.6 & 2.3 & $(14.0-23.1)$ \\
\hline Charleston, West Virginia & 862 & 29.3 & 2.2 & $(25.0-33.5)$ \\
\hline Charleston-North Charleston, South Carolina & 1,358 & 21.9 & 1.7 & $(18.6-25.2)$ \\
\hline Charlotte-Concord-Gastonia, North Carolina-South Carolina & 2,062 & 19.2 & 1.2 & $(16.8-21.7)$ \\
\hline Chicago-Naperville-Elgin, Illinois-Indiana-Wisconsin & 3,924 & 16.2 & 0.9 & $(14.5-17.9)$ \\
\hline Cincinnati, Ohio-Kentucky-Indiana & 1,978 & 23.9 & 1.8 & $(20.4-27.5)$ \\
\hline Claremont-Lebanon, New Hampshire-Vermont & 1,626 & 22.2 & 1.9 & $(18.4-26.0)$ \\
\hline Cleveland-Elyria, Ohio & 944 & 19.1 & 2.1 & $(15.1-23.2)$ \\
\hline College Station-Bryan, Texas & 556 & 11.2 & 2.4 & $(6.6-15.9)$ \\
\hline Colorado Springs, Colorado & 1,195 & 18.1 & 1.6 & $(15.0-21.1)$ \\
\hline Columbia, South Carolina & 1,173 & 21.0 & 1.7 & $(17.7-24.3)$ \\
\hline Columbus, Ohio & 1,603 & 19.4 & 1.6 & $(16.2-22.6)$ \\
\hline Corpus Christi, Texas & 592 & 15.5 & 2.7 & $(10.3-20.8)$ \\
\hline Dallas-Plano-Irving, Texas§ & 1,221 & 12.1 & 1.5 & $(9.1-15.0)$ \\
\hline Dayton, Ohio & 556 & 22.4 & 2.8 & $(16.9-27.8)$ \\
\hline Denver-Aurora-Lakewood, Colorado & 5,303 & 15.6 & 0.7 & $(14.3-16.9)$ \\
\hline Des Moines-West Des Moines, lowa & 1,282 & 18.6 & 1.7 & $(15.3-22.0)$ \\
\hline Duluth, Minnesota-Wisconsin & 920 & 24.9 & 2.1 & $(20.8-29.1)$ \\
\hline El Paso, Texas & 668 & 13.3 & 2.0 & $(9.3-17.3)$ \\
\hline Evansville, Indiana-Kentucky & 626 & 21.6 & 3.0 & $(15.6-27.5)$ \\
\hline Fargo, North Dakota-Minnesota & 1,106 & 15.2 & 1.6 & $(12.1-18.3)$ \\
\hline Fayetteville-Springdale-Rogers, Arkansas-Missouri & 779 & 21.8 & 2.6 & $(16.8-26.8)$ \\
\hline Fort Wayne, Indiana & 828 & 22.4 & 2.4 & $(17.8-27.1)$ \\
\hline Fort Worth-Arlington, Texas ${ }^{\S}$ & 720 & 15.4 & 2.1 & $(11.4-19.5)$ \\
\hline Grand Island, Nebraska & 1,014 & 17.0 & 2.0 & $(13.1-20.9)$ \\
\hline Grand Rapids-Wyoming, Michigan & 872 & 18.6 & 2.0 & $(14.7-22.5)$ \\
\hline Greensboro-High Point, North Carolina & 495 & 20.2 & 2.6 & $(15.2-25.3)$ \\
\hline Greenville-Anderson-Mauldin, South Carolina & 1,445 & 18.0 & 1.4 & $(15.2-20.7)$ \\
\hline Hagerstown-Martinsburg, Maryland-West Virginia & 765 & 24.6 & 3.2 & $(18.4-30.8)$ \\
\hline Hartford-West Hartford-East Hartford, Connecticut & 2,487 & 15.6 & 1.1 & $(13.4-17.9)$ \\
\hline Hilton Head Island-Bluffton-Beaufort, South Carolina & 527 & 21.4 & 4.1 & $(13.5-29.4)$ \\
\hline Houston-The Woodlands-Sugar Land, Texas & 2,003 & 11.9 & 1.1 & $(9.6-14.1)$ \\
\hline Huntington-Ashland, West Virginia-Kentucky-Ohio & 1,214 & 26.3 & 2.0 & $(22.3-30.3)$ \\
\hline Idaho Falls, Idaho & 499 & 10.4 & 1.8 & $(6.9-13.9)$ \\
\hline Indianapolis-Carmel-Anderson, Indiana & 3,443 & 20.6 & 1.1 & $(18.5-22.8)$ \\
\hline Jacksonville, Florida & 636 & 19.9 & 2.4 & $(15.2-24.7)$ \\
\hline Kansas City, Missouri-Kansas & 4,696 & 18.4 & 1.1 & $(16.2-20.6)$ \\
\hline Kingsport-Bristol-Bristol, Tennessee-Virginia & 481 & 27.4 & 3.6 & $(20.4-34.5)$ \\
\hline Knoxville, Tennessee & 536 & 24.4 & 3.3 & $(18.0-30.8)$ \\
\hline Lafayette, Louisiana & 534 & 25.1 & 2.4 & $(20.3-29.9)$ \\
\hline Lexington-Fayette, Kentucky & 602 & 28.4 & 2.4 & $(23.6-33.2)$ \\
\hline Lincoln, Nebraska & 1,964 & 18.3 & 1.2 & $(16.0-20.7)$ \\
\hline
\end{tabular}

See table footnotes on page 79. 
TABLE 34. (Continued) Age-adjusted* prevalence estimates of current smoking ${ }^{\dagger}$ among adults aged $\geq 18$ years, by metropolitan and micropolitan statistical area - Behavioral Risk Factor Surveillance System, United States, 2014

\begin{tabular}{|c|c|c|c|c|}
\hline MMSA & $\begin{array}{l}\text { Sample } \\
\text { size }\end{array}$ & $\%$ & SE & $95 \% \mathrm{Cl}$ \\
\hline Little Rock-North Little Rock-Conway, Arkansas & 1,118 & 22.2 & 2.0 & $(18.3-26.1)$ \\
\hline Logan, Utah-Idaho & 602 & 5.0 & 1.0 & $(3.0-6.9)$ \\
\hline Los Angeles-Long Beach-Anaheim, California & 2,165 & 12.8 & 0.9 & $(10.9-14.6)$ \\
\hline Louisville-Jefferson County, Kentucky-Indiana & 2,350 & 21.5 & 1.7 & $(18.1-24.9)$ \\
\hline Madison, Wisconsin & 528 & 11.5 & 2.0 & $(7.5-15.4)$ \\
\hline Memphis, Tennessee-Mississippi-Arkansas & 823 & 18.7 & 2.4 & $(14.1-23.4)$ \\
\hline Miami-Fort Lauderdale-West Palm Beach, Florida & 2,020 & 14.9 & 1.3 & $(12.4-17.4)$ \\
\hline Milwaukee-Waukesha-West Allis, Wisconsin & 1,284 & 17.5 & 1.7 & $(14.2-20.9)$ \\
\hline Minneapolis-St. Paul-Bloomington, Minnesota-Wisconsin & 8,452 & 15.1 & 0.5 & $(14.2-16.1)$ \\
\hline Minot, North Dakota & 574 & 23.9 & 2.8 & $(18.3-29.4)$ \\
\hline Montgomery, Alabama & 491 & 21.2 & 2.7 & $(15.9-26.5)$ \\
\hline $\begin{array}{l}\text { Montgomery County-Bucks County-Chester County, } \\
\text { Pennsylvania§ }\end{array}$ & 780 & 13.3 & 1.8 & $(9.9-16.8)$ \\
\hline $\begin{array}{l}\text { Myrtle Beach-Conway-North Myrtle Beach, South Carolina- } \\
\text { North Carolina }\end{array}$ & 958 & 21.3 & 2.0 & $(17.4-25.2)$ \\
\hline Nashville-Davidson County-Murfreesboro-Franklin, Tennessee & 756 & 23.4 & 2.2 & $(19.0-27.8)$ \\
\hline Nassau County-Suffolk County, New York ${ }^{\S}$ & 722 & 12.9 & 1.7 & $(9.7-16.2)$ \\
\hline Newark, New Jersey-Pennsylvania ${ }^{\S}$ & 3,867 & 16.0 & 1.0 & $(13.9-18.0)$ \\
\hline New Orleans-Metairie, Louisiana & 1,856 & 21.9 & 1.3 & $(19.3-24.4)$ \\
\hline New York-Jersey City-White Plains, New York-New Jersey ${ }^{\S}$ & 6,982 & 13.1 & 0.6 & $(11.9-14.3)$ \\
\hline Norfolk, Nebraska & 953 & 19.8 & 1.7 & $(16.5-23.1)$ \\
\hline North Platte, Nebraska & 928 & 26.8 & 2.1 & $(22.7-30.8)$ \\
\hline North Port-Sarasota-Bradenton, Florida & 488 & 23.6 & 4.1 & $(15.6-31.7)$ \\
\hline Oakland-Hayward-Berkeley, California§ & 661 & 10.1 & 1.5 & $(7.3-13.0)$ \\
\hline Ogden-Clearfield, Utah & 2,829 & 10.8 & 0.7 & $(9.4-12.2)$ \\
\hline Oklahoma City, Oklahoma & 2,366 & 18.7 & 1.1 & $(16.6-20.8)$ \\
\hline Omaha-Council Bluffs, Nebraska-lowa & 4,725 & 17.6 & 0.8 & $(15.9-19.2)$ \\
\hline Orlando-Kissimmee-Sanford, Florida & 901 & 16.4 & 1.8 & $(13.0-19.9)$ \\
\hline Philadelphia, Pennsylvania ${ }^{\S}$ & 1,427 & 22.0 & 1.7 & $(18.8-25.3)$ \\
\hline Phoenix-Mesa-Scottsdale, Arizona & 8,831 & 16.9 & 0.7 & $(15.5-18.2)$ \\
\hline Pittsburgh, Pennsylvania & 2,301 & 21.0 & 1.3 & $(18.4-23.5)$ \\
\hline Ponce, Puerto Rico & 524 & 13.6 & 2.3 & $(9.1-18.1)$ \\
\hline Portland-South Portland, Maine & 2,665 & 16.6 & 1.3 & $(14.2-19.1)$ \\
\hline Portland-Vancouver-Hillsboro, Oregon-Washington & 2,699 & 14.9 & 1.0 & $(13.0-16.9)$ \\
\hline Providence-Warwick, Rhode Island-Massachusetts & 7,648 & 17.6 & 0.9 & $(15.8-19.4)$ \\
\hline Provo-Orem, Utah & 2,077 & 5.3 & 0.6 & $(4.2-6.4)$ \\
\hline Raleigh, North Carolina & 698 & 15.8 & 1.8 & $(12.4-19.3)$ \\
\hline Rapid City, South Dakota & 1,370 & 20.6 & 1.8 & $(17.0-24.2)$ \\
\hline Reno, Nevada & 1,169 & 15.0 & 1.5 & $(12.0-18.0)$ \\
\hline Richmond, Virginia & 1,404 & 20.0 & 1.5 & $(17.0-23.0)$ \\
\hline Riverside-San Bernardino-Ontario, California & 884 & 14.2 & 1.5 & $(11.3-17.2)$ \\
\hline Roanoke, Virginia & 510 & 24.5 & 3.1 & $(18.5-30.6)$ \\
\hline Rochester, Minnesota & 684 & 15.6 & 2.0 & $(11.7-19.4)$ \\
\hline Rockingham County-Strafford County, New Hampshire $§$ & 1,367 & 18.8 & 1.7 & $(15.4-22.2)$ \\
\hline Sacramento-Roseville-Arden-Arcade, California & 605 & 13.8 & 1.7 & $(10.4-17.2)$ \\
\hline St. Cloud, Minnesota & 535 & 16.5 & 2.0 & $(12.5-20.4)$ \\
\hline St. Louis, Missouri-Illinois & 1,845 & 17.5 & 1.5 & $(14.5-20.4)$ \\
\hline Salisbury, Maryland-Delaware & 1,896 & 21.6 & 1.9 & $(17.9-25.3)$ \\
\hline Salt Lake City, Utah & 5,197 & 10.9 & 0.5 & $(9.9-12.0)$ \\
\hline San Antonio-New Braunfels, Texas & 2,169 & 13.2 & 1.0 & $(11.2-15.2)$ \\
\hline San Juan-Carolina-Caguas, Puerto Rico & 3,717 & 11.6 & 0.7 & $(10.1-13.0)$ \\
\hline Scottsbluff, Nebraska & 879 & 21.6 & 2.0 & $(17.7-25.5)$ \\
\hline Seattle-Bellevue-Everett, Washington ${ }^{\S}$ & 3,555 & 12.8 & 0.9 & $(11.1-14.5)$ \\
\hline Shreveport-Bossier City, Louisiana & 528 & 25.6 & 2.6 & $(20.5-30.7)$ \\
\hline Silver Spring-Frederick-Rockville, Maryland $\$$ & 2,314 & 8.4 & 1.1 & $(6.1-10.6)$ \\
\hline Sioux City, lowa-Nebraska-South Dakota & 1,109 & 20.5 & 2.5 & $(15.6-25.3)$ \\
\hline Sioux Falls, South Dakota & 1,314 & 17.2 & 1.8 & $(13.8-20.7)$ \\
\hline Spartanburg, South Carolina & 538 & 28.8 & 3.3 & $(22.3-35.3)$ \\
\hline Spokane-Spokane Valley, Washington & 708 & 19.8 & 2.4 & $(15.2-24.5)$ \\
\hline Springfield, Massachusetts & 1,026 & 20.3 & 2.2 & $(16.0-24.5)$ \\
\hline Tampa-St. Petersburg-Clearwater, Florida & 1,497 & 22.8 & 1.8 & $(19.2-26.4)$ \\
\hline
\end{tabular}

See table footnotes on page 79. 
TABLE 34. (Continued) Age-adjusted* prevalence estimates of current smoking ${ }^{\dagger}$ among adults aged $\geq 18$ years, by metropolitan and micropolitan statistical area - Behavioral Risk Factor Surveillance System, United States, 2014

\begin{tabular}{|c|c|c|c|c|}
\hline MMSA & $\begin{array}{l}\text { Sample } \\
\text { size }\end{array}$ & $\%$ & SE & $95 \% \mathrm{Cl}$ \\
\hline Toledo, Ohio & 635 & 18.2 & 2.6 & $(13.1-23.2)$ \\
\hline Topeka, Kansas & 1,390 & 21.9 & 1.6 & $(18.8-24.9)$ \\
\hline Tulsa, Oklahoma & 1,972 & 18.3 & 1.2 & $(16.0-20.7)$ \\
\hline Tuscaloosa, Alabama & 697 & 21.5 & 2.2 & $(17.3-25.8)$ \\
\hline Virginia Beach-Norfolk-Newport News, Virginia-North Carolina & 1,805 & 22.1 & 1.5 & $(19.1-25.1)$ \\
\hline Warren-Troy-Farmington Hills, Michigan ${ }^{\S}$ & 2,063 & 21.5 & 1.4 & $(18.7-24.3)$ \\
\hline $\begin{array}{l}\text { Washington-Arlington-Alexandria, District of Columbia- } \\
\text { Virginia-Maryland-West Virginia§ }\end{array}$ & 7,907 & 13.4 & 0.7 & $(12.0-14.8)$ \\
\hline Wichita, Kansas & 2,621 & 19.3 & 1.0 & $(17.3-21.2)$ \\
\hline Wichita Falls, Texas & 513 & 13.0 & 2.4 & $(8.3-17.7)$ \\
\hline Wilmington, Delaware-Maryland-New Jersey ${ }^{\S}$ & 2,660 & 18.7 & 1.3 & $(16.2-21.2)$ \\
\hline Worcester, Massachusetts-Connecticut & 2,293 & 18.1 & 1.4 & $(15.4-20.8)$ \\
\hline Youngstown-Warren-Boardman, Ohio-Pennsylvania & 503 & 27.5 & 3.7 & $(20.1-34.8)$ \\
\hline Median & & 18.6 & & \\
\hline Range & & $5.0-29.3$ & & \\
\hline
\end{tabular}

Abbreviations: $\mathrm{Cl}=$ confidence interval; $\mathrm{MMSA}=$ metropolitan and micropolitan statistical areas; $\mathrm{SE}=$ standard error.

${ }^{*}$ Age adjusted to the 2000 U.S. standard population.

${ }^{\dagger}$ Current smoking is defined as having smoked at least 100 cigarettes and smoking daily or some days during the period of the survey.

$\S$ Metropolitan division. 
TABLE 35. Age-adjusted* prevalence estimates of adults aged $\geq 18$ years who reported binge drinking ${ }^{\dagger}$ during the past 30 days, by state/ territory - Behavioral Risk Factor Surveillance System, United States, 2013

\begin{tabular}{|c|c|c|c|c|}
\hline State/Territory & $\begin{array}{l}\text { Sample } \\
\text { size }\end{array}$ & $\%$ & SE & $95 \% \mathrm{Cl}$ \\
\hline Alabama & 6,263 & 11.8 & 0.7 & $(10.5-13.2)$ \\
\hline Alaska & 4,390 & 19.6 & 0.9 & $(17.8-21.3)$ \\
\hline Arizona & 4,047 & 14.0 & 1.1 & $(11.8-16.1)$ \\
\hline Arkansas & 5,042 & 13.4 & 0.8 & $(11.8-15.1)$ \\
\hline California & 9,945 & 17.9 & 0.6 & $(16.8-19.0)$ \\
\hline Colorado & 12,602 & 19.0 & 0.5 & $(18.0-20.0)$ \\
\hline Connecticut & 7,354 & 19.8 & 0.8 & $(18.2-21.3)$ \\
\hline Delaware & 4,972 & 18.2 & 0.8 & $(16.6-19.9)$ \\
\hline District of Columbia & 4,591 & 21.6 & 1.0 & $(19.5-23.6)$ \\
\hline Florida & 32,367 & 17.2 & 0.6 & (16.1-18.3) \\
\hline Georgia & 7,612 & 13.3 & 0.6 & $(12.2-14.4)$ \\
\hline Hawaii & 7,584 & 19.4 & 0.7 & $(18.0-20.8)$ \\
\hline Idaho & 5,426 & 15.4 & 0.8 & $(13.8-17.0)$ \\
\hline Illinois & 5,462 & 22.6 & 0.9 & $(20.8-24.4)$ \\
\hline Indiana & 9,910 & 16.0 & 0.5 & $(14.9-17.0)$ \\
\hline lowa & 7,903 & 23.4 & 0.7 & $(22.0-24.9)$ \\
\hline Kansas & 22,508 & 16.2 & 0.3 & $(15.5-16.8)$ \\
\hline Kentucky & 10,350 & 14.4 & 0.6 & $(13.2-15.5)$ \\
\hline Louisiana & 5,032 & 16.9 & 1.0 & $(14.9-18.8)$ \\
\hline Maine & 7,849 & 19.6 & 0.7 & $(18.2-21.0)$ \\
\hline Maryland & 12,363 & 14.9 & 0.5 & $(13.8-15.9)$ \\
\hline Massachusetts & 14,040 & 20.8 & 0.6 & $(19.6-22.0)$ \\
\hline Michigan & 12,368 & 19.9 & 0.6 & $(18.8-21.0)$ \\
\hline Minnesota & 13,576 & 21.9 & 0.6 & $(20.6-23.1)$ \\
\hline Mississippi & 7,113 & 12.9 & 0.7 & $(11.6-14.2)$ \\
\hline Missouri & 6,898 & 18.3 & 0.8 & (16.7-19.9) \\
\hline Montana & 9,362 & 22.6 & 0.7 & $(21.3-23.9)$ \\
\hline Nebraska & 16,500 & 21.1 & 0.6 & $(20.0-22.2)$ \\
\hline Nevada & 4,881 & 15.7 & 1.0 & $(13.7-17.7)$ \\
\hline New Hampshire & 6,165 & 18.4 & 0.8 & $(16.8-19.9)$ \\
\hline New Jersey & 12,293 & 17.4 & 0.5 & $(16.4-18.5)$ \\
\hline New Mexico & 8,914 & 15.3 & 0.6 & $(14.1-16.5)$ \\
\hline New York & 8,471 & 18.3 & 0.6 & $(17.1-19.5)$ \\
\hline North Carolina & 8,470 & 13.8 & 0.6 & $(12.7-14.9)$ \\
\hline North Dakota & 7,504 & 25.2 & 0.8 & $(23.6-26.7)$ \\
\hline Ohio & 11,350 & 18.4 & 0.6 & (17.2-19.6) \\
\hline Oklahoma & 8,027 & 13.4 & 0.6 & $(12.2-14.5)$ \\
\hline Oregon & 5,661 & 17.6 & 0.7 & $(16.1-19.0)$ \\
\hline Pennsylvania & 10,921 & 18.8 & 0.5 & $(17.7-19.8)$ \\
\hline Rhode Island & 6,133 & 19.1 & 0.8 & $(17.5-20.7)$ \\
\hline South Carolina & 10,300 & 15.9 & 0.6 & $(14.8-17.1)$ \\
\hline South Dakota & 6,640 & 20.6 & 0.9 & $(18.9-22.4)$ \\
\hline Tennessee & 5,334 & 10.5 & 0.7 & $(9.1-11.8)$ \\
\hline Texas & 10,353 & 16.7 & 0.6 & $(15.6-17.9)$ \\
\hline Utah & 12,354 & 11.9 & 0.4 & $(11.1-12.6)$ \\
\hline Vermont & 6,166 & 19.1 & 0.8 & $(17.5-20.7)$ \\
\hline Virginia & 7,961 & 16.8 & 0.6 & $(15.5-18.0)$ \\
\hline Washington & 10,889 & 17.5 & 0.5 & (16.4-18.5) \\
\hline West Virginia & 5,765 & 12.4 & 0.6 & $(11.2-13.7)$ \\
\hline Wisconsin & 6,160 & 24.2 & 0.9 & $(22.4-26.0)$ \\
\hline Wyoming & 6,228 & 17.7 & 0.8 & $(16.0-19.3)$ \\
\hline Guam & 1,787 & 20.5 & 1.3 & $(18.0-23.0)$ \\
\hline Puerto Rico & 5,884 & 15.6 & 0.7 & $(14.2-17.0)$ \\
\hline Median & & 17.7 & & \\
\hline Range & & $10.5-25.2$ & & \\
\hline
\end{tabular}

Abbreviations: $\mathrm{Cl}=$ confidence interval; $\mathrm{SE}=$ standard error.

* Age adjusted to the 2000 U.S. standard population.

† For men, having five or more drinks on at least one occasion; for women, having four or more drinks on at least one occasion. 
TABLE 36. Age-adjusted* prevalence estimates of adults aged $\geq 18$ years who reported binge drinking ${ }^{\dagger}$ during the past 30 days, by metropolitan and micropolitan statistical area - Behavioral Risk Factor Surveillance System, United States, 2013

\begin{tabular}{|c|c|c|c|c|}
\hline MMSA & $\begin{array}{l}\text { Sample } \\
\text { size }\end{array}$ & $\%$ & SE & $95 \% \mathrm{Cl}$ \\
\hline Aguadilla-Isabela, Puerto Rico & 583 & 14.1 & 2.4 & $(9.4-18.9)$ \\
\hline Akron, Ohio & 657 & 20.4 & 2.7 & $(15.2-25.7)$ \\
\hline Albuquerque, New Mexico & 1,994 & 15.5 & 1.1 & $(13.3-17.8)$ \\
\hline Allentown-Bethlehem-Easton, Pennsylvania-New Jersey & 968 & 18.8 & 2.0 & $(14.8-22.7)$ \\
\hline Anchorage, Alaska & 1,463 & 18.0 & 1.4 & $(15.4-20.7)$ \\
\hline Atlanta-Sandy Springs-Roswell, Georgia & 3,288 & 13.8 & 0.9 & $(12.1-15.5)$ \\
\hline Augusta-Richmond County, Georgia-South Carolina & 866 & 18.9 & 2.4 & $(14.3-23.6)$ \\
\hline Austin-Round Rock, Texas & 885 & 22.4 & 2.0 & $(18.6-26.3)$ \\
\hline Baltimore-Columbia-Towson, Maryland & 4,516 & 16.3 & 0.9 & $(14.5-18.1)$ \\
\hline Baton Rouge, Louisiana & 889 & 20.7 & 2.3 & $(16.1-25.3)$ \\
\hline Billings, Montana & 786 & 21.6 & 1.7 & $(18.2-24.9)$ \\
\hline Birmingham-Hoover, Alabama & 1,303 & 14.4 & 1.5 & $(11.5-17.4)$ \\
\hline Bismarck, North Dakota & 990 & 25.1 & 1.9 & $(21.3-28.8)$ \\
\hline Boise City, Idaho & 1,434 & 16.9 & 1.5 & $(14.0-19.7)$ \\
\hline Boston, Massachusetts $§$ & 3,789 & 22.9 & 1.1 & $(20.7-25.2)$ \\
\hline Buffalo-Cheektowaga-Niagara Falls, New York & 484 & 25.3 & 2.9 & $(19.6-30.9)$ \\
\hline Burlington-South Burlington, Vermont & 1,577 & 20.0 & 1.4 & $(17.3-22.8)$ \\
\hline Cambridge-Newton-Framingham, Massachusetts $§$ & 4,544 & 20.6 & 1.0 & $(18.5-22.6)$ \\
\hline Camden, New Jersey ${ }^{\S}$ & 1,730 & 18.4 & 1.4 & $(15.8-21.1)$ \\
\hline Cedar Rapids, lowa & 629 & 21.8 & 2.4 & $(17.0-26.6)$ \\
\hline Charleston, West Virginia & 798 & 15.7 & 1.8 & $(12.1-19.3)$ \\
\hline Charleston-North Charleston, South Carolina & 1,486 & 20.2 & 1.5 & $(17.3-23.1)$ \\
\hline Charlotte-Concord-Gastonia, North Carolina-South Carolina & 1,869 & 14.9 & 1.2 & $(12.6-17.2)$ \\
\hline Chattanooga, Tennessee-Georgia & 524 & 9.6 & 2.3 & $(5.0-14.1)$ \\
\hline Chicago-Naperville-Elgin, Illinois-Indiana-Wisconsin & 3,227 & 23.5 & 1.2 & $(21.1-25.8)$ \\
\hline Cincinnati, Ohio-Kentucky-Indiana & 2,466 & 19.5 & 1.4 & $(16.9-22.2)$ \\
\hline Claremont-Lebanon, New Hampshire-Vermont & 1,620 & 15.2 & 1.8 & $(11.7-18.6)$ \\
\hline Cleveland-Elyria, Ohio & 1,033 & 17.2 & 1.8 & $(13.7-20.8)$ \\
\hline Colorado Springs, Colorado & 1,282 & 15.2 & 1.4 & $(12.5-17.8)$ \\
\hline Columbia, South Carolina & 1,403 & 18.5 & 1.6 & $(15.5-21.6)$ \\
\hline Columbus, Ohio & 1,766 & 18.4 & 1.2 & $(16.1-20.8)$ \\
\hline Crestview-Fort Walton Beach-Destin, Florida & 1,022 & 19.0 & 2.0 & $(15.1-23.0)$ \\
\hline Dallas-Plano-Irving, Texas ${ }^{\S}$ & 857 & 17.4 & 1.7 & $(14.1-20.7)$ \\
\hline Davenport-Moline-Rock Island, lowa-Illinois & 659 & 22.8 & 2.7 & $(17.6-28.0)$ \\
\hline Dayton, Ohio & 797 & 17.7 & 2.1 & $(13.5-21.9)$ \\
\hline Deltona-Daytona Beach-Ormond Beach, Florida & 1,052 & 18.2 & 2.4 & $(13.4-23.0)$ \\
\hline Denver-Aurora-Lakewood, Colorado & 5,281 & 20.2 & 0.7 & $(18.8-21.7)$ \\
\hline Des Moines-West Des Moines, lowa & 1,310 & 20.0 & 1.6 & $(16.9-23.2)$ \\
\hline Duluth, Minnesota-Wisconsin & 677 & 21.8 & 2.8 & $(16.2-27.3)$ \\
\hline Durham-Chapel Hill, North Carolina & 596 & 14.8 & 2.2 & $(10.4-19.2)$ \\
\hline El Paso, Texas & 713 & 15.1 & 2.0 & $(11.1-19.1)$ \\
\hline Evansville, Indiana-Kentucky & 557 & 16.2 & 2.6 & $(11.1-21.2)$ \\
\hline Fargo, North Dakota-Minnesota & 1,147 & 24.8 & 1.8 & $(21.2-28.3)$ \\
\hline Fayetteville-Springdale-Rogers, Arkansas-Missouri & 787 & 18.0 & 2.3 & $(13.5-22.4)$ \\
\hline Fort Smith, Arkansas-Oklahoma & 485 & 12.6 & 2.4 & $(8.0-17.3)$ \\
\hline Fort Wayne, Indiana & 748 & 15.2 & 2.0 & $(11.4-19.1)$ \\
\hline Fort Worth-Arlington, Texas ${ }^{\S}$ & 782 & 14.7 & 1.9 & $(11.1-18.4)$ \\
\hline Gainesville, Florida & 993 & 17.2 & 2.3 & $(12.6-21.8)$ \\
\hline Grand Forks, North Dakota-Minnesota & 484 & 21.0 & 3.0 & $(15.1-26.9)$ \\
\hline Grand Island, Nebraska & 773 & 20.2 & 2.4 & $(15.5-24.9)$ \\
\hline Grand Rapids-Wyoming, Michigan & 1,313 & 20.3 & 1.5 & $(17.4-23.2)$ \\
\hline Greensboro-High Point, North Carolina & 636 & 12.0 & 1.7 & $(8.6-15.4)$ \\
\hline Greenville-Anderson-Mauldin, South Carolina & 1,284 & 14.3 & 1.4 & $(11.5-17.1)$ \\
\hline Gulfport-Biloxi-Pascagoula, Mississippi & 733 & 15.5 & 1.8 & $(11.9-19.1)$ \\
\hline Hagerstown-Martinsburg, Maryland-West Virginia & 736 & 13.3 & 2.6 & $(8.3-18.3)$ \\
\hline Hartford-West Hartford-East Hartford, Connecticut & 2,714 & 19.1 & 1.3 & $(16.5-21.7)$ \\
\hline Hilton Head Island-Bluffton-Beaufort, South Carolina & 777 & 20.9 & 2.8 & $(15.4-26.4)$ \\
\hline Houston-The Woodlands-Sugar Land, Texas & 1,302 & 15.5 & 1.4 & $(12.8-18.3)$ \\
\hline Huntington-Ashland, West Virginia-Kentucky-Ohio & 1,136 & 12.8 & 1.5 & $(10.0-15.7)$ \\
\hline Idaho Falls, Idaho & 492 & 8.5 & 1.7 & $(5.1-11.8)$ \\
\hline Indianapolis-Carmel-Anderson, Indiana & 2,430 & 15.0 & 1.0 & $(13.0-16.9)$ \\
\hline Jackson, Mississippi & 759 & 11.9 & 1.7 & $(8.5-15.3)$ \\
\hline
\end{tabular}

See table footnotes on page 83 . 
TABLE 36. (Continued) Age-adjusted* prevalence estimates of adults aged $\geq 18$ years who reported binge drinking ${ }^{\dagger}$ during the past 30 days, by metropolitan and micropolitan statistical area - Behavioral Risk Factor Surveillance System, United States, 2013

\begin{tabular}{|c|c|c|c|c|}
\hline MMSA & $\begin{array}{l}\text { Sample } \\
\text { size }\end{array}$ & $\%$ & SE & $95 \% \mathrm{Cl}$ \\
\hline Jacksonville, Florida & 2,737 & 16.6 & 1.2 & $(14.3-19.0)$ \\
\hline Kansas City, Missouri-Kansas & 7,178 & 17.8 & 1.0 & $(15.9-19.8)$ \\
\hline Kingsport-Bristol-Bristol, Tennessee-Virginia & 507 & 12.4 & 2.9 & $(6.8-18.1)$ \\
\hline Knoxville, Tennessee & 603 & 9.3 & 1.6 & $(6.1-12.4)$ \\
\hline Lansing-East Lansing, Michigan & 668 & 24.6 & 2.3 & $(20.1-29.1)$ \\
\hline Lexington-Fayette, Kentucky & 608 & 16.3 & 1.8 & $(12.8-19.8)$ \\
\hline Lincoln, Nebraska & 1,807 & 23.0 & 1.2 & $(20.5-25.4)$ \\
\hline Little Rock-North Little Rock-Conway, Arkansas & 1,099 & 14.0 & 1.6 & $(10.8-17.2)$ \\
\hline Logan, Utah-Idaho & 622 & 9.0 & 1.4 & $(6.3-11.8)$ \\
\hline Los Angeles-Long Beach-Anaheim, California & 2,573 & 17.2 & 1.0 & $(15.1-19.2)$ \\
\hline Louisville-Jefferson County, Kentucky-Indiana & 2,029 & 18.1 & 1.4 & $(15.3-20.8)$ \\
\hline Lubbock, Texas & 514 & 18.5 & 2.8 & $(13.0-24.1)$ \\
\hline Manhattan, Kansas & 642 & 22.1 & 2.0 & $(18.1-26.0)$ \\
\hline Memphis, Tennessee-Mississippi-Arkansas & 1,125 & 11.8 & 1.5 & $(8.8-14.7)$ \\
\hline Miami-Fort Lauderdale-West Palm Beach, Florida & 2,077 & 18.0 & 1.5 & $(15.2-20.9)$ \\
\hline Milwaukee-Waukesha-West Allis, Wisconsin & 1,170 & 21.6 & 2.0 & $(17.6-25.6)$ \\
\hline Minneapolis-St. Paul-Bloomington, Minnesota-Wisconsin & 8,611 & 22.6 & 0.9 & $(20.9-24.4)$ \\
\hline Minot, North Dakota & 626 & 24.7 & 2.3 & $(20.2-29.1)$ \\
\hline $\begin{array}{l}\text { Montgomery County-Bucks County-Chester County, } \\
\text { Pennsylvania§ }\end{array}$ & 930 & 22.6 & 1.8 & $(19.1-26.2)$ \\
\hline $\begin{array}{l}\text { Myrtle Beach-Conway-North Myrtle Beach, South Carolina- } \\
\text { North Carolina }\end{array}$ & 746 & 16.7 & 2.2 & $(12.4-21.0)$ \\
\hline Nashville-Davidson County-Murfreesboro-Franklin, Tennessee & 985 & 13.3 & 1.4 & $(10.5-16.0)$ \\
\hline Nassau County-Suffolk County, New York ${ }^{\S}$ & 879 & 18.7 & 1.9 & $(14.9-22.5)$ \\
\hline Newark, New Jersey-Pennsylvania ${ }^{\S}$ & 3,768 & 16.8 & 0.9 & $(15.0-18.7)$ \\
\hline New Orleans-Metairie, Louisiana & 1,230 & 19.6 & 2.2 & $(15.3-23.9)$ \\
\hline New York-Jersey City-White Plains, New York-New Jersey $§$ & 8,295 & 17.5 & 0.6 & $(16.3-18.8)$ \\
\hline Norfolk, Nebraska & 641 & 19.3 & 2.0 & $(15.4-23.1)$ \\
\hline North Platte, Nebraska & 689 & 18.9 & 2.3 & $(14.4-23.4)$ \\
\hline North Port-Sarasota-Bradenton, Florida & 1,015 & 20.3 & 2.2 & $(16.0-24.5)$ \\
\hline Oakland-Hayward-Berkeley, California ${ }^{\S}$ & 620 & 16.2 & 2.1 & $(12.2-20.3)$ \\
\hline Ogden-Clearfield, Utah & 2,397 & 12.0 & 0.9 & $(10.3-13.7)$ \\
\hline Oklahoma City, Oklahoma & 2,581 & 14.7 & 1.1 & $(12.6-16.8)$ \\
\hline Omaha-Council Bluffs, Nebraska-lowa & 3,016 & 20.9 & 1.0 & $(18.9-23.0)$ \\
\hline Orlando-Kissimmee-Sanford, Florida & 2,154 & 15.4 & 1.3 & $(12.9-17.8)$ \\
\hline Panama City, Florida & 978 & 19.2 & 2.1 & $(15.0-23.3)$ \\
\hline Pensacola-Ferry Pass-Brent, Florida & 1,245 & 14.7 & 1.4 & $(11.9-17.4)$ \\
\hline Philadelphia, Pennsylvania ${ }^{\S}$ & 1,678 & 19.2 & 1.4 & $(16.5-21.9)$ \\
\hline Phoenix-Mesa-Scottsdale, Arizona & 1,474 & 14.4 & 1.5 & $(11.4-17.4)$ \\
\hline Pittsburgh, Pennsylvania & 2,248 & 21.5 & 1.3 & $(19.0-24.1)$ \\
\hline Ponce, Puerto Rico & 521 & 14.2 & 2.2 & $(9.9-18.5)$ \\
\hline Portland-South Portland, Maine & 2,549 & 21.6 & 1.3 & $(19.1-24.1)$ \\
\hline Portland-Vancouver-Hillsboro, Oregon-Washington & 3,118 & 16.9 & 0.9 & $(15.1-18.7)$ \\
\hline Port St. Lucie, Florida & 956 & 15.9 & 2.3 & $(11.3-20.4)$ \\
\hline Providence-Warwick, Rhode Island-Massachusetts & 7,795 & 19.5 & 1.0 & $(17.6-21.4)$ \\
\hline Provo-Orem, Utah & 1,806 & 7.2 & 0.7 & $(5.7-8.6)$ \\
\hline Raleigh, North Carolina & 640 & 16.6 & 1.6 & $(13.4-19.8)$ \\
\hline Rapid City, South Dakota & 841 & 20.0 & 2.0 & $(16.1-23.8)$ \\
\hline Reno, Nevada & 1,756 & 19.9 & 1.3 & $(17.3-22.4)$ \\
\hline Richmond, Virginia & 1,224 & 20.6 & 1.6 & $(17.4-23.8)$ \\
\hline Riverside-San Bernardino-Ontario, California & 1,194 & 16.4 & 1.4 & $(13.7-19.2)$ \\
\hline Rochester, New York & 492 & 15.1 & 2.2 & $(10.7-19.5)$ \\
\hline Rockingham County-Strafford County, New Hampshire ${ }^{\S}$ & 1,585 & 20.0 & 1.5 & $(17.0-23.0)$ \\
\hline Sacramento-Roseville-Arden-Arcade, California & 794 & 19.5 & 2.0 & $(15.6-23.4)$ \\
\hline St. Louis, Missouri-Illinois & 1,991 & 22.1 & 1.6 & $(19.0-25.1)$ \\
\hline Salem, Oregon & 497 & 15.3 & 2.7 & $(10.1-20.5)$ \\
\hline Salina, Kansas & 510 & 13.8 & 2.2 & $(9.6-18.1)$ \\
\hline Salisbury, Maryland-Delaware & 1,956 & 15.4 & 1.5 & $(12.5-18.4)$ \\
\hline Salt Lake City, Utah & 4,480 & 14.2 & 0.7 & $(12.8-15.5)$ \\
\hline San Antonio-New Braunfels, Texas & 902 & 19.7 & 2.0 & $(15.9-23.6)$ \\
\hline San Francisco-Redwood City-South San Francisco, California $\$$ & 461 & 23.9 & 2.8 & $(18.5-29.4)$ \\
\hline San Jose-Sunnyvale-Santa Clara, California & 533 & 15.9 & 2.0 & $(12.0-19.8)$ \\
\hline
\end{tabular}

See table footnotes on page 83. 
TABLE 36. (Continued) Age-adjusted* prevalence estimates of adults aged $\geq 18$ years who reported binge drinking ${ }^{\dagger}$ during the past 30 days, by metropolitan and micropolitan statistical area - Behavioral Risk Factor Surveillance System, United States, 2013

\begin{tabular}{|c|c|c|c|c|}
\hline MMSA & $\begin{array}{l}\text { Sample } \\
\text { size }\end{array}$ & $\%$ & SE & $95 \% \mathrm{Cl}$ \\
\hline San Juan-Carolina-Caguas, Puerto Rico & 3,568 & 16.4 & 0.9 & $(14.6-18.1)$ \\
\hline Scottsbluff, Nebraska & 691 & 15.7 & 2.3 & $(11.3-20.2)$ \\
\hline Scranton-Wilkes-Barre-Hazleton, Pennsylvania & 543 & 23.8 & 2.9 & $(18.1-29.4)$ \\
\hline Seattle-Bellevue-Everett, Washington ${ }^{\S}$ & 3,667 & 18.3 & 0.9 & $(16.6-20.0)$ \\
\hline Shreveport-Bossier City, Louisiana & 551 & 17.8 & 3.1 & $(11.6-23.9)$ \\
\hline Silver Spring-Frederick-Rockville, Maryland ${ }^{\S}$ & 2,314 & 14.8 & 1.1 & $(12.6-16.9)$ \\
\hline Sioux City, lowa-Nebraska-South Dakota & 1,013 & 21.9 & 2.8 & $(16.5-27.3)$ \\
\hline Sioux Falls, South Dakota & 966 & 22.2 & 1.9 & $(18.5-26.0)$ \\
\hline Spartanburg, South Carolina & 571 & 8.8 & 2.0 & $(4.9-12.7)$ \\
\hline Spokane-Spokane Valley, Washington & 844 & 17.8 & 2.0 & $(14.0-21.7)$ \\
\hline Springfield, Massachusetts & 1,466 & 18.5 & 2.0 & $(14.6-22.5)$ \\
\hline Tallahassee, Florida & 1,771 & 17.4 & 1.9 & $(13.6-21.1)$ \\
\hline Tampa-St. Petersburg-Clearwater, Florida & 2,088 & 17.1 & 1.3 & $(14.6-19.5)$ \\
\hline Toledo, Ohio & 951 & 20.1 & 2.2 & $(15.9-24.4)$ \\
\hline Topeka, Kansas & 2,325 & 14.7 & 1.1 & $(12.6-16.9)$ \\
\hline Tulsa, Oklahoma & 1,948 & 12.5 & 1.1 & $(10.2-14.7)$ \\
\hline Virginia Beach-Norfolk-Newport News, Virginia-North Carolina & 1,572 & 17.5 & 1.4 & $(14.7-20.2)$ \\
\hline Warren-Troy-Farmington Hills, Michigan ${ }^{\S}$ & 2,181 & 21.1 & 1.3 & $(18.7-23.6)$ \\
\hline $\begin{array}{l}\text { Washington-Arlington-Alexandria, District of Columbia- } \\
\text { Virginia-Maryland-West Virginias }\end{array}$ & 8,483 & 16.5 & 0.8 & $(14.9-18.1)$ \\
\hline Wichita, Kansas & 4,769 & 15.5 & 0.7 & $(14.1-17.0)$ \\
\hline Wilmington, Delaware-Maryland-New Jersey $§$ & 3,132 & 17.9 & 1.0 & $(15.9-19.9)$ \\
\hline Winston-Salem, North Carolina & 661 & 12.2 & 2.1 & $(8.1-16.3)$ \\
\hline Worcester, Massachusetts-Connecticut & 2,614 & 20.0 & 1.4 & $(17.3-22.7)$ \\
\hline Median & & 17.8 & & \\
\hline Range & & $7.2-25.3$ & & \\
\hline
\end{tabular}

Abbreviations: $\mathrm{Cl}$ = confidence interval; MMSA = metropolitan and micropolitan statistical areas; $\mathrm{SE}=$ standard error.

* Age adjusted to the 2000 U.S. standard population.

† For men, having five or more drinks on one occasion; for women, having four or more drinks on one occasion.

$\S$ Metropolitan division. 
TABLE 37. Age-adjusted* prevalence estimates of adults aged $\geq 18$ years who reported binge drinking ${ }^{\dagger}$ during the past 30 days, by state/territory - Behavioral Risk Factor Surveillance System, United States, 2014

\begin{tabular}{|c|c|c|c|c|}
\hline State/Territory & $\begin{array}{l}\text { Sample } \\
\text { size }\end{array}$ & $\%$ & SE & $95 \% \mathrm{Cl}$ \\
\hline Alabama & 8,230 & 12.9 & 0.6 & $(11.6-14.1)$ \\
\hline Alaska & 4,134 & 20.0 & 0.9 & $(18.2-21.7)$ \\
\hline Arizona & 13,743 & 15.7 & 0.6 & $(14.5-17.0)$ \\
\hline Arkansas & 4,846 & 14.3 & 1.0 & $(12.4-16.2)$ \\
\hline California & 7,279 & 16.0 & 0.6 & $(14.9-17.1)$ \\
\hline Colorado & 12,113 & 17.9 & 0.5 & $(17.0-18.9)$ \\
\hline Connecticut & 7,305 & 17.4 & 0.7 & $(16.0-18.9)$ \\
\hline Delaware & 4,101 & 17.0 & 1.0 & $(15.0-19.0)$ \\
\hline District of Columbia & 3,750 & 24.1 & 1.2 & $(21.6-26.5)$ \\
\hline Florida & 8,965 & 16.5 & 0.6 & $(15.3-17.8)$ \\
\hline Georgia & 5,854 & 14.6 & 0.7 & $(13.1-16.0)$ \\
\hline Hawaii & 6,812 & 21.0 & 0.8 & $(19.5-22.5)$ \\
\hline Idaho & 5,178 & 15.5 & 0.9 & $(13.8-17.2)$ \\
\hline Illinois & 4,797 & 21.0 & 0.9 & $(19.3-22.7)$ \\
\hline Indiana & 10,804 & 15.6 & 0.5 & $(14.5-16.6)$ \\
\hline lowa & 7,655 & 23.1 & 0.7 & $(21.6-24.5)$ \\
\hline Kansas & 12,989 & 16.8 & 0.5 & $(15.9-17.7)$ \\
\hline Kentucky & 10,428 & 13.6 & 0.7 & $(12.3-14.9)$ \\
\hline Louisiana & 6,365 & 16.5 & 0.7 & $(15.2-17.8)$ \\
\hline Maine & 8,705 & 19.6 & 0.7 & $(18.2-21.1)$ \\
\hline Maryland & 11,829 & 16.2 & 0.7 & $(14.8-17.6)$ \\
\hline Massachusetts & 14,343 & 18.7 & 0.6 & (17.5-19.8) \\
\hline Michigan & 8,162 & 20.3 & 0.7 & $(19.0-21.6)$ \\
\hline Minnesota & 15,544 & 20.5 & 0.4 & $(19.7-21.4)$ \\
\hline Mississippi & 3,993 & 13.6 & 0.9 & $(11.9-15.4)$ \\
\hline Missouri & 6,765 & 16.1 & 0.8 & $(14.6-17.6)$ \\
\hline Montana & 7,164 & 20.8 & 0.8 & $(19.1-22.4)$ \\
\hline Nebraska & 21,443 & 21.5 & 0.5 & $(20.5-22.5)$ \\
\hline Nevada & 3,558 & 16.7 & 1.1 & $(14.6-18.9)$ \\
\hline New Hampshire & 5,762 & 18.8 & 0.9 & (16.9-20.6) \\
\hline New Jersey & 11,968 & 17.0 & 0.6 & $(15.9-18.2)$ \\
\hline New Mexico & 8,238 & 14.6 & 0.7 & $(13.2-16.0)$ \\
\hline New York & 6,263 & 15.8 & 0.6 & $(14.6-17.1)$ \\
\hline North Carolina & 6,797 & 14.5 & 0.6 & $(13.3-15.7)$ \\
\hline North Dakota & 7,353 & 25.1 & 0.9 & $(23.2-26.9)$ \\
\hline Ohio & 10,499 & 19.6 & 0.7 & $(18.2-21.0)$ \\
\hline Oklahoma & 8,076 & 13.6 & 0.6 & $(12.5-14.8)$ \\
\hline Oregon & 4,893 & 17.9 & 0.8 & (16.3-19.5) \\
\hline Pennsylvania & 10,315 & 18.3 & 0.6 & $(17.0-19.5)$ \\
\hline Rhode Island & 5,952 & 19.6 & 0.9 & $(17.8-21.4)$ \\
\hline South Carolina & 10,468 & 15.1 & 0.6 & $(14.0-16.2)$ \\
\hline South Dakota & 7,032 & 18.5 & 0.9 & (16.8-20.3) \\
\hline Tennessee & 4,766 & 11.4 & 0.8 & (9.7-13.0) \\
\hline Texas & 14,193 & 16.4 & 0.5 & $(15.4-17.5)$ \\
\hline Utah & 14,350 & 11.1 & 0.3 & $(10.4-11.7)$ \\
\hline Vermont & 6,078 & 20.1 & 0.7 & $(18.7-21.6)$ \\
\hline Virginia & 8,959 & 16.0 & 0.6 & $(14.9-17.2)$ \\
\hline Washington & 9,656 & 18.0 & 0.6 & (16.8-19.2) \\
\hline West Virginia & 6,002 & 10.7 & 0.6 & (9.5-11.8) \\
\hline Wisconsin & 6,641 & 23.2 & 0.8 & $(21.6-24.8)$ \\
\hline Wyoming & 5,965 & 18.1 & 1.1 & $(16.0-20.2)$ \\
\hline Guam & 2,326 & 22.2 & 1.2 & $(19.8-24.6)$ \\
\hline Puerto Rico & 5,830 & 14.1 & 0.6 & $(12.8-15.3)$ \\
\hline Median & & 17.0 & & \\
\hline Range & & $10.7-25.1$ & & \\
\hline
\end{tabular}

Abbreviations: $\mathrm{Cl}=$ confidence interval; $\mathrm{SE}=$ standard error.

* Age adjusted to the 2000 U.S. standard population.

† For men, having five or more drinks on one occasion; for women, having four or more drinks on one occasion. 
TABLE 38. Age-adjusted* prevalence estimates of adults aged $\geq 18$ years who reported binge drinking ${ }^{\dagger}$ during the past 30 days, by metropolitan and micropolitan statistical area - Behavioral Risk Factor Surveillance System, United States, 2014

\begin{tabular}{|c|c|c|c|c|}
\hline MMSA & $\begin{array}{l}\text { Sample } \\
\text { size }\end{array}$ & $\%$ & SE & $95 \% \mathrm{Cl}$ \\
\hline Aberdeen, South Dakota & 587 & 21.3 & 2.9 & $(15.5-27.0)$ \\
\hline Aguadilla-Isabela, Puerto Rico & 537 & 11.1 & 1.9 & $(7.3-14.9)$ \\
\hline Albuquerque, New Mexico & 1,638 & 13.5 & 1.3 & $(11.0-15.9)$ \\
\hline Allentown-Bethlehem-Easton, Pennsylvania-New Jersey & 1,027 & 17.2 & 2.3 & $(12.7-21.7)$ \\
\hline Anchorage, Alaska & 1,685 & 19.4 & 1.3 & $(16.9-21.9)$ \\
\hline Atlanta-Sandy Springs-Roswell, Georgia & 2,557 & 15.7 & 1.0 & $(13.7-17.7)$ \\
\hline Augusta-Richmond County, Georgia-South Carolina & 827 & 11.6 & 2.3 & $(7.2-16.1)$ \\
\hline Austin-Round Rock, Texas & 2,099 & 20.4 & 1.3 & $(17.9-22.9)$ \\
\hline Baltimore-Columbia-Towson, Maryland & 4,320 & 17.3 & 1.1 & $(15.1-19.6)$ \\
\hline Baton Rouge, Louisiana & 880 & 17.9 & 1.8 & $(14.5-21.4)$ \\
\hline Berlin, New Hampshire-Vermont & 510 & 16.1 & 3.3 & $(9.7-22.5)$ \\
\hline Billings, Montana & 764 & 20.7 & 2.2 & $(16.4-24.9)$ \\
\hline Birmingham-Hoover, Alabama & 1,494 & 13.7 & 1.3 & $(11.1-16.3)$ \\
\hline Bismarck, North Dakota & 977 & 21.6 & 2.2 & $(17.3-25.9)$ \\
\hline Boise City, Idaho & 1,274 & 14.3 & 1.6 & $(11.1-17.5)$ \\
\hline Boston, Massachusetts $§$ & 4,149 & 19.4 & 1.1 & $(17.3-21.5)$ \\
\hline Burlington-South Burlington, Vermont & 1,867 & 22.2 & 1.3 & $(19.7-24.7)$ \\
\hline Cambridge-Newton-Framingham, Massachusetts ${ }^{\S}$ & 4,762 & 18.5 & 0.9 & $(16.6-20.3)$ \\
\hline Camden, New Jersey ${ }^{\S}$ & 1,592 & 18.5 & 1.7 & $(15.3-21.8)$ \\
\hline Cedar Rapids, lowa & 610 & 20.9 & 2.4 & $(16.2-25.5)$ \\
\hline Charleston, West Virginia & 849 & 10.8 & 1.5 & $(7.9-13.8)$ \\
\hline Charleston-North Charleston, South Carolina & 1,325 & 19.7 & 1.6 & $(16.6-22.7)$ \\
\hline Charlotte-Concord-Gastonia, North Carolina-South Carolina & 2,007 & 14.8 & 1.1 & $(12.6-17.0)$ \\
\hline Chicago-Naperville-Elgin, Illinois-Indiana-Wisconsin & 3,884 & 21.0 & 1.0 & $(19.0-23.0)$ \\
\hline Cincinnati, Ohio-Kentucky-Indiana & 1,935 & 21.1 & 1.8 & $(17.5-24.6)$ \\
\hline Claremont-Lebanon, New Hampshire-Vermont & 1,609 & 16.6 & 1.6 & $(13.4-19.8)$ \\
\hline Cleveland-Elyria, Ohio & 927 & 24.4 & 2.2 & $(20.1-28.7)$ \\
\hline College Station-Bryan, Texas & 549 & 12.4 & 2.5 & $(7.5-17.3)$ \\
\hline Colorado Springs, Colorado & 1,175 & 12.2 & 1.3 & $(9.7-14.7)$ \\
\hline Columbia, South Carolina & 1,155 & 17.0 & 1.6 & $(13.9-20.0)$ \\
\hline Columbus, Ohio & 1,581 & 18.9 & 1.6 & $(15.8-22.0)$ \\
\hline Corpus Christi, Texas & 570 & 16.7 & 3.6 & $(9.7-23.7)$ \\
\hline Dallas-Plano-Irving, Texas ${ }^{\S}$ & 1,192 & 18.2 & 1.8 & $(14.7-21.7)$ \\
\hline Dayton, Ohio & 550 & 16.7 & 2.5 & $(11.9-21.6)$ \\
\hline Denver-Aurora-Lakewood, Colorado & 5,229 & 19.4 & 0.7 & $(18.0-20.8)$ \\
\hline Des Moines-West Des Moines, lowa & 1,266 & 21.2 & 1.8 & $(17.7-24.8)$ \\
\hline Duluth, Minnesota-Wisconsin & 895 & 22.2 & 2.0 & $(18.3-26.2)$ \\
\hline El Paso, Texas & 655 & 18.9 & 2.4 & $(14.2-23.7)$ \\
\hline Evansville, Indiana-Kentucky & 615 & 16.2 & 3.0 & $(10.3-22.1)$ \\
\hline Fargo, North Dakota-Minnesota & 1,092 & 26.3 & 2.0 & $(22.4-30.1)$ \\
\hline Fayetteville-Springdale-Rogers, Arkansas-Missouri & 766 & 15.2 & 2.3 & $(10.7-19.8)$ \\
\hline Fort Wayne, Indiana & 809 & 14.0 & 2.0 & $(10.1-17.9)$ \\
\hline Fort Worth-Arlington, Texas ${ }^{\S}$ & 705 & 13.2 & 2.0 & $(9.3-17.2)$ \\
\hline Grand Island, Nebraska & 1,003 & 18.5 & 2.2 & $(14.2-22.7)$ \\
\hline Grand Rapids-Wyoming, Michigan & 869 & 21.1 & 2.0 & $(17.1-25.1)$ \\
\hline Greensboro-High Point, North Carolina & 482 & 15.2 & 2.3 & $(10.6-19.7)$ \\
\hline Greenville-Anderson-Mauldin, South Carolina & 1,421 & 11.9 & 1.1 & $(9.7-14.2)$ \\
\hline Hagerstown-Martinsburg, Maryland-West Virginia & 748 & 15.8 & 3.2 & $(9.6-22.0)$ \\
\hline Hartford-West Hartford-East Hartford, Connecticut & 2,443 & 17.9 & 1.2 & $(15.5-20.4)$ \\
\hline Hilton Head Island-Bluffton-Beaufort, South Carolina & 514 & 18.3 & 2.9 & $(12.6-24.0)$ \\
\hline Houston-The Woodlands-Sugar Land, Texas & 1,963 & 15.3 & 1.3 & $(12.8-17.9)$ \\
\hline Huntington-Ashland, West Virginia-Kentucky-Ohio & 1,202 & 10.2 & 1.5 & $(7.3-13.1)$ \\
\hline Idaho Falls, Idaho & 494 & 12.4 & 2.1 & $(8.2-16.5)$ \\
\hline Indianapolis-Carmel-Anderson, Indiana & 3,390 & 14.7 & 0.9 & $(12.9-16.6)$ \\
\hline Jacksonville, Florida & 619 & 17.2 & 2.4 & $(12.5-22.0)$ \\
\hline Kansas City, Missouri-Kansas & 4,640 & 16.4 & 1.0 & $(14.4-18.4)$ \\
\hline Kingsport-Bristol-Bristol, Tennessee-Virginia & 477 & 10.3 & 2.6 & $(5.2-15.4)$ \\
\hline Knoxville, Tennessee & 517 & 7.2 & 1.8 & $(3.8-10.7)$ \\
\hline Lafayette, Louisiana & 523 & 17.9 & 2.1 & $(13.8-22.0)$ \\
\hline Lexington-Fayette, Kentucky & 585 & 11.9 & 1.8 & $(8.4-15.4)$ \\
\hline Lincoln, Nebraska & 1,937 & 23.2 & 1.3 & $(20.6-25.8)$ \\
\hline
\end{tabular}

See table footnotes on page 87. 
TABLE 38. (Continued) Age-adjusted* prevalence estimates of adults aged $\geq 18$ years who reported binge drinking ${ }^{\dagger}$ during the past 30 days, by metropolitan and micropolitan statistical area - Behavioral Risk Factor Surveillance System, United States, 2014

\begin{tabular}{|c|c|c|c|c|}
\hline MMSA & $\begin{array}{l}\text { Sample } \\
\text { size }\end{array}$ & $\%$ & SE & $95 \% \mathrm{Cl}$ \\
\hline Little Rock-North Little Rock-Conway, Arkansas & 1,100 & 13.8 & 1.7 & $(10.4-17.2)$ \\
\hline Logan, Utah-Idaho & 602 & 6.9 & 1.2 & $(4.5-9.3)$ \\
\hline Los Angeles-Long Beach-Anaheim, California & 1,943 & 15.0 & 1.0 & $(13.0-16.9)$ \\
\hline Louisville-Jefferson County, Kentucky-Indiana & 2,281 & 16.7 & 1.5 & $(13.7-19.6)$ \\
\hline Madison, Wisconsin & 518 & 23.8 & 2.3 & $(19.2-28.3)$ \\
\hline Memphis, Tennessee-Mississippi-Arkansas & 813 & 9.4 & 1.7 & $(6.1-12.7)$ \\
\hline Miami-Fort Lauderdale-West Palm Beach, Florida & 1,963 & 14.2 & 1.2 & $(11.8-16.7)$ \\
\hline Milwaukee-Waukesha-West Allis, Wisconsin & 1,267 & 23.2 & 1.9 & $(19.5-26.8)$ \\
\hline Minneapolis-St. Paul-Bloomington, Minnesota-Wisconsin & 8,309 & 20.0 & 0.6 & $(18.9-21.1)$ \\
\hline Minot, North Dakota & 569 & 20.6 & 2.6 & $(15.5-25.7)$ \\
\hline Montgomery, Alabama & 486 & 16.2 & 2.7 & $(10.9-21.5)$ \\
\hline $\begin{array}{l}\text { Montgomery County-Bucks County-Chester County, } \\
\text { Pennsylvania§ }\end{array}$ & 768 & 21.3 & 2.3 & $(16.8-25.8)$ \\
\hline $\begin{array}{l}\text { Myrtle Beach-Conway-North Myrtle Beach, South Carolina- } \\
\text { North Carolina }\end{array}$ & 945 & 16.4 & 1.9 & $(12.6-20.2)$ \\
\hline Nashville-Davidson County-Murfreesboro-Franklin, Tennessee & 734 & 17.0 & 2.3 & $(12.4-21.6)$ \\
\hline Nassau County-Suffolk County, New York ${ }^{\S}$ & 709 & 17.9 & 2.2 & $(13.5-22.3)$ \\
\hline Newark, New Jersey-Pennsylvania§ & 3,818 & 16.4 & 1.0 & $(14.4-18.5)$ \\
\hline New Orleans-Metairie, Louisiana & 1,800 & 18.4 & 1.3 & $(15.9-20.9)$ \\
\hline New York-Jersey City-White Plains, New York-New Jersey $§$ & 6,813 & 15.7 & 0.7 & $(14.4-17.0)$ \\
\hline Norfolk, Nebraska & 940 & 24.4 & 1.9 & $(20.7-28.0)$ \\
\hline North Platte, Nebraska & 913 & 19.3 & 1.9 & $(15.6-23.0)$ \\
\hline North Port-Sarasota-Bradenton, Florida & 472 & 11.1 & 2.5 & $(6.2-15.9)$ \\
\hline Oakland-Hayward-Berkeley, California $\$$ & 596 & 15.3 & 2.1 & $(11.2-19.4)$ \\
\hline Ogden-Clearfield, Utah & 2,814 & 10.1 & 0.7 & $(8.7-11.4)$ \\
\hline Oklahoma City, Oklahoma & 2,326 & 13.9 & 1.0 & $(11.9-15.9)$ \\
\hline Omaha-Council Bluffs, Nebraska-lowa & 4,667 & 20.8 & 0.9 & $(19.0-22.6)$ \\
\hline Orlando-Kissimmee-Sanford, Florida & 876 & 14.8 & 1.8 & $(11.4-18.3)$ \\
\hline Philadelphia, Pennsylvania§ & 1,382 & 20.3 & 1.6 & $(17.1-23.5)$ \\
\hline Phoenix-Mesa-Scottsdale, Arizona & 8,689 & 16.2 & 0.7 & $(14.8-17.7)$ \\
\hline Pittsburgh, Pennsylvania & 2,260 & 20.6 & 1.3 & $(18.0-23.1)$ \\
\hline Ponce, Puerto Rico & 517 & 16.7 & 2.5 & $(11.8-21.6)$ \\
\hline Portland-South Portland, Maine & 2,625 & 20.5 & 1.3 & $(17.9-23.0)$ \\
\hline Portland-Vancouver-Hillsboro, Oregon-Washington & 2,666 & 16.8 & 1.0 & $(14.7-18.8)$ \\
\hline Providence-Warwick, Rhode Island-Massachusetts & 7,476 & 19.5 & 0.9 & $(17.7-21.4)$ \\
\hline Provo-Orem, Utah & 2,063 & 6.7 & 0.6 & $(5.5-8.0)$ \\
\hline Raleigh, North Carolina & 681 & 16.6 & 1.9 & $(12.9-20.3)$ \\
\hline Rapid City, South Dakota & 1,351 & 15.5 & 1.6 & $(12.4-18.6)$ \\
\hline Reno, Nevada & 1,154 & 21.7 & 1.8 & $(18.1-25.3)$ \\
\hline Richmond, Virginia & 1,397 & 16.5 & 1.5 & $(13.7-19.4)$ \\
\hline Riverside-San Bernardino-Ontario, California & 769 & 15.6 & 1.8 & $(12.1-19.0)$ \\
\hline Roanoke, Virginia & 496 & 16.9 & 2.7 & $(11.7-22.2)$ \\
\hline Rochester, Minnesota & 666 & 19.5 & 1.9 & $(15.8-23.1)$ \\
\hline Rockingham County-Strafford County, New Hampshire & 1,337 & 20.1 & 1.8 & $(16.6-23.7)$ \\
\hline Sacramento-Roseville-Arden-Arcade, California & 545 & 17.9 & 2.3 & $(13.5-22.4)$ \\
\hline St. Cloud, Minnesota & 522 & 20.7 & 2.0 & $(16.7-24.7)$ \\
\hline St. Louis, Missouri-Illinois & 1,822 & 18.1 & 1.5 & $(15.2-21.0)$ \\
\hline Salisbury, Maryland-Delaware & 1,862 & 13.7 & 1.9 & $(10.0-17.4)$ \\
\hline Salt Lake City, Utah & 5,149 & 13.9 & 0.6 & $(12.7-15.0)$ \\
\hline San Antonio-New Braunfels, Texas & 2,107 & 17.3 & 1.2 & $(14.9-19.7)$ \\
\hline San Juan-Carolina-Caguas, Puerto Rico & 3,663 & 14.1 & 0.8 & $(12.6-15.7)$ \\
\hline Scottsbluff, Nebraska & 869 & 17.5 & 1.9 & $(13.8-21.3)$ \\
\hline Seattle-Bellevue-Everett, Washington ${ }^{\S}$ & 3,533 & 18.0 & 0.9 & $(16.1-19.8)$ \\
\hline Shreveport-Bossier City, Louisiana & 518 & 16.1 & 2.3 & $(11.6-20.5)$ \\
\hline Silver Spring-Frederick-Rockville, Maryland ${ }^{\S}$ & 2,261 & 14.9 & 1.5 & $(12.0-17.8)$ \\
\hline Sioux City, lowa-Nebraska-South Dakota & 1,093 & 21.2 & 2.7 & $(16.0-26.5)$ \\
\hline Sioux Falls, South Dakota & 1,289 & 18.1 & 1.8 & $(14.6-21.5)$ \\
\hline Spartanburg, South Carolina & 534 & 11.1 & 2.2 & $(6.7-15.5)$ \\
\hline Spokane-Spokane Valley, Washington & 698 & 22.3 & 2.5 & $(17.4-27.2)$ \\
\hline Springfield, Massachusetts & 1,012 & 19.6 & 2.1 & $(15.6-23.7)$ \\
\hline Tampa-St. Petersburg-Clearwater, Florida & 1,459 & 20.5 & 1.8 & $(17.1-24.0)$ \\
\hline Toledo, Ohio & 624 & 18.8 & 2.5 & $(13.9-23.7)$ \\
\hline
\end{tabular}

See table footnotes on page 87. 
TABLE 38. (Continued) Age-adjusted* prevalence estimates of adults aged $\geq 18$ years who reported binge drinking ${ }^{\dagger}$ during the past 30 days, by metropolitan and micropolitan statistical area - Behavioral Risk Factor Surveillance System, United States, 2014

\begin{tabular}{|c|c|c|c|c|}
\hline MMSA & $\begin{array}{l}\text { Sample } \\
\text { size }\end{array}$ & $\%$ & SE & $95 \% \mathrm{Cl}$ \\
\hline Topeka, Kansas & 1,375 & 16.9 & 1.4 & $(14.1-19.7)$ \\
\hline Tulsa, Oklahoma & 1,944 & 14.0 & 1.1 & $(11.8-16.3)$ \\
\hline Tuscaloosa, Alabama & 685 & 12.0 & 1.8 & $(8.5-15.4)$ \\
\hline Virginia Beach-Norfolk-Newport News, Virginia-North Carolina & 1,777 & 16.4 & 1.3 & $(13.8-19.0)$ \\
\hline Warren-Troy-Farmington Hills, Michigan ${ }^{\S}$ & 2,043 & 20.2 & 1.3 & $(17.7-22.7)$ \\
\hline $\begin{array}{l}\text { Washington-Arlington-Alexandria, District of Columbia- } \\
\text { Virginia-Maryland-West Virginias }\end{array}$ & 7,724 & 16.6 & 0.8 & $(15.1-18.2)$ \\
\hline Wichita, Kansas & 2,586 & 14.4 & 0.9 & $(12.6-16.1)$ \\
\hline Wichita Falls, Texas & 507 & 12.5 & 3.4 & $(5.9-19.1)$ \\
\hline Wilmington, Delaware-Maryland-New Jersey & 2,630 & 18.2 & 1.3 & $(15.6-20.7)$ \\
\hline Worcester, Massachusetts-Connecticut & 2,248 & 17.7 & 1.3 & $(15.1-20.3)$ \\
\hline Youngstown-Warren-Boardman, Ohio-Pennsylvania & 496 & 17.1 & 3.2 & $(10.9-23.3)$ \\
\hline $\begin{array}{l}\text { Median } \\
\text { Range }\end{array}$ & & $\begin{array}{c}17.0 \\
6.7-26.3\end{array}$ & & \\
\hline
\end{tabular}

Abbreviations: $\mathrm{Cl}=$ confidence interval; $\mathrm{MMSA}=$ metropolitan and micropolitan statistical areas; $\mathrm{SE}=$ standard error.

* Age adjusted to the 2000 U.S. standard population.

† For men, having five or more drinks on one occasion; for women, having four or more drinks on one occasion.

$\S$ Metropolitan division. 
TABLE 39. Age-adjusted* prevalence estimates of adults aged $\geq 18$ years with obesity, ${ }^{\dagger}$ by state/territory - Behavioral Risk Factor Surveillance System, United States, 2013

\begin{tabular}{|c|c|c|c|c|}
\hline State/Territory & $\begin{array}{l}\text { Sample } \\
\text { size }\end{array}$ & $\%$ & SE & $95 \% \mathrm{Cl}$ \\
\hline Alabama & 6,244 & 32.0 & 0.9 & $(30.3-33.8)$ \\
\hline Alaska & 4,396 & 28.2 & 1.0 & $(26.2-30.1)$ \\
\hline Arizona & 4,029 & 26.6 & 1.4 & $(24.0-29.3)$ \\
\hline Arkansas & 5,023 & 34.9 & 1.1 & $(32.8-37.0)$ \\
\hline California & 10,669 & 23.8 & 0.6 & $(22.7-24.9)$ \\
\hline Colorado & 12,920 & 21.0 & 0.5 & $(20.1-22.0)$ \\
\hline Connecticut & 7,309 & 24.5 & 0.8 & $(22.9-26.0)$ \\
\hline Delaware & 4,893 & 30.4 & 0.9 & $(28.5-32.2)$ \\
\hline District of Columbia & 4,705 & 23.1 & 1.0 & $(21.2-25.0)$ \\
\hline Florida & 32,552 & 26.1 & 0.6 & $(24.9-27.2)$ \\
\hline Georgia & 7,606 & 29.9 & 0.7 & $(28.5-31.4)$ \\
\hline Hawaii & 7,609 & 21.9 & 0.7 & $(20.5-23.4)$ \\
\hline Idaho & 5,315 & 29.2 & 0.9 & $(27.4-31.0)$ \\
\hline Illinois & 5,490 & 28.9 & 0.9 & $(27.1-30.6)$ \\
\hline Indiana & 9,770 & 31.2 & 0.6 & $(30.0-32.5)$ \\
\hline lowa & 7,672 & 30.8 & 0.7 & $(29.4-32.3)$ \\
\hline Kansas & 21,991 & 29.7 & 0.4 & $(28.9-30.4)$ \\
\hline Kentucky & 10,325 & 32.6 & 0.7 & $(31.2-34.1)$ \\
\hline Louisiana & 5,014 & 32.6 & 1.1 & $(30.5-34.8)$ \\
\hline Maine & 7,791 & 28.0 & 0.7 & $(26.5-29.4)$ \\
\hline Maryland & 12,169 & 27.6 & 0.7 & $(26.3-28.9)$ \\
\hline Massachusetts & 13,883 & 22.9 & 0.6 & $(21.8-24.1)$ \\
\hline Michigan & 12,177 & 30.8 & 0.6 & $(29.6-32.0)$ \\
\hline Minnesota & 13,450 & 24.9 & 0.7 & $(23.5-26.2)$ \\
\hline Mississippi & 7,032 & 34.8 & 0.9 & $(33.1-36.5)$ \\
\hline Missouri & 6,783 & 30.2 & 0.9 & (28.4-31.9) \\
\hline Montana & 9,323 & 24.0 & 0.6 & $(22.8-25.3)$ \\
\hline Nebraska & 16,250 & 29.1 & 0.6 & $(27.9-30.3)$ \\
\hline Nevada & 4,871 & 26.1 & 1.2 & $(23.8-28.4)$ \\
\hline New Hampshire & 6,141 & 26.1 & 0.8 & $(24.5-27.7)$ \\
\hline New Jersey & 12,256 & 25.7 & 0.6 & (24.5-26.9) \\
\hline New Mexico & 8,914 & 26.3 & 0.7 & $(24.9-27.7)$ \\
\hline New York & 8,465 & 24.8 & 0.6 & $(23.6-26.1)$ \\
\hline North Carolina & 8,292 & 28.9 & 0.7 & $(27.5-30.3)$ \\
\hline North Dakota & 7,413 & 30.5 & 0.8 & $(29.0-32.1)$ \\
\hline Ohio & 11,260 & 29.8 & 0.7 & $(28.5-31.1)$ \\
\hline Oklahoma & 7,875 & 32.2 & 0.7 & $(30.8-33.7)$ \\
\hline Oregon & 5,668 & 26.0 & 0.8 & $(24.4-27.7)$ \\
\hline Pennsylvania & 10,857 & 29.3 & 0.6 & $(28.1-30.6)$ \\
\hline Rhode Island & 6,191 & 27.0 & 0.8 & (25.4-28.6) \\
\hline South Carolina & 10,227 & 31.5 & 0.7 & $(30.2-32.9)$ \\
\hline South Dakota & 6,531 & 29.4 & 1.0 & $(27.4-31.3)$ \\
\hline Tennessee & 5,452 & 33.4 & 1.0 & $(31.5-35.3)$ \\
\hline Texas & 10,085 & 30.6 & 0.7 & $(29.2-32.0)$ \\
\hline Utah & 12,027 & 24.4 & 0.5 & $(23.4-25.3)$ \\
\hline Vermont & 6,106 & 24.1 & 0.8 & $(22.6-25.6)$ \\
\hline Virginia & 7,941 & 26.7 & 0.7 & $(25.4-28.0)$ \\
\hline Washington & 10,554 & 26.6 & 0.6 & (25.4-27.8) \\
\hline West Virginia & 5,636 & 35.2 & 0.8 & $(33.5-36.8)$ \\
\hline Wisconsin & 6,188 & 29.0 & 0.9 & $(27.2-30.8)$ \\
\hline Wyoming & 6,147 & 27.6 & 0.9 & $(25.8-29.3)$ \\
\hline Guam & 1,803 & 26.6 & 1.4 & (23.9-29.4) \\
\hline Puerto Rico & 5,762 & 27.8 & 0.8 & $(26.2-29.3)$ \\
\hline Median & & 28.2 & & \\
\hline Range & & $21.0-35.2$ & & \\
\hline
\end{tabular}

Abbreviations: $\mathrm{Cl}=$ confidence interval; $\mathrm{SE}=$ standard error.

* Age adjusted to the 2000 U.S. standard population.

† Body mass index $\geq 30$. 
TABLE 40. Age-adjusted* prevalence estimates of adults aged $\geq 18$ years with obesity, ${ }^{\dagger}$ by metropolitan and micropolitan statistical area Behavioral Risk Factor Surveillance System, United States, 2013

\begin{tabular}{|c|c|c|c|c|}
\hline MMSA & $\begin{array}{l}\text { Sample } \\
\text { size }\end{array}$ & $\%$ & SE & $95 \% \mathrm{Cl}$ \\
\hline Aguadilla-Isabela, Puerto Rico & 569 & 24.4 & 2.3 & $(19.8-29.0)$ \\
\hline Akron, Ohio & 643 & 32.3 & 2.9 & $(26.6-38.0)$ \\
\hline Albuquerque, New Mexico & 1,994 & 23.6 & 1.3 & $(21.0-26.1)$ \\
\hline Allentown-Bethlehem-Easton, Pennsylvania-New Jersey & 960 & 28.6 & 2.3 & $(24.0-33.1)$ \\
\hline Anchorage, Alaska & 1,455 & 26.9 & 1.5 & $(23.9-29.9)$ \\
\hline Atlanta-Sandy Springs-Roswell, Georgia & 3,258 & 26.2 & 1.0 & $(24.2-28.2)$ \\
\hline Augusta-Richmond County, Georgia-South Carolina & 866 & 36.1 & 2.7 & $(30.8-41.5)$ \\
\hline Austin-Round Rock, Texas & 869 & 25.1 & 2.0 & $(21.1-29.1)$ \\
\hline Baltimore-Columbia-Towson, Maryland & 4,465 & 27.2 & 1.0 & $(25.3-29.2)$ \\
\hline Baton Rouge, Louisiana & 878 & 34.4 & 2.6 & $(29.4-39.5)$ \\
\hline Billings, Montana & 783 & 25.4 & 1.8 & $(21.9-28.8)$ \\
\hline Birmingham-Hoover, Alabama & 1,312 & 32.3 & 1.8 & $(28.8-35.7)$ \\
\hline Bismarck, North Dakota & 976 & 29.3 & 1.9 & $(25.6-33.1)$ \\
\hline Boise City, Idaho & 1,404 & 27.7 & 1.7 & $(24.4-31.0)$ \\
\hline Boston, Massachusetts $§$ & 3,725 & 22.0 & 1.1 & $(19.8-24.1)$ \\
\hline Buffalo-Cheektowaga-Niagara Falls, New York & 487 & 23.3 & 2.4 & $(18.5-28.0)$ \\
\hline Burlington-South Burlington, Vermont & 1,562 & 21.9 & 1.3 & $(19.4-24.5)$ \\
\hline Cambridge-Newton-Framingham, Massachusetts $§$ & 4,521 & 20.9 & 1.0 & $(19.0-22.8)$ \\
\hline Camden, New Jersey ${ }^{\S}$ & 1,726 & 28.1 & 1.5 & $(25.1-31.1)$ \\
\hline Cedar Rapids, lowa & 609 & 34.6 & 2.7 & $(29.3-39.8)$ \\
\hline Charleston, West Virginia & 777 & 35.4 & 2.3 & $(30.9-39.8)$ \\
\hline Charleston-North Charleston, South Carolina & 1,494 & 27.2 & 1.6 & $(24.1-30.2)$ \\
\hline Charlotte-Concord-Gastonia, North Carolina-South Carolina & 1,823 & 28.3 & 1.4 & $(25.5-31.1)$ \\
\hline Chattanooga, Tennessee-Georgia & 535 & 25.7 & 2.7 & $(20.4-31.0)$ \\
\hline Chicago-Naperville-Elgin, Illinois-Indiana-Wisconsin & 3,231 & 28.7 & 1.2 & $(26.4-31.0)$ \\
\hline Cincinnati, Ohio-Kentucky-Indiana & 2,465 & 30.8 & 1.5 & $(28.0-33.7)$ \\
\hline Claremont-Lebanon, New Hampshire-Vermont & 1,618 & 25.3 & 1.9 & $(21.7-29.0)$ \\
\hline Cleveland-Elyria, Ohio & 1,034 & 27.1 & 1.9 & $(23.3-30.8)$ \\
\hline Colorado Springs, Colorado & 1,315 & 23.7 & 1.5 & $(20.7-26.6)$ \\
\hline Columbia, South Carolina & 1,380 & 31.8 & 1.8 & $(28.3-35.3)$ \\
\hline Columbus, Ohio & 1,752 & 31.4 & 1.5 & $(28.6-34.3)$ \\
\hline Crestview-Fort Walton Beach-Destin, Florida & 1,031 & 25.5 & 2.1 & $(21.4-29.7)$ \\
\hline Dallas-Plano-Irving, Texas ${ }^{\S}$ & 829 & 25.8 & 1.8 & $(22.2-29.4)$ \\
\hline Davenport-Moline-Rock Island, lowa-Illinois & 646 & 32.6 & 2.8 & $(27.2-38.0)$ \\
\hline Dayton, Ohio & 795 & 31.0 & 2.4 & $(26.2-35.8)$ \\
\hline Deltona-Daytona Beach-Ormond Beach, Florida & 1,059 & 23.0 & 2.3 & $(18.5-27.6)$ \\
\hline Denver-Aurora-Lakewood, Colorado & 5,408 & 20.4 & 0.7 & $(19.0-21.7)$ \\
\hline Des Moines-West Des Moines, lowa & 1,276 & 29.6 & 1.8 & $(26.1-33.1)$ \\
\hline Duluth, Minnesota-Wisconsin & 660 & 21.3 & 2.4 & $(16.5-26.0)$ \\
\hline Durham-Chapel Hill, North Carolina & 577 & 23.7 & 2.3 & $(19.1-28.3)$ \\
\hline El Paso, Texas & 692 & 30.4 & 2.3 & $(25.9-34.8)$ \\
\hline Evansville, Indiana-Kentucky & 545 & 33.9 & 3.1 & $(27.9-39.9)$ \\
\hline Fargo, North Dakota-Minnesota & 1,130 & 27.2 & 1.9 & $(23.5-30.9)$ \\
\hline Fayetteville-Springdale-Rogers, Arkansas-Missouri & 782 & 29.0 & 2.5 & $(24.1-33.9)$ \\
\hline Fort Smith, Arkansas-Oklahoma & 478 & 35.5 & 3.1 & $(29.4-41.7)$ \\
\hline Fort Wayne, Indiana & 742 & 25.2 & 2.0 & $(21.2-29.2)$ \\
\hline Fort Worth-Arlington, Texas ${ }^{\S}$ & 756 & 31.1 & 2.5 & $(26.3-36.0)$ \\
\hline Gainesville, Florida & 982 & 25.1 & 2.3 & $(20.6-29.6)$ \\
\hline Grand Forks, North Dakota-Minnesota & 476 & 29.4 & 3.3 & $(22.8-35.9)$ \\
\hline Grand Island, Nebraska & 756 & 35.5 & 2.8 & $(29.9-41.0)$ \\
\hline Grand Rapids-Wyoming, Michigan & 1,285 & 28.8 & 1.7 & $(25.5-32.1)$ \\
\hline Greensboro-High Point, North Carolina & 619 & 30.8 & 2.5 & $(26.0-35.7)$ \\
\hline Greenville-Anderson-Mauldin, South Carolina & 1,274 & 28.7 & 1.8 & $(25.3-32.2)$ \\
\hline Gulfport-Biloxi-Pascagoula, Mississippi & 734 & 33.3 & 2.4 & $(28.6-37.9)$ \\
\hline Hagerstown-Martinsburg, Maryland-West Virginia & 726 & 34.8 & 3.0 & $(28.9-40.8)$ \\
\hline Hartford-West Hartford-East Hartford, Connecticut & 2,686 & 26.8 & 1.4 & $(24.1-29.5)$ \\
\hline Hilton Head Island-Bluffton-Beaufort, South Carolina & 795 & 25.6 & 2.8 & $(20.0-31.1)$ \\
\hline Houston-The Woodlands-Sugar Land, Texas & 1,289 & 26.3 & 1.8 & $(22.8-29.7)$ \\
\hline Huntington-Ashland, West Virginia-Kentucky-Ohio & 1,102 & 37.1 & 1.9 & $(33.4-40.9)$ \\
\hline Idaho Falls, Idaho & 486 & 35.7 & 3.0 & $(29.7-41.6)$ \\
\hline Indianapolis-Carmel-Anderson, Indiana & 2,409 & 31.8 & 1.3 & $(29.3-34.2)$ \\
\hline
\end{tabular}

See table footnotes on page 91. 
TABLE 40. (Continued) Age-adjusted* prevalence estimates of adults aged $\geq 18$ years with obesity, ${ }^{\dagger}$ by metropolitan and micropolitan statistical area - Behavioral Risk Factor Surveillance System, United States, 2013

\begin{tabular}{|c|c|c|c|c|}
\hline MMSA & $\begin{array}{l}\text { Sample } \\
\text { size }\end{array}$ & $\%$ & SE & $95 \% \mathrm{Cl}$ \\
\hline Jackson, Mississippi & 757 & 31.1 & 2.3 & $(26.6-35.6)$ \\
\hline Jacksonville, Florida & 2,751 & 28.4 & 1.4 & $(25.7-31.1)$ \\
\hline Kansas City, Missouri-Kansas & 7,031 & 29.2 & 1.1 & $(27.0-31.3)$ \\
\hline Kingsport-Bristol-Bristol, Tennessee-Virginia & 509 & 29.3 & 3.2 & $(23.1-35.6)$ \\
\hline Knoxville, Tennessee & 616 & 31.3 & 2.6 & $(26.2-36.5)$ \\
\hline Lansing-East Lansing, Michigan & 651 & 27.2 & 2.1 & $(23.0-31.3)$ \\
\hline Lexington-Fayette, Kentucky & 610 & 32.9 & 2.5 & $(28.0-37.8)$ \\
\hline Lincoln, Nebraska & 1,785 & 25.5 & 1.2 & $(23.2-27.8)$ \\
\hline Little Rock-North Little Rock-Conway, Arkansas & 1,092 & 32.9 & 2.1 & $(28.8-37.0)$ \\
\hline Logan, Utah-Idaho & 608 & 23.1 & 2.1 & $(19.0-27.1)$ \\
\hline Los Angeles-Long Beach-Anaheim, California & 2,782 & 22.4 & 1.0 & $(20.4-24.5)$ \\
\hline Louisville-Jefferson County, Kentucky-Indiana & 2,021 & 29.5 & 1.6 & $(26.3-32.7)$ \\
\hline Lubbock, Texas & 500 & 33.2 & 3.2 & $(27.0-39.5)$ \\
\hline Manhattan, Kansas & 632 & 22.9 & 1.9 & $(19.1-26.7)$ \\
\hline Memphis, Tennessee-Mississippi-Arkansas & 1,141 & 32.4 & 2.1 & $(28.2-36.5)$ \\
\hline Miami-Fort Lauderdale-West Palm Beach, Florida & 2,107 & 23.6 & 1.5 & $(20.7-26.6)$ \\
\hline Milwaukee-Waukesha-West Allis, Wisconsin & 1,182 & 27.6 & 2.1 & $(23.5-31.7)$ \\
\hline Minneapolis-St. Paul-Bloomington, Minnesota-Wisconsin & 8,556 & 23.7 & 0.9 & $(21.9-25.4)$ \\
\hline Minot, North Dakota & 625 & 26.4 & 2.2 & $(22.2-30.7)$ \\
\hline $\begin{array}{l}\text { Montgomery County-Bucks County-Chester County, } \\
\text { Pennsylvania }\end{array}$ & 937 & 22.7 & 1.7 & $(19.4-26.0)$ \\
\hline $\begin{array}{l}\text { Myrtle Beach-Conway-North Myrtle Beach, South Carolina- } \\
\text { North Carolina }\end{array}$ & 736 & 32.1 & 2.6 & $(27.0-37.2)$ \\
\hline Nashville-Davidson County-Murfreesboro-Franklin, Tennessee & 980 & 33.4 & 2.0 & $(29.4-37.4)$ \\
\hline Nassau County-Suffolk County, New York ${ }^{\S}$ & 888 & 24.3 & 1.9 & $(20.6-28.0)$ \\
\hline Newark, New Jersey-Pennsylvania ${ }^{\S}$ & 3,762 & 23.7 & 1.0 & $(21.7-25.7)$ \\
\hline New Orleans-Metairie, Louisiana & 1,236 & 30.2 & 2.1 & $(26.1-34.4)$ \\
\hline New York-Jersey City-White Plains, New York-New Jersey ${ }^{\S}$ & 8,284 & 23.7 & 0.7 & $(22.3-25.0)$ \\
\hline Norfolk, Nebraska & 623 & 34.8 & 2.3 & $(30.3-39.4)$ \\
\hline North Platte, Nebraska & 686 & 31.7 & 2.8 & $(26.2-37.1)$ \\
\hline North Port-Sarasota-Bradenton, Florida & 1,024 & 23.9 & 2.3 & $(19.4-28.5)$ \\
\hline Oakland-Hayward-Berkeley, California§ & 657 & 19.5 & 2.0 & $(15.6-23.3)$ \\
\hline Ogden-Clearfield, Utah & 2,307 & 24.5 & 1.0 & $(22.5-26.6)$ \\
\hline Oklahoma City, Oklahoma & 2,510 & 29.4 & 1.2 & $(27.1-31.7)$ \\
\hline Omaha-Council Bluffs, Nebraska-lowa & 2,972 & 30.2 & 1.2 & $(27.9-32.5)$ \\
\hline Orlando-Kissimmee-Sanford, Florida & 2,161 & 25.8 & 1.4 & $(23.0-28.6)$ \\
\hline Panama City, Florida & 978 & 28.5 & 2.2 & $(24.1-32.9)$ \\
\hline Pensacola-Ferry Pass-Brent, Florida & 1,263 & 26.4 & 1.6 & $(23.2-29.6)$ \\
\hline Philadelphia, Pennsylvania§ & 1,685 & 27.3 & 1.5 & $(24.4-30.2)$ \\
\hline Phoenix-Mesa-Scottsdale, Arizona & 1,470 & 25.1 & 1.9 & $(21.4-28.7)$ \\
\hline Pittsburgh, Pennsylvania & 2,247 & 29.2 & 1.4 & $(26.5-31.8)$ \\
\hline Ponce, Puerto Rico & 507 & 28.7 & 2.7 & $(23.4-34.0)$ \\
\hline Portland-South Portland, Maine & 2,555 & 24.6 & 1.2 & $(22.2-27.0)$ \\
\hline Portland-Vancouver-Hillsboro, Oregon-Washington & 3,094 & 24.7 & 1.1 & $(22.5-26.8)$ \\
\hline Port St. Lucie, Florida & 985 & 23.2 & 2.5 & $(18.3-28.0)$ \\
\hline Providence-Warwick, Rhode Island-Massachusetts & 7,821 & 27.3 & 1.0 & $(25.4-29.2)$ \\
\hline Provo-Orem, Utah & 1,735 & 24.0 & 1.2 & $(21.7-26.3)$ \\
\hline Raleigh, North Carolina & 624 & 25.0 & 2.0 & $(21.1-29.0)$ \\
\hline Rapid City, South Dakota & 832 & 28.7 & 2.2 & $(24.3-33.0)$ \\
\hline Reno, Nevada & 1,745 & 23.5 & 1.5 & $(20.6-26.3)$ \\
\hline Richmond, Virginia & 1,222 & 29.1 & 1.8 & $(25.7-32.6)$ \\
\hline Riverside-San Bernardino-Ontario, California & 1,273 & 29.1 & 1.6 & $(25.9-32.3)$ \\
\hline Rochester, New York & 480 & 26.7 & 2.4 & $(21.9-31.5)$ \\
\hline Rockingham County-Strafford County, New Hampshire $§$ & 1,566 & 26.1 & 1.5 & $(23.1-29.1)$ \\
\hline Sacramento-Roseville-Arden-Arcade, California & 834 & 25.7 & 2.2 & $(21.3-30.0)$ \\
\hline St. Louis, Missouri-Illinois & 1,966 & 26.9 & 1.5 & $(24.0-29.9)$ \\
\hline Salem, Oregon & 496 & 33.9 & 3.3 & $(27.5-40.3)$ \\
\hline Salina, Kansas & 497 & 34.1 & 2.8 & $(28.7-39.5)$ \\
\hline Salisbury, Maryland-Delaware & 1,923 & 31.3 & 1.7 & $(27.9-34.7)$ \\
\hline Salt Lake City, Utah & 4,395 & 25.0 & 0.8 & $(23.4-26.6)$ \\
\hline San Antonio-New Braunfels, Texas & 884 & 35.6 & 2.2 & $(31.4-39.9)$ \\
\hline
\end{tabular}

See table footnotes on page 91. 
TABLE 40. (Continued) Age-adjusted* prevalence estimates of adults aged $\geq 18$ years with obesity, ${ }^{\dagger}$ by metropolitan and micropolitan statistical area - Behavioral Risk Factor Surveillance System, United States, 2013

\begin{tabular}{|c|c|c|c|c|}
\hline MMSA & $\begin{array}{l}\text { Sample } \\
\text { size }\end{array}$ & $\%$ & SE & $95 \% \mathrm{Cl}$ \\
\hline San Francisco-Redwood City-South San Francisco, California ${ }^{\S}$ & 497 & 12.1 & 1.7 & $(8.9-15.4)$ \\
\hline San Jose-Sunnyvale-Santa Clara, California & 577 & 18.3 & 2.3 & $(13.8-22.7)$ \\
\hline San Juan-Carolina-Caguas, Puerto Rico & 3,511 & 28.5 & 1.0 & $(26.4-30.5)$ \\
\hline Scottsbluff, Nebraska & 683 & 37.0 & 2.8 & $(31.5-42.4)$ \\
\hline Scranton-Wilkes-Barre-Hazleton, Pennsylvania & 540 & 30.3 & 3.0 & $(24.4-36.1)$ \\
\hline Seattle-Bellevue-Everett, Washington & 3,570 & 23.2 & 0.9 & $(21.4-24.9)$ \\
\hline Shreveport-Bossier City, Louisiana & 543 & 33.9 & 3.4 & $(27.2-40.6)$ \\
\hline Silver Spring-Frederick-Rockville, Maryland ${ }^{\S}$ & 2,241 & 19.8 & 1.2 & $(17.3-22.2)$ \\
\hline Sioux City, lowa-Nebraska-South Dakota & 984 & 36.9 & 3.2 & $(30.5-43.2)$ \\
\hline Sioux Falls, South Dakota & 949 & 27.0 & 2.0 & $(23.1-30.9)$ \\
\hline Spartanburg, South Carolina & 566 & 30.7 & 3.0 & $(24.8-36.6)$ \\
\hline Spokane-Spokane Valley, Washington & 815 & 29.8 & 2.3 & $(25.3-34.2)$ \\
\hline Springfield, Massachusetts & 1,449 & 25.7 & 1.9 & $(21.9-29.5)$ \\
\hline Tallahassee, Florida & 1,769 & 29.6 & 2.1 & $(25.5-33.7)$ \\
\hline Tampa-St. Petersburg-Clearwater, Florida & 2,117 & 27.3 & 1.4 & $(24.5-30.0)$ \\
\hline Toledo, Ohio & 933 & 30.0 & 2.2 & $(25.6-34.4)$ \\
\hline Topeka, Kansas & 2,268 & 32.3 & 1.3 & $(29.7-34.9)$ \\
\hline Tulsa, Oklahoma & 1,899 & 32.3 & 1.5 & $(29.4-35.3)$ \\
\hline Virginia Beach-Norfolk-Newport News, Virginia-North Carolina & 1,573 & 27.9 & 1.6 & $(24.8-31.0)$ \\
\hline Warren-Troy-Farmington Hills, Michigan ${ }^{\S}$ & 2,172 & 29.3 & 1.4 & $(26.6-32.0)$ \\
\hline $\begin{array}{l}\text { Washington-Arlington-Alexandria, District of Columbia- } \\
\text { Virginia-Maryland-West Virginias }\end{array}$ & 8,558 & 24.3 & 0.8 & $(22.6-25.9)$ \\
\hline Wichita, Kansas & 4,689 & 31.3 & 0.9 & $(29.6-33.0)$ \\
\hline Wilmington, Delaware-Maryland-New Jersey ${ }^{\S}$ & 3,066 & 29.6 & 1.2 & $(27.3-31.9)$ \\
\hline Winston-Salem, North Carolina & 652 & 30.6 & 2.7 & $(25.4-35.8)$ \\
\hline Worcester, Massachusetts-Connecticut & 2,595 & 26.5 & 1.4 & $(23.7-29.2)$ \\
\hline Median & & 28.3 & & \\
\hline Range & & $12.1-37.1$ & & \\
\hline
\end{tabular}

Abbreviations: $\mathrm{Cl}$ = confidence interval; MMSA = metropolitan and micropolitan statistical areas; $\mathrm{SE}=$ standard error.

* Age adjusted to the 2000 U.S. standard population.

† Body mass index $\geq 30$.

$\S$ Metropolitan division. 
TABLE 41. Age-adjusted* prevalence estimates of adults aged $\geq 18$ years with obesity, ${ }^{\dagger}$ by state/territory - Behavioral Risk Factor Surveillance System, United States, 2014

\begin{tabular}{|c|c|c|c|c|}
\hline State/Territory & $\begin{array}{c}\text { Sample } \\
\text { size }\end{array}$ & $\%$ & SE & $95 \% \mathrm{Cl}$ \\
\hline Alabama & 8,190 & 33.3 & 0.8 & $(31.7-34.8)$ \\
\hline Alaska & 4,142 & 29.3 & 1.0 & $(27.3-31.2)$ \\
\hline Arizona & 13,849 & 28.9 & 0.7 & $(27.5-30.2)$ \\
\hline Arkansas & 4,886 & 35.9 & 1.2 & $(33.6-38.2)$ \\
\hline California & 8,025 & 24.4 & 0.6 & $(23.2-25.6)$ \\
\hline Colorado & 12,567 & 21.0 & 0.5 & $(20.0-21.9)$ \\
\hline Connecticut & 7,237 & 25.4 & 0.8 & (23.9-26.9) \\
\hline Delaware & 3,983 & 30.0 & 1.1 & $(27.8-32.2)$ \\
\hline District of Columbia & 3,787 & 22.0 & 1.1 & $(19.9-24.1)$ \\
\hline Florida & 9,156 & 26.1 & 0.7 & $(24.7-27.4)$ \\
\hline Georgia & 5,844 & 30.1 & 0.8 & $(28.5-31.8)$ \\
\hline Hawaii & 6,905 & 22.4 & 0.7 & $(20.9-23.8)$ \\
\hline Idaho & 5,125 & 28.8 & 1.0 & $(26.9-30.7)$ \\
\hline Illinois & 4,835 & 28.7 & 0.9 & $(26.9-30.5)$ \\
\hline Indiana & 10,773 & 32.3 & 0.6 & $(31.1-33.6)$ \\
\hline lowa & 7,572 & 30.3 & 0.7 & $(28.8-31.7)$ \\
\hline Kansas & 12,825 & 30.9 & 0.5 & $(29.8-31.9)$ \\
\hline Kentucky & 10,442 & 31.3 & 0.8 & $(29.7-32.8)$ \\
\hline Louisiana & 6,366 & 34.6 & 0.8 & $(33.0-36.2)$ \\
\hline Maine & 8,705 & 27.5 & 0.7 & $(26.1-28.9)$ \\
\hline Maryland & 11,713 & 29.0 & 0.8 & $(27.4-30.6)$ \\
\hline Massachusetts & 14,394 & 22.6 & 0.6 & $(21.5-23.7)$ \\
\hline Michigan & 8,024 & 29.8 & 0.7 & $(28.5-31.2)$ \\
\hline Minnesota & 15,309 & 27.1 & 0.5 & $(26.2-28.0)$ \\
\hline Mississippi & 3,975 & 35.5 & 1.1 & $(33.3-37.7)$ \\
\hline Missouri & 6,695 & 30.0 & 0.9 & $(28.3-31.8)$ \\
\hline Montana & 7,064 & 26.0 & 0.8 & $(24.4-27.7)$ \\
\hline Nebraska & 21,130 & 29.9 & 0.5 & $(28.8-30.9)$ \\
\hline Nevada & 3,555 & 27.7 & 1.2 & $(25.3-30.2)$ \\
\hline New Hampshire & 5,730 & 26.8 & 0.9 & $(25.0-28.5)$ \\
\hline New Jersey & 11,955 & 26.3 & 0.6 & $(25.0-27.5)$ \\
\hline New Mexico & 8,284 & 28.6 & 0.8 & $(27.0-30.2)$ \\
\hline New York & 6,325 & 26.4 & 0.7 & $(25.0-27.9)$ \\
\hline North Carolina & 6,621 & 29.5 & 0.7 & $(28.1-30.9)$ \\
\hline North Dakota & 7,269 & 32.1 & 0.9 & $(30.2-33.9)$ \\
\hline Ohio & 10,313 & 31.7 & 0.8 & $(30.2-33.2)$ \\
\hline Oklahoma & 8,009 & 32.7 & 0.7 & $(31.3-34.0)$ \\
\hline Oregon & 4,919 & 27.5 & 0.9 & $(25.7-29.3)$ \\
\hline Pennsylvania & 10,374 & 29.8 & 0.7 & $(28.4-31.1)$ \\
\hline Rhode Island & 5,969 & 26.7 & 0.9 & $(25.0-28.5)$ \\
\hline South Carolina & 10,393 & 31.8 & 0.7 & $(30.5-33.1)$ \\
\hline South Dakota & 6,960 & 29.5 & 1.0 & $(27.5-31.5)$ \\
\hline Tennessee & 4,793 & 30.9 & 1.0 & $(28.9-33.0)$ \\
\hline Texas & 14,058 & 31.5 & 0.7 & $(30.2-32.9)$ \\
\hline Utah & 13,965 & 25.9 & 0.4 & $(25.1-26.8)$ \\
\hline Vermont & 6,068 & 24.1 & 0.7 & $(22.7-25.5)$ \\
\hline Virginia & 8,879 & 28.0 & 0.6 & $(26.7-29.2)$ \\
\hline Washington & 9,444 & 26.8 & 0.7 & $(25.5-28.1)$ \\
\hline West Virginia & 5,846 & 35.5 & 0.8 & $(33.9-37.2)$ \\
\hline Wisconsin & 6,655 & 30.8 & 0.9 & $(29.1-32.5)$ \\
\hline Wyoming & 5,973 & 29.3 & 1.1 & $(27.2-31.5)$ \\
\hline Guam & 2,352 & 27.7 & 1.2 & $(25.3-30.2)$ \\
\hline Puerto Rico & 5,743 & 28.0 & 0.8 & $(26.5-29.6)$ \\
\hline Median & & 29.0 & & \\
\hline Range & & $21.0-35.9$ & & \\
\hline
\end{tabular}

Abbreviations: $\mathrm{Cl}=$ confidence interval; $\mathrm{SE}=$ standard error.

* Age adjusted to the 2000 U.S. standard population.

† Body mass index $\geq 30$. 
TABLE 42. Age-adjusted* prevalence estimates of adults aged $\geq 18$ years with obesity, ${ }^{\dagger}$ by metropolitan and micropolitan statistical area Behavioral Risk Factor Surveillance System, United States, 2014

\begin{tabular}{|c|c|c|c|c|}
\hline MMSA & $\begin{array}{c}\text { Sample } \\
\text { size }\end{array}$ & $\%$ & SE & $95 \% \mathrm{Cl}$ \\
\hline Aberdeen, South Dakota & 573 & 33.2 & 3.0 & $(27.3-39.1)$ \\
\hline Aguadilla-Isabela, Puerto Rico & 522 & 29.7 & 2.6 & $(24.6-34.7)$ \\
\hline Albuquerque, New Mexico & 1,668 & 27.6 & 1.5 & $(24.6-30.6)$ \\
\hline Allentown-Bethlehem-Easton, Pennsylvania-New Jersey & 1,018 & 30.1 & 2.5 & $(25.2-35.1)$ \\
\hline Anchorage, Alaska & 1,689 & 29.5 & 1.4 & $(26.7-32.2)$ \\
\hline Atlanta-Sandy Springs-Roswell, Georgia & 2,568 & 28.0 & 1.1 & $(25.7-30.2)$ \\
\hline Augusta-Richmond County, Georgia-South Carolina & 832 & 28.6 & 2.7 & $(23.3-33.9)$ \\
\hline Austin-Round Rock, Texas & 2,084 & 25.7 & 1.3 & $(23.2-28.2)$ \\
\hline Baltimore-Columbia-Towson, Maryland & 4,298 & 29.9 & 1.3 & $(27.4-32.5)$ \\
\hline Baton Rouge, Louisiana & 868 & 35.5 & 2.1 & $(31.4-39.6)$ \\
\hline Berlin, New Hampshire-Vermont & 511 & 33.1 & 6.3 & $(20.8-45.4)$ \\
\hline Billings, Montana & 754 & 28.7 & 2.3 & $(24.2-33.2)$ \\
\hline Birmingham-Hoover, Alabama & 1,488 & 32.4 & 1.7 & $(29.2-35.7)$ \\
\hline Bismarck, North Dakota & 962 & 31.0 & 2.4 & $(26.4-35.7)$ \\
\hline Boise City, Idaho & 1,267 & 30.0 & 1.9 & $(26.3-33.7)$ \\
\hline Boston, Massachusetts $§$ & 4,161 & 22.3 & 1.0 & $(20.3-24.2)$ \\
\hline Burlington-South Burlington, Vermont & 1,852 & 20.3 & 1.1 & $(18.1-22.5)$ \\
\hline Cambridge-Newton-Framingham, Massachusetts $§$ & 4,774 & 21.8 & 1.0 & $(19.9-23.7)$ \\
\hline Camden, New Jersey ${ }^{\S}$ & 1,608 & 28.9 & 1.8 & $(25.4-32.4)$ \\
\hline Cedar Rapids, lowa & 586 & 33.6 & 2.7 & $(28.3-38.8)$ \\
\hline Charleston, West Virginia & 830 & 34.6 & 2.2 & $(30.3-38.8)$ \\
\hline Charleston-North Charleston, South Carolina & 1,319 & 31.7 & 1.8 & $(28.1-35.2)$ \\
\hline Charlotte-Concord-Gastonia, North Carolina-South Carolina & 1,967 & 26.6 & 1.4 & $(23.8-29.3)$ \\
\hline Chicago-Naperville-Elgin, Illinois-Indiana-Wisconsin & 3,906 & 27.2 & 1.0 & $(25.2-29.3)$ \\
\hline Cincinnati, Ohio-Kentucky-Indiana & 1,911 & 31.7 & 1.8 & $(28.1-35.3)$ \\
\hline Claremont-Lebanon, New Hampshire-Vermont & 1,587 & 22.8 & 1.5 & $(19.9-25.6)$ \\
\hline Cleveland-Elyria, Ohio & 917 & 28.0 & 2.1 & $(23.9-32.1)$ \\
\hline College Station-Bryan, Texas & 530 & 34.0 & 4.0 & $(26.3-41.8)$ \\
\hline Colorado Springs, Colorado & 1,226 & 24.2 & 1.6 & $(21.1-27.4)$ \\
\hline Columbia, South Carolina & 1,130 & 33.0 & 1.8 & $(29.4-36.6)$ \\
\hline Columbus, Ohio & 1,578 & 31.9 & 1.8 & $(28.5-35.4)$ \\
\hline Corpus Christi, Texas & 576 & 42.5 & 4.1 & $(34.4-50.6)$ \\
\hline Dallas-Plano-Irving, Texas ${ }^{\S}$ & 1,192 & 29.0 & 2.0 & $(25.0-32.9)$ \\
\hline Dayton, Ohio & 538 & 36.5 & 2.9 & $(30.8-42.2)$ \\
\hline Denver-Aurora-Lakewood, Colorado & 5,400 & 19.8 & 0.7 & $(18.5-21.1)$ \\
\hline Des Moines-West Des Moines, lowa & 1,254 & 27.5 & 1.8 & $(24.0-30.9)$ \\
\hline Duluth, Minnesota-Wisconsin & 893 & 28.3 & 2.1 & $(24.2-32.4)$ \\
\hline El Paso, Texas & 645 & 33.8 & 2.6 & $(28.8-38.9)$ \\
\hline Evansville, Indiana-Kentucky & 603 & 34.6 & 3.5 & $(27.7-41.6)$ \\
\hline Fargo, North Dakota-Minnesota & 1,083 & 27.7 & 1.8 & $(24.3-31.2)$ \\
\hline Fayetteville-Springdale-Rogers, Arkansas-Missouri & 758 & 31.5 & 2.9 & $(25.8-37.2)$ \\
\hline Fort Wayne, Indiana & 797 & 31.9 & 2.5 & $(27.0-36.8)$ \\
\hline Fort Worth-Arlington, Texas ${ }^{\S}$ & 698 & 30.7 & 2.5 & $(25.8-35.6)$ \\
\hline Grand Island, Nebraska & 981 & 35.6 & 2.6 & $(30.6-40.6)$ \\
\hline Grand Rapids-Wyoming, Michigan & 849 & 26.0 & 1.8 & $(22.5-29.5)$ \\
\hline Greensboro-High Point, North Carolina & 462 & 34.9 & 3.2 & $(28.7-41.1)$ \\
\hline Greenville-Anderson-Mauldin, South Carolina & 1,402 & 31.4 & 1.7 & $(28.1-34.8)$ \\
\hline Hagerstown-Martinsburg, Maryland-West Virginia & 739 & 28.8 & 2.8 & $(23.2-34.3)$ \\
\hline Hartford-West Hartford-East Hartford, Connecticut & 2,441 & 24.9 & 1.1 & $(22.6-27.1)$ \\
\hline Hilton Head Island-Bluffton-Beaufort, South Carolina & 524 & 22.4 & 2.6 & $(17.3-27.5)$ \\
\hline Houston-The Woodlands-Sugar Land, Texas & 1,943 & 31.0 & 1.7 & $(27.7-34.4)$ \\
\hline Huntington-Ashland, West Virginia-Kentucky-Ohio & 1,168 & 35.7 & 2.1 & $(31.6-39.7)$ \\
\hline Idaho Falls, Idaho & 487 & 32.9 & 2.8 & $(27.3-38.4)$ \\
\hline Indianapolis-Carmel-Anderson, Indiana & 3,391 & 31.9 & 1.2 & $(29.6-34.2)$ \\
\hline Jacksonville, Florida & 631 & 29.8 & 2.7 & $(24.6-35.0)$ \\
\hline Kansas City, Missouri-Kansas & 4,576 & 30.0 & 1.2 & $(27.6-32.4)$ \\
\hline Kingsport-Bristol-Bristol, Tennessee-Virginia & 477 & 29.3 & 3.6 & $(22.1-36.4)$ \\
\hline Knoxville, Tennessee & 531 & 30.7 & 3.3 & $(24.2-37.2)$ \\
\hline Lafayette, Louisiana & 518 & 33.2 & 2.6 & $(28.1-38.4)$ \\
\hline Lexington-Fayette, Kentucky & 592 & 25.9 & 2.3 & $(21.5-30.4)$ \\
\hline Lincoln, Nebraska & 1,909 & 25.5 & 1.3 & $(22.9-28.0)$ \\
\hline
\end{tabular}

See table footnotes on page 95 . 
TABLE 42. (Continued) Age-adjusted* prevalence estimates of adults aged $\geq 18$ years with obesity, ${ }^{\dagger}$ by metropolitan and micropolitan statistical area - Behavioral Risk Factor Surveillance System, United States, 2014

\begin{tabular}{|c|c|c|c|c|}
\hline MMSA & $\begin{array}{l}\text { Sample } \\
\text { size }\end{array}$ & $\%$ & SE & $95 \% \mathrm{Cl}$ \\
\hline Little Rock-North Little Rock-Conway, Arkansas & 1,096 & 32.1 & 2.1 & $(27.9-36.2)$ \\
\hline Logan, Utah-Idaho & 578 & 22.8 & 1.9 & $(19.0-26.5)$ \\
\hline Los Angeles-Long Beach-Anaheim, California & 2,170 & 22.5 & 1.1 & $(20.3-24.7)$ \\
\hline Louisville-Jefferson County, Kentucky-Indiana & 2,323 & 30.5 & 1.8 & $(27.0-33.9)$ \\
\hline Madison, Wisconsin & 517 & 26.8 & 2.4 & $(22.0-31.6)$ \\
\hline Memphis, Tennessee-Mississippi-Arkansas & 826 & 34.1 & 2.8 & $(28.7-39.6)$ \\
\hline Miami-Fort Lauderdale-West Palm Beach, Florida & 2,027 & 24.3 & 1.4 & $(21.6-27.1)$ \\
\hline Milwaukee-Waukesha-West Allis, Wisconsin & 1,285 & 30.6 & 2.0 & $(26.7-34.6)$ \\
\hline Minneapolis-St. Paul-Bloomington, Minnesota-Wisconsin & 8,147 & 24.8 & 0.6 & $(23.6-25.9)$ \\
\hline Minot, North Dakota & 559 & 30.3 & 3.0 & $(24.5-36.1)$ \\
\hline Montgomery, Alabama & 482 & 33.2 & 2.9 & $(27.6-38.8)$ \\
\hline $\begin{array}{l}\text { Montgomery County-Bucks County-Chester County, } \\
\text { Pennsylvania§ }\end{array}$ & 765 & 23.4 & 2.1 & $(19.2-27.6)$ \\
\hline $\begin{array}{l}\text { Myrtle Beach-Conway-North Myrtle Beach, South Carolina- } \\
\text { North Carolina }\end{array}$ & 935 & 30.0 & 2.2 & $(25.7-34.3)$ \\
\hline Nashville-Davidson County-Murfreesboro-Franklin, Tennessee & 743 & 27.2 & 2.1 & $(23.1-31.3)$ \\
\hline Nassau County-Suffolk County, New York ${ }^{\S}$ & 706 & 23.7 & 2.1 & $(19.6-27.9)$ \\
\hline Newark, New Jersey-Pennsylvania§ & 3,796 & 26.1 & 1.1 & $(24.0-28.3)$ \\
\hline New Orleans-Metairie, Louisiana & 1,814 & 33.9 & 1.5 & $(31.0-36.8)$ \\
\hline New York-Jersey City-White Plains, New York-New Jersey ${ }^{\S}$ & 6,868 & 24.4 & 0.8 & $(22.9-25.9)$ \\
\hline Norfolk, Nebraska & 917 & 31.0 & 2.0 & $(27.2-34.9)$ \\
\hline North Platte, Nebraska & 921 & 31.9 & 2.1 & $(27.9-36.0)$ \\
\hline North Port-Sarasota-Bradenton, Florida & 477 & 26.9 & 4.3 & $(18.4-35.3)$ \\
\hline Oakland-Hayward-Berkeley, California§ & 647 & 24.4 & 2.2 & $(20.1-28.7)$ \\
\hline Ogden-Clearfield, Utah & 2,742 & 27.8 & 1.0 & $(25.9-29.7)$ \\
\hline Oklahoma City, Oklahoma & 2,295 & 30.6 & 1.2 & $(28.2-33.0)$ \\
\hline Omaha-Council Bluffs, Nebraska-lowa & 4,578 & 29.3 & 1.0 & $(27.4-31.2)$ \\
\hline Orlando-Kissimmee-Sanford, Florida & 897 & 26.3 & 2.0 & $(22.4-30.2)$ \\
\hline Philadelphia, Pennsylvania ${ }^{\S}$ & 1,429 & 29.9 & 1.8 & $(26.3-33.5)$ \\
\hline Phoenix-Mesa-Scottsdale, Arizona & 8,730 & 28.6 & 0.8 & $(27.1-30.2)$ \\
\hline Pittsburgh, Pennsylvania & 2,292 & 31.4 & 1.5 & $(28.5-34.3)$ \\
\hline Ponce, Puerto Rico & 511 & 30.9 & 2.8 & $(25.5-36.4)$ \\
\hline Portland-South Portland, Maine & 2,613 & 24.0 & 1.2 & $(21.6-26.5)$ \\
\hline Portland-Vancouver-Hillsboro, Oregon-Washington & 2,675 & 24.1 & 1.2 & $(21.7-26.4)$ \\
\hline Providence-Warwick, Rhode Island-Massachusetts & 7,503 & 26.5 & 0.9 & $(24.7-28.3)$ \\
\hline Provo-Orem, Utah & 1,995 & 25.6 & 1.1 & $(23.5-27.8)$ \\
\hline Raleigh, North Carolina & 660 & 23.9 & 2.0 & $(20.1-27.8)$ \\
\hline Rapid City, South Dakota & 1,337 & 26.9 & 2.1 & $(22.7-31.0)$ \\
\hline Reno, Nevada & 1,146 & 19.7 & 1.6 & $(16.7-22.8)$ \\
\hline Richmond, Virginia & 1,364 & 28.6 & 1.6 & $(25.4-31.7)$ \\
\hline Riverside-San Bernardino-Ontario, California & 859 & 27.7 & 2.0 & $(23.9-31.6)$ \\
\hline Roanoke, Virginia & 500 & 27.5 & 2.9 & $(21.8-33.2)$ \\
\hline Rochester, Minnesota & 667 & 24.1 & 1.9 & $(20.4-27.8)$ \\
\hline Rockingham County-Strafford County, New Hampshire $§$ & 1,350 & 26.2 & 1.7 & $(22.9-29.5)$ \\
\hline Sacramento-Roseville-Arden-Arcade, California & 594 & 26.1 & 2.3 & $(21.5-30.7)$ \\
\hline St. Cloud, Minnesota & 525 & 29.3 & 2.3 & $(24.9-33.8)$ \\
\hline St. Louis, Missouri-Illinois & 1,807 & 27.6 & 1.7 & $(24.2-31.0)$ \\
\hline Salisbury, Maryland-Delaware & 1,804 & 32.5 & 2.1 & $(28.3-36.6)$ \\
\hline Salt Lake City, Utah & 5,010 & 26.5 & 0.7 & $(25.0-27.9)$ \\
\hline San Antonio-New Braunfels, Texas & 2,117 & 30.2 & 1.3 & $(27.6-32.8)$ \\
\hline San Juan-Carolina-Caguas, Puerto Rico & 3,615 & 27.3 & 0.9 & $(25.4-29.1)$ \\
\hline Scottsbluff, Nebraska & 854 & 35.1 & 2.4 & $(30.4-39.8)$ \\
\hline Seattle-Bellevue-Everett, Washington ${ }^{\S}$ & 3,459 & 22.7 & 1.0 & $(20.8-24.6)$ \\
\hline Shreveport-Bossier City, Louisiana & 515 & 36.0 & 3.0 & $(30.2-41.8)$ \\
\hline Silver Spring-Frederick-Rockville, Maryland ${ }^{\S}$ & 2,227 & 21.5 & 1.5 & $(18.5-24.5)$ \\
\hline Sioux City, lowa-Nebraska-South Dakota & 1,069 & 30.5 & 2.7 & $(25.1-35.8)$ \\
\hline Sioux Falls, South Dakota & 1,260 & 27.3 & 2.0 & $(23.4-31.1)$ \\
\hline Spartanburg, South Carolina & 531 & 28.5 & 3.0 & $(22.6-34.4)$ \\
\hline Spokane-Spokane Valley, Washington & 688 & 31.1 & 2.4 & $(26.3-35.9)$ \\
\hline Springfield, Massachusetts & 1,024 & 23.9 & 2.1 & $(19.8-28.0)$ \\
\hline Tampa-St. Petersburg-Clearwater, Florida & 1,476 & 28.7 & 1.8 & $(25.1-32.3)$ \\
\hline
\end{tabular}

See table footnotes on page 95. 
TABLE 42. (Continued) Age-adjusted* prevalence estimates of adults aged $\geq 18$ years with obesity, ${ }^{\dagger}$ by metropolitan and micropolitan statistical area - Behavioral Risk Factor Surveillance System, United States, 2014

\begin{tabular}{|c|c|c|c|c|}
\hline MMSA & $\begin{array}{l}\text { Sample } \\
\text { size }\end{array}$ & $\%$ & SE & $95 \% \mathrm{Cl}$ \\
\hline Toledo, Ohio & 619 & 36.7 & 2.8 & $(31.3-42.2)$ \\
\hline Topeka, Kansas & 1,360 & 32.9 & 1.7 & $(29.6-36.3)$ \\
\hline Tulsa, Oklahoma & 1,916 & 30.2 & 1.4 & $(27.6-32.9)$ \\
\hline Tuscaloosa, Alabama & 680 & 32.2 & 2.2 & $(27.8-36.5)$ \\
\hline Virginia Beach-Norfolk-Newport News, Virginia-North Carolina & 1,771 & 31.0 & 1.5 & $(28.0-34.0)$ \\
\hline Warren-Troy-Farmington Hills, Michigan ${ }^{\S}$ & 2,012 & 27.8 & 1.4 & $(25.1-30.6)$ \\
\hline $\begin{array}{l}\text { Washington-Arlington-Alexandria, District of Columbia- } \\
\text { Virginia-Maryland-West Virginia } \$\end{array}$ & 7,725 & 24.1 & 0.9 & $(22.4-25.8)$ \\
\hline Wichita, Kansas & 2,561 & 32.0 & 1.1 & $(29.8-34.2)$ \\
\hline Wichita Falls, Texas & 502 & 30.8 & 4.5 & $(22.0-39.7)$ \\
\hline Wilmington, Delaware-Maryland-New Jersey ${ }^{\S}$ & 2,575 & 30.6 & 1.4 & $(27.8-33.4)$ \\
\hline Worcester, Massachusetts-Connecticut & 2,239 & 25.4 & 1.4 & $(22.8-28.1)$ \\
\hline Youngstown-Warren-Boardman, Ohio-Pennsylvania & 495 & 29.5 & 3.4 & $(22.8-36.1)$ \\
\hline Median & & 29.3 & & \\
\hline Range & & $19.7-42.5$ & & \\
\hline
\end{tabular}

Abbreviations: $\mathrm{Cl}=$ confidence interval; $\mathrm{MMSA}=$ metropolitan and micropolitan statistical areas; $\mathrm{SE}=$ standard error.

* Age adjusted to the 2000 U.S. standard population.

${ }^{\dagger}$ Body mass index $\geq 30$.

$\S$ Metropolitan division. 
TABLE 43. Age-adjusted* prevalence estimates of adults aged $\geq 45$ years who have ever been told by a health professional they have diabetes, ${ }^{\dagger}$ by state/territory — Behavioral Risk Factor Surveillance System, United States, 2013

\begin{tabular}{|c|c|c|c|c|}
\hline State/Territory & $\begin{array}{l}\text { Sample } \\
\text { size }\end{array}$ & $\%$ & SE & $95 \% \mathrm{Cl}$ \\
\hline Alabama & 4,837 & 21.5 & 0.8 & (19.9-23.1) \\
\hline Alaska & 2,715 & 13.7 & 1.0 & $(11.7-15.7)$ \\
\hline Arizona & 3,063 & 17.9 & 1.3 & $(15.3-20.6)$ \\
\hline Arkansas & 3,945 & 17.5 & 0.9 & $(15.8-19.2)$ \\
\hline California & 6,863 & 18.2 & 0.7 & (16.8-19.6) \\
\hline Colorado & 9,231 & 11.1 & 0.4 & $(10.3-12.0)$ \\
\hline Connecticut & 5,341 & 13.0 & 0.6 & $(11.8-14.2)$ \\
\hline Delaware & 3,678 & 17.1 & 0.8 & $(15.6-18.7)$ \\
\hline District of Columbia & 3,478 & 16.0 & 0.9 & $(14.2-17.9)$ \\
\hline Florida & 26,015 & 17.2 & 0.6 & $(16.1-18.3)$ \\
\hline Georgia & 5,396 & 18.7 & 0.7 & $(17.3-20.1)$ \\
\hline Hawaii & 4,776 & 14.0 & 0.8 & $(12.4-15.7)$ \\
\hline Idaho & 3,880 & 13.5 & 0.8 & $(12.1-15.0)$ \\
\hline Illinois & 3,912 & 16.6 & 0.8 & $(15.0-18.3)$ \\
\hline Indiana & 7,375 & 18.3 & 0.6 & $(17.2-19.4)$ \\
\hline lowa & 5,918 & 14.8 & 0.6 & (13.7-15.9) \\
\hline Kansas & 16,075 & 15.7 & 0.3 & $(15.0-16.3)$ \\
\hline Kentucky & 7,792 & 16.8 & 0.6 & $(15.6-18.1)$ \\
\hline Louisiana & 3,803 & 19.6 & 0.9 & $(17.8-21.4)$ \\
\hline Maine & 6,015 & 14.3 & 0.6 & $(13.1-15.4)$ \\
\hline Maryland & 9,246 & 16.2 & 0.6 & $(15.1-17.3)$ \\
\hline Massachusetts & 10,399 & 13.8 & 0.5 & $(12.7-14.9)$ \\
\hline Michigan & 8,954 & 16.3 & 0.5 & $(15.2-17.3)$ \\
\hline Minnesota & 9,688 & 12.6 & 0.7 & $(11.2-14.0)$ \\
\hline Mississippi & 5,497 & 20.1 & 0.7 & (18.6-21.6) \\
\hline Missouri & 5,057 & 14.8 & 0.7 & $(13.4-16.1)$ \\
\hline Montana & 6,703 & 11.6 & 0.5 & $(10.6-12.6)$ \\
\hline Nebraska & 12,159 & 15.1 & 0.5 & $(14.0-16.2)$ \\
\hline Nevada & 3,441 & 16.3 & 1.4 & $(13.6-18.9)$ \\
\hline New Hampshire & 4,743 & 14.4 & 0.7 & $(13.1-15.7)$ \\
\hline New Jersey & 8,465 & 15.5 & 0.6 & $(14.2-16.7)$ \\
\hline New Mexico & 6,323 & 17.1 & 0.8 & (15.6-18.6) \\
\hline New York & 5,673 & 18.0 & 0.7 & (16.5-19.4) \\
\hline North Carolina & 6,032 & 18.8 & 0.7 & $(17.4-20.1)$ \\
\hline North Dakota & 5,335 & 15.0 & 0.7 & $(13.7-16.3)$ \\
\hline Ohio & 8,426 & 16.5 & 0.6 & $(15.4-17.7)$ \\
\hline Oklahoma & 5,816 & 17.8 & 0.6 & $(16.6-19.0)$ \\
\hline Oregon & 4,239 & 14.9 & 0.8 & (13.4-16.4) \\
\hline Pennsylvania & 7,956 & 15.8 & 0.6 & (14.7-16.8) \\
\hline Rhode Island & 4,636 & 14.7 & 0.7 & (13.4-16.0) \\
\hline South Carolina & 7,664 & 20.1 & 0.7 & (18.8-21.4) \\
\hline South Dakota & 4,652 & 14.2 & 0.9 & $(12.5-15.9)$ \\
\hline Tennessee & 3,815 & 21.1 & 1.0 & $(19.2-23.0)$ \\
\hline Texas & 7,098 & 19.3 & 0.8 & $(17.7-20.8)$ \\
\hline Utah & 7,395 & 14.5 & 0.5 & $(13.4-15.5)$ \\
\hline Vermont & 4,673 & 12.2 & 0.6 & $(11.0-13.3)$ \\
\hline Virginia & 5,648 & 16.5 & 0.7 & $(15.2-17.8)$ \\
\hline Washington & 7,837 & 14.2 & 0.5 & $(13.2-15.3)$ \\
\hline West Virginia & 4,063 & 19.5 & 0.7 & (18.1-20.9) \\
\hline Wisconsin & 4,630 & 13.5 & 0.8 & $(11.9-15.0)$ \\
\hline Wyoming & 4,938 & 13.2 & 0.6 & $(12.0-14.4)$ \\
\hline Guam & 761 & 27.5 & 2.4 & $(22.8-32.3)$ \\
\hline Puerto Rico & 3,905 & 24.7 & 0.8 & $(23.1-26.4)$ \\
\hline Median & & 16.2 & & \\
\hline Range & & $11.1-27.5$ & & \\
\hline
\end{tabular}

Abbreviations: $\mathrm{Cl}=$ confidence interval; $\mathrm{SE}=$ standard error

* Age adjusted to the 2000 U.S. standard population.

† Excluding prediabetes or borderline diabetes and diabetes during pregnancy for women. 
TABLE 44. Age-adjusted* prevalence estimates of adults aged $\geq 45$ years who have ever been told by a health professional they have diabetes, ${ }^{\dagger}$ by metropolitan and micropolitan statistical area - Behavioral Risk Factor Surveillance System, United States, 2013

\begin{tabular}{|c|c|c|c|c|}
\hline MMSA & $\begin{array}{l}\text { Sample } \\
\text { size }\end{array}$ & $\%$ & SE & $95 \% \mathrm{Cl}$ \\
\hline Aguadilla-Isabela, Puerto Rico & 397 & 24.8 & 2.6 & $(19.6-29.9)$ \\
\hline Akron, Ohio & 529 & 14.5 & 2.0 & $(10.6-18.3)$ \\
\hline Albuquerque, New Mexico & 1,311 & 16.4 & 1.5 & $(13.4-19.4)$ \\
\hline Allentown-Bethlehem-Easton, Pennsylvania-New Jersey & 701 & 16.0 & 2.3 & $(11.4-20.6)$ \\
\hline Anchorage, Alaska & 898 & 14.5 & 1.6 & $(11.3-17.7)$ \\
\hline Atlanta-Sandy Springs-Roswell, Georgia & 2,187 & 17.6 & 1.1 & $(15.4-19.9)$ \\
\hline Augusta-Richmond County, Georgia-South Carolina & 650 & 22.0 & 2.3 & $(17.5-26.5)$ \\
\hline Austin-Round Rock, Texas & 585 & 16.2 & 2.2 & $(11.9-20.4)$ \\
\hline Baltimore-Columbia-Towson, Maryland & 3,305 & 16.0 & 0.9 & $(14.3-17.6)$ \\
\hline Baton Rouge, Louisiana & 645 & 23.0 & 2.4 & $(18.4-27.7)$ \\
\hline Billings, Montana & 478 & 11.0 & 1.5 & $(8.1-14.0)$ \\
\hline Birmingham-Hoover, Alabama & 954 & 19.6 & 1.6 & $(16.5-22.7)$ \\
\hline Bismarck, North Dakota & 684 & 12.9 & 1.6 & $(9.7-16.0)$ \\
\hline Boise City, Idaho & 970 & 13.0 & 1.4 & $(10.3-15.8)$ \\
\hline Boston, Massachusetts ${ }^{\S}$ & 2,722 & 14.4 & 1.0 & $(12.4-16.5)$ \\
\hline Buffalo-Cheektowaga-Niagara Falls, New York & 352 & 10.6 & 1.9 & $(7.0-14.2)$ \\
\hline Burlington-South Burlington, Vermont & 1,067 & 11.3 & 1.1 & $(9.0-13.5)$ \\
\hline Cambridge-Newton-Framingham, Massachusetts $\S$ & 3,266 & 13.1 & 1.0 & $(11.1-15.1)$ \\
\hline Camden, New Jersey ${ }^{\S}$ & 1,211 & 16.6 & 1.3 & $(14.0-19.2)$ \\
\hline Cedar Rapids, lowa & 460 & 15.4 & 1.9 & $(11.8-19.1)$ \\
\hline Charleston, West Virginia & 576 & 21.5 & 2.0 & $(17.7-25.3)$ \\
\hline Charleston-North Charleston, South Carolina & 1,061 & 20.6 & 1.8 & $(17.0-24.1)$ \\
\hline Charlotte-Concord-Gastonia, North Carolina-South Carolina & 1,267 & 19.1 & 1.4 & $(16.2-21.9)$ \\
\hline Chattanooga, Tennessee-Georgia & 423 & 23.8 & 2.9 & $(18.2-29.5)$ \\
\hline Chicago-Naperville-Elgin, Illinois-Indiana-Wisconsin & 2,253 & 16.7 & 1.1 & $(14.4-18.9)$ \\
\hline Cincinnati, Ohio-Kentucky-Indiana & 1,806 & 15.7 & 1.2 & $(13.4-18.0)$ \\
\hline Claremont-Lebanon, New Hampshire-Vermont & 1,253 & 14.3 & 2.0 & $(10.4-18.2)$ \\
\hline Cleveland-Elyria, Ohio & 787 & 17.8 & 1.8 & $(14.4-21.3)$ \\
\hline Colorado Springs, Colorado & 920 & 11.6 & 1.3 & $(9.0-14.3)$ \\
\hline Columbia, South Carolina & 960 & 16.0 & 1.8 & $(12.4-19.5)$ \\
\hline Columbus, Ohio & 1,158 & 17.3 & 1.5 & $(14.3-20.3)$ \\
\hline Crestview-Fort Walton Beach-Destin, Florida & 771 & 15.2 & 1.5 & $(12.2-18.2)$ \\
\hline Dallas-Plano-Irving, Texas ${ }^{\S}$ & 514 & 15.0 & 1.9 & $(11.3-18.7)$ \\
\hline Davenport-Moline-Rock Island, lowa-Illinois & 494 & 18.6 & 2.7 & $(13.3-23.8)$ \\
\hline Dayton, Ohio & 611 & 15.4 & 1.7 & $(12.0-18.7)$ \\
\hline Deltona-Daytona Beach-Ormond Beach, Florida & 901 & 15.0 & 1.8 & $(11.4-18.5)$ \\
\hline Denver-Aurora-Lakewood, Colorado & 3,583 & 11.2 & 0.7 & $(9.9-12.5)$ \\
\hline Des Moines-West Des Moines, lowa & 960 & 15.0 & 1.3 & $(12.4-17.6)$ \\
\hline Duluth, Minnesota-Wisconsin & 505 & 16.2 & 2.9 & $(10.5-22.0)$ \\
\hline Durham-Chapel Hill, North Carolina & 421 & 14.5 & 2.2 & $(10.2-18.7)$ \\
\hline El Paso, Texas & 479 & 22.2 & 2.3 & $(17.7-26.6)$ \\
\hline Evansville, Indiana-Kentucky & 428 & 21.9 & 2.8 & $(16.4-27.4)$ \\
\hline Fargo, North Dakota-Minnesota & 718 & 13.4 & 1.8 & $(9.9-16.9)$ \\
\hline Fayetteville-Springdale-Rogers, Arkansas-Missouri & 569 & 12.3 & 1.7 & $(8.9-15.6)$ \\
\hline Fort Smith, Arkansas-Oklahoma & 382 & 18.6 & 2.6 & $(13.5-23.7)$ \\
\hline Fort Wayne, Indiana & 549 & 15.5 & 1.8 & $(11.9-19.1)$ \\
\hline Fort Worth-Arlington, Texas ${ }^{\S}$ & 564 & 24.0 & 2.7 & $(18.8-29.2)$ \\
\hline Gainesville, Florida & 745 & 14.0 & 2.1 & $(10.0-18.0)$ \\
\hline Grand Forks, North Dakota-Minnesota & 340 & 16.7 & 3.2 & $(10.4-23.0)$ \\
\hline Grand Island, Nebraska & 595 & 15.4 & 1.8 & $(11.8-19.0)$ \\
\hline Grand Rapids-Wyoming, Michigan & 880 & 14.7 & 1.4 & $(11.9-17.5)$ \\
\hline Greensboro-High Point, North Carolina & 453 & 20.5 & 2.4 & $(15.8-25.3)$ \\
\hline Greenville-Anderson-Mauldin, South Carolina & 922 & 18.9 & 1.6 & $(15.8-22.1)$ \\
\hline Gulfport-Biloxi-Pascagoula, Mississippi & 548 & 18.7 & 1.9 & $(15.1-22.4)$ \\
\hline Hagerstown-Martinsburg, Maryland-West Virginia & 559 & 17.2 & 1.9 & $(13.4-20.9)$ \\
\hline Hartford-West Hartford-East Hartford, Connecticut & 1,991 & 12.0 & 1.0 & $(10.1-14.0)$ \\
\hline Hilton Head Island-Bluffton-Beaufort, South Carolina & 673 & 14.7 & 1.9 & $(11.0-18.4)$ \\
\hline Houston-The Woodlands-Sugar Land, Texas & 757 & 19.5 & 2.4 & $(14.7-24.2)$ \\
\hline Huntington-Ashland, West Virginia-Kentucky-Ohio & 793 & 23.2 & 1.8 & $(19.6-26.8)$ \\
\hline Idaho Falls, Idaho & 320 & 13.5 & 2.4 & $(8.8-18.2)$ \\
\hline Indianapolis-Carmel-Anderson, Indiana & 1,734 & 18.4 & 1.1 & $(16.2-20.5)$ \\
\hline
\end{tabular}

See table footnotes on page 99. 
TABLE 44. (Continued) Age-adjusted* prevalence estimates of adults aged $\geq 45$ years who have ever been told by a health professional they have diabetes, ${ }^{\dagger}$ by metropolitan and micropolitan statistical area - Behavioral Risk Factor Surveillance System, United States, 2013

\begin{tabular}{|c|c|c|c|c|}
\hline MMSA & $\begin{array}{l}\text { Sample } \\
\text { size }\end{array}$ & $\%$ & SE & $95 \% \mathrm{Cl}$ \\
\hline Jackson, Mississippi & 536 & 12.8 & 1.8 & $(9.4-16.3)$ \\
\hline Jacksonville, Florida & 2,009 & 19.7 & 1.5 & $(16.7-22.7)$ \\
\hline Kansas City, Missouri-Kansas & 5,092 & 14.0 & 1.0 & $(12.0-16.0)$ \\
\hline Kingsport-Bristol-Bristol, Tennessee-Virginia & 397 & 21.9 & 3.0 & $(16.1-27.8)$ \\
\hline Knoxville, Tennessee & 409 & 20.4 & 2.9 & $(14.7-26.0)$ \\
\hline Lansing-East Lansing, Michigan & 462 & 18.4 & 2.2 & $(14.0-22.8)$ \\
\hline Lexington-Fayette, Kentucky & 372 & 15.1 & 2.4 & $(10.3-19.8)$ \\
\hline Lincoln, Nebraska & 1,087 & 14.6 & 1.2 & $(12.3-16.9)$ \\
\hline Little Rock-North Little Rock-Conway, Arkansas & 822 & 19.2 & 2.1 & $(15.1-23.3)$ \\
\hline Logan, Utah-Idaho & 356 & 12.8 & 2.3 & $(8.2-17.4)$ \\
\hline Los Angeles-Long Beach-Anaheim, California & 1,708 & 22.1 & 1.5 & $(19.1-25.1)$ \\
\hline Louisville-Jefferson County, Kentucky-Indiana & 1,525 & 15.0 & 1.4 & $(12.2-17.7)$ \\
\hline Lubbock, Texas & 400 & 16.8 & 2.2 & $(12.6-21.1)$ \\
\hline Manhattan, Kansas & 380 & 12.2 & 1.8 & $(8.6-15.8)$ \\
\hline Memphis, Tennessee-Mississippi-Arkansas & 811 & 22.8 & 2.2 & $(18.6-27.1)$ \\
\hline Miami-Fort Lauderdale-West Palm Beach, Florida & 1,561 & 16.8 & 1.6 & $(13.7-19.9)$ \\
\hline Milwaukee-Waukesha-West Allis, Wisconsin & 887 & 13.5 & 1.8 & $(10.0-17.1)$ \\
\hline Minneapolis-St. Paul-Bloomington, Minnesota-Wisconsin & 6,093 & 12.3 & 1.0 & $(10.4-14.2)$ \\
\hline Minot, North Dakota & 408 & 16.4 & 2.3 & $(11.9-20.9)$ \\
\hline $\begin{array}{l}\text { Montgomery County-Bucks County-Chester County, } \\
\text { Pennsylvania }\end{array}$ & 626 & 10.8 & 1.3 & $(8.2-13.4)$ \\
\hline $\begin{array}{l}\text { Myrtle Beach-Conway-North Myrtle Beach, South Carolina- } \\
\text { North Carolina }\end{array}$ & 571 & 14.7 & 1.8 & $(11.2-18.2)$ \\
\hline Nashville-Davidson County-Murfreesboro-Franklin, Tennessee & 646 & 16.3 & 1.9 & $(12.7-19.9)$ \\
\hline Nassau County-Suffolk County, New York ${ }^{\S}$ & 604 & 14.5 & 1.8 & $(10.9-18.1)$ \\
\hline Newark, New Jersey-Pennsylvania ${ }^{\S}$ & 2,610 & 13.4 & 0.9 & $(11.6-15.3)$ \\
\hline New Orleans-Metairie, Louisiana & 919 & 19.6 & 2.1 & $(15.5-23.7)$ \\
\hline New York-Jersey City-White Plains, New York-New Jersey ${ }^{\S}$ & 5,323 & 19.7 & 0.9 & $(17.9-21.6)$ \\
\hline Norfolk, Nebraska & 448 & 18.1 & 2.2 & $(13.8-22.5)$ \\
\hline North Platte, Nebraska & 530 & 16.5 & 2.1 & $(12.3-20.7)$ \\
\hline North Port-Sarasota-Bradenton, Florida & 856 & 12.6 & 1.6 & $(9.4-15.7)$ \\
\hline Oakland-Hayward-Berkeley, California ${ }^{\S}$ & 391 & 14.9 & 2.4 & $(10.2-19.6)$ \\
\hline Ogden-Clearfield, Utah & 1,394 & 15.3 & 1.1 & $(13.1-17.6)$ \\
\hline Oklahoma City, Oklahoma & 1,770 & 17.2 & 1.1 & $(15.1-19.3)$ \\
\hline Omaha-Council Bluffs, Nebraska-lowa & 2,009 & 16.9 & 1.2 & $(14.6-19.2)$ \\
\hline Orlando-Kissimmee-Sanford, Florida & 1,575 & 19.8 & 1.5 & $(16.9-22.8)$ \\
\hline Panama City, Florida & 801 & 18.7 & 2.3 & $(14.2-23.3)$ \\
\hline Pensacola-Ferry Pass-Brent, Florida & 878 & 17.6 & 1.6 & $(14.4-20.8)$ \\
\hline Philadelphia, Pennsylvania§ & 1,161 & 16.8 & 1.5 & $(13.9-19.7)$ \\
\hline Phoenix-Mesa-Scottsdale, Arizona & 1,056 & 18.4 & 2.0 & $(14.6-22.3)$ \\
\hline Pittsburgh, Pennsylvania & 1,698 & 15.3 & 1.2 & $(12.9-17.6)$ \\
\hline Ponce, Puerto Rico & 333 & 23.2 & 2.7 & $(17.9-28.6)$ \\
\hline Portland-South Portland, Maine & 1,930 & 11.5 & 0.9 & $(9.8-13.1)$ \\
\hline Portland-Vancouver-Hillsboro, Oregon-Washington & 2,257 & 15.2 & 1.1 & $(13.1-17.3)$ \\
\hline Port St. Lucie, Florida & 831 & 15.7 & 1.7 & $(12.3-19.1)$ \\
\hline Providence-Warwick, Rhode Island-Massachusetts & 5,942 & 14.2 & 0.8 & $(12.7-15.7)$ \\
\hline Provo-Orem, Utah & 857 & 14.0 & 1.4 & $(11.4-16.7)$ \\
\hline Raleigh, North Carolina & 360 & 19.3 & 2.4 & $(14.7-24.0)$ \\
\hline Rapid City, South Dakota & 601 & 13.3 & 1.7 & $(10.0-16.5)$ \\
\hline Reno, Nevada & 1,208 & 13.8 & 1.3 & $(11.2-16.4)$ \\
\hline Richmond, Virginia & 858 & 17.1 & 1.8 & $(13.5-20.6)$ \\
\hline Riverside-San Bernardino-Ontario, California & 814 & 20.1 & 1.8 & $(16.5-23.7)$ \\
\hline Rochester, New York & 336 & 14.1 & 1.9 & $(10.3-17.9)$ \\
\hline Rockingham County-Strafford County, New Hampshire ${ }^{\S}$ & 1,178 & 14.2 & 1.2 & $(11.9-16.6)$ \\
\hline Sacramento-Roseville-Arden-Arcade, California & 566 & 15.8 & 2.6 & $(10.7-20.8)$ \\
\hline St. Louis, Missouri-Illinois & 1,438 & 15.4 & 1.3 & $(12.9-17.9)$ \\
\hline Salem, Oregon & 379 & 15.2 & 2.2 & $(10.9-19.5)$ \\
\hline Salina, Kansas & 369 & 17.8 & 2.2 & $(13.4-22.1)$ \\
\hline Salisbury, Maryland-Delaware & 1,598 & 19.2 & 1.4 & $(16.5-21.9)$ \\
\hline Salt Lake City, Utah & 2,689 & 15.3 & 0.9 & $(13.6-17.0)$ \\
\hline San Antonio-New Braunfels, Texas & 613 & 18.9 & 2.3 & $(14.5-23.3)$ \\
\hline
\end{tabular}

See table footnotes on page 99. 
TABLE 44. (Continued) Age-adjusted* prevalence estimates of adults aged $\geq 45$ years who have ever been told by a health professional they have diabetes, ${ }^{\dagger}$ by metropolitan and micropolitan statistical area - Behavioral Risk Factor Surveillance System, United States, 2013

\begin{tabular}{|c|c|c|c|c|}
\hline MMSA & $\begin{array}{l}\text { Sample } \\
\text { size }\end{array}$ & $\%$ & SE & $95 \% \mathrm{Cl}$ \\
\hline San Francisco-Redwood City-South San Francisco, California ${ }^{\S}$ & 303 & 11.2 & 2.9 & $(5.6-16.8)$ \\
\hline San Jose-Sunnyvale-Santa Clara, California & 336 & 14.1 & 2.6 & $(9.0-19.2)$ \\
\hline San Juan-Carolina-Caguas, Puerto Rico & 2,338 & 23.7 & 1.0 & $(21.7-25.7)$ \\
\hline Scottsbluff, Nebraska & 547 & 14.0 & 1.7 & $(10.7-17.3)$ \\
\hline Scranton-Wilkes-Barre-Hazleton, Pennsylvania & 427 & 12.8 & 1.8 & $(9.4-16.3)$ \\
\hline Seattle-Bellevue-Everett, Washington ${ }^{\S}$ & 2,449 & 13.2 & 0.9 & $(11.4-14.9)$ \\
\hline Shreveport-Bossier City, Louisiana & 437 & 14.7 & 1.9 & $(11.0-18.4)$ \\
\hline Silver Spring-Frederick-Rockville, Maryland ${ }^{\S}$ & 1,651 & 13.6 & 1.2 & $(11.3-15.9)$ \\
\hline Sioux City, lowa-Nebraska-South Dakota & 750 & 17.4 & 2.3 & $(12.8-22.0)$ \\
\hline Sioux Falls, South Dakota & 596 & 15.3 & 2.0 & $(11.3-19.3)$ \\
\hline Spartanburg, South Carolina & 453 & 22.7 & 2.8 & $(17.3-28.2)$ \\
\hline Spokane-Spokane Valley, Washington & 630 & 13.8 & 1.7 & $(10.5-17.2)$ \\
\hline Springfield, Massachusetts & 1,133 & 14.7 & 1.6 & $(11.6-17.8)$ \\
\hline Tallahassee, Florida & 1,385 & 17.8 & 1.8 & $(14.3-21.3)$ \\
\hline Tampa-St. Petersburg-Clearwater, Florida & 1,586 & 17.0 & 1.3 & $(14.5-19.6)$ \\
\hline Toledo, Ohio & 711 & 15.5 & 1.7 & $(12.1-18.9)$ \\
\hline Topeka, Kansas & 1,707 & 15.8 & 1.1 & $(13.7-17.9)$ \\
\hline Tulsa, Oklahoma & 1,427 & 16.9 & 1.2 & $(14.5-19.2)$ \\
\hline Virginia Beach-Norfolk-Newport News, Virginia-North Carolina & 1,114 & 18.1 & 1.7 & $(14.8-21.3)$ \\
\hline Warren-Troy-Farmington Hills, Michigan ${ }^{\S}$ & 1,596 & 15.6 & 1.1 & $(13.4-17.8)$ \\
\hline $\begin{array}{l}\text { Washington-Arlington-Alexandria, District of Columbia- } \\
\text { Virginia-Maryland-West Virginia\$ }\end{array}$ & 6,065 & 15.0 & 0.9 & $(13.1-16.8)$ \\
\hline Wichita, Kansas & 3,382 & 17.9 & 0.8 & $(16.4-19.5)$ \\
\hline Wilmington, Delaware-Maryland-New Jersey ${ }^{\S}$ & 2,239 & 15.2 & 1.0 & $(13.3-17.1)$ \\
\hline Winston-Salem, North Carolina & 513 & 16.9 & 1.9 & $(13.2-20.5)$ \\
\hline Worcester, Massachusetts-Connecticut & 1,891 & 15.9 & 1.4 & $(13.2-18.6)$ \\
\hline $\begin{array}{l}\text { Median } \\
\text { Range }\end{array}$ & & $\begin{array}{c}15.8 \\
10.6-24.8\end{array}$ & & \\
\hline
\end{tabular}

Abbreviations: $\mathrm{Cl}=$ confidence interval; $\mathrm{MMSA}=$ metropolitan and micropolitan statistical areas; $\mathrm{SE}=$ standard error.

* Age adjusted to the 2000 U.S. standard population.

† Excluding prediabetes or borderline diabetes and diabetes during pregnancy for women.

$\S$ Metropolitan division. 
TABLE 45. Age-adjusted* prevalence estimates of adults aged $\geq 45$ years who have ever been told by a health professional they have some form of arthritis, ${ }^{\dagger}$ by state/territory - Behavioral Risk Factor Surveillance System, United States, 2013

\begin{tabular}{|c|c|c|c|c|}
\hline State/Territory & $\begin{array}{l}\text { Sample } \\
\text { size }\end{array}$ & $\%$ & SE & $95 \% \mathrm{Cl}$ \\
\hline Alabama & 4,976 & 48.8 & 1.0 & $(46.9-50.7)$ \\
\hline Alaska & 2,786 & 38.6 & 1.3 & $(36.0-41.3)$ \\
\hline Arizona & 3,112 & 39.0 & 1.5 & $(36.1-41.9)$ \\
\hline Arkansas & 4,006 & 43.7 & 1.1 & $(41.5-45.8)$ \\
\hline California & 7,150 & 35.9 & 0.8 & $(34.3-37.4)$ \\
\hline Colorado & 9,389 & 36.5 & 0.6 & $(35.3-37.7)$ \\
\hline Connecticut & 5,432 & 37.2 & 0.9 & $(35.4-38.9)$ \\
\hline Delaware & 3,755 & 40.3 & 1.1 & $(38.3-42.4)$ \\
\hline District of Columbia & 3,542 & 35.9 & 1.2 & $(33.6-38.1)$ \\
\hline Florida & 26,506 & 37.6 & 0.7 & $(36.3-39.0)$ \\
\hline Georgia & 5,548 & 40.4 & 0.9 & $(38.7-42.1)$ \\
\hline Hawaii & 5,126 & 30.6 & 1.0 & $(28.7-32.5)$ \\
\hline Idaho & 3,960 & 37.9 & 1.1 & $(35.8-40.0)$ \\
\hline Illinois & 3,977 & 39.4 & 1.1 & $(37.3-41.5)$ \\
\hline Indiana & 7,485 & 42.6 & 0.7 & $(41.3-44.0)$ \\
\hline lowa & 6,023 & 36.9 & 0.8 & $(35.4-38.4)$ \\
\hline Kansas & 16,437 & 37.4 & 0.4 & $(36.6-38.3)$ \\
\hline Kentucky & 8,060 & 46.2 & 0.9 & $(44.5-47.9)$ \\
\hline Louisiana & 3,976 & 41.0 & 1.2 & $(38.8-43.3)$ \\
\hline Maine & 6,118 & 42.4 & 0.8 & $(40.8-44.0)$ \\
\hline Maryland & 9,552 & 38.2 & 0.7 & $(36.9-39.6)$ \\
\hline Massachusetts & 10,636 & 38.0 & 0.7 & $(36.6-39.4)$ \\
\hline Michigan & 9,124 & 46.3 & 0.7 & $(45.0-47.7)$ \\
\hline Minnesota & 9,912 & 31.0 & 0.9 & $(29.2-32.9)$ \\
\hline Mississippi & 5,603 & 45.9 & 1.0 & $(44.0-47.8)$ \\
\hline Missouri & 5,196 & 42.8 & 1.0 & $(40.7-44.8)$ \\
\hline Montana & 6,837 & 39.0 & 0.8 & $(37.4-40.7)$ \\
\hline Nebraska & 12,400 & 39.0 & 0.7 & $(37.6-40.3)$ \\
\hline Nevada & 3,495 & 33.1 & 1.4 & $(30.3-35.8)$ \\
\hline New Hampshire & 4,823 & 39.7 & 0.9 & $(37.9-41.4)$ \\
\hline New Jersey & 8,730 & 35.0 & 0.7 & $(33.6-36.5)$ \\
\hline New Mexico & 6,445 & 37.8 & 0.9 & $(36.1-39.5)$ \\
\hline New York & 5,783 & 39.7 & 0.8 & $(38.0-41.3)$ \\
\hline North Carolina & 6,179 & 40.7 & 0.8 & $(39.1-42.4)$ \\
\hline North Dakota & 5,429 & 40.1 & 0.9 & $(38.3-41.8)$ \\
\hline Ohio & 8,608 & 43.4 & 0.8 & $(41.9-44.9)$ \\
\hline Oklahoma & 5,942 & 40.6 & 0.8 & $(39.0-42.1)$ \\
\hline Oregon & 4,320 & 40.0 & 0.9 & $(38.2-41.9)$ \\
\hline Pennsylvania & 8,103 & 43.2 & 0.7 & $(41.8-44.6)$ \\
\hline Rhode Island & 4,729 & 41.3 & 0.9 & $(39.6-43.0)$ \\
\hline South Carolina & 7,818 & 44.8 & 0.8 & $(43.2-46.4)$ \\
\hline South Dakota & 4,719 & 38.3 & 1.2 & $(35.9-40.7)$ \\
\hline Tennessee & 4,254 & 39.8 & 1.1 & $(37.6-41.9)$ \\
\hline Texas & 7,211 & 35.5 & 0.9 & $(33.7-37.2)$ \\
\hline Utah & 7,533 & 35.9 & 0.7 & $(34.6-37.3)$ \\
\hline Vermont & 4,721 & 40.3 & 0.9 & $(38.7-42.0)$ \\
\hline Virginia & 5,800 & 40.2 & 0.8 & (38.5-41.8) \\
\hline Washington & 7,995 & 41.0 & 0.7 & $(39.6-42.5)$ \\
\hline West Virginia & 4,147 & 51.0 & 0.9 & $(49.2-52.8)$ \\
\hline Wisconsin & 4,687 & 38.0 & 1.1 & $(35.8-40.2)$ \\
\hline Wyoming & 4,996 & 38.3 & 0.9 & $(36.5-40.0)$ \\
\hline Guam & 810 & 32.0 & 2.2 & $(27.7-36.2)$ \\
\hline Puerto Rico & 4,011 & 39.0 & 1.0 & $(37.1-40.8)$ \\
\hline Median & & 39.4 & & \\
\hline Range & & $30.6-51.0$ & & \\
\hline
\end{tabular}

Abbreviations: $\mathrm{Cl}=$ confidence interval; $\mathrm{SE}=$ standard error.

* Age adjusted to the 2000 U.S. standard population.

† Including arthritis, rheumatoid arthritis, gout, lupus, or fibromyalgia. 
TABLE 46. Age-adjusted* prevalence estimates of adults aged $\geq 45$ years who have ever been told by a health professional they have some form of arthritis, ${ }^{\dagger}$ by metropolitan and micropolitan statistical area - Behavioral Risk Factor Surveillance System, United States, 2013

\begin{tabular}{|c|c|c|c|c|}
\hline MMSA & $\begin{array}{l}\text { Sample } \\
\text { size }\end{array}$ & $\%$ & SE & $95 \% \mathrm{Cl}$ \\
\hline Aguadilla-Isabela, Puerto Rico & 406 & 36.1 & 2.8 & $(30.5-41.6)$ \\
\hline Akron, Ohio & 539 & 41.7 & 2.7 & $(36.4-47.0)$ \\
\hline Albuquerque, New Mexico & 1,337 & 38.3 & 1.7 & $(35.0-41.6)$ \\
\hline Allentown-Bethlehem-Easton, Pennsylvania-New Jersey & 719 & 42.5 & 2.8 & $(37.1-47.9)$ \\
\hline Anchorage, Alaska & 925 & 38.0 & 2.1 & $(34.0-42.0)$ \\
\hline Atlanta-Sandy Springs-Roswell, Georgia & 2,250 & 36.8 & 1.3 & $(34.2-39.3)$ \\
\hline Augusta-Richmond County, Georgia-South Carolina & 669 & 42.8 & 2.7 & $(37.5-48.0)$ \\
\hline Austin-Round Rock, Texas & 592 & 35.2 & 2.7 & $(29.8-40.6)$ \\
\hline Baltimore-Columbia-Towson, Maryland & 3,428 & 38.8 & 1.1 & $(36.6-41.0)$ \\
\hline Baton Rouge, Louisiana & 671 & 42.1 & 2.5 & $(37.3-47.0)$ \\
\hline Billings, Montana & 492 & 39.6 & 2.4 & $(34.9-44.3)$ \\
\hline Birmingham-Hoover, Alabama & 984 & 48.7 & 1.9 & $(44.9-52.5)$ \\
\hline Bismarck, North Dakota & 692 & 39.9 & 2.2 & $(35.6-44.2)$ \\
\hline Boise City, Idaho & 988 & 34.9 & 2.0 & $(31.0-38.7)$ \\
\hline Boston, Massachusetts ${ }^{\S}$ & 2,785 & 37.6 & 1.4 & $(34.9-40.4)$ \\
\hline Buffalo-Cheektowaga-Niagara Falls, New York & 365 & 42.0 & 3.2 & $(35.7-48.4)$ \\
\hline Burlington-South Burlington, Vermont & 1,074 & 39.0 & 1.7 & $(35.6-42.4)$ \\
\hline Cambridge-Newton-Framingham, Massachusetts ${ }^{\S}$ & 3,341 & 34.7 & 1.2 & $(32.4-37.1)$ \\
\hline Camden, New Jersey ${ }^{\S}$ & 1,246 & 38.6 & 1.8 & $(35.1-42.0)$ \\
\hline Cedar Rapids, lowa & 468 & 33.6 & 2.5 & $(28.8-38.5)$ \\
\hline Charleston, West Virginia & 585 & 50.0 & 2.4 & $(45.3-54.8)$ \\
\hline Charleston-North Charleston, South Carolina & 1,082 & 39.3 & 2.0 & $(35.5-43.2)$ \\
\hline Charlotte-Concord-Gastonia, North Carolina-South Carolina & 1,291 & 39.4 & 1.8 & $(35.9-43.0)$ \\
\hline Chattanooga, Tennessee-Georgia & 459 & 36.6 & 2.9 & $(30.8-42.3)$ \\
\hline Chicago-Naperville-Elgin, Illinois-Indiana-Wisconsin & 2,278 & 38.8 & 1.4 & $(36.0-41.5)$ \\
\hline Cincinnati, Ohio-Kentucky-Indiana & 1,846 & 41.2 & 1.6 & $(38.1-44.3)$ \\
\hline Claremont-Lebanon, New Hampshire-Vermont & 1,260 & 37.8 & 1.8 & $(34.3-41.3)$ \\
\hline Cleveland-Elyria, Ohio & 800 & 43.8 & 2.2 & $(39.5-48.2)$ \\
\hline Colorado Springs, Colorado & 934 & 38.8 & 1.9 & $(35.1-42.5)$ \\
\hline Columbia, South Carolina & 983 & 44.4 & 2.3 & $(39.9-48.8)$ \\
\hline Columbus, Ohio & 1,185 & 42.0 & 1.8 & $(38.6-45.5)$ \\
\hline Crestview-Fort Walton Beach-Destin, Florida & 781 & 40.0 & 2.2 & $(35.6-44.3)$ \\
\hline Dallas-Plano-Irving, Texas $§$ & 523 & 27.6 & 2.1 & $(23.4-31.8)$ \\
\hline Davenport-Moline-Rock Island, lowa-Illinois & 506 & 37.8 & 2.7 & $(32.6-43.0)$ \\
\hline Dayton, Ohio & 631 & 42.0 & 2.5 & $(37.2-46.9)$ \\
\hline Deltona-Daytona Beach-Ormond Beach, Florida & 917 & 44.2 & 2.5 & $(39.4-49.1)$ \\
\hline Denver-Aurora-Lakewood, Colorado & 3,654 & 36.3 & 0.9 & $(34.4-38.1)$ \\
\hline Des Moines-West Des Moines, lowa & 971 & 39.5 & 1.9 & $(35.7-43.3)$ \\
\hline Duluth, Minnesota-Wisconsin & 515 & 39.7 & 3.8 & $(32.3-47.1)$ \\
\hline Durham-Chapel Hill, North Carolina & 430 & 36.9 & 2.7 & $(31.7-42.2)$ \\
\hline El Paso, Texas & 483 & 35.7 & 2.8 & $(30.2-41.1)$ \\
\hline Evansville, Indiana-Kentucky & 437 & 44.3 & 3.0 & $(38.4-50.1)$ \\
\hline Fargo, North Dakota-Minnesota & 737 & 39.2 & 2.7 & $(33.9-44.5)$ \\
\hline Fayetteville-Springdale-Rogers, Arkansas-Missouri & 582 & 39.1 & 2.7 & $(33.8-44.4)$ \\
\hline Fort Smith, Arkansas-Oklahoma & 380 & 40.7 & 3.2 & $(34.6-46.9)$ \\
\hline Fort Wayne, Indiana & 562 & 42.2 & 2.5 & $(37.3-47.1)$ \\
\hline Fort Worth-Arlington, Texas ${ }^{\S}$ & 567 & 37.4 & 2.9 & $(31.7-43.1)$ \\
\hline Gainesville, Florida & 762 & 40.7 & 2.7 & $(35.4-46.0)$ \\
\hline Grand Forks, North Dakota-Minnesota & 344 & 35.7 & 3.7 & $(28.5-43.0)$ \\
\hline Grand Island, Nebraska & 610 & 42.9 & 2.5 & $(37.9-47.9)$ \\
\hline Grand Rapids-Wyoming, Michigan & 899 & 42.6 & 2.0 & $(38.6-46.6)$ \\
\hline Greensboro-High Point, North Carolina & 461 & 41.4 & 2.9 & $(35.7-47.2)$ \\
\hline Greenville-Anderson-Mauldin, South Carolina & 936 & 43.5 & 2.1 & $(39.5-47.5)$ \\
\hline Gulfport-Biloxi-Pascagoula, Mississippi & 560 & 44.1 & 2.6 & $(39.0-49.2)$ \\
\hline Hagerstown-Martinsburg, Maryland-West Virginia & 578 & 45.5 & 2.6 & $(40.5-50.6)$ \\
\hline Hartford-West Hartford-East Hartford, Connecticut & 2,022 & 37.8 & 1.5 & $(34.9-40.7)$ \\
\hline Hilton Head Island-Bluffton-Beaufort, South Carolina & 679 & 37.6 & 2.6 & $(32.6-42.6)$ \\
\hline Houston-The Woodlands-Sugar Land, Texas & 774 & 35.9 & 2.5 & $(30.9-40.8)$ \\
\hline Huntington-Ashland, West Virginia-Kentucky-Ohio & 817 & 52.4 & 2.2 & $(48.2-56.7)$ \\
\hline Idaho Falls, Idaho & 333 & 40.6 & 3.6 & $(33.5-47.6)$ \\
\hline Indianapolis-Carmel-Anderson, Indiana & 1,759 & 41.4 & 1.4 & $(38.7-44.0)$ \\
\hline
\end{tabular}

See table footnotes on page 103. 
TABLE 46. (Continued) Age-adjusted* prevalence estimates of adults aged $\geq 45$ years who have ever been told by a health professional they have some form of arthritis, ${ }^{\dagger}$ by metropolitan and micropolitan statistical area - Behavioral Risk Factor Surveillance System, United States, 2013

\begin{tabular}{|c|c|c|c|c|}
\hline MMSA & $\begin{array}{l}\text { Sample } \\
\text { size }\end{array}$ & $\%$ & SE & $95 \% \mathrm{Cl}$ \\
\hline Jackson, Mississippi & 545 & 43.3 & 2.8 & $(37.8-48.8)$ \\
\hline Jacksonville, Florida & 2,057 & 39.6 & 1.7 & $(36.3-42.9)$ \\
\hline Kansas City, Missouri-Kansas & 5,189 & 42.1 & 1.6 & $(39.0-45.2)$ \\
\hline Kingsport-Bristol-Bristol, Tennessee-Virginia & 440 & 48.5 & 3.5 & $(41.6-55.5)$ \\
\hline Knoxville, Tennessee & 467 & 43.1 & 3.3 & $(36.6-49.5)$ \\
\hline Lansing-East Lansing, Michigan & 467 & 46.4 & 2.7 & $(41.0-51.8)$ \\
\hline Lexington-Fayette, Kentucky & 381 & 38.8 & 3.2 & $(32.6-45.1)$ \\
\hline Lincoln, Nebraska & 1,104 & 36.7 & 1.6 & $(33.5-39.9)$ \\
\hline Little Rock-North Little Rock-Conway, Arkansas & 841 & 41.7 & 2.2 & $(37.3-46.0)$ \\
\hline Logan, Utah-Idaho & 362 & 34.8 & 3.0 & $(28.9-40.7)$ \\
\hline Los Angeles-Long Beach-Anaheim, California & 1,786 & 34.1 & 1.6 & $(31.0-37.1)$ \\
\hline Louisville-Jefferson County, Kentucky-Indiana & 1,579 & 43.2 & 2.1 & $(39.0-47.3)$ \\
\hline Lubbock, Texas & 406 & 34.9 & 2.8 & $(29.4-40.5)$ \\
\hline Manhattan, Kansas & 388 & 34.4 & 2.6 & $(29.3-39.4)$ \\
\hline Memphis, Tennessee-Mississippi-Arkansas & 847 & 37.5 & 2.2 & $(33.1-41.8)$ \\
\hline Miami-Fort Lauderdale-West Palm Beach, Florida & 1,593 & 31.7 & 1.8 & $(28.2-35.2)$ \\
\hline Milwaukee-Waukesha-West Allis, Wisconsin & 900 & 38.9 & 2.6 & $(33.8-44.0)$ \\
\hline Minneapolis-St. Paul-Bloomington, Minnesota-Wisconsin & 6,240 & 29.7 & 1.2 & $(27.3-32.1)$ \\
\hline Minot, North Dakota & 418 & 34.2 & 2.7 & $(28.8-39.6)$ \\
\hline $\begin{array}{l}\text { Montgomery County-Bucks County-Chester County, } \\
\text { Pennsylvania§ }\end{array}$ & 638 & 36.9 & 2.2 & $(32.7-41.2)$ \\
\hline $\begin{array}{l}\text { Myrtle Beach-Conway-North Myrtle Beach, South Carolina- } \\
\text { North Carolina }\end{array}$ & 583 & 52.0 & 3.0 & $(46.1-57.9)$ \\
\hline Nashville-Davidson County-Murfreesboro-Franklin, Tennessee & 699 & 35.5 & 2.3 & $(30.9-40.0)$ \\
\hline Nassau County-Suffolk County, New York ${ }^{\S}$ & 610 & 36.5 & 2.3 & $(31.9-41.0)$ \\
\hline Newark, New Jersey-Pennsylvania ${ }^{\S}$ & 2,693 & 31.5 & 1.2 & $(29.1-33.9)$ \\
\hline New Orleans-Metairie, Louisiana & 960 & 39.4 & 2.6 & $(34.3-44.5)$ \\
\hline New York-Jersey City-White Plains, New York-New Jersey & 5,441 & 36.3 & 1.0 & $(34.3-38.2)$ \\
\hline Norfolk, Nebraska & 452 & 39.7 & 2.6 & $(34.7-44.7)$ \\
\hline North Platte, Nebraska & 539 & 40.1 & 2.6 & $(34.9-45.3)$ \\
\hline North Port-Sarasota-Bradenton, Florida & 862 & 37.9 & 2.3 & $(33.4-42.5)$ \\
\hline Oakland-Hayward-Berkeley, California ${ }^{\S}$ & 408 & 32.4 & 2.9 & $(26.8-38.1)$ \\
\hline Ogden-Clearfield, Utah & 1,424 & 38.4 & 1.5 & $(35.4-41.3)$ \\
\hline Oklahoma City, Oklahoma & 1,813 & 39.2 & 1.4 & $(36.5-41.9)$ \\
\hline Omaha-Council Bluffs, Nebraska-lowa & 2,050 & 40.0 & 1.4 & $(37.2-42.7)$ \\
\hline Orlando-Kissimmee-Sanford, Florida & 1,611 & 37.3 & 1.7 & $(33.9-40.6)$ \\
\hline Panama City, Florida & 806 & 46.8 & 2.8 & $(41.3-52.3)$ \\
\hline Pensacola-Ferry Pass-Brent, Florida & 894 & 41.1 & 2.1 & $(37.1-45.2)$ \\
\hline Philadelphia, Pennsylvania§ & 1,183 & 45.8 & 2.0 & $(41.9-49.7)$ \\
\hline Phoenix-Mesa-Scottsdale, Arizona & 1,077 & 38.8 & 2.1 & $(34.6-43.0)$ \\
\hline Pittsburgh, Pennsylvania & 1,725 & 45.2 & 1.5 & $(42.2-48.2)$ \\
\hline Ponce, Puerto Rico & 342 & 34.4 & 3.1 & $(28.4-40.5)$ \\
\hline Portland-South Portland, Maine & 1,973 & 39.3 & 1.4 & $(36.6-42.1)$ \\
\hline Portland-Vancouver-Hillsboro, Oregon-Washington & 2,294 & 38.4 & 1.3 & $(35.9-40.9)$ \\
\hline Port St. Lucie, Florida & 840 & 40.3 & 2.4 & $(35.6-45.0)$ \\
\hline Providence-Warwick, Rhode Island-Massachusetts & 6,061 & 42.1 & 1.1 & $(39.9-44.4)$ \\
\hline Provo-Orem, Utah & 876 & 34.5 & 1.8 & $(31.0-38.1)$ \\
\hline Raleigh, North Carolina & 369 & 33.9 & 3.0 & $(28.0-39.9)$ \\
\hline Rapid City, South Dakota & 611 & 44.2 & 2.9 & $(38.6-49.9)$ \\
\hline Reno, Nevada & 1,221 & 32.7 & 1.8 & $(29.1-36.2)$ \\
\hline Richmond, Virginia & 883 & 38.5 & 2.1 & $(34.4-42.6)$ \\
\hline Riverside-San Bernardino-Ontario, California & 850 & 40.5 & 2.1 & $(36.5-44.6)$ \\
\hline Rochester, New York & 344 & 45.0 & 3.4 & $(38.3-51.6)$ \\
\hline Rockingham County-Strafford County, New Hampshire $§$ & 1,195 & 41.5 & 1.8 & $(38.0-44.9)$ \\
\hline Sacramento-Roseville-Arden-Arcade, California & 587 & 37.0 & 2.8 & $(31.6-42.4)$ \\
\hline St. Louis, Missouri-Illinois & 1,477 & 42.6 & 1.9 & $(38.9-46.3)$ \\
\hline Salem, Oregon & 394 & 45.1 & 3.2 & $(38.9-51.4)$ \\
\hline Salina, Kansas & 377 & 32.5 & 2.7 & $(27.1-37.8)$ \\
\hline Salisbury, Maryland-Delaware & 1,640 & 43.4 & 1.7 & $(40.0-46.7)$ \\
\hline Salt Lake City, Utah & 2,737 & 35.4 & 1.1 & (33.2-37.5) \\
\hline San Antonio-New Braunfels, Texas & 626 & 37.2 & 2.4 & $(32.6-41.8)$ \\
\hline
\end{tabular}

See table footnotes on page 103. 
TABLE 46. (Continued) Age-adjusted* prevalence estimates of adults aged $\geq 45$ years who have ever been told by a health professional they have some form of arthritis, ${ }^{\dagger}$ by metropolitan and micropolitan statistical area - Behavioral Risk Factor Surveillance System, United States, 2013

\begin{tabular}{|c|c|c|c|c|}
\hline MMSA & $\begin{array}{l}\text { Sample } \\
\text { size }\end{array}$ & $\%$ & SE & $95 \% \mathrm{Cl}$ \\
\hline San Francisco-Redwood City-South San Francisco, California ${ }^{\S}$ & 321 & 31.4 & 3.3 & $(24.9-38.0)$ \\
\hline San Jose-Sunnyvale-Santa Clara, California & 353 & 32.5 & 3.2 & $(26.3-38.7)$ \\
\hline San Juan-Carolina-Caguas, Puerto Rico & 2,404 & 39.6 & 1.2 & $(37.2-42.0)$ \\
\hline Scottsbluff, Nebraska & 558 & 43.6 & 2.8 & $(38.1-49.1)$ \\
\hline Scranton-Wilkes-Barre-Hazleton, Pennsylvania & 432 & 45.8 & 3.0 & $(40.0-51.6)$ \\
\hline Seattle-Bellevue-Everett, Washington $\$$ & 2,499 & 37.5 & 1.2 & $(35.2-39.9)$ \\
\hline Shreveport-Bossier City, Louisiana & 445 & 41.5 & 3.1 & $(35.5-47.5)$ \\
\hline Silver Spring-Frederick-Rockville, Maryland ${ }^{\S}$ & 1,710 & 34.4 & 1.4 & $(31.5-37.2)$ \\
\hline Sioux City, lowa-Nebraska-South Dakota & 768 & 29.2 & 2.9 & $(23.4-35.0)$ \\
\hline Sioux Falls, South Dakota & 604 & 34.3 & 2.5 & $(29.3-39.3)$ \\
\hline Spartanburg, South Carolina & 465 & 48.8 & 3.4 & $(42.3-55.4)$ \\
\hline Spokane-Spokane Valley, Washington & 640 & 42.6 & 2.6 & $(37.6-47.6)$ \\
\hline Springfield, Massachusetts & 1,167 & 40.5 & 2.2 & $(36.2-44.7)$ \\
\hline Tallahassee, Florida & 1,423 & 38.9 & 2.3 & $(34.3-43.4)$ \\
\hline Tampa-St. Petersburg-Clearwater, Florida & 1,607 & 41.1 & 1.8 & $(37.7-44.5)$ \\
\hline Toledo, Ohio & 735 & 48.2 & 2.5 & $(43.3-53.1)$ \\
\hline Topeka, Kansas & 1,761 & 39.4 & 1.4 & $(36.8-42.1)$ \\
\hline Tulsa, Oklahoma & 1,465 & 39.5 & 1.6 & $(36.4-42.6)$ \\
\hline Virginia Beach-Norfolk-Newport News, Virginia-North Carolina & 1,142 & 39.7 & 1.8 & $(36.1-43.3)$ \\
\hline Warren-Troy-Farmington Hills, Michigan ${ }^{\S}$ & 1,615 & 42.5 & 1.5 & $(39.6-45.3)$ \\
\hline $\begin{array}{l}\text { Washington-Arlington-Alexandria, District of Columbia- } \\
\text { Virginia-Maryland-West Virginias }\end{array}$ & 6,199 & 36.3 & 1.1 & $(34.1-38.4)$ \\
\hline Wichita, Kansas & 3,445 & 36.6 & 1.0 & $(34.7-38.5)$ \\
\hline Wilmington, Delaware-Maryland-New Jersey ${ }^{\S}$ & 2,279 & 40.1 & 1.3 & $(37.6-42.7)$ \\
\hline Winston-Salem, North Carolina & 527 & 43.5 & 2.7 & $(38.1-48.9)$ \\
\hline Worcester, Massachusetts-Connecticut & 1,926 & 40.8 & 1.8 & $(37.3-44.4)$ \\
\hline Median & & 39.4 & & \\
\hline Range & & $27.6-52.4$ & & \\
\hline
\end{tabular}

Abbreviations: $\mathrm{Cl}=$ confidence interval; $\mathrm{MMSA}=$ micropolitan and metropolitan statistical areas; $\mathrm{SE}=$ standard error.

* Age adjusted to the 2000 U.S. standard population.

† Including arthritis, rheumatoid arthritis, gout, lupus, or fibromyalgia.

$\S$ Metropolitan division. 
TABLE 47. Age-adjusted* prevalence estimates of adults aged $\geq 45$ years who have ever been told by a health professional they have some form of arthritis, ${ }^{\dagger}$ by state/territory - Behavioral Risk Factor Surveillance System, United States, 2014

\begin{tabular}{|c|c|c|c|c|}
\hline State/Territory & $\begin{array}{l}\text { Sample } \\
\text { size }\end{array}$ & $\%$ & SE & $95 \% \mathrm{Cl}$ \\
\hline Alabama & 6,457 & 50.5 & 0.8 & $(48.9-52.2)$ \\
\hline Alaska & 2,855 & 36.7 & 1.3 & $(34.3-39.2)$ \\
\hline Arizona & 11,683 & 38.0 & 0.7 & $(36.7-39.3)$ \\
\hline Arkansas & 4,182 & 46.0 & 1.1 & $(43.7-48.2)$ \\
\hline California & 5,302 & 34.1 & 0.8 & $(32.5-35.7)$ \\
\hline Colorado & 9,458 & 37.4 & 0.6 & $(36.2-38.5)$ \\
\hline Connecticut & 5,850 & 36.4 & 0.8 & $(34.8-38.0)$ \\
\hline Delaware & 3,249 & 41.3 & 1.1 & $(39.1-43.5)$ \\
\hline District of Columbia & 3,032 & 36.1 & 1.3 & $(33.6-38.6)$ \\
\hline Florida & 7,529 & 39.5 & 0.8 & $(38.0-41.0)$ \\
\hline Georgia & 4,638 & 41.8 & 0.9 & $(40.0-43.6)$ \\
\hline Hawaii & 4,807 & 31.2 & 1.0 & $(29.3-33.1)$ \\
\hline Idaho & 3,919 & 37.7 & 1.1 & $(35.6-39.8)$ \\
\hline Illinois & 3,558 & 39.9 & 1.0 & $(37.8-41.9)$ \\
\hline Indiana & 8,602 & 43.5 & 0.7 & $(42.2-44.8)$ \\
\hline lowa & 6,043 & 38.4 & 0.8 & $(36.9-39.9)$ \\
\hline Kansas & 9,639 & 39.8 & 0.6 & (38.7-41.0) \\
\hline Kentucky & 8,581 & 49.2 & 0.9 & $(47.5-51.0)$ \\
\hline Louisiana & 4,743 & 41.8 & 0.9 & $(40.1-43.5)$ \\
\hline Maine & 7,093 & 43.8 & 0.8 & $(42.3-45.4)$ \\
\hline Maryland & 9,764 & 40.0 & 0.8 & (38.4-41.5) \\
\hline Massachusetts & 11,655 & 41.9 & 0.7 & $(40.5-43.3)$ \\
\hline Michigan & 6,210 & 46.5 & 0.8 & $(45.0-48.0)$ \\
\hline Minnesota & 10,987 & 33.9 & 0.5 & $(32.9-34.9)$ \\
\hline Mississippi & 3,152 & 46.1 & 1.2 & $(43.8-48.5)$ \\
\hline Missouri & 5,395 & 42.1 & 1.0 & $(40.2-44.0)$ \\
\hline Montana & 5,697 & 37.9 & 0.9 & $(36.0-39.7)$ \\
\hline Nebraska & 16,061 & 38.0 & 0.6 & $(36.9-39.1)$ \\
\hline Nevada & 2,636 & 36.5 & 1.4 & $(33.8-39.3)$ \\
\hline New Hampshire & 4,699 & 39.4 & 0.9 & (37.6-41.3) \\
\hline New Jersey & 9,243 & 35.1 & 0.7 & $(33.7-36.6)$ \\
\hline New Mexico & 6,482 & 38.0 & 0.9 & $(36.3-39.8)$ \\
\hline New York & 4,684 & 38.2 & 0.8 & $(36.5-39.8)$ \\
\hline North Carolina & 4,923 & 43.1 & 0.8 & $(41.4-44.7)$ \\
\hline North Dakota & 5,815 & 40.4 & 0.9 & $(38.5-42.2)$ \\
\hline Ohio & 8,379 & 44.9 & 0.8 & $(43.3-46.5)$ \\
\hline Oklahoma & 6,095 & 41.8 & 0.7 & $(40.3-43.2)$ \\
\hline Oregon & 3,881 & 38.2 & 1.0 & $(36.2-40.2)$ \\
\hline Pennsylvania & 8,210 & 44.7 & 0.7 & $(43.3-46.2)$ \\
\hline Rhode Island & 4,912 & 41.9 & 0.9 & $(40.1-43.6)$ \\
\hline South Carolina & 8,087 & 44.6 & 0.7 & $(43.2-46.1)$ \\
\hline South Dakota & 5,207 & 39.6 & 1.1 & (37.3-41.8) \\
\hline Tennessee & 3,964 & 47.8 & 1.1 & (45.7-49.9) \\
\hline Texas & 10,621 & 34.3 & 0.8 & $(32.7-35.8)$ \\
\hline Utah & 8,548 & 36.8 & 0.6 & $(35.6-38.0)$ \\
\hline Vermont & 4,589 & 40.4 & 0.8 & $(38.8-42.1)$ \\
\hline Virginia & 6,751 & 41.5 & 0.8 & $(39.9-43.0)$ \\
\hline Washington & 7,413 & 39.7 & 0.7 & $(38.3-41.2)$ \\
\hline West Virginia & 4,508 & 54.7 & 0.9 & $(53.0-56.5)$ \\
\hline Wisconsin & 5,067 & 38.9 & 0.9 & (37.1-40.8) \\
\hline Wyoming & 5,163 & 37.9 & 1.0 & (35.9-40.0) \\
\hline Guam & 1,200 & 31.8 & 1.9 & $(28.1-35.5)$ \\
\hline Puerto Rico & 4,072 & 40.4 & 0.9 & $(38.6-42.3)$ \\
\hline Median & & 39.8 & & \\
\hline Range & & $31.2-54.7$ & & \\
\hline
\end{tabular}

Abbreviations: $\mathrm{Cl}=$ confidence interval; $\mathrm{SE}=$ standard error.

* Age adjusted to the 2000 U.S. standard population.

† Including arthritis, rheumatoid arthritis, gout, lupus, or fibromyalgia. 
TABLE 48. Age-adjusted* prevalence estimates of adults aged $\geq 45$ years who have ever been told by a health professional they have some form of arthritis, ${ }^{\dagger}$ by metropolitan and micropolitan statistical area - Behavioral Risk Factor Surveillance System, United States, 2014

\begin{tabular}{|c|c|c|c|c|}
\hline MMSA & $\begin{array}{l}\text { Sample } \\
\text { size }\end{array}$ & $\%$ & SE & $95 \% \mathrm{Cl}$ \\
\hline Aberdeen, South Dakota & 615 & 45.5 & 2.7 & $(40.2-50.9)$ \\
\hline Aguadilla-Isabela, Puerto Rico & 541 & 40.9 & 3.3 & (34.4-47.3) \\
\hline Albuquerque, New Mexico & 1,786 & 37.6 & 1.7 & $(34.3-40.9)$ \\
\hline Allentown-Bethlehem-Easton, Pennsylvania-New Jersey & 1,089 & 42.4 & 2.4 & $(37.7-47.1)$ \\
\hline Anchorage, Alaska & 1,771 & 35.9 & 1.8 & $(32.3-39.5)$ \\
\hline Atlanta-Sandy Springs-Roswell, Georgia & 2,767 & 36.7 & 1.4 & $(34.1-39.4)$ \\
\hline Augusta-Richmond County, Georgia-South Carolina & 882 & 44.4 & 3.4 & $(37.8-51.0)$ \\
\hline Austin-Round Rock, Texas & 2,241 & 31.2 & 1.5 & $(28.2-34.1)$ \\
\hline Baltimore-Columbia-Towson, Maryland & 4,597 & 40.2 & 1.3 & $(37.7-42.7)$ \\
\hline Baton Rouge, Louisiana & 919 & 37.8 & 2.2 & $(33.5-42.1)$ \\
\hline Berlin, New Hampshire-Vermont & 538 & 41.7 & 3.0 & $(35.8-47.5)$ \\
\hline Billings, Montana & 809 & 37.3 & 2.4 & $(32.5-42.1)$ \\
\hline Birmingham-Hoover, Alabama & 1,570 & 46.2 & 1.9 & $(42.5-49.9)$ \\
\hline Bismarck, North Dakota & 1,032 & 40.1 & 2.3 & $(35.6-44.5)$ \\
\hline Boise City, Idaho & 1,345 & 35.9 & 2.0 & (31.9-39.9) \\
\hline Boston, Massachusetts ${ }^{\S}$ & 4,529 & 39.1 & 1.2 & $(36.7-41.5)$ \\
\hline Burlington-South Burlington, Vermont & 1,979 & 39.6 & 1.6 & $(36.5-42.6)$ \\
\hline Cambridge-Newton-Framingham, Massachusetts ${ }^{\S}$ & 5,149 & 40.2 & 1.2 & $(37.8-42.5)$ \\
\hline Camden, New Jersey ${ }^{\S}$ & 1,711 & 39.9 & 1.7 & $(36.6-43.2)$ \\
\hline Cedar Rapids, lowa & 639 & 35.7 & 2.4 & $(30.9-40.5)$ \\
\hline Charleston, West Virginia & 872 & 53.7 & 2.5 & $(48.8-58.6)$ \\
\hline Charleston-North Charleston, South Carolina & 1,398 & 41.3 & 1.9 & $(37.5-45.0)$ \\
\hline Charlotte-Concord-Gastonia, North Carolina-South Carolina & 2,147 & 41.1 & 1.7 & $(37.7-44.4)$ \\
\hline Chicago-Naperville-Elgin, Illinois-Indiana-Wisconsin & 4,118 & 39.4 & 1.3 & $(36.9-41.9)$ \\
\hline Cincinnati, Ohio-Kentucky-Indiana & 2,037 & 43.9 & 1.9 & $(40.2-47.6)$ \\
\hline Claremont-Lebanon, New Hampshire-Vermont & 1,679 & 41.6 & 1.8 & $(38.2-45.1)$ \\
\hline Cleveland-Elyria, Ohio & 961 & 43.8 & 2.4 & $(39.0-48.5)$ \\
\hline College Station-Bryan, Texas & 572 & 28.4 & 3.9 & $(20.8-36.0)$ \\
\hline Colorado Springs, Colorado & 1,292 & 38.5 & 1.9 & $(34.8-42.3)$ \\
\hline Columbia, South Carolina & 1,201 & 43.5 & 2.1 & (39.4-47.6) \\
\hline Columbus, Ohio & 1,654 & 44.5 & 1.9 & $(40.7-48.3)$ \\
\hline Corpus Christi, Texas & 619 & 39.9 & 3.6 & $(32.7-47.0)$ \\
\hline Dallas-Plano-Irving, Texas ${ }^{\S}$ & 1,290 & 32.6 & 2.4 & $(27.9-37.3)$ \\
\hline Dayton, Ohio & 562 & 47.1 & 3.3 & $(40.5-53.6)$ \\
\hline Denver-Aurora-Lakewood, Colorado & 5,760 & 36.5 & 0.9 & $(34.7-38.2)$ \\
\hline Des Moines-West Des Moines, lowa & 1,353 & 43.2 & 2.0 & $(39.4-47.1)$ \\
\hline Duluth, Minnesota-Wisconsin & 945 & 45.0 & 2.7 & $(39.8-50.2)$ \\
\hline El Paso, Texas & 721 & 37.0 & 2.8 & $(31.5-42.4)$ \\
\hline Evansville, Indiana-Kentucky & 656 & 48.0 & 2.9 & $(42.4-53.6)$ \\
\hline Fargo, North Dakota-Minnesota & 1,147 & 39.5 & 2.3 & $(35.0-43.9)$ \\
\hline Fayetteville-Springdale-Rogers, Arkansas-Missouri & 811 & 42.5 & 2.9 & $(36.8-48.1)$ \\
\hline Fort Wayne, Indiana & 855 & 39.8 & 2.4 & $(35.1-44.5)$ \\
\hline Fort Worth-Arlington, Texas ${ }^{\S}$ & 755 & 32.5 & 2.5 & $(27.6-37.3)$ \\
\hline Grand Island, Nebraska & 1,051 & 41.8 & 2.3 & $(37.3-46.3)$ \\
\hline Grand Rapids-Wyoming, Michigan & 894 & 48.7 & 2.5 & $(43.8-53.7)$ \\
\hline Greensboro-High Point, North Carolina & 521 & 39.3 & 2.8 & $(33.8-44.7)$ \\
\hline Greenville-Anderson-Mauldin, South Carolina & 1,484 & 44.4 & 2.1 & $(40.3-48.5)$ \\
\hline Hagerstown-Martinsburg, Maryland-West Virginia & 782 & 49.5 & 2.7 & $(44.2-54.9)$ \\
\hline Hartford-West Hartford-East Hartford, Connecticut & 2,627 & 37.3 & 1.4 & $(34.6-40.1)$ \\
\hline Hilton Head Island-Bluffton-Beaufort, South Carolina & 551 & 43.3 & 3.2 & $(36.9-49.6)$ \\
\hline Houston-The Woodlands-Sugar Land, Texas & 2,148 & 32.3 & 2.1 & $(28.2-36.4)$ \\
\hline Huntington-Ashland, West Virginia-Kentucky-Ohio & 1,243 & 50.5 & 2.0 & $(46.5-54.4)$ \\
\hline Idaho Falls, Idaho & 516 & 37.9 & 3.1 & $(31.8-44.0)$ \\
\hline Indianapolis-Carmel-Anderson, Indiana & 3,587 & 43.6 & 1.2 & $(41.2-46.0)$ \\
\hline Jacksonville, Florida & 670 & 42.4 & 2.8 & $(37.0-47.8)$ \\
\hline Kansas City, Missouri-Kansas & 4,857 & 38.7 & 1.3 & $(36.1-41.3)$ \\
\hline Kingsport-Bristol-Bristol, Tennessee-Virginia & 503 & 54.1 & 3.8 & $(46.6-61.6)$ \\
\hline Knoxville, Tennessee & 559 & 49.7 & 3.1 & $(43.6-55.8)$ \\
\hline Lafayette, Louisiana & 562 & 39.3 & 2.8 & $(33.9-44.7)$ \\
\hline Lexington-Fayette, Kentucky & 622 & 42.2 & 3.4 & $(35.6-48.9)$ \\
\hline Lincoln, Nebraska & 1,995 & 36.8 & 1.6 & $(33.6-39.9)$ \\
\hline
\end{tabular}

See table footnotes on page 107. 
TABLE 48. (Continued) Age-adjusted* prevalence estimates of adults aged $\geq 45$ years who have ever been told by a health professional they have some form of arthritis, ${ }^{\dagger}$ by metropolitan and micropolitan statistical area - Behavioral Risk Factor Surveillance System, United States, 2014

\begin{tabular}{|c|c|c|c|c|}
\hline MMSA & $\begin{array}{l}\text { Sample } \\
\text { size }\end{array}$ & $\%$ & SE & $95 \% \mathrm{Cl}$ \\
\hline Little Rock-North Little Rock-Conway, Arkansas & 1,179 & 43.2 & 2.3 & $(38.6-47.8)$ \\
\hline Logan, Utah-Idaho & 623 & 36.7 & 2.8 & $(31.1-42.2)$ \\
\hline Los Angeles-Long Beach-Anaheim, California & 2,431 & 31.6 & 1.5 & $(28.6-34.6)$ \\
\hline Louisville-Jefferson County, Kentucky-Indiana & 2,449 & 46.9 & 1.9 & $(43.1-50.6)$ \\
\hline Madison, Wisconsin & 549 & 36.5 & 3.0 & $(30.6-42.4)$ \\
\hline Memphis, Tennessee-Mississippi-Arkansas & 879 & 41.9 & 2.8 & $(36.5-47.4)$ \\
\hline Miami-Fort Lauderdale-West Palm Beach, Florida & 2,205 & 34.4 & 1.6 & $(31.3-37.4)$ \\
\hline Milwaukee-Waukesha-West Allis, Wisconsin & 1,352 & 39.2 & 2.0 & $(35.2-43.2)$ \\
\hline Minneapolis-St. Paul-Bloomington, Minnesota-Wisconsin & 8,724 & 32.4 & 0.7 & $(31.0-33.8)$ \\
\hline Minot, North Dakota & 592 & 41.2 & 3.1 & $(35.2-47.2)$ \\
\hline Montgomery, Alabama & 514 & 54.7 & 3.3 & $(48.2-61.2)$ \\
\hline $\begin{array}{l}\text { Montgomery County-Bucks County-Chester County, } \\
\text { Pennsylvania§ }\end{array}$ & 801 & 37.8 & 2.1 & $(33.6-42.0)$ \\
\hline $\begin{array}{l}\text { Myrtle Beach-Conway-North Myrtle Beach, South Carolina- } \\
\text { North Carolina }\end{array}$ & 992 & 46.0 & 2.3 & $(41.4-50.5)$ \\
\hline Nashville-Davidson County-Murfreesboro-Franklin, Tennessee & 798 & 43.0 & 2.6 & $(37.9-48.2)$ \\
\hline Nassau County-Suffolk County, New York ${ }^{\S}$ & 766 & 35.7 & 2.3 & $(31.3-40.2)$ \\
\hline Newark, New Jersey-Pennsylvania§ & 4,122 & 32.3 & 1.2 & $(30.0-34.7)$ \\
\hline New Orleans-Metairie, Louisiana & 1,905 & 38.5 & 1.6 & $(35.4-41.6)$ \\
\hline New York-Jersey City-White Plains, New York-New Jersey ${ }^{\S}$ & 7,495 & 34.8 & 0.9 & $(33.0-36.7)$ \\
\hline Norfolk, Nebraska & 995 & 36.2 & 2.0 & $(32.3-40.2)$ \\
\hline North Platte, Nebraska & 962 & 43.6 & 2.3 & $(39.2-48.1)$ \\
\hline North Port-Sarasota-Bradenton, Florida & 512 & 38.6 & 3.3 & $(32.2-45.0)$ \\
\hline Oakland-Hayward-Berkeley, California§ & 697 & 32.3 & 2.7 & $(27.1-37.5)$ \\
\hline Ogden-Clearfield, Utah & 2,913 & 37.4 & 1.3 & $(34.8-40.0)$ \\
\hline Oklahoma City, Oklahoma & 2,422 & 39.1 & 1.4 & $(36.3-41.8)$ \\
\hline Omaha-Council Bluffs, Nebraska-lowa & 4,853 & 37.8 & 1.1 & $(35.7-40.0)$ \\
\hline Orlando-Kissimmee-Sanford, Florida & 952 & 40.3 & 2.4 & $(35.5-45.0)$ \\
\hline Philadelphia, Pennsylvania ${ }^{\S}$ & 1,513 & 45.2 & 2.0 & $(41.2-49.2)$ \\
\hline Phoenix-Mesa-Scottsdale, Arizona & 9,356 & 37.6 & 0.8 & $(36.1-39.1)$ \\
\hline Pittsburgh, Pennsylvania & 2,408 & 47.3 & 1.5 & $(44.4-50.2)$ \\
\hline Ponce, Puerto Rico & 529 & 40.8 & 3.1 & $(34.8-46.8)$ \\
\hline Portland-South Portland, Maine & 2,743 & 42.3 & 1.4 & $(39.5-45.0)$ \\
\hline Portland-Vancouver-Hillsboro, Oregon-Washington & 2,833 & 36.4 & 1.3 & $(33.9-38.9)$ \\
\hline Providence-Warwick, Rhode Island-Massachusetts & 8,061 & 43.8 & 1.0 & $(41.8-45.9)$ \\
\hline Provo-Orem, Utah & 2,143 & 36.5 & 1.7 & (33.1-39.8) \\
\hline Raleigh, North Carolina & 721 & 36.3 & 2.5 & $(31.4-41.2)$ \\
\hline Rapid City, South Dakota & 1,414 & 41.8 & 2.3 & $(37.3-46.3)$ \\
\hline Reno, Nevada & 1,192 & 37.2 & 1.9 & $(33.4-41.0)$ \\
\hline Richmond, Virginia & 1,456 & 41.6 & 2.0 & $(37.6-45.5)$ \\
\hline Riverside-San Bernardino-Ontario, California & 938 & 36.4 & 2.4 & $(31.8-41.1)$ \\
\hline Roanoke, Virginia & 531 & 38.6 & 2.9 & $(32.8-44.3)$ \\
\hline Rochester, Minnesota & 695 & 31.4 & 2.3 & $(26.9-35.9)$ \\
\hline Rockingham County-Strafford County, New Hampshire ${ }^{\S}$ & 1,434 & 37.7 & 1.7 & $(34.3-41.1)$ \\
\hline Sacramento-Roseville-Arden-Arcade, California & 642 & 36.8 & 3.0 & $(31.0-42.6)$ \\
\hline St. Cloud, Minnesota & 559 & 34.4 & 2.8 & $(28.9-39.8)$ \\
\hline St. Louis, Missouri-Illinois & 1,924 & 40.1 & 1.8 & $(36.5-43.7)$ \\
\hline Salisbury, Maryland-Delaware & 1,956 & 44.3 & 1.8 & $(40.8-47.8)$ \\
\hline Salt Lake City, Utah & 5,396 & 35.9 & 1.0 & $(33.9-37.9)$ \\
\hline San Antonio-New Braunfels, Texas & 2,262 & 33.7 & 1.5 & $(30.8-36.5)$ \\
\hline San Juan-Carolina-Caguas, Puerto Rico & 3,735 & 40.2 & 1.2 & $(37.9-42.6)$ \\
\hline Scottsbluff, Nebraska & 903 & 41.7 & 2.6 & $(36.7-46.7)$ \\
\hline Seattle-Bellevue-Everett, Washington ${ }^{\S}$ & 3,681 & 37.5 & 1.1 & $(35.3-39.8)$ \\
\hline Shreveport-Bossier City, Louisiana & 548 & 44.6 & 3.1 & $(38.5-50.7)$ \\
\hline Silver Spring-Frederick-Rockville, Maryland $§$ & 2,365 & 33.7 & 1.7 & $(30.3-37.0)$ \\
\hline Sioux City, lowa-Nebraska-South Dakota & 1,139 & 35.3 & 3.0 & $(29.4-41.1)$ \\
\hline Sioux Falls, South Dakota & 1,338 & 37.6 & 2.2 & $(33.3-41.9)$ \\
\hline Spartanburg, South Carolina & 560 & 50.0 & 3.2 & $(43.7-56.3)$ \\
\hline Spokane-Spokane Valley, Washington & 723 & 41.8 & 2.6 & $(36.7-46.9)$ \\
\hline Springfield, Massachusetts & 1,094 & 51.5 & 2.7 & $(46.1-56.8)$ \\
\hline Tampa-St. Petersburg-Clearwater, Florida & 1,574 & 41.3 & 1.9 & $(37.6-45.0)$ \\
\hline
\end{tabular}

See table footnotes on page 107. 
TABLE 48. (Continued) Age-adjusted* prevalence estimates of adults aged $\geq 45$ years who have ever been told by a health professional they have some form of arthritis, ${ }^{\dagger}$ by metropolitan and micropolitan statistical area - Behavioral Risk Factor Surveillance System, United States, 2014

\begin{tabular}{|c|c|c|c|c|}
\hline MMSA & $\begin{array}{l}\text { Sample } \\
\text { size }\end{array}$ & $\%$ & SE & $95 \% \mathrm{Cl}$ \\
\hline Toledo, Ohio & 649 & 46.7 & 2.8 & $(41.2-52.2)$ \\
\hline Topeka, Kansas & 1,434 & 40.8 & 1.8 & $(37.3-44.3)$ \\
\hline Tulsa, Oklahoma & 2,021 & 38.1 & 1.4 & $(35.3-40.8)$ \\
\hline Tuscaloosa, Alabama & 719 & 47.8 & 2.7 & $(42.4-53.1)$ \\
\hline Virginia Beach-Norfolk-Newport News, Virginia-North Carolina & 1,876 & 42.3 & 1.7 & $(39.0-45.7)$ \\
\hline Warren-Troy-Farmington Hills, Michigan ${ }^{\S}$ & 2,100 & 43.2 & 1.5 & $(40.2-46.1)$ \\
\hline $\begin{array}{l}\text { Washington-Arlington-Alexandria, District of Columbia- } \\
\text { Virginia-Maryland-West Virginias }\end{array}$ & 8,238 & 38.9 & 1.1 & $(36.7-41.1)$ \\
\hline Wichita, Kansas & 2,725 & 41.0 & 1.3 & $(38.5-43.5)$ \\
\hline Wichita Falls, Texas & 543 & 45.0 & 3.0 & $(39.1-50.8)$ \\
\hline Wilmington, Delaware-Maryland-New Jersey ${ }^{\S}$ & 2,749 & 39.8 & 1.4 & $(37.1-42.5)$ \\
\hline Worcester, Massachusetts-Connecticut & 2,456 & 40.5 & 1.7 & $(37.2-43.8)$ \\
\hline Youngstown-Warren-Boardman, Ohio-Pennsylvania & 522 & 44.9 & 3.1 & $(38.7-51.0)$ \\
\hline Median & & 40.1 & & \\
\hline Range & & $28.4-54.7$ & & \\
\hline
\end{tabular}

Abbreviations: $\mathrm{Cl}=$ confidence interval; $\mathrm{MMSA}=$ metropolitan and micropolitan statistical areas; $\mathrm{SE}=$ standard error.

${ }^{*}$ Age adjusted to the 2000 U.S. standard population.

${ }^{\dagger}$ Including arthritis, rheumatoid arthritis, gout, lupus, or fibromyalgia.

$\S$ Metropolitan division. 
TABLE 49. Age-adjusted* prevalence estimates of adults aged $\geq 18$ years who have ever been told by a health professional they have a depressive disorder, ${ }^{\dagger}$ by state/territory - Behavioral Risk Factor Surveillance System, United States, 2013

\begin{tabular}{|c|c|c|c|c|}
\hline State/Territory & $\begin{array}{c}\text { Sample } \\
\text { size }\end{array}$ & $\%$ & SE & $95 \% \mathrm{Cl}$ \\
\hline Alabama & 6,467 & 21.4 & 0.7 & (19.9-22.8) \\
\hline Alaska & 4,561 & 16.1 & 0.8 & $(14.6-17.6)$ \\
\hline Arizona & 4,223 & 17.9 & 1.0 & (15.9-19.9) \\
\hline Arkansas & 5,240 & 23.2 & 0.9 & (21.4-24.9) \\
\hline California & 11,495 & 12.9 & 0.4 & $(12.0-13.7)$ \\
\hline Colorado & 13,572 & 18.0 & 0.5 & $(17.1-18.9)$ \\
\hline Connecticut & 7,673 & 17.2 & 0.6 & $(16.0-18.5)$ \\
\hline Delaware & 5,181 & 17.6 & 0.8 & $(16.0-19.1)$ \\
\hline District of Columbia & 4,903 & 20.8 & 0.9 & $(18.9-22.6)$ \\
\hline Florida & 33,983 & 16.7 & 0.5 & $(15.8-17.6)$ \\
\hline Georgia & 8,095 & 17.2 & 0.6 & $(16.0-18.3)$ \\
\hline Hawaii & 7,834 & 11.4 & 0.5 & $(10.4-12.4)$ \\
\hline Idaho & 5,604 & 20.8 & 0.8 & $(19.2-22.4)$ \\
\hline Illinois & 5,599 & 15.4 & 0.7 & $(14.0-16.7)$ \\
\hline Indiana & 10,289 & 19.5 & 0.6 & (18.4-20.6) \\
\hline lowa & 8,138 & 19.4 & 0.6 & (18.1-20.6) \\
\hline Kansas & 23,166 & 18.1 & 0.3 & $(17.4-18.7)$ \\
\hline Kentucky & 10,973 & 20.1 & 0.6 & $(18.8-21.3)$ \\
\hline Louisiana & 5,226 & 18.6 & 0.9 & $(16.8-20.4)$ \\
\hline Maine & 8,070 & 23.8 & 0.7 & $(22.4-25.2)$ \\
\hline Maryland & 12,957 & 15.8 & 0.5 & $(14.8-16.8)$ \\
\hline Massachusetts & 14,997 & 19.7 & 0.6 & (18.6-20.8) \\
\hline Michigan & 12,704 & 21.2 & 0.5 & $(20.1-22.2)$ \\
\hline Minnesota & 14,284 & 18.4 & 0.6 & $(17.3-19.6)$ \\
\hline Mississippi & 7,415 & 19.1 & 0.7 & $(17.7-20.4)$ \\
\hline Missouri & 7,088 & 21.3 & 0.7 & $(19.8-22.7)$ \\
\hline Montana & 9,652 & 21.1 & 0.6 & $(19.9-22.4)$ \\
\hline Nebraska & 17,065 & 18.3 & 0.5 & $(17.3-19.4)$ \\
\hline Nevada & 5,075 & 17.6 & 1.0 & $(15.6-19.5)$ \\
\hline New Hampshire & 6,430 & 22.4 & 0.8 & $(20.9-24.0)$ \\
\hline New Jersey & 13,339 & 13.7 & 0.5 & $(12.8-14.6)$ \\
\hline New Mexico & 9,286 & 19.4 & 0.6 & (18.1-20.6) \\
\hline New York & 8,923 & 16.0 & 0.6 & $(14.9-17.1)$ \\
\hline North Carolina & 8,825 & 18.5 & 0.6 & (17.4-19.6) \\
\hline North Dakota & 7,767 & 16.9 & 0.7 & $(15.6-18.2)$ \\
\hline Ohio & 11,913 & 20.3 & 0.6 & $(19.2-21.5)$ \\
\hline Oklahoma & 8,214 & 23.3 & 0.6 & $(22.1-24.6)$ \\
\hline Oregon & 5,913 & 26.8 & 0.8 & $(25.1-28.4)$ \\
\hline Pennsylvania & 11,363 & 18.2 & 0.5 & $(17.2-19.2)$ \\
\hline Rhode Island & 6,509 & 22.3 & 0.8 & $(20.8-23.8)$ \\
\hline South Carolina & 10,621 & 19.4 & 0.6 & $(18.3-20.6)$ \\
\hline South Dakota & 6,854 & 14.6 & 0.7 & $(13.2-16.0)$ \\
\hline Tennessee & 5,786 & 19.0 & 0.8 & $(17.5-20.5)$ \\
\hline Texas & 10,837 & 15.9 & 0.5 & $(14.8-16.9)$ \\
\hline Utah & 12,718 & 21.8 & 0.5 & $(20.9-22.7)$ \\
\hline Vermont & 6,360 & 23.1 & 0.8 & $(21.6-24.7)$ \\
\hline Virginia & 8,423 & 16.3 & 0.5 & $(15.2-17.3)$ \\
\hline Washington & 11,112 & 23.4 & 0.6 & $(22.2-24.5)$ \\
\hline West Virginia & 5,876 & 22.0 & 0.7 & $(20.7-23.3)$ \\
\hline Wisconsin & 6,565 & 18.1 & 0.8 & $(16.5-19.7)$ \\
\hline Wyoming & 6,435 & 18.7 & 0.7 & $(17.2-20.1)$ \\
\hline Guam & 1,887 & 8.6 & 0.8 & $(7.0-10.2)$ \\
\hline Puerto Rico & 6,000 & 18.3 & 0.6 & $(17.0-19.5)$ \\
\hline Median & & 18.6 & & \\
\hline Range & & $8.6-26.8$ & & \\
\hline
\end{tabular}

Abbreviations: $\mathrm{Cl}=$ confidence interval; $\mathrm{SE}=$ standard error.

* Age adjusted to the 2000 U.S. standard population.

† Including depression, major depression, dysthymia, or minor depression. 
TABLE 50. Age-adjusted* prevalence estimates of adults aged $\geq 18$ years who have ever been told by a health professional they have a depressive disorder, ${ }^{\dagger}$ by metropolitan and micropolitan statistical area - Behavioral Risk Factor Surveillance System, United States, 2013

\begin{tabular}{|c|c|c|c|c|}
\hline MMSA & $\begin{array}{l}\text { Sample } \\
\text { size }\end{array}$ & $\%$ & SE & $95 \% \mathrm{Cl}$ \\
\hline Aguadilla-Isabela, Puerto Rico & 593 & 16.5 & 1.9 & $(12.8-20.2)$ \\
\hline Akron, Ohio & 690 & 23.4 & 2.8 & $(18.0-28.8)$ \\
\hline Albuquerque, New Mexico & 2,078 & 20.3 & 1.1 & $(18.0-22.5)$ \\
\hline Allentown-Bethlehem-Easton, Pennsylvania-New Jersey & 1,028 & 19.0 & 2.0 & $(15.1-22.9)$ \\
\hline Anchorage, Alaska & 1,516 & 16.2 & 1.2 & $(13.9-18.6)$ \\
\hline Atlanta-Sandy Springs-Roswell, Georgia & 3,498 & 15.4 & 0.8 & $(13.8-17.0)$ \\
\hline Augusta-Richmond County, Georgia-South Carolina & 907 & 16.5 & 1.9 & $(12.8-20.3)$ \\
\hline Austin-Round Rock, Texas & 929 & 17.0 & 1.6 & $(13.8-20.2)$ \\
\hline Baltimore-Columbia-Towson, Maryland & 4,753 & 17.4 & 0.8 & $(15.8-19.1)$ \\
\hline Baton Rouge, Louisiana & 923 & 15.8 & 1.8 & $(12.2-19.3)$ \\
\hline Billings, Montana & 816 & 23.7 & 1.7 & $(20.3-27.1)$ \\
\hline Birmingham-Hoover, Alabama & 1,348 & 21.2 & 1.5 & $(18.3-24.0)$ \\
\hline Bismarck, North Dakota & 1,034 & 16.7 & 1.5 & $(13.8-19.6)$ \\
\hline Boise City, Idaho & 1,479 & 20.8 & 1.5 & $(18.0-23.7)$ \\
\hline Boston, Massachusetts ${ }^{\S}$ & 4,041 & 17.9 & 0.9 & $(16.0-19.7)$ \\
\hline Buffalo-Cheektowaga-Niagara Falls, New York & 502 & 13.6 & 1.9 & $(9.9-17.3)$ \\
\hline Burlington-South Burlington, Vermont & 1,624 & 23.2 & 1.4 & $(20.5-25.9)$ \\
\hline Cambridge-Newton-Framingham, Massachusetts ${ }^{\S}$ & 4,878 & 17.6 & 0.9 & $(15.9-19.4)$ \\
\hline Camden, New Jersey ${ }^{\S}$ & 1,860 & 17.6 & 1.3 & $(15.1-20.0)$ \\
\hline Cedar Rapids, lowa & 647 & 21.1 & 2.3 & $(16.5-25.7)$ \\
\hline Charleston, West Virginia & 817 & 24.0 & 1.9 & $(20.3-27.8)$ \\
\hline Charleston-North Charleston, South Carolina & 1,539 & 18.5 & 1.4 & $(15.8-21.2)$ \\
\hline Charlotte-Concord-Gastonia, North Carolina-South Carolina & 1,945 & 18.0 & 1.1 & $(15.8-20.3)$ \\
\hline Chattanooga, Tennessee-Georgia & 575 & 19.5 & 2.6 & $(14.4-24.7)$ \\
\hline Chicago-Naperville-Elgin, Illinois-Indiana-Wisconsin & 3,337 & 14.9 & 0.9 & $(13.2-16.7)$ \\
\hline Cincinnati, Ohio-Kentucky-Indiana & 2,601 & 20.1 & 1.2 & $(17.7-22.5)$ \\
\hline Claremont-Lebanon, New Hampshire-Vermont & 1,675 & 23.3 & 1.8 & $(19.8-26.7)$ \\
\hline Cleveland-Elyria, Ohio & 1,104 & 17.0 & 1.7 & $(13.7-20.3)$ \\
\hline Colorado Springs, Colorado & 1,369 & 20.8 & 1.4 & $(18.1-23.6)$ \\
\hline Columbia, South Carolina & 1,435 & 18.7 & 1.5 & $(15.7-21.7)$ \\
\hline Columbus, Ohio & 1,853 & 23.3 & 1.3 & $(20.8-25.9)$ \\
\hline Crestview-Fort Walton Beach-Destin, Florida & 1,076 & 18.7 & 1.8 & $(15.1-22.3)$ \\
\hline Dallas-Plano-Irving, Texas ${ }^{\S}$ & 899 & 13.6 & 1.3 & $(11.1-16.2)$ \\
\hline Davenport-Moline-Rock Island, lowa-Illinois & 671 & 18.0 & 2.2 & $(13.6-22.4)$ \\
\hline Dayton, Ohio & 838 & 20.6 & 2.2 & $(16.3-24.9)$ \\
\hline Deltona-Daytona Beach-Ormond Beach, Florida & 1,108 & 15.8 & 2.0 & $(11.8-19.7)$ \\
\hline Denver-Aurora-Lakewood, Colorado & 5,685 & 17.6 & 0.6 & $(16.4-18.9)$ \\
\hline Des Moines-West Des Moines, lowa & 1,343 & 19.8 & 1.5 & $(16.9-22.7)$ \\
\hline Duluth, Minnesota-Wisconsin & 700 & 18.0 & 2.3 & $(13.4-22.6)$ \\
\hline Durham-Chapel Hill, North Carolina & 614 & 15.7 & 2.0 & $(11.7-19.7)$ \\
\hline El Paso, Texas & 752 & 14.5 & 1.7 & $(11.2-17.8)$ \\
\hline Evansville, Indiana-Kentucky & 574 & 22.4 & 2.4 & $(17.7-27.1)$ \\
\hline Fargo, North Dakota-Minnesota & 1,179 & 21.5 & 1.7 & $(18.1-24.9)$ \\
\hline Fayetteville-Springdale-Rogers, Arkansas-Missouri & 817 & 22.8 & 2.2 & $(18.5-27.0)$ \\
\hline Fort Smith, Arkansas-Oklahoma & 499 & 28.5 & 2.8 & $(22.9-34.0)$ \\
\hline Fort Wayne, Indiana & 779 & 17.8 & 1.9 & $(14.1-21.4)$ \\
\hline Fort Worth-Arlington, Texas ${ }^{\S}$ & 808 & 18.5 & 1.9 & $(14.8-22.1)$ \\
\hline Gainesville, Florida & 1,027 & 17.5 & 2.0 & $(13.7-21.4)$ \\
\hline Grand Forks, North Dakota-Minnesota & 502 & 18.1 & 2.8 & $(12.7-23.5)$ \\
\hline Grand Island, Nebraska & 797 & 18.9 & 2.0 & $(15.0-22.8)$ \\
\hline Grand Rapids-Wyoming, Michigan & 1,340 & 21.3 & 1.5 & $(18.4-24.3)$ \\
\hline Greensboro-High Point, North Carolina & 663 & 21.1 & 2.1 & $(16.9-25.2)$ \\
\hline Greenville-Anderson-Mauldin, South Carolina & 1,340 & 22.5 & 1.7 & $(19.1-25.8)$ \\
\hline Gulfport-Biloxi-Pascagoula, Mississippi & 768 & 24.5 & 2.1 & $(20.4-28.7)$ \\
\hline Hagerstown-Martinsburg, Maryland-West Virginia & 765 & 24.7 & 2.7 & $(19.4-30.1)$ \\
\hline Hartford-West Hartford-East Hartford, Connecticut & 2,825 & 19.0 & 1.1 & $(16.7-21.2)$ \\
\hline Hilton Head Island-Bluffton-Beaufort, South Carolina & 821 & 12.4 & 1.8 & $(9.0-15.9)$ \\
\hline Houston-The Woodlands-Sugar Land, Texas & 1,382 & 13.7 & 1.3 & $(11.2-16.3)$ \\
\hline Huntington-Ashland, West Virginia-Kentucky-Ohio & 1,170 & 25.1 & 1.7 & $(21.8-28.3)$ \\
\hline Idaho Falls, Idaho & 506 & 28.2 & 2.9 & $(22.6-33.8)$ \\
\hline Indianapolis-Carmel-Anderson, Indiana & 2,529 & 20.8 & 1.1 & $(18.7-22.9)$ \\
\hline
\end{tabular}

See table footnotes on page 111. 
TABLE 50. (Continued) Age-adjusted* prevalence estimates of adults aged $\geq 18$ years who have ever been told by a health professional they have a depressive disorder, ${ }^{\dagger}$ by metropolitan and micropolitan statistical area - Behavioral Risk Factor Surveillance System, United States, 2013

\begin{tabular}{|c|c|c|c|c|}
\hline MMSA & $\begin{array}{l}\text { Sample } \\
\text { size }\end{array}$ & $\%$ & SE & $95 \% \mathrm{Cl}$ \\
\hline Jackson, Mississippi & 798 & 15.3 & 1.6 & $(12.3-18.4)$ \\
\hline Jacksonville, Florida & 2,859 & 20.2 & 1.3 & $(17.7-22.6)$ \\
\hline Kansas City, Missouri-Kansas & 7,389 & 19.3 & 0.9 & $(17.4-21.1)$ \\
\hline Kingsport-Bristol-Bristol, Tennessee-Virginia & 532 & 25.0 & 3.3 & $(18.5-31.6)$ \\
\hline Knoxville, Tennessee & 649 & 21.0 & 2.0 & $(17.0-25.0)$ \\
\hline Lansing-East Lansing, Michigan & 683 & 20.4 & 1.9 & $(16.6-24.2)$ \\
\hline Lexington-Fayette, Kentucky & 636 & 17.3 & 1.8 & $(13.8-20.8)$ \\
\hline Lincoln, Nebraska & 1,862 & 17.3 & 1.0 & $(15.3-19.2)$ \\
\hline Little Rock-North Little Rock-Conway, Arkansas & 1,140 & 22.6 & 1.8 & $(19.2-26.1)$ \\
\hline Logan, Utah-Idaho & 641 & 20.2 & 2.0 & $(16.3-24.1)$ \\
\hline Los Angeles-Long Beach-Anaheim, California & 3,034 & 11.9 & 0.8 & $(10.3-13.5)$ \\
\hline Louisville-Jefferson County, Kentucky-Indiana & 2,144 & 18.3 & 1.4 & $(15.7-21.0)$ \\
\hline Lubbock, Texas & 530 & 20.1 & 2.6 & $(15.0-25.2)$ \\
\hline Manhattan, Kansas & 662 & 23.2 & 1.9 & $(19.4-27.0)$ \\
\hline Memphis, Tennessee-Mississippi-Arkansas & 1,202 & 12.4 & 1.3 & $(10.0-14.9)$ \\
\hline Miami-Fort Lauderdale-West Palm Beach, Florida & 2,194 & 12.9 & 1.1 & $(10.7-15.0)$ \\
\hline Milwaukee-Waukesha-West Allis, Wisconsin & 1,266 & 20.3 & 1.9 & $(16.6-24.1)$ \\
\hline Minneapolis-St. Paul-Bloomington, Minnesota-Wisconsin & 9,100 & 18.1 & 0.7 & $(16.6-19.5)$ \\
\hline Minot, North Dakota & 651 & 12.4 & 1.7 & $(9.0-15.8)$ \\
\hline $\begin{array}{l}\text { Montgomery County-Bucks County-Chester County, } \\
\text { Pennsylvania§ }\end{array}$ & 970 & 16.5 & 1.5 & $(13.6-19.5)$ \\
\hline $\begin{array}{l}\text { Myrtle Beach-Conway-North Myrtle Beach, South Carolina- } \\
\text { North Carolina }\end{array}$ & 767 & 19.7 & 2.1 & $(15.6-23.7)$ \\
\hline Nashville-Davidson County-Murfreesboro-Franklin, Tennessee & 1,063 & 16.7 & 1.5 & $(13.7-19.7)$ \\
\hline Nassau County-Suffolk County, New York ${ }^{\S}$ & 937 & 13.8 & 1.5 & $(10.9-16.6)$ \\
\hline Newark, New Jersey-Pennsylvania§ & 4,106 & 12.6 & 0.8 & $(11.0-14.1)$ \\
\hline New Orleans-Metairie, Louisiana & 1,283 & 17.9 & 1.8 & $(14.4-21.5)$ \\
\hline New York-Jersey City-White Plains, New York-New Jersey ${ }^{\S}$ & 8,912 & 13.8 & 0.6 & $(12.7-14.9)$ \\
\hline Norfolk, Nebraska & 674 & 19.0 & 1.8 & $(15.4-22.6)$ \\
\hline North Platte, Nebraska & 713 & 23.1 & 2.4 & $(18.4-27.8)$ \\
\hline North Port-Sarasota-Bradenton, Florida & 1,087 & 22.7 & 2.1 & $(18.5-26.8)$ \\
\hline Oakland-Hayward-Berkeley, California§ & 699 & 12.1 & 1.4 & (9.4-14.9) \\
\hline Ogden-Clearfield, Utah & 2,443 & 22.5 & 1.0 & $(20.5-24.4)$ \\
\hline Oklahoma City, Oklahoma & 2,634 & 20.1 & 1.0 & $(18.1-22.0)$ \\
\hline Omaha-Council Bluffs, Nebraska-lowa & 3,115 & 20.2 & 1.0 & $(18.2-22.2)$ \\
\hline Orlando-Kissimmee-Sanford, Florida & 2,267 & 16.6 & 1.2 & $(14.3-19.0)$ \\
\hline Panama City, Florida & 1,020 & 19.8 & 2.0 & $(16.0-23.7)$ \\
\hline Pensacola-Ferry Pass-Brent, Florida & 1,307 & 18.2 & 1.3 & $(15.6-20.8)$ \\
\hline Philadelphia, Pennsylvania $§$ & 1,768 & 19.5 & 1.4 & $(16.7-22.2)$ \\
\hline Phoenix-Mesa-Scottsdale, Arizona & 1,544 & 17.2 & 1.4 & $(14.5-19.9)$ \\
\hline Pittsburgh, Pennsylvania & 2,352 & 16.3 & 1.1 & $(14.2-18.4)$ \\
\hline Ponce, Puerto Rico & 531 & 17.2 & 2.3 & $(12.6-21.8)$ \\
\hline Portland-South Portland, Maine & 2,634 & 23.0 & 1.2 & $(20.7-25.4)$ \\
\hline Portland-Vancouver-Hillsboro, Oregon-Washington & 3,226 & 25.7 & 1.1 & $(23.6-27.8)$ \\
\hline Port St. Lucie, Florida & 1,023 & 15.2 & 1.8 & $(11.6-18.8)$ \\
\hline Providence-Warwick, Rhode Island-Massachusetts & 8,279 & 23.1 & 0.9 & $(21.3-24.9)$ \\
\hline Provo-Orem, Utah & 1,856 & 20.2 & 1.1 & $(18.0-22.4)$ \\
\hline Raleigh, North Carolina & 675 & 14.3 & 1.5 & $(11.4-17.3)$ \\
\hline Rapid City, South Dakota & 868 & 18.7 & 1.9 & $(15.0-22.4)$ \\
\hline Reno, Nevada & 1,803 & 15.6 & 1.2 & $(13.3-17.9)$ \\
\hline Richmond, Virginia & 1,306 & 18.7 & 1.5 & $(15.9-21.6)$ \\
\hline Riverside-San Bernardino-Ontario, California & 1,369 & 13.0 & 1.1 & $(10.9-15.1)$ \\
\hline Rochester, New York & 507 & 21.0 & 2.5 & $(16.2-25.9)$ \\
\hline Rockingham County-Strafford County, New Hampshire $§$ & 1,656 & 22.8 & 1.5 & $(19.8-25.8)$ \\
\hline Sacramento-Roseville-Arden-Arcade, California & 890 & 17.5 & 1.9 & $(13.8-21.1)$ \\
\hline St. Louis, Missouri-Illinois & 2,052 & 18.8 & 1.2 & $(16.4-21.2)$ \\
\hline Salem, Oregon & 523 & 26.7 & 2.8 & $(21.1-32.2)$ \\
\hline Salina, Kansas & 524 & 17.4 & 2.1 & $(13.3-21.5)$ \\
\hline Salisbury, Maryland-Delaware & 2,052 & 16.6 & 1.4 & $(13.8-19.4)$ \\
\hline Salt Lake City, Utah & 4,657 & 22.5 & 0.8 & $(21.0-24.0)$ \\
\hline San Antonio-New Braunfels, Texas & 936 & 17.6 & 1.6 & $(14.5-20.7)$ \\
\hline
\end{tabular}

See table footnotes on page 111. 
TABLE 50. (Continued) Age-adjusted* prevalence estimates of adults aged $\geq 18$ years who have ever been told by a health professional they have a depressive disorder, ${ }^{\dagger}$ by metropolitan and micropolitan statistical area - Behavioral Risk Factor Surveillance System, United States, 2013

\begin{tabular}{|c|c|c|c|c|}
\hline MMSA & $\begin{array}{l}\text { Sample } \\
\text { size }\end{array}$ & $\%$ & SE & $95 \% \mathrm{Cl}$ \\
\hline San Francisco-Redwood City-South San Francisco, California ${ }^{\S}$ & 538 & 16.2 & 2.1 & $(12.1-20.2)$ \\
\hline San Jose-Sunnyvale-Santa Clara, California & 625 & 7.7 & 1.2 & $(5.4-10.0)$ \\
\hline San Juan-Carolina-Caguas, Puerto Rico & 3,648 & 19.0 & 0.8 & $(17.4-20.6)$ \\
\hline Scottsbluff, Nebraska & 710 & 21.0 & 2.3 & $(16.5-25.6)$ \\
\hline Scranton-Wilkes-Barre-Hazleton, Pennsylvania & 562 & 22.4 & 2.7 & $(17.1-27.7)$ \\
\hline Seattle-Bellevue-Everett, Washington $\S$ & 3,757 & 23.1 & 0.9 & $(21.3-24.8)$ \\
\hline Shreveport-Bossier City, Louisiana & 572 & 15.9 & 2.1 & $(11.8-20.0)$ \\
\hline Silver Spring-Frederick-Rockville, Maryland ${ }^{\S}$ & 2,415 & 14.6 & 1.0 & $(12.7-16.6)$ \\
\hline Sioux City, lowa-Nebraska-South Dakota & 1,052 & 15.3 & 2.3 & $(10.8-19.8)$ \\
\hline Sioux Falls, South Dakota & 1,006 & 13.1 & 1.4 & $(10.4-15.9)$ \\
\hline Spartanburg, South Carolina & 592 & 17.0 & 2.3 & $(12.6-21.5)$ \\
\hline Spokane-Spokane Valley, Washington & 859 & 21.1 & 2.0 & $(17.2-25.0)$ \\
\hline Springfield, Massachusetts & 1,575 & 23.2 & 1.9 & $(19.5-26.9)$ \\
\hline Tallahassee, Florida & 1,852 & 17.8 & 1.6 & $(14.6-21.0)$ \\
\hline Tampa-St. Petersburg-Clearwater, Florida & 2,202 & 19.5 & 1.3 & $(17.0-22.0)$ \\
\hline Toledo, Ohio & 993 & 19.6 & 1.9 & $(15.9-23.3)$ \\
\hline Topeka, Kansas & 2,397 & 20.9 & 1.1 & $(18.7-23.0)$ \\
\hline Tulsa, Oklahoma & 1,993 & 26.1 & 1.4 & $(23.3-28.9)$ \\
\hline Virginia Beach-Norfolk-Newport News, Virginia-North Carolina & 1,677 & 15.6 & 1.2 & $(13.3-17.9)$ \\
\hline Warren-Troy-Farmington Hills, Michigan $\$$ & 2,253 & 19.5 & 1.2 & $(17.2-21.9)$ \\
\hline $\begin{array}{l}\text { Washington-Arlington-Alexandria, District of Columbia- } \\
\text { Virginia-Maryland-West Virginia§ }\end{array}$ & 8,983 & 14.0 & 0.7 & $(12.7-15.3)$ \\
\hline Wichita, Kansas & 4,910 & 19.0 & 0.7 & $(17.6-20.4)$ \\
\hline Wilmington, Delaware-Maryland-New Jersey ${ }^{\S}$ & 3,271 & 17.0 & 0.9 & $(15.2-18.7)$ \\
\hline Winston-Salem, North Carolina & 693 & 23.2 & 2.4 & $(18.6-27.9)$ \\
\hline Worcester, Massachusetts-Connecticut & 2,755 & 22.6 & 1.4 & $(19.9-25.3)$ \\
\hline Median & & 18.7 & & \\
\hline Range & & $7.7-28.5$ & & \\
\hline
\end{tabular}

Abbreviations: $\mathrm{Cl}=$ confidence interval; $\mathrm{MMSA}=$ metropolitan and micropolitan statistical areas; $\mathrm{SE}=$ standard error.

${ }^{*}$ Age adjusted to the 2000 U.S. standard population.

† Including depression, major depression, dysthymia, or minor depression.

$\S$ Metropolitan division. 
TABLE 51. Age-adjusted* prevalence estimates of adults aged $\geq 18$ years who have ever been told by a health professional they have a depressive disorder, ${ }^{\dagger}$ by state/territory - Behavioral Risk Factor Surveillance System, United States, 2014

\begin{tabular}{|c|c|c|c|c|}
\hline State/Territory & $\begin{array}{l}\text { Sample } \\
\text { size }\end{array}$ & $\%$ & SE & $95 \% \mathrm{Cl}$ \\
\hline Alabama & 8,611 & 21.1 & 0.6 & (19.9-22.4) \\
\hline Alaska & 4,373 & 15.4 & 0.8 & (13.9-16.9) \\
\hline Arizona & 14,801 & 18.7 & 0.6 & $(17.6-19.8)$ \\
\hline Arkansas & 5,225 & 21.5 & 0.9 & $(19.6-23.3)$ \\
\hline California & 8,781 & 13.1 & 0.4 & $(12.3-14.0)$ \\
\hline Colorado & 13,331 & 16.9 & 0.4 & $(16.1-17.7)$ \\
\hline Connecticut & 7,914 & 18.2 & 0.7 & $(16.9-19.5)$ \\
\hline Delaware & 4,285 & 18.3 & 0.9 & $(16.4-20.1)$ \\
\hline District of Columbia & 4,054 & 18.1 & 1.0 & $(16.1-20.2)$ \\
\hline Florida & 9,766 & 15.9 & 0.5 & (14.9-17.0) \\
\hline Georgia & 6,322 & 18.1 & 0.7 & $(16.8-19.4)$ \\
\hline Hawaii & 7,217 & 10.7 & 0.5 & (9.7-11.7) \\
\hline Idaho & 5,468 & 19.6 & 0.8 & $(18.0-21.2)$ \\
\hline Illinois & 5,040 & 16.6 & 0.7 & $(15.2-18.1)$ \\
\hline Indiana & 11,473 & 20.8 & 0.6 & (19.7-21.9) \\
\hline lowa & 8,095 & 18.9 & 0.6 & $(17.7-20.2)$ \\
\hline Kansas & 13,676 & 18.6 & 0.4 & (17.7-19.4) \\
\hline Kentucky & 11,144 & 23.9 & 0.8 & $(22.4-25.4)$ \\
\hline Louisiana & 6,753 & 18.5 & 0.6 & $(17.3-19.7)$ \\
\hline Maine & 9,094 & 24.2 & 0.7 & $(22.7-25.6)$ \\
\hline Maryland & 12,520 & 15.9 & 0.6 & $(14.7-17.2)$ \\
\hline Massachusetts & 15,545 & 21.7 & 0.6 & $(20.6-22.8)$ \\
\hline Michigan & 8,439 & 20.4 & 0.6 & $(19.2-21.7)$ \\
\hline Minnesota & 16,359 & 18.3 & 0.4 & $(17.5-19.0)$ \\
\hline Mississippi & 4,184 & 19.8 & 0.9 & (18.0-21.6) \\
\hline Missouri & 7,046 & 21.9 & 0.8 & (20.3-23.4) \\
\hline Montana & 7,460 & 20.6 & 0.8 & $(19.1-22.1)$ \\
\hline Nebraska & 22,346 & 17.8 & 0.5 & (16.9-18.7) \\
\hline Nevada & 3,734 & 15.8 & 1.0 & (13.9-17.7) \\
\hline New Hampshire & 6,159 & 21.7 & 0.9 & (19.9-23.4) \\
\hline New Jersey & 12,977 & 13.1 & 0.5 & $(12.2-14.0)$ \\
\hline New Mexico & 8,919 & 21.0 & 0.7 & $(19.6-22.4)$ \\
\hline New York & 6,834 & 15.7 & 0.6 & $(14.6-16.9)$ \\
\hline North Carolina & 7,254 & 18.8 & 0.6 & (17.7-19.9) \\
\hline North Dakota & 7,756 & 17.3 & 0.7 & (15.9-18.8) \\
\hline Ohio & 10,893 & 21.1 & 0.7 & $(19.7-22.5)$ \\
\hline Oklahoma & 8,413 & 21.7 & 0.6 & (20.5-22.9) \\
\hline Oregon & 5,197 & 24.1 & 0.8 & $(22.5-25.8)$ \\
\hline Pennsylvania & 10,949 & 19.9 & 0.6 & (18.7-21.0) \\
\hline Rhode Island & 6,413 & 20.7 & 0.8 & $(19.1-22.3)$ \\
\hline South Carolina & 10,952 & 19.7 & 0.5 & (18.6-20.7) \\
\hline South Dakota & 7,369 & 17.0 & 0.9 & $(15.3-18.7)$ \\
\hline Tennessee & 5,115 & 21.2 & 0.9 & $(19.5-22.9)$ \\
\hline Texas & 15,343 & 14.5 & 0.5 & $(13.6-15.4)$ \\
\hline Utah & 14,959 & 20.7 & 0.4 & (19.9-21.4) \\
\hline Vermont & 6,443 & 22.1 & 0.7 & $(20.8-23.5)$ \\
\hline Virginia & 9,433 & 17.4 & 0.6 & (16.3-18.5) \\
\hline Washington & 10,047 & 21.4 & 0.6 & (20.3-22.6) \\
\hline West Virginia & 6,178 & 23.8 & 0.7 & $(22.4-25.2)$ \\
\hline Wisconsin & 7,020 & 16.9 & 0.7 & $(15.5-18.3)$ \\
\hline Wyoming & 6,380 & 19.0 & 0.9 & (17.2-20.9) \\
\hline Guam & 2,506 & 8.9 & 0.8 & (7.3-10.6) \\
\hline Puerto Rico & 5,981 & 17.9 & 0.6 & $(16.7-19.1)$ \\
\hline Median & & 18.8 & & \\
\hline Range & & $8.9-24.2$ & & \\
\hline
\end{tabular}

Abbreviations: $\mathrm{Cl}=$ confidence interval; $\mathrm{SE}=$ standard error.

* Age adjusted to the 2000 U.S. standard population.

† Including depression, major depression, dysthymia, or minor depression. 
TABLE 52. Age-adjusted* prevalence estimates of adults aged $\geq 18$ years who have ever been told by a health professional they have a depressive disorder, ${ }^{\dagger}$ by metropolitan and micropolitan statistical area - Behavioral Risk Factor Surveillance System, United States, 2014

\begin{tabular}{|c|c|c|c|c|}
\hline MMSA & $\begin{array}{l}\text { Sample } \\
\text { size }\end{array}$ & $\%$ & SE & $95 \% \mathrm{Cl}$ \\
\hline Aberdeen, South Dakota & 617 & 19.4 & 3.0 & $(13.5-25.3)$ \\
\hline Aguadilla-Isabela, Puerto Rico & 544 & 17.4 & 1.9 & $(13.8-21.1)$ \\
\hline Albuquerque, New Mexico & 1,787 & 22.6 & 1.4 & $(20.0-25.3)$ \\
\hline Allentown-Bethlehem-Easton, Pennsylvania-New Jersey & 1,092 & 18.6 & 2.1 & $(14.5-22.7)$ \\
\hline Anchorage, Alaska & 1,781 & 16.0 & 1.1 & $(13.7-18.2)$ \\
\hline Atlanta-Sandy Springs-Roswell, Georgia & 2,767 & 17.1 & 1.0 & $(15.2-19.0)$ \\
\hline Augusta-Richmond County, Georgia-South Carolina & 881 & 20.2 & 2.6 & $(15.0-25.3)$ \\
\hline Austin-Round Rock, Texas & 2,248 & 15.2 & 1.0 & $(13.3-17.1)$ \\
\hline Baltimore-Columbia-Towson, Maryland & 4,595 & 17.5 & 1.1 & $(15.4-19.6)$ \\
\hline Baton Rouge, Louisiana & 918 & 17.5 & 1.6 & $(14.4-20.5)$ \\
\hline Berlin, New Hampshire-Vermont & 540 & 18.8 & 3.2 & $(12.6-25.0)$ \\
\hline Billings, Montana & 805 & 23.3 & 2.0 & $(19.3-27.3)$ \\
\hline Birmingham-Hoover, Alabama & 1,570 & 22.2 & 1.4 & $(19.5-24.9)$ \\
\hline Bismarck, North Dakota & 1,035 & 17.0 & 1.9 & $(13.3-20.6)$ \\
\hline Boise City, Idaho & 1,353 & 20.7 & 1.6 & $(17.6-23.8)$ \\
\hline Boston, Massachusetts $§$ & 4,517 & 20.9 & 1.1 & $(18.8-23.1)$ \\
\hline Burlington-South Burlington, Vermont & 1,970 & 22.4 & 1.2 & $(20.0-24.8)$ \\
\hline Cambridge-Newton-Framingham, Massachusetts ${ }^{\S}$ & 5,150 & 20.0 & 0.9 & $(18.2-21.8)$ \\
\hline Camden, New Jersey ${ }^{\S}$ & 1,710 & 14.7 & 1.3 & $(12.1-17.2)$ \\
\hline Cedar Rapids, lowa & 640 & 22.4 & 2.3 & $(17.9-26.8)$ \\
\hline Charleston, West Virginia & 876 & 27.3 & 2.1 & $(23.2-31.4)$ \\
\hline Charleston-North Charleston, South Carolina & 1,393 & 19.8 & 1.5 & $(16.8-22.8)$ \\
\hline Charlotte-Concord-Gastonia, North Carolina-South Carolina & 2,146 & 18.1 & 1.1 & $(15.9-20.2)$ \\
\hline Chicago-Naperville-Elgin, Illinois-Indiana-Wisconsin & 4,116 & 15.8 & 0.9 & $(14.1-17.5)$ \\
\hline Cincinnati, Ohio-Kentucky-Indiana & 2,032 & 23.9 & 1.8 & $(20.5-27.4)$ \\
\hline Claremont-Lebanon, New Hampshire-Vermont & 1,678 & 22.6 & 1.8 & $(19.0-26.1)$ \\
\hline Cleveland-Elyria, Ohio & 964 & 20.6 & 2.1 & $(16.4-24.7)$ \\
\hline College Station-Bryan, Texas & 576 & 15.1 & 2.5 & $(10.2-20.0)$ \\
\hline Colorado Springs, Colorado & 1,291 & 18.9 & 1.4 & $(16.2-21.6)$ \\
\hline Columbia, South Carolina & 1,202 & 18.3 & 1.4 & $(15.6-21.1)$ \\
\hline Columbus, Ohio & 1,652 & 18.6 & 1.4 & $(15.9-21.2)$ \\
\hline Corpus Christi, Texas & 618 & 17.5 & 2.4 & $(12.7-22.2)$ \\
\hline Dallas-Plano-Irving, Texas ${ }^{\S}$ & 1,292 & 13.2 & 1.4 & $(10.5-15.9)$ \\
\hline Dayton, Ohio & 563 & 28.3 & 2.8 & $(22.7-33.8)$ \\
\hline Denver-Aurora-Lakewood, Colorado & 5,771 & 16.6 & 0.6 & $(15.4-17.8)$ \\
\hline Des Moines-West Des Moines, lowa & 1,348 & 19.2 & 1.6 & $(16.1-22.3)$ \\
\hline Duluth, Minnesota-Wisconsin & 948 & 20.9 & 1.9 & $(17.3-24.6)$ \\
\hline El Paso, Texas & 720 & 14.8 & 1.6 & $(11.6-18.0)$ \\
\hline Evansville, Indiana-Kentucky & 655 & 25.5 & 3.1 & $(19.3-31.6)$ \\
\hline Fargo, North Dakota-Minnesota & 1,148 & 19.9 & 1.6 & $(16.7-23.1)$ \\
\hline Fayetteville-Springdale-Rogers, Arkansas-Missouri & 815 & 17.4 & 1.8 & $(13.8-20.9)$ \\
\hline Fort Wayne, Indiana & 858 & 21.6 & 2.2 & $(17.2-25.9)$ \\
\hline Fort Worth-Arlington, Texas ${ }^{\S}$ & 755 & 17.1 & 2.0 & $(13.1-21.1)$ \\
\hline Grand Island, Nebraska & 1,056 & 15.5 & 1.6 & $(12.3-18.6)$ \\
\hline Grand Rapids-Wyoming, Michigan & 895 & 21.0 & 2.0 & $(17.0-24.9)$ \\
\hline Greensboro-High Point, North Carolina & 521 & 17.6 & 2.1 & $(13.5-21.7)$ \\
\hline Greenville-Anderson-Mauldin, South Carolina & 1,485 & 19.5 & 1.3 & $(16.9-22.1)$ \\
\hline Hagerstown-Martinsburg, Maryland-West Virginia & 783 & 21.6 & 2.9 & $(15.9-27.4)$ \\
\hline Hartford-West Hartford-East Hartford, Connecticut & 2,634 & 17.8 & 1.0 & $(15.7-19.8)$ \\
\hline Hilton Head Island-Bluffton-Beaufort, South Carolina & 552 & 16.7 & 3.6 & $(9.7-23.8)$ \\
\hline Houston-The Woodlands-Sugar Land, Texas & 2,147 & 13.0 & 1.1 & $(10.8-15.1)$ \\
\hline Huntington-Ashland, West Virginia-Kentucky-Ohio & 1,243 & 25.2 & 1.9 & $(21.5-28.8)$ \\
\hline Idaho Falls, Idaho & 517 & 20.0 & 2.2 & $(15.6-24.4)$ \\
\hline Indianapolis-Carmel-Anderson, Indiana & 3,588 & 21.4 & 1.0 & $(19.4-23.4)$ \\
\hline Jacksonville, Florida & 668 & 14.7 & 1.9 & $(11.0-18.4)$ \\
\hline Kansas City, Missouri-Kansas & 4,856 & 19.8 & 1.1 & $(17.7-21.9)$ \\
\hline Kingsport-Bristol-Bristol, Tennessee-Virginia & 506 & 27.5 & 3.3 & $(21.1-34.0)$ \\
\hline Knoxville, Tennessee & 561 & 28.1 & 3.3 & $(21.7-34.5)$ \\
\hline Lafayette, Louisiana & 562 & 13.9 & 1.7 & $(10.6-17.2)$ \\
\hline Lexington-Fayette, Kentucky & 620 & 22.5 & 2.2 & $(18.2-26.8)$ \\
\hline Lincoln, Nebraska & 2,005 & 19.2 & 1.2 & $(16.8-21.5)$ \\
\hline
\end{tabular}

See table footnotes on page 115. 
TABLE 52. (Continued) Age-adjusted* prevalence estimates of adults aged $\geq 18$ years who have ever been told by a health professional they have a depressive disorder, ${ }^{\dagger}$ by metropolitan and micropolitan statistical area — Behavioral Risk Factor Surveillance System, United States, 2014

\begin{tabular}{|c|c|c|c|c|}
\hline MMSA & $\begin{array}{l}\text { Sample } \\
\text { size }\end{array}$ & $\%$ & SE & $95 \% \mathrm{Cl}$ \\
\hline Little Rock-North Little Rock-Conway, Arkansas & 1,174 & 23.9 & 1.9 & $(20.2-27.7)$ \\
\hline Logan, Utah-Idaho & 622 & 19.3 & 1.8 & $(15.8-22.7)$ \\
\hline Los Angeles-Long Beach-Anaheim, California & 2,441 & 11.7 & 0.8 & $(10.2-13.2)$ \\
\hline Louisville-Jefferson County, Kentucky-Indiana & 2,453 & 22.6 & 1.7 & $(19.2-26.0)$ \\
\hline Madison, Wisconsin & 549 & 16.7 & 2.0 & $(12.7-20.7)$ \\
\hline Memphis, Tennessee-Mississippi-Arkansas & 878 & 13.8 & 1.8 & $(10.3-17.2)$ \\
\hline Miami-Fort Lauderdale-West Palm Beach, Florida & 2,213 & 12.6 & 1.0 & $(10.7-14.5)$ \\
\hline Milwaukee-Waukesha-West Allis, Wisconsin & 1,351 & 18.6 & 1.8 & $(15.1-22.0)$ \\
\hline Minneapolis-St. Paul-Bloomington, Minnesota-Wisconsin & 8,729 & 18.0 & 0.5 & $(17.0-19.0)$ \\
\hline Minot, North Dakota & 595 & 17.9 & 2.4 & $(13.2-22.7)$ \\
\hline Montgomery, Alabama & 513 & 16.5 & 2.0 & $(12.6-20.3)$ \\
\hline $\begin{array}{l}\text { Montgomery County-Bucks County-Chester County, } \\
\text { Pennsylvania§ }\end{array}$ & 800 & 16.6 & 2.0 & $(12.7-20.6)$ \\
\hline $\begin{array}{l}\text { Myrtle Beach-Conway-North Myrtle Beach, South Carolina- } \\
\text { North Carolina }\end{array}$ & 995 & 20.5 & 1.9 & $(16.9-24.2)$ \\
\hline Nashville-Davidson County-Murfreesboro-Franklin, Tennessee & 800 & 18.8 & 1.9 & $(15.2-22.5)$ \\
\hline Nassau County-Suffolk County, New York ${ }^{\S}$ & 770 & 11.2 & 1.6 & $(8.1-14.3)$ \\
\hline Newark, New Jersey-Pennsylvania§ & 4,124 & 12.0 & 0.8 & $(10.5-13.6)$ \\
\hline New Orleans-Metairie, Louisiana & 1,908 & 19.2 & 1.2 & $(16.9-21.5)$ \\
\hline New York-Jersey City-White Plains, New York-New Jersey ${ }^{\S}$ & 7,503 & 14.3 & 0.6 & $(13.1-15.4)$ \\
\hline Norfolk, Nebraska & 996 & 16.7 & 1.6 & $(13.5-19.9)$ \\
\hline North Platte, Nebraska & 962 & 19.8 & 1.7 & $(16.4-23.2)$ \\
\hline North Port-Sarasota-Bradenton, Florida & 511 & 22.4 & 3.9 & $(14.9-30.0)$ \\
\hline Oakland-Hayward-Berkeley, California§ & 694 & 14.5 & 1.6 & $(11.4-17.6)$ \\
\hline Ogden-Clearfield, Utah & 2,917 & 21.3 & 0.9 & $(19.6-23.0)$ \\
\hline Oklahoma City, Oklahoma & 2,429 & 20.0 & 1.0 & $(18.0-22.0)$ \\
\hline Omaha-Council Bluffs, Nebraska-lowa & 4,858 & 18.4 & 0.8 & $(16.8-20.1)$ \\
\hline Orlando-Kissimmee-Sanford, Florida & 950 & 16.5 & 1.7 & $(13.2-19.7)$ \\
\hline Philadelphia, Pennsylvania ${ }^{\S}$ & 1,509 & 18.4 & 1.4 & $(15.6-21.2)$ \\
\hline Phoenix-Mesa-Scottsdale, Arizona & 9,357 & 17.5 & 0.6 & $(16.3-18.8)$ \\
\hline Pittsburgh, Pennsylvania & 2,413 & 19.8 & 1.2 & $(17.5-22.1)$ \\
\hline Ponce, Puerto Rico & 530 & 16.4 & 2.1 & $(12.2-20.5)$ \\
\hline Portland-South Portland, Maine & 2,744 & 21.8 & 1.2 & $(19.3-24.2)$ \\
\hline Portland-Vancouver-Hillsboro, Oregon-Washington & 2,832 & 22.9 & 1.1 & $(20.7-25.0)$ \\
\hline Providence-Warwick, Rhode Island-Massachusetts & 8,063 & 21.8 & 0.9 & $(20.0-23.6)$ \\
\hline Provo-Orem, Utah & 2,142 & 18.4 & 0.9 & $(16.5-20.2)$ \\
\hline Raleigh, North Carolina & 719 & 17.5 & 1.8 & $(13.9-21.0)$ \\
\hline Rapid City, South Dakota & 1,414 & 17.3 & 1.6 & $(14.1-20.5)$ \\
\hline Reno, Nevada & 1,192 & 16.7 & 1.5 & $(13.8-19.7)$ \\
\hline Richmond, Virginia & 1,457 & 15.5 & 1.2 & $(13.1-17.8)$ \\
\hline Riverside-San Bernardino-Ontario, California & 937 & 10.5 & 1.1 & $(8.4-12.6)$ \\
\hline Roanoke, Virginia & 533 & 19.7 & 2.6 & $(14.5-24.8)$ \\
\hline Rochester, Minnesota & 696 & 14.9 & 1.6 & $(11.7-18.1)$ \\
\hline Rockingham County-Strafford County, New Hampshire $§$ & 1,439 & 21.8 & 1.7 & $(18.4-25.1)$ \\
\hline Sacramento-Roseville-Arden-Arcade, California & 640 & 13.9 & 1.7 & $(10.7-17.2)$ \\
\hline St. Cloud, Minnesota & 559 & 17.5 & 1.9 & $(13.8-21.2)$ \\
\hline St. Louis, Missouri-Illinois & 1,916 & 20.0 & 1.4 & $(17.2-22.8)$ \\
\hline Salisbury, Maryland-Delaware & 1,953 & 20.6 & 2.0 & $(16.7-24.6)$ \\
\hline Salt Lake City, Utah & 5,400 & 21.8 & 0.6 & $(20.6-23.1)$ \\
\hline San Antonio-New Braunfels, Texas & 2,268 & 14.7 & 1.0 & $(12.8-16.6)$ \\
\hline San Juan-Carolina-Caguas, Puerto Rico & 3,743 & 18.0 & 0.8 & $(16.5-19.5)$ \\
\hline Scottsbluff, Nebraska & 906 & 23.5 & 2.1 & $(19.4-27.6)$ \\
\hline Seattle-Bellevue-Everett, Washington ${ }^{\S}$ & 3,684 & 19.8 & 0.9 & $(18.1-21.6)$ \\
\hline Shreveport-Bossier City, Louisiana & 550 & 17.4 & 2.0 & $(13.5-21.2)$ \\
\hline Silver Spring-Frederick-Rockville, Maryland ${ }^{\S}$ & 2,371 & 15.2 & 1.4 & $(12.5-18.0)$ \\
\hline Sioux City, lowa-Nebraska-South Dakota & 1,141 & 16.8 & 2.2 & $(12.4-21.2)$ \\
\hline Sioux Falls, South Dakota & 1,338 & 19.7 & 1.9 & $(16.0-23.5)$ \\
\hline Spartanburg, South Carolina & 562 & 25.8 & 3.0 & $(19.8-31.7)$ \\
\hline Spokane-Spokane Valley, Washington & 723 & 22.1 & 2.2 & $(17.7-26.4)$ \\
\hline Springfield, Massachusetts & 1,098 & 29.5 & 2.3 & $(25.1-34.0)$ \\
\hline Tampa-St. Petersburg-Clearwater, Florida & 1,576 & 18.1 & 1.5 & $(15.2-21.1)$ \\
\hline
\end{tabular}
See table footnotes on page 115. 
TABLE 52. (Continued) Age-adjusted* prevalence estimates of adults aged $\geq 18$ years who have ever been told by a health professional they have a depressive disorder, ${ }^{\dagger}$ by metropolitan and micropolitan statistical area - Behavioral Risk Factor Surveillance System, United States, 2014

\begin{tabular}{|c|c|c|c|c|}
\hline MMSA & $\begin{array}{l}\text { Sample } \\
\text { size }\end{array}$ & $\%$ & SE & $95 \% \mathrm{Cl}$ \\
\hline Toledo, Ohio & 646 & 25.1 & 2.8 & $(19.7-30.6)$ \\
\hline Topeka, Kansas & 1,439 & 19.0 & 1.3 & $(16.4-21.5)$ \\
\hline Tulsa, Oklahoma & 2,023 & 21.4 & 1.2 & $(19.0-23.7)$ \\
\hline Tuscaloosa, Alabama & 718 & 20.2 & 2.1 & $(16.2-24.3)$ \\
\hline Virginia Beach-Norfolk-Newport News, Virginia-North Carolina & 1,878 & 16.7 & 1.3 & $(14.2-19.2)$ \\
\hline Warren-Troy-Farmington Hills, Michigan ${ }^{\S}$ & 2,105 & 18.6 & 1.3 & $(16.1-21.2)$ \\
\hline $\begin{array}{l}\text { Washington-Arlington-Alexandria, District of Columbia- } \\
\text { Virginia-Maryland-West Virginias }\end{array}$ & 8,261 & 13.4 & 0.7 & $(12.1-14.7)$ \\
\hline Wichita, Kansas & 2,720 & 20.9 & 1.0 & $(19.0-22.8)$ \\
\hline Wichita Falls, Texas & 542 & 16.7 & 2.8 & $(11.1-22.2)$ \\
\hline Wilmington, Delaware-Maryland-New Jersey ${ }^{\S}$ & 2,758 & 18.9 & 1.2 & $(16.5-21.2)$ \\
\hline Worcester, Massachusetts-Connecticut & 2,446 & 22.6 & 1.4 & $(19.9-25.2)$ \\
\hline Youngstown-Warren-Boardman, Ohio-Pennsylvania & 521 & 19.3 & 2.8 & $(13.8-24.8)$ \\
\hline $\begin{array}{l}\text { Median } \\
\text { Range }\end{array}$ & & $\begin{array}{c}18.8 \\
10.5-29.5\end{array}$ & & \\
\hline
\end{tabular}

Abbreviations: $\mathrm{Cl}=$ confidence interval; $\mathrm{MMSA}=$ metropolitan and micropolitan statistical areas; $\mathrm{SE}=$ standard error.

${ }^{*}$ Age adjusted to the 2000 U.S. standard population.

${ }^{\dagger}$ Including depression, major depression, dysthymia, or minor depression.

$\S$ Metropolitan division. 
TABLE 53. Age-adjusted* prevalence estimates of adults aged $\geq 45$ years who have ever been told by a health professional they have had a coronary heart disease ${ }^{\dagger}$ by state/territory - Behavioral Risk Factor Surveillance System, United States, 2013

\begin{tabular}{|c|c|c|c|c|}
\hline State/Territory & $\begin{array}{l}\text { Sample } \\
\text { size }\end{array}$ & $\%$ & SE & $95 \% \mathrm{Cl}$ \\
\hline Alabama & 4,900 & 14.0 & 0.6 & $(12.7-15.3)$ \\
\hline Alaska & 2,775 & 11.5 & 0.9 & $(9.7-13.3)$ \\
\hline Arizona & 3,094 & 10.8 & 0.9 & $(9.0-12.7)$ \\
\hline Arkansas & 3,956 & 13.3 & 0.7 & $(11.9-14.7)$ \\
\hline California & 7,123 & 8.8 & 0.5 & (7.8-9.7) \\
\hline Colorado & 9,372 & 8.2 & 0.4 & $(7.5-8.9)$ \\
\hline Connecticut & 5,392 & 9.8 & 0.5 & $(8.8-10.9)$ \\
\hline Delaware & 3,736 & 11.4 & 0.6 & $(10.1-12.7)$ \\
\hline District of Columbia & 3,534 & 10.1 & 0.8 & $(8.7-11.6)$ \\
\hline Florida & 26,393 & 12.2 & 0.5 & $(11.3-13.1)$ \\
\hline Georgia & 5,529 & 10.9 & 0.5 & $(9.9-12.0)$ \\
\hline Hawaii & 5,100 & 7.4 & 0.5 & $(6.3-8.5)$ \\
\hline Idaho & 3,932 & 9.2 & 0.6 & $(8.0-10.4)$ \\
\hline Illinois & 3,962 & 10.5 & 0.6 & $(9.3-11.8)$ \\
\hline Indiana & 7,430 & 12.5 & 0.5 & $(11.6-13.4)$ \\
\hline lowa & 5,996 & 11.2 & 0.5 & $(10.3-12.1)$ \\
\hline Kansas & 16,354 & 11.3 & 0.3 & (10.7-11.8) \\
\hline Kentucky & 8,019 & 14.9 & 0.6 & $(13.7-16.2)$ \\
\hline Louisiana & 3,951 & 13.9 & 0.8 & $(12.4-15.4)$ \\
\hline Maine & 6,098 & 11.6 & 0.5 & $(10.5-12.6)$ \\
\hline Maryland & 9,525 & 9.8 & 0.4 & $(8.9-10.7)$ \\
\hline Massachusetts & 10,620 & 9.3 & 0.4 & (8.4-10.1) \\
\hline Michigan & 9,082 & 12.9 & 0.5 & $(12.0-13.8)$ \\
\hline Minnesota & 9,900 & 8.5 & 0.6 & $(7.3-9.6)$ \\
\hline Mississippi & 5,525 & 13.3 & 0.7 & $(12.0-14.6)$ \\
\hline Missouri & 5,152 & 13.2 & 0.7 & (11.9-14.6) \\
\hline Montana & 6,810 & 9.7 & 0.5 & $(8.7-10.7)$ \\
\hline Nebraska & 12,314 & 10.5 & 0.4 & $(9.7-11.3)$ \\
\hline Nevada & 3,487 & 10.8 & 0.9 & $(9.1-12.6)$ \\
\hline New Hampshire & 4,815 & 10.2 & 0.6 & $(9.0-11.4)$ \\
\hline New Jersey & 8,685 & 10.4 & 0.5 & $(9.4-11.4)$ \\
\hline New Mexico & 6,418 & 9.2 & 0.4 & $(8.4-10.1)$ \\
\hline New York & 5,773 & 12.3 & 0.6 & $(11.1-13.5)$ \\
\hline North Carolina & 6,141 & 11.7 & 0.5 & (10.7-12.7) \\
\hline North Dakota & 5,397 & 10.9 & 0.5 & $(9.9-12.0)$ \\
\hline Ohio & 8,544 & 12.7 & 0.5 & $(11.7-13.8)$ \\
\hline Oklahoma & 5,933 & 13.3 & 0.5 & $(12.3-14.3)$ \\
\hline Oregon & 4,308 & 10.1 & 0.6 & $(8.9-11.2)$ \\
\hline Pennsylvania & 8,067 & 11.9 & 0.5 & $(11.0-12.8)$ \\
\hline Rhode Island & 4,713 & 11.0 & 0.6 & $(9.9-12.1)$ \\
\hline South Carolina & 7,740 & 12.3 & 0.5 & $(11.3-13.3)$ \\
\hline South Dakota & 4,708 & 12.4 & 0.8 & $(10.8-14.0)$ \\
\hline Tennessee & 4,249 & 16.9 & 0.8 & $(15.2-18.5)$ \\
\hline Texas & 7,175 & 11.0 & 0.6 & $(9.9-12.2)$ \\
\hline Utah & 7,488 & 9.6 & 0.4 & $(8.8-10.4)$ \\
\hline Vermont & 4,719 & 9.9 & 0.5 & (8.9-10.9) \\
\hline Virginia & 5,766 & 10.6 & 0.5 & $(9.6-11.5)$ \\
\hline Washington & 7,958 & 10.5 & 0.5 & (9.6-11.4) \\
\hline West Virginia & 4,134 & 17.5 & 0.7 & $(16.1-18.8)$ \\
\hline Wisconsin & 4,686 & 11.1 & 0.8 & (9.6-12.6) \\
\hline Wyoming & 4,987 & 10.6 & 0.5 & $(9.6-11.7)$ \\
\hline Guam & 806 & 14.3 & 1.8 & $(10.7-17.8)$ \\
\hline Puerto Rico & 4,033 & 16.1 & 0.7 & $(14.7-17.6)$ \\
\hline Median & & 11.0 & & \\
\hline Range & & $7.4-17.5$ & & \\
\hline
\end{tabular}

Abbreviations: $\mathrm{Cl}=$ confidence interval; $\mathrm{SE}=$ standard error.

* Age adjusted to the 2000 U.S. standard population.

${ }^{\dagger}$ Including heart attack (also known as myocardial infarction) or angina. 
TABLE 54. Age-adjusted* prevalence estimates of adults aged $\geq 45$ years who have ever been told by a health professional they have had a coronary heart disease, ${ }^{\dagger}$ by metropolitan and micropolitan statistical area - Behavioral Risk Factor Surveillance System, United States, 2013

\begin{tabular}{|c|c|c|c|c|}
\hline MMSA & $\begin{array}{l}\text { Sample } \\
\text { size }\end{array}$ & $\%$ & SE & $95 \% \mathrm{Cl}$ \\
\hline Aguadilla-Isabela, Puerto Rico & 408 & 15.6 & 2.2 & $(11.2-20.0)$ \\
\hline Akron, Ohio & 535 & 13.8 & 2.4 & $(9.1-18.5)$ \\
\hline Albuquerque, New Mexico & 1,333 & 7.6 & 0.8 & $(6.0-9.1)$ \\
\hline Allentown-Bethlehem-Easton, Pennsylvania-New Jersey & 716 & 14.4 & 1.8 & $(10.8-18.0)$ \\
\hline Anchorage, Alaska & 921 & 11.7 & 1.4 & $(8.9-14.4)$ \\
\hline Atlanta-Sandy Springs-Roswell, Georgia & 2,243 & 10.3 & 0.9 & $(8.6-12.0)$ \\
\hline Augusta-Richmond County, Georgia-South Carolina & 669 & 12.7 & 2.0 & $(8.8-16.7)$ \\
\hline Austin-Round Rock, Texas & 587 & 8.8 & 1.6 & $(5.7-12.0)$ \\
\hline Baltimore-Columbia-Towson, Maryland & 3,411 & 11.1 & 0.7 & $(9.7-12.6)$ \\
\hline Baton Rouge, Louisiana & 670 & 12.8 & 1.5 & $(9.9-15.7)$ \\
\hline Billings, Montana & 492 & 10.6 & 1.5 & $(7.7-13.5)$ \\
\hline Birmingham-Hoover, Alabama & 974 & 12.8 & 1.3 & $(10.3-15.2)$ \\
\hline Bismarck, North Dakota & 690 & 10.3 & 1.3 & $(7.7-12.9)$ \\
\hline Boise City, Idaho & 984 & 8.8 & 1.1 & $(6.7-11.0)$ \\
\hline Boston, Massachusetts $§$ & 2,780 & 9.6 & 0.9 & $(7.9-11.3)$ \\
\hline Buffalo-Cheektowaga-Niagara Falls, New York & 362 & 12.0 & 2.1 & $(7.9-16.1)$ \\
\hline Burlington-South Burlington, Vermont & 1,074 & 9.4 & 1.1 & $(7.3-11.4)$ \\
\hline Cambridge-Newton-Framingham, Massachusetts ${ }^{\S}$ & 3,333 & 8.3 & 0.7 & $(7.0-9.6)$ \\
\hline Camden, New Jersey ${ }^{\S}$ & 1,246 & 11.3 & 1.2 & $(8.9-13.6)$ \\
\hline Cedar Rapids, lowa & 463 & 10.9 & 1.6 & $(7.8-13.9)$ \\
\hline Charleston, West Virginia & 581 & 16.1 & 1.6 & $(12.8-19.3)$ \\
\hline Charleston-North Charleston, South Carolina & 1,066 & 11.2 & 1.5 & $(8.3-14.2)$ \\
\hline Charlotte-Concord-Gastonia, North Carolina-South Carolina & 1,284 & 13.5 & 1.3 & $(11.0-16.1)$ \\
\hline Chattanooga, Tennessee-Georgia & 458 & 14.4 & 2.1 & $(10.2-18.6)$ \\
\hline Chicago-Naperville-Elgin, Illinois-Indiana-Wisconsin & 2,262 & 9.9 & 0.9 & $(8.2-11.5)$ \\
\hline Cincinnati, Ohio-Kentucky-Indiana & 1,837 & 12.7 & 1.1 & $(10.6-14.9)$ \\
\hline Claremont-Lebanon, New Hampshire-Vermont & 1,260 & 10.6 & 2.0 & $(6.7-14.6)$ \\
\hline Cleveland-Elyria, Ohio & 793 & 10.7 & 1.5 & $(7.8-13.6)$ \\
\hline Colorado Springs, Colorado & 933 & 6.7 & 1.1 & $(4.6-8.8)$ \\
\hline Columbia, South Carolina & 972 & 10.2 & 1.2 & $(7.8-12.7)$ \\
\hline Columbus, Ohio & 1,174 & 12.4 & 1.2 & $(10.1-14.7)$ \\
\hline Crestview-Fort Walton Beach-Destin, Florida & 778 & 12.2 & 1.5 & $(9.2-15.1)$ \\
\hline Dallas-Plano-Irving, Texas $§$ & 521 & 11.9 & 1.8 & $(8.5-15.3)$ \\
\hline Davenport-Moline-Rock Island, lowa-Illinois & 502 & 14.1 & 1.9 & $(10.4-17.9)$ \\
\hline Dayton, Ohio & 627 & 12.2 & 1.6 & $(9.2-15.3)$ \\
\hline Deltona-Daytona Beach-Ormond Beach, Florida & 915 & 10.6 & 1.3 & $(8.0-13.2)$ \\
\hline Denver-Aurora-Lakewood, Colorado & 3,655 & 7.4 & 0.5 & $(6.4-8.4)$ \\
\hline Des Moines-West Des Moines, lowa & 967 & 11.3 & 1.1 & $(9.1-13.5)$ \\
\hline Duluth, Minnesota-Wisconsin & 516 & 13.2 & 2.8 & $(7.7-18.7)$ \\
\hline Durham-Chapel Hill, North Carolina & 430 & 7.8 & 1.4 & $(5.1-10.5)$ \\
\hline El Paso, Texas & 481 & 7.7 & 1.7 & $(4.4-11.0)$ \\
\hline Evansville, Indiana-Kentucky & 436 & 11.6 & 2.1 & $(7.5-15.8)$ \\
\hline Fargo, North Dakota-Minnesota & 733 & 11.2 & 1.8 & $(7.6-14.7)$ \\
\hline Fayetteville-Springdale-Rogers, Arkansas-Missouri & 574 & 10.5 & 1.4 & $(7.8-13.2)$ \\
\hline Fort Smith, Arkansas-Oklahoma & 380 & 15.2 & 2.2 & $(10.9-19.4)$ \\
\hline Fort Wayne, Indiana & 559 & 11.7 & 1.5 & $(8.7-14.7)$ \\
\hline Fort Worth-Arlington, Texas ${ }^{\S}$ & 569 & 11.7 & 2.0 & $(7.8-15.6)$ \\
\hline Gainesville, Florida & 763 & 9.1 & 1.4 & $(6.5-11.8)$ \\
\hline Grand Forks, North Dakota-Minnesota & 343 & 9.7 & 1.8 & $(6.2-13.2)$ \\
\hline Grand Island, Nebraska & 605 & 8.5 & 1.2 & $(6.1-10.8)$ \\
\hline Grand Rapids-Wyoming, Michigan & 896 & 12.5 & 1.4 & $(9.7-15.2)$ \\
\hline Greensboro-High Point, North Carolina & 455 & 10.9 & 1.7 & $(7.6-14.3)$ \\
\hline Greenville-Anderson-Mauldin, South Carolina & 927 & 13.2 & 1.3 & $(10.6-15.9)$ \\
\hline Gulfport-Biloxi-Pascagoula, Mississippi & 553 & 14.1 & 1.6 & $(10.9-17.2)$ \\
\hline Hagerstown-Martinsburg, Maryland-West Virginia & 578 & 14.2 & 2.0 & $(10.2-18.1)$ \\
\hline Hartford-West Hartford-East Hartford, Connecticut & 2,002 & 10.2 & 0.9 & $(8.4-12.1)$ \\
\hline Hilton Head Island-Bluffton-Beaufort, South Carolina & 676 & 10.1 & 1.4 & $(7.4-12.8)$ \\
\hline Houston-The Woodlands-Sugar Land, Texas & 767 & 11.5 & 1.8 & $(8.1-15.0)$ \\
\hline Huntington-Ashland, West Virginia-Kentucky-Ohio & 820 & 20.7 & 1.8 & $(17.1-24.2)$ \\
\hline Idaho Falls, Idaho & 331 & 8.7 & 1.9 & $(5.0-12.5)$ \\
\hline Indianapolis-Carmel-Anderson, Indiana & 1,751 & 12.5 & 0.9 & $(10.7-14.3)$ \\
\hline
\end{tabular}

See table footnotes on page 119. 
TABLE 54. (Continued) Age-adjusted* prevalence estimates of adults aged $\geq 45$ years who have ever been told by a health professional they have had a coronary heart disease, ${ }^{\dagger}$ by metropolitan and micropolitan statistical area - Behavioral Risk Factor Surveillance System, United States, 2013

\begin{tabular}{|c|c|c|c|c|}
\hline MMSA & $\begin{array}{l}\text { Sample } \\
\text { size }\end{array}$ & $\%$ & SE & $95 \% \mathrm{Cl}$ \\
\hline Jackson, Mississippi & 539 & 11.3 & 1.7 & $(7.9-14.7)$ \\
\hline Jacksonville, Florida & 2,049 & 13.0 & 1.2 & $(10.7-15.3)$ \\
\hline Kansas City, Missouri-Kansas & 5,183 & 11.0 & 0.9 & $(9.2-12.7)$ \\
\hline Kingsport-Bristol-Bristol, Tennessee-Virginia & 440 & 17.3 & 3.1 & $(11.2-23.4)$ \\
\hline Knoxville, Tennessee & 465 & 15.6 & 2.1 & $(11.5-19.7)$ \\
\hline Lansing-East Lansing, Michigan & 464 & 7.8 & 1.5 & $(4.8-10.7)$ \\
\hline Lexington-Fayette, Kentucky & 379 & 13.9 & 2.6 & $(8.8-19.1)$ \\
\hline Lincoln, Nebraska & 1,109 & 11.4 & 1.1 & $(9.3-13.5)$ \\
\hline Little Rock-North Little Rock-Conway, Arkansas & 823 & 14.7 & 1.7 & $(11.4-18.0)$ \\
\hline Logan, Utah-Idaho & 362 & 8.0 & 1.5 & $(5.0-11.0)$ \\
\hline Los Angeles-Long Beach-Anaheim, California & 1,775 & 8.0 & 0.9 & $(6.2-9.9)$ \\
\hline Louisville-Jefferson County, Kentucky-Indiana & 1,569 & 13.2 & 1.5 & $(10.3-16.0)$ \\
\hline Lubbock, Texas & 408 & 8.8 & 1.6 & $(5.7-11.8)$ \\
\hline Manhattan, Kansas & 388 & 8.5 & 1.6 & $(5.4-11.6)$ \\
\hline Memphis, Tennessee-Mississippi-Arkansas & 843 & 16.9 & 2.3 & $(12.5-21.4)$ \\
\hline Miami-Fort Lauderdale-West Palm Beach, Florida & 1,589 & 10.8 & 1.3 & $(8.3-13.4)$ \\
\hline Milwaukee-Waukesha-West Allis, Wisconsin & 899 & 11.6 & 1.8 & $(8.0-15.2)$ \\
\hline Minneapolis-St. Paul-Bloomington, Minnesota-Wisconsin & 6,234 & 9.3 & 0.8 & $(7.7-10.9)$ \\
\hline Minot, North Dakota & 413 & 7.8 & 1.4 & $(5.0-10.7)$ \\
\hline $\begin{array}{l}\text { Montgomery County-Bucks County-Chester County, } \\
\text { Pennsylvania§ }\end{array}$ & 639 & 9.0 & 1.1 & $(6.8-11.3)$ \\
\hline $\begin{array}{l}\text { Myrtle Beach-Conway-North Myrtle Beach, South Carolina- } \\
\text { North Carolina }\end{array}$ & 576 & 17.1 & 2.4 & $(12.4-21.8)$ \\
\hline Nashville-Davidson County-Murfreesboro-Franklin, Tennessee & 698 & 16.0 & 1.8 & $(12.4-19.6)$ \\
\hline Nassau County-Suffolk County, New York ${ }^{\S}$ & 610 & 12.1 & 1.6 & $(9.1-15.2)$ \\
\hline Newark, New Jersey-Pennsylvania ${ }^{\S}$ & 2,674 & 9.2 & 0.9 & $(7.5-10.9)$ \\
\hline New Orleans-Metairie, Louisiana & 959 & 12.3 & 1.6 & $(9.1-15.5)$ \\
\hline New York-Jersey City-White Plains, New York-New Jersey ${ }^{\S}$ & 5,420 & 11.5 & 0.7 & $(10.1-13.0)$ \\
\hline Norfolk, Nebraska & 446 & 17.1 & 2.0 & $(13.2-21.0)$ \\
\hline North Platte, Nebraska & 531 & 12.4 & 1.7 & $(9.1-15.6)$ \\
\hline North Port-Sarasota-Bradenton, Florida & 861 & 13.1 & 1.3 & $(10.4-15.7)$ \\
\hline Oakland-Hayward-Berkeley, California§ & 409 & 6.9 & 1.5 & $(4.1-9.8)$ \\
\hline Ogden-Clearfield, Utah & 1,422 & 8.1 & 0.8 & $(6.5-9.7)$ \\
\hline Oklahoma City, Oklahoma & 1,809 & 12.6 & 0.9 & $(10.8-14.3)$ \\
\hline Omaha-Council Bluffs, Nebraska-lowa & 2,050 & 10.4 & 0.9 & $(8.7-12.2)$ \\
\hline Orlando-Kissimmee-Sanford, Florida & 1,597 & 11.0 & 1.1 & $(8.9-13.2)$ \\
\hline Panama City, Florida & 805 & 16.0 & 1.9 & $(12.2-19.8)$ \\
\hline Pensacola-Ferry Pass-Brent, Florida & 889 & 12.4 & 1.3 & $(9.9-14.9)$ \\
\hline Philadelphia, Pennsylvania§ & 1,181 & 12.0 & 1.2 & $(9.6-14.5)$ \\
\hline Phoenix-Mesa-Scottsdale, Arizona & 1,073 & 10.2 & 1.4 & $(7.6-12.9)$ \\
\hline Pittsburgh, Pennsylvania & 1,717 & 11.1 & 0.9 & $(9.3-12.8)$ \\
\hline Ponce, Puerto Rico & 342 & 20.9 & 2.8 & $(15.4-26.4)$ \\
\hline Portland-South Portland, Maine & 1,967 & 10.1 & 0.8 & $(8.4-11.8)$ \\
\hline Portland-Vancouver-Hillsboro, Oregon-Washington & 2,293 & 8.8 & 0.8 & $(7.2-10.4)$ \\
\hline Port St. Lucie, Florida & 831 & 11.1 & 1.3 & $(8.4-13.7)$ \\
\hline Providence-Warwick, Rhode Island-Massachusetts & 6,046 & 10.7 & 0.6 & $(9.5-12.0)$ \\
\hline Provo-Orem, Utah & 875 & 8.7 & 1.1 & $(6.6-10.8)$ \\
\hline Raleigh, North Carolina & 372 & 9.6 & 1.8 & $(6.1-13.1)$ \\
\hline Rapid City, South Dakota & 611 & 12.6 & 1.7 & $(9.3-15.8)$ \\
\hline Reno, Nevada & 1,219 & 10.8 & 1.1 & $(8.5-13.0)$ \\
\hline Richmond, Virginia & 885 & 10.8 & 1.3 & $(8.3-13.2)$ \\
\hline Riverside-San Bernardino-Ontario, California & 848 & 12.9 & 1.5 & $(9.9-15.9)$ \\
\hline Rochester, New York & 342 & 15.7 & 2.9 & $(10.0-21.4)$ \\
\hline Rockingham County-Strafford County, New Hampshire ${ }^{\S}$ & 1,188 & 10.6 & 1.1 & $(8.5-12.7)$ \\
\hline Sacramento-Roseville-Arden-Arcade, California & 586 & 8.0 & 1.3 & $(5.4-10.6)$ \\
\hline St. Louis, Missouri-Illinois & 1,467 & 13.2 & 1.3 & $(10.6-15.7)$ \\
\hline Salem, Oregon & 392 & 10.4 & 2.3 & $(5.9-14.9)$ \\
\hline Salina, Kansas & 377 & 10.8 & 1.7 & $(7.5-14.0)$ \\
\hline Salisbury, Maryland-Delaware & 1,638 & 13.8 & 1.2 & $(11.5-16.0)$ \\
\hline Salt Lake City, Utah & 2,716 & 10.2 & 0.7 & $(8.7-11.6)$ \\
\hline
\end{tabular}

See table footnotes on page 119. 
TABLE 54. (Continued) Age-adjusted* prevalence estimates of adults aged $\geq 45$ years who have ever been told by a health professional they have had a coronary heart disease ${ }^{\dagger}$ by metropolitan and micropolitan statistical area - Behavioral Risk Factor Surveillance System, United States, 2013

\begin{tabular}{|c|c|c|c|c|}
\hline MMSA & $\begin{array}{l}\text { Sample } \\
\text { size }\end{array}$ & $\%$ & SE & $95 \% \mathrm{Cl}$ \\
\hline San Antonio-New Braunfels, Texas & 621 & 9.1 & 1.3 & $(6.6-11.7)$ \\
\hline San Francisco-Redwood City-South San Francisco, California ${ }^{\S}$ & 321 & 6.2 & 1.7 & $(2.9-9.5)$ \\
\hline San Jose-Sunnyvale-Santa Clara, California & 351 & 7.3 & 1.5 & $(4.3-10.3)$ \\
\hline San Juan-Carolina-Caguas, Puerto Rico & 2,422 & 15.1 & 0.9 & $(13.2-16.9)$ \\
\hline Scottsbluff, Nebraska & 557 & 13.3 & 1.7 & $(10.0-16.6)$ \\
\hline Scranton-Wilkes-Barre-Hazleton, Pennsylvania & 432 & 10.9 & 1.7 & $(7.6-14.2)$ \\
\hline Seattle-Bellevue-Everett, Washington $\S$ & 2,487 & 9.7 & 0.8 & $(8.1-11.2)$ \\
\hline Shreveport-Bossier City, Louisiana & 445 & 16.0 & 2.4 & $(11.3-20.6)$ \\
\hline Silver Spring-Frederick-Rockville, Maryland $d^{\S}$ & 1,706 & 7.1 & 0.9 & $(5.4-8.8)$ \\
\hline Sioux City, lowa-Nebraska-South Dakota & 762 & 10.4 & 1.8 & $(6.8-13.9)$ \\
\hline Sioux Falls, South Dakota & 608 & 9.7 & 1.5 & $(6.7-12.6)$ \\
\hline Spartanburg, South Carolina & 462 & 12.5 & 2.1 & $(8.3-16.6)$ \\
\hline Spokane-Spokane Valley, Washington & 634 & 9.6 & 1.4 & $(6.8-12.4)$ \\
\hline Springfield, Massachusetts & 1,161 & 10.1 & 1.5 & $(7.3-13.0)$ \\
\hline Tallahassee, Florida & 1,424 & 9.6 & 1.3 & $(7.1-12.2)$ \\
\hline Tampa-St. Petersburg-Clearwater, Florida & 1,603 & 13.4 & 1.2 & $(11.0-15.7)$ \\
\hline Toledo, Ohio & 726 & 15.1 & 1.9 & $(11.4-18.8)$ \\
\hline Topeka, Kansas & 1,754 & 11.4 & 0.8 & $(9.8-13.1)$ \\
\hline Tulsa, Oklahoma & 1,462 & 12.2 & 1.0 & $(10.3-14.2)$ \\
\hline Virginia Beach-Norfolk-Newport News, Virginia-North Carolina & 1,137 & 10.1 & 1.1 & $(8.1-12.2)$ \\
\hline Warren-Troy-Farmington Hills, Michigan ${ }^{\S}$ & 1,612 & 12.0 & 1.0 & $(10.0-14.0)$ \\
\hline $\begin{array}{l}\text { Washington-Arlington-Alexandria, District of Columbia- } \\
\text { Virginia-Maryland-West Virginias }\end{array}$ & 6,186 & 8.0 & 0.7 & $(6.7-9.4)$ \\
\hline Wichita, Kansas & 3,425 & 11.9 & 0.6 & $(10.6-13.1)$ \\
\hline Wilmington, Delaware-Maryland-New Jersey ${ }^{\S}$ & 2,268 & 10.8 & 0.8 & $(9.3-12.4)$ \\
\hline Winston-Salem, North Carolina & 523 & 9.0 & 1.3 & $(6.5-11.5)$ \\
\hline Worcester, Massachusetts-Connecticut & 1,925 & 9.4 & 0.9 & $(7.6-11.2)$ \\
\hline Median & & 11.1 & & \\
\hline Range & & $6.2-20.9$ & & \\
\hline
\end{tabular}

Abbreviations: $\mathrm{Cl}=$ confidence interval; $\mathrm{MMSA}=$ metropolitan and micropolitan statistical areas; $\mathrm{SE}=$ standard error.

* Age adjusted to the 2000 U.S. standard population.

${ }^{\dagger}$ Including heart attack (also known as myocardial infarction) or angina.

$\S$ Metropolitan division. 
TABLE 55. Age-adjusted* prevalence estimates of adults aged $\geq 45$ years who have ever been told by a health professional they have had a coronary heart disease, ${ }^{\dagger}$ by state/territory - Behavioral Risk Factor Surveillance System, United States, 2014

\begin{tabular}{|c|c|c|c|c|}
\hline State/Territory & $\begin{array}{c}\text { Sample } \\
\text { size }\end{array}$ & $\%$ & SE & $95 \% \mathrm{Cl}$ \\
\hline Alabama & 6,387 & 14.6 & 0.6 & (13.4-15.8) \\
\hline Alaska & 2,854 & 8.9 & 0.8 & (7.4-10.5) \\
\hline Arizona & 11,619 & 10.5 & 0.4 & $(9.7-11.2)$ \\
\hline Arkansas & 4,148 & 15.9 & 0.8 & $(14.4-17.5)$ \\
\hline California & 5,291 & 10.2 & 0.5 & $(9.1-11.2)$ \\
\hline Colorado & 9,435 & 8.6 & 0.4 & $(7.9-9.3)$ \\
\hline Connecticut & 5,831 & 9.4 & 0.5 & $(8.4-10.3)$ \\
\hline Delaware & 3,231 & 11.9 & 0.7 & (10.4-13.3) \\
\hline District of Columbia & 3,024 & 8.3 & 0.7 & (6.9-9.6) \\
\hline Florida & 7,520 & 12.1 & 0.5 & $(11.2-13.1)$ \\
\hline Georgia & 4,615 & 12.2 & 0.6 & $(11.0-13.5)$ \\
\hline Hawaii & 4,795 & 8.0 & 0.6 & (6.9-9.2) \\
\hline Idaho & 3,906 & 9.2 & 0.5 & $(8.2-10.3)$ \\
\hline Illinois & 3,549 & 10.2 & 0.6 & $(9.0-11.4)$ \\
\hline Indiana & 8,550 & 12.5 & 0.4 & $(11.6-13.3)$ \\
\hline lowa & 5,999 & 10.7 & 0.5 & (9.7-11.6) \\
\hline Kansas & 9,549 & 10.7 & 0.4 & $(10.0-11.4)$ \\
\hline Kentucky & 8,527 & 15.6 & 0.6 & (14.4-16.9) \\
\hline Louisiana & 4,719 & 15.5 & 0.6 & $(14.3-16.7)$ \\
\hline Maine & 7,063 & 11.8 & 0.5 & $(10.7-12.8)$ \\
\hline Maryland & 9,723 & 8.9 & 0.4 & (8.0-9.7) \\
\hline Massachusetts & 11,616 & 10.9 & 0.5 & $(10.0-11.8)$ \\
\hline Michigan & 6,182 & 13.2 & 0.5 & $(12.1-14.2)$ \\
\hline Minnesota & 10,977 & 9.6 & 0.3 & $(8.9-10.2)$ \\
\hline Mississippi & 3,117 & 13.0 & 0.7 & $(11.5-14.4)$ \\
\hline Missouri & 5,359 & 12.9 & 0.7 & $(11.5-14.2)$ \\
\hline Montana & 5,653 & 10.0 & 0.5 & (8.9-11.0) \\
\hline Nebraska & 15,937 & 10.0 & 0.3 & (9.4-10.6) \\
\hline Nevada & 2,625 & 12.2 & 1.0 & $(10.3-14.2)$ \\
\hline New Hampshire & 4,688 & 10.1 & 0.6 & (9.0-11.2) \\
\hline New Jersey & 9,223 & 11.2 & 0.5 & $(10.2-12.1)$ \\
\hline New Mexico & 6,462 & 9.8 & 0.5 & (8.9-10.8) \\
\hline New York & 4,658 & 10.1 & 0.5 & $(9.1-11.2)$ \\
\hline North Carolina & 4,888 & 13.6 & 0.6 & $(12.5-14.8)$ \\
\hline North Dakota & 5,791 & 11.3 & 0.6 & $(10.2-12.4)$ \\
\hline Ohio & 8,314 & 13.2 & 0.6 & $(12.1-14.3)$ \\
\hline Oklahoma & 6,070 & 14.4 & 0.5 & $(13.3-15.4)$ \\
\hline Oregon & 3,856 & 9.9 & 0.6 & $(8.8-11.0)$ \\
\hline Pennsylvania & 8,164 & 11.7 & 0.4 & $(10.8-12.5)$ \\
\hline Rhode Island & 4,906 & 10.3 & 0.5 & (9.3-11.4) \\
\hline South Carolina & 8,018 & 12.2 & 0.5 & $(11.2-13.1)$ \\
\hline South Dakota & 5,178 & 12.1 & 0.7 & $(10.6-13.5)$ \\
\hline Tennessee & 3,943 & 14.9 & 0.7 & (13.4-16.3) \\
\hline Texas & 10,559 & 11.2 & 0.5 & $(10.1-12.2)$ \\
\hline Utah & 8,507 & 9.1 & 0.4 & (8.3-9.8) \\
\hline Vermont & 4,573 & 9.8 & 0.5 & (8.8-10.8) \\
\hline Virginia & 6,723 & 11.2 & 0.5 & $(10.2-12.1)$ \\
\hline Washington & 7,373 & 10.0 & 0.4 & (9.2-10.9) \\
\hline West Virginia & 4,497 & 16.1 & 0.6 & (14.8-17.3) \\
\hline Wisconsin & 5,057 & 9.5 & 0.6 & (8.3-10.6) \\
\hline Wyoming & 5,138 & 11.0 & 0.6 & $(9.9-12.1)$ \\
\hline Guam & 1,193 & 10.7 & 1.5 & (7.8-13.6) \\
\hline Puerto Rico & 4,075 & 17.1 & 0.8 & $(15.6-18.6)$ \\
\hline Median & & 11.0 & & \\
\hline Range & & $8.0-17.1$ & & \\
\hline
\end{tabular}

Abbreviations: $\mathrm{Cl}=$ confidence interval; $\mathrm{SE}=$ standard error.

* Age adjusted to the 2000 U.S. standard population.

† Including heart attack (also known as myocardial infarction) or angina. 
TABLE 56. Age-adjusted* prevalence estimates of adults aged $\geq 45$ years who have ever been told by a health professional they have had a coronary heart disease, ${ }^{\dagger}$ by metropolitan and micropolitan statistical area — Behavioral Risk Factor Surveillance System, United States, 2014

\begin{tabular}{|c|c|c|c|c|}
\hline MMSA & $\begin{array}{c}\text { Sample } \\
\text { size }\end{array}$ & $\%$ & SE & $95 \% \mathrm{Cl}$ \\
\hline Aberdeen, South Dakota & 457 & 14.2 & 1.9 & $(10.5-17.8)$ \\
\hline Aguadilla-Isabela, Puerto Rico & 360 & 15.9 & 2.6 & $(10.8-21.1)$ \\
\hline Albuquerque, New Mexico & 1,252 & 9.0 & 0.9 & $(7.1-10.8)$ \\
\hline Allentown-Bethlehem-Easton, Pennsylvania-New Jersey & 876 & 11.6 & 1.6 & $(8.4-14.8)$ \\
\hline Anchorage, Alaska & 1,126 & 8.8 & 1.2 & $(6.5-11.2)$ \\
\hline Atlanta-Sandy Springs-Roswell, Georgia & 1,893 & 10.4 & 1.0 & $(8.5-12.3)$ \\
\hline Augusta-Richmond County, Georgia-South Carolina & 630 & 8.7 & 1.3 & $(6.1-11.3)$ \\
\hline Austin-Round Rock, Texas & 1,515 & 9.4 & 0.9 & $(7.5-11.2)$ \\
\hline Baltimore-Columbia-Towson, Maryland & 3,483 & 8.0 & 0.6 & $(6.9-9.2)$ \\
\hline Baton Rouge, Louisiana & 604 & 14.8 & 1.6 & $(11.7-18.0)$ \\
\hline Berlin, New Hampshire-Vermont & 443 & 15.8 & 2.3 & $(11.2-20.3)$ \\
\hline Billings, Montana & 586 & 9.3 & 1.7 & $(6.0-12.6)$ \\
\hline Birmingham-Hoover, Alabama & 1,102 & 12.9 & 1.3 & $(10.4-15.4)$ \\
\hline Bismarck, North Dakota & 803 & 9.4 & 1.1 & $(7.2-11.6)$ \\
\hline Boise City, Idaho & 956 & 9.5 & 1.1 & $(7.3-11.6)$ \\
\hline Boston, Massachusetts ${ }^{\S}$ & 3,350 & 11.0 & 0.8 & $(9.4-12.6)$ \\
\hline Burlington-South Burlington, Vermont & 1,298 & 8.2 & 0.9 & $(6.4-10.1)$ \\
\hline Cambridge-Newton-Framingham, Massachusetts ${ }^{\S}$ & 3,821 & 9.7 & 0.7 & $(8.4-11.0)$ \\
\hline Camden, New Jersey ${ }^{\S}$ & 1,285 & 11.4 & 1.1 & $(9.2-13.7)$ \\
\hline Cedar Rapids, lowa & 463 & 11.6 & 1.9 & $(7.8-15.4)$ \\
\hline Charleston, West Virginia & 621 & 16.8 & 1.7 & $(13.4-20.2)$ \\
\hline Charleston-North Charleston, South Carolina & 983 & 10.1 & 1.1 & $(7.8-12.3)$ \\
\hline Charlotte-Concord-Gastonia, North Carolina-South Carolina & 1,449 & 12.7 & 1.1 & $(10.5-15.0)$ \\
\hline Chicago-Naperville-Elgin, Illinois-Indiana-Wisconsin & 2,836 & 8.6 & 0.7 & (7.3-9.9) \\
\hline Cincinnati, Ohio-Kentucky-Indiana & 1,468 & 12.7 & 1.3 & $(10.1-15.3)$ \\
\hline Claremont-Lebanon, New Hampshire-Vermont & 1,282 & 9.7 & 1.0 & $(7.7-11.7)$ \\
\hline Cleveland-Elyria, Ohio & 723 & 11.2 & 1.3 & $(8.7-13.8)$ \\
\hline College Station-Bryan, Texas & 436 & 10.3 & 2.3 & $(5.8-14.8)$ \\
\hline Colorado Springs, Colorado & 889 & 8.9 & 1.1 & $(6.7-11.1)$ \\
\hline Columbia, South Carolina & 817 & 11.5 & 1.3 & $(9.0-14.0)$ \\
\hline Columbus, Ohio & 1,173 & 12.8 & 1.3 & $(10.2-15.4)$ \\
\hline Corpus Christi, Texas & 500 & 12.3 & 2.8 & $(6.8-17.7)$ \\
\hline Dallas-Plano-Irving, Texas $§$ & 902 & 10.3 & 1.7 & $(7.1-13.6)$ \\
\hline Dayton, Ohio & 421 & 17.1 & 2.6 & $(12.0-22.2)$ \\
\hline Denver-Aurora-Lakewood, Colorado & 3,838 & 8.1 & 0.5 & $(7.1-9.1)$ \\
\hline Des Moines-West Des Moines, lowa & 972 & 9.6 & 1.2 & $(7.2-11.9)$ \\
\hline Duluth, Minnesota-Wisconsin & 675 & 12.2 & 1.7 & $(9.0-15.5)$ \\
\hline El Paso, Texas & 515 & 9.6 & 1.5 & $(6.6-12.6)$ \\
\hline Evansville, Indiana-Kentucky & 543 & 15.2 & 2.3 & $(10.6-19.8)$ \\
\hline Fargo, North Dakota-Minnesota & 817 & 10.2 & 1.3 & $(7.6-12.7)$ \\
\hline Fayetteville-Springdale-Rogers, Arkansas-Missouri & 629 & 13.5 & 1.6 & $(10.3-16.6)$ \\
\hline Fort Wayne, Indiana & 658 & 11.0 & 1.4 & $(8.2-13.7)$ \\
\hline Fort Worth-Arlington, Texas $§$ & 539 & 11.5 & 1.6 & $(8.4-14.6)$ \\
\hline Grand Island, Nebraska & 789 & 10.4 & 1.2 & $(8.0-12.7)$ \\
\hline Grand Rapids-Wyoming, Michigan & 628 & 11.4 & 1.7 & $(8.1-14.6)$ \\
\hline Greensboro-High Point, North Carolina & 357 & 10.0 & 1.7 & $(6.7-13.2)$ \\
\hline Greenville-Anderson-Mauldin, South Carolina & 1,034 & 13.9 & 1.7 & $(10.7-17.2)$ \\
\hline Hagerstown-Martinsburg, Maryland-West Virginia & 636 & 14.6 & 1.9 & $(10.8-18.4)$ \\
\hline Hartford-West Hartford-East Hartford, Connecticut & 1,925 & 10.0 & 0.9 & $(8.3-11.7)$ \\
\hline Hilton Head Island-Bluffton-Beaufort, South Carolina & 440 & 11.8 & 2.2 & $(7.5-16.0)$ \\
\hline Houston-The Woodlands-Sugar Land, Texas & 1,438 & 10.7 & 1.5 & $(7.8-13.7)$ \\
\hline Huntington-Ashland, West Virginia-Kentucky-Ohio & 941 & 19.0 & 1.6 & $(15.9-22.1)$ \\
\hline Idaho Falls, Idaho & 341 & 9.6 & 1.9 & $(5.8-13.3)$ \\
\hline Indianapolis-Carmel-Anderson, Indiana & 2,668 & 12.1 & 0.8 & $(10.6-13.6)$ \\
\hline Jacksonville, Florida & 477 & 13.6 & 2.2 & $(9.4-17.9)$ \\
\hline Kansas City, Missouri-Kansas & 3,503 & 10.3 & 0.8 & $(8.8-11.8)$ \\
\hline Kingsport-Bristol-Bristol, Tennessee-Virginia & 404 & 15.0 & 2.1 & $(11.0-19.1)$ \\
\hline Knoxville, Tennessee & 445 & 14.5 & 2.0 & $(10.5-18.5)$ \\
\hline Lafayette, Louisiana & 383 & 16.4 & 2.2 & $(12.1-20.7)$ \\
\hline Lexington-Fayette, Kentucky & 388 & 16.4 & 2.9 & $(10.7-22.2)$ \\
\hline Lincoln, Nebraska & 1,217 & 8.3 & 0.9 & $(6.5-10.0)$ \\
\hline
\end{tabular}

See table footnotes on page 123. 
TABLE 56. (Continued) Age-adjusted* prevalence estimates of adults aged $\geq 45$ years who have ever been told by a health professional they have had a coronary heart disease, ${ }^{\dagger}$ by metropolitan and micropolitan statistical area - Behavioral Risk Factor Surveillance System, United States, 2014

\begin{tabular}{|c|c|c|c|c|}
\hline MMSA & $\begin{array}{l}\text { Sample } \\
\text { size }\end{array}$ & $\%$ & SE & $95 \% \mathrm{Cl}$ \\
\hline Little Rock-North Little Rock-Conway, Arkansas & 891 & 15.7 & 1.8 & $(12.2-19.1)$ \\
\hline Logan, Utah-Idaho & 333 & 8.4 & 1.5 & $(5.4-11.4)$ \\
\hline Los Angeles-Long Beach-Anaheim, California & 1,338 & 10.0 & 0.9 & $(8.1-11.8)$ \\
\hline Louisville-Jefferson County, Kentucky-Indiana & 1,949 & 14.0 & 1.2 & $(11.6-16.3)$ \\
\hline Madison, Wisconsin & 350 & 7.7 & 1.7 & $(4.3-11.1)$ \\
\hline Memphis, Tennessee-Mississippi-Arkansas & 653 & 12.3 & 1.7 & $(9.0-15.6)$ \\
\hline Miami-Fort Lauderdale-West Palm Beach, Florida & 1,636 & 10.7 & 1.0 & $(8.7-12.6)$ \\
\hline Milwaukee-Waukesha-West Allis, Wisconsin & 968 & 8.4 & 1.1 & $(6.1-10.6)$ \\
\hline Minneapolis-St. Paul-Bloomington, Minnesota-Wisconsin & 5,682 & 9.1 & 0.5 & $(8.2-10.0)$ \\
\hline Minot, North Dakota & 428 & 12.0 & 1.8 & $(8.4-15.6)$ \\
\hline Montgomery, Alabama & 360 & 14.9 & 2.2 & $(10.5-19.3)$ \\
\hline $\begin{array}{l}\text { Montgomery County-Bucks County-Chester County, } \\
\text { Pennsylvania§ }\end{array}$ & 585 & 9.5 & 1.4 & $(6.7-12.3)$ \\
\hline $\begin{array}{l}\text { Myrtle Beach-Conway-North Myrtle Beach, South Carolina- } \\
\text { North Carolina }\end{array}$ & 764 & 14.2 & 1.7 & $(10.9-17.5)$ \\
\hline Nashville-Davidson County-Murfreesboro-Franklin, Tennessee & 563 & 14.7 & 1.8 & $(11.1-18.3)$ \\
\hline Nassau County-Suffolk County, New York ${ }^{\S}$ & 551 & 9.9 & 1.4 & $(7.1-12.7)$ \\
\hline Newark, New Jersey-Pennsylvania ${ }^{\S}$ & 2,952 & 11.2 & 0.9 & $(9.4-12.9)$ \\
\hline New Orleans-Metairie, Louisiana & 1,345 & 15.0 & 1.2 & $(12.7-17.3)$ \\
\hline New York-Jersey City-White Plains, New York-New Jersey ${ }^{\S}$ & 4,776 & 9.7 & 0.6 & $(8.6-10.9)$ \\
\hline Norfolk, Nebraska & 696 & 11.1 & 1.4 & $(8.3-13.9)$ \\
\hline North Platte, Nebraska & 706 & 13.1 & 1.4 & $(10.3-15.8)$ \\
\hline North Port-Sarasota-Bradenton, Florida & 440 & 12.5 & 2.1 & $(8.3-16.7)$ \\
\hline Oakland-Hayward-Berkeley, California§ & 434 & 9.1 & 1.7 & $(5.8-12.4)$ \\
\hline Ogden-Clearfield, Utah & 1,594 & 8.0 & 0.7 & $(6.6-9.4)$ \\
\hline Oklahoma City, Oklahoma & 1,640 & 13.9 & 0.9 & $(12.1-15.8)$ \\
\hline Omaha-Council Bluffs, Nebraska-lowa & 3,285 & 9.5 & 0.7 & $(8.2-10.8)$ \\
\hline Orlando-Kissimmee-Sanford, Florida & 661 & 14.6 & 1.6 & $(11.4-17.8)$ \\
\hline Philadelphia, Pennsylvania ${ }^{\S}$ & 1,089 & 11.1 & 1.3 & $(8.5-13.7)$ \\
\hline Phoenix-Mesa-Scottsdale, Arizona & 7,202 & 10.5 & 0.5 & $(9.6-11.4)$ \\
\hline Pittsburgh, Pennsylvania & 1,810 & 12.8 & 0.9 & $(10.9-14.6)$ \\
\hline Ponce, Puerto Rico & 375 & 19.2 & 2.8 & $(13.7-24.6)$ \\
\hline Portland-South Portland, Maine & 2,177 & 9.9 & 0.9 & $(8.2-11.7)$ \\
\hline Portland-Vancouver-Hillsboro, Oregon-Washington & 2,042 & 8.8 & 0.7 & $(7.4-10.3)$ \\
\hline Providence-Warwick, Rhode Island-Massachusetts & 6,192 & 11.9 & 0.8 & $(10.4-13.4)$ \\
\hline Provo-Orem, Utah & 975 & 7.6 & 0.9 & $(5.8-9.3)$ \\
\hline Raleigh, North Carolina & 402 & 12.3 & 1.8 & $(8.8-15.8)$ \\
\hline Rapid City, South Dakota & 1,035 & 14.7 & 1.7 & $(11.4-17.9)$ \\
\hline Reno, Nevada & 850 & 9.4 & 1.1 & $(7.2-11.6)$ \\
\hline Richmond, Virginia & 1,014 & 12.0 & 1.3 & $(9.4-14.6)$ \\
\hline Riverside-San Bernardino-Ontario, California & 573 & 10.7 & 1.6 & $(7.5-13.8)$ \\
\hline Roanoke, Virginia & 405 & 13.1 & 2.2 & $(8.8-17.4)$ \\
\hline Rochester, Minnesota & 458 & 8.3 & 1.4 & $(5.6-11.0)$ \\
\hline Rockingham County-Strafford County, New Hampshire ${ }^{\S}$ & 1,081 & 11.7 & 1.1 & $(9.5-13.8)$ \\
\hline Sacramento-Roseville-Arden-Arcade, California & 411 & 13.6 & 2.8 & $(8.1-19.2)$ \\
\hline St. Cloud, Minnesota & 338 & 8.7 & 1.7 & $(5.3-12.0)$ \\
\hline St. Louis, Missouri-Illinois & 1,403 & 12.7 & 1.3 & $(10.1-15.3)$ \\
\hline Salisbury, Maryland-Delaware & 1,605 & 13.2 & 1.0 & $(11.3-15.1)$ \\
\hline Salt Lake City, Utah & 3,000 & 9.2 & 0.6 & $(8.0-10.4)$ \\
\hline San Antonio-New Braunfels, Texas & 1,619 & 10.9 & 1.0 & $(8.8-12.9)$ \\
\hline San Juan-Carolina-Caguas, Puerto Rico & 2,562 & 17.4 & 1.0 & $(15.5-19.3)$ \\
\hline Scottsbluff, Nebraska & 703 & 14.8 & 1.9 & $(11.0-18.5)$ \\
\hline Seattle-Bellevue-Everett, Washington ${ }^{\S}$ & 2,603 & 9.2 & 0.7 & $(7.9-10.6)$ \\
\hline Shreveport-Bossier City, Louisiana & 378 & 12.1 & 1.9 & $(8.3-15.9)$ \\
\hline Silver Spring-Frederick-Rockville, Maryland ${ }^{\S}$ & 1,779 & 8.2 & 1.1 & $(6.0-10.4)$ \\
\hline Sioux City, lowa-Nebraska-South Dakota & 871 & 10.7 & 2.1 & $(6.6-14.9)$ \\
\hline Sioux Falls, South Dakota & 905 & 11.2 & 1.2 & $(8.9-13.5)$ \\
\hline Spartanburg, South Carolina & 432 & 11.5 & 1.8 & $(8.0-15.0)$ \\
\hline Spokane-Spokane Valley, Washington & 546 & 10.1 & 1.6 & $(6.9-13.2)$ \\
\hline Springfield, Massachusetts & 785 & 13.3 & 1.9 & $(9.7-17.0)$ \\
\hline
\end{tabular}

See table footnotes on page 123. 
TABLE 56. (Continued) Age-adjusted* prevalence estimates of adults aged $\geq 45$ years who have ever been told by a health professional they have had a coronary heart disease, ${ }^{\dagger}$ by metropolitan and micropolitan statistical area - Behavioral Risk Factor Surveillance System, United States, 2014

\begin{tabular}{|c|c|c|c|c|}
\hline MMSA & $\begin{array}{l}\text { Sample } \\
\text { size }\end{array}$ & $\%$ & SE & $95 \% \mathrm{Cl}$ \\
\hline Tampa-St. Petersburg-Clearwater, Florida & 1,219 & 11.7 & 1.2 & $(9.4-14.1)$ \\
\hline Toledo, Ohio & 498 & 17.4 & 2.3 & $(12.9-21.9)$ \\
\hline Topeka, Kansas & 1,034 & 8.9 & 0.9 & $(7.1-10.7)$ \\
\hline Tulsa, Oklahoma & 1,462 & 13.9 & 1.1 & $(11.8-16.0)$ \\
\hline Tuscaloosa, Alabama & 490 & 12.8 & 1.9 & $(9.0-16.6)$ \\
\hline Virginia Beach-Norfolk-Newport News, Virginia-North Carolina & 1,339 & 11.0 & 1.1 & $(8.9-13.1)$ \\
\hline Warren-Troy-Farmington Hills, Michigan $\S$ & 1,538 & 12.7 & 1.0 & $(10.7-14.7)$ \\
\hline $\begin{array}{l}\text { Washington-Arlington-Alexandria, District of Columbia- } \\
\text { Virginia-Maryland-West Virginia§ }\end{array}$ & 5,922 & 8.0 & 0.6 & $(6.8-9.2)$ \\
\hline Wichita, Kansas & 1,872 & 11.2 & 0.8 & $(9.6-12.7)$ \\
\hline Wichita Falls, Texas & 474 & 12.4 & 2.4 & $(7.7-17.1)$ \\
\hline Wilmington, Delaware-Maryland-New Jersey ${ }^{\S}$ & 2,051 & 12.1 & 1.0 & $(10.1-14.1)$ \\
\hline Worcester, Massachusetts-Connecticut & 1,783 & 10.8 & 1.1 & $(8.7-12.9)$ \\
\hline Youngstown-Warren-Boardman, Ohio-Pennsylvania & 416 & 11.4 & 1.8 & $(7.9-14.9)$ \\
\hline Median & & 11.3 & & \\
\hline Range & & $7.6-19.2$ & & \\
\hline
\end{tabular}

Abbreviations: $\mathrm{Cl}=$ confidence interval; $\mathrm{MMSA}=$ metropolitan and micropolitan statistical areas; $\mathrm{SE}=$ standard error.

* Age adjusted to the 2000 U.S. standard population.

+ Including heart attack (also known as myocardial infarction) or angina.

$\S$ Metropolitan division. 
TABLE 57. Age-adjusted* prevalence estimates of adults aged $\geq 45$ years who have ever been told by a health professional they have had a stroke, by state/territory - Behavioral Risk Factor Surveillance System, United States, 2013

\begin{tabular}{|c|c|c|c|c|}
\hline State/Territory & $\begin{array}{l}\text { Sample } \\
\text { size }\end{array}$ & $\%$ & SE & $95 \% \mathrm{Cl}$ \\
\hline Alabama & 4,962 & 7.4 & 0.5 & $(6.4-8.4)$ \\
\hline Alaska & 2,798 & 5.0 & 0.6 & $(3.8-6.2)$ \\
\hline Arizona & 3,118 & 4.5 & 0.5 & $(3.5-5.5)$ \\
\hline Arkansas & 4,020 & 6.6 & 0.5 & $(5.6-7.6)$ \\
\hline California & 7,156 & 4.0 & 0.3 & $(3.5-4.6)$ \\
\hline Colorado & 9,423 & 3.2 & 0.2 & $(2.8-3.6)$ \\
\hline Connecticut & 5,454 & 3.8 & 0.3 & $(3.2-4.5)$ \\
\hline Delaware & 3,762 & 5.7 & 0.5 & $(4.7-6.7)$ \\
\hline District of Columbia & 3,552 & 5.9 & 0.6 & $(4.7-7.0)$ \\
\hline Florida & 26,625 & 5.3 & 0.3 & $(4.8-5.9)$ \\
\hline Georgia & 5,570 & 5.0 & 0.4 & $(4.3-5.7)$ \\
\hline Hawaii & 5,137 & 4.1 & 0.4 & $(3.4-4.9)$ \\
\hline Idaho & 3,972 & 3.6 & 0.4 & $(2.9-4.4)$ \\
\hline Illinois & 3,976 & 4.6 & 0.5 & $(3.7-5.6)$ \\
\hline Indiana & 7,517 & 5.2 & 0.3 & $(4.6-5.8)$ \\
\hline lowa & 6,040 & 4.5 & 0.3 & $(3.9-5.2)$ \\
\hline Kansas & 16,476 & 4.9 & 0.2 & $(4.5-5.3)$ \\
\hline Kentucky & 8,090 & 6.5 & 0.4 & $(5.7-7.4)$ \\
\hline Louisiana & 3,985 & 6.3 & 0.6 & $(5.0-7.5)$ \\
\hline Maine & 6,142 & 3.7 & 0.3 & $(3.1-4.3)$ \\
\hline Maryland & 9,567 & 5.1 & 0.3 & $(4.5-5.8)$ \\
\hline Massachusetts & 10,675 & 3.8 & 0.3 & $(3.3-4.4)$ \\
\hline Michigan & 9,156 & 5.5 & 0.3 & $(4.9-6.2)$ \\
\hline Minnesota & 9,932 & 3.5 & 0.4 & $(2.8-4.3)$ \\
\hline Mississippi & 5,612 & 7.5 & 0.5 & $(6.5-8.5)$ \\
\hline Missouri & 5,218 & 5.2 & 0.4 & $(4.4-6.0)$ \\
\hline Montana & 6,849 & 5.1 & 0.4 & $(4.3-5.8)$ \\
\hline Nebraska & 12,454 & 4.1 & 0.2 & $(3.6-4.5)$ \\
\hline Nevada & 3,504 & 5.1 & 0.6 & $(4.0-6.3)$ \\
\hline New Hampshire & 4,832 & 3.5 & 0.3 & $(2.9-4.1)$ \\
\hline New Jersey & 8,751 & 4.3 & 0.3 & $(3.6-4.9)$ \\
\hline New Mexico & 6,463 & 4.0 & 0.3 & $(3.4-4.6)$ \\
\hline New York & 5,819 & 4.0 & 0.4 & $(3.3-4.7)$ \\
\hline North Carolina & 6,206 & 5.9 & 0.4 & $(5.1-6.6)$ \\
\hline North Dakota & 5,449 & 4.2 & 0.3 & $(3.5-4.9)$ \\
\hline Ohio & 8,620 & 5.9 & 0.4 & $(5.1-6.6)$ \\
\hline Oklahoma & 5,969 & 6.0 & 0.4 & $(5.3-6.7)$ \\
\hline Oregon & 4,323 & 5.3 & 0.4 & $(4.5-6.2)$ \\
\hline Pennsylvania & 8,122 & 5.0 & 0.3 & $(4.4-5.6)$ \\
\hline Rhode Island & 4,753 & 4.1 & 0.4 & $(3.3-4.8)$ \\
\hline South Carolina & 7,838 & 6.1 & 0.4 & $(5.3-6.9)$ \\
\hline South Dakota & 4,742 & 4.5 & 0.5 & $(3.6-5.4)$ \\
\hline Tennessee & 4,266 & 6.3 & 0.5 & $(5.3-7.4)$ \\
\hline Texas & 7,241 & 4.5 & 0.4 & $(3.8-5.2)$ \\
\hline Utah & 7,557 & 4.2 & 0.3 & $(3.6-4.8)$ \\
\hline Vermont & 4,739 & 4.3 & 0.4 & $(3.6-5.1)$ \\
\hline Virginia & 5,813 & 4.7 & 0.4 & $(4.0-5.5)$ \\
\hline Washington & 8,025 & 4.5 & 0.3 & $(3.9-5.1)$ \\
\hline West Virginia & 4,170 & 6.2 & 0.4 & $(5.4-7.0)$ \\
\hline Wisconsin & 4,701 & 3.8 & 0.4 & $(2.9-4.6)$ \\
\hline Wyoming & 5,011 & 4.7 & 0.4 & $(4.0-5.4)$ \\
\hline Guam & 815 & 4.9 & 0.9 & $(3.1-6.6)$ \\
\hline Puerto Rico & 4,050 & 3.1 & 0.3 & $(2.5-3.7)$ \\
\hline Median & & 4.7 & & \\
\hline Range & & $3.1-7.5$ & & \\
\hline
\end{tabular}

Abbreviations: $\mathrm{Cl}=$ confidence interval; $\mathrm{SE}=$ standard error

* Age adjusted to the 2000 U.S. standard population. 
TABLE 58. Age-adjusted* prevalence estimates of adults aged $\geq 45$ years who have ever been told by a health professional they have had a stroke, by metropolitan and micropolitan statistical area - Behavioral Risk Factor Surveillance System, United States, 2013

\begin{tabular}{|c|c|c|c|c|}
\hline MMSA & $\begin{array}{c}\text { Sample } \\
\text { size }\end{array}$ & $\%$ & SE & $95 \% \mathrm{Cl}$ \\
\hline Aguadilla-Isabela, Puerto Rico & 410 & $N / A^{\S}$ & $N / A^{\S}$ & $(\mathrm{N} / \mathrm{A}-\mathrm{N} / \mathrm{A})^{\S}$ \\
\hline Akron, Ohio & 539 & 7.3 & 1.4 & $(4.6-9.9)$ \\
\hline Albuquerque, New Mexico & 1,337 & 3.1 & 0.5 & $(2.1-4.1)$ \\
\hline Allentown-Bethlehem-Easton, Pennsylvania-New Jersey & 721 & 5.1 & 1.1 & $(2.9-7.3)$ \\
\hline Anchorage, Alaska & 924 & 5.3 & 1.0 & $(3.5-7.2)$ \\
\hline Atlanta-Sandy Springs-Roswell, Georgia & 2,256 & 4.7 & 0.5 & $(3.7-5.8)$ \\
\hline Augusta-Richmond County, Georgia-South Carolina & 675 & 5.7 & 1.4 & $(3.0-8.4)$ \\
\hline Austin-Round Rock, Texas & 593 & 6.3 & 1.6 & $(3.1-9.5)$ \\
\hline Baltimore-Columbia-Towson, Maryland & 3,431 & 6.1 & 0.6 & $(5.0-7.3)$ \\
\hline Baton Rouge, Louisiana & 673 & 6.3 & 1.1 & $(4.2-8.4)$ \\
\hline Billings, Montana & 489 & 7.2 & 1.3 & $(4.7-9.8)$ \\
\hline Birmingham-Hoover, Alabama & 979 & 6.6 & 0.9 & $(4.8-8.5)$ \\
\hline Bismarck, North Dakota & 697 & 4.8 & 1.0 & $(2.9-6.8)$ \\
\hline Boise City, Idaho & 990 & 2.9 & 0.6 & $(1.7-4.1)$ \\
\hline Boston, Massachusetts ${ }^{\dagger}$ & 2,801 & 3.8 & 0.5 & $(2.7-4.8)$ \\
\hline Buffalo-Cheektowaga-Niagara Falls, New York & 365 & 5.1 & 1.4 & $(2.4-7.8)$ \\
\hline Burlington-South Burlington, Vermont & 1,078 & 4.6 & 0.9 & $(2.9-6.3)$ \\
\hline Cambridge-Newton-Framingham, Massachusetts ${ }^{\dagger}$ & 3,337 & 3.6 & 0.5 & $(2.6-4.5)$ \\
\hline Camden, New Jersey ${ }^{\dagger}$ & 1,251 & 6.7 & 1.1 & $(4.6-8.8)$ \\
\hline Cedar Rapids, lowa & 467 & 5.2 & 1.3 & $(2.6-7.8)$ \\
\hline Charleston, West Virginia & 586 & 7.8 & 1.3 & $(5.3-10.3)$ \\
\hline Charleston-North Charleston, South Carolina & 1,080 & 8.5 & 1.4 & $(5.7-11.3)$ \\
\hline Charlotte-Concord-Gastonia, North Carolina-South Carolina & 1,297 & 5.0 & 0.7 & $(3.6-6.4)$ \\
\hline Chattanooga, Tennessee-Georgia & 462 & 3.2 & 0.8 & $(1.7-4.7)$ \\
\hline Chicago-Naperville-Elgin, Illinois-Indiana-Wisconsin & 2,286 & 4.7 & 0.7 & $(3.4-6.1)$ \\
\hline Cincinnati, Ohio-Kentucky-Indiana & 1,848 & 5.4 & 0.7 & $(4.0-6.7)$ \\
\hline Claremont-Lebanon, New Hampshire-Vermont & 1,267 & 2.5 & 0.5 & $(1.6-3.3)$ \\
\hline Cleveland-Elyria, Ohio & 800 & 4.5 & 0.9 & $(2.7-6.3)$ \\
\hline Colorado Springs, Colorado & 935 & 3.1 & 0.7 & $(1.6-4.5)$ \\
\hline Columbia, South Carolina & 987 & 4.7 & 0.9 & $(2.9-6.6)$ \\
\hline Columbus, Ohio & 1,186 & 5.2 & 0.8 & $(3.7-6.8)$ \\
\hline Crestview-Fort Walton Beach-Destin, Florida & 787 & 6.1 & 1.2 & $(3.8-8.5)$ \\
\hline Dallas-Plano-Irving, Texas ${ }^{\dagger}$ & 525 & 3.0 & 0.8 & $(1.4-4.6)$ \\
\hline Davenport-Moline-Rock Island, lowa-Illinois & 506 & $N / A^{\S}$ & $N / A^{\S}$ & $(N / A-N / A)^{\S}$ \\
\hline Dayton, Ohio & 628 & 5.8 & 1.2 & $(3.4-8.3)$ \\
\hline Deltona-Daytona Beach-Ormond Beach, Florida & 926 & 4.1 & 1.0 & $(2.1-6.1)$ \\
\hline Denver-Aurora-Lakewood, Colorado & 3,670 & 3.0 & 0.3 & $(2.3-3.6)$ \\
\hline Des Moines-West Des Moines, lowa & 975 & 4.4 & 0.9 & $(2.7-6.0)$ \\
\hline Duluth, Minnesota-Wisconsin & 517 & $N / A^{\S}$ & $N / A^{\S}$ & $(\mathrm{N} / \mathrm{A}-\mathrm{N} / \mathrm{A})^{\S}$ \\
\hline Durham-Chapel Hill, North Carolina & 432 & 5.5 & 1.5 & $(2.6-8.5)$ \\
\hline El Paso, Texas & 482 & $N / A^{\S}$ & $N / A^{\S}$ & $(\mathrm{N} / \mathrm{A}-\mathrm{N} / \mathrm{A})^{\S}$ \\
\hline Evansville, Indiana-Kentucky & 439 & 4.0 & 0.9 & $(2.1-5.8)$ \\
\hline Fargo, North Dakota-Minnesota & 737 & 4.1 & 1.0 & $(2.2-6.1)$ \\
\hline Fayetteville-Springdale-Rogers, Arkansas-Missouri & 581 & 6.1 & 1.1 & $(3.9-8.3)$ \\
\hline Fort Smith, Arkansas-Oklahoma & 386 & 8.4 & 2.0 & $(4.5-12.3)$ \\
\hline Fort Wayne, Indiana & 565 & 3.9 & 0.9 & $(2.2-5.6)$ \\
\hline Fort Worth-Arlington, Texas ${ }^{\dagger}$ & 571 & 8.1 & 1.9 & $(4.4-11.9)$ \\
\hline Gainesville, Florida & 769 & 4.9 & 1.1 & $(2.7-7.1)$ \\
\hline Grand Forks, North Dakota-Minnesota & 344 & $N / A^{\S}$ & $N / A^{\S}$ & $(\mathrm{N} / \mathrm{A}-\mathrm{N} / \mathrm{A})^{\S}$ \\
\hline Grand Island, Nebraska & 611 & 2.3 & 0.7 & $(1.0-3.7)$ \\
\hline Grand Rapids-Wyoming, Michigan & 900 & 4.3 & 0.8 & $(2.8-5.8)$ \\
\hline Greensboro-High Point, North Carolina & 460 & 5.8 & 1.4 & $(3.0-8.6)$ \\
\hline Greenville-Anderson-Mauldin, South Carolina & 941 & 4.9 & 0.8 & $(3.2-6.5)$ \\
\hline Gulfport-Biloxi-Pascagoula, Mississippi & 561 & 5.8 & 1.0 & $(3.8-7.8)$ \\
\hline Hagerstown-Martinsburg, Maryland-West Virginia & 580 & 3.4 & 0.7 & $(1.9-4.9)$ \\
\hline Hartford-West Hartford-East Hartford, Connecticut & 2,025 & 3.9 & 0.6 & $(2.8-5.0)$ \\
\hline Hilton Head Island-Bluffton-Beaufort, South Carolina & 682 & 3.7 & 1.1 & $(1.6-5.9)$ \\
\hline Houston-The Woodlands-Sugar Land, Texas & 774 & 3.0 & 0.9 & $(1.2-4.7)$ \\
\hline Huntington-Ashland, West Virginia-Kentucky-Ohio & 826 & 7.5 & 1.1 & $(5.3-9.6)$ \\
\hline Idaho Falls, Idaho & 336 & $N / A^{\S}$ & $N / A^{\S}$ & $(\mathrm{N} / \mathrm{A}-\mathrm{N} / \mathrm{A})^{\S}$ \\
\hline Indianapolis-Carmel-Anderson, Indiana & 1,764 & 4.6 & 0.6 & $(3.5-5.7)$ \\
\hline
\end{tabular}

See table footnotes on page 127. 
TABLE 58. (Continued) Age-adjusted* prevalence estimates of adults aged $\geq 45$ years who have ever been told by a health professional they have had a stroke, by metropolitan and micropolitan statistical area - Behavioral Risk Factor Surveillance System, United States, 2013

\begin{tabular}{|c|c|c|c|c|}
\hline MMSA & $\begin{array}{l}\text { Sample } \\
\text { size }\end{array}$ & $\%$ & SE & $95 \% \mathrm{Cl}$ \\
\hline Jackson, Mississippi & 545 & 6.5 & 1.5 & $(3.6-9.3)$ \\
\hline Jacksonville, Florida & 2,066 & 6.6 & 1.0 & $(4.7-8.5)$ \\
\hline Kansas City, Missouri-Kansas & 5,205 & 3.5 & 0.4 & $(2.7-4.3)$ \\
\hline Kingsport-Bristol-Bristol, Tennessee-Virginia & 443 & $\mathrm{~N} / \mathrm{A}^{\S}$ & $N / A^{\S}$ & $(\mathrm{N} / \mathrm{A}-\mathrm{N} / \mathrm{A})^{\S}$ \\
\hline Knoxville, Tennessee & 466 & 6.4 & 1.4 & $(3.6-9.1)$ \\
\hline Lansing-East Lansing, Michigan & 469 & 5.7 & 1.4 & $(2.9-8.5)$ \\
\hline Lexington-Fayette, Kentucky & 380 & 6.1 & 1.4 & $(3.3-8.8)$ \\
\hline Lincoln, Nebraska & 1,114 & 3.9 & 0.6 & $(2.8-5.1)$ \\
\hline Little Rock-North Little Rock-Conway, Arkansas & 839 & 6.3 & 1.0 & $(4.4-8.3)$ \\
\hline Logan, Utah-Idaho & 362 & $N / A^{\S}$ & $N / A^{\S}$ & $(\mathrm{N} / \mathrm{A}-\mathrm{N} / \mathrm{A})^{\S}$ \\
\hline Los Angeles-Long Beach-Anaheim, California & 1,784 & 3.5 & 0.5 & $(2.5-4.5)$ \\
\hline Louisville-Jefferson County, Kentucky-Indiana & 1,582 & 7.1 & 1.2 & $(4.9-9.4)$ \\
\hline Lubbock, Texas & 406 & 5.3 & 1.3 & $(2.7-7.8)$ \\
\hline Manhattan, Kansas & 388 & 4.8 & 1.3 & $(2.3-7.3)$ \\
\hline Memphis, Tennessee-Mississippi-Arkansas & 852 & 6.5 & 1.2 & $(4.1-8.9)$ \\
\hline Miami-Fort Lauderdale-West Palm Beach, Florida & 1,604 & 3.7 & 0.6 & $(2.5-4.8)$ \\
\hline Milwaukee-Waukesha-West Allis, Wisconsin & 903 & 4.4 & 1.0 & $(2.3-6.4)$ \\
\hline Minneapolis-St. Paul-Bloomington, Minnesota-Wisconsin & 6,248 & 3.6 & 0.5 & $(2.6-4.7)$ \\
\hline Minot, North Dakota & 417 & 3.4 & 0.9 & $(1.6-5.1)$ \\
\hline $\begin{array}{l}\text { Montgomery County-Bucks County-Chester County, } \\
\text { Pennsylvania }^{\dagger}\end{array}$ & 642 & 3.1 & 0.7 & $(1.7-4.5)$ \\
\hline $\begin{array}{l}\text { Myrtle Beach-Conway-North Myrtle Beach, South Carolina- } \\
\text { North Carolina }\end{array}$ & 584 & 5.1 & 1.2 & $(2.7-7.5)$ \\
\hline Nashville-Davidson County-Murfreesboro-Franklin, Tennessee & 702 & 5.8 & 1.0 & $(3.7-7.8)$ \\
\hline Nassau County-Suffolk County, New York ${ }^{\dagger}$ & 613 & 2.7 & 0.7 & $(1.3-4.2)$ \\
\hline Newark, New Jersey-Pennsylvania ${ }^{\dagger}$ & 2,699 & 3.2 & 0.5 & $(2.2-4.3)$ \\
\hline New Orleans-Metairie, Louisiana & 962 & 6.6 & 1.8 & $(3.0-10.2)$ \\
\hline New York-Jersey City-White Plains, New York-New Jersey ${ }^{\dagger}$ & 5,471 & 4.1 & 0.4 & $(3.2-5.0)$ \\
\hline Norfolk, Nebraska & 454 & 6.6 & 1.6 & $(3.6-9.7)$ \\
\hline North Platte, Nebraska & 538 & 5.6 & 1.1 & $(3.5-7.7)$ \\
\hline North Port-Sarasota-Bradenton, Florida & 867 & 4.5 & 0.7 & $(3.1-6.0)$ \\
\hline Oakland-Hayward-Berkeley, California ${ }^{\dagger}$ & 409 & 4.5 & 1.2 & $(2.2-6.9)$ \\
\hline Ogden-Clearfield, Utah & 1,430 & 3.9 & 0.6 & $(2.7-5.1)$ \\
\hline Oklahoma City, Oklahoma & 1,820 & 5.6 & 0.6 & $(4.4-6.9)$ \\
\hline Omaha-Council Bluffs, Nebraska-lowa & 2,064 & 3.8 & 0.5 & $(2.8-4.8)$ \\
\hline Orlando-Kissimmee-Sanford, Florida & 1,613 & 5.5 & 0.8 & $(4.0-7.1)$ \\
\hline Panama City, Florida & 810 & 5.3 & 1.0 & $(3.4-7.3)$ \\
\hline Pensacola-Ferry Pass-Brent, Florida & 898 & 5.2 & 0.8 & $(3.6-6.7)$ \\
\hline Philadelphia, Pennsylvania ${ }^{\dagger}$ & 1,188 & 6.4 & 0.9 & $(4.7-8.1)$ \\
\hline Phoenix-Mesa-Scottsdale, Arizona & 1,078 & 4.1 & 0.7 & $(2.6-5.5)$ \\
\hline Pittsburgh, Pennsylvania & 1,726 & 4.5 & 0.6 & $(3.2-5.7)$ \\
\hline Ponce, Puerto Rico & 345 & 3.1 & 0.9 & $(1.3-4.8)$ \\
\hline Portland-South Portland, Maine & 1,977 & 3.0 & 0.5 & $(2.1-4.0)$ \\
\hline Portland-Vancouver-Hillsboro, Oregon-Washington & 2,301 & 4.4 & 0.5 & $(3.4-5.5)$ \\
\hline Port St. Lucie, Florida & 842 & 5.0 & 1.2 & $(2.5-7.4)$ \\
\hline Providence-Warwick, Rhode Island-Massachusetts & 6,092 & 4.1 & 0.4 & $(3.3-4.9)$ \\
\hline Provo-Orem, Utah & 878 & 4.4 & 0.8 & $(2.9-6.0)$ \\
\hline Raleigh, North Carolina & 372 & 6.8 & 1.8 & $(3.3-10.2)$ \\
\hline Rapid City, South Dakota & 612 & 3.5 & 0.8 & $(2.0-5.1)$ \\
\hline Reno, Nevada & 1,223 & 3.2 & 0.6 & $(2.1-4.4)$ \\
\hline Richmond, Virginia & 889 & 4.5 & 0.9 & $(2.8-6.2)$ \\
\hline Riverside-San Bernardino-Ontario, California & 851 & 5.8 & 1.1 & $(3.5-8.0)$ \\
\hline Rochester, New York & 343 & 7.4 & 2.0 & $(3.4-11.3)$ \\
\hline Rockingham County-Strafford County, New Hampshire ${ }^{\dagger}$ & 1,196 & 3.7 & 0.6 & $(2.5-5.0)$ \\
\hline Sacramento-Roseville-Arden-Arcade, California & 589 & 2.8 & 0.7 & $(1.5-4.2)$ \\
\hline St. Louis, Missouri-Illinois & 1,477 & 5.5 & 0.8 & $(4.0-7.1)$ \\
\hline Salem, Oregon & 396 & 5.7 & 1.3 & $(3.2-8.2)$ \\
\hline Salina, Kansas & 381 & 8.1 & 1.6 & $(4.9-11.2)$ \\
\hline Salisbury, Maryland-Delaware & 1,641 & 5.2 & 0.7 & $(3.7-6.6)$ \\
\hline Salt Lake City, Utah & 2,746 & 4.4 & 0.5 & $(3.5-5.4)$ \\
\hline San Antonio-New Braunfels, Texas & 628 & 4.3 & 0.9 & $(2.4-6.1)$ \\
\hline
\end{tabular}

See table footnotes on page 127. 
TABLE 58. (Continued) Age-adjusted* prevalence estimates of adults aged $\geq 45$ years who have ever been told by a health professional they have had a stroke, by metropolitan and micropolitan statistical area — Behavioral Risk Factor Surveillance System, United States, 2013

\begin{tabular}{|c|c|c|c|c|}
\hline MMSA & $\begin{array}{l}\text { Sample } \\
\text { size }\end{array}$ & $\%$ & SE & $95 \% \mathrm{Cl}$ \\
\hline San Francisco-Redwood City-South San Francisco, California ${ }^{\dagger}$ & 323 & $N / A^{\S}$ & $N / A^{\S}$ & $(N / A-N / A)^{\S}$ \\
\hline San Jose-Sunnyvale-Santa Clara, California & 354 & $N / A^{\S}$ & $N / A^{\S}$ & $(\mathrm{N} / \mathrm{A}-\mathrm{N} / \mathrm{A})^{\S}$ \\
\hline San Juan-Carolina-Caguas, Puerto Rico & 2,430 & 3.2 & 0.4 & $(2.4-4.0)$ \\
\hline Scottsbluff, Nebraska & 561 & 3.4 & 0.8 & $(1.8-5.0)$ \\
\hline Scranton-Wilkes-Barre-Hazleton, Pennsylvania & 435 & 4.4 & 1.1 & $(2.4-6.5)$ \\
\hline Seattle-Bellevue-Everett, Washington ${ }^{\dagger}$ & 2,505 & 4.0 & 0.5 & $(3.1-4.9)$ \\
\hline Shreveport-Bossier City, Louisiana & 449 & 6.5 & 1.8 & $(3.0-10.1)$ \\
\hline Silver Spring-Frederick-Rockville, Maryland ${ }^{\dagger}$ & 1,712 & 3.3 & 0.6 & $(2.1-4.4)$ \\
\hline Sioux City, lowa-Nebraska-South Dakota & 768 & 6.1 & 1.5 & $(3.2-9.0)$ \\
\hline Sioux Falls, South Dakota & 608 & 5.3 & 1.2 & $(2.9-7.8)$ \\
\hline Spartanburg, South Carolina & 466 & 9.4 & 2.3 & $(5.0-13.8)$ \\
\hline Spokane-Spokane Valley, Washington & 643 & 4.4 & 0.9 & $(2.6-6.2)$ \\
\hline Springfield, Massachusetts & 1,170 & 3.9 & 0.9 & $(2.2-5.6)$ \\
\hline Tallahassee, Florida & 1,428 & 5.4 & 1.2 & $(3.1-7.7)$ \\
\hline Tampa-St. Petersburg-Clearwater, Florida & 1,614 & 6.4 & 0.9 & $(4.6-8.1)$ \\
\hline Toledo, Ohio & 735 & 4.5 & 0.9 & $(2.8-6.2)$ \\
\hline Topeka, Kansas & 1,769 & 4.6 & 0.6 & $(3.4-5.7)$ \\
\hline Tulsa, Oklahoma & 1,469 & 5.3 & 0.7 & $(4.0-6.6)$ \\
\hline Virginia Beach-Norfolk-Newport News, Virginia-North Carolina & 1,144 & 5.4 & 0.8 & $(3.8-6.9)$ \\
\hline Warren-Troy-Farmington Hills, Michigan ${ }^{\dagger}$ & 1,623 & 6.1 & 0.8 & $(4.6-7.6)$ \\
\hline $\begin{array}{l}\text { Washington-Arlington-Alexandria, District of Columbia- } \\
\text { Virginia-Maryland-West Virginia }^{\dagger}\end{array}$ & 6,218 & 4.9 & 0.6 & $(3.7-6.1)$ \\
\hline Wichita, Kansas & 3,455 & 5.7 & 0.5 & $(4.8-6.6)$ \\
\hline Wilmington, Delaware-Maryland-New Jersey ${ }^{\dagger}$ & 2,283 & 6.1 & 0.6 & $(4.9-7.4)$ \\
\hline Winston-Salem, North Carolina & 527 & 5.2 & 1.1 & $(3.0-7.4)$ \\
\hline Worcester, Massachusetts-Connecticut & 1,946 & 4.1 & 0.7 & $(2.8-5.4)$ \\
\hline $\begin{array}{l}\text { Median } \\
\text { Range }\end{array}$ & & $\begin{array}{c}4.9 \\
2.3-9.4\end{array}$ & & \\
\hline
\end{tabular}

Abbreviations: $\mathrm{Cl}=$ confidence interval; $\mathrm{MMSA}=$ metropolitan and micropolitan statistical areas; $\mathrm{N} / \mathrm{A}=$ not available; $\mathrm{SE}=$ standard error.

* Age adjusted to the 2000 U.S. standard population.

${ }^{\dagger}$ Metropolitan division.

$\S$ Estimate not available if the unweighted sample size for the denominator was $<50$ or if the relative standard error is $>30 \%$. 
TABLE 59. Age-adjusted* prevalence estimates of adults aged $\geq 45$ years who have ever been told by a health professional they have had a stroke, by state/territory - Behavioral Risk Factor Surveillance System, United States, 2014

\begin{tabular}{|c|c|c|c|c|}
\hline State/Territory & $\begin{array}{l}\text { Sample } \\
\text { size }\end{array}$ & $\%$ & SE & $95 \% \mathrm{Cl}$ \\
\hline Alabama & 6,467 & 7.3 & 0.4 & $(6.5-8.2)$ \\
\hline Alaska & 2,875 & 5.0 & 0.6 & $(3.9-6.2)$ \\
\hline Arizona & 11,694 & 4.8 & 0.3 & $(4.3-5.4)$ \\
\hline Arkansas & 4,191 & 7.6 & 0.6 & $(6.4-8.8)$ \\
\hline California & 5,319 & 4.5 & 0.4 & $(3.8-5.3)$ \\
\hline Colorado & 9,495 & 3.3 & 0.2 & $(2.8-3.7)$ \\
\hline Connecticut & 5,870 & 4.1 & 0.4 & $(3.3-4.8)$ \\
\hline Delaware & 3,256 & 5.3 & 0.6 & $(4.2-6.4)$ \\
\hline District of Columbia & 3,045 & 6.0 & 0.6 & $(4.8-7.1)$ \\
\hline Florida & 7,564 & 4.7 & 0.3 & $(4.1-5.4)$ \\
\hline Georgia & 4,637 & 6.2 & 0.5 & $(5.2-7.2)$ \\
\hline Hawaii & 4,818 & 4.8 & 0.4 & $(3.9-5.6)$ \\
\hline Idaho & 3,922 & 4.6 & 0.4 & $(3.7-5.4)$ \\
\hline Illinois & 3,556 & 5.0 & 0.5 & $(4.1-5.9)$ \\
\hline Indiana & 8,628 & 5.2 & 0.3 & $(4.6-5.8)$ \\
\hline lowa & 6,046 & 4.0 & 0.3 & $(3.4-4.6)$ \\
\hline Kansas & 9,651 & 5.0 & 0.3 & $(4.5-5.5)$ \\
\hline Kentucky & 8,605 & 6.4 & 0.5 & $(5.5-7.3)$ \\
\hline Louisiana & 4,759 & 6.6 & 0.5 & $(5.7-7.5)$ \\
\hline Maine & 7,110 & 4.6 & 0.3 & $(4.0-5.3)$ \\
\hline Maryland & 9,784 & 5.3 & 0.5 & $(4.4-6.3)$ \\
\hline Massachusetts & 11,670 & 4.3 & 0.3 & $(3.7-4.9)$ \\
\hline Michigan & 6,219 & 5.0 & 0.4 & $(4.3-5.7)$ \\
\hline Minnesota & 11,020 & 3.7 & 0.2 & $(3.3-4.1)$ \\
\hline Mississippi & 3,157 & 6.1 & 0.5 & $(5.2-7.1)$ \\
\hline Missouri & 5,400 & 6.3 & 0.5 & $(5.4-7.2)$ \\
\hline Montana & 5,708 & 4.2 & 0.4 & $(3.4-4.9)$ \\
\hline Nebraska & 16,096 & 4.4 & 0.3 & $(3.9-4.9)$ \\
\hline Nevada & 2,642 & 5.5 & 0.6 & $(4.3-6.8)$ \\
\hline New Hampshire & 4,716 & 3.5 & 0.3 & $(2.8-4.1)$ \\
\hline New Jersey & 9,282 & 4.2 & 0.3 & $(3.7-4.8)$ \\
\hline New Mexico & 6,485 & 4.6 & 0.4 & $(3.8-5.4)$ \\
\hline New York & 4,692 & 4.1 & 0.4 & $(3.4-4.8)$ \\
\hline North Carolina & 4,936 & 6.4 & 0.4 & $(5.6-7.2)$ \\
\hline North Dakota & 5,827 & 4.2 & 0.4 & $(3.4-5.0)$ \\
\hline Ohio & 8,368 & 5.7 & 0.4 & $(4.9-6.5)$ \\
\hline Oklahoma & 6,112 & 5.7 & 0.4 & $(5.0-6.4)$ \\
\hline Oregon & 3,884 & 5.0 & 0.4 & $(4.1-5.8)$ \\
\hline Pennsylvania & 8,217 & 5.1 & 0.3 & $(4.5-5.7)$ \\
\hline Rhode Island & 4,934 & 3.8 & 0.4 & $(3.1-4.5)$ \\
\hline South Carolina & 8,115 & 5.6 & 0.3 & $(4.9-6.2)$ \\
\hline South Dakota & 5,224 & 4.5 & 0.5 & $(3.6-5.4)$ \\
\hline Tennessee & 3,965 & 7.0 & 0.5 & $(6.0-8.0)$ \\
\hline Texas & 10,641 & 5.1 & 0.4 & $(4.3-5.8)$ \\
\hline Utah & 8,557 & 4.5 & 0.3 & $(3.9-5.1)$ \\
\hline Vermont & 4,593 & 3.6 & 0.3 & $(3.0-4.3)$ \\
\hline Virginia & 6,763 & 4.7 & 0.3 & $(4.1-5.4)$ \\
\hline Washington & 7,418 & 4.5 & 0.3 & $(3.9-5.1)$ \\
\hline West Virginia & 4,525 & 6.5 & 0.4 & $(5.6-7.3)$ \\
\hline Wisconsin & 5,084 & 4.5 & 0.5 & $(3.6-5.5)$ \\
\hline Wyoming & 5,182 & 4.4 & 0.4 & $(3.7-5.1)$ \\
\hline Guam & 1,202 & 8.0 & 1.1 & $(5.8-10.2)$ \\
\hline Puerto Rico & 4,085 & 3.6 & 0.4 & $(2.9-4.3)$ \\
\hline Median & & 4.8 & & \\
\hline Range & & $3.3-8.0$ & & \\
\hline
\end{tabular}

Abbreviations: $\mathrm{Cl}=$ confidence interval; $\mathrm{SE}=$ standard error.

* Age adjusted to the 2000 U.S. standard population. 
TABLE 60. Age-adjusted* prevalence estimates of adults aged $\geq 45$ years who have ever been told by a health professional they have had a stroke, by metropolitan and micropolitan statistical area - Behavioral Risk Factor Surveillance System, United States, 2014

\begin{tabular}{|c|c|c|c|c|}
\hline MMSA & $\begin{array}{l}\text { Sample } \\
\text { size }\end{array}$ & $\%$ & SE & $95 \% \mathrm{Cl}$ \\
\hline Aberdeen, South Dakota & 461 & 6.1 & 1.7 & $(2.8-9.4)$ \\
\hline Aguadilla-Isabela, Puerto Rico & 360 & $N / A^{\S}$ & $N / A^{\S}$ & $(N / A-N / A)^{\S}$ \\
\hline Albuquerque, New Mexico & 1,254 & 4.7 & 0.8 & $(3.1-6.3)$ \\
\hline Allentown-Bethlehem-Easton, Pennsylvania-New Jersey & 882 & 4.5 & 1.1 & $(2.3-6.6)$ \\
\hline Anchorage, Alaska & 1,133 & 4.9 & 1.0 & $(3.0-6.8)$ \\
\hline Atlanta-Sandy Springs-Roswell, Georgia & 1,899 & 5.0 & 0.7 & $(3.6-6.3)$ \\
\hline Augusta-Richmond County, Georgia-South Carolina & 637 & 3.9 & 0.9 & $(2.2-5.7)$ \\
\hline Austin-Round Rock, Texas & 1,524 & 3.8 & 0.6 & $(2.6-5.0)$ \\
\hline Baltimore-Columbia-Towson, Maryland & 3,509 & 5.8 & 0.8 & $(4.3-7.3)$ \\
\hline Baton Rouge, Louisiana & 604 & 7.7 & 1.3 & $(5.2-10.2)$ \\
\hline Berlin, New Hampshire-Vermont & 446 & 9.0 & 2.0 & $(5.0-12.9)$ \\
\hline Billings, Montana & 593 & 3.6 & 1.1 & $(1.5-5.7)$ \\
\hline Birmingham-Hoover, Alabama & 1,116 & 6.2 & 1.0 & $(4.3-8.2)$ \\
\hline Bismarck, North Dakota & 808 & 4.1 & 1.0 & $(2.1-6.2)$ \\
\hline Boise City, Idaho & 963 & 4.4 & 0.8 & $(2.8-6.0)$ \\
\hline Boston, Massachusetts $^{\dagger}$ & 3,363 & 3.7 & 0.5 & $(2.7-4.8)$ \\
\hline Burlington-South Burlington, Vermont & 1,296 & 2.6 & 0.6 & $(1.3-3.8)$ \\
\hline Cambridge-Newton-Framingham, Massachusetts ${ }^{\dagger}$ & 3,839 & 4.2 & 0.5 & $(3.2-5.2)$ \\
\hline Camden, New Jersey ${ }^{\dagger}$ & 1,293 & 4.9 & 0.7 & $(3.5-6.4)$ \\
\hline Cedar Rapids, lowa & 471 & 4.9 & 1.3 & $(2.4-7.5)$ \\
\hline Charleston, West Virginia & 622 & 8.4 & 1.4 & $(5.6-11.2)$ \\
\hline Charleston-North Charleston, South Carolina & 995 & 3.8 & 0.6 & $(2.5-5.0)$ \\
\hline Charlotte-Concord-Gastonia, North Carolina-South Carolina & 1,464 & 7.0 & 1.0 & $(5.0-8.9)$ \\
\hline Chicago-Naperville-Elgin, Illinois-Indiana-Wisconsin & 2,852 & 5.3 & 0.6 & $(4.1-6.4)$ \\
\hline Cincinnati, Ohio-Kentucky-Indiana & 1,478 & 4.8 & 0.8 & $(3.3-6.4)$ \\
\hline Claremont-Lebanon, New Hampshire-Vermont & 1,287 & 3.9 & 0.6 & $(2.7-5.0)$ \\
\hline Cleveland-Elyria, Ohio & 725 & 6.1 & 1.1 & $(3.9-8.2)$ \\
\hline College Station-Bryan, Texas & 441 & $N / A^{\S}$ & $N / A^{\S}$ & $(\mathrm{N} / \mathrm{A}-\mathrm{N} / \mathrm{A})^{\S}$ \\
\hline Colorado Springs, Colorado & 893 & 2.6 & 0.6 & $(1.4-3.7)$ \\
\hline Columbia, South Carolina & 823 & 6.4 & 1.4 & $(3.7-9.1)$ \\
\hline Columbus, Ohio & 1,179 & 5.5 & 0.9 & $(3.8-7.2)$ \\
\hline Corpus Christi, Texas & 506 & 6.0 & 1.5 & $(3.1-8.9)$ \\
\hline Dallas-Plano-Irving, Texas $^{\dagger}$ & 913 & 5.4 & 1.1 & $(3.2-7.7)$ \\
\hline Dayton, Ohio & 424 & 8.6 & 2.3 & $(4.1-13.2)$ \\
\hline Denver-Aurora-Lakewood, Colorado & 3,856 & 3.1 & 0.3 & $(2.5-3.7)$ \\
\hline Des Moines-West Des Moines, lowa & 981 & 3.2 & 0.6 & $(2.1-4.4)$ \\
\hline Duluth, Minnesota-Wisconsin & 683 & 5.8 & 1.3 & $(3.3-8.4)$ \\
\hline El Paso, Texas & 516 & 3.4 & 0.9 & $(1.6-5.2)$ \\
\hline Evansville, Indiana-Kentucky & 548 & 6.2 & 1.4 & $(3.5-9.0)$ \\
\hline Fargo, North Dakota-Minnesota & 827 & 2.4 & 0.6 & $(1.3-3.5)$ \\
\hline Fayetteville-Springdale-Rogers, Arkansas-Missouri & 636 & 7.7 & 1.4 & $(5.0-10.4)$ \\
\hline Fort Wayne, Indiana & 658 & 7.0 & 1.5 & $(4.2-9.9)$ \\
\hline Fort Worth-Arlington, Texas ${ }^{\dagger}$ & 540 & 4.6 & 1.0 & $(2.6-6.6)$ \\
\hline Grand Island, Nebraska & 792 & 6.3 & 1.4 & $(3.6-9.0)$ \\
\hline Grand Rapids-Wyoming, Michigan & 633 & $N / A^{\S}$ & $N / A^{\S}$ & $(N / A-N / A)^{\S}$ \\
\hline Greensboro-High Point, North Carolina & 363 & 2.8 & 0.8 & $(1.3-4.3)$ \\
\hline Greenville-Anderson-Mauldin, South Carolina & 1,044 & 4.6 & 0.7 & $(3.1-6.0)$ \\
\hline Hagerstown-Martinsburg, Maryland-West Virginia & 639 & 6.1 & 1.3 & $(3.5-8.6)$ \\
\hline Hartford-West Hartford-East Hartford, Connecticut & 1,940 & 4.9 & 0.7 & $(3.5-6.2)$ \\
\hline Hilton Head Island-Bluffton-Beaufort, South Carolina & 445 & 3.3 & 0.9 & $(1.6-5.0)$ \\
\hline Houston-The Woodlands-Sugar Land, Texas & 1,446 & 6.5 & 1.2 & $(4.2-8.8)$ \\
\hline Huntington-Ashland, West Virginia-Kentucky-Ohio & 945 & 8.3 & 1.1 & $(6.1-10.5)$ \\
\hline Idaho Falls, Idaho & 341 & $N / A^{\S}$ & $N / A^{\S}$ & $(\mathrm{N} / \mathrm{A}-\mathrm{N} / \mathrm{A})^{\S}$ \\
\hline Indianapolis-Carmel-Anderson, Indiana & 2,692 & 5.6 & 0.6 & $(4.5-6.7)$ \\
\hline Jacksonville, Florida & 481 & 5.9 & 1.3 & $(3.5-8.4)$ \\
\hline Kansas City, Missouri-Kansas & 3,538 & 6.0 & 0.6 & $(4.7-7.2)$ \\
\hline Kingsport-Bristol-Bristol, Tennessee-Virginia & 404 & 4.2 & 1.0 & $(2.3-6.1)$ \\
\hline Knoxville, Tennessee & 449 & 6.6 & 1.5 & $(3.7-9.5)$ \\
\hline Lafayette, Louisiana & 390 & 4.5 & 1.3 & $(2.0-7.0)$ \\
\hline Lexington-Fayette, Kentucky & 392 & $N / A^{\S}$ & $N / A^{\S}$ & $(\mathrm{N} / \mathrm{A}-\mathrm{N} / \mathrm{A})^{\S}$ \\
\hline Lincoln, Nebraska & 1,233 & 4.1 & 0.6 & $(2.9-5.3)$ \\
\hline
\end{tabular}

See table footnotes on page 131. 
TABLE 60. (Continued) Age-adjusted* prevalence estimates of adults aged $\geq 45$ years who have ever been told by a health professional they have had a stroke, by metropolitan and micropolitan statistical area - Behavioral Risk Factor Surveillance System, United States, 2014

\begin{tabular}{|c|c|c|c|c|}
\hline MMSA & $\begin{array}{l}\text { Sample } \\
\text { size }\end{array}$ & $\%$ & SE & $95 \% \mathrm{Cl}$ \\
\hline Little Rock-North Little Rock-Conway, Arkansas & 896 & 7.3 & 1.5 & $(4.4-10.2)$ \\
\hline Logan, Utah-Idaho & 334 & $N / A^{\S}$ & $N / A^{\S}$ & $(\mathrm{N} / \mathrm{A}-\mathrm{N} / \mathrm{A})^{\S}$ \\
\hline Los Angeles-Long Beach-Anaheim, California & 1,343 & 5.0 & 0.8 & $(3.5-6.5)$ \\
\hline Louisville-Jefferson County, Kentucky-Indiana & 1,971 & 4.8 & 0.7 & $(3.5-6.1)$ \\
\hline Madison, Wisconsin & 352 & $N / A^{\S}$ & $N / A^{\S}$ & $(\mathrm{N} / \mathrm{A}-\mathrm{N} / \mathrm{A})^{\S}$ \\
\hline Memphis, Tennessee-Mississippi-Arkansas & 654 & 5.6 & 1.4 & $(2.9-8.3)$ \\
\hline Miami-Fort Lauderdale-West Palm Beach, Florida & 1,646 & 3.0 & 0.5 & $(2.0-4.1)$ \\
\hline Milwaukee-Waukesha-West Allis, Wisconsin & 975 & 5.4 & 1.2 & $(3.1-7.7)$ \\
\hline Minneapolis-St. Paul-Bloomington, Minnesota-Wisconsin & 5,706 & 3.5 & 0.3 & $(3.0-4.1)$ \\
\hline Minot, North Dakota & 430 & 6.0 & 1.6 & $(2.9-9.1)$ \\
\hline Montgomery, Alabama & 368 & 7.8 & 1.8 & $(4.3-11.3)$ \\
\hline $\begin{array}{l}\text { Montgomery County-Bucks County-Chester County, } \\
\text { Pennsylvania }{ }^{\dagger}\end{array}$ & 582 & 3.5 & 0.8 & $(1.8-5.1)$ \\
\hline $\begin{array}{l}\text { Myrtle Beach-Conway-North Myrtle Beach, South Carolina- } \\
\text { North Carolina }\end{array}$ & 771 & 5.2 & 1.2 & $(2.8-7.6)$ \\
\hline Nashville-Davidson County-Murfreesboro-Franklin, Tennessee & 567 & 5.9 & 1.1 & $(3.8-8.0)$ \\
\hline Nassau County-Suffolk County, New York ${ }^{\dagger}$ & 555 & 3.6 & 0.9 & $(1.9-5.3)$ \\
\hline Newark, New Jersey-Pennsylvania ${ }^{\dagger}$ & 2,973 & 4.2 & 0.5 & $(3.2-5.2)$ \\
\hline New Orleans-Metairie, Louisiana & 1,353 & 6.1 & 1.0 & $(4.2-8.0)$ \\
\hline New York-Jersey City-White Plains, New York-New Jersey ${ }^{\dagger}$ & 4,814 & 3.6 & 0.4 & $(2.9-4.4)$ \\
\hline Norfolk, Nebraska & 701 & 3.4 & 0.8 & $(1.8-5.0)$ \\
\hline North Platte, Nebraska & 715 & 5.3 & 0.9 & $(3.5-7.1)$ \\
\hline North Port-Sarasota-Bradenton, Florida & 443 & 4.3 & 1.1 & $(2.2-6.4)$ \\
\hline Oakland-Hayward-Berkeley, California ${ }^{\dagger}$ & 435 & $\mathrm{~N} / \mathrm{A}^{\S}$ & $N / A^{\S}$ & $(\mathrm{N} / \mathrm{A}-\mathrm{N} / \mathrm{A})^{\S}$ \\
\hline Ogden-Clearfield, Utah & 1,602 & 4.5 & 0.6 & $(3.3-5.8)$ \\
\hline Oklahoma City, Oklahoma & 1,645 & 5.1 & 0.6 & $(3.9-6.2)$ \\
\hline Omaha-Council Bluffs, Nebraska-lowa & 3,304 & 4.4 & 0.4 & $(3.5-5.2)$ \\
\hline Orlando-Kissimmee-Sanford, Florida & 665 & 5.7 & 1.1 & $(3.5-7.9)$ \\
\hline Philadelphia, Pennsylvania ${ }^{\dagger}$ & 1,094 & 6.3 & 1.1 & $(4.2-8.4)$ \\
\hline Phoenix-Mesa-Scottsdale, Arizona & 7,246 & 4.8 & 0.3 & $(4.1-5.4)$ \\
\hline Pittsburgh, Pennsylvania & 1,825 & 5.5 & 0.6 & $(4.2-6.7)$ \\
\hline Ponce, Puerto Rico & 377 & $N / A^{\S}$ & $N / A^{\S}$ & $(N / A-N / A)^{\S}$ \\
\hline Portland-South Portland, Maine & 2,188 & 4.3 & 0.6 & $(3.1-5.5)$ \\
\hline Portland-Vancouver-Hillsboro, Oregon-Washington & 2,052 & 5.2 & 0.6 & $(4.1-6.3)$ \\
\hline Providence-Warwick, Rhode Island-Massachusetts & 6,230 & 4.6 & 0.6 & $(3.4-5.7)$ \\
\hline Provo-Orem, Utah & 978 & 4.0 & 0.7 & $(2.7-5.3)$ \\
\hline Raleigh, North Carolina & 405 & 4.3 & 1.2 & $(2.0-6.6)$ \\
\hline Rapid City, South Dakota & 1,043 & 5.8 & 1.0 & $(3.9-7.7)$ \\
\hline Reno, Nevada & 855 & 5.3 & 0.9 & $(3.5-7.1)$ \\
\hline Richmond, Virginia & 1,020 & 4.6 & 0.8 & $(3.0-6.1)$ \\
\hline Riverside-San Bernardino-Ontario, California & 578 & 6.2 & 1.2 & $(3.8-8.6)$ \\
\hline Roanoke, Virginia & 405 & 5.5 & 1.5 & $(2.5-8.4)$ \\
\hline Rochester, Minnesota & 457 & $\mathrm{~N} / \mathrm{A}^{\S}$ & $N / A^{\S}$ & $(\mathrm{N} / \mathrm{A}-\mathrm{N} / \mathrm{A})^{\S}$ \\
\hline Rockingham County-Strafford County, New Hampshire ${ }^{\dagger}$ & 1,088 & 2.7 & 0.6 & $(1.5-3.9)$ \\
\hline Sacramento-Roseville-Arden-Arcade, California & 414 & 2.3 & 0.7 & $(0.9-3.6)$ \\
\hline St. Cloud, Minnesota & 339 & $\mathrm{~N} / \mathrm{A}^{\S}$ & $N / A^{\S}$ & $(\mathrm{N} / \mathrm{A}-\mathrm{N} / \mathrm{A})^{\S}$ \\
\hline St. Louis, Missouri-Illinois & 1,408 & 5.1 & 0.8 & $(3.5-6.8)$ \\
\hline Salisbury, Maryland-Delaware & 1,625 & 5.0 & 0.6 & $(3.7-6.2)$ \\
\hline Salt Lake City, Utah & 3,019 & 4.6 & 0.5 & $(3.6-5.5)$ \\
\hline San Antonio-New Braunfels, Texas & 1,625 & 4.1 & 0.6 & $(3.0-5.3)$ \\
\hline San Juan-Carolina-Caguas, Puerto Rico & 2,568 & 4.3 & 0.5 & $(3.3-5.3)$ \\
\hline Scottsbluff, Nebraska & 709 & 6.1 & 1.4 & $(3.3-8.9)$ \\
\hline Seattle-Bellevue-Everett, Washington ${ }^{\dagger}$ & 2,621 & 3.7 & 0.4 & $(2.9-4.5)$ \\
\hline Shreveport-Bossier City, Louisiana & 378 & 7.8 & 1.5 & $(4.9-10.7)$ \\
\hline Silver Spring-Frederick-Rockville, Maryland ${ }^{\dagger}$ & 1,791 & $N / A^{\S}$ & $N / A^{\S}$ & $(\mathrm{N} / \mathrm{A}-\mathrm{N} / \mathrm{A})^{\S}$ \\
\hline Sioux City, lowa-Nebraska-South Dakota & 883 & 5.4 & 1.3 & $(2.9-7.9)$ \\
\hline Sioux Falls, South Dakota & 909 & 4.0 & 0.7 & $(2.7-5.3)$ \\
\hline Spartanburg, South Carolina & 439 & 8.7 & 1.7 & $(5.4-11.9)$ \\
\hline Spokane-Spokane Valley, Washington & 553 & 6.5 & 1.2 & $(4.1-8.9)$ \\
\hline Springfield, Massachusetts & 788 & 6.8 & 1.6 & $(3.6-10.0)$ \\
\hline Tampa-St. Petersburg-Clearwater, Florida & 1,220 & 4.7 & 0.7 & $(3.4-6.1)$ \\
\hline Toledo, Ohio & 499 & 5.5 & 1.2 & $(3.2-7.8)$ \\
\hline
\end{tabular}

See table footnotes on page 131. 
TABLE 60. (Continued) Age-adjusted* prevalence estimates of adults aged $\geq 45$ years who have ever been told by a health professional they have had a stroke, by metropolitan and micropolitan statistical area - Behavioral Risk Factor Surveillance System, United States, 2014

\begin{tabular}{|c|c|c|c|c|}
\hline MMSA & $\begin{array}{l}\text { Sample } \\
\text { size }\end{array}$ & $\%$ & SE & $95 \% \mathrm{Cl}$ \\
\hline Topeka, Kansas & 1,045 & 3.7 & 0.6 & $(2.5-4.8)$ \\
\hline Tulsa, Oklahoma & 1,477 & 6.5 & 0.7 & $(5.0-7.9)$ \\
\hline Tuscaloosa, Alabama & 494 & 7.6 & 1.8 & $(4.2-11.1)$ \\
\hline Virginia Beach-Norfolk-Newport News, Virginia-North Carolina & 1,341 & 5.6 & 0.9 & $(3.9-7.3)$ \\
\hline Warren-Troy-Farmington Hills, Michigan ${ }^{\dagger}$ & 1,544 & 5.2 & 0.8 & $(3.7-6.8)$ \\
\hline $\begin{array}{l}\text { Washington-Arlington-Alexandria, District of Columbia- } \\
\text { Virginia-Maryland-West Virginia }^{\dagger}\end{array}$ & 5,949 & 4.8 & 0.6 & $(3.7-5.9)$ \\
\hline Wichita, Kansas & 1,890 & 5.1 & 0.6 & $(4.0-6.3)$ \\
\hline Wichita Falls, Texas & 476 & 4.9 & 1.1 & $(2.7-7.1)$ \\
\hline Wilmington, Delaware-Maryland-New Jersey ${ }^{\dagger}$ & 2,065 & 6.3 & 0.8 & $(4.7-7.8)$ \\
\hline Worcester, Massachusetts-Connecticut & 1,794 & 3.2 & 0.6 & $(2.0-4.3)$ \\
\hline Youngstown-Warren-Boardman, Ohio-Pennsylvania & 419 & 5.2 & 1.3 & $(2.7-7.6)$ \\
\hline Median & & 5.0 & & \\
\hline Range & & $2.3-9.0$ & & \\
\hline
\end{tabular}

Abbreviations: $\mathrm{Cl}=$ confidence interval; $\mathrm{MMSA}=$ metropolitan and micropolitan statistical areas; $\mathrm{N} / \mathrm{A}=$ not available; $\mathrm{SE}=$ standard error.

${ }^{*}$ Age adjusted to the 2000 U.S. standard population.

† Metropolitan division.

$\S$ Estimate not available if the unweighted sample size for the denominator was $<50$ or if the relative standard error is $>30 \%$. 
TABLE 61. Age-adjusted* prevalence estimates of adults aged $\geq 18$ years who have ever been told by a health professional they have high blood pressure, ${ }^{\dagger}$ by state/territory - Behavioral Risk Factor Surveillance System, United States, 2013

\begin{tabular}{|c|c|c|c|c|}
\hline State/Territory & $\begin{array}{l}\text { Sample } \\
\text { size }\end{array}$ & $\%$ & SE & $95 \% \mathrm{Cl}$ \\
\hline Alabama & 6,492 & 37.5 & 0.8 & $(35.9-39.1)$ \\
\hline Alaska & 4,564 & 29.9 & 0.9 & $(28.2-31.6)$ \\
\hline Arizona & 4,242 & 28.8 & 1.2 & $(26.6-31.1)$ \\
\hline Arkansas & 5,245 & 36.0 & 0.9 & $(34.2-37.9)$ \\
\hline California & 11,502 & 28.1 & 0.5 & $(27.1-29.1)$ \\
\hline Colorado & 13,615 & 25.7 & 0.4 & $(24.8-26.6)$ \\
\hline Connecticut & 7,680 & 28.2 & 0.7 & $(27.0-29.5)$ \\
\hline Delaware & 5,191 & 32.4 & 0.8 & $(30.8-34.0)$ \\
\hline District of Columbia & 4,913 & 30.0 & 0.8 & $(28.4-31.7)$ \\
\hline Florida & 34,074 & 30.3 & 0.5 & $(29.3-31.3)$ \\
\hline Georgia & 8,116 & 34.3 & 0.6 & $(33.0-35.5)$ \\
\hline Hawaii & 7,831 & 26.1 & 0.7 & $(24.8-27.4)$ \\
\hline Idaho & 5,611 & 27.7 & 0.7 & $(26.2-29.2)$ \\
\hline Illinois & 5,603 & 28.6 & 0.8 & $(27.0-30.1)$ \\
\hline Indiana & 10,298 & 31.3 & 0.5 & $(30.3-32.4)$ \\
\hline lowa & 8,139 & 28.6 & 0.6 & $(27.4-29.7)$ \\
\hline Kansas & 23,221 & 29.3 & 0.3 & $(28.7-29.9)$ \\
\hline Kentucky & 10,992 & 36.5 & 0.6 & $(35.2-37.7)$ \\
\hline Louisiana & 5,240 & 38.0 & 0.9 & $(36.2-39.9)$ \\
\hline Maine & 8,079 & 29.1 & 0.6 & $(27.9-30.4)$ \\
\hline Maryland & 12,971 & 30.8 & 0.5 & $(29.7-31.8)$ \\
\hline Massachusetts & 15,012 & 27.2 & 0.5 & $(26.2-28.2)$ \\
\hline Michigan & 12,728 & 31.7 & 0.5 & $(30.6-32.7)$ \\
\hline Minnesota & 14,290 & 25.2 & 0.6 & $(24.0-26.4)$ \\
\hline Mississippi & 7,440 & 38.0 & 0.7 & $(36.6-39.5)$ \\
\hline Missouri & 7,104 & 29.5 & 0.7 & $(28.1-31.0)$ \\
\hline Montana & 9,672 & 26.3 & 0.6 & $(25.2-27.3)$ \\
\hline Nebraska & 17,085 & 28.3 & 0.5 & $(27.3-29.3)$ \\
\hline Nevada & 5,090 & 29.1 & 1.0 & $(27.1-31.1)$ \\
\hline New Hampshire & 6,449 & 27.0 & 0.7 & $(25.7-28.3)$ \\
\hline New Jersey & 13,347 & 28.4 & 0.5 & $(27.4-29.4)$ \\
\hline New Mexico & 9,289 & 27.4 & 0.6 & $(26.2-28.5)$ \\
\hline New York & 8,942 & 29.5 & 0.6 & $(28.3-30.6)$ \\
\hline North Carolina & 8,841 & 33.2 & 0.6 & $(32.0-34.4)$ \\
\hline North Dakota & 7,788 & 27.7 & 0.6 & $(26.5-28.9)$ \\
\hline Ohio & 11,934 & 30.5 & 0.5 & $(29.4-31.5)$ \\
\hline Oklahoma & 8,224 & 35.4 & 0.6 & $(34.2-36.7)$ \\
\hline Oregon & 5,934 & 29.5 & 0.8 & $(28.0-31.1)$ \\
\hline Pennsylvania & 11,382 & 30.3 & 0.5 & $(29.3-31.4)$ \\
\hline Rhode Island & 6,509 & 31.1 & 0.7 & $(29.7-32.5)$ \\
\hline South Carolina & 10,675 & 35.6 & 0.6 & $(34.4-36.8)$ \\
\hline South Dakota & 6,883 & 27.9 & 0.8 & $(26.4-29.4)$ \\
\hline Tennessee & 5,798 & 36.1 & 0.8 & $(34.5-37.7)$ \\
\hline Texas & 10,865 & 31.0 & 0.6 & $(29.8-32.2)$ \\
\hline Utah & 12,730 & 25.8 & 0.4 & $(24.9-26.6)$ \\
\hline Vermont & 6,377 & 27.6 & 0.7 & $(26.4-28.9)$ \\
\hline Virginia & 8,443 & 30.9 & 0.6 & $(29.7-32.1)$ \\
\hline Washington & 11,140 & 28.9 & 0.5 & $(27.8-29.9)$ \\
\hline West Virginia & 5,879 & 36.7 & 0.7 & $(35.3-38.1)$ \\
\hline Wisconsin & 6,575 & 29.6 & 0.8 & $(28.0-31.2)$ \\
\hline Wyoming & 6,434 & 26.8 & 0.7 & $(25.5-28.1)$ \\
\hline Guam & 1,889 & 31.5 & 1.3 & $(28.9-34.1)$ \\
\hline Puerto Rico & 5,996 & 40.1 & 0.8 & $(38.6-41.6)$ \\
\hline Median & & 29.5 & & \\
\hline Range & & $25.2-40.1$ & & \\
\hline
\end{tabular}

Abbreviations: $\mathrm{Cl}=$ confidence interval; $\mathrm{SE}=$ standard error

* Age adjusted to the 2000 U.S. standard population.

† Excluding pregnant women. 
TABLE 62. Age-adjusted* prevalence estimates of adults aged $\geq 18$ years who have ever been told by a health professional they have high blood pressure, ${ }^{\dagger}$ by metropolitan and micropolitan statistical area - Behavioral Risk Factor Surveillance System, United States, 2013

\begin{tabular}{|c|c|c|c|c|}
\hline MMSA & $\begin{array}{l}\text { Sample } \\
\text { size }\end{array}$ & $\%$ & SE & $95 \% \mathrm{Cl}$ \\
\hline Aguadilla-Isabela, Puerto Rico & 591 & 37.6 & 2.3 & $(33.1-42.0)$ \\
\hline Akron, Ohio & 691 & 28.3 & 2.2 & $(24.0-32.7)$ \\
\hline Albuquerque, New Mexico & 2,078 & 25.2 & 1.1 & $(23.1-27.2)$ \\
\hline Allentown-Bethlehem-Easton, Pennsylvania-New Jersey & 1,029 & 32.9 & 2.0 & $(29.0-36.7)$ \\
\hline Anchorage, Alaska & 1,521 & 29.8 & 1.3 & $(27.2-32.4)$ \\
\hline Atlanta-Sandy Springs-Roswell, Georgia & 3,506 & 30.8 & 0.9 & $(29.1-32.6)$ \\
\hline Augusta-Richmond County, Georgia-South Carolina & 908 & 40.2 & 2.4 & $(35.4-44.9)$ \\
\hline Austin-Round Rock, Texas & 935 & 27.8 & 1.8 & $(24.3-31.3)$ \\
\hline Baltimore-Columbia-Towson, Maryland & 4,751 & 30.8 & 0.8 & $(29.2-32.4)$ \\
\hline Baton Rouge, Louisiana & 927 & 39.7 & 2.3 & $(35.2-44.2)$ \\
\hline Billings, Montana & 817 & 26.8 & 1.6 & $(23.6-29.9)$ \\
\hline Birmingham-Hoover, Alabama & 1,352 & 35.6 & 1.5 & $(32.6-38.6)$ \\
\hline Bismarck, North Dakota & 1,034 & 27.9 & 1.5 & $(25.0-30.7)$ \\
\hline Boise City, Idaho & 1,483 & 28.7 & 1.4 & $(25.9-31.4)$ \\
\hline Boston, Massachusetts $§$ & 4,057 & 27.4 & 0.9 & $(25.6-29.3)$ \\
\hline Buffalo-Cheektowaga-Niagara Falls, New York & 505 & 26.5 & 2.3 & $(21.9-31.0)$ \\
\hline Burlington-South Burlington, Vermont & 1,630 & 26.5 & 1.2 & $(24.2-28.8)$ \\
\hline Cambridge-Newton-Framingham, Massachusetts ${ }^{\S}$ & 4,880 & 26.8 & 0.9 & $(25.0-28.5)$ \\
\hline Camden, New Jersey ${ }^{\S}$ & 1,862 & 30.4 & 1.2 & $(28.1-32.6)$ \\
\hline Cedar Rapids, lowa & 648 & 27.6 & 2.1 & $(23.5-31.8)$ \\
\hline Charleston, West Virginia & 817 & 38.6 & 1.9 & $(34.8-42.3)$ \\
\hline Charleston-North Charleston, South Carolina & 1,546 & 31.0 & 1.4 & $(28.3-33.7)$ \\
\hline Charlotte-Concord-Gastonia, North Carolina-South Carolina & 1,951 & 35.2 & 1.3 & $(32.6-37.7)$ \\
\hline Chattanooga, Tennessee-Georgia & 575 & 37.1 & 2.7 & $(31.8-42.3)$ \\
\hline Chicago-Naperville-Elgin, Illinois-Indiana-Wisconsin & 3,339 & 27.0 & 1.0 & $(25.1-29.0)$ \\
\hline Cincinnati, Ohio-Kentucky-Indiana & 2,601 & 31.8 & 1.2 & $(29.5-34.1)$ \\
\hline Claremont-Lebanon, New Hampshire-Vermont & 1,683 & 26.7 & 1.4 & $(23.9-29.5)$ \\
\hline Cleveland-Elyria, Ohio & 1,104 & 27.4 & 1.4 & $(24.6-30.2)$ \\
\hline Colorado Springs, Colorado & 1,373 & 28.9 & 1.4 & $(26.1-31.8)$ \\
\hline Columbia, South Carolina & 1,444 & 31.5 & 1.5 & $(28.6-34.3)$ \\
\hline Columbus, Ohio & 1,858 & 31.7 & 1.2 & $(29.3-34.1)$ \\
\hline Crestview-Fort Walton Beach-Destin, Florida & 1,079 & 34.2 & 2.0 & $(30.3-38.1)$ \\
\hline Dallas-Plano-Irving, Texas ${ }^{\S}$ & 901 & 29.3 & 1.6 & $(26.2-32.4)$ \\
\hline Davenport-Moline-Rock Island, lowa-Illinois & 672 & 28.8 & 2.1 & $(24.7-32.9)$ \\
\hline Dayton, Ohio & 836 & 30.3 & 1.9 & $(26.6-34.1)$ \\
\hline Deltona-Daytona Beach-Ormond Beach, Florida & 1,105 & 30.9 & 2.2 & $(26.6-35.2)$ \\
\hline Denver-Aurora-Lakewood, Colorado & 5,702 & 25.6 & 0.6 & $(24.4-26.8)$ \\
\hline Des Moines-West Des Moines, lowa & 1,344 & 30.8 & 1.5 & $(27.8-33.7)$ \\
\hline Duluth, Minnesota-Wisconsin & 701 & 22.2 & 2.1 & $(18.0-26.4)$ \\
\hline Durham-Chapel Hill, North Carolina & 616 & 28.9 & 2.1 & $(24.8-33.0)$ \\
\hline El Paso, Texas & 752 & 27.6 & 1.8 & $(24.0-31.2)$ \\
\hline Evansville, Indiana-Kentucky & 575 & 35.9 & 2.8 & $(30.3-41.5)$ \\
\hline Fargo, North Dakota-Minnesota & 1,182 & 30.2 & 1.7 & $(26.9-33.4)$ \\
\hline Fayetteville-Springdale-Rogers, Arkansas-Missouri & 819 & 30.6 & 2.2 & $(26.2-35.0)$ \\
\hline Fort Smith, Arkansas-Oklahoma & 496 & 42.2 & 3.1 & $(36.1-48.3)$ \\
\hline Fort Wayne, Indiana & 780 & 32.3 & 2.2 & $(28.0-36.6)$ \\
\hline Fort Worth-Arlington, Texas ${ }^{\S}$ & 809 & 32.5 & 2.1 & $(28.3-36.7)$ \\
\hline Gainesville, Florida & 1,034 & 30.8 & 2.2 & $(26.6-35.0)$ \\
\hline Grand Forks, North Dakota-Minnesota & 503 & 28.7 & 2.8 & $(23.2-34.2)$ \\
\hline Grand Island, Nebraska & 796 & 30.1 & 2.0 & $(26.2-34.0)$ \\
\hline Grand Rapids-Wyoming, Michigan & 1,342 & 31.1 & 1.4 & $(28.3-33.8)$ \\
\hline Greensboro-High Point, North Carolina & 665 & 34.8 & 2.0 & $(30.8-38.7)$ \\
\hline Greenville-Anderson-Mauldin, South Carolina & 1,340 & 33.1 & 1.5 & $(30.1-36.1)$ \\
\hline Gulfport-Biloxi-Pascagoula, Mississippi & 771 & 36.8 & 2.0 & $(32.9-40.7)$ \\
\hline Hagerstown-Martinsburg, Maryland-West Virginia & 767 & 29.8 & 2.5 & $(24.9-34.6)$ \\
\hline Hartford-West Hartford-East Hartford, Connecticut & 2,830 & 29.1 & 1.1 & $(26.9-31.2)$ \\
\hline Hilton Head Island-Bluffton-Beaufort, South Carolina & 824 & 28.8 & 2.1 & $(24.7-33.0)$ \\
\hline Houston-The Woodlands-Sugar Land, Texas & 1,382 & 31.6 & 1.6 & $(28.4-34.7)$ \\
\hline Huntington-Ashland, West Virginia-Kentucky-Ohio & 1,174 & 39.3 & 1.6 & $(36.2-42.4)$ \\
\hline Idaho Falls, Idaho & 508 & 28.9 & 2.3 & $(24.4-33.5)$ \\
\hline Indianapolis-Carmel-Anderson, Indiana & 2,533 & 30.9 & 1.0 & $(28.9-32.9)$ \\
\hline Jackson, Mississippi & 802 & 34.7 & 1.9 & $(31.0-38.4)$ \\
\hline
\end{tabular}

See table footnotes on page 135. 
TABLE 62. (Continued) Age-adjusted* prevalence estimates of adults aged $\geq 18$ years who have ever been told by a health professional they have high blood pressure, ${ }^{\dagger}$ by metropolitan and micropolitan statistical area - Behavioral Risk Factor Surveillance System, United States, 2013

\begin{tabular}{|c|c|c|c|c|}
\hline MMSA & $\begin{array}{l}\text { Sample } \\
\text { size }\end{array}$ & $\%$ & SE & $95 \% \mathrm{Cl}$ \\
\hline Jacksonville, Florida & 2,876 & 31.9 & 1.2 & $(29.5-34.2)$ \\
\hline Kansas City, Missouri-Kansas & 7,406 & 28.3 & 1.0 & $(26.4-30.1)$ \\
\hline Kingsport-Bristol-Bristol, Tennessee-Virginia & 537 & 38.1 & 3.3 & $(31.6-44.6)$ \\
\hline Knoxville, Tennessee & 651 & 31.7 & 2.0 & $(27.8-35.6)$ \\
\hline Lansing-East Lansing, Michigan & 686 & 28.8 & 1.7 & $(25.4-32.2)$ \\
\hline Lexington-Fayette, Kentucky & 636 & 33.2 & 2.0 & $(29.3-37.0)$ \\
\hline Lincoln, Nebraska & 1,872 & 26.5 & 1.1 & $(24.4-28.6)$ \\
\hline Little Rock-North Little Rock-Conway, Arkansas & 1,141 & 34.5 & 1.8 & $(31.0-37.9)$ \\
\hline Logan, Utah-Idaho & 639 & 23.2 & 1.9 & $(19.5-26.9)$ \\
\hline Los Angeles-Long Beach-Anaheim, California & 3,039 & 28.3 & 0.9 & $(26.5-30.2)$ \\
\hline Louisville-Jefferson County, Kentucky-Indiana & 2,146 & 34.2 & 1.4 & $(31.5-36.9)$ \\
\hline Lubbock, Texas & 529 & 28.9 & 2.3 & $(24.4-33.5)$ \\
\hline Manhattan, Kansas & 662 & 26.8 & 1.7 & $(23.5-30.1)$ \\
\hline Memphis, Tennessee-Mississippi-Arkansas & 1,205 & 36.6 & 1.7 & $(33.3-40.0)$ \\
\hline Miami-Fort Lauderdale-West Palm Beach, Florida & 2,203 & 27.7 & 1.3 & $(25.1-30.3)$ \\
\hline Milwaukee-Waukesha-West Allis, Wisconsin & 1,266 & 33.1 & 1.9 & $(29.4-36.8)$ \\
\hline Minneapolis-St. Paul-Bloomington, Minnesota-Wisconsin & 9,099 & 23.7 & 0.8 & $(22.2-25.2)$ \\
\hline Minot, North Dakota & 652 & 27.2 & 1.9 & $(23.5-30.8)$ \\
\hline $\begin{array}{l}\text { Montgomery County-Bucks County-Chester County, } \\
\text { Pennsylvania§ }\end{array}$ & 970 & 29.0 & 1.6 & $(25.9-32.0)$ \\
\hline $\begin{array}{l}\text { Myrtle Beach-Conway-North Myrtle Beach, South Carolina- } \\
\text { North Carolina }\end{array}$ & 772 & 33.0 & 2.0 & $(29.0-37.0)$ \\
\hline Nashville-Davidson County-Murfreesboro-Franklin, Tennessee & 1,062 & 35.6 & 1.7 & $(32.1-39.0)$ \\
\hline Nassau County-Suffolk County, New York ${ }^{\S}$ & 938 & 29.0 & 1.8 & $(25.4-32.5)$ \\
\hline Newark, New Jersey-Pennsylvania ${ }^{\S}$ & 4,108 & 28.0 & 0.9 & $(26.3-29.8)$ \\
\hline New Orleans-Metairie, Louisiana & 1,284 & 35.6 & 1.9 & $(31.8-39.3)$ \\
\hline New York-Jersey City-White Plains, New York-New Jersey & 8,924 & 29.3 & 0.6 & $(28.1-30.5)$ \\
\hline Norfolk, Nebraska & 670 & 30.3 & 2.0 & $(26.5-34.2)$ \\
\hline North Platte, Nebraska & 719 & 30.5 & 2.0 & $(26.7-34.3)$ \\
\hline North Port-Sarasota-Bradenton, Florida & 1,090 & 27.5 & 1.8 & $(23.9-31.1)$ \\
\hline Oakland-Hayward-Berkeley, California ${ }^{\S}$ & 699 & 25.6 & 2.0 & $(21.6-29.5)$ \\
\hline Ogden-Clearfield, Utah & 2,447 & 28.7 & 0.9 & $(26.8-30.5)$ \\
\hline Oklahoma City, Oklahoma & 2,641 & 33.8 & 1.0 & $(31.8-35.9)$ \\
\hline Omaha-Council Bluffs, Nebraska-lowa & 3,118 & 28.3 & 1.0 & $(26.4-30.2)$ \\
\hline Orlando-Kissimmee-Sanford, Florida & 2,273 & 31.4 & 1.4 & $(28.8-34.1)$ \\
\hline Panama City, Florida & 1,025 & 35.7 & 2.2 & $(31.4-40.0)$ \\
\hline Pensacola-Ferry Pass-Brent, Florida & 1,316 & 31.6 & 1.4 & $(28.8-34.4)$ \\
\hline Philadelphia, Pennsylvania $\$$ & 1,767 & 32.2 & 1.4 & $(29.4-35.1)$ \\
\hline Phoenix-Mesa-Scottsdale, Arizona & 1,546 & 28.1 & 1.6 & $(24.9-31.3)$ \\
\hline Pittsburgh, Pennsylvania & 2,359 & 28.9 & 1.1 & $(26.7-31.1)$ \\
\hline Ponce, Puerto Rico & 530 & 39.0 & 2.5 & $(34.2-43.9)$ \\
\hline Portland-South Portland, Maine & 2,633 & 27.7 & 1.1 & $(25.6-29.9)$ \\
\hline Portland-Vancouver-Hillsboro, Oregon-Washington & 3,238 & 27.2 & 1.0 & $(25.3-29.1)$ \\
\hline Port St. Lucie, Florida & 1,021 & 29.8 & 2.3 & $(25.2-34.4)$ \\
\hline Providence-Warwick, Rhode Island-Massachusetts & 8,280 & 29.9 & 0.8 & $(28.4-31.5)$ \\
\hline Provo-Orem, Utah & 1,854 & 23.4 & 1.0 & $(21.4-25.4)$ \\
\hline Raleigh, North Carolina & 675 & 29.8 & 1.8 & $(26.2-33.4)$ \\
\hline Rapid City, South Dakota & 871 & 30.8 & 1.9 & $(27.0-34.6)$ \\
\hline Reno, Nevada & 1,817 & 26.3 & 1.1 & $(24.2-28.5)$ \\
\hline Richmond, Virginia & 1,310 & 35.4 & 1.6 & $(32.2-38.6)$ \\
\hline Riverside-San Bernardino-Ontario, California & 1,369 & 30.6 & 1.5 & $(27.7-33.5)$ \\
\hline Rochester, New York & 508 & 30.7 & 2.2 & $(26.4-34.9)$ \\
\hline Rockingham County-Strafford County, New Hampshire ${ }^{\S}$ & 1,660 & 27.9 & 1.3 & $(25.3-30.5)$ \\
\hline Sacramento-Roseville-Arden-Arcade, California & 889 & 28.4 & 1.7 & $(25.0-31.7)$ \\
\hline St. Louis, Missouri-Illinois & 2,059 & 30.2 & 1.3 & $(27.7-32.7)$ \\
\hline Salem, Oregon & 527 & 31.2 & 2.9 & $(25.6-36.9)$ \\
\hline Salina, Kansas & 524 & 32.2 & 2.3 & $(27.7-36.7)$ \\
\hline Salisbury, Maryland-Delaware & 2,062 & 32.3 & 1.4 & $(29.6-35.0)$ \\
\hline Salt Lake City, Utah & 4,666 & 25.8 & 0.7 & $(24.4-27.2)$ \\
\hline San Antonio-New Braunfels, Texas & 937 & 31.9 & 1.6 & $(28.7-35.1)$ \\
\hline San Francisco-Redwood City-South San Francisco, California & 540 & 24.2 & 2.2 & $(20.0-28.5)$ \\
\hline San Jose-Sunnyvale-Santa Clara, California & 624 & 26.2 & 2.4 & $(21.5-30.9)$ \\
\hline
\end{tabular}

See table footnotes on page 135. 
TABLE 62. (Continued) Age-adjusted* prevalence estimates of adults aged $\geq 18$ years who have ever been told by a health professional they have high blood pressure, ${ }^{\dagger}$ by metropolitan and micropolitan statistical area — Behavioral Risk Factor Surveillance System, United States, 2013

\begin{tabular}{|c|c|c|c|c|}
\hline MMSA & $\begin{array}{l}\text { Sample } \\
\text { size }\end{array}$ & $\%$ & SE & $95 \% \mathrm{Cl}$ \\
\hline San Juan-Carolina-Caguas, Puerto Rico & 3,647 & 40.6 & 1.0 & $(38.7-42.5)$ \\
\hline Scottsbluff, Nebraska & 713 & 33.1 & 2.4 & $(28.4-37.9)$ \\
\hline Scranton-Wilkes-Barre-Hazleton, Pennsylvania & 566 & 34.0 & 2.6 & $(29.0-39.1)$ \\
\hline Seattle-Bellevue-Everett, Washington ${ }^{\S}$ & 3,769 & 27.9 & 0.9 & $(26.3-29.6)$ \\
\hline Shreveport-Bossier City, Louisiana & 571 & 40.5 & 3.0 & $(34.6-46.4)$ \\
\hline Silver Spring-Frederick-Rockville, Maryland ${ }^{\S}$ & 2,417 & 25.0 & 1.0 & $(23.1-27.0)$ \\
\hline Sioux City, lowa-Nebraska-South Dakota & 1,054 & 33.7 & 2.7 & $(28.4-39.0)$ \\
\hline Sioux Falls, South Dakota & 1,007 & 27.4 & 1.6 & $(24.2-30.6)$ \\
\hline Spartanburg, South Carolina & 595 & 34.4 & 2.6 & $(29.4-39.4)$ \\
\hline Spokane-Spokane Valley, Washington & 862 & 29.1 & 1.9 & $(25.3-32.9)$ \\
\hline Springfield, Massachusetts & 1,566 & 31.6 & 2.0 & $(27.6-35.6)$ \\
\hline Tallahassee, Florida & 1,850 & 30.8 & 1.5 & $(27.8-33.8)$ \\
\hline Tampa-St. Petersburg-Clearwater, Florida & 2,208 & 32.4 & 1.3 & $(29.9-34.8)$ \\
\hline Toledo, Ohio & 998 & 29.9 & 1.8 & $(26.4-33.5)$ \\
\hline Topeka, Kansas & 2,401 & 30.7 & 1.0 & $(28.7-32.7)$ \\
\hline Tulsa, Oklahoma & 1,995 & 35.7 & 1.3 & $(33.1-38.2)$ \\
\hline Virginia Beach-Norfolk-Newport News, Virginia-North Carolina & 1,676 & 31.1 & 1.4 & $(28.4-33.8)$ \\
\hline Warren-Troy-Farmington Hills, Michigan ${ }^{\S}$ & 2,254 & 29.5 & 1.1 & $(27.3-31.6)$ \\
\hline $\begin{array}{l}\text { Washington-Arlington-Alexandria, District of Columbia- } \\
\text { Virginia-Maryland-West Virginia } \$\end{array}$ & 9,000 & 29.1 & 0.8 & $(27.6-30.6)$ \\
\hline Wichita, Kansas & 4,923 & 31.1 & 0.7 & $(29.7-32.6)$ \\
\hline Wilmington, Delaware-Maryland-New Jersey ${ }^{\S}$ & 3,272 & 32.2 & 0.9 & $(30.4-34.1)$ \\
\hline Winston-Salem, North Carolina & 695 & 33.2 & 2.4 & $(28.4-37.9)$ \\
\hline Worcester, Massachusetts-Connecticut & 2,756 & 25.7 & 1.1 & $(23.6-27.8)$ \\
\hline $\begin{array}{l}\text { Median } \\
\text { Range }\end{array}$ & & $\begin{array}{c}30.4 \\
22.2-42.2\end{array}$ & & \\
\hline
\end{tabular}

Abbreviations: $\mathrm{Cl}=$ confidence interval; $\mathrm{MMSA}=$ metropolitan and micropolitan statistical areas; $\mathrm{SE}=$ standard error.

* Age adjusted to the 2000 U.S. standard population.

† Excluding pregnant women.

$\S$ Metropolitan division. 
TABLE 63. Age-adjusted* prevalence estimates of adults aged $\geq 18$ years who have ever been told by a health professional they have high blood cholesterol, by state/territory - Behavioral Risk Factor Surveillance System, United States, 2013

\begin{tabular}{|c|c|c|c|c|}
\hline State/Territory & $\begin{array}{l}\text { Sample } \\
\text { size }\end{array}$ & $\%$ & SE & $95 \% \mathrm{Cl}$ \\
\hline Alabama & 5,632 & 38.4 & 1.0 & $(36.4-40.4)$ \\
\hline Alaska & 3,616 & 35.0 & 1.1 & $(32.8-37.2)$ \\
\hline Arizona & 3,553 & 35.0 & 1.5 & $(32.1-38.0)$ \\
\hline Arkansas & 4,466 & 36.1 & 1.1 & $(33.9-38.3)$ \\
\hline California & 9,466 & 34.1 & 0.7 & $(32.8-35.4)$ \\
\hline Colorado & 11,709 & 31.4 & 0.6 & $(30.3-32.5)$ \\
\hline Connecticut & 6,823 & 32.8 & 0.8 & $(31.2-34.5)$ \\
\hline Delaware & 4,547 & 35.0 & 1.0 & $(33.1-36.9)$ \\
\hline District of Columbia & 4,481 & 33.3 & 1.1 & $(31.2-35.4)$ \\
\hline Florida & 30,216 & 34.3 & 0.6 & $(33.1-35.6)$ \\
\hline Georgia & 6,953 & 34.5 & 0.8 & $(33.1-36.0)$ \\
\hline Hawaii & 6,338 & 30.4 & 0.8 & $(28.7-32.0)$ \\
\hline Idaho & 4,555 & 32.9 & 1.1 & $(30.8-34.9)$ \\
\hline Illinois & 4,821 & 32.0 & 1.0 & $(30.1-33.9)$ \\
\hline Indiana & 8,848 & 34.7 & 0.7 & $(33.3-36.1)$ \\
\hline lowa & 7,018 & 35.2 & 0.8 & $(33.6-36.8)$ \\
\hline Kansas & 19,337 & 33.0 & 0.4 & $(32.2-33.9)$ \\
\hline Kentucky & 9,563 & 38.0 & 0.8 & $(36.5-39.5)$ \\
\hline Louisiana & 4,585 & 36.4 & 1.2 & $(34.1-38.7)$ \\
\hline Maine & 7,221 & 33.1 & 0.8 & $(31.6-34.6)$ \\
\hline Maryland & 11,613 & 33.1 & 0.7 & $(31.7-34.4)$ \\
\hline Massachusetts & 13,375 & 32.5 & 0.6 & $(31.2-33.8)$ \\
\hline Michigan & 11,093 & 34.8 & 0.6 & $(33.6-36.0)$ \\
\hline Minnesota & 12,421 & 29.5 & 0.8 & $(28.0-31.0)$ \\
\hline Mississippi & 6,309 & 37.1 & 0.9 & $(35.2-38.9)$ \\
\hline Missouri & 6,020 & 33.0 & 1.0 & $(31.1-34.9)$ \\
\hline Montana & 7,945 & 29.2 & 0.7 & $(27.8-30.6)$ \\
\hline Nebraska & 14,365 & 32.2 & 0.6 & $(31.0-33.5)$ \\
\hline Nevada & 4,237 & 34.3 & 1.4 & $(31.6-37.0)$ \\
\hline New Hampshire & 5,809 & 31.9 & 0.9 & $(30.2-33.5)$ \\
\hline New Jersey & 11,482 & 34.8 & 0.7 & $(33.5-36.2)$ \\
\hline New Mexico & 7,515 & 31.8 & 0.8 & $(30.2-33.4)$ \\
\hline New York & 7,752 & 34.9 & 0.7 & $(33.5-36.4)$ \\
\hline North Carolina & 7,611 & 36.2 & 0.8 & $(34.7-37.7)$ \\
\hline North Dakota & 6,433 & 31.3 & 0.8 & $(29.7-32.9)$ \\
\hline Ohio & 10,252 & 32.3 & 0.7 & $(31.0-33.7)$ \\
\hline Oklahoma & 6,981 & 35.8 & 0.8 & $(34.2-37.3)$ \\
\hline Oregon & 5,040 & 31.7 & 0.9 & $(29.9-33.6)$ \\
\hline Pennsylvania & 9,752 & 33.1 & 0.7 & $(31.7-34.4)$ \\
\hline Rhode Island & 5,895 & 33.9 & 0.9 & $(32.1-35.6)$ \\
\hline South Carolina & 9,378 & 37.1 & 0.8 & $(35.6-38.6)$ \\
\hline South Dakota & 5,644 & 30.5 & 1.0 & $(28.5-32.5)$ \\
\hline Tennessee & 5,012 & 34.0 & 1.0 & $(32.1-35.9)$ \\
\hline Texas & 9,026 & 34.6 & 0.8 & $(33.0-36.1)$ \\
\hline Utah & 10,134 & 31.2 & 0.5 & $(30.2-32.3)$ \\
\hline Vermont & 5,650 & 28.8 & 0.8 & $(27.3-30.3)$ \\
\hline Virginia & 7,410 & 34.7 & 0.8 & $(33.2-36.2)$ \\
\hline Washington & 9,524 & 32.2 & 0.7 & $(30.9-33.5)$ \\
\hline West Virginia & 5,031 & 36.7 & 0.9 & $(35.0-38.4)$ \\
\hline Wisconsin & 5,663 & 31.2 & 1.0 & $(29.3-33.2)$ \\
\hline Wyoming & 5,629 & 30.5 & 0.9 & $(28.7-32.3)$ \\
\hline Guam & 1,341 & 36.0 & 1.6 & $(32.8-39.2)$ \\
\hline Puerto Rico & 5,092 & 34.5 & 0.9 & $(32.8-36.2)$ \\
\hline Median & & 33.9 & & \\
\hline Range & & $28.8-38.4$ & & \\
\hline
\end{tabular}

Abbreviations: $\mathrm{Cl}=$ confidence interval; $\mathrm{SE}=$ standard error.

${ }^{*}$ Age adjusted to the 2000 U.S. standard population. 
TABLE 64. Age-adjusted* prevalence estimates of adults aged $\geq 18$ years who have ever been told by a health professional they have high blood cholesterol, by metropolitan and micropolitan statistical area - Behavioral Risk Factor Surveillance System, United States, 2013

\begin{tabular}{|c|c|c|c|c|}
\hline MMSA & $\begin{array}{c}\text { Sample } \\
\text { size }\end{array}$ & $\%$ & SE & $95 \% \mathrm{Cl}$ \\
\hline Aguadilla-Isabela, Puerto Rico & 517 & 31.4 & 2.7 & $(26.2-36.6)$ \\
\hline Akron, Ohio & 600 & 32.6 & 3.1 & $(26.5-38.7)$ \\
\hline Albuquerque, New Mexico & 1,663 & 32.1 & 1.5 & $(29.1-35.1)$ \\
\hline Allentown-Bethlehem-Easton, Pennsylvania-New Jersey & 876 & 35.0 & 2.5 & $(30.0-39.9)$ \\
\hline Anchorage, Alaska & 1,246 & 34.6 & 1.7 & $(31.3-37.8)$ \\
\hline Atlanta-Sandy Springs-Roswell, Georgia & 2,991 & 33.4 & 1.1 & $(31.3-35.5)$ \\
\hline Augusta-Richmond County, Georgia-South Carolina & 816 & 34.5 & 2.4 & $(29.7-39.2)$ \\
\hline Austin-Round Rock, Texas & 784 & 32.4 & 1.9 & $(28.7-36.1)$ \\
\hline Baltimore-Columbia-Towson, Maryland & 4,273 & 31.4 & 1.0 & $(29.4-33.3)$ \\
\hline Baton Rouge, Louisiana & 806 & 39.6 & 2.9 & $(33.9-45.4)$ \\
\hline Billings, Montana & 655 & 28.4 & 1.8 & $(24.8-32.0)$ \\
\hline Birmingham-Hoover, Alabama & 1,175 & 37.5 & 2.0 & $(33.6-41.4)$ \\
\hline Bismarck, North Dakota & 883 & 31.5 & 1.9 & $(27.7-35.3)$ \\
\hline Boise City, Idaho & 1,235 & 31.9 & 1.8 & $(28.4-35.4)$ \\
\hline Boston, Massachusetts ${ }^{\dagger}$ & 3,597 & 30.9 & 1.1 & $(28.7-33.2)$ \\
\hline Buffalo-Cheektowaga-Niagara Falls, New York & 442 & 28.9 & 2.6 & $(23.7-34.0)$ \\
\hline Burlington-South Burlington, Vermont & 1,430 & 26.3 & 1.3 & $(23.7-28.8)$ \\
\hline Cambridge-Newton-Framingham, Massachusetts ${ }^{\dagger}$ & 4,339 & 31.7 & 1.1 & $(29.6-33.9)$ \\
\hline Camden, New Jersey ${ }^{\dagger}$ & 1,633 & 33.7 & 1.5 & $(30.7-36.6)$ \\
\hline Cedar Rapids, lowa & 568 & 36.5 & 2.9 & $(30.8-42.2)$ \\
\hline Charleston, West Virginia & 728 & 35.2 & 2.0 & $(31.3-39.1)$ \\
\hline Charleston-North Charleston, South Carolina & 1,370 & 33.9 & 1.6 & $(30.8-37.0)$ \\
\hline Charlotte-Concord-Gastonia, North Carolina-South Carolina & 1,671 & 38.7 & 1.6 & $(35.6-41.8)$ \\
\hline Chattanooga, Tennessee-Georgia & 516 & 32.9 & 2.8 & $(27.4-38.3)$ \\
\hline Chicago-Naperville-Elgin, Illinois-Indiana-Wisconsin & 2,864 & 30.5 & 1.2 & $(28.2-32.8)$ \\
\hline Cincinnati, Ohio-Kentucky-Indiana & 2,246 & 32.5 & 1.4 & $(29.9-35.2)$ \\
\hline Claremont-Lebanon, New Hampshire-Vermont & 1,484 & 29.4 & 2.2 & $(25.1-33.6)$ \\
\hline Cleveland-Elyria, Ohio & 969 & 29.7 & 1.9 & $(25.9-33.5)$ \\
\hline Colorado Springs, Colorado & 1,193 & 33.5 & 1.7 & $(30.2-36.8)$ \\
\hline Columbia, South Carolina & 1,266 & 34.6 & 1.9 & $(30.8-38.4)$ \\
\hline Columbus, Ohio & 1,550 & 35.4 & 1.6 & $(32.4-38.5)$ \\
\hline Crestview-Fort Walton Beach-Destin, Florida & 909 & 33.3 & 2.4 & $(28.6-38.0)$ \\
\hline Dallas-Plano-Irving, Texas ${ }^{\dagger}$ & 735 & 34.3 & 2.1 & $(30.1-38.5)$ \\
\hline Davenport-Moline-Rock Island, lowa-Illinois & 589 & 35.0 & 2.7 & $(29.7-40.3)$ \\
\hline Dayton, Ohio & 744 & 33.4 & 2.8 & $(28.0-38.9)$ \\
\hline Deltona-Daytona Beach-Ormond Beach, Florida & 1,002 & 38.6 & 3.2 & $(32.3-44.9)$ \\
\hline Denver-Aurora-Lakewood, Colorado & 4,911 & 31.5 & 0.8 & $(30.0-33.0)$ \\
\hline Des Moines-West Des Moines, lowa & 1,188 & 36.4 & 1.8 & $(32.9-40.0)$ \\
\hline Duluth, Minnesota-Wisconsin & 610 & 28.0 & 2.3 & $(23.5-32.6)$ \\
\hline Durham-Chapel Hill, North Carolina & 540 & 30.0 & 2.4 & $(25.3-34.8)$ \\
\hline El Paso, Texas & 606 & 31.9 & 2.5 & $(27.1-36.7)$ \\
\hline Evansville, Indiana-Kentucky & 511 & 38.2 & 3.5 & (31.4-44.9) \\
\hline Fargo, North Dakota-Minnesota & 987 & 32.8 & 2.8 & $(27.3-38.3)$ \\
\hline Fayetteville-Springdale-Rogers, Arkansas-Missouri & 662 & 30.3 & 2.4 & $(25.5-35.1)$ \\
\hline Fort Smith, Arkansas-Oklahoma & 420 & 34.5 & 3.4 & $(27.8-41.3)$ \\
\hline Fort Wayne, Indiana & 655 & 36.6 & 2.8 & $(31.1-42.1)$ \\
\hline Fort Worth-Arlington, Texas ${ }^{\dagger}$ & 721 & 32.8 & 2.2 & $(28.4-37.2)$ \\
\hline Gainesville, Florida & 918 & 31.7 & 2.5 & $(26.8-36.7)$ \\
\hline Grand Forks, North Dakota-Minnesota & 416 & 29.8 & 3.5 & $(22.9-36.7)$ \\
\hline Grand Island, Nebraska & 685 & 33.7 & 2.8 & $(28.2-39.2)$ \\
\hline Grand Rapids-Wyoming, Michigan & 1,180 & 33.5 & 1.7 & $(30.3-36.7)$ \\
\hline Greensboro-High Point, North Carolina & 580 & 37.6 & 2.7 & $(32.4-42.8)$ \\
\hline Greenville-Anderson-Mauldin, South Carolina & 1,165 & 35.8 & 1.9 & $(32.1-39.4)$ \\
\hline Gulfport-Biloxi-Pascagoula, Mississippi & 647 & 36.9 & 2.6 & $(31.7-42.1)$ \\
\hline Hagerstown-Martinsburg, Maryland-West Virginia & 678 & 34.8 & 2.8 & $(29.3-40.3)$ \\
\hline Hartford-West Hartford-East Hartford, Connecticut & 2,536 & 34.1 & 1.3 & $(31.5-36.6)$ \\
\hline Hilton Head Island-Bluffton-Beaufort, South Carolina & 750 & 34.8 & 2.7 & $(29.6-40.0)$ \\
\hline Houston-The Woodlands-Sugar Land, Texas & 1,171 & 34.2 & 1.9 & $(30.4-38.0)$ \\
\hline Huntington-Ashland, West Virginia-Kentucky-Ohio & 992 & 36.2 & 1.9 & $(32.5-40.0)$ \\
\hline Idaho Falls, Idaho & 390 & 36.3 & 3.6 & $(29.1-43.4)$ \\
\hline Indianapolis-Carmel-Anderson, Indiana & 2,176 & 34.7 & 1.4 & $(32.0-37.4)$ \\
\hline
\end{tabular}

See table footnotes on page 139. 
TABLE 64. (Continued) Age-adjusted* prevalence estimates of adults aged $\geq 18$ years who have ever been told by a health professional they have high blood cholesterol, by metropolitan and micropolitan statistical area — Behavioral Risk Factor Surveillance System, United States, 2013

\begin{tabular}{|c|c|c|c|c|}
\hline MMSA & $\begin{array}{l}\text { Sample } \\
\text { size }\end{array}$ & $\%$ & SE & $95 \% \mathrm{Cl}$ \\
\hline Jackson, Mississippi & 675 & 36.6 & 2.6 & $(31.6-41.7)$ \\
\hline Jacksonville, Florida & 2,532 & 33.1 & 1.4 & $(30.4-35.7)$ \\
\hline Kansas City, Missouri-Kansas & 6,383 & 34.8 & 1.2 & $(32.3-37.2)$ \\
\hline Kingsport-Bristol-Bristol, Tennessee-Virginia & 486 & 32.6 & 3.2 & $(26.3-38.8)$ \\
\hline Knoxville, Tennessee & 558 & 33.3 & 2.6 & $(28.2-38.4)$ \\
\hline Lansing-East Lansing, Michigan & 588 & 34.6 & 2.4 & $(29.9-39.3)$ \\
\hline Lexington-Fayette, Kentucky & 513 & 38.7 & 2.9 & $(33.1-44.3)$ \\
\hline Lincoln, Nebraska & 1,528 & 32.0 & 1.3 & $(29.4-34.5)$ \\
\hline Little Rock-North Little Rock-Conway, Arkansas & 995 & 39.1 & 2.3 & $(34.6-43.6)$ \\
\hline Logan, Utah-Idaho & 472 & 30.7 & 2.5 & $(25.8-35.6)$ \\
\hline Los Angeles-Long Beach-Anaheim, California & 2,511 & 36.4 & 1.3 & $(33.9-38.9)$ \\
\hline Louisville-Jefferson County, Kentucky-Indiana & 1,881 & 36.2 & 1.6 & $(33.0-39.4)$ \\
\hline Lubbock, Texas & 455 & 36.2 & 3.8 & $(28.7-43.6)$ \\
\hline Manhattan, Kansas & 514 & 30.2 & 2.1 & $(26.0-34.4)$ \\
\hline Memphis, Tennessee-Mississippi-Arkansas & 1,052 & 33.8 & 1.8 & $(30.3-37.3)$ \\
\hline Miami-Fort Lauderdale-West Palm Beach, Florida & 1,936 & 31.6 & 1.6 & $(28.5-34.8)$ \\
\hline Milwaukee-Waukesha-West Allis, Wisconsin & 1,083 & 31.5 & 2.2 & $(27.2-35.8)$ \\
\hline Minneapolis-St. Paul-Bloomington, Minnesota-Wisconsin & 8,025 & 28.9 & 1.0 & $(27.0-30.8)$ \\
\hline Minot, North Dakota & 533 & 29.8 & 2.3 & $(25.2-34.4)$ \\
\hline $\begin{array}{l}\text { Montgomery County-Bucks County-Chester County, } \\
\text { Pennsylvania }^{\dagger}\end{array}$ & 846 & 32.5 & 1.9 & $(28.7-36.2)$ \\
\hline $\begin{array}{l}\text { Myrtle Beach-Conway-North Myrtle Beach, South Carolina- } \\
\text { North Carolina }\end{array}$ & 665 & 38.4 & 3.0 & $(32.6-44.3)$ \\
\hline Nashville-Davidson County-Murfreesboro-Franklin, Tennessee & 901 & 33.2 & 1.9 & $(29.4-37.0)$ \\
\hline Nassau County-Suffolk County, New York ${ }^{\dagger}$ & 828 & 37.4 & 2.3 & $(32.9-41.9)$ \\
\hline Newark, New Jersey-Pennsylvania ${ }^{\dagger}$ & 3,550 & 34.5 & 1.3 & $(32.0-36.9)$ \\
\hline New Orleans-Metairie, Louisiana & 1,127 & 33.9 & 2.3 & $(29.3-38.5)$ \\
\hline New York-Jersey City-White Plains, New York-New Jersey ${ }^{\dagger}$ & 7,644 & 35.1 & 0.8 & $(33.5-36.7)$ \\
\hline Norfolk, Nebraska & 562 & 36.0 & 2.6 & $(30.9-41.2)$ \\
\hline North Platte, Nebraska & 608 & 37.4 & 3.1 & $(31.3-43.4)$ \\
\hline North Port-Sarasota-Bradenton, Florida & 958 & 38.0 & 2.6 & $(32.8-43.2)$ \\
\hline Oakland-Hayward-Berkeley, California ${ }^{\dagger}$ & 568 & 32.7 & 2.5 & $(27.8-37.6)$ \\
\hline Ogden-Clearfield, Utah & 2,008 & 31.9 & 1.1 & $(29.7-34.1)$ \\
\hline Oklahoma City, Oklahoma & 2,246 & 36.6 & 1.4 & $(33.9-39.4)$ \\
\hline Omaha-Council Bluffs, Nebraska-lowa & 2,660 & 32.9 & 1.2 & $(30.6-35.3)$ \\
\hline Orlando-Kissimmee-Sanford, Florida & 2,013 & 36.0 & 1.6 & $(32.9-39.1)$ \\
\hline Panama City, Florida & 908 & 35.4 & 2.4 & $(30.6-40.1)$ \\
\hline Pensacola-Ferry Pass-Brent, Florida & 1,103 & 32.2 & 1.8 & $(28.7-35.6)$ \\
\hline Philadelphia, Pennsylvania ${ }^{\dagger}$ & 1,503 & 33.4 & 1.8 & $(29.9-36.8)$ \\
\hline Phoenix-Mesa-Scottsdale, Arizona & 1,293 & 36.1 & 2.1 & $(32.0-40.3)$ \\
\hline Pittsburgh, Pennsylvania & 2,044 & 30.7 & 1.5 & $(27.8-33.6)$ \\
\hline Ponce, Puerto Rico & 445 & 31.0 & 2.6 & $(25.9-36.1)$ \\
\hline Portland-South Portland, Maine & 2,351 & 32.1 & 1.4 & $(29.4-34.7)$ \\
\hline Portland-Vancouver-Hillsboro, Oregon-Washington & 2,769 & 31.3 & 1.1 & $(29.1-33.6)$ \\
\hline Port St. Lucie, Florida & 938 & 33.8 & 2.9 & $(28.1-39.4)$ \\
\hline Providence-Warwick, Rhode Island-Massachusetts & 7,502 & 35.6 & 1.1 & (33.4-37.8) \\
\hline Provo-Orem, Utah & 1,379 & 30.0 & 1.4 & $(27.3-32.6)$ \\
\hline Raleigh, North Carolina & 550 & 31.6 & 2.1 & $(27.5-35.8)$ \\
\hline Rapid City, South Dakota & 734 & 30.9 & 2.4 & $(26.3-35.5)$ \\
\hline Reno, Nevada & 1,518 & 31.6 & 1.5 & $(28.7-34.6)$ \\
\hline Richmond, Virginia & 1,154 & 33.3 & 1.8 & $(29.7-36.9)$ \\
\hline Riverside-San Bernardino-Ontario, California & 1,108 & 33.4 & 1.8 & $(30.0-36.9)$ \\
\hline Rochester, New York & 448 & 29.6 & 2.5 & $(24.8-34.5)$ \\
\hline Rockingham County-Strafford County, New Hampshire ${ }^{\dagger}$ & 1,505 & 32.2 & 1.5 & $(29.1-35.2)$ \\
\hline Sacramento-Roseville-Arden-Arcade, California & 745 & 30.4 & 2.2 & $(26.1-34.6)$ \\
\hline St. Louis, Missouri-Illinois & 1,776 & 31.6 & 1.6 & $(28.4-34.8)$ \\
\hline Salem, Oregon & 450 & 33.7 & 4.0 & $(25.9-41.5)$ \\
\hline Salina, Kansas & 428 & 33.2 & 3.0 & $(27.2-39.1)$ \\
\hline Salisbury, Maryland-Delaware & 1,846 & 36.6 & 2.0 & $(32.7-40.5)$ \\
\hline Salt Lake City, Utah & 3,755 & 31.5 & 0.9 & $(29.7-33.3)$ \\
\hline San Antonio-New Braunfels, Texas & 786 & 34.6 & 2.2 & $(30.2-39.0)$ \\
\hline
\end{tabular}

See table footnotes on page 139. 
TABLE 64. (Continued) Age-adjusted* prevalence estimates of adults aged $\geq 18$ years who have ever been told by a health professional they have high blood cholesterol, by metropolitan and micropolitan statistical area — Behavioral Risk Factor Surveillance System, United States, 2013

\begin{tabular}{|c|c|c|c|c|}
\hline MMSA & $\begin{array}{l}\text { Sample } \\
\text { size }\end{array}$ & $\%$ & SE & $95 \% \mathrm{Cl}$ \\
\hline San Francisco-Redwood City-South San Francisco, California ${ }^{\dagger}$ & 449 & 36.2 & 3.1 & $(30.1-42.2)$ \\
\hline San Jose-Sunnyvale-Santa Clara, California & 517 & 31.6 & 2.6 & $(26.5-36.7)$ \\
\hline San Juan-Carolina-Caguas, Puerto Rico & 3,069 & 35.3 & 1.1 & $(33.0-37.5)$ \\
\hline Scottsbluff, Nebraska & 597 & 30.5 & 2.5 & $(25.7-35.4)$ \\
\hline Scranton-Wilkes-Barre-Hazleton, Pennsylvania & 491 & 33.5 & 3.2 & $(27.2-39.8)$ \\
\hline Seattle-Bellevue-Everett, Washington ${ }^{\dagger}$ & 3,224 & 31.2 & 1.0 & $(29.2-33.3)$ \\
\hline Shreveport-Bossier City, Louisiana & 499 & 38.7 & 3.7 & $(31.5-45.8)$ \\
\hline Silver Spring-Frederick-Rockville, Maryland ${ }^{\dagger}$ & 2,156 & 34.3 & 1.5 & $(31.4-37.3)$ \\
\hline Sioux City, lowa-Nebraska-South Dakota & 845 & 31.4 & 3.0 & $(25.5-37.2)$ \\
\hline Sioux Falls, South Dakota & 826 & 29.8 & 2.0 & $(25.8-33.8)$ \\
\hline Spartanburg, South Carolina & 508 & 37.4 & 3.7 & $(30.2-44.7)$ \\
\hline Spokane-Spokane Valley, Washington & 732 & 33.6 & 2.7 & $(28.4-38.9)$ \\
\hline Springfield, Massachusetts & 1,397 & 32.2 & 2.1 & $(28.0-36.3)$ \\
\hline Tallahassee, Florida & 1,664 & 34.0 & 1.9 & $(30.2-37.8)$ \\
\hline Tampa-St. Petersburg-Clearwater, Florida & 1,944 & 37.1 & 1.7 & $(33.9-40.4)$ \\
\hline Toledo, Ohio & 864 & 30.4 & 2.2 & $(26.2-34.6)$ \\
\hline Topeka, Kansas & 2,044 & 34.4 & 1.4 & $(31.7-37.0)$ \\
\hline Tulsa, Oklahoma & 1,707 & 35.9 & 1.6 & $(32.9-39.0)$ \\
\hline Virginia Beach-Norfolk-Newport News, Virginia-North Carolina & 1,481 & 31.5 & 1.4 & $(28.7-34.4)$ \\
\hline Warren-Troy-Farmington Hills, Michigan ${ }^{\dagger}$ & 2,016 & 34.3 & 1.3 & $(31.7-36.9)$ \\
\hline $\begin{array}{l}\text { Washington-Arlington-Alexandria, District of Columbia- } \\
\text { Virginia-Maryland-West Virginiat }\end{array}$ & 8,058 & 34.5 & 1.1 & $(32.5-36.6)$ \\
\hline Wichita, Kansas & 4,104 & 34.5 & 1.0 & $(32.6-36.4)$ \\
\hline Wilmington, Delaware-Maryland-New Jersey ${ }^{\dagger}$ & 2,835 & 34.8 & 1.2 & $(32.3-37.2)$ \\
\hline Winston-Salem, North Carolina & 617 & 38.3 & 2.7 & $(33.1-43.6)$ \\
\hline Worcester, Massachusetts-Connecticut & 2,419 & 35.9 & 1.6 & $(32.8-39.0)$ \\
\hline Median & & 33.5 & & \\
\hline Range & & $26.3-39.6$ & & \\
\hline
\end{tabular}

Abbreviations: $\mathrm{Cl}=$ confidence interval; $\mathrm{MMSA}=$ metropolitan and micropolitan statistical areas; $\mathrm{SE}=$ standard error.

* Age adjusted to the 2000 U.S. standard population.

† Metropolitan division. 


The Morbidity and Mortality Weekly Report (MMWR) Series is prepared by the Centers for Disease Control and Prevention (CDC) and is available free of charge in electronic format. To receive an electronic copy each week, visit $M M W R$ 's free subscription page at https://www.cdc.gov/mmwr $/ \mathrm{mmwrsubscribe.html}$. Paper copy subscriptions are available through the Superintendent of Documents, U.S. Government Printing Office, Washington, DC 20402; telephone 202-512-1800

Readers who have difficulty accessing this PDF file may access the HTML file at https://www.cdc.gov/mmwr/volumes/66/ss/ss6616a1.htm?s_ cid=ss6616a1_w. Address all inquiries about the $M M W R$ Series, including material to be considered for publication, to Executive Editor, $M M W R$ Series, Mailstop E-90, CDC, 1600 Clifton Rd., N.E., Atlanta, GA 30329-4027 or to mmwrq@cdc.gov.

All material in the MMWR Series is in the public domain and may be used and reprinted without permission; citation as to source, however, is appreciated.

Use of trade names and commercial sources is for identification only and does not imply endorsement by the U.S. Department of Health and Human Services.

References to non-CDC sites on the Internet are provided as a service to $M M W R$ readers and do not constitute or imply endorsement of these organizations or their programs by CDC or the U.S. Department of Health and Human Services. CDC is not responsible for the content of these sites. URL addresses listed in $M M W R$ were current as of the date of publication.

ISSN: 1546-0738 (Print) 